\title{
Tailoring Surface Adsorption and Reactivity of Fullerene-Based Compounds: A Theoretical Probe into $\mathrm{C}_{2}$-Gas-Fullerene Surface Interactions
}

\author{
Manjusha C. Padole, Parag A. Deshpande* \\ Quantum and Molecular Engineering Laboratory, \\ Department of Chemical Engineering, \\ Indian Institute of Technology Kharagpur, \\ Kharagpur 721302, India
}

Supporting Information

*Corresponding author: Parag A. Deshpande

Email: parag@che.iitkgp.ernet.in

Tel. : (+91) 3222283916 
Table S1: Natural bond orbital analysis with bond index, bond order and hybrids for $\mathrm{C}_{60}, \mathrm{C}_{59} \mathrm{Pd}$ and $\mathrm{C}_{59} \mathrm{Ni}$.

\begin{tabular}{|c|c|c|c|c|}
\hline System & Bond & Bond index & Bond order & Hybrid $(\mathrm{AO}(\%))$ \\
\hline \multirow{3}{*}{$\mathrm{C}_{60}$} & M-C5 & 1.388 & 1.138 & $\mathrm{sp}^{1.78}(\mathrm{~s}(36.00 \%) \mathrm{p}(63.96 \%))-\mathrm{sp}^{1.78}(\mathrm{~s}(36.00 \%) \mathrm{p}(63.96 \%))$ \\
\hline & $\mathrm{M}-\mathrm{C} 1$ & 1.158 & 0.996 & $\mathrm{sp}^{2.14}(\mathrm{~s}(31.88 \%) \mathrm{p}(68.08 \%))-\mathrm{sp}^{2.14}(\mathrm{~s}(31.88 \%) \mathrm{p}(68.08 \%))$ \\
\hline & $\mathrm{M}-\mathrm{C} 6$ & 1.158 & 0.996 & $\mathrm{sp}^{2.14}(\mathrm{~s}(31.88 \%) \mathrm{p}(68.08 \%))-\mathrm{sp}^{2.14}(\mathrm{~s}(31.88 \%) \mathrm{p}(68.08 \%))$ \\
\hline \multirow{4}{*}{$\mathrm{C}_{59} \mathrm{Pd}$} & M-C5 & 0.823 & 0.606 & $\mathrm{sp}^{0.02} \mathrm{~d}^{1.53}(\mathrm{~s}(39.19 \%) \mathrm{p}(0.77 \%) \mathrm{d}(60.03 \%))-\mathrm{sp}^{5.13} \mathrm{~d}^{0.01}(\mathrm{~s}(16.31 \%) \mathrm{p}(83.59 \%) \mathrm{d}(0.11 \%))$ \\
\hline & & & & $\mathrm{sp}^{2.4} \mathrm{~d}^{0.93}(\mathrm{~s}(23.08 \%) \mathrm{p}(55.36 \%) \mathrm{d}(21.56 \%))-\mathrm{sp}^{8.70} \mathrm{~d}^{0.00}(\mathrm{~s}(10.31 \%) \mathrm{p}(89.66 \%) \mathrm{d}(0.03 \%))$ \\
\hline & $\mathrm{M}-\mathrm{C} 1$ & 0.638 & 0.511 & $\mathrm{sp}^{1.19} \mathrm{~d}^{3.25}(\mathrm{~s}(18.39 \%) \mathrm{p}(21.86 \%) \mathrm{d}(59.74 \%))-\mathrm{sp}^{4.34} \mathrm{~d}^{0.00}(\mathrm{~s}(18.71 \%) \mathrm{p}(81.23 \%) \mathrm{d}(0.06 \%))$ \\
\hline & $\mathrm{M}-\mathrm{C} 6$ & 0.638 & 0.511 & $\mathrm{sp}^{1.19} \mathrm{~d}^{3.25}(\mathrm{~s}(18.39 \%) \mathrm{p}(21.86 \%) \mathrm{d}(59.74 \%))-\mathrm{sp}^{4.34} \mathrm{~d}^{0.00}(\mathrm{~s}(18.71 \%) \mathrm{p}(81.23 \%) \mathrm{d}(0.06 \%))$ \\
\hline \multirow{3}{*}{$\mathrm{C}_{59} \mathrm{Ni}$} & & & & - \\
\hline & $\mathrm{M}-\mathrm{C} 1$ & 0.664 & 0.539 & $\mathrm{sp}^{0.18} \mathrm{~d}^{1.18}(\mathrm{~s}(42.34 \%) \mathrm{p}(7.51 \%) \mathrm{d}(50.15 \%))-\mathrm{sp}^{3.79} \mathrm{~d}^{0.00}(\mathrm{~s}(20.85 \%) \mathrm{p}(79.11 \%) \mathrm{d}(0.04 \%))$ \\
\hline & $\mathrm{M}-\mathrm{C} 6$ & 0.664 & 0.539 & $\mathrm{sp}^{0.18} \mathrm{~d}^{1.18}(\mathrm{~s}(42.34 \%) \mathrm{p}(7.51 \%) \mathrm{d}(50.15 \%))-\mathrm{sp}^{3.79} \mathrm{~d}^{0.00}(\mathrm{~s}(20.85 \%) \mathrm{p}(79.11 \%) \mathrm{d}(0.04 \%))$ \\
\hline
\end{tabular}


Table S2: Second order interaction energy $\mathrm{E}^{2}(\mathrm{kcal} / \mathrm{mol})$ between donor and acceptor orbitals for pure and metal substituted fullerene $\mathrm{C}_{59} \mathrm{M}(\mathrm{M}=\mathrm{C} / \mathrm{Pd} / \mathrm{Ni})$.

\begin{tabular}{|c|c|c|c|c|c|}
\hline System & Donor & acceptor & $E^{2}$ & $\mathrm{E}_{j}-\mathrm{E}_{i}$ & $F(i, j)$ \\
\hline \multirow{3}{*}{$\mathrm{C}_{60}$} & $\sigma-\mathrm{M}-\mathrm{C} 5$ & $\sigma^{*}$-M-C1 & 2.9 & 1.19 & 0.052 \\
\hline & $\sigma-\mathrm{M}-\mathrm{C} 5$ & $\sigma^{*}-\mathrm{M}-\mathrm{C} 6$ & 2.9 & 1.19 & 0.052 \\
\hline & $\sigma-\mathrm{M}-\mathrm{C} 1$ & $\sigma^{*}$-M-C6 & 1.6 & 1.14 & 0.039 \\
\hline \multirow{8}{*}{$\mathrm{C}_{59} \mathrm{Pd}$} & $\sigma-\mathrm{M}-\mathrm{C} 5$ & $\sigma^{*}-\mathrm{M}-\mathrm{C} 1$ & 96.9 & 0.5 & 0.199 \\
\hline & $\sigma-\mathrm{M}-\mathrm{C} 5$ & $\sigma^{*}$-M-C6 & 96.9 & 0.5 & 0.199 \\
\hline & $\sigma-\mathrm{M}-\mathrm{C} 1$ & $\sigma^{*}-\mathrm{M}-\mathrm{C} 6$ & 119.3 & 0.51 & 0.222 \\
\hline & $\sigma$-M-C5 & $\mathrm{LP}^{*}(5) \mathrm{M}$ & 42.5 & 0.77 & 0.172 \\
\hline & $\sigma-\mathrm{M}-\mathrm{C} 1$ & $\mathrm{LP}^{*}(4) \mathrm{M}$ & 1.5 & 0.43 & 0.024 \\
\hline & $\sigma-\mathrm{M}-\mathrm{C} 1$ & $\mathrm{LP}^{*}(5) \mathrm{M}$ & 29.7 & 0.79 & 0.146 \\
\hline & $\sigma-\mathrm{M}-\mathrm{C} 6$ & $\mathrm{LP}^{*}(4) \mathrm{M}$ & 1.5 & 0.43 & 0.024 \\
\hline & $\sigma-\mathrm{M}-\mathrm{C} 6$ & $\mathrm{LP}^{*}(5) \mathrm{M}$ & 29.7 & 0.79 & 0.146 \\
\hline \multirow{7}{*}{$\mathrm{C}_{59} \mathrm{Ni}$} & $\sigma-\mathrm{M}-\mathrm{C} 1$ & $\sigma^{*}$-M-C6 & 131.5 & 0.65 & 0.263 \\
\hline & $\sigma-\mathrm{M}-\mathrm{C} 1$ & $\mathrm{LP}^{*}(6) \mathrm{M}$ & 1.8 & 0.42 & 0.026 \\
\hline & $\sigma-\mathrm{M}-\mathrm{C} 1$ & $\mathrm{LP}^{*}(7) \mathrm{M}$ & 31.2 & 0.58 & 0.127 \\
\hline & $\sigma-\mathrm{M}-\mathrm{C} 2$ & $\mathrm{LP}^{*}(2) \mathrm{C} 5$ & 41.4 & 0.2 & 0.09 \\
\hline & $\sigma-\mathrm{M}-\mathrm{C} 6$ & $\mathrm{LP}^{*}(6) \mathrm{M}$ & 1.8 & 0.42 & 0.026 \\
\hline & $\sigma-\mathrm{M}-\mathrm{C} 6$ & $\mathrm{LP}^{*}(7) \mathrm{M}$ & 31.2 & 0.58 & 0.127 \\
\hline & $\sigma$-M-C6 & $\mathrm{LP}^{*}(2) \mathrm{C} 5$ & 41.4 & 0.2 & 0.09 \\
\hline
\end{tabular}


Table S3: Adsorption energies $(\Delta E ; \mathrm{T}=298 \mathrm{~K}, \mathrm{P}=1 \mathrm{~atm})$ and $\mathrm{C}-\mathrm{C}$ bond lengths of $\mathrm{C}_{2} \mathrm{H}_{2}$ over different adsorption sites on $\mathrm{C}_{60}$ determined using different functionals. $\Delta E$ are given in $\mathrm{kcal} / \mathrm{mol}$ and the bond lengths are given in $\AA$.

\begin{tabular}{ccccccc}
\hline Adsorption site & \multicolumn{2}{c}{ B3LYP } & \multicolumn{2}{c}{ B97D } & \multicolumn{2}{c}{ wB97XD } \\
\hline & $\Delta E$ & C-C & $\Delta E$ & C-C & $\Delta E$ & C-C \\
\hline $\mathrm{T}$ & 61.4 & 1.32 & 2.6 & 1.21 & - & - \\
$\mathrm{HH}$ & -24.5 & 1.33 & -15.4 & 1.34 & -23.9 & 1.33 \\
$\mathrm{PH}$ & -3.3 & 1.32 & 6.5 & 1.55 & 9.6 & 1.53 \\
Free & - & 1.21 & - & 1.2 & - & 1.2 \\
\hline
\end{tabular}


Table S4: Adsorption energies $(\Delta E ; \mathrm{T}=298 \mathrm{~K}, \mathrm{P}=1 \mathrm{~atm})$ and $\mathrm{C}-\mathrm{C}$ bond lengths of $\mathrm{C}_{2} \mathrm{H}_{2}$ over different adsorption sites on $\mathrm{C}_{59} \mathrm{M}(\mathrm{M}=\mathrm{Pd} / \mathrm{Ni})$ determined using different functionals. $\Delta E$ are given in $\mathrm{kcal} / \mathrm{mol}$ and the bond lengths are given in $\AA$.

\begin{tabular}{ccccccccccccccc}
\hline Adsorption site & \multicolumn{4}{c}{ B3LYP } & \multicolumn{4}{c}{ B97D } & \multicolumn{5}{c}{ wB97XD } \\
\hline & \multicolumn{2}{c}{$\mathrm{Pd}$} & \multicolumn{2}{c}{$\mathrm{Ni}$} & \multicolumn{2}{c}{$\mathrm{Pd}$} & \multicolumn{2}{c}{$\mathrm{Ni}$} & \multicolumn{3}{c}{$\mathrm{Pd}$} & \multicolumn{2}{c}{$\mathrm{Ni}$} \\
\hline & $\Delta E$ & $\mathrm{C}-\mathrm{C}$ & $\Delta E$ & $\mathrm{C}-\mathrm{C}$ & $\Delta E$ & $\mathrm{C}-\mathrm{C}$ & $\Delta E$ & $\mathrm{C}-\mathrm{C}$ & $\Delta E$ & $\mathrm{C}-\mathrm{C}$ & $\Delta E$ & $\mathrm{C}-\mathrm{C}$ \\
\hline $\mathrm{TM}$ & -13.1 & 1.23 & -22.1 & 1.26 & -9.4 & 1.25 & -15.7 & 1.27 & -10.2 & 1.23 & -18.0 & 1.26 \\
$\mathrm{TC} 1$ & - & - & - & - & - & - & - & - & - & - & - & - \\
$\mathrm{TC} 5$ & - & - & - & - & - & - & - & - & - & - & - & - \\
BMC1 & -22.6 & 1.35 & -27.6 & 1.36 & -13.9 & 1.36 & -21.5 & 1.37 & -19.7 & 1.35 & -23.5 & 1.36 \\
BMC5 & -40.5 & 1.36 & -44.1 & 1.36 & -28.9 & 1.36 & -32.7 & 1.37 & -39.1 & 1.35 & -41.6 & 1.36 \\
BC1C2 & -35.6 & 1.35 & -36.8 & 1.35 & -27.4 & 1.36 & -27.8 & 1.36 & -34.7 & 1.34 & -36.4 & 1.4 \\
BC2C3 & -16.0 & 1.33 & -17.2 & 1.33 & -8.3 & 1.34 & -8.7 & 1.34 & -15.2 & 1.33 & -16.6 & 1.33 \\
BC3C4 & -28.0 & 1.33 & -29.7 & 1.33 & -18.9 & 1.34 & -19.6 & 1.34 & -28.6 & 1.33 & -30.5 & 1.33 \\
BC4C5 & -28.7 & 1.35 & -28.5 & 1.35 & -22.6 & 1.36 & -22.1 & 1.36 & -25.9 & 1.34 & -25.8 & 1.34 \\
BC6C7 & -23.3 & 1.36 & -23.0 & 1.35 & -19.2 & 1.37 & -18.5 & 1.37 & -19.5 & 1.35 & -19.1 & 1.35 \\
BC7C8 & - & - & - & - & - & - & - & - & - & - & - & - \\
\hline
\end{tabular}


Table S5: Adsorption energies $(\Delta E ; \mathrm{T}=298 \mathrm{~K}, \mathrm{P}=1 \mathrm{~atm})$ and $\mathrm{C}-\mathrm{C}$ bond lengths of $\mathrm{C}_{2} \mathrm{H}_{4}$ over different adsorption sites on $\mathrm{C}_{60}$ determined using different functionals. $\Delta E$ are given in $\mathrm{kcal} / \mathrm{mol}$ and the bond lengths are given in $\AA$.

\begin{tabular}{ccccccc}
\hline Adsorption site & \multicolumn{2}{c}{ B3LYP } & \multicolumn{2}{c}{ B97D } & \multicolumn{2}{c}{ wB97XD } \\
\hline & $\Delta E$ & C-C & $\Delta E$ & C-C & $\Delta E$ & C-C \\
\hline $\mathrm{T}$ & -0.4 & 1.33 & 6.5 & 1.34 & 7.6 & 1.33 \\
$\mathrm{HH}$ & -12.2 & 1.55 & 0.5 & 1.55 & -9.7 & 1.54 \\
$\mathrm{PH}$ & 0.01 & 1.55 & 17.5 & 1.56 & 12.2 & 1.56 \\
Free & - & 1.33 & - & 1.34 & - & 1.33 \\
\hline
\end{tabular}


Table S6: Adsorption energies $(\Delta E ; \mathrm{T}=298 \mathrm{~K}, \mathrm{P}=1 \mathrm{~atm})$ and $\mathrm{C}-\mathrm{C}$ bond lengths of $\mathrm{C}_{2} \mathrm{H}_{4}$ over different adsorption sites on $\mathrm{C}_{59} \mathrm{M}(\mathrm{M}=\mathrm{Pd} / \mathrm{Ni})$ determined using different functionals. $\Delta E$ are given in $\mathrm{kcal} / \mathrm{mol}$ and the bond lengths are given in $\AA$.

\begin{tabular}{ccccccccccccccc}
\hline Adsorption site & \multicolumn{4}{c}{ B3LYP } & \multicolumn{4}{c}{ B97D } & \multicolumn{5}{c}{ wB97XD } \\
\hline & \multicolumn{2}{c}{$\mathrm{Pd}$} & \multicolumn{2}{c}{$\mathrm{Ni}$} & \multicolumn{2}{c}{$\mathrm{Pd}$} & \multicolumn{2}{c}{$\mathrm{Ni}$} & \multicolumn{3}{c}{$\mathrm{Pd}$} & \multicolumn{2}{c}{$\mathrm{Ni}$} \\
\hline & $\Delta E$ & $\mathrm{C}-\mathrm{C}$ & $\Delta E$ & $\mathrm{C}-\mathrm{C}$ & $\Delta E$ & $\mathrm{C}-\mathrm{C}$ & $\Delta E$ & $\mathrm{C}-\mathrm{C}$ & $\Delta E$ & $\mathrm{C}-\mathrm{C}$ & $\Delta E$ & $\mathrm{C}-\mathrm{C}$ \\
\hline $\mathrm{TM}$ & -15.5 & 1.37 & -17.9 & 1.37 & -7.8 & 1.38 & -10.5 & 1.38 & -8.0 & 1.36 & -11.7 & 1.37 \\
$\mathrm{TC} 1$ & - & - & - & - & - & - & - & - & - & - & - & - \\
$\mathrm{TC} 5$ & - & - & - & - & - & - & - & - & - & - & - & - \\
BMC1 & -7.0 & 1.53 & -8.4 & 1.54 & 3.11 & 1.54 & -0.1 & 1.54 & -3.4 & 1.53 & - & - \\
BMC5 & -23.3 & 1.53 & - & - & -10.7 & 1.54 & - & - & -22.1 & 1.52 & - & - \\
BC1C2 & -19.1 & 1.54 & -19.9 & 1.55 & -7.7 & 1.55 & -7.4 & 1.55 & -17.5 & 1.54 & -18.7 & 1.54 \\
BC2C3 & -4.5 & 1.54 & -6.0 & 1.54 & 6.5 & 1.55 & 6.0 & 1.55 & -2.0 & 1.54 & -3.6 & 1.54 \\
BC3C4 & -15.2 & 1.54 & -17.0 & 1.54 & -2.7 & 1.55 & -3.3 & 1.55 & -14.4 & 1.54 & -16.2 & 1.54 \\
BC4C5 & -12.8 & 1.54 & -13.5 & 1.54 & -3.3 & 1.55 & -3.2 & 1.55 & -9.2 & 1.54 & -9.7 & 1.54 \\
BC6C7 & -3.4 & 1.41 & -4.4 & 1.35 & 4.4 & 1.55 & 4.5 & 1.56 & 0.6 & 1.55 & -0.2 & 1.55 \\
BC7C8 & - & - & - & - & - & - & - & - & - & - & - & - \\
\hline
\end{tabular}


Table S7: Adsorption energies $(\Delta E ; \mathrm{T}=298 \mathrm{~K}, \mathrm{P}=1 \mathrm{~atm})$ and $\mathrm{C}-\mathrm{C}$ bond lengths of $\mathrm{C}_{2} \mathrm{H}_{6}$ over different adsorption sites on $\mathrm{C}_{60}$ determined using different functionals. $\Delta E$ are given in $\mathrm{kcal} / \mathrm{mol}$ and the bond lengths are given in $\AA$.

\begin{tabular}{cccccccc}
\hline Adsorption site & \multicolumn{2}{c}{ B3LYP } & \multicolumn{2}{c}{ B97D } & \multicolumn{2}{c}{ wB97XD } \\
\hline & $\Delta E$ & C-C & $\Delta E$ & C-C & $\Delta E$ & C-C \\
\hline $\mathrm{T}$ & -0.2 & 1.53 & 8.8 & 1.54 & 9.0 & 1.53 \\
$\mathrm{HH}$ & -2.0 & 1.53 & - & - & - & - \\
$\mathrm{PH}$ & -0.5 & 1.53 & - & - & - & - \\
Free & - & 1.53 & - & 1.54 & - & 1.53 \\
\hline
\end{tabular}


Table S8: Adsorption energies $(\Delta E ; \mathrm{T}=298 \mathrm{~K}, \mathrm{P}=1 \mathrm{~atm})$ and $\mathrm{C}-\mathrm{C}$ bond lengths of $\mathrm{C}_{2} \mathrm{H}_{6}$ over different adsorption sites on $\mathrm{C}_{59} \mathrm{M}(\mathrm{M}=\mathrm{Pd} / \mathrm{Ni})$ determined using different functionals. $\Delta E$ are given in $\mathrm{kcal} / \mathrm{mol}$ and the bond lengths are given in $\AA$.

\begin{tabular}{cccccccccccccc}
\hline Adsorption site & \multicolumn{3}{c}{ B3LYP } & \multicolumn{4}{c}{ B97D } & \multicolumn{3}{c}{ wB97XD } \\
\hline & \multicolumn{3}{c}{$\mathrm{Pd}$} & \multicolumn{2}{c}{$\mathrm{Ni}$} & \multicolumn{2}{c}{$\mathrm{Pd}$} & \multicolumn{2}{c}{$\mathrm{Ni}$} & \multicolumn{2}{c}{$\mathrm{Pd}$} & $\mathrm{Ni}$ \\
\hline & $\Delta E$ & $\mathrm{C}-\mathrm{C}$ & $\Delta E$ & $\mathrm{C}-\mathrm{C}$ & $\Delta E$ & $\mathrm{C}-\mathrm{C}$ & $\Delta E$ & $\mathrm{C}-\mathrm{C}$ & $\Delta E$ & $\mathrm{C}-\mathrm{C}$ & $\Delta E$ & $\mathrm{C}-\mathrm{C}$ \\
\hline $\mathrm{TM}$ & -2.6 & 1.53 & -3.4 & 1.53 & 3.1 & 1.54 & 2.5 & 1.54 & 6.1 & 1.53 & 3.24 & 1.53 \\
$\mathrm{TC} 1$ & - & - & - & - & - & - & - & - & - & - & - & - \\
$\mathrm{TC} 5$ & - & - & - & - & - & - & - & - & - & - & - & - \\
$\mathrm{BMC} 1$ & - & - & - & - & - & - & - & - & - & - & - & - \\
$\mathrm{BMC} 5$ & - & - & - & - & - & - & - & - & - & - & - & - \\
$\mathrm{BC} 1 \mathrm{C} 2$ & - & - & - & - & - & - & - & - & - & - & - & - \\
$\mathrm{BC} 2 \mathrm{C} 3$ & - & - & -0.5 & 1.53 & - & - & - & - & - & - & - & - \\
$\mathrm{BC} 3 \mathrm{C} 4$ & -0.8 & 1.53 & -0.7 & 1.53 & - & - & - & - & - & - & - & - \\
$\mathrm{BC} 4 \mathrm{C} 5$ & - & - & - & - & - & - & - & - & - & - & - & - \\
$\mathrm{BC} 6 \mathrm{C} 7$ & - & - & - & - & - & - & - & - & - & - & - & - \\
$\mathrm{BC} 7 \mathrm{C} 8$ & - & - & -0.7 & 1.53 & - & - & - & - & - & - & - & - \\
\hline
\end{tabular}


Table S9: Second order interaction energy $\mathrm{E}^{2}(\mathrm{kcal} / \mathrm{mol})$ between donar and acceptor orbitals for adsorption of $\mathrm{C}_{2} \mathrm{H}_{2}$ and $\mathrm{C}_{2} \mathrm{H}_{4}$ over $\mathrm{C}_{60} . \mathrm{M}=\mathrm{C}$

\begin{tabular}{|c|c|c|c|c|c|}
\hline \multicolumn{3}{|c|}{$\mathrm{C}_{2} \mathrm{H}_{2}$} & \multicolumn{3}{|c|}{$\mathrm{C}_{2} \mathrm{H}_{4}$} \\
\hline Donar & acceptor & $\mathrm{E}^{2}$ & Donar & acceptor & $\mathrm{E}^{2}$ \\
\hline$\sigma-\mathrm{M}-\mathrm{C} 1$ & $\sigma *-\mathrm{M}-\mathrm{C} 5$ & 0.79 & $\sigma-\mathrm{M}-\mathrm{C} 1$ & $\sigma *-\mathrm{M}-\mathrm{C} 5$ & 1.41 \\
\hline$\sigma$-M-C6 & $\sigma *-\mathrm{M}-\mathrm{C} 5$ & 0.70 & $\sigma$-C4-C5 & $\sigma *-\mathrm{M}-\mathrm{C} 5$ & 1.41 \\
\hline$\sigma-\mathrm{C} 1-\mathrm{C} 8$ & $\sigma *-\mathrm{M}-\mathrm{C} 5$ & 1.37 & $\sigma$-M-C5 & $\sigma *-\mathrm{C} 1-\mathrm{C} 2$ & 1.17 \\
\hline$\sigma-\mathrm{M}-\mathrm{C} 5$ & $\sigma *-\mathrm{M}-\mathrm{C} 1$ & 1.08 & $\sigma$-M-C5 & $\sigma *-\mathrm{C} 4-\mathrm{C} 5$ & 1.17 \\
\hline$\sigma$-M-C5 & $\sigma *-\mathrm{C} 1-\mathrm{C} 8$ & 1.20 & $\sigma$-M-C5 & $\sigma *-\mathrm{Ca} 1-\mathrm{H} 1$ & 0.88 \\
\hline$\sigma-\mathrm{M}-\mathrm{C} 5$ & $\sigma *-\mathrm{Ca} 1-\mathrm{H} 1$ & 4.85 & $\sigma$-M-C5 & $\sigma *-\mathrm{Ca} 1-\mathrm{H} 2$ & 0.88 \\
\hline$\sigma-\mathrm{M}-\mathrm{C} 5$ & $\sigma *-\mathrm{Ca} 2-\mathrm{H} 2$ & 4.85 & $\sigma-\mathrm{M}-\mathrm{C} 5$ & $\sigma *-\mathrm{Ca} 2-\mathrm{H} 3$ & 0.88 \\
\hline$\sigma$-Ca1-Ca2 & $\sigma *-\mathrm{M}-\mathrm{C} 1$ & 1.67 & $\sigma-\mathrm{M}-\mathrm{C} 5$ & $\sigma *-\mathrm{Ca} 2-\mathrm{H} 4$ & 0.88 \\
\hline$\sigma$-Ca1-Ca2 & $\sigma *-\mathrm{M}-\mathrm{C} 5$ & 1.67 & $\sigma$-Ca1-Ca2 & $\sigma *-\mathrm{M}-\mathrm{C} 1$ & 1.53 \\
\hline$\sigma$-Ca1-Ca2 & $\sigma *-\mathrm{M}-\mathrm{Ca} 2$ & 1.02 & $\sigma$-Ca1-Ca2 & $\sigma *-\mathrm{C} 4-\mathrm{C} 5$ & 1.53 \\
\hline$\sigma$-Ca1-Ca2 & $\sigma *$-Ca1-H1 & 2.18 & $\sigma$-Ca1-H1 & $\sigma *-\mathrm{M}-\mathrm{C} 1$ & 1.47 \\
\hline$\sigma-\mathrm{Ca} 1-\mathrm{Ca} 2$ & $\sigma *-\mathrm{Ca} 2-\mathrm{H} 2$ & 2.18 & $\sigma-\mathrm{Ca} 2-\mathrm{H} 2$ & $\sigma *-\mathrm{M}-\mathrm{C} 1$ & 1.47 \\
\hline$\pi$-Ca1-Ca2 & $\sigma *-\mathrm{M}-\mathrm{C} 1$ & 3.78 & $\sigma-\mathrm{M}-\mathrm{Ca} 2$ & $\sigma *-\mathrm{C} 5-\mathrm{M}$ & 1.07 \\
\hline$\pi-\mathrm{Ca} 1-\mathrm{Ca} 2$ & $\sigma *-\mathrm{M}-\mathrm{C} 5$ & 3.78 & $\sigma$-C5-Ca1 & $\sigma *-\mathrm{Ca} 1-\mathrm{H} 1$ & 1.05 \\
\hline$\sigma$-Ca1-H1 & $\sigma *-\mathrm{M}-\mathrm{Ca} 2$ & 0.98 & $\sigma-\mathrm{C} 5-\mathrm{Ca} 1$ & $\sigma *-\mathrm{Ca} 1-\mathrm{H} 2$ & 1.05 \\
\hline$\sigma-\mathrm{Ca} 1-\mathrm{H} 1$ & $\sigma *-\mathrm{Ca} 1-\mathrm{Ca} 2$ & 1.74 & $\sigma-\mathrm{C} 5-\mathrm{Ca} 2$ & $\sigma *-\mathrm{C} 5-\mathrm{M}$ & 1.22 \\
\hline$\sigma-\mathrm{Ca} 2-\mathrm{H} 2$ & $\sigma *-\mathrm{M}-\mathrm{Ca} 2$ & 0.98 & $\sigma-\mathrm{C} 5-\mathrm{Ca} 2$ & $\sigma *-\mathrm{Ca} 2-\mathrm{H} 3$ & 1.05 \\
\hline$\sigma$-Ca1-H2 & $\sigma *-\mathrm{Ca} 1-\mathrm{Ca} 2$ & 1.74 & $\sigma$-C5-Ca2 & $\sigma *-\mathrm{Ca} 2-\mathrm{H} 4$ & 1.05 \\
\hline$\sigma$-Ca1-M & $\sigma *-\mathrm{M}-\mathrm{C} 2$ & 1.23 & & & \\
\hline$\sigma-\mathrm{Ca} 1-\mathrm{M}$ & $\sigma *-\mathrm{Ca} 1-\mathrm{Ca} 2$ & 1.35 & & & \\
\hline$\sigma-\mathrm{Ca} 1-\mathrm{M}$ & $\sigma *-\mathrm{Ca} 1-\mathrm{H} 1$ & 0.61 & & & \\
\hline$\sigma$-Ca1-H1 & $\sigma *-\mathrm{Ca} 2-\mathrm{H} 2$ & 7.44 & & & \\
\hline
\end{tabular}


Table S10: Second order interaction energy $\mathrm{E}^{2}(\mathrm{kcal} / \mathrm{mol})$ between donar and acceptor orbitals for adsorption of $\mathrm{C}_{2} \mathrm{H}_{2}$ and $\mathrm{C}_{2} \mathrm{H}_{4}$ over metal substituted fullerene $\mathrm{C}_{59} \mathrm{M}(\mathrm{M}=\mathrm{Pd} / \mathrm{Ni})$.

\begin{tabular}{|c|c|c|c|c|c|c|c|c|c|c|c|}
\hline \multicolumn{6}{|c|}{$\mathrm{C}_{2} \mathrm{H}_{2}$} & \multicolumn{6}{|c|}{$\mathrm{C}_{2} \mathrm{H}_{4}$} \\
\hline \multicolumn{3}{|c|}{$\mathrm{C}_{59} \mathrm{Pd}$} & \multicolumn{3}{|c|}{$\mathrm{C}_{59} \mathrm{Ni}$} & \multicolumn{3}{|c|}{$\mathrm{C}_{59} \mathrm{Pd}$} & \multicolumn{3}{|c|}{$\mathrm{C}_{59} \mathrm{Ni}$} \\
\hline Donar & acceptor & $E^{2}$ & Donar & acceptor & $\mathrm{E}^{2}$ & Donar & acceptor & $E^{2}$ & Donar & acceptor & $\mathrm{E}^{2}$ \\
\hline$\sigma-\mathrm{M}-\mathrm{C} 1$ & $\sigma *-\mathrm{M}-\mathrm{C} 5$ & 18.92 & $\sigma-\mathrm{M}-\mathrm{C} 1$ & $\sigma *$-M-C5 & 20.86 & $\sigma-\mathrm{M}-\mathrm{C} 1$ & $\sigma *-\mathrm{M}-\mathrm{C} 5$ & 14.13 & $\sigma-\mathrm{C} 7-\mathrm{C} 8$ & $\sigma *-\mathrm{C} 1-\mathrm{C} 2$ & 1.27 \\
\hline$\sigma-\mathrm{M}-\mathrm{C} 6$ & $\sigma *-\mathrm{M}-\mathrm{C} 5$ & 85.22 & $\sigma-\mathrm{M}-\mathrm{C} 1$ & $\sigma *-\mathrm{M}-\mathrm{C} 5$ & 102.23 & $\sigma-\mathrm{M}-\mathrm{C} 6$ & $\sigma *-\mathrm{M}-\mathrm{C} 5$ & 41.76 & $\sigma-\mathrm{C} 1-\mathrm{C} 8$ & $\sigma *-\mathrm{C} 1-\mathrm{C} 2$ & 0.66 \\
\hline$\sigma-\mathrm{M}-\mathrm{C} 5$ & $\sigma *-\mathrm{M}-\mathrm{C} 5$ & 42.35 & $\sigma-\mathrm{M}-\mathrm{C} 5$ & $\sigma *-\mathrm{M}-\mathrm{C} 5$ & 70.81 & $\sigma-\mathrm{M}-\mathrm{C} 5$ & $\sigma *-\mathrm{M}-\mathrm{C} 5$ & 35.17 & $\sigma-\mathrm{C} 2-\mathrm{C} 3$ & $\sigma *-\mathrm{C} 1-\mathrm{C} 2$ & 0.82 \\
\hline$\sigma-\mathrm{M}-\mathrm{Ca} 2$ & $\sigma *-\mathrm{M}-\mathrm{C} 5$ & 6.45 & $\sigma-\mathrm{M}-\mathrm{Ca} 2$ & $\sigma *-\mathrm{M}-\mathrm{C} 5$ & 2.74 & $\sigma-\mathrm{M}-\mathrm{Ca} 2$ & $\sigma *-\mathrm{M}-\mathrm{C} 5$ & 24.63 & $\sigma-\mathrm{C} 1-\mathrm{C} 2$ & $\mathrm{LP}^{*}(5) \mathrm{M}$ & 1.28 \\
\hline$\sigma-\mathrm{Ca} 1-\mathrm{Ca} 2$ & $\sigma *-\mathrm{M}-\mathrm{C} 5$ & 4.06 & $\sigma-\mathrm{Ca} 1-\mathrm{Ca} 2$ & $\sigma *-\mathrm{M}-\mathrm{C} 5$ & 0.73 & $\sigma-\mathrm{Ca} 1-\mathrm{Ca} 2$ & $\sigma *-\mathrm{M}-\mathrm{C} 5$ & 0.84 & $\sigma-\mathrm{C} 1-\mathrm{C} 2$ & $\mathrm{LP}^{*}(6) \mathrm{M}$ & 6.47 \\
\hline$\sigma$-Ca1-H1 & $\sigma *-\mathrm{M}-\mathrm{C} 5$ & 2.48 & $\pi-\mathrm{Ca} 1-\mathrm{Ca} 2$ & $\sigma *-\mathrm{M}-\mathrm{C} 5$ & 4.15 & $\sigma-\mathrm{Ca} 1-\mathrm{H} 1$ & $\sigma *-\mathrm{M}-\mathrm{C} 5$ & 2.49 & $\sigma-\mathrm{C} 1-\mathrm{C} 2$ & $\sigma *-\mathrm{M}-\mathrm{C} 1$ & 1.07 \\
\hline$\sigma-\mathrm{Ca} 2-\mathrm{H} 2$ & $\sigma *-\mathrm{M}-\mathrm{C} 5$ & 2.29 & $\sigma-\mathrm{Ca} 1-\mathrm{H} 1$ & $\sigma *-\mathrm{M}-\mathrm{C} 5$ & 1.82 & $\sigma-\mathrm{Ca} 1-\mathrm{H} 2$ & $\sigma *-\mathrm{M}-\mathrm{C} 5$ & 1.12 & $\sigma-\mathrm{C} 1-\mathrm{C} 2$ & $\sigma *-\mathrm{M}-\mathrm{C} 6$ & 0.67 \\
\hline$\sigma-\mathrm{M}-\mathrm{C} 1$ & $\mathrm{LP}^{*}(4) \mathrm{M}$ & 1.98 & $\sigma-\mathrm{Ca} 2-\mathrm{H} 2$ & $\sigma *-\mathrm{M}-\mathrm{C} 5$ & 2.86 & $\sigma-\mathrm{Ca} 2-\mathrm{H} 3$ & $\sigma *-\mathrm{M}-\mathrm{C} 5$ & 1.21 & $\sigma-\mathrm{C} 1-\mathrm{C} 2$ & $\sigma *-\mathrm{C} 1-\mathrm{C} 8$ & 1.13 \\
\hline$\sigma-\mathrm{M}-\mathrm{C} 1$ & $\mathrm{LP}^{*}(5) \mathrm{M}$ & 5.98 & $\sigma-\mathrm{M}-\mathrm{C} 1$ & $\mathrm{LP}^{*}(4) \mathrm{M}$ & 0.82 & $\sigma-\mathrm{Ca} 2-\mathrm{H} 4$ & $\sigma *-\mathrm{M}-\mathrm{C} 5$ & 1.41 & $\sigma-\mathrm{C} 1-\mathrm{C} 2$ & $\sigma *-\mathrm{C} 2-\mathrm{C} 3$ & 1.55 \\
\hline$\sigma-\mathrm{M}-\mathrm{C} 5$ & $\mathrm{LP}^{*}(4) \mathrm{M}$ & 2.01 & $\sigma-\mathrm{M}-\mathrm{C} 1$ & $\mathrm{LP}^{*}(5) \mathrm{M}$ & 2.87 & $\sigma-\mathrm{M}-\mathrm{C} 5$ & $\mathrm{LP}^{*}(4) \mathrm{M}$ & 2.34 & $\sigma-\mathrm{C} 1-\mathrm{C} 2$ & $\sigma *-\mathrm{Ca} 1-\mathrm{H} 1$ & 0.64 \\
\hline$\sigma-\mathrm{M}-\mathrm{C} 5$ & $\mathrm{LP}^{*}(5) \mathrm{M}$ & 19.56 & $\sigma-\mathrm{M}-\mathrm{C} 5$ & $\mathrm{LP}^{*}(5) \mathrm{M}$ & 17.76 & $\sigma-\mathrm{M}-\mathrm{C} 5$ & $\mathrm{LP}^{*}(5) \mathrm{M}$ & 12.05 & $\sigma-\mathrm{C} 1-\mathrm{C} 2$ & $\sigma *-\mathrm{Ca} 1-\mathrm{H} 2$ & 1.14 \\
\hline$\sigma-\mathrm{M}-\mathrm{C} 5$ & $\sigma *-\mathrm{M}-\mathrm{C} 1$ & 44.94 & $\sigma-\mathrm{M}-\mathrm{C} 5$ & $\sigma *-\mathrm{M}-\mathrm{C} 1$ & 76.27 & $\sigma-\mathrm{M}-\mathrm{C} 5$ & $\sigma *-\mathrm{M}-\mathrm{C} 1$ & 20.44 & $\sigma-\mathrm{C} 1-\mathrm{C} 2$ & $\sigma *-\mathrm{Ca} 2-\mathrm{H} 3$ & 0.97 \\
\hline$\pi$-M-C5 & $\sigma *-\mathrm{M}-\mathrm{C} 6$ & 86.93 & $\sigma-\mathrm{M}-\mathrm{C} 5$ & $\sigma *-\mathrm{M}-\mathrm{C} 6$ & 120.25 & $\sigma-\mathrm{M}-\mathrm{C} 5$ & $\sigma *-\mathrm{M}-\mathrm{C} 6$ & 39.78 & $\sigma-\mathrm{C} 1-\mathrm{C} 2$ & $\sigma *-\mathrm{Ca} 2-\mathrm{H} 4$ & 0.83 \\
\hline$\sigma-\mathrm{M}-\mathrm{C} 5$ & $\sigma *-\mathrm{M}-\mathrm{Ca} 2$ & 60.18 & $\sigma-\mathrm{M}-\mathrm{C} 5$ & $\sigma *-\mathrm{M}-\mathrm{C} 5$ & 70.81 & $\sigma-\mathrm{M}-\mathrm{C} 5$ & $\sigma *-\mathrm{M}-\mathrm{C} 5$ & 35.17 & $\sigma-\mathrm{C} 1-\mathrm{Ca} 2$ & $\mathrm{LP}^{*}(5) \mathrm{M}$ & 0.75 \\
\hline$\sigma-\mathrm{M}-\mathrm{C} 5$ & $\sigma *-\mathrm{M}-\mathrm{C} 5$ & 42.35 & $\sigma-\mathrm{M}-\mathrm{C} 5$ & $\sigma *-\mathrm{M}-\mathrm{Ca} 2$ & 44.30 & $\sigma-\mathrm{M}-\mathrm{C} 5$ & $\sigma *-\mathrm{M}-\mathrm{Ca} 2$ & 106.06 & $\sigma-\mathrm{C} 1-\mathrm{Ca} 2$ & $\mathrm{LP}^{*}(6) \mathrm{M}$ & 4.61 \\
\hline$\sigma-\mathrm{M}-\mathrm{C} 5$ & $\sigma *-\mathrm{Ca} 1-\mathrm{Ca} 2$ & 0.91 & $\sigma-\mathrm{M}-\mathrm{C} 5$ & $\sigma *-\mathrm{Ca} 1-\mathrm{Ca} 2$ & 0.81 & $\sigma-\mathrm{M}-\mathrm{C} 5$ & $\sigma *-\mathrm{Ca} 1-\mathrm{Ca} 2$ & 1.45 & $\sigma-\mathrm{C} 1-\mathrm{Ca} 2$ & $\mathrm{LP}^{*}(7) \mathrm{M}$ & 0.73 \\
\hline$\sigma-\mathrm{M}-\mathrm{C} 5$ & $\pi *-\mathrm{Ca} 1-\mathrm{Ca} 2$ & 2.26 & $\sigma-\mathrm{M}-\mathrm{C} 5$ & $\pi *-\mathrm{Ca} 1-\mathrm{Ca} 2$ & 1.76 & $\sigma-\mathrm{M}-\mathrm{C} 5$ & $\sigma *-\mathrm{Ca} 1-\mathrm{H} 1$ & 3.10 & $\sigma-\mathrm{C} 1-\mathrm{Ca} 2$ & $\sigma-\mathrm{M}-\mathrm{C} 1$ & 1.08 \\
\hline$\sigma-\mathrm{M}-\mathrm{C} 5$ & $\sigma *-\mathrm{Ca} 1-\mathrm{H} 1$ & 4.88 & $\sigma-\mathrm{M}-\mathrm{C} 5$ & $\sigma *-\mathrm{Ca} 1-\mathrm{H} 1$ & 5.16 & $\sigma-\mathrm{M}-\mathrm{C} 5$ & $\sigma *-\mathrm{Ca} 1-\mathrm{H} 2$ & 0.61 & $\sigma-\mathrm{C} 1-\mathrm{Ca} 2$ & $\sigma-\mathrm{C} 1-\mathrm{C} 8$ & 1.02 \\
\hline$\sigma-\mathrm{M}-\mathrm{C} 5$ & $\sigma *-\mathrm{Ca} 2-\mathrm{H} 2$ & 1.18 & $\sigma-\mathrm{M}-\mathrm{Ca} 2$ & $\mathrm{LP}^{*}(4) \mathrm{M}$ & 3.13 & $\sigma-\mathrm{M}-\mathrm{C} 5$ & $\sigma *-\mathrm{Ca} 2-\mathrm{H} 3$ & 0.76 & $\sigma-\mathrm{C} 1-\mathrm{Ca} 2$ & $\sigma-\mathrm{C} 2-\mathrm{C} 3$ & 2.04 \\
\hline$\sigma-\mathrm{M}-\mathrm{Ca} 2$ & $\mathrm{LP}^{*}(4) \mathrm{M}$ & 1.62 & $\sigma-\mathrm{M}-\mathrm{Ca} 2$ & $\sigma-\mathrm{M}-\mathrm{C} 1$ & 10.08 & $\sigma-\mathrm{M}-\mathrm{C} 5$ & $\sigma *-\mathrm{Ca} 2-\mathrm{H} 4$ & 1.52 & $\sigma-\mathrm{C} 1-\mathrm{Ca} 2$ & $\sigma$-Ca1-H1 & 0.84 \\
\hline$\sigma-\mathrm{M}-\mathrm{Ca} 2$ & $\sigma *-\mathrm{M}-\mathrm{C} 1$ & 1.69 & $\sigma-\mathrm{M}-\mathrm{Ca} 2$ & $\sigma-\mathrm{M}-\mathrm{C} 6$ & 1.09 & $\sigma-\mathrm{M}-\mathrm{Ca} 2$ & $\mathrm{LP}^{*}(5) \mathrm{M}$ & 1.06 & $\sigma-\mathrm{C} 1-\mathrm{Ca} 2$ & $\sigma-\mathrm{Ca} 1-\mathrm{H} 2$ & 1.17 \\
\hline$\sigma-\mathrm{M}-\mathrm{Ca} 2$ & $\sigma *-\mathrm{Ca} 1-\mathrm{Ca} 2$ & 1.47 & $\sigma-\mathrm{M}-\mathrm{Ca} 2$ & $\sigma-\mathrm{M}-\mathrm{C} 5$ & 2.74 & $\sigma-\mathrm{M}-\mathrm{Ca} 2$ & $\sigma *-\mathrm{M}-\mathrm{C} 1$ & 11.36 & $\sigma-\mathrm{C} 2-\mathrm{Ca} 1$ & $\sigma-\mathrm{M}-\mathrm{C} 1$ & 1.57 \\
\hline$\sigma-\mathrm{M}-\mathrm{Ca} 2$ & $\pi *-\mathrm{Ca} 1-\mathrm{Ca} 2$ & 3.13 & $\sigma-\mathrm{M}-\mathrm{Ca} 2$ & $\sigma-\mathrm{C} 5-\mathrm{Ca} 1$ & 1.7 & $\sigma-\mathrm{M}-\mathrm{Ca} 2$ & $\sigma *-\mathrm{M}-\mathrm{C} 6$ & 1.25 & $\sigma-\mathrm{C} 1-\mathrm{Ca} 2$ & $\sigma-\mathrm{C} 1-\mathrm{C} 8$ & 2.36 \\
\hline$\sigma-\mathrm{M}-\mathrm{Ca} 2$ & $\sigma *-\mathrm{Ca} 1-\mathrm{H} 1$ & 11.99 & $\sigma-\mathrm{M}-\mathrm{Ca} 2$ & $\sigma-\mathrm{Ca} 1-\mathrm{Ca} 2$ & 1.22 & $\sigma-\mathrm{M}-\mathrm{Ca} 2$ & $\sigma *-\mathrm{M}-\mathrm{C} 5$ & 24.63 & $\sigma-\mathrm{C} 1-\mathrm{Ca} 2$ & $\sigma-\mathrm{C} 2-\mathrm{C} 3$ & 0.98 \\
\hline$\sigma-\mathrm{M}-\mathrm{Ca} 2$ & $\sigma *-\mathrm{Ca} 2-\mathrm{H} 2$ & 1.10 & $\sigma-\mathrm{M}-\mathrm{Ca} 2$ & $\sigma$-Ca1-H1 & 12.79 & $\sigma-\mathrm{M}-\mathrm{Ca} 2$ & $\sigma *-\mathrm{M}-\mathrm{Ca} 2$ & 1.62 & $\sigma-\mathrm{C} 1-\mathrm{Ca} 2$ & $\sigma-\mathrm{Ca} 2-\mathrm{H} 3$ & 1.18 \\
\hline$\sigma-\mathrm{C} 5-\mathrm{Ca} 1$ & $\mathrm{LP}^{*}(4) \mathrm{M}$ & 2.30 & $\sigma-\mathrm{M}-\mathrm{Ca} 2$ & $\sigma-\mathrm{Ca} 2-\mathrm{H} 2$ & 1.28 & $\sigma-\mathrm{M}-\mathrm{Ca} 2$ & $\sigma *-\mathrm{C} 5-\mathrm{Ca} 1$ & 0.7 & $\sigma-\mathrm{C} 1-\mathrm{Ca} 2$ & $\sigma-\mathrm{Ca} 2-\mathrm{H} 4$ & 0.97 \\
\hline$\sigma-\mathrm{C} 5-\mathrm{Ca} 1$ & $\mathrm{LP}^{*}(5) \mathrm{M}$ & 7.88 & $\sigma-\mathrm{C} 5-\mathrm{Ca} 1$ & $\mathrm{LP}^{*}(4) \mathrm{M}$ & 7.53 & $\sigma-\mathrm{M}-\mathrm{Ca} 2$ & $\sigma *-\mathrm{Ca} 1-\mathrm{Ca} 2$ & 1.19 & $\sigma-\mathrm{Ca} 1-\mathrm{Ca} 2$ & $\sigma-\mathrm{M}-\mathrm{C} 1$ & 0.79 \\
\hline$\sigma-\mathrm{C} 5-\mathrm{Ca} 1$ & $\sigma *-\mathrm{M}-\mathrm{C} 5$ & 0.57 & $\sigma-\mathrm{C} 5-\mathrm{Ca} 1$ & $\mathrm{LP}^{*}(5) \mathrm{M}$ & 12.28 & $\sigma-\mathrm{M}-\mathrm{Ca} 2$ & $\sigma *-\mathrm{Ca} 1-\mathrm{H} 1$ & 4.11 & $\sigma-\mathrm{Ca} 1-\mathrm{Ca} 2$ & $\sigma-\mathrm{C} 1-\mathrm{C} 8$ & 2.81 \\
\hline$\sigma-\mathrm{C} 5-\mathrm{Ca} 1$ & $\sigma *-\mathrm{Ca} 1-\mathrm{Ca} 2$ & 1.69 & $\sigma-\mathrm{C} 5-\mathrm{Ca} 1$ & $\sigma-\mathrm{M}-\mathrm{C} 1$ & 1.08 & $\sigma-\mathrm{M}-\mathrm{Ca} 2$ & $\sigma *-\mathrm{Ca} 1-\mathrm{H} 2$ & 0.77 & $\sigma-\mathrm{Ca} 1-\mathrm{Ca} 2$ & $\sigma-\mathrm{C} 1-\mathrm{Ca} 2$ & 0.79 \\
\hline$\sigma-\mathrm{C} 5-\mathrm{Ca} 1$ & $\sigma *-\mathrm{Ca} 2-\mathrm{H} 2$ & 3.08 & $\sigma-\mathrm{C} 5-\mathrm{Ca} 1$ & $\sigma-\mathrm{Ca} 1-\mathrm{Ca} 2$ & 1.62 & $\sigma-\mathrm{C} 5-\mathrm{Ca} 1$ & $\mathrm{LP}^{*}(5)$ & 4.53 & $\sigma-\mathrm{Ca} 1-\mathrm{Ca} 2$ & $\sigma-\mathrm{C} 2-\mathrm{C} 3$ & 1.76 \\
\hline$\sigma-\mathrm{Ca} 1-\mathrm{Ca} 2$ & $\mathrm{LP}^{*}(4) \mathrm{M}$ & 1.34 & $\sigma-\mathrm{C} 5-\mathrm{Ca} 1$ & $\sigma-\mathrm{Ca} 1-\mathrm{H} 1$ & 0.55 & $\sigma-\mathrm{C} 5-\mathrm{Ca} 1$ & $\sigma *-\mathrm{Ca} 1-\mathrm{Ca} 2$ & 0.74 & $\sigma-\mathrm{Ca} 1-\mathrm{Ca} 2$ & $\sigma-\mathrm{Ca} 2-\mathrm{H} 3$ & 0.64 \\
\hline$\sigma-\mathrm{Ca} 1-\mathrm{Ca} 2$ & $\mathrm{LP}^{*}(5) \mathrm{M}$ & 1.54 & $\sigma-\mathrm{C} 5-\mathrm{Ca} 1$ & $\sigma-\mathrm{Ca} 2-\mathrm{H} 2$ & 2.81 & $\sigma-\mathrm{C} 5-\mathrm{Ca} 1$ & $\sigma *-\mathrm{Ca} 2-\mathrm{H} 2$ & 1.27 & $\sigma-\mathrm{Ca} 1-\mathrm{Ca} 2$ & $\sigma-\mathrm{Ca} 2-\mathrm{H} 4$ & 0.51 \\
\hline$\pi$-Ca1-Ca2 & $\sigma *-\mathrm{M}-\mathrm{C} 1$ & 4.40 & $\sigma-\mathrm{Ca} 1-\mathrm{Ca} 2$ & $\mathrm{LP}^{*}(4) \mathrm{M}$ & 5.45 & $\sigma-\mathrm{Ca} 1-\mathrm{Ca} 2$ & $\sigma *-\mathrm{M}-\mathrm{C} 5$ & 0.84 & $\sigma-\mathrm{Ca} 2-\mathrm{H} 3$ & $\sigma-\mathrm{Ca} 2-\mathrm{H} 4$ & 1.12 \\
\hline$\pi-\mathrm{Ca} 1-\mathrm{Ca} 2$ & $\sigma *-\mathrm{M}-\mathrm{C} 5$ & 4.06 & $\sigma-\mathrm{Ca} 1-\mathrm{Ca} 2$ & $\mathrm{LP}^{*}(5) \mathrm{M}$ & 2.52 & $\sigma-\mathrm{Ca} 1-\mathrm{Ca} 2$ & $\sigma *-\mathrm{C} 5-\mathrm{Ca} 1$ & 0.75 & $\sigma-\mathrm{Ca} 1-\mathrm{H} 2$ & $\sigma-\mathrm{C} 2-\mathrm{C} 3$ & 1.09 \\
\hline$\pi$-Ca1-H1 & $\sigma *-\mathrm{M}-\mathrm{C} 5$ & 2.48 & $\pi-\mathrm{Ca} 1-\mathrm{Ca} 2$ & $\mathrm{LP}^{*}(4) \mathrm{M}$ & 22.24 & $\sigma-\mathrm{Ca} 1-\mathrm{Ca} 2$ & $\sigma *-\mathrm{Ca} 1-\mathrm{H} 1$ & 0.81 & $\sigma-\mathrm{Ca} 1-\mathrm{H} 2$ & $\sigma-\mathrm{Ca} 2-\mathrm{H} 3$ & 0.80 \\
\hline$\pi$-Ca1-H1 & $\sigma *-\mathrm{M}-\mathrm{Ca} 2$ & 2.53 & $\pi-\mathrm{Ca} 1-\mathrm{Ca} 2$ & $\mathrm{LP}^{*}(5) \mathrm{M}$ & 0.69 & $\sigma-\mathrm{Ca} 1-\mathrm{Ca} 2$ & $\sigma *-\mathrm{Ca} 1-\mathrm{H} 2$ & 0.60 & $\sigma-\mathrm{Ca} 2-\mathrm{H} 3$ & $\mathrm{LP}^{*}(6) \mathrm{M}$ & 1.89 \\
\hline$\pi$-Ca1-H1 & $\sigma *-\mathrm{Ca} 1-\mathrm{Ca} 2$ & 1.22 & $\pi-\mathrm{Ca} 1-\mathrm{Ca} 2$ & $\mathrm{M}-\mathrm{C} 1$ & 1.33 & $\sigma-\mathrm{Ca} 1-\mathrm{H} 1$ & $\sigma *-\mathrm{M}-\mathrm{C} 5$ & 2.49 & $\sigma-\mathrm{Ca} 2-\mathrm{H} 3$ & $\mathrm{LP}^{*}(7) \mathrm{M}$ & 2.05 \\
\hline$\pi$-Ca2-H2 & $\mathrm{LP}^{*}(4) \mathrm{M}$ & 0.62 & $\pi-\mathrm{Ca} 1-\mathrm{Ca} 2$ & $\sigma-\mathrm{M}-\mathrm{C} 5$ & 0.73 & $\sigma$-Ca1-H1 & $\sigma *-\mathrm{M}-\mathrm{Ca} 2$ & 1.51 & $\sigma-\mathrm{Ca} 2-\mathrm{H} 3$ & $\sigma-\mathrm{M}-\mathrm{C} 1$ & 1.29 \\
\hline$\pi-\mathrm{Ca} 2-\mathrm{H} 2$ & $\mathrm{LP}^{*}(5) \mathrm{M}$ & 0.92 & $\sigma-\mathrm{Ca} 1-\mathrm{Ca} 2$ & $\sigma-\mathrm{M}-\mathrm{Ca} 2$ & 0.93 & $\sigma-\mathrm{Ca} 1-\mathrm{H} 2$ & $\sigma *-\mathrm{M}-\mathrm{C} 5$ & 1.12 & $\sigma-\mathrm{Ca} 2-\mathrm{H} 3$ & $\sigma-\mathrm{C} 1-\mathrm{C} 8$ & 1.2 \\
\hline$\pi-\mathrm{Ca} 2-\mathrm{H} 2$ & $\sigma *-\mathrm{M}-\mathrm{C} 1$ & 0.83 & $\sigma-\mathrm{Ca} 1-\mathrm{Ca} 2$ & $\sigma-\mathrm{Ca} 1-\mathrm{H} 1$ & 2.26 & $\sigma-\mathrm{Ca} 1-\mathrm{H} 2$ & $\sigma *-\mathrm{M}-\mathrm{Ca} 2$ & 0.75 & $\sigma-\mathrm{Ca} 2-\mathrm{H} 3$ & $\sigma-\mathrm{Ca} 1-\mathrm{H} 4$ & 0.75 \\
\hline$\pi-\mathrm{Ca} 2-\mathrm{H} 2$ & $\sigma *-\mathrm{M}-\mathrm{C} 5$ & 2.29 & $\sigma-\mathrm{Ca} 1-\mathrm{Ca} 2$ & $\sigma-\mathrm{C} 5-\mathrm{Ca} 1$ & 1.37 & $\sigma-\mathrm{Ca} 1-\mathrm{H} 2$ & $\sigma *-\mathrm{Ca} 2-\mathrm{H} 3$ & 1.7 & $\sigma-\mathrm{Ca} 2-\mathrm{H} 4$ & $\sigma-\mathrm{M}-\mathrm{C} 1$ & 1.64 \\
\hline$\pi$-Ca2-H2 & $\sigma *-\mathrm{M}-\mathrm{Ca} 2$ & 0.65 & $\sigma-\mathrm{Ca} 1-\mathrm{H} 1$ & $\mathrm{LP}^{*}(4) \mathrm{M}$ & 1.39 & $\sigma-\mathrm{Ca} 2-\mathrm{H} 3$ & $\mathrm{LP}^{*}(4) \mathrm{M}$ & 0.83 & $\sigma-\mathrm{Ca} 2-\mathrm{H} 4$ & $\sigma-\mathrm{C} 1-\mathrm{Ca} 2$ & 0.67 \\
\hline$\pi$-Ca2-H2 & $\sigma *-\mathrm{C} 5-\mathrm{Ca} 1$ & 3.53 & $\sigma-\mathrm{Ca} 1-\mathrm{H} 1$ & $\mathrm{LP}^{*}(5) \mathrm{M}$ & 0.52 & $\sigma-\mathrm{Ca} 2-\mathrm{H} 3$ & $\sigma *-\mathrm{M}-\mathrm{C} 5$ & 1.21 & $\sigma-\mathrm{Ca} 2-\mathrm{H} 4$ & $\sigma-\mathrm{Ca} 1-\mathrm{H} 1$ & 0.92 \\
\hline$\pi$-Ca2-H2 & $\sigma *-\mathrm{Ca} 1-\mathrm{Ca} 2$ & 0.52 & $\sigma-\mathrm{Ca} 1-\mathrm{H} 1$ & $\sigma-\mathrm{M}-\mathrm{C} 5$ & 1.82 & $\sigma-\mathrm{Ca} 2-\mathrm{H} 3$ & $\sigma *-\mathrm{M}-\mathrm{Ca} 2$ & 0.72 & & & \\
\hline \multirow[t]{9}{*}{$\pi-\mathrm{Ca} 2-\mathrm{H} 2$} & $\pi *-\mathrm{Ca} 1-\mathrm{Ca} 2$ & 0.99 & $\sigma-\mathrm{Ca} 1-\mathrm{H} 1$ & $\sigma-\mathrm{M}-\mathrm{Ca} 2$ & 2.42 & $\sigma-\mathrm{Ca} 2-\mathrm{H} 3$ & $\sigma *-\mathrm{Ca} 1-\mathrm{H} 2$ & 1.42 & & & \\
\hline & & & $\sigma$-Ca1-H1 & $\sigma-\mathrm{Ca} 1-\mathrm{Ca} 2$ & 0.99 & $\sigma-\mathrm{Ca} 2-\mathrm{H} 4$ & $\sigma *-\mathrm{M}-\mathrm{C} 5$ & 1.41 & & & \\
\hline & & & $\sigma-\mathrm{Ca} 2-\mathrm{H} 2$ & $\mathrm{LP}^{*}(4) \mathrm{M}$ & 3.45 & $\sigma-\mathrm{Ca} 2-\mathrm{H} 4$ & $\sigma *-\mathrm{M}-\mathrm{Ca} 2$ & 0.83 & & & \\
\hline & & & $\sigma-\mathrm{Ca} 2-\mathrm{H} 2$ & $\mathrm{LP}^{*}(5) \mathrm{M}$ & 1.17 & $\sigma-\mathrm{Ca} 2-\mathrm{H} 4$ & $\sigma *-\mathrm{C} 5-\mathrm{Ca} 1$ & 0.62 & & & \\
\hline & & & $\sigma-\mathrm{Ca} 2-\mathrm{H} 2$ & $\sigma-\mathrm{M}-\mathrm{C} 1$ & 2.89 & $\sigma-\mathrm{Ca} 2-\mathrm{H} 4$ & $\sigma *-\mathrm{Ca} 1-\mathrm{H} 1$ & 0.68 & & & \\
\hline & & & $\sigma-\mathrm{Ca} 2-\mathrm{H} 2$ & $\sigma$-M-C5 & 2.86 & & & & & & \\
\hline & & & $\sigma-\mathrm{Ca} 2-\mathrm{H} 2$ & $\sigma-\mathrm{M}-\mathrm{Ca} 2$ & 2.58 & & & & & & \\
\hline & & & $\sigma-\mathrm{Ca} 2-\mathrm{H} 2$ & $\sigma-\mathrm{C} 5-\mathrm{Ca} 1$ & 3.58 & & & & & & \\
\hline & & & $\sigma-\mathrm{Ca} 2-\mathrm{H} 1$ & $\sigma-\mathrm{Ca} 1-\mathrm{Ca} 2$ & 0.64 & & & & & & \\
\hline
\end{tabular}


Table S11: Natural population analysis for electron occupancy of "d" orbitals of isolated Pd and $\mathrm{Ni}$ atoms, $\mathrm{Pd}$ atom in $\mathrm{C}_{59} \mathrm{Pd}$ and $\mathrm{Ni}$ atom in $\mathrm{C}_{59} \mathrm{Ni}$.

\begin{tabular}{ccccc}
\hline "d" orbital & Isolated $\mathrm{Pd}$ & $\mathrm{Pd}$ in $\mathrm{C}_{59} \mathrm{Pd}$ & Isolated $\mathrm{Ni}$ & $\mathrm{Ni}$ in $\mathrm{C}_{59} \mathrm{Ni}$ \\
\hline $\mathrm{dxy}$ & 2.00000 & 1.67868 & 1.99997 & 1.69591 \\
$\mathrm{dxz}$ & 2.00000 & 1.66545 & 1.99997 & 1.66654 \\
$\mathrm{dyz}$ & 2.00000 & 1.77917 & 1.99998 & 1.68369 \\
$\mathrm{dx}^{2} \mathrm{y}^{2}$ & 2.00000 & 1.94039 & 1.32120 & 1.95746 \\
$\mathrm{dz}^{2}$ & 2.00000 & 1.89640 & 1.77372 & 1.89568 \\
\hline
\end{tabular}


Figure S1: Density of states of $\mathrm{C}_{2} \mathrm{H}_{2}-\mathrm{C}_{60}$ adsorption complex with different adsorption sites. (a) T, (b) HH and (c) PH.

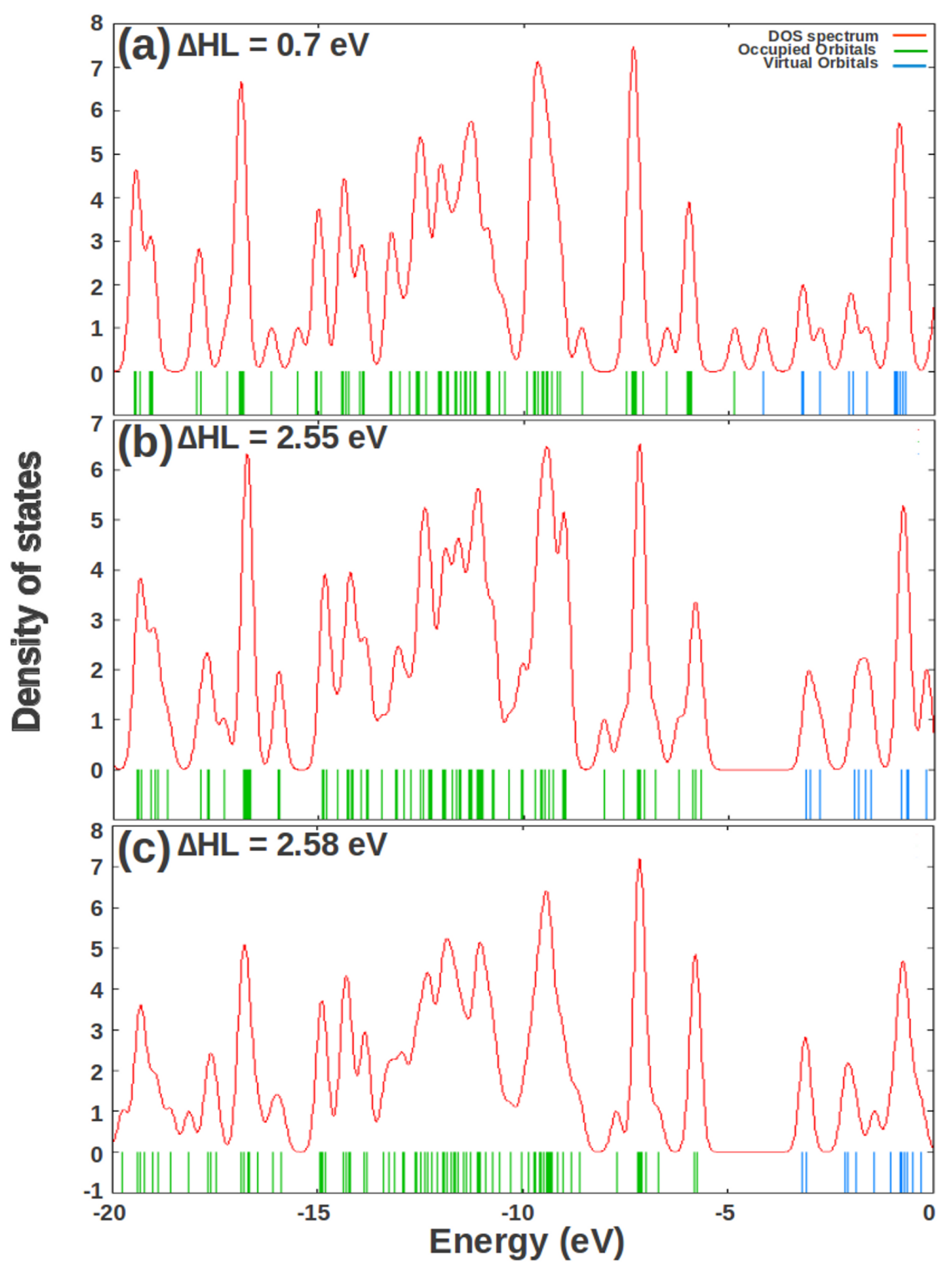


Figure S2: Variation of Gibbs free energy $(\Delta G)$ as a function of distance of adsorption site from the dopant metal for adsorption of $\mathrm{C}_{2} \mathrm{H}_{2}$. The dotted curve shows the adsorption energy over metal free $\mathrm{C}_{60}$.

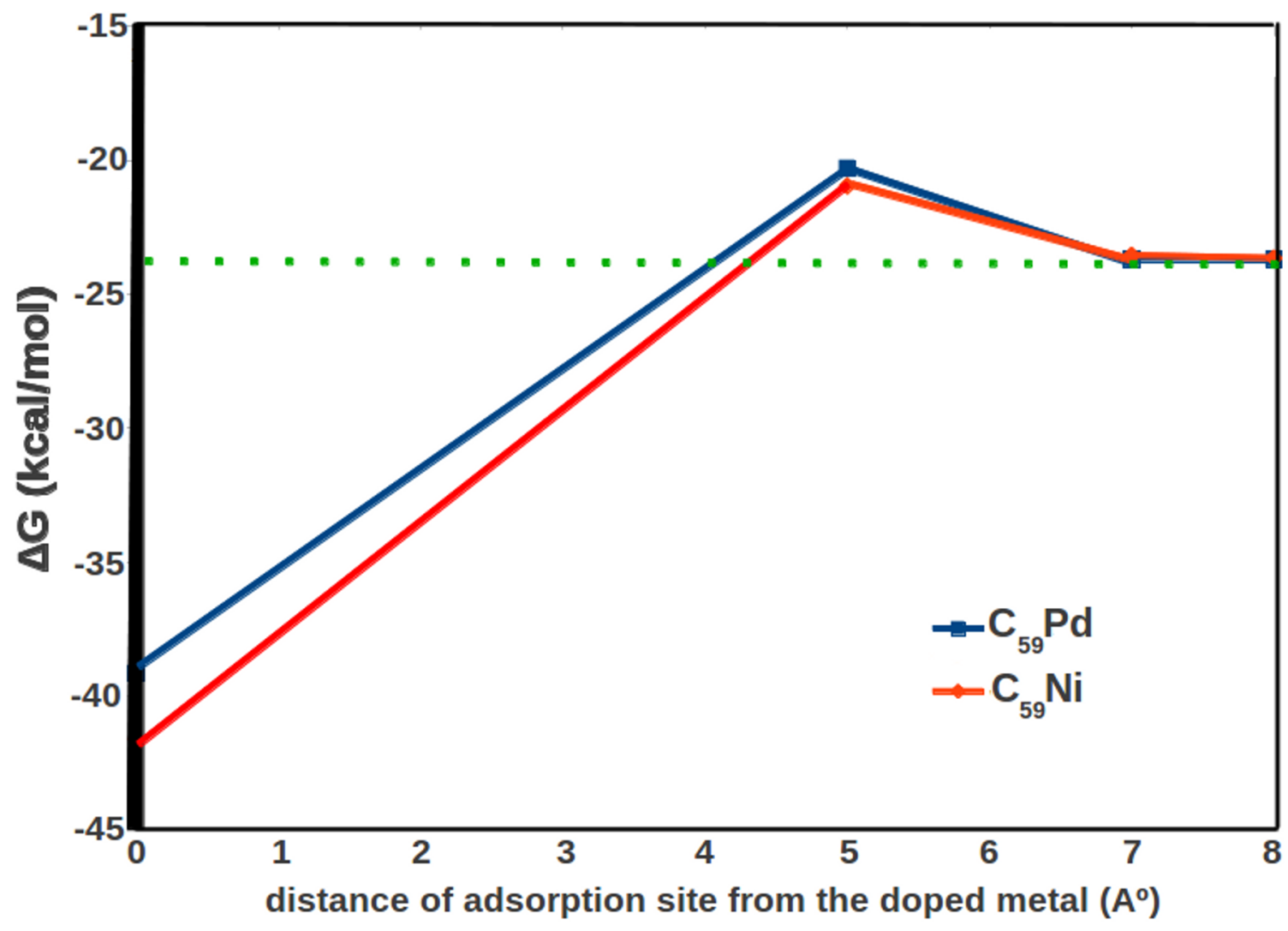




\section{Coordinates of all the intermediate and transition state structures observed during acetylene hydrogenation}

(0) For pure fullerene $\left(C_{60}\right)$

\begin{tabular}{|c|c|c|c|}
\hline C & -1.80016 & -3.02789 & -0.00262 \\
\hline $\mathrm{C}$ & -0.96759 & -3.17531 & -1.17893 \\
\hline U & 0.39052 & -3.42241 & -0.73843 \\
\hline $\mathrm{C}$ & 0.39712 & -3.42759 & 0.71037 \\
\hline U & -0.95691 & -3.18368 & 1.16498 \\
\hline $\mathrm{C}$ & -1.17852 & -2.41326 & 2.27981 \\
\hline $\mathrm{C}$ & -0.05847 & -1.84365 & 3.00116 \\
\hline $\mathrm{C}$ & 1.22476 & -2.07463 & 2.57023 \\
\hline C & 1.45863 & -2.88783 & 1.39407 \\
\hline C & 2.57206 & -2.31313 & 0.66665 \\
\hline $\mathrm{C}$ & 2.56581 & -2.30822 & -0.70656 \\
\hline $\mathrm{C}$ & 1.44578 & -2.87774 & -1.42789 \\
\hline $\mathrm{C}$ & 1.20123 & -2.05617 & -2.59604 \\
\hline $\mathrm{C}$ & -0.08585 & -1.82216 & -3.01360 \\
\hline $\mathrm{C}$ & -1.19931 & -2.39695 & -2.28617 \\
\hline $\mathrm{C}$ & -2.27646 & -1.42794 & -2.27781 \\
\hline $\mathrm{C}$ & -1.82887 & -0.25449 & -3.0002 \\
\hline $\mathrm{C}$ & -0.47494 & -0.49814 & -3.4548 \\
\hline $\mathrm{C}$ & 0.44343 & 0.52281 & -3.45540 \\
\hline $\mathrm{C}$ & 1.80138 & 0.27599 & -3.01480 \\
\hline $\mathrm{C}$ & 2.17008 & -0.97893 & -2.5965 \\
\hline $\mathrm{C}$ & 3.01353 & -1.13473 & -1.4288 \\
\hline $\mathrm{C}$ & 3.44398 & -0.02738 & -0.7402 \\
\hline $\mathrm{C}$ & 3.45058 & -0.03256 & 0.7086 \\
\hline $\mathrm{C}$ & 3.02638 & -1.14481 & 1.39318 \\
\hline $\mathrm{C}$ & 2.19360 & -0.997 & 2.5695 \\
\hline $\mathrm{C}$ & 1.82877 & 0.25450 & 3.00016 \\
\hline $\mathrm{C}$ & 0.47489 & 0.49812 & 3.45486 \\
\hline $\mathrm{C}$ & -0.44348 & -0.52283 & 3.4554 \\
\hline $\mathrm{C}$ & -1.80149 & -0.27598 & 3.0148 \\
\hline $\mathrm{C}$ & -2.17012 & 0.97892 & 2.59657 \\
\hline $\mathrm{C}$ & -1.20122 & 2.05617 & 2.5960 \\
\hline $\mathrm{C}$ & 0.08584 & 1.82215 & 3.01358 \\
\hline $\mathrm{C}$ & 1.19931 & 2.39693 & 2.28627 \\
\hline $\mathrm{C}$ & 2.27638 & 1.42794 & 2.27775 \\
\hline $\mathrm{C}$ & 3.06573 & 1.28832 & 1.1628 \\
\hline $\mathrm{C}$ & 2.82105 & 2.10973 & -0.00531 \\
\hline $\mathrm{C}$ & 3.05506 & 1.29670 & -1.18146 \\
\hline $\mathrm{C}$ & 2.25560 & 1.44425 & -2.28812 \\
\hline $\mathrm{C}$ & 1.17852 & 2.41325 & -2.2799 \\
\hline $\mathrm{C}$ & 0.05846 & 1.84364 & -3.0011 \\
\hline $\mathrm{C}$ & -1.22474 & 2.07463 & -2.5702 \\
\hline
\end{tabular}




$\begin{array}{lrrr}\mathrm{C} & -2.19364 & 0.99738 & -2.56964 \\ \mathrm{C} & -3.02635 & 1.14478 & -1.39318 \\ \mathrm{C} & -2.57198 & 2.31311 & -0.66663 \\ \mathrm{C} & -1.45850 & 2.88772 & -1.39406 \\ \mathrm{C} & -0.39699 & 3.42755 & -0.71037 \\ \mathrm{C} & 0.95686 & 3.18375 & -1.16509 \\ \mathrm{C} & 1.80029 & 3.02822 & 0.00262 \\ \mathrm{C} & 0.96754 & 3.17538 & 1.17905 \\ \mathrm{C} & -0.39039 & 3.42237 & 0.73843 \\ \mathrm{C} & -1.44565 & 2.87764 & 1.42788 \\ \mathrm{C} & -2.56573 & 2.30820 & 0.70653 \\ \mathrm{C} & -3.01350 & 1.13469 & 1.42882 \\ \mathrm{C} & -3.44410 & 0.02734 & 0.74023 \\ \mathrm{C} & -3.45069 & 0.03252 & -0.70862 \\ \mathrm{C} & -3.06570 & -1.28827 & -1.16278 \\ \mathrm{C} & -2.82112 & -2.10975 & 0.00530 \\ \mathrm{C} & -3.05503 & -1.29664 & 1.18141 \\ \mathrm{C} & -2.25567 & -1.44425 & 2.28818\end{array}$

Intermediate structures observed over $C_{60}$ during acetylene hydrogenation.

(1) Intermediate IM1 $\left(\mathrm{C}_{2} \mathrm{H}_{2} \mathrm{C}_{60}\right)$

$\begin{array}{lrrr}C & 3.55332 & -0.81670 & -0.00014 \\ C & 2.83994 & -1.46055 & 1.17964 \\ C & 2.08105 & -2.59721 & 0.72662 \\ C & 2.08101 & -2.59715 & -0.72694 \\ C & 2.83986 & -1.46045 & -1.17990 \\ C & 2.39928 & -0.74195 & -2.27558 \\ C & 1.22066 & -1.17581 & -2.99672 \\ C & 0.51375 & -2.31407 & -2.59101 \\ C & 0.95327 & -3.04170 & -1.42481 \\ C & -0.22225 & -3.49322 & -0.69742 \\ C & -0.22221 & -3.49328 & 0.69715 \\ C & 0.95335 & -3.04183 & 1.42451 \\ C & 0.51389 & -2.31428 & 2.59079 \\ C & 1.22083 & -1.17606 & 2.99656 \\ C & 2.39941 & -0.74214 & 2.27539 \\ C & 2.39940 & 0.74194 & 2.27544 \\ C & 1.22083 & 1.17581 & 2.99666 \\ C & 0.49547 & -0.00014 & 3.44226 \\ C & -0.89942 & -0.00014 & 3.46448 \\ C & -1.63178 & -1.17722 & 3.02519 \\ C & -0.93818 & -2.31114 & 2.59798 \\ C & -1.39457 & -3.04057 & 1.42522 \\ C & -2.52291 & -2.60482 & 0.72710 \\ C & -2.52295 & -2.60476 & -0.72718 \\ C & -1.39465 & -3.04045 & -1.42539\end{array}$




\begin{tabular}{|c|c|c|c|}
\hline $\mathrm{C}$ & -0.93832 & -2.31092 & -2.59812 \\
\hline $\mathrm{C}$ & -1.63194 & -1.17697 & -3.02521 \\
\hline $\mathrm{C}$ & -0.89960 & 0.00014 & -3.46444 \\
\hline C & 0.49529 & 0.00014 & -3.44229 \\
\hline $\mathrm{C}$ & 1.22067 & 1.17606 & -2.99663 \\
\hline $\mathrm{C}$ & 0.51375 & 2.31428 & -2.59082 \\
\hline $\mathrm{C}$ & -0.93832 & 2.31114 & -2.59793 \\
\hline $\mathrm{C}$ & -1.63194 & 1.17722 & -3.02511 \\
\hline C & -2.80697 & 0.72797 & -2.30336 \\
\hline $\mathrm{C}$ & -2.80697 & -0.72778 & -2.30342 \\
\hline $\mathrm{C}$ & -3.24372 & -1.42613 & -1.17545 \\
\hline $\mathrm{C}$ & -3.69127 & -0.69798 & 0.00007 \\
\hline $\mathrm{C}$ & -3.24366 & -1.42622 & 1.17551 \\
\hline $\mathrm{C}$ & -2.80684 & -0.72797 & 2.30351 \\
\hline $\mathrm{C}$ & -2.80684 & 0.72778 & 2.30357 \\
\hline $\mathrm{C}$ & -1.63178 & 1.17697 & 3.02529 \\
\hline $\mathrm{C}$ & -0.93818 & 2.31092 & 2.59817 \\
\hline $\mathrm{C}$ & 0.51389 & 2.31407 & 2.59098 \\
\hline $\mathrm{C}$ & 0.95335 & 3.04170 & 1.42476 \\
\hline $\mathrm{C}$ & -0.22221 & 3.49322 & 0.69743 \\
\hline $\mathrm{C}$ & -1.39457 & 3.04045 & 1.42547 \\
\hline $\mathrm{C}$ & -2.52291 & 2.60476 & 0.72732 \\
\hline $\mathrm{C}$ & -3.24366 & 1.42613 & 1.17562 \\
\hline $\mathrm{C}$ & -3.69127 & 0.69798 & 0.00013 \\
\hline $\mathrm{C}$ & -3.24372 & 1.42622 & -1.17534 \\
\hline $\mathrm{C}$ & -2.52295 & 2.60482 & -0.72697 \\
\hline $\mathrm{C}$ & -1.39465 & 3.04057 & -1.42515 \\
\hline $\mathrm{C}$ & -0.22225 & 3.49328 & -0.69714 \\
\hline $\mathrm{C}$ & 0.95328 & 3.04183 & -1.42457 \\
\hline $\mathrm{C}$ & 2.08101 & 2.59721 & -0.72674 \\
\hline $\mathrm{C}$ & 2.08105 & 2.59715 & 0.72683 \\
\hline $\mathrm{C}$ & 2.83992 & 1.46044 & 1.17974 \\
\hline $\mathrm{C}$ & 3.55332 & 0.81670 & -0.00006 \\
\hline $\mathrm{C}$ & 2.83988 & 1.46055 & -1.17980 \\
\hline $\mathrm{C}$ & 2.39929 & 0.74214 & -2.27552 \\
\hline $\mathrm{C}$ & 5.07179 & 0.66836 & -0.00001 \\
\hline $\mathrm{C}$ & 5.07179 & -0.66836 & -0.00017 \\
\hline $\mathrm{H}$ & 5.84953 & 1.42585 & 0.00012 \\
\hline $\mathrm{H}$ & 5.84954 & -1.42583 & -0.00023 \\
\hline
\end{tabular}

(2) Intermediate IM2 $\left(\mathrm{C}_{2} \mathrm{H}_{2} \_2 \mathrm{H} \_\mathrm{C}_{60}\right)$
$\begin{array}{llll}\text { C } & -3.46363 & -1.05150 & -0.09716\end{array}$
$\begin{array}{llll}\text { C } & -2.73743 & -1.43205 & -1.37630\end{array}$
C $\quad-1.89863 \quad-2.58884 \quad-1.14786$
$\begin{array}{llll}\text { C } & -1.85942 & -2.83980 & 0.28022\end{array}$
$\begin{array}{llll}\text { C } & -2.66784 & -1.84271 & 0.93231\end{array}$
$\begin{array}{llll}\text { C } & -2.25050 & -1.31015 & 2.13388\end{array}$ 


\begin{tabular}{|c|c|c|c|}
\hline C & -1.03660 & -1.79098 & 2.75908 \\
\hline C & -0.26938 & -2.78911 & 2.14880 \\
\hline C & -0.69159 & -3.32871 & 0.87724 \\
\hline $\mathrm{C}$ & 0.49262 & -3.56570 & 0.06705 \\
\hline $\mathrm{C}$ & 0.45744 & -3.32300 & -1.30387 \\
\hline $\mathrm{C}$ & -0.76386 & -2.83375 & -1.92645 \\
\hline $\mathrm{C}$ & -0.39818 & -1.88272 & -2.95236 \\
\hline $\mathrm{C}$ & -1.18352 & -0.74038 & -3.13679 \\
\hline C & -2.35511 & -0.49873 & -2.31548 \\
\hline $\mathrm{C}$ & -2.46257 & 0.94724 & -2.08203 \\
\hline $\mathrm{C}$ & -1.38326 & 1.55821 & -2.68808 \\
\hline $\mathrm{C}$ & -0.55903 & 0.53738 & -3.35797 \\
\hline $\mathrm{C}$ & 0.82071 & 0.64061 & -3.39775 \\
\hline $\mathrm{C}$ & 1.64406 & -0.53166 & -3.19605 \\
\hline $\mathrm{C}$ & 1.04442 & -1.77927 & -2.97685 \\
\hline C & 1.57955 & -2.66666 & -1.95739 \\
\hline C & 2.69117 & -2.27800 & -1.20829 \\
\hline $\mathrm{C}$ & 2.73020 & -2.53195 & 0.22439 \\
\hline $\mathrm{C}$ & 1.65197 & -3.16319 & 0.85063 \\
\hline C & 1.17898 & -2.68553 & 2.13536 \\
\hline C & 1.80775 & -1.59360 & 2.74740 \\
\hline $\mathrm{C}$ & 1.01044 & -0.56596 & 3.39467 \\
\hline $\mathrm{C}$ & -0.37969 & -0.66150 & 3.39312 \\
\hline $\mathrm{C}$ & -1.19188 & 0.51894 & 3.15337 \\
\hline $\mathrm{C}$ & -0.56914 & 1.75614 & 2.92621 \\
\hline $\mathrm{C}$ & 0.88033 & 1.85769 & 2.93209 \\
\hline $\mathrm{C}$ & 1.65453 & 0.72001 & 3.15721 \\
\hline C & 2.84292 & 0.48390 & 2.36036 \\
\hline C & 2.93168 & -0.94765 & 2.10349 \\
\hline $\mathrm{C}$ & 3.38406 & -1.40634 & 0.86272 \\
\hline $\mathrm{C}$ & 3.75596 & -0.45261 & -0.16954 \\
\hline $\mathrm{C}$ & 3.32066 & -0.99048 & -1.44947 \\
\hline $\mathrm{C}$ & 2.80707 & -0.13444 & -2.42447 \\
\hline $\mathrm{C}$ & 2.71874 & 1.29575 & -2.16341 \\
\hline $\mathrm{C}$ & 1.49454 & 1.78343 & -2.75735 \\
\hline $\mathrm{C}$ & 0.75392 & 2.77070 & -2.12896 \\
\hline $\mathrm{C}$ & -0.71930 & 2.69274 & -2.07474 \\
\hline C & -1.15850 & 3.19471 & -0.86939 \\
\hline $\mathrm{C}$ & 0.01670 & 3.55897 & -0.07542 \\
\hline $\mathrm{C}$ & 1.20612 & 3.32294 & -0.87538 \\
\hline $\mathrm{C}$ & 2.38090 & 2.84762 & -0.28183 \\
\hline $\mathrm{C}$ & 3.15536 & 1.81536 & -0.93994 \\
\hline $\mathrm{C}$ & 3.67612 & 0.91758 & 0.07946 \\
\hline $\mathrm{C}$ & 3.21274 & 1.39745 & 1.37192 \\
\hline $\mathrm{C}$ & 2.41486 & 2.59129 & 1.14593 \\
\hline $\mathrm{C}$ & 1.26630 & 2.81671 & 1.91142 \\
\hline $\mathrm{C}$ & 0.05420 & 3.31438 & 1.28683 \\
\hline C & -1.08595 & 2.63193 & 1.92258 \\
\hline $\mathrm{C}$ & -2.20102 & 2.25906 & 1.17740 \\
\hline
\end{tabular}




$\begin{array}{lrrr}\mathrm{C} & -2.47169 & 2.78668 & -0.22964 \\ \mathrm{C} & -3.24633 & 1.57201 & -0.95381 \\ \mathrm{C} & -3.61764 & 0.54570 & 0.20051 \\ \mathrm{C} & -2.83248 & 1.01504 & 1.40270 \\ \mathrm{C} & -2.33379 & 0.14522 & 2.37609 \\ \mathrm{C} & -5.10176 & 0.22176 & 0.33747 \\ \mathrm{C} & -4.98562 & -1.07366 & 0.03109 \\ \mathrm{H} & -5.94129 & 0.86191 & 0.59507 \\ \mathrm{H} & -5.68478 & -1.90006 & -0.05314 \\ \mathrm{H} & -4.18158 & 1.97814 & -1.35660 \\ \mathrm{H} & -3.16243 & 3.64076 & -0.19917\end{array}$

(3) Intermediate IM3 $\left(\mathrm{C}_{2} \mathrm{H}_{3} \_\mathrm{H} \_\mathrm{C}_{60}\right)$

\begin{tabular}{|c|c|c|c|}
\hline C & -3.46489 & -0.96809 & -0.22364 \\
\hline C & -2.73042 & -1.31976 & -1.51353 \\
\hline $\mathrm{C}$ & -1.93122 & -2.49960 & -1.30034 \\
\hline C & -1.94205 & -2.81825 & 0.11750 \\
\hline C & -2.74513 & -1.83070 & 0.79419 \\
\hline C & -2.34857 & -1.33300 & 2.02532 \\
\hline C & -1.16109 & -1.86763 & 2.66118 \\
\hline $\mathrm{C}$ & -0.39957 & -2.85832 & 2.03012 \\
\hline $\mathrm{C}$ & -0.79456 & -3.34031 & 0.72513 \\
\hline $\mathrm{C}$ & 0.40862 & -3.56657 & -0.05948 \\
\hline $\mathrm{C}$ & 0.41718 & -3.26310 & -1.42315 \\
\hline C & -0.77122 & -2.71988 & -2.05290 \\
\hline C & -0.35746 & -1.73465 & -3.02496 \\
\hline C & -1.11404 & -0.56326 & -3.17757 \\
\hline $\mathrm{C}$ & -2.30964 & -0.35486 & -2.40223 \\
\hline $\mathrm{C}$ & -2.38228 & 1.09099 & -2.07212 \\
\hline $\mathrm{C}$ & -1.21786 & 1.73224 & -2.69223 \\
\hline $\mathrm{C}$ & -0.44462 & 0.71844 & -3.35175 \\
\hline $\mathrm{C}$ & 0.95972 & 0.77575 & -3.37158 \\
\hline C & 1.72994 & -0.43210 & -3.17614 \\
\hline C & 1.08838 & -1.66798 & -3.01514 \\
\hline C & 1.57096 & -2.61051 & -2.01805 \\
\hline C & 2.67218 & -2.28621 & -1.22255 \\
\hline $\mathrm{C}$ & 2.65951 & -2.59587 & 0.19617 \\
\hline C & 1.55124 & -3.23275 & 0.76787 \\
\hline C & 1.05040 & -2.78959 & 2.05869 \\
\hline $\mathrm{C}$ & 1.68246 & -1.73601 & 2.72578 \\
\hline C & 0.89313 & -0.71803 & 3.39637 \\
\hline C & -0.49841 & -0.78134 & 3.35802 \\
\hline $\mathrm{C}$ & -1.27827 & 0.42747 & 3.14979 \\
\hline $\mathrm{C}$ & -0.62102 & 1.65284 & 2.98602 \\
\hline $\mathrm{C}$ & 0.82678 & 1.72135 & 3.02868 \\
\hline $\mathrm{C}$ & 1.57197 & 0.56008 & 3.22696 \\
\hline C & 2.77718 & 0.32836 & 2.45424 \\
\hline
\end{tabular}




$\begin{array}{lrrr}\mathrm{C} & 2.84147 & -1.08871 & 2.13856 \\ \mathrm{C} & 3.32269 & -1.51330 & 0.89728 \\ \mathrm{C} & 3.74584 & -0.52897 & -0.08642 \\ \mathrm{C} & 3.34114 & -1.00629 & -1.39832 \\ \mathrm{C} & 2.88468 & -0.10312 & -2.35898 \\ \mathrm{C} & 2.82267 & 1.31797 & -2.05007 \\ \mathrm{C} & 1.63383 & 1.85892 & -2.67685 \\ \mathrm{C} & 0.89027 & 2.84447 & -2.02818 \\ \mathrm{C} & -0.56928 & 2.79009 & -2.02618 \\ \mathrm{C} & -1.05015 & 3.26764 & -0.77781 \\ \mathrm{C} & 0.07676 & 3.55064 & 0.04779 \\ \mathrm{C} & 1.29657 & 3.31878 & -0.72736 \\ \mathrm{C} & 2.43415 & 2.79631 & -0.11443 \\ \mathrm{C} & 3.21545 & 1.77605 & -0.78994 \\ \mathrm{C} & 3.68122 & 0.83228 & 0.21202 \\ \mathrm{C} & 3.18534 & 1.27359 & 1.50719 \\ \mathrm{C} & 2.42051 & 2.48704 & 1.30688 \\ \mathrm{C} & 1.25338 & 2.71160 & 2.04991 \\ \mathrm{C} & 0.07321 & 3.26246 & 1.42409 \\ \mathrm{C} & -1.08794 & 2.58952 & 1.99844 \\ \mathrm{C} & -2.19842 & 2.27361 & 1.21858 \\ \mathrm{C} & -2.38202 & 2.81542 & -0.20812 \\ \mathrm{C} & -2.91374 & 1.54550 & -0.90739 \\ \mathrm{C} & -3.56787 & 0.62109 & 0.10096 \\ \mathrm{C} & -2.86272 & 1.03254 & 1.38044 \\ \mathrm{C} & -2.41187 & 0.10781 & 2.32778 \\ \mathrm{C} & -5.13019 & 0.45270 & 0.10540 \\ \mathrm{C} & -4.95187 & -0.99411 & -0.16582 \\ \mathrm{H} & -5.64450 & -1.82878 & -0.19816 \\ \mathrm{H} & -5.58770 & 0.71076 & 1.07551 \\ \mathrm{H} & -5.67407 & 1.03313 & -0.66241 \\ \mathrm{H} & -3.13645 & 3.62083 & -0.23965\end{array}$

(4) Intermediate IM4 $\left(\mathrm{CHCH}_{3} \mathrm{C}_{60}\right)$

$\begin{array}{lrrr}\mathrm{C} & -3.52746 & -1.08721 & 0.48491 \\ \mathrm{C} & -2.70427 & -1.25228 & 1.68240 \\ \mathrm{C} & -2.35649 & -0.00069 & 2.39023 \\ \mathrm{C} & -2.70429 & 1.25124 & 1.68294 \\ \mathrm{C} & -3.52747 & 1.08677 & 0.48538 \\ \mathrm{C} & -2.98564 & 1.54130 & -0.72088 \\ \mathrm{C} & -2.03405 & 2.62576 & -0.69123 \\ \mathrm{C} & -1.51142 & 3.07902 & 0.51522 \\ \mathrm{C} & -1.80822 & 2.32453 & 1.72046 \\ \mathrm{C} & -0.61465 & 2.30621 & 2.52718 \\ \mathrm{C} & -0.31005 & 1.16446 & 3.25518 \\ \mathrm{C} & -1.17709 & -0.00086 & 3.17134 \\ \mathrm{C} & -0.31002 & -1.16616 & 3.25459\end{array}$




\begin{tabular}{|c|c|c|c|}
\hline C & -0.61459 & -2.30757 & 2.52602 \\
\hline $\mathrm{C}$ & -1.80817 & -2.32555 & 1.71936 \\
\hline $\mathrm{C}$ & -1.51132 & -3.07935 & 0.51364 \\
\hline $\mathrm{C}$ & -0.12710 & -3.51177 & 0.57435 \\
\hline $\mathrm{C}$ & 0.42796 & -3.04631 & 1.83239 \\
\hline $\mathrm{C}$ & 1.74868 & -2.60794 & 1.89754 \\
\hline $\mathrm{C}$ & 2.07898 & -1.42465 & 2.67564 \\
\hline $\mathrm{C}$ & 1.07275 & -0.72443 & 3.34516 \\
\hline $\mathrm{C}$ & 1.07273 & 0.72272 & 3.34553 \\
\hline $\mathrm{C}$ & 2.07895 & 1.42332 & 2.67638 \\
\hline $\mathrm{C}$ & 1.74861 & 2.60700 & 1.89889 \\
\hline $\mathrm{C}$ & 0.42787 & 3.04536 & 1.83396 \\
\hline $\mathrm{C}$ & -0.12719 & 3.51146 & 0.57617 \\
\hline $\mathrm{C}$ & 0.66027 & 3.50056 & -0.57802 \\
\hline $\mathrm{C}$ & 0.09683 & 3.04079 & -1.83730 \\
\hline $\mathrm{C}$ & -1.22393 & 2.59444 & -1.88084 \\
\hline $\mathrm{C}$ & -1.59890 & 1.40769 & -2.62204 \\
\hline C & -0.60949 & 0.72359 & -3.32436 \\
\hline $\mathrm{C}$ & 0.77382 & 1.17606 & -3.28660 \\
\hline $\mathrm{C}$ & 1.12389 & 2.31014 & -2.55370 \\
\hline C & 2.32595 & 2.30929 & -1.73679 \\
\hline C & 2.03737 & 3.04154 & -0.51329 \\
\hline $\mathrm{C}$ & 2.57262 & 2.60651 & 0.70175 \\
\hline $\mathrm{C}$ & 3.41811 & 1.42529 & 0.74255 \\
\hline $\mathrm{C}$ & 3.11785 & 0.69753 & 1.96575 \\
\hline $\mathrm{C}$ & 3.11785 & -0.69846 & 1.96538 \\
\hline $\mathrm{C}$ & 3.41815 & -1.42558 & 0.74181 \\
\hline $\mathrm{C}$ & 2.57269 & -2.60679 & 0.70039 \\
\hline $\mathrm{C}$ & 2.03746 & -3.04121 & -0.51486 \\
\hline $\mathrm{C}$ & 0.66036 & -3.50025 & -0.57983 \\
\hline $\mathrm{C}$ & 0.09693 & -3.03985 & -1.83886 \\
\hline $\mathrm{C}$ & 1.12397 & -2.30879 & -2.55491 \\
\hline C & 2.32602 & -2.30833 & -1.73798 \\
\hline C & 3.13931 & -1.17542 & -1.69785 \\
\hline $\mathrm{C}$ & 3.69859 & -0.72647 & -0.43344 \\
\hline $\mathrm{C}$ & 3.69858 & 0.72680 & -0.43306 \\
\hline $\mathrm{C}$ & 3.13927 & 1.17639 & -1.69725 \\
\hline $\mathrm{C}$ & 2.78770 & 0.00068 & -2.47697 \\
\hline C & 1.62690 & 0.00086 & -3.25504 \\
\hline $\mathrm{C}$ & 0.77385 & -1.17434 & -3.28720 \\
\hline $\mathrm{C}$ & -0.60947 & -0.72187 & -3.32474 \\
\hline $\mathrm{C}$ & -1.59883 & -1.40635 & -2.62271 \\
\hline $\mathrm{C}$ & -1.22385 & -2.59347 & -1.88213 \\
\hline C & -2.03394 & -2.62540 & -0.69255 \\
\hline $\mathrm{C}$ & -2.98555 & -1.54088 & -0.72164 \\
\hline $\mathrm{C}$ & -2.67045 & -0.70715 & -1.89643 \\
\hline $\mathrm{C}$ & -2.67055 & 0.70809 & -1.89614 \\
\hline $\mathrm{C}$ & -5.65208 & 0.00019 & -0.52162 \\
\hline $\mathrm{C}$ & -4.55722 & -0.00025 & 0.54325 \\
\hline
\end{tabular}




$\begin{array}{llll}\mathrm{H} & -5.02239 & -0.00044 & 1.53403 \\ \mathrm{H} & -6.28219 & -0.88771 & -0.40484 \\ \mathrm{H} & -5.25502 & 0.00123 & -1.53863 \\ \mathrm{H} & -6.28297 & 0.88733 & -0.40328\end{array}$

(5) Intermediate IM5 $\left(\mathrm{C}_{2} \mathrm{H}_{4} \mathrm{C}_{60}\right)$

\begin{tabular}{|c|c|c|c|}
\hline C & -3.01328 & 1.24182 & 0.00028 \\
\hline $\mathrm{C}$ & -3.01412 & 0.38772 & -1.17573 \\
\hline C & -3.01602 & -0.99459 & -0.72688 \\
\hline $\mathrm{C}$ & -3.01601 & -0.99491 & 0.72648 \\
\hline $\mathrm{C}$ & -3.01411 & 0.38721 & 1.17592 \\
\hline $\mathrm{C}$ & -2.28044 & 0.75274 & 2.30540 \\
\hline $\mathrm{C}$ & -1.51722 & -0.24899 & 3.03170 \\
\hline $\mathrm{C}$ & -1.51887 & -1.57604 & 2.59980 \\
\hline $\mathrm{C}$ & -2.28419 & -1.95692 & 1.42418 \\
\hline $\mathrm{C}$ & -1.52144 & -2.95836 & 0.69719 \\
\hline $\mathrm{C}$ & -1.52145 & -2.95806 & -0.69844 \\
\hline C & -2.28420 & -1.95631 & -1.42500 \\
\hline $\mathrm{C}$ & -1.51889 & -1.57492 & -2.60047 \\
\hline $\mathrm{C}$ & -1.51724 & -0.24769 & -3.03179 \\
\hline $\mathrm{C}$ & -2.28046 & 0.75373 & -2.30506 \\
\hline $\mathrm{C}$ & -1.51398 & 1.98884 & -2.30475 \\
\hline $\mathrm{C}$ & -0.27805 & 1.75128 & -3.03191 \\
\hline $\mathrm{C}$ & -0.27985 & 0.36857 & -3.48056 \\
\hline $\mathrm{C}$ & 0.90613 & -0.36702 & -3.48082 \\
\hline $\mathrm{C}$ & 0.90434 & -1.74979 & -3.03280 \\
\hline $\mathrm{C}$ & -0.28392 & -2.34133 & -2.60096 \\
\hline $\mathrm{C}$ & -0.28519 & -3.19593 & -1.42511 \\
\hline $\mathrm{C}$ & 0.90181 & -3.42484 & -0.72753 \\
\hline $\mathrm{C}$ & 0.90182 & -3.42515 & 0.72606 \\
\hline $\mathrm{C}$ & -0.28518 & -3.19654 & 1.42374 \\
\hline $\mathrm{C}$ & -0.28390 & -2.34244 & 2.59995 \\
\hline $\mathrm{C}$ & 0.90437 & -1.75109 & 3.03205 \\
\hline $\mathrm{C}$ & 0.90616 & -0.36851 & 3.48066 \\
\hline $\mathrm{C}$ & -0.27982 & 0.36707 & 3.48073 \\
\hline $\mathrm{C}$ & -0.27803 & 1.74998 & 3.03267 \\
\hline $\mathrm{C}$ & 0.91019 & 2.34166 & 2.60097 \\
\hline $\mathrm{C}$ & 2.14514 & 1.57506 & 2.60031 \\
\hline $\mathrm{C}$ & 2.14352 & 0.24783 & 3.03163 \\
\hline $\mathrm{C}$ & 2.90664 & -0.75343 & 2.30497 \\
\hline $\mathrm{C}$ & 2.14030 & -1.98851 & 2.30467 \\
\hline C & 2.13908 & -2.80845 & 1.17534 \\
\hline $\mathrm{C}$ & 2.90407 & -2.42764 & -0.00053 \\
\hline $\mathrm{C}$ & 2.13907 & -2.80795 & -1.17656 \\
\hline $\mathrm{C}$ & 2.14028 & -1.98752 & -2.30554 \\
\hline $\mathrm{C}$ & 2.90662 & -0.75244 & -2.30531 \\
\hline $\mathrm{C}$ & 2.14350 & 0.24913 & -3.03154 \\
\hline
\end{tabular}




$\begin{array}{lrrr}\mathrm{C} & 2.14512 & 1.57618 & -2.59965 \\ \mathrm{C} & 0.91017 & 2.34278 & -2.59997 \\ \mathrm{C} & 0.91140 & 3.19683 & -1.42386 \\ \mathrm{C} & 2.14759 & 2.95841 & -0.69717 \\ \mathrm{C} & 2.91011 & 1.95693 & -1.42406 \\ \mathrm{C} & 3.64302 & 0.99539 & -0.72657 \\ \mathrm{C} & 3.64116 & -0.38697 & -1.17608 \\ \mathrm{C} & 3.64010 & -1.24168 & -0.00028 \\ \mathrm{C} & 3.64117 & -0.38748 & 1.17589 \\ \mathrm{C} & 3.64302 & 0.99507 & 0.72698 \\ \mathrm{C} & 2.91012 & 1.95632 & 1.42488 \\ \mathrm{C} & 2.14760 & 2.95811 & 0.69842 \\ \mathrm{C} & 0.91141 & 3.19621 & 1.42523 \\ \mathrm{C} & -0.27558 & 3.42500 & 0.72759 \\ \mathrm{C} & -0.27559 & 3.42531 & -0.72612 \\ \mathrm{C} & -1.51285 & 2.80886 & -1.17543 \\ \mathrm{C} & -2.27802 & 2.42808 & 0.00053 \\ \mathrm{C} & -1.51284 & 2.80836 & 1.17665 \\ \mathrm{C} & -1.51396 & 1.98785 & 2.30561 \\ \mathrm{C} & -7.05010 & 0.66333 & 0.00000 \\ \mathrm{C} & -7.04218 & -0.66841 & 0.00001 \\ \mathrm{H} & -7.03977 & -1.24184 & -0.92398 \\ \mathrm{H} & -7.03989 & -1.24184 & 0.92399 \\ \mathrm{H} & -7.05414 & 1.23664 & -0.92404 \\ \mathrm{H} & -7.05426 & 1.23664 & 0.92405\end{array}$

(6) Intermediate IM6 $\left(\mathrm{C}_{2} \mathrm{H}_{4} \_2 \mathrm{H}_{-} \mathrm{C}_{60}\right)$

$\begin{array}{lrrr}C & -2.77932 & 1.05846 & 1.37531 \\ C & -2.14250 & 2.29598 & 1.14425 \\ C & -2.40951 & 2.81085 & -0.26617 \\ C & -3.19902 & 1.59268 & -0.97668 \\ C & -3.58501 & 0.58226 & 0.19207 \\ C & -3.45063 & -0.99759 & -0.10310 \\ C & -2.70777 & -1.39856 & -1.36218 \\ C & -2.32181 & -0.48891 & -2.31935 \\ C & -2.41513 & 0.95842 & -2.09826 \\ C & -1.32770 & 1.55115 & -2.70906 \\ C & -0.65182 & 2.68293 & -2.10546 \\ C & -1.08970 & 3.20068 & -0.90648 \\ C & 0.08832 & 3.55837 & -0.11212 \\ C & 0.12094 & 3.32945 & 1.25310 \\ C & -1.02800 & 2.66648 & 1.89237 \\ C & -0.52453 & 1.79601 & 2.90717 \\ C & 0.92564 & 1.87937 & 2.91563 \\ C & 1.32565 & 2.82317 & 1.88590 \\ C & 2.47271 & 2.57513 & 1.12563 \\ C & 2.44502 & 2.81718 & -0.30470\end{array}$




\begin{tabular}{|c|c|c|c|}
\hline C & 1.27727 & 3.30028 & -0.90617 \\
\hline $\mathrm{C}$ & 0.82256 & 2.74189 & -2.15589 \\
\hline $\mathrm{C}$ & 1.55299 & 1.73940 & -2.77219 \\
\hline $\mathrm{C}$ & 0.86739 & 0.59906 & -3.40404 \\
\hline $\mathrm{C}$ & -0.51411 & 0.51402 & -3.36775 \\
\hline C & -1.15361 & -0.75418 & -3.13687 \\
\hline $\mathrm{C}$ & -0.38373 & -1.90458 & -2.93664 \\
\hline C & -0.76437 & -2.83920 & -1.90036 \\
\hline $\mathrm{C}$ & -1.89525 & -2.56954 & -1.12468 \\
\hline $\mathrm{C}$ & -1.86946 & -2.80695 & 0.30470 \\
\hline C & -0.70916 & -3.30622 & 0.90914 \\
\hline C & 0.47445 & -3.56617 & 0.10396 \\
\hline $\mathrm{C}$ & 0.44798 & -3.33877 & -1.26991 \\
\hline $\mathrm{C}$ & 1.58022 & -2.70335 & -1.92700 \\
\hline $\mathrm{C}$ & 1.05938 & -1.81958 & -2.95744 \\
\hline C & 1.67503 & -0.58139 & -3.18781 \\
\hline $\mathrm{C}$ & 2.84056 & -0.19119 & -2.41613 \\
\hline $\mathrm{C}$ & 2.76928 & 1.24253 & -2.16961 \\
\hline $\mathrm{C}$ & 3.20853 & 1.76882 & -0.95013 \\
\hline C & 3.71540 & 0.87495 & 0.07971 \\
\hline $\mathrm{C}$ & 3.25497 & 1.37361 & 1.36593 \\
\hline $\mathrm{C}$ & 2.87136 & 0.47475 & 2.36267 \\
\hline $\mathrm{C}$ & 1.68462 & 0.73397 & 3.15468 \\
\hline $\mathrm{C}$ & 1.02398 & -0.54182 & 3.40354 \\
\hline $\mathrm{C}$ & 1.80930 & -1.58576 & 2.76784 \\
\hline $\mathrm{C}$ & 2.94262 & -0.96030 & 2.12027 \\
\hline $\mathrm{C}$ & 3.39226 & -1.43708 & 0.88534 \\
\hline $\mathrm{C}$ & 3.77890 & -0.49862 & -0.15531 \\
\hline $\mathrm{C}$ & 3.34047 & -1.04374 & -1.43113 \\
\hline $\mathrm{C}$ & 2.69400 & -2.32074 & -1.17856 \\
\hline $\mathrm{C}$ & 2.72542 & -2.56054 & 0.25671 \\
\hline C & 1.63743 & -3.17171 & 0.88634 \\
\hline $\mathrm{C}$ & 1.16800 & -2.67594 & 2.16540 \\
\hline $\mathrm{C}$ & -0.28156 & -2.76157 & 2.17659 \\
\hline $\mathrm{C}$ & -1.03728 & -1.74790 & 2.77421 \\
\hline $\mathrm{C}$ & -0.36729 & -0.62037 & 3.39896 \\
\hline $\mathrm{C}$ & -1.16242 & 0.56834 & 3.14562 \\
\hline $\mathrm{C}$ & -2.30405 & 0.20103 & 2.36736 \\
\hline $\mathrm{C}$ & -2.24305 & -1.25796 & 2.13923 \\
\hline $\mathrm{C}$ & -2.67120 & -1.79135 & 0.94228 \\
\hline $\mathrm{C}$ & -5.01466 & -1.09807 & -0.08256 \\
\hline $\mathrm{C}$ & -5.11742 & 0.35879 & 0.42558 \\
\hline $\mathrm{H}$ & -5.38642 & 0.43018 & 1.48350 \\
\hline $\mathrm{H}$ & -5.77848 & 1.02136 & -0.14275 \\
\hline $\mathrm{H}$ & -5.41991 & -1.87260 & 0.57404 \\
\hline $\mathrm{H}$ & -5.42497 & -1.24134 & -1.08651 \\
\hline $\mathrm{H}$ & -4.12750 & 2.00859 & -1.38544 \\
\hline $\mathrm{H}$ & -3.09227 & 3.67155 & -0.24520 \\
\hline
\end{tabular}


(7) Intermediate IM7 $\left(\mathrm{C}_{2} \mathrm{H}_{5} \_\mathrm{HC} 8 \_\mathrm{C}_{60}\right)$

\begin{tabular}{|c|c|c|c|}
\hline $\mathrm{C}$ & -3.53265 & -0.34725 & -0.37011 \\
\hline C & -2.77564 & -1.04923 & -1.51651 \\
\hline C & -2.20767 & -2.28835 & -1.05737 \\
\hline C & -2.33669 & -2.34226 & 0.38973 \\
\hline $\mathrm{C}$ & -2.98961 & -1.14376 & 0.83943 \\
\hline $\mathrm{C}$ & -2.57594 & -0.54897 & 2.01588 \\
\hline $\mathrm{C}$ & -1.53192 & -1.15254 & 2.81232 \\
\hline $\mathrm{C}$ & -0.92382 & -2.34186 & 2.39856 \\
\hline C & -1.33672 & -2.95459 & 1.15867 \\
\hline C & -0.15708 & -3.50176 & 0.50793 \\
\hline C & -0.03007 & -3.44812 & -0.87799 \\
\hline C & -1.07839 & -2.83528 & -1.68026 \\
\hline C & -0.45463 & -2.12360 & -2.77238 \\
\hline $\mathrm{C}$ & -0.99136 & -0.89875 & -3.18741 \\
\hline $\mathrm{C}$ & -2.16662 & -0.36072 & -2.54359 \\
\hline $\mathrm{C}$ & -2.01845 & 1.11050 & -2.48626 \\
\hline $\mathrm{C}$ & -0.77903 & 1.45110 & -3.05507 \\
\hline $\mathrm{C}$ & -0.11848 & 0.21480 & -3.49436 \\
\hline $\mathrm{C}$ & 1.25718 & 0.06246 & -3.38106 \\
\hline C & 1.81944 & -1.20096 & -2.94865 \\
\hline $\mathrm{C}$ & 0.98031 & -2.28154 & -2.65143 \\
\hline $\mathrm{C}$ & 1.24733 & -3.09883 & -1.47965 \\
\hline $\mathrm{C}$ & 2.34601 & -2.81753 & -0.66575 \\
\hline $\mathrm{C}$ & 2.21673 & -2.87984 & 0.78145 \\
\hline $\mathrm{C}$ & 0.98944 & -3.21804 & 1.35852 \\
\hline $\mathrm{C}$ & 0.51425 & -2.50104 & 2.52484 \\
\hline $\mathrm{C}$ & 1.28553 & -1.46830 & 3.07016 \\
\hline C & 0.64852 & -0.23689 & 3.51263 \\
\hline C & -0.73027 & -0.08280 & 3.38603 \\
\hline C & -1.29089 & 1.18869 & 2.94984 \\
\hline $\mathrm{C}$ & -0.43040 & 2.26061 & 2.64674 \\
\hline $\mathrm{C}$ & 0.99903 & 2.08845 & 2.75780 \\
\hline $\mathrm{C}$ & 1.53698 & 0.87099 & 3.19547 \\
\hline $\mathrm{C}$ & 2.71221 & 0.32855 & 2.55377 \\
\hline $\mathrm{C}$ & 2.55725 & -1.12130 & 2.47535 \\
\hline $\mathrm{C}$ & 3.01649 & -1.81301 & 1.35189 \\
\hline $\mathrm{C}$ & 3.64650 & -1.08683 & 0.25999 \\
\hline $\mathrm{C}$ & 3.22615 & -1.70662 & -0.98718 \\
\hline $\mathrm{C}$ & 2.96675 & -0.91437 & -2.10659 \\
\hline $\mathrm{C}$ & 3.13028 & 0.52985 & -2.02639 \\
\hline $\mathrm{C}$ & 2.07743 & 1.14167 & -2.80810 \\
\hline $\mathrm{C}$ & 1.48502 & 2.31954 & -2.38271 \\
\hline $\mathrm{C}$ & 0.02769 & 2.49943 & -2.48094 \\
\hline $\mathrm{C}$ & -0.40288 & 3.21594 & -1.37100 \\
\hline $\mathrm{C}$ & 0.73015 & 3.48441 & -0.51125 \\
\hline
\end{tabular}




$\begin{array}{lrrr}\mathrm{C} & 1.91869 & 2.94286 & -1.15177 \\ \mathrm{C} & 2.92406 & 2.35341 & -0.38406 \\ \mathrm{C} & 3.54746 & 1.12293 & -0.83131 \\ \mathrm{C} & 3.80197 & 0.29670 & 0.33701 \\ \mathrm{C} & 3.32789 & 1.02186 & 1.50680 \\ \mathrm{C} & 2.78591 & 2.28782 & 1.06047 \\ \mathrm{C} & 1.63918 & 2.81526 & 1.67141 \\ \mathrm{C} & 0.60429 & 3.43431 & 0.87486 \\ \mathrm{C} & -0.69398 & 3.08088 & 1.48540 \\ \mathrm{C} & -1.79231 & 2.82192 & 0.68864 \\ \mathrm{C} & -1.80701 & 3.08166 & -0.81992 \\ \mathrm{C} & -2.48658 & 1.79253 & -1.32562 \\ \mathrm{C} & -3.05269 & 1.09563 & -0.27561 \\ \mathrm{C} & -2.63234 & 1.69481 & 0.98096 \\ \mathrm{C} & -2.42848 & 0.91679 & 2.12426 \\ \mathrm{C} & -5.89723 & 0.14553 & 0.59428 \\ \mathrm{C} & -5.07995 & -0.46561 & -0.54665 \\ \mathrm{H} & -5.32135 & -1.53072 & -0.65109 \\ \mathrm{H} & -5.34270 & 0.01452 & -1.49753 \\ \mathrm{H} & -5.65671 & -0.32432 & 1.55414 \\ \mathrm{H} & -6.96783 & 0.00431 & 0.41063 \\ \mathrm{H} & -5.71358 & 1.22137 & 0.68983 \\ \mathrm{H} & -2.41596 & 3.96707 & -1.07071 \\ & & & \end{array}$

(8) Intermediate IM8 $\left(\mathrm{C}_{2} \mathrm{H}_{5} \_\mathrm{HC} 1 \_\mathrm{C}_{60}\right)$

$\begin{array}{lrrr}C & -3.57942 & -0.08803 & -0.43197 \\ C & -2.76373 & -0.14239 & -1.74914 \\ C & -2.21455 & -1.45933 & -1.95226 \\ C & -2.36741 & -2.21462 & -0.72341 \\ C & -3.02252 & -1.36913 & 0.24848 \\ C & -2.59086 & -1.41410 & 1.55953 \\ C & -1.54901 & -2.33599 & 1.96930 \\ C & -0.95461 & -3.19025 & 1.03243 \\ C & -1.37814 & -3.12958 & -0.34735 \\ C & -0.20312 & -3.32326 & -1.19296 \\ C & -0.07580 & -2.61582 & -2.38459 \\ C & -1.11156 & -1.67178 & -2.78359 \\ C & -0.47334 & -0.53788 & -3.41430 \\ C & -0.98054 & 0.74616 & -3.20557 \\ C & -2.13863 & 0.94805 & -2.34289 \\ C & -1.97977 & 2.20680 & -1.66169 \\ C & -0.72695 & 2.74603 & -2.00079 \\ C & -0.08968 & 1.85279 & -2.98149 \\ C & 1.28646 & 1.64720 & -2.96314 \\ C & 1.82186 & 0.31210 & -3.17303 \\ C & 0.95481 & -0.76325 & -3.40321 \\ C & 1.20550 & -2.04671 & -2.76368\end{array}$




\begin{tabular}{|c|c|c|c|}
\hline $\mathrm{C}$ & 2.31026 & -2.20095 & -1.92483 \\
\hline $\mathrm{C}$ & 2.17718 & -2.93768 & -0.67733 \\
\hline $\mathrm{C}$ & 0.94456 & -3.49032 & -0.32185 \\
\hline $\mathrm{C}$ & 0.47749 & -3.40530 & 1.05417 \\
\hline $\mathrm{C}$ & 1.26550 & -2.75747 & 2.01278 \\
\hline $\mathrm{C}$ & 0.64389 & -1.87152 & 2.98352 \\
\hline $\mathrm{C}$ & -0.73179 & -1.66500 & 2.95295 \\
\hline $\mathrm{C}$ & -1.27795 & -0.31729 & 3.14815 \\
\hline $\mathrm{C}$ & -0.38420 & 0.76902 & 3.38344 \\
\hline $\mathrm{C}$ & 1.04312 & 0.53285 & 3.42216 \\
\hline $\mathrm{C}$ & 1.55144 & -0.75453 & 3.22278 \\
\hline $\mathrm{C}$ & 2.71848 & -0.95230 & 2.39105 \\
\hline $\mathrm{C}$ & 2.54734 & -2.19175 & 1.64606 \\
\hline $\mathrm{C}$ & 2.99330 & -2.27410 & 0.32329 \\
\hline $\mathrm{C}$ & 3.63828 & -1.13522 & -0.30492 \\
\hline $\mathrm{C}$ & 3.20928 & -1.08380 & -1.69141 \\
\hline $\mathrm{C}$ & 2.97159 & 0.14699 & -2.30868 \\
\hline $\mathrm{C}$ & 3.14459 & 1.38550 & -1.55942 \\
\hline $\mathrm{C}$ & 2.11455 & 2.31750 & -1.97002 \\
\hline $\mathrm{C}$ & 1.51650 & 3.16377 & -1.02699 \\
\hline $\mathrm{C}$ & 0.09062 & 3.40707 & -1.03900 \\
\hline $\mathrm{C}$ & -0.36594 & 3.49417 & 0.32761 \\
\hline $\mathrm{C}$ & 0.78506 & 3.31178 & 1.20035 \\
\hline $\mathrm{C}$ & 1.95478 & 3.11406 & 0.35972 \\
\hline $\mathrm{C}$ & 2.95067 & 2.22241 & 0.75240 \\
\hline $\mathrm{C}$ & 3.55892 & 1.33537 & -0.22666 \\
\hline $\mathrm{C}$ & 3.80138 & 0.05413 & 0.41195 \\
\hline $\mathrm{C}$ & 3.33598 & 0.14635 & 1.78384 \\
\hline $\mathrm{C}$ & 2.81810 & 1.48653 & 2.00061 \\
\hline $\mathrm{C}$ & 1.69805 & 1.67685 & 2.80692 \\
\hline $\mathrm{C}$ & 0.66321 & 2.61556 & 2.40048 \\
\hline $\mathrm{C}$ & -0.61623 & 2.03894 & 2.76393 \\
\hline $\mathrm{C}$ & -1.70512 & 2.19319 & 1.88197 \\
\hline $\mathrm{C}$ & -1.60149 & 2.95589 & 0.70081 \\
\hline $\mathrm{C}$ & -2.61832 & 2.42187 & -0.31776 \\
\hline $\mathrm{C}$ & -3.11679 & 1.13573 & 0.34459 \\
\hline $\mathrm{C}$ & -2.62094 & 1.07160 & 1.63111 \\
\hline $\mathrm{C}$ & -2.40728 & -0.16852 & 2.31810 \\
\hline $\mathrm{C}$ & -5.97338 & -0.18335 & 0.56843 \\
\hline $\mathrm{C}$ & -5.11444 & -0.14107 & -0.69795 \\
\hline $\mathrm{H}$ & -5.31549 & -1.02069 & -1.32282 \\
\hline $\mathrm{H}$ & -5.37660 & 0.73713 & -1.30230 \\
\hline $\mathrm{H}$ & -5.75335 & -1.07213 & 1.16990 \\
\hline $\mathrm{H}$ & -7.03677 & -0.20883 & 0.30760 \\
\hline $\mathrm{H}$ & -5.80160 & 0.69789 & 1.19601 \\
\hline $\mathrm{H}$ & -3.47160 & 3.12274 & -0.41465 \\
\hline
\end{tabular}


(9) Intermediate IM9 $\left(\mathrm{C}_{2} \mathrm{H}_{6} \mathrm{C}_{60}\right)$

$\begin{array}{lrrr}\text { C } & -3.01564 & -0.96625 & -0.51740 \\ \mathrm{C} & -2.40598 & -1.32343 & -1.78772 \\ \mathrm{C} & -1.59237 & -2.50929 & -1.57797 \\ \mathrm{C} & -1.69870 & -2.88530 & -0.17809 \\ \mathrm{C} & -2.57805 & -1.93162 & 0.47711 \\ \mathrm{C} & -2.30862 & -1.51643 & 1.78193 \\ \mathrm{C} & -1.14800 & -2.03728 & 2.48527 \\ \mathrm{C} & -0.30327 & -2.95284 & 1.85586 \\ \mathrm{C} & -0.58439 & -3.38519 & 0.49710 \\ \mathrm{C} & 0.68312 & -3.52987 & -0.19965 \\ \mathrm{C} & 0.78552 & -3.16878 & -1.54363 \\ \mathrm{C} & -0.37522 & -2.64784 & -2.24679 \\ \mathrm{C} & 0.07894 & -1.60617 & -3.15303 \\ \mathrm{C} & -0.70207 & -0.46736 & -3.35461 \\ \mathrm{C} & -1.96944 & -0.32286 & -2.65767 \\ \mathrm{C} & -2.12539 & 1.07587 & -2.29404 \\ \mathrm{C} & -0.95393 & 1.79582 & -2.76575 \\ \mathrm{C} & -0.07420 & 0.84221 & -3.42106 \\ \mathrm{C} & 1.30959 & 0.96032 & -3.28386 \\ \mathrm{C} & 2.12300 & -0.22607 & -3.07402 \\ \mathrm{C} & 1.51999 & -1.48304 & -3.00959 \\ \mathrm{C} & 1.95669 & -2.44841 & -2.01499 \\ \mathrm{C} & 2.97930 & -2.11884 & -1.12429 \\ \mathrm{C} & 2.87244 & -2.49527 & 0.27559 \\ \mathrm{C} & 1.74773 & -3.18656 & 0.72844 \\ \mathrm{C} & 1.13800 & -2.82979 & 1.99874 \\ \mathrm{C} & 1.67752 & -1.79603 & 2.76576 \\ \mathrm{C} & 0.79778 & -0.84246 & 3.42098 \\ \mathrm{C} & -0.58602 & -0.96067 & 3.28380 \\ \mathrm{C} & -1.39940 & 0.22571 & 3.07399 \\ \mathrm{C} & -0.79625 & 1.48267 & 3.00936 \\ \mathrm{C} & 0.64477 & 1.60595 & 3.15295 \\ \mathrm{C} & 1.42571 & 0.46709 & 3.35462 \\ \mathrm{C} & 2.69305 & 0.32252 & 2.65773 \\ \mathrm{C} & 2.84910 & -1.07605 & 2.29412 \\ \mathrm{C} & 3.43459 & -1.41881 & 1.07433 \\ \mathrm{C} & 3.88885 & -0.37708 & 0.16792 \\ \mathrm{C} & 3.60771 & -0.80994 & -1.19122 \\ \mathrm{C} & 3.18756 & 0.11750 & -2.14616 \\ \mathrm{C} & 3.03235 & 1.51627 & -1.78225 \\ \mathrm{C} & 1.87165 & 2.03703 & -2.48542 \\ \mathrm{C} & 1.02691 & 2.95265 & -1.85593 \\ \mathrm{C} & -0.41435 & 2.82955 & -1.99875 \\ \mathrm{C} & -1.02399 & 3.18627 & -0.72842 \\ \mathrm{C} & 0.04059 & 3.52971 & 0.19967 \\ \mathrm{C} & 1.30806 & 3.38506 & -0.49710 \\ \mathrm{C} & 2.42246 & 2.88529 & 0.17799\end{array}$




$\begin{array}{lrrr}\mathrm{C} & 3.30195 & 1.93153 & -0.47744 \\ \mathrm{C} & 3.73867 & 0.96576 & 0.51724 \\ \mathrm{C} & 3.12946 & 1.32283 & 1.78779 \\ \mathrm{C} & 2.31602 & 2.50904 & 1.57806 \\ \mathrm{C} & 1.09896 & 2.64769 & 2.24691 \\ \mathrm{C} & -0.06178 & 3.16853 & 1.54364 \\ \mathrm{C} & -1.23299 & 2.44816 & 2.01495 \\ \mathrm{C} & -2.25561 & 2.11854 & 1.12425 \\ \mathrm{C} & -2.14871 & 2.49499 & -0.27557 \\ \mathrm{C} & -2.71087 & 1.41856 & -1.07423 \\ \mathrm{C} & -3.16502 & 0.37703 & -0.16821 \\ \mathrm{C} & -2.88462 & 0.80987 & 1.19141 \\ \mathrm{C} & -2.46385 & -0.11789 & 2.14607 \\ \mathrm{C} & -7.25843 & -0.76254 & -0.01178 \\ \mathrm{C} & -7.21506 & 0.76732 & 0.01304 \\ \mathrm{H} & -6.40122 & 1.13166 & 0.65001 \\ \mathrm{H} & -8.15119 & 1.18909 & 0.39719 \\ \mathrm{H} & -7.05622 & 1.17650 & -0.99129 \\ \mathrm{H} & -7.42912 & -1.17363 & 0.98994 \\ \mathrm{H} & -6.31519 & -1.17824 & -0.38481 \\ \mathrm{H} & -8.06179 & -1.12975 & -0.66096\end{array}$

Intermediate structures observed over impure fullerene during acetylene hydrogenation.

\section{For Palladium doped fullerene}

(0) Catalyst $\mathrm{C}_{59} \mathrm{Pd}$

$\begin{array}{rrrr}\text { Pd } & -4.09506 & -0.01574 & 0.00000 \\ \text { C } & -2.85450 & -0.97039 & -1.25504 \\ \text { C } & -2.29347 & -2.18904 & -0.72881 \\ \text { C } & -2.29347 & -2.18904 & 0.72882 \\ \text { C } & -2.85449 & -0.97038 & 1.25505 \\ \text { C } & -2.21468 & -0.25407 & 2.24904 \\ \text { C } & -1.13594 & -0.89271 & 2.97032 \\ \text { C } & -0.66125 & -2.14940 & 2.57456 \\ \text { C } & -1.23190 & -2.79974 & 1.42094 \\ \text { C } & -0.15085 & -3.44798 & 0.69546 \\ \text { C } & -0.15085 & -3.44798 & -0.69545 \\ \text { C } & -1.23190 & -2.79975 & -1.42093 \\ \text { C } & -0.66125 & -2.14941 & -2.57455 \\ \text { C } & -1.13595 & -0.89272 & -2.97032 \\ \text { C } & -2.21468 & -0.25407 & -2.24904 \\ \text { C } & -1.95759 & 1.21399 & -2.24596 \\ \text { C } & -0.71749 & 1.41665 & -2.95829\end{array}$




\begin{tabular}{|c|c|c|c|}
\hline $\mathrm{C}$ & -0.21604 & 0.13221 & -3.41467 \\
\hline $\mathrm{C}$ & 1.15317 & -0.12596 & -3.45237 \\
\hline $\mathrm{C}$ & 1.65466 & -1.42175 & -3.01761 \\
\hline $\mathrm{C}$ & 0.76615 & -2.41264 & -2.59201 \\
\hline $\mathrm{C}$ & 1.08431 & -3.21428 & -1.42329 \\
\hline $\mathrm{C}$ & 2.27389 & -2.99271 & -0.72717 \\
\hline $\mathrm{C}$ & 2.27389 & -2.99271 & 0.72718 \\
\hline $\mathrm{C}$ & 1.08431 & -3.21428 & 1.42331 \\
\hline $\mathrm{C}$ & 0.76616 & -2.41263 & 2.59201 \\
\hline $\mathrm{C}$ & 1.65466 & -1.42174 & 3.01761 \\
\hline $\mathrm{C}$ & 1.15317 & -0.12595 & 3.45237 \\
\hline $\mathrm{C}$ & -0.21604 & 0.13222 & 3.41467 \\
\hline $\mathrm{C}$ & -0.71749 & 1.41666 & 2.95828 \\
\hline $\mathrm{C}$ & 0.19735 & 2.41375 & 2.58396 \\
\hline $\mathrm{C}$ & 1.61860 & 2.13556 & 2.59264 \\
\hline $\mathrm{C}$ & 2.09401 & 0.89336 & 3.02374 \\
\hline $\mathrm{C}$ & 3.16316 & 0.23355 & 2.30252 \\
\hline $\mathrm{C}$ & 2.89434 & -1.19840 & 2.30224 \\
\hline $\mathrm{C}$ & 3.19788 & -1.96601 & 1.17465 \\
\hline $\mathrm{C}$ & 3.77421 & -1.33484 & 0.00000 \\
\hline $\mathrm{C}$ & 3.19788 & -1.96601 & -1.17465 \\
\hline $\mathrm{C}$ & 2.89434 & -1.19841 & -2.30224 \\
\hline $\mathrm{C}$ & 3.16316 & 0.23354 & -2.30253 \\
\hline $\mathrm{C}$ & 2.09401 & 0.89335 & -3.02374 \\
\hline $\mathrm{C}$ & 1.61860 & 2.13555 & -2.59265 \\
\hline $\mathrm{C}$ & 0.19735 & 2.41375 & -2.58397 \\
\hline $\mathrm{C}$ & -0.09119 & 3.22232 & -1.42485 \\
\hline $\mathrm{C}$ & 1.14866 & 3.44535 & -0.69764 \\
\hline $\mathrm{C}$ & 2.21057 & 2.77148 & -1.42467 \\
\hline $\mathrm{C}$ & 3.23663 & 2.13096 & -0.72764 \\
\hline $\mathrm{C}$ & 3.72517 & 0.83799 & -1.17527 \\
\hline $\mathrm{C}$ & 4.02726 & 0.03929 & -0.00000 \\
\hline $\mathrm{C}$ & 3.72517 & 0.83799 & 1.17527 \\
\hline $\mathrm{C}$ & 3.23663 & 2.13096 & 0.72763 \\
\hline $\mathrm{C}$ & 2.21057 & 2.77148 & 1.42466 \\
\hline $\mathrm{C}$ & 1.14867 & 3.44536 & 0.69763 \\
\hline $\mathrm{C}$ & -0.09119 & 3.22233 & 1.42484 \\
\hline $\mathrm{C}$ & -1.27612 & 3.01018 & 0.72324 \\
\hline $\mathrm{C}$ & -1.27612 & 3.01017 & -0.72325 \\
\hline $\mathrm{C}$ & -2.26542 & 2.02933 & -1.15054 \\
\hline $\mathrm{C}$ & -3.11023 & 1.71301 & -0.00000 \\
\hline $\mathrm{C}$ & -2.26542 & 2.02934 & 1.15053 \\
\hline $\mathrm{C}$ & -1.95759 & 1.21400 & 2.24596 \\
\hline
\end{tabular}

(1) Intermediate IM1 $\left(\mathrm{C}_{2} \mathrm{H}_{2} \mathrm{C}_{59} \mathrm{Pd}\right)$

$\begin{array}{cccc}\text { Pd } & -3.93027 & -0.58408 & -0.06245 \\ \text { C } & -2.50719 & -1.38749 & -1.29897\end{array}$




\begin{tabular}{|c|c|c|c|}
\hline $\mathrm{C}$ & -1.78313 & -2.51904 & -0.76625 \\
\hline $\mathrm{C}$ & -1.82239 & -2.50256 & 0.69136 \\
\hline $\mathrm{C}$ & -2.56254 & -1.36553 & 1.18556 \\
\hline $\mathrm{C}$ & -2.04885 & -0.56846 & 2.18431 \\
\hline $\mathrm{C}$ & -0.90458 & -1.04058 & 2.92875 \\
\hline $\mathrm{C}$ & -0.26226 & -2.23362 & 2.57912 \\
\hline $\mathrm{C}$ & -0.71255 & -2.96426 & 1.41954 \\
\hline $\mathrm{C}$ & 0.46043 & -3.48181 & 0.73539 \\
\hline $\mathrm{C}$ & 0.49836 & -3.50014 & -0.65623 \\
\hline $\mathrm{C}$ & -0.63536 & -3.00038 & -1.41394 \\
\hline $\mathrm{C}$ & -0.12472 & -2.29762 & -2.56608 \\
\hline $\mathrm{C}$ & -0.74982 & -1.11549 & -2.97872 \\
\hline $\mathrm{C}$ & -1.93479 & -0.63033 & -2.30473 \\
\hline $\mathrm{C}$ & -1.85236 & 0.87199 & -2.30579 \\
\hline $\mathrm{C}$ & -0.62158 & 1.22209 & -2.99468 \\
\hline $\mathrm{C}$ & 0.04779 & 0.00866 & -3.41566 \\
\hline $\mathrm{C}$ & 1.43946 & -0.07296 & -3.42734 \\
\hline $\mathrm{C}$ & 2.09273 & -1.28657 & -2.96500 \\
\hline $\mathrm{C}$ & 1.32426 & -2.37432 & -2.54288 \\
\hline $\mathrm{C}$ & 1.71263 & -3.11785 & -1.35602 \\
\hline $\mathrm{C}$ & 2.84571 & -2.73779 & -0.63379 \\
\hline $\mathrm{C}$ & 2.80627 & -2.71950 & 0.82035 \\
\hline $\mathrm{C}$ & 1.63528 & -3.08173 & 1.48949 \\
\hline $\mathrm{C}$ & 1.18543 & -2.30971 & 2.63563 \\
\hline $\mathrm{C}$ & 1.93037 & -1.21025 & 3.07036 \\
\hline $\mathrm{C}$ & 1.25481 & 0.01509 & 3.46782 \\
\hline $\mathrm{C}$ & -0.13370 & 0.09660 & 3.38124 \\
\hline $\mathrm{C}$ & -0.77937 & 1.29834 & 2.89388 \\
\hline $\mathrm{C}$ & 0.00788 & 2.39655 & 2.52792 \\
\hline $\mathrm{C}$ & 1.45591 & 2.30932 & 2.58659 \\
\hline $\mathrm{C}$ & 2.06900 & 1.14163 & 3.04503 \\
\hline $\mathrm{C}$ & 3.23563 & 0.61489 & 2.36604 \\
\hline $\mathrm{C}$ & 3.14919 & -0.83936 & 2.38148 \\
\hline $\mathrm{C}$ & 3.57856 & -1.57709 & 1.27484 \\
\hline $\mathrm{C}$ & 4.09829 & -0.88997 & 0.10480 \\
\hline $\mathrm{C}$ & 3.64229 & -1.60665 & -1.07451 \\
\hline $\mathrm{C}$ & 3.27367 & -0.89764 & -2.22038 \\
\hline $\mathrm{C}$ & 3.35930 & 0.55668 & -2.23592 \\
\hline $\mathrm{C}$ & 2.23015 & 1.06413 & -2.98874 \\
\hline $\mathrm{C}$ & 1.59463 & 2.24347 & -2.59454 \\
\hline $\mathrm{C}$ & 0.14561 & 2.32984 & -2.61464 \\
\hline $\mathrm{C}$ & -0.27111 & 3.11950 & -1.48326 \\
\hline $\mathrm{C}$ & 0.91242 & 3.50420 & -0.73276 \\
\hline $\mathrm{C}$ & 2.06954 & 2.96434 & -1.42346 \\
\hline $\mathrm{C}$ & 3.15032 & 2.46853 & -0.69040 \\
\hline $\mathrm{C}$ & 3.80874 & 1.24268 & -1.10508 \\
\hline $\mathrm{C}$ & 4.18076 & 0.50397 & 0.08976 \\
\hline $\mathrm{C}$ & 3.74617 & 1.27215 & 1.24414 \\
\hline $\mathrm{C}$ & 3.11134 & 2.48691 & 0.76371 \\
\hline
\end{tabular}




$\begin{array}{lrrr}\mathrm{C} & 1.99320 & 3.00061 & 1.42495 \\ \mathrm{C} & 0.87434 & 3.52194 & 0.66030 \\ \mathrm{C} & -0.34836 & 3.15256 & 1.35526 \\ \mathrm{C} & -1.48901 & 2.78958 & 0.63772 \\ \mathrm{C} & -1.44695 & 2.77379 & -0.81939 \\ \mathrm{C} & -2.29997 & 1.70176 & -1.28594 \\ \mathrm{C} & -3.21165 & 1.36322 & -0.13180 \\ \mathrm{C} & -2.35944 & 1.71935 & 1.07730 \\ \mathrm{C} & -1.96962 & 0.92966 & 2.14712 \\ \mathrm{C} & -4.66112 & 1.75286 & -0.13536 \\ \mathrm{C} & -5.38447 & 0.78974 & 0.49137 \\ \mathrm{H} & -4.99672 & 2.64500 & -0.67509 \\ \mathrm{H} & -6.47251 & 0.83483 & 0.55471\end{array}$

(2) Intermediate IM2 $\left(\mathrm{C}_{2} \mathrm{H}_{2} \_2 \mathrm{H}_{-} \mathrm{C}_{59} \mathrm{Pd}\right)$

$\begin{array}{cccc}\text { Pd } & -4.00694 & -0.45491 & -0.16908 \\ \mathrm{C} & -2.54695 & -1.02106 & -1.47982 \\ \mathrm{C} & -1.85228 & -2.23968 & -1.11414 \\ \mathrm{C} & -1.91494 & -2.48190 & 0.29246 \\ \mathrm{C} & -2.83982 & -1.56904 & 1.09021 \\ \mathrm{C} & -2.05863 & -0.72743 & 2.06691 \\ \mathrm{C} & -0.96842 & -1.37927 & 2.76392 \\ \mathrm{C} & -0.36852 & -2.51888 & 2.20772 \\ \mathrm{C} & -0.84183 & -3.08039 & 0.94308 \\ \mathrm{C} & 0.31833 & -3.52897 & 0.21142 \\ \mathrm{C} & 0.37533 & -3.35543 & -1.16880 \\ \mathrm{C} & -0.71706 & -2.68196 & -1.83192 \\ \mathrm{C} & -0.16894 & -1.84798 & -2.86277 \\ \mathrm{C} & -0.73489 & -0.58172 & -3.07585 \\ \mathrm{C} & -1.90916 & -0.14221 & -2.35688 \\ \mathrm{C} & -1.76103 & 1.34240 & -2.16220 \\ \mathrm{C} & -0.48998 & 1.71876 & -2.78529 \\ \mathrm{C} & 0.12230 & 0.54621 & -3.36646 \\ \mathrm{C} & 1.51002 & 0.39148 & -3.39319 \\ \mathrm{C} & 2.09906 & -0.90806 & -3.11457 \\ \mathrm{C} & 1.27429 & -2.00370 & -2.85953 \\ \mathrm{C} & 1.61534 & -2.93653 & -1.79676 \\ \mathrm{C} & 2.75474 & -2.72454 & -1.01884 \\ \mathrm{C} & 2.69455 & -2.91463 & 0.42360 \\ \mathrm{C} & 1.50051 & -3.30369 & 1.02635 \\ \mathrm{C} & 1.07175 & -2.68717 & 2.26985 \\ \mathrm{C} & 1.86554 & -1.70368 & 2.86558 \\ \mathrm{C} & 1.24973 & -0.52144 & 3.44512 \\ \mathrm{C} & -0.13608 & -0.36384 & 3.37357 \\ \mathrm{C} & -0.71579 & 0.91375 & 3.04131 \\ \mathrm{C} & 0.13026 & 1.99458 & 2.82721 \\ \mathrm{C} & 1.56919 & 1.85133 & 2.89816\end{array}$




$\begin{array}{lrrr}\mathrm{C} & 2.12378 & 0.60407 & 3.19256 \\ \mathrm{C} & 3.27779 & 0.12771 & 2.44921 \\ \mathrm{C} & 3.11904 & -1.30461 & 2.25042 \\ \mathrm{C} & 3.51692 & -1.88921 & 1.04668 \\ \mathrm{C} & 4.08773 & -1.06953 & -0.00856 \\ \mathrm{C} & 3.60760 & -1.58081 & -1.28102 \\ \mathrm{C} & 3.29011 & -0.69327 & -2.31564 \\ \mathrm{C} & 3.44879 & 0.73724 & -2.11614 \\ \mathrm{C} & 2.35680 & 1.40821 & -2.79733 \\ \mathrm{C} & 1.77674 & 2.54735 & -2.24026 \\ \mathrm{C} & 0.33406 & 2.72315 & -2.26376 \\ \mathrm{C} & -0.05256 & 3.36137 & -1.03153 \\ \mathrm{C} & 1.13527 & 3.55244 & -0.22197 \\ \mathrm{C} & 2.27432 & 3.06370 & -0.97219 \\ \mathrm{C} & 3.31601 & 2.41120 & -0.30856 \\ \mathrm{C} & 3.91899 & 1.22698 & -0.89299 \\ \mathrm{C} & 4.23815 & 0.30558 & 0.18143 \\ \mathrm{C} & 3.82540 & 0.91552 & 1.43615 \\ \mathrm{C} & 3.25676 & 2.21924 & 1.13430 \\ \mathrm{C} & 2.15557 & 2.68275 & 1.85507 \\ \mathrm{C} & 1.07721 & 3.36473 & 1.16193 \\ \mathrm{C} & -0.16566 & 2.92861 & 1.75583 \\ \mathrm{C} & -1.29231 & 2.73027 & 0.97487 \\ \mathrm{C} & -1.25886 & 2.98972 & -0.44344 \\ \mathrm{C} & -2.20366 & 2.06891 & -1.05605 \\ \mathrm{C} & -3.10804 & 1.55510 & 0.03211 \\ \mathrm{C} & -2.25771 & 1.65783 & 1.21659 \\ \mathrm{C} & -1.91913 & 0.68702 & 2.21184 \\ \mathrm{C} & -4.59633 & 1.81455 & 0.06637 \\ \mathrm{C} & -5.36271 & 0.84183 & 0.61595 \\ \mathrm{H} & -4.94422 & 2.73883 & -0.41118 \\ \mathrm{H} & -6.43022 & 0.90786 & 0.80203 \\ \mathrm{H} & -5.01382 & -1.69289 & -0.21328 \\ & -3.57258 & -2.16543 & 1.65246\end{array}$

(3) Intermediate IM3 $\left(\mathrm{C}_{2} \mathrm{H}_{3} \_\mathrm{HC} 1 \_\mathrm{C}_{59} \mathrm{Pd}\right)$

$\begin{array}{cccc}\text { Pd } & -4.16840 & -0.46905 & -0.37848 \\ \text { C } & -2.55967 & -1.09261 & -1.40928 \\ \text { C } & -1.78772 & -2.29029 & -1.08776 \\ \text { C } & -1.72469 & -2.69667 & 0.34026 \\ \text { C } & -2.52466 & -1.92772 & 1.28231 \\ \text { C } & -1.86527 & -1.20017 & 2.26529 \\ \text { C } & -0.61196 & -1.72265 & 2.73807 \\ \text { C } & 0.01083 & -2.79205 & 2.09833 \\ \text { C } & -0.52163 & -3.23265 & 0.82588 \\ \text { C } & 0.60364 & -3.54013 & -0.02449 \\ \text { C } & 0.53174 & -3.23603 & -1.37457\end{array}$




\begin{tabular}{|c|c|c|c|}
\hline $\mathrm{C}$ & -0.65898 & -2.58596 & -1.89731 \\
\hline $\mathrm{C}$ & -0.21619 & -1.66774 & -2.92086 \\
\hline $\mathrm{C}$ & -0.86014 & -0.43740 & -3.06012 \\
\hline $\mathrm{C}$ & -2.01289 & -0.14002 & -2.26328 \\
\hline $\mathrm{C}$ & -1.94531 & 1.33526 & -1.94769 \\
\hline $\mathrm{C}$ & -0.75431 & 1.85204 & -2.60061 \\
\hline $\mathrm{C}$ & -0.09797 & 0.76972 & -3.30275 \\
\hline $\mathrm{C}$ & 1.28938 & 0.71501 & -3.40278 \\
\hline $\mathrm{C}$ & 1.97014 & -0.56098 & -3.24937 \\
\hline $\mathrm{C}$ & 1.23029 & -1.72493 & -3.01808 \\
\hline $\mathrm{C}$ & 1.69850 & -2.69927 & -2.05320 \\
\hline $\mathrm{C}$ & 2.88199 & -2.47329 & -1.34630 \\
\hline $\mathrm{C}$ & 2.94840 & -2.78227 & 0.07381 \\
\hline $\mathrm{C}$ & 1.83079 & -3.31003 & 0.71906 \\
\hline $\mathrm{C}$ & 1.45848 & -2.83506 & 2.03873 \\
\hline $\mathrm{C}$ & 2.21581 & -1.83675 & 2.65845 \\
\hline $\mathrm{C}$ & 1.55137 & -0.74681 & 3.35522 \\
\hline $\mathrm{C}$ & 0.15820 & -0.68723 & 3.36521 \\
\hline $\mathrm{C}$ & -0.55983 & 0.55425 & 3.17809 \\
\hline $\mathrm{C}$ & 0.19083 & 1.71925 & 3.00476 \\
\hline $\mathrm{C}$ & 1.64338 & 1.67765 & 2.97437 \\
\hline $\mathrm{C}$ & 2.31509 & 0.46505 & 3.13740 \\
\hline $\mathrm{C}$ & 3.44540 & 0.13524 & 2.29119 \\
\hline $\mathrm{C}$ & 3.38037 & -1.28881 & 1.99055 \\
\hline $\mathrm{C}$ & 3.74098 & -1.75215 & 0.72243 \\
\hline $\mathrm{C}$ & 4.17042 & -0.80995 & -0.29820 \\
\hline $\mathrm{C}$ & 3.64227 & -1.25853 & -1.57580 \\
\hline $\mathrm{C}$ & 3.19421 & -0.32184 & -2.51182 \\
\hline $\mathrm{C}$ & 3.27185 & 1.10132 & -2.21167 \\
\hline $\mathrm{C}$ & 2.09775 & 1.74257 & -2.76981 \\
\hline $\mathrm{C}$ & 1.47732 & 2.79243 & -2.08915 \\
\hline $\mathrm{C}$ & 0.02732 & 2.85761 & -2.01523 \\
\hline $\mathrm{C}$ & -0.32827 & 3.35140 & -0.70871 \\
\hline $\mathrm{C}$ & 0.89067 & 3.57632 & 0.04409 \\
\hline $\mathrm{C}$ & 2.01300 & 3.23936 & -0.81200 \\
\hline $\mathrm{C}$ & 3.13932 & 2.61740 & -0.26885 \\
\hline $\mathrm{C}$ & 3.78405 & 1.52976 & -0.98420 \\
\hline $\mathrm{C}$ & 4.23769 & 0.55444 & -0.00810 \\
\hline $\mathrm{C}$ & 3.86809 & 1.03701 & 1.31244 \\
\hline $\mathrm{C}$ & 3.18615 & 2.30980 & 1.15245 \\
\hline $\mathrm{C}$ & 2.09729 & 2.62644 & 1.97062 \\
\hline $\mathrm{C}$ & 0.92759 & 3.26971 & 1.40230 \\
\hline $\mathrm{C}$ & -0.24579 & 2.69916 & 2.04707 \\
\hline $\mathrm{C}$ & -1.40677 & 2.45140 & 1.32299 \\
\hline $\mathrm{C}$ & -1.46032 & 2.82849 & -0.08236 \\
\hline $\mathrm{C}$ & -2.34111 & 1.90449 & -0.74394 \\
\hline $\mathrm{C}$ & -3.16620 & 1.24054 & 0.33211 \\
\hline $\mathrm{C}$ & -2.24785 & 1.27489 & 1.54643 \\
\hline $\mathrm{C}$ & -1.81603 & 0.29581 & 2.45581 \\
\hline
\end{tabular}




$\begin{array}{llll}\mathrm{C} & -4.60489 & 1.53971 & 0.39643 \\ \mathrm{C} & -5.47065 & 0.87889 & 1.23716 \\ \mathrm{H} & -5.00772 & 2.18574 & -0.38220 \\ \mathrm{H} & -6.54015 & 1.04679 & 1.16289 \\ \mathrm{H} & -5.11625 & 0.33819 & 2.11079 \\ \mathrm{H} & -3.57883 & -1.74906 & 1.07264\end{array}$

(4) Intermediate IM4 ( $\left.\mathrm{CHCH}_{3} \mathrm{C}_{59} \mathrm{Pd}\right)$

$\begin{array}{cccc}\text { Pd } & -3.82882 & -0.86933 & -0.10246 \\ \mathrm{C} & -2.38477 & -1.73278 & -1.17108 \\ \mathrm{C} & -1.59533 & -2.74878 & -0.50737 \\ \mathrm{C} & -1.66022 & -2.57881 & 0.93548 \\ \mathrm{C} & -2.49586 & -1.44470 & 1.27272 \\ \mathrm{C} & -2.03467 & -0.48958 & 2.16920 \\ \mathrm{C} & -0.87451 & -0.80667 & 2.98000 \\ \mathrm{C} & -0.15275 & -1.99048 & 2.78247 \\ \mathrm{C} & -0.53214 & -2.87827 & 1.71211 \\ \mathrm{C} & 0.68182 & -3.39268 & 1.10346 \\ \mathrm{C} & 0.74370 & -3.55423 & -0.27819 \\ \mathrm{C} & -0.40686 & -3.20723 & -1.09310 \\ \mathrm{C} & 0.07415 & -2.58526 & -2.30122 \\ \mathrm{C} & -0.61515 & -1.48606 & -2.82908 \\ \mathrm{C} & -1.83800 & -1.00368 & -2.21774 \\ \mathrm{C} & -1.84304 & 0.49901 & -2.36963 \\ \mathrm{C} & -0.61788 & 0.83861 & -3.08502 \\ \mathrm{C} & 0.12229 & -0.36946 & -3.37420 \\ \mathrm{C} & 1.51762 & -0.37375 & -3.37699 \\ \mathrm{C} & 2.23426 & -1.49293 & -2.78883 \\ \mathrm{C} & 1.52504 & -2.57404 & -2.26194 \\ \mathrm{C} & 1.94168 & -3.16952 & -1.00279 \\ \mathrm{C} & 3.03848 & -2.64786 & -0.31376 \\ \mathrm{C} & 2.97400 & -2.47950 & 1.13036 \\ \mathrm{C} & 1.81543 & -2.83925 & 1.82222 \\ \mathrm{C} & 1.29639 & -1.97435 & 2.86914 \\ \mathrm{C} & 1.96843 & -0.79415 & 3.19280 \\ \mathrm{C} & 1.21446 & 0.42256 & 3.44294 \\ \mathrm{C} & -0.17505 & 0.41193 & 3.31689 \\ \mathrm{C} & -0.87347 & 1.51433 & 2.69471 \\ \mathrm{C} & -0.14037 & 2.61760 & 2.24315 \\ \mathrm{C} & 1.30857 & 2.62130 & 2.33433 \\ \mathrm{C} & 1.97453 & 1.54330 & 2.91894 \\ \mathrm{C} & 3.18411 & 1.01842 & 2.31687 \\ \mathrm{C} & 3.17983 & -0.42794 & 2.48543 \\ & 3.67086 & -1.25158 & 1.46921 \\ \mathrm{C} & 3.16972 & -0.66206 & 0.23784 \\ \mathrm{C} & & -1.52365 & -0.86368 \\ \mathrm{C} & & -2.08160\end{array}$




\begin{tabular}{lrrr}
$\mathrm{C}$ & 3.38724 & 0.48515 & -2.24991 \\
$\mathrm{C}$ & 2.24051 & 0.84506 & -3.06054 \\
$\mathrm{C}$ & 1.53588 & 2.02089 & -2.79896 \\
$\mathrm{C}$ & 0.08434 & 2.02328 & -2.83637 \\
$\mathrm{C}$ & -0.38745 & 2.91421 & -1.80765 \\
$\mathrm{C}$ & 0.76386 & 3.44118 & -1.09458 \\
$\mathrm{C}$ & 1.95659 & 2.89038 & -1.71096 \\
$\mathrm{C}$ & 3.05115 & 2.53576 & -0.91853 \\
$\mathrm{C}$ & 3.78117 & 1.31102 & -1.19414 \\
$\mathrm{C}$ & 4.17413 & 0.72460 & 0.07618 \\
$\mathrm{C}$ & 3.67748 & 1.58326 & 1.13817 \\
$\mathrm{C}$ & 2.98742 & 2.70456 & 0.52561 \\
$\mathrm{C}$ & 1.83207 & 3.22200 & 1.11663 \\
$\mathrm{C}$ & 0.70300 & 3.60307 & 0.28819 \\
$\mathrm{C}$ & -0.51143 & 3.24376 & 1.00016 \\
$\mathrm{C}$ & -1.62360 & 2.76336 & 0.31593 \\
$\mathrm{C}$ & -1.55949 & 2.59220 & -1.13071 \\
$\mathrm{C}$ & -2.36568 & 1.43691 & -1.48301 \\
$\mathrm{C}$ & -3.31808 & 1.26675 & -0.31665 \\
$\mathrm{C}$ & -2.47162 & 1.71574 & 0.85512 \\
$\mathrm{C}$ & -2.03554 & 1.00865 & 1.97327 \\
$\mathrm{C}$ & -4.69883 & 1.35828 & -0.40754 \\
$\mathrm{C}$ & -5.61604 & 1.76204 & 0.72128 \\
$\mathrm{H}$ & -5.12747 & 1.35300 & -1.40993 \\
$\mathrm{H}$ & -5.94767 & 2.80199 & 0.58626 \\
$\mathrm{H}$ & -5.11848 & 1.69741 & 1.69347 \\
$\mathrm{H}$ & -6.52131 & 1.14486 & 0.75311 \\
& & & \\
\hline
\end{tabular}

(5) Intermediate IM5 $\left(\mathrm{C}_{2} \mathrm{H}_{4} \_\mathrm{C}_{59} \mathrm{Pd}\right)$

$\begin{array}{rrrr}\text { Pd } & -3.88412 & -0.69663 & -0.02875 \\ \mathrm{C} & -2.43583 & -1.58230 & -1.18687 \\ \mathrm{C} & -1.66979 & -2.66094 & -0.61372 \\ \mathrm{C} & -1.69774 & -2.54726 & 0.83205 \\ \mathrm{C} & -2.46070 & -1.38569 & 1.22958 \\ \mathrm{C} & -1.98303 & -0.52131 & 2.19516 \\ \mathrm{C} & -0.82930 & -0.92459 & 2.97360 \\ \mathrm{C} & -0.14625 & -2.11312 & 2.68932 \\ \mathrm{C} & -0.57411 & -2.92874 & 1.58141 \\ \mathrm{C} & 0.61246 & -3.44820 & 0.92023 \\ \mathrm{C} & 0.64449 & -3.54396 & -0.46897 \\ \mathrm{C} & -0.51139 & -3.13206 & -1.24645 \\ \mathrm{C} & -0.03688 & -2.46270 & -2.43208 \\ \mathrm{C} & -0.70861 & -1.32240 & -2.89162 \\ \mathrm{C} & -1.90136 & -0.83955 & -2.22367 \\ \mathrm{C} & -1.87261 & 0.66215 & -2.29764 \\ \mathrm{C} & -0.66105 & 1.01485 & -3.02297 \\ \mathrm{C} & 0.04436 & -0.19464 & -3.39163\end{array}$




\begin{tabular}{|c|c|c|c|}
\hline C & 1.43870 & -0.22980 & -3.41917 \\
\hline $\mathrm{C}$ & 2.13867 & -1.39457 & -2.90250 \\
\hline $\mathrm{C}$ & 1.41421 & -2.48604 & -2.41837 \\
\hline $\mathrm{C}$ & 1.83850 & -3.15302 & -1.19794 \\
\hline $\mathrm{C}$ & 2.96055 & -2.69300 & -0.50586 \\
\hline $\mathrm{C}$ & 2.92875 & -2.59707 & 0.94546 \\
\hline $\mathrm{C}$ & 1.77490 & -2.96273 & 1.64245 \\
\hline $\mathrm{C}$ & 1.30353 & -2.14165 & 2.74564 \\
\hline $\mathrm{C}$ & 2.01346 & -0.99696 & 3.11521 \\
\hline $\mathrm{C}$ & 1.29969 & 0.22461 & 3.45143 \\
\hline $\mathrm{C}$ & -0.09187 & 0.25719 & 3.36594 \\
\hline $\mathrm{C}$ & -0.77409 & 1.41301 & 2.82470 \\
\hline $\mathrm{C}$ & -0.02601 & 2.51797 & 2.40348 \\
\hline $\mathrm{C}$ & 1.42412 & 2.47609 & 2.45300 \\
\hline $\mathrm{C}$ & 2.07578 & 1.35297 & 2.96661 \\
\hline $\mathrm{C}$ & 3.25523 & 0.82849 & 2.30698 \\
\hline $\mathrm{C}$ & 3.21735 & -0.62452 & 2.40037 \\
\hline $\mathrm{C}$ & 3.66408 & -1.40603 & 1.33159 \\
\hline $\mathrm{C}$ & 4.15339 & -0.76639 & 0.12203 \\
\hline $\mathrm{C}$ & 3.71392 & -1.56007 & -1.01324 \\
\hline $\mathrm{C}$ & 3.31271 & -0.92664 & -2.19198 \\
\hline $\mathrm{C}$ & 3.34761 & 0.52660 & -2.28557 \\
\hline $\mathrm{C}$ & 2.19514 & 0.95442 & -3.05316 \\
\hline $\mathrm{C}$ & 1.52263 & 2.13041 & -2.71480 \\
\hline $\mathrm{C}$ & 0.07160 & 2.16837 & -2.72241 \\
\hline $\mathrm{C}$ & -0.36062 & 3.00664 & -1.63315 \\
\hline $\mathrm{C}$ & 0.81662 & 3.47197 & -0.91932 \\
\hline $\mathrm{C}$ & 1.98424 & 2.93017 & -1.59085 \\
\hline $\mathrm{C}$ & 3.08732 & 2.51135 & -0.84328 \\
\hline $\mathrm{C}$ & 3.78302 & 1.28727 & -1.19773 \\
\hline $\mathrm{C}$ & 4.18791 & 0.62644 & 0.03168 \\
\hline $\mathrm{C}$ & 3.73658 & 1.44086 & 1.14738 \\
\hline $\mathrm{C}$ & 3.05973 & 2.60736 & 0.60851 \\
\hline $\mathrm{C}$ & 1.93163 & 3.12109 & 1.25176 \\
\hline $\mathrm{C}$ & 0.79118 & 3.56704 & 0.47119 \\
\hline $\mathrm{C}$ & -0.41407 & 3.20429 & 1.19663 \\
\hline $\mathrm{C}$ & -1.55257 & 2.77839 & 0.51210 \\
\hline $\mathrm{C}$ & -1.52135 & 2.66779 & -0.94005 \\
\hline $\mathrm{C}$ & -2.34276 & 1.53261 & -1.32361 \\
\hline $\mathrm{C}$ & -3.27169 & 1.28909 & -0.14613 \\
\hline $\mathrm{C}$ & -2.39341 & 1.71030 & 1.01465 \\
\hline $\mathrm{C}$ & -1.95659 & 0.97426 & 2.10299 \\
\hline $\mathrm{C}$ & -4.68347 & 1.91513 & -0.18163 \\
\hline $\mathrm{C}$ & -5.46588 & 0.69881 & 0.31513 \\
\hline $\mathrm{H}$ & -5.71425 & 0.71339 & 1.37724 \\
\hline $\mathrm{H}$ & -6.31497 & 0.39801 & -0.30503 \\
\hline $\mathrm{H}$ & -4.75793 & 2.82429 & 0.42969 \\
\hline $\mathrm{H}$ & -4.94965 & 2.17673 & -1.21074 \\
\hline
\end{tabular}


(6) Intermediate IM6 $\left(\mathrm{C}_{2} \mathrm{H}_{4} \_\mathrm{HC} 1 \_\mathrm{C}_{59} \mathrm{Pd}\right)$

\begin{tabular}{|c|c|c|c|}
\hline $\mathrm{Pd}$ & -3.91881 & -0.51407 & -0.26968 \\
\hline $\mathrm{C}$ & -2.42721 & -1.21865 & -1.47597 \\
\hline $\mathrm{C}$ & -1.73178 & -2.37597 & -1.03068 \\
\hline $\mathrm{C}$ & -2.02142 & -2.79027 & 0.42820 \\
\hline $\mathrm{C}$ & -2.84449 & -1.61172 & 1.15382 \\
\hline $\mathrm{C}$ & -2.07410 & -0.75626 & 2.07871 \\
\hline $\mathrm{C}$ & -0.96239 & -1.28405 & 2.72894 \\
\hline $\mathrm{C}$ & -0.30120 & -2.48619 & 2.29250 \\
\hline $\mathrm{C}$ & -0.71037 & -3.10974 & 1.12509 \\
\hline C & 0.48633 & -3.53857 & 0.40997 \\
\hline $\mathrm{C}$ & 0.55705 & -3.40129 & -0.96536 \\
\hline $\mathrm{C}$ & -0.56549 & -2.78770 & -1.67903 \\
\hline $\mathrm{C}$ & -0.02184 & -2.01106 & -2.75096 \\
\hline $\mathrm{C}$ & -0.63269 & -0.79739 & -3.09769 \\
\hline $\mathrm{C}$ & -1.82311 & -0.38291 & -2.42072 \\
\hline $\mathrm{C}$ & -1.74286 & 1.11003 & -2.27438 \\
\hline C & -0.48943 & 1.52421 & -2.89634 \\
\hline $\mathrm{C}$ & 0.18674 & 0.35215 & -3.41249 \\
\hline C & 1.57673 & 0.25604 & -3.39564 \\
\hline $\mathrm{C}$ & 2.21128 & -1.00299 & -3.03086 \\
\hline $\mathrm{C}$ & 1.42674 & -2.11218 & -2.71707 \\
\hline $\mathrm{C}$ & 1.78844 & -2.97270 & -1.60505 \\
\hline $\mathrm{C}$ & 2.91521 & -2.67967 & -0.83388 \\
\hline $\mathrm{C}$ & 2.84433 & -2.80443 & 0.61206 \\
\hline $\mathrm{C}$ & 1.65317 & -3.22280 & 1.21577 \\
\hline $\mathrm{C}$ & 1.16838 & -2.55699 & 2.39805 \\
\hline $\mathrm{C}$ & 1.89458 & -1.51184 & 2.94831 \\
\hline C & 1.20477 & -0.31198 & 3.44392 \\
\hline C & -0.17217 & -0.20910 & 3.33049 \\
\hline $\mathrm{C}$ & -0.79518 & 1.03075 & 2.96349 \\
\hline $\mathrm{C}$ & 0.00437 & 2.15067 & 2.72348 \\
\hline $\mathrm{C}$ & 1.44594 & 2.05104 & 2.81109 \\
\hline C & 2.03972 & 0.83445 & 3.16829 \\
\hline C & 3.22524 & 0.36657 & 2.47556 \\
\hline $\mathrm{C}$ & 3.13687 & -1.08301 & 2.34723 \\
\hline C & 3.60274 & -1.71500 & 1.18926 \\
\hline C & 4.15474 & -0.91665 & 0.10344 \\
\hline C & 3.72550 & -1.51398 & -1.14969 \\
\hline C & 3.38068 & -0.69545 & -2.22747 \\
\hline $\mathrm{C}$ & 3.47139 & 0.75273 & -2.10321 \\
\hline $\mathrm{C}$ & 2.36338 & 1.33680 & -2.83014 \\
\hline C & 1.72338 & 2.48076 & -2.34231 \\
\hline C & 0.27407 & 2.58220 & -2.39460 \\
\hline C & -0.16904 & 3.25791 & -1.20210 \\
\hline $\mathrm{C}$ & 0.99678 & 3.56111 & -0.38953 \\
\hline C & 2.17114 & 3.07965 & -1.09930 \\
\hline
\end{tabular}




$\begin{array}{lrrr}\mathrm{C} & 3.23070 & 2.50799 & -0.38793 \\ \mathrm{C} & 3.89471 & 1.32483 & -0.89984 \\ \mathrm{C} & 4.23377 & 0.47072 & 0.22609 \\ \mathrm{C} & 3.76692 & 1.12622 & 1.43846 \\ \mathrm{C} & 3.15084 & 2.38748 & 1.06048 \\ \mathrm{C} & 2.01979 & 2.84692 & 1.73800 \\ \mathrm{C} & 0.92475 & 3.44979 & 0.99624 \\ \mathrm{C} & -0.31735 & 3.01995 & 1.61663 \\ \mathrm{C} & -1.42945 & 2.72124 & 0.83699 \\ \mathrm{C} & -1.36405 & 2.85490 & -0.60584 \\ \mathrm{C} & -2.22205 & 1.83636 & -1.18878 \\ \mathrm{C} & -3.16189 & 1.39467 & -0.08212 \\ \mathrm{C} & -2.32000 & 1.60969 & 1.14261 \\ \mathrm{C} & -1.96816 & 0.71987 & 2.14695 \\ \mathrm{C} & -4.61485 & 1.93368 & 0.03130 \\ \mathrm{C} & -5.30797 & 0.75058 & 0.73293 \\ \mathrm{H} & -5.24778 & 0.80063 & 1.82121 \\ \mathrm{H} & -6.33838 & 0.58019 & 0.40503 \\ \mathrm{H} & -4.63548 & 2.90592 & 0.54967 \\ \mathrm{H} & -5.02158 & 2.09813 & -0.97388 \\ \mathrm{H} & -3.77330 & -1.99795 & 1.59313 \\ \mathrm{H} & -2.68189 & -3.66851 & 0.45791\end{array}$

(7) Intermediate IM7 $\left(\mathrm{CH}_{2} \mathrm{CH}_{3} \_\mathrm{HC} 8 \_\mathrm{C}_{59} \mathrm{Pd}\right)$

$\begin{array}{cccc}\text { Pd } & -3.81589 & -0.74305 & -0.25760 \\ \mathrm{C} & -2.33327 & -1.25692 & -1.44573 \\ \mathrm{C} & -1.66690 & -2.43921 & -1.03208 \\ \mathrm{C} & -1.93079 & -2.92279 & 0.41836 \\ \mathrm{C} & -2.65501 & -1.69701 & 1.07615 \\ \mathrm{C} & -2.01861 & -0.90559 & 2.00736 \\ \mathrm{C} & -0.87888 & -1.41251 & 2.73325 \\ \mathrm{C} & -0.19289 & -2.55592 & 2.27275 \\ \mathrm{C} & -0.59980 & -3.22187 & 1.09323 \\ \mathrm{C} & 0.58493 & -3.53952 & 0.36229 \\ \mathrm{C} & 0.64417 & -3.39798 & -1.02833 \\ \mathrm{C} & -0.49454 & -2.79723 & -1.71429 \\ \mathrm{C} & 0.01354 & -1.96608 & -2.77039 \\ \mathrm{C} & -0.63338 & -0.76914 & -3.09712 \\ \mathrm{C} & -1.82222 & -0.37056 & -2.39491 \\ \mathrm{C} & -1.78640 & 1.11024 & -2.26146 \\ \mathrm{C} & -0.55198 & 1.56064 & -2.88364 \\ \mathrm{C} & 0.15059 & 0.41113 & -3.41120 \\ \mathrm{C} & 1.54240 & 0.35462 & -3.39098 \\ \mathrm{C} & 2.21534 & -0.89040 & -3.04663 \\ \mathrm{C} & 1.46262 & -2.02634 & -2.74737 \\ \mathrm{C} & 1.85102 & -2.89812 & -1.65202 \\ \mathrm{C} & 2.97996 & -2.58242 & -0.88530\end{array}$




\begin{tabular}{|c|c|c|c|}
\hline C & 2.92139 & -2.72817 & 0.56046 \\
\hline $\mathrm{C}$ & 1.75602 & -3.19462 & 1.16671 \\
\hline $\mathrm{C}$ & 1.26715 & -2.57768 & 2.37272 \\
\hline $\mathrm{C}$ & 1.95904 & -1.51063 & 2.94181 \\
\hline $\mathrm{C}$ & 1.23460 & -0.35716 & 3.45911 \\
\hline $\mathrm{C}$ & -0.15349 & -0.31274 & 3.32502 \\
\hline C & -0.81818 & 0.91492 & 2.94314 \\
\hline C & -0.06473 & 2.07282 & 2.74486 \\
\hline $\mathrm{C}$ & 1.38709 & 2.02815 & 2.84290 \\
\hline $\mathrm{C}$ & 2.02094 & 0.83167 & 3.17832 \\
\hline C & 3.22004 & 0.41932 & 2.47620 \\
\hline $\mathrm{C}$ & 3.18010 & -1.02981 & 2.32997 \\
\hline $\mathrm{C}$ & 3.65141 & -1.62500 & 1.15531 \\
\hline C & 4.16932 & -0.79639 & 0.07906 \\
\hline C & 3.74529 & -1.38867 & -1.18162 \\
\hline C & 3.37864 & -0.56189 & -2.24839 \\
\hline C & 3.42660 & 0.88579 & -2.09798 \\
\hline $\mathrm{C}$ & 2.29912 & 1.45070 & -2.81351 \\
\hline $\mathrm{C}$ & 1.62988 & 2.56805 & -2.30567 \\
\hline C & 0.18060 & 2.63069 & -2.35718 \\
\hline C & -0.27945 & 3.26930 & -1.14786 \\
\hline $\mathrm{C}$ & 0.87953 & 3.58731 & -0.33072 \\
\hline $\mathrm{C}$ & 2.06447 & 3.16093 & -1.05187 \\
\hline C & 3.14091 & 2.60499 & -0.35349 \\
\hline C & 3.84022 & 1.45209 & -0.88943 \\
\hline $\mathrm{C}$ & 4.20969 & 0.59052 & 0.22219 \\
\hline $\mathrm{C}$ & 3.73202 & 1.21239 & 1.44684 \\
\hline $\mathrm{C}$ & 3.07314 & 2.45732 & 1.09202 \\
\hline $\mathrm{C}$ & 1.92576 & 2.85981 & 1.78191 \\
\hline $\mathrm{C}$ & 0.80956 & 3.43981 & 1.05373 \\
\hline $\mathrm{C}$ & -0.41662 & 2.95547 & 1.66267 \\
\hline $\mathrm{C}$ & -1.53596 & 2.66219 & 0.88458 \\
\hline C & -1.46010 & 2.82377 & -0.55612 \\
\hline C & -2.28835 & 1.79963 & -1.16573 \\
\hline $\mathrm{C}$ & -3.21520 & 1.27204 & -0.10792 \\
\hline $\mathrm{C}$ & -2.38205 & 1.50660 & 1.15094 \\
\hline $\mathrm{C}$ & -1.97264 & 0.59406 & 2.11312 \\
\hline $\mathrm{C}$ & -4.62029 & 1.90864 & -0.08312 \\
\hline $\mathrm{C}$ & -5.57612 & 1.29201 & 0.95347 \\
\hline $\mathrm{H}$ & -4.49433 & 2.97948 & 0.14692 \\
\hline $\mathrm{H}$ & -5.05913 & 1.86328 & -1.08836 \\
\hline $\mathrm{H}$ & -2.60394 & -3.78778 & 0.43791 \\
\hline $\mathrm{H}$ & -5.82848 & 0.23719 & 0.73761 \\
\hline $\mathrm{H}$ & -6.53105 & 1.82784 & 0.96536 \\
\hline $\mathrm{H}$ & -5.14772 & 1.32873 & 1.95989 \\
\hline
\end{tabular}


(8) Intermediate IM8 $\left(\mathrm{CH}_{2} \mathrm{CH}_{3} \_\mathrm{HC} 1 \_\mathrm{C}_{59} \mathrm{Pd}\right)$

$\begin{array}{crrr}\text { Pd } & -4.13986 & -0.68279 & -0.42943 \\ \mathrm{C} & -2.47837 & -1.36650 & -1.24365 \\ \mathrm{C} & -1.66982 & -2.47948 & -0.75702 \\ \mathrm{C} & -1.57996 & -2.71875 & 0.71074 \\ \mathrm{C} & -2.39300 & -1.87552 & 1.55575 \\ \mathrm{C} & -1.76344 & -1.03595 & 2.45602 \\ \mathrm{C} & -0.49074 & -1.44012 & 2.97561 \\ \mathrm{C} & 0.17367 & -2.54691 & 2.44663 \\ \mathrm{C} & -0.34997 & -3.15008 & 1.23994 \\ \mathrm{C} & 0.78019 & -3.50829 & 0.41668 \\ \mathrm{C} & 0.68362 & -3.36875 & -0.95798 \\ \mathrm{C} & -0.53538 & -2.82846 & -1.54092 \\ \mathrm{C} & -0.13352 & -2.03697 & -2.68066 \\ \mathrm{C} & -0.82731 & -0.86519 & -2.98325 \\ \mathrm{C} & -1.98558 & -0.51211 & -2.22064 \\ \mathrm{C} & -1.98290 & 0.98574 & -2.09111 \\ \mathrm{C} & -0.81631 & 1.46640 & -2.80793 \\ \mathrm{C} & -0.11678 & 0.33389 & -3.37756 \\ \mathrm{C} & 1.27149 & 0.32327 & -3.47540 \\ \mathrm{C} & 2.00385 & -0.89565 & -3.16742 \\ \mathrm{C} & 1.31346 & -2.05077 & -2.78613 \\ \mathrm{C} & 1.82396 & -2.87744 & -1.71237 \\ \mathrm{C} & 3.00274 & -2.52096 & -1.05298 \\ \mathrm{C} & 3.09214 & -2.65098 & 0.39312 \\ \mathrm{C} & 2.00350 & -3.14304 & 1.11143 \\ \mathrm{C} & 1.62164 & -2.53094 & 2.36986 \\ \mathrm{C} & 2.33850 & -1.43220 & 2.85411 \\ \mathrm{C} & 1.63173 & -0.29358 & 3.42222 \\ \mathrm{C} & 0.23802 & -0.29597 & 3.44716 \\ \mathrm{C} & -0.53680 & 0.87809 & 3.10351 \\ \mathrm{C} & 0.15713 & 2.04503 & 2.78037 \\ \mathrm{C} & 1.60959 & 2.06582 & 2.74042 \\ \mathrm{C} & 2.33785 & 0.91442 & 3.04591 \\ \mathrm{C} & 3.47583 & 0.53265 & 2.23268 \\ \mathrm{C} & 3.47265 & -0.91949 & 2.10960 \\ \mathrm{C} & 3.84423 & -1.51757 & 0.90213 \\ \mathrm{C} & 4.22636 & -0.69008 & -0.22998 \\ \mathrm{C} & 3.71037 & -1.31299 & -1.43769 \\ \mathrm{C} & 3.22010 & -0.51772 & -2.47716 \\ \mathrm{C} & 3.23758 & 0.93418 & -2.35490 \\ \mathrm{C} & 2.03653 & 1.45366 & -2.97850 \\ \mathrm{C} & 1.37266 & 2.55090 & -2.42335 \\ \mathrm{C} & -0.07802 & 2.56525 & -2.34926 \\ \mathrm{C} & -0.45354 & 3.18665 & -1.10400 \\ \mathrm{C} & 0.75635 & 3.55470 & -0.39385 \\ \mathrm{C} & 1.89134 & 3.16956 & -1.21280 \\ \mathrm{C} & 3.04601 & 2.66695 & -0.60923 \\ & & & \\ & & & \end{array}$




$\begin{array}{lrrr}\mathrm{C} & 3.73524 & 1.52980 & -1.19328 \\ \mathrm{C} & 4.23513 & 0.70115 & -0.10952 \\ \mathrm{C} & 3.85174 & 1.32520 & 1.14596 \\ \mathrm{C} & 3.11205 & 2.53673 & 0.83837 \\ \mathrm{C} & 2.01313 & 2.90149 & 1.62186 \\ \mathrm{C} & 0.81145 & 3.41459 & 0.99041 \\ \mathrm{C} & -0.33077 & 2.87078 & 1.71056 \\ \mathrm{C} & -1.47897 & 2.47463 & 1.02989 \\ \mathrm{C} & -1.55686 & 2.68595 & -0.40809 \\ \mathrm{C} & -2.39423 & 1.64866 & -0.94579 \\ \mathrm{C} & -3.22771 & 1.08461 & 0.19590 \\ \mathrm{C} & -2.25668 & 1.28764 & 1.39079 \\ \mathrm{C} & -1.77932 & 0.47319 & 2.42781 \\ \mathrm{C} & -4.55336 & 1.87472 & 0.40102 \\ \mathrm{C} & -5.57623 & 1.15161 & 1.29501 \\ \mathrm{H} & -4.32061 & 2.85345 & 0.84537 \\ \mathrm{H} & -4.99889 & 2.07966 & -0.57931 \\ \mathrm{H} & -3.40115 & -1.64520 & 1.24147 \\ \mathrm{H} & -5.94129 & 0.20298 & 0.85430 \\ \mathrm{H} & -6.46787 & 1.77111 & 1.44114 \\ \mathrm{H} & -5.15401 & 0.91381 & 2.27635\end{array}$

(9) Intermediate IM9 $\left(\mathrm{CH}_{3} \mathrm{CH}_{3} \mathrm{C}_{59} \mathrm{Pd}\right)$

$\begin{array}{crrr}\text { Pd } & 3.73610 & -0.88000 & -0.00221 \\ \mathrm{C} & 2.68946 & 0.29541 & -1.25898 \\ \mathrm{C} & 2.39274 & 1.60509 & -0.73745 \\ \mathrm{C} & 2.39453 & 1.61003 & 0.71954 \\ \mathrm{C} & 2.69250 & 0.30391 & 1.24917 \\ \mathrm{C} & 1.92485 & -0.25807 & 2.24999 \\ \mathrm{C} & 1.00229 & 0.59305 & 2.97109 \\ \mathrm{C} & 0.79543 & 1.91838 & 2.56980 \\ \mathrm{C} & 1.48500 & 2.43095 & 1.41123 \\ \mathrm{C} & 0.55959 & 3.28426 & 0.68324 \\ \mathrm{C} & 0.55793 & 3.27957 & -0.70796 \\ \mathrm{C} & 1.48153 & 2.42133 & -1.43244 \\ \mathrm{C} & 0.78922 & 1.90106 & -2.58591 \\ \mathrm{C} & 0.99514 & 0.57307 & -2.97883 \\ \mathrm{C} & 1.91943 & -0.27322 & -2.25423 \\ \mathrm{C} & 1.36861 & -1.65505 & -2.24890 \\ \mathrm{C} & 0.11228 & -1.60197 & -2.95904 \\ \mathrm{C} & -0.11569 & -0.24319 & -3.41947 \\ \mathrm{C} & -1.40364 & 0.29020 & -3.45374 \\ \mathrm{C} & -1.62758 & 1.66200 & -3.02211 \\ \mathrm{C} & -0.55437 & 2.45175 & -2.60215 \\ \mathrm{C} & -0.69934 & 3.30401 & -1.43470 \\ \mathrm{C} & -1.90806 & 3.33391 & -0.73672 \\ \mathrm{C} & -1.90633 & 3.33882 & 0.71754\end{array}$




\begin{tabular}{|c|c|c|c|}
\hline C & -0.69593 & 3.31358 & 1.41282 \\
\hline $\mathrm{C}$ & -0.54813 & 2.46915 & 2.58558 \\
\hline $\mathrm{C}$ & -1.62032 & 1.68221 & 3.01337 \\
\hline $\mathrm{C}$ & -1.39533 & 0.31334 & 3.45368 \\
\hline $\mathrm{C}$ & -0.10746 & -0.22028 & 3.41983 \\
\hline $\mathrm{C}$ & 0.11942 & -1.58212 & 2.96801 \\
\hline $\mathrm{C}$ & -0.98022 & -2.37163 & 2.59539 \\
\hline $\mathrm{C}$ & -2.31460 & -1.80830 & 2.60303 \\
\hline $\mathrm{C}$ & -2.52519 & -0.49326 & 3.02920 \\
\hline $\mathrm{C}$ & -3.43683 & 0.36951 & 2.30485 \\
\hline $\mathrm{C}$ & -2.88094 & 1.71628 & 2.29984 \\
\hline $\mathrm{C}$ & -3.02122 & 2.52556 & 1.16921 \\
\hline $\mathrm{C}$ & -3.71651 & 2.02248 & -0.00301 \\
\hline $\mathrm{C}$ & -3.02402 & 2.51765 & -1.18025 \\
\hline $\mathrm{C}$ & -2.88648 & 1.70083 & -2.30579 \\
\hline $\mathrm{C}$ & -3.44239 & 0.35409 & -2.30043 \\
\hline $\mathrm{C}$ & -2.53248 & -0.51353 & -3.02117 \\
\hline $\mathrm{C}$ & -2.32086 & -1.82568 & -2.58672 \\
\hline $\mathrm{C}$ & -0.98645 & -2.38897 & -2.57854 \\
\hline $\mathrm{C}$ & -0.86644 & -3.23273 & -1.41411 \\
\hline $\mathrm{C}$ & -2.12433 & -3.19504 & -0.68523 \\
\hline $\mathrm{C}$ & -3.02779 & -2.32221 & -1.41439 \\
\hline $\mathrm{C}$ & -3.89868 & -1.48229 & -0.71847 \\
\hline $\mathrm{C}$ & -4.11372 & -0.11841 & -1.17060 \\
\hline $\mathrm{C}$ & -4.24490 & 0.72911 & 0.00198 \\
\hline $\mathrm{C}$ & -4.11093 & -0.11056 & 1.17987 \\
\hline $\mathrm{C}$ & -3.89693 & -1.47743 & 0.73631 \\
\hline $\mathrm{C}$ & -3.02433 & -2.31264 & 1.43572 \\
\hline $\mathrm{C}$ & -2.12265 & -3.19036 & 0.71025 \\
\hline $\mathrm{C}$ & -0.86302 & -3.22317 & 1.43634 \\
\hline $\mathrm{C}$ & 0.33932 & -3.25802 & 0.73251 \\
\hline $\mathrm{C}$ & 0.33759 & -3.26290 & -0.71293 \\
\hline $\mathrm{C}$ & 1.50488 & -2.50400 & -1.14406 \\
\hline $\mathrm{C}$ & 2.39152 & -2.34270 & 0.00424 \\
\hline $\mathrm{C}$ & 1.50766 & -2.49628 & 1.15576 \\
\hline $\mathrm{C}$ & 1.37403 & -1.63993 & 2.25521 \\
\hline $\mathrm{C}$ & 6.43087 & 0.52081 & 0.00302 \\
\hline $\mathrm{C}$ & 6.61180 & 2.04104 & 0.00416 \\
\hline $\mathrm{H}$ & 7.16398 & 2.37113 & -0.88222 \\
\hline $\mathrm{H}$ & 7.16892 & 2.36906 & 0.88823 \\
\hline $\mathrm{H}$ & 5.64491 & 2.55549 & 0.00751 \\
\hline $\mathrm{H}$ & 7.38754 & -0.01316 & -0.00063 \\
\hline $\mathrm{H}$ & 5.88383 & 0.19446 & -0.89586 \\
\hline $\mathrm{H}$ & 5.88889 & 0.19218 & 0.90392 \\
\hline
\end{tabular}




\section{For Nickel doped fullerene}

(0) Catalyst $\mathrm{C}_{59} \mathrm{Ni}$

\begin{tabular}{|c|c|c|c|}
\hline $\mathrm{Ni}$ & 4.22667 & 0.02143 & 0.00002 \\
\hline $\mathrm{C}$ & 3.14334 & -0.92016 & 1.27797 \\
\hline C & 2.54637 & -2.13210 & 0.74041 \\
\hline C & 2.54643 & -2.13267 & -0.73895 \\
\hline $\mathrm{C}$ & 3.14357 & -0.92119 & -1.27735 \\
\hline $\mathrm{C}$ & 2.45325 & -0.23397 & -2.24715 \\
\hline C & 1.34009 & -0.85289 & -2.91736 \\
\hline $\mathrm{C}$ & 0.90321 & -2.10334 & -2.55343 \\
\hline $\mathrm{C}$ & 1.48764 & -2.73372 & -1.39514 \\
\hline C & 0.42585 & -3.40310 & -0.68427 \\
\hline $\mathrm{C}$ & 0.42581 & -3.40264 & 0.68649 \\
\hline C & 1.48756 & -2.73273 & 1.39696 \\
\hline $\mathrm{C}$ & 0.90306 & -2.10162 & 2.55481 \\
\hline C & 1.33990 & -0.85094 & 2.91799 \\
\hline $\mathrm{C}$ & 2.45306 & -0.23241 & 2.24742 \\
\hline C & 2.12424 & 1.25217 & 2.17702 \\
\hline C & 0.86489 & 1.42480 & 2.89643 \\
\hline C & 0.39520 & 0.14245 & 3.35434 \\
\hline C & -0.94448 & -0.14604 & 3.41872 \\
\hline $\mathrm{C}$ & -1.41657 & -1.44840 & 2.99775 \\
\hline $\mathrm{C}$ & -0.51480 & -2.39277 & 2.56926 \\
\hline $\mathrm{C}$ & -0.81281 & -3.20400 & 1.40965 \\
\hline $\mathrm{C}$ & -1.99035 & -3.01937 & 0.72619 \\
\hline $\mathrm{C}$ & -1.99031 & -3.01985 & -0.72435 \\
\hline C & -0.81273 & -3.20494 & -1.40763 \\
\hline $\mathrm{C}$ & -0.51464 & -2.39447 & -2.56776 \\
\hline C & -1.41637 & -1.45036 & -2.99690 \\
\hline C & -0.94424 & -0.14827 & -3.41867 \\
\hline $\mathrm{C}$ & 0.39544 & 0.14024 & -3.35437 \\
\hline C & 0.86512 & 1.42287 & -2.89721 \\
\hline C & -0.06171 & 2.37284 & -2.52855 \\
\hline C & -1.47747 & 2.08126 & -2.56995 \\
\hline C & -1.90295 & 0.84217 & -2.98373 \\
\hline C & -2.96575 & 0.15943 & -2.28050 \\
\hline $\mathrm{C}$ & -2.65584 & -1.25748 & -2.27868 \\
\hline C & -2.93863 & -2.02375 & -1.17182 \\
\hline C & -3.52042 & -1.40278 & 0.00034 \\
\hline $\mathrm{C}$ & -2.93871 & -2.02299 & 1.17294 \\
\hline C & -2.65599 & -1.25600 & 2.27932 \\
\hline C & -2.96591 & 0.16091 & 2.28021 \\
\hline C & -1.90316 & 0.84411 & 2.98308 \\
\hline C & -1.47767 & 2.08295 & 2.56854 \\
\hline $\mathrm{C}$ & -0.06192 & 2.37452 & 2.52708 \\
\hline C & 0.18848 & 3.23383 & 1.39493 \\
\hline
\end{tabular}




$\begin{array}{lrrr}\mathrm{C} & -1.05895 & 3.41505 & 0.68483 \\ \mathrm{C} & -2.09024 & 2.70449 & 1.41019 \\ \mathrm{C} & -3.08347 & 2.04831 & 0.72399 \\ \mathrm{C} & -3.52780 & 0.74230 & 1.16899 \\ \mathrm{C} & -3.81762 & -0.06001 & -0.00010 \\ \mathrm{C} & -3.52772 & 0.74154 & -1.16970 \\ \mathrm{C} & -3.08342 & 2.04784 & -0.72551 \\ \mathrm{C} & -2.09012 & 2.70356 & -1.41206 \\ \mathrm{C} & -1.05889 & 3.41459 & -0.68708 \\ \mathrm{C} & 0.18861 & 3.23288 & -1.39695 \\ \mathrm{C} & 1.36290 & 3.07384 & -0.72415 \\ \mathrm{C} & 1.36284 & 3.07437 & 0.72234 \\ \mathrm{C} & 2.41054 & 2.16139 & 1.15699 \\ \mathrm{C} & 3.20478 & 1.79911 & -0.00035 \\ \mathrm{C} & 2.41062 & 2.16051 & -1.15806 \\ \mathrm{C} & 2.12440 & 1.25066 & -2.17758\end{array}$

(1) Intermediate IM1 $\left(\mathrm{C}_{2} \mathrm{H}_{2} \_\mathrm{C}_{59} \mathrm{Ni}\right)$

$\begin{array}{cccc}\mathrm{Ni} & -3.95335 & -0.67118 & -0.14296 \\ \mathrm{C} & -2.65029 & -1.26526 & -1.41088 \\ \mathrm{C} & -1.93350 & -2.44811 & -0.99104 \\ \mathrm{C} & -1.98863 & -2.57413 & 0.46255 \\ \mathrm{C} & -2.74582 & -1.49120 & 1.03845 \\ \mathrm{C} & -2.25341 & -0.79837 & 2.12098 \\ \mathrm{C} & -1.10789 & -1.31622 & 2.82873 \\ \mathrm{C} & -0.44813 & -2.46663 & 2.38062 \\ \mathrm{C} & -0.88286 & -3.09480 & 1.15570 \\ \mathrm{C} & 0.30062 & -3.53419 & 0.43568 \\ \mathrm{C} & 0.34799 & -3.42923 & -0.95261 \\ \mathrm{C} & -0.78383 & -2.87329 & -1.67461 \\ \mathrm{C} & -0.26943 & -2.07576 & -2.76205 \\ \mathrm{C} & -0.90374 & -0.87200 & -3.08849 \\ \mathrm{C} & -2.09268 & -0.45246 & -2.38077 \\ \mathrm{C} & -2.02910 & 1.04089 & -2.27081 \\ \mathrm{C} & -0.80078 & 1.46404 & -2.91487 \\ \mathrm{C} & -0.11308 & 0.29379 & -3.42464 \\ \mathrm{C} & 1.27898 & 0.22803 & -3.42111 \\ \mathrm{C} & 1.94073 & -1.01354 & -3.05321 \\ \mathrm{C} & 1.17953 & -2.14055 & -2.73030 \\ \mathrm{C} & 1.56440 & -2.97709 & -1.60584 \\ \mathrm{C} & 2.68778 & -2.64853 & -0.84426 \\ \mathrm{C} & 2.63738 & -2.75608 & 0.60539 \\ \mathrm{C} & 1.46624 & -3.18860 & 1.23058 \\ \mathrm{C} & 0.99946 & -2.52602 & 2.43709 \\ \mathrm{C} & 1.72746 & -1.45872 & 2.97185 \\ \mathrm{C} & 1.03314 & -0.28005 & 3.46692\end{array}$




\begin{tabular}{lrrr}
$\mathrm{C}$ & -0.35560 & -0.21006 & 3.38094 \\
$\mathrm{C}$ & -1.01597 & 1.02117 & 2.99010 \\
$\mathrm{C}$ & -0.24003 & 2.15419 & 2.71589 \\
$\mathrm{C}$ & 1.20851 & 2.08338 & 2.78139 \\
$\mathrm{C}$ & 1.83440 & 0.88907 & 3.14455 \\
$\mathrm{C}$ & 3.01253 & 0.43754 & 2.43371 \\
$\mathrm{C}$ & 2.94469 & -1.01438 & 2.32527 \\
$\mathrm{C}$ & 3.39235 & -1.64895 & 1.16322 \\
$\mathrm{C}$ & 3.91201 & -0.85771 & 0.06090 \\
$\mathrm{C}$ & 3.47520 & -1.47603 & -1.17982 \\
$\mathrm{C}$ & 3.10847 & -0.67612 & -2.26504 \\
$\mathrm{C}$ & 3.17744 & 0.77541 & -2.15565 \\
$\mathrm{C}$ & 2.05054 & 1.33285 & -2.87482 \\
$\mathrm{C}$ & 1.39736 & 2.46769 & -2.38776 \\
$\mathrm{C}$ & -0.05159 & 2.53897 & -2.41955 \\
$\mathrm{C}$ & -0.49234 & 3.21644 & -1.22551 \\
$\mathrm{C}$ & 0.67794 & 3.55069 & -0.43072 \\
$\mathrm{C}$ & 1.84988 & 3.08887 & -1.15274 \\
$\mathrm{C}$ & 2.92977 & 2.54501 & -0.45359 \\
$\mathrm{C}$ & 3.60727 & 1.36681 & -0.96510 \\
$\mathrm{C}$ & 3.97770 & 0.53345 & 0.16570 \\
$\mathrm{C}$ & 3.52328 & 1.19482 & 1.37690 \\
$\mathrm{C}$ & 2.87661 & 2.43825 & 0.99616 \\
$\mathrm{C}$ & 1.74471 & 2.87746 & 1.68735 \\
$\mathrm{C}$ & 0.62516 & 3.44539 & 0.95774 \\
$\mathrm{C}$ & -0.59818 & 2.99662 & 1.60494 \\
$\mathrm{C}$ & -1.72200 & 2.67068 & 0.84396 \\
$\mathrm{C}$ & -1.66662 & 2.79153 & -0.60585 \\
$\mathrm{C}$ & -2.49759 & 1.75711 & -1.17728 \\
$\mathrm{C}$ & -3.37128 & 1.23684 & -0.07519 \\
$\mathrm{C}$ & -2.57599 & 1.55060 & 1.17935 \\
$\mathrm{C}$ & -2.19077 & 0.69545 & 2.20244 \\
$\mathrm{C}$ & -4.85023 & 1.34039 & -0.10471 \\
& -5.43492 & 0.28882 & 0.53765 \\
$\mathrm{H}$ & -6.51837 & 0.19045 & 0.61631 \\
\hline
\end{tabular}

(2) Intermediate IM2 $\left(\mathrm{C}_{2} \mathrm{H}_{2} \_2 \mathrm{H} \_\mathrm{C}_{59} \mathrm{Ni}\right)$

$\begin{array}{rrrr}\mathrm{Ni} & -4.01712 & -0.50104 & -0.25486 \\ \mathrm{C} & -2.69373 & -0.88364 & -1.58320 \\ \mathrm{C} & -2.00165 & -2.13569 & -1.34424 \\ \mathrm{C} & -2.08138 & -2.51731 & 0.03128 \\ \mathrm{C} & -3.02242 & -1.68179 & 0.88302 \\ \mathrm{C} & -2.27020 & -0.95632 & 1.96262 \\ \mathrm{C} & -1.18588 & -1.65717 & 2.61721 \\ \mathrm{C} & -0.56301 & -2.72740 & 1.95653 \\ \mathrm{C} & -1.01423 & -3.17115 & 0.63762\end{array}$




\begin{tabular}{|c|c|c|c|}
\hline C & 0.16090 & -3.53520 & -0.11787 \\
\hline C & 0.23283 & -3.23226 & -1.47481 \\
\hline C & -0.85821 & -2.50879 & -2.08868 \\
\hline $\mathrm{C}$ & -0.30388 & -1.58514 & -3.03700 \\
\hline $\mathrm{C}$ & -0.88253 & -0.31213 & -3.15369 \\
\hline $\mathrm{C}$ & -2.06718 & 0.05006 & -2.41077 \\
\hline $\mathrm{C}$ & -1.93930 & 1.50709 & -2.09132 \\
\hline $\mathrm{C}$ & -0.66850 & 1.95818 & -2.65502 \\
\hline $\mathrm{C}$ & -0.03313 & 0.84758 & -3.32874 \\
\hline $\mathrm{C}$ & 1.35642 & 0.71040 & -3.33802 \\
\hline C & 1.95664 & -0.60236 & -3.16608 \\
\hline C & 1.14084 & -1.72507 & -3.02379 \\
\hline $\mathrm{C}$ & 1.47677 & -2.74582 & -2.04373 \\
\hline $\mathrm{C}$ & 2.60394 & -2.59538 & -1.23406 \\
\hline $\mathrm{C}$ & 2.52734 & -2.91771 & 0.18350 \\
\hline C & 1.33032 & -3.37445 & 0.73027 \\
\hline $\mathrm{C}$ & 0.87895 & -2.88202 & 2.02001 \\
\hline $\mathrm{C}$ & 1.65127 & -1.94810 & 2.71513 \\
\hline C & 1.01251 & -0.83274 & 3.39623 \\
\hline C & -0.37454 & -0.68898 & 3.32598 \\
\hline C & -0.96886 & 0.60733 & 3.10322 \\
\hline $\mathrm{C}$ & -0.13622 & 1.71382 & 2.99283 \\
\hline $\mathrm{C}$ & 1.30368 & 1.58424 & 3.06898 \\
\hline C & 1.87292 & 0.32292 & 3.25718 \\
\hline $\mathrm{C}$ & 3.04321 & -0.06845 & 2.48958 \\
\hline $\mathrm{C}$ & 2.90694 & -1.47827 & 2.15762 \\
\hline $\mathrm{C}$ & 3.32766 & -1.94416 & 0.91031 \\
\hline C & 3.90165 & -1.02464 & -0.05672 \\
\hline C & 3.44623 & -1.42298 & -1.37775 \\
\hline $\mathrm{C}$ & 3.13228 & -0.44790 & -2.33131 \\
\hline $\mathrm{C}$ & 3.26886 & 0.96004 & -1.99945 \\
\hline $\mathrm{C}$ & 2.17939 & 1.67803 & -2.63576 \\
\hline $\mathrm{C}$ & 1.57536 & 2.75411 & -1.98488 \\
\hline $\mathrm{C}$ & 0.13135 & 2.91497 & -2.01839 \\
\hline $\mathrm{C}$ & -0.28629 & 3.42449 & -0.73614 \\
\hline $\mathrm{C}$ & 0.88597 & 3.55501 & 0.10804 \\
\hline $\mathrm{C}$ & 2.04460 & 3.15550 & -0.66554 \\
\hline $\mathrm{C}$ & 3.08540 & 2.45858 & -0.04778 \\
\hline $\mathrm{C}$ & 3.71396 & 1.34137 & -0.72922 \\
\hline $\mathrm{C}$ & 4.03010 & 0.32900 & 0.26084 \\
\hline $\mathrm{C}$ & 3.59162 & 0.81566 & 1.55984 \\
\hline $\mathrm{C}$ & 3.00817 & 2.13384 & 1.37008 \\
\hline $\mathrm{C}$ & 1.89007 & 2.51426 & 2.11287 \\
\hline $\mathrm{C}$ & 0.81036 & 3.23983 & 1.46766 \\
\hline $\mathrm{C}$ & -0.43352 & 2.73150 & 2.00037 \\
\hline $\mathrm{C}$ & -1.54480 & 2.58551 & 1.18474 \\
\hline $\mathrm{C}$ & -1.49232 & 2.97363 & -0.20296 \\
\hline $\mathrm{C}$ & -2.40226 & 2.09710 & -0.91786 \\
\hline $\mathrm{C}$ & -3.28371 & 1.42371 & 0.08905 \\
\hline
\end{tabular}




$\begin{array}{lrrr}\mathrm{C} & -2.48522 & 1.47190 & 1.30977 \\ \mathrm{C} & -2.15436 & 0.43823 & 2.23902 \\ \mathrm{C} & -4.77248 & 1.47408 & 0.05855 \\ \mathrm{C} & -5.40670 & 0.39008 & 0.58278 \\ \mathrm{H} & -5.23477 & 2.32667 & -0.45183 \\ \mathrm{H} & -6.46523 & 0.28796 & 0.79568 \\ \mathrm{H} & -3.78431 & -2.31709 & 1.35719 \\ \mathrm{H} & -4.94830 & -1.62239 & -0.49248\end{array}$

(3) Intermediate IM3 $\left(\mathrm{C}_{2} \mathrm{H}_{3} \_\mathrm{H} \_\mathrm{C}_{59} \mathrm{Ni}\right)$

$\begin{array}{lrrr}\mathrm{Ni} & -3.96868 & -0.51983 & -0.42539 \\ \mathrm{C} & -2.60315 & -0.86341 & -1.64434 \\ \mathrm{C} & -1.92034 & -2.13745 & -1.48863 \\ \mathrm{C} & -2.00352 & -2.67014 & -0.12652 \\ \mathrm{C} & -2.89444 & -1.89542 & 0.75672 \\ \mathrm{C} & -2.29631 & -1.28677 & 1.87957 \\ \mathrm{C} & -1.10706 & -1.90817 & 2.42151 \\ \mathrm{C} & -0.44794 & -2.94332 & 1.76071 \\ \mathrm{C} & -0.87397 & -3.28070 & 0.41607 \\ \mathrm{C} & 0.31601 & -3.53620 & -0.36456 \\ \mathrm{C} & 0.36966 & -3.11968 & -1.68887 \\ \mathrm{C} & -0.75733 & -2.39588 & -2.24985 \\ \mathrm{C} & -0.22600 & -1.38366 & -3.12964 \\ \mathrm{C} & -0.84286 & -0.12969 & -3.19093 \\ \mathrm{C} & -2.03274 & 0.13586 & -2.42306 \\ \mathrm{C} & -1.96806 & 1.58004 & -2.00942 \\ \mathrm{C} & -0.72202 & 2.11477 & -2.52822 \\ \mathrm{C} & -0.03845 & 1.07439 & -3.26902 \\ \mathrm{C} & 1.35245 & 0.99148 & -3.26504 \\ \mathrm{C} & 1.99750 & -0.30829 & -3.17614 \\ \mathrm{C} & 1.22273 & -1.46955 & -3.11664 \\ \mathrm{C} & 1.59498 & -2.54368 & -2.21399 \\ \mathrm{C} & 2.71519 & -2.40755 & -1.39037 \\ \mathrm{C} & 2.65392 & -2.84113 & -0.00243 \\ \mathrm{C} & 1.47532 & -3.39313 & 0.49865 \\ \mathrm{C} & 0.99837 & -3.02173 & 1.81941 \\ \mathrm{C} & 1.72071 & -2.10276 & 2.58487 \\ \mathrm{C} & 1.02061 & -1.06195 & 3.31736 \\ \mathrm{C} & -0.36814 & -0.96311 & 3.21399 \\ \mathrm{C} & -1.03354 & 0.31348 & 3.09361 \\ \mathrm{C} & -0.24563 & 1.46721 & 3.08885 \\ \mathrm{C} & 1.20205 & 1.38439 & 3.17302 \\ \mathrm{C} & 1.82776 & 0.14120 & 3.27873 \\ \mathrm{C} & 3.02072 & -0.14413 & 2.50316 \\ \mathrm{C} & 2.95170 & -1.53320 & 2.06999 \\ & 3.40853 & -1.89271 & 0.79963\end{array}$




$\begin{array}{lrrr}\mathrm{C} & 3.94126 & -0.87690 & -0.09345 \\ \mathrm{C} & 3.51275 & -1.19619 & -1.44453 \\ \mathrm{C} & 3.16196 & -0.16823 & -2.32498 \\ \mathrm{C} & 3.24193 & 1.21930 & -1.89268 \\ \mathrm{C} & 2.12913 & 1.93862 & -2.48297 \\ \mathrm{C} & 1.48016 & 2.94352 & -1.76426 \\ \mathrm{C} & 0.03069 & 3.04681 & -1.80075 \\ \mathrm{C} & -0.42037 & 3.44075 & -0.48944 \\ \mathrm{C} & 0.73825 & 3.55814 & 0.37591 \\ \mathrm{C} & 1.91925 & 3.26230 & -0.41361 \\ \mathrm{C} & 2.98262 & 2.56289 & 0.16085 \\ \mathrm{C} & 3.66062 & 1.52387 & -0.59497 \\ \mathrm{C} & 4.01095 & 0.45422 & 0.32286 \\ \mathrm{C} & 3.54184 & 0.82795 & 1.64692 \\ \mathrm{C} & 2.90536 & 2.12988 & 1.54805 \\ \mathrm{C} & 1.76076 & 2.40563 & 2.30079 \\ \mathrm{C} & 0.65898 & 3.13488 & 1.70166 \\ \mathrm{C} & -0.57561 & 2.54805 & 2.19538 \\ \mathrm{C} & -1.67741 & 2.40998 & 1.36054 \\ \mathrm{C} & -1.61757 & 2.91182 & -0.00358 \\ \mathrm{C} & -2.46619 & 2.07038 & -0.81000 \\ \mathrm{C} & -3.33720 & 1.29258 & 0.12870 \\ \mathrm{C} & -2.55134 & 1.24343 & 1.40342 \\ \mathrm{C} & -2.22209 & 0.16340 & 2.24309 \\ \mathrm{C} & -4.77653 & 1.24276 & 0.05530 \\ \mathrm{C} & -5.48934 & 0.36248 & 0.86530 \\ \mathrm{H} & -5.26256 & 1.71827 & -0.79606 \\ \mathrm{H} & -5.10474 & 0.05967 & 1.83673 \\ \mathrm{H} & -6.55337 & 0.22005 & 0.70447 \\ \mathrm{H} & -4.01368 & -1.97313 & 0.61531\end{array}$

(4) Intermediate IM4 $\left(\mathrm{CHCH}_{3} \mathrm{C}_{59} \mathrm{Ni}\right)$

$\begin{array}{lrrr}\mathrm{Ni} & -3.76055 & -0.99902 & -0.17993 \\ \mathrm{C} & -2.45814 & -1.80903 & -1.22345 \\ \mathrm{C} & -1.64564 & -2.83155 & -0.60434 \\ \mathrm{C} & -1.73712 & -2.69911 & 0.84082 \\ \mathrm{C} & -2.60964 & -1.58979 & 1.15831 \\ \mathrm{C} & -2.21885 & -0.66389 & 2.11765 \\ \mathrm{C} & -1.06506 & -0.95405 & 2.94749 \\ \mathrm{C} & -0.28726 & -2.09866 & 2.72729 \\ \mathrm{C} & -0.61388 & -2.97702 & 1.63051 \\ \mathrm{C} & 0.62968 & -3.42132 & 1.02374 \\ \mathrm{C} & 0.71685 & -3.54710 & -0.36152 \\ \mathrm{C} & -0.43667 & -3.23296 & -1.18717 \\ \mathrm{C} & 0.03232 & -2.56079 & -2.37412 \\ \mathrm{C} & -0.69927 & -1.48267 & -2.89042\end{array}$




\begin{tabular}{|c|c|c|c|}
\hline $\mathrm{C}$ & -1.94293 & -1.06282 & -2.27513 \\
\hline $\mathrm{C}$ & -2.01311 & 0.43645 & -2.40285 \\
\hline $\mathrm{C}$ & -0.80219 & 0.84882 & -3.09500 \\
\hline $\mathrm{C}$ & -0.00463 & -0.32049 & -3.39955 \\
\hline $\mathrm{C}$ & 1.38877 & -0.26066 & -3.37729 \\
\hline $\mathrm{C}$ & 2.14676 & -1.35967 & -2.80255 \\
\hline $\mathrm{C}$ & 1.47984 & -2.48432 & -2.31119 \\
\hline $\mathrm{C}$ & 1.90542 & -3.09004 & -1.05940 \\
\hline $\mathrm{C}$ & 2.96825 & -2.53550 & -0.34318 \\
\hline $\mathrm{C}$ & 2.87784 & -2.40507 & 1.10326 \\
\hline $\mathrm{C}$ & 1.72784 & -2.83353 & 1.77010 \\
\hline C & 1.15801 & -2.01874 & 2.83127 \\
\hline $\mathrm{C}$ & 1.77220 & -0.81730 & 3.19154 \\
\hline $\mathrm{C}$ & 0.96150 & 0.35787 & 3.46268 \\
\hline $\mathrm{C}$ & -0.42458 & 0.28747 & 3.32338 \\
\hline $\mathrm{C}$ & -1.16408 & 1.37531 & 2.71914 \\
\hline $\mathrm{C}$ & -0.47762 & 2.51982 & 2.29596 \\
\hline $\mathrm{C}$ & 0.96860 & 2.58577 & 2.40437 \\
\hline $\mathrm{C}$ & 1.67538 & 1.52476 & 2.97282 \\
\hline $\mathrm{C}$ & 2.91386 & 1.06922 & 2.37412 \\
\hline $\mathrm{C}$ & 2.97337 & -0.37982 & 2.50889 \\
\hline $\mathrm{C}$ & 3.51403 & -1.15568 & 1.48042 \\
\hline $\mathrm{C}$ & 4.00141 & -0.51485 & 0.27031 \\
\hline $\mathrm{C}$ & 3.65963 & -1.36661 & -0.85629 \\
\hline $\mathrm{C}$ & 3.25945 & -0.79356 & -2.06621 \\
\hline $\mathrm{C}$ & 3.19990 & 0.65544 & -2.20100 \\
\hline $\mathrm{C}$ & 2.04995 & 0.98290 & -3.01998 \\
\hline $\mathrm{C}$ & 1.28930 & 2.11969 & -2.74112 \\
\hline $\mathrm{C}$ & -0.16005 & 2.05776 & -2.80169 \\
\hline $\mathrm{C}$ & -0.68775 & 2.89661 & -1.75635 \\
\hline C & 0.42810 & 3.45777 & -1.01306 \\
\hline $\mathrm{C}$ & 1.65405 & 2.97928 & -1.62558 \\
\hline $\mathrm{C}$ & 2.75317 & 2.65563 & -0.82645 \\
\hline $\mathrm{C}$ & 3.54216 & 1.47261 & -1.12058 \\
\hline $\mathrm{C}$ & 3.94474 & 0.87417 & 0.14107 \\
\hline $\mathrm{C}$ & 3.39585 & 1.68384 & 1.21574 \\
\hline $\mathrm{C}$ & 2.66290 & 2.78674 & 0.62003 \\
\hline C & 1.47760 & 3.23611 & 1.20669 \\
\hline $\mathrm{C}$ & 0.34191 & 3.58358 & 0.37217 \\
\hline $\mathrm{C}$ & -0.86349 & 3.15319 & 1.06075 \\
\hline $\mathrm{C}$ & -1.94231 & 2.63435 & 0.34828 \\
\hline $\mathrm{C}$ & -1.85204 & 2.50189 & -1.10089 \\
\hline C & -2.58845 & 1.31609 & -1.49119 \\
\hline $\mathrm{C}$ & -3.51159 & 1.01402 & -0.33399 \\
\hline $\mathrm{C}$ & -2.73695 & 1.53241 & 0.85305 \\
\hline $\mathrm{C}$ & -2.28551 & 0.83376 & 1.96877 \\
\hline $\mathrm{C}$ & -4.88458 & 0.82729 & -0.42228 \\
\hline C & -5.86043 & 1.00794 & 0.71647 \\
\hline $\mathrm{H}$ & -5.30976 & 0.76406 & -1.42543 \\
\hline
\end{tabular}




$\begin{array}{llll}\mathrm{H} & -6.42988 & 1.93865 & 0.58249 \\ \mathrm{H} & -5.35169 & 1.06460 & 1.68311 \\ \mathrm{H} & -6.59420 & 0.19358 & 0.75826\end{array}$

(5) Intermediate IM5 $\left(\mathrm{C}_{2} \mathrm{H}_{4} \_\mathrm{C}_{59} \mathrm{Ni}\right)$

$\begin{array}{lrrr}\mathrm{Ni} & -3.94124 & 0.28522 & 0.00005 \\ \mathrm{C} & -2.84956 & -0.71598 & -1.18756 \\ \mathrm{C} & -2.38862 & -2.00074 & -0.72538 \\ \mathrm{C} & -2.38859 & -2.00068 & 0.72558 \\ \mathrm{C} & -2.84949 & -0.71588 & 1.18769 \\ \mathrm{C} & -2.18418 & -0.06679 & 2.21705 \\ \mathrm{C} & -1.15471 & -0.78084 & 2.95117 \\ \mathrm{C} & -0.76629 & -2.07020 & 2.57544 \\ \mathrm{C} & -1.37852 & -2.68478 & 1.42197 \\ \mathrm{C} & -0.34699 & -3.40449 & 0.69754 \\ \mathrm{C} & -0.34701 & -3.40453 & -0.69734 \\ \mathrm{C} & -1.37857 & -2.68487 & -1.42177 \\ \mathrm{C} & -0.76637 & -2.07034 & -2.57529 \\ \mathrm{C} & -1.15482 & -0.78100 & -2.95107 \\ \mathrm{C} & -2.18427 & -0.06693 & -2.21696 \\ \mathrm{C} & -1.80920 & 1.37170 & -2.21829 \\ \mathrm{C} & -0.56249 & 1.48864 & -2.95033 \\ \mathrm{C} & -0.16148 & 0.17304 & -3.40466 \\ \mathrm{C} & 1.18629 & -0.18197 & -3.45029 \\ \mathrm{C} & 1.59804 & -1.50855 & -3.02066 \\ \mathrm{C} & 0.64120 & -2.43095 & -2.59191 \\ \mathrm{C} & 0.90264 & -3.25497 & -1.42391 \\ \mathrm{C} & 2.10478 & -3.11865 & -0.72754 \\ \mathrm{C} & 2.10480 & -3.11861 & 0.72765 \\ \mathrm{C} & 0.90268 & -3.25490 & 1.42407 \\ \mathrm{C} & 0.64129 & -2.43081 & 2.59203 \\ \mathrm{C} & 1.59814 & -1.50839 & 3.02071 \\ \mathrm{C} & 1.18641 & -0.18179 & 3.45028 \\ \mathrm{C} & -0.16136 & 0.17322 & 3.40469 \\ \mathrm{C} & -0.56238 & 1.48881 & 2.95030 \\ \mathrm{C} & 0.41652 & 2.41592 & 2.57277 \\ \mathrm{C} & 1.81682 & 2.04467 & 2.59050 \\ \mathrm{C} & 2.19635 & 0.76942 & 3.01758 \\ \mathrm{C} & 3.22117 & 0.03639 & 2.30189 \\ \mathrm{C} & 2.84980 & -1.37175 & 2.30171 \\ \mathrm{C} & 3.09901 & -2.15964 & 1.17518 \\ \mathrm{C} & 2.84971 & -1.56760 & -0.00001 \\ \mathrm{C} & -1.37189 & 0.03627 & -2.30198 \\ \mathrm{C} & 0.76926 & -3.01767\end{array}$




$\begin{array}{lrrr}\mathrm{C} & 1.81673 & 2.04453 & -2.59063 \\ \mathrm{C} & 0.41643 & 2.41578 & -2.57288 \\ \mathrm{C} & 0.18856 & 3.25866 & -1.41884 \\ \mathrm{C} & 1.44414 & 3.39160 & -0.69793 \\ \mathrm{C} & 2.45314 & 2.63957 & -1.42470 \\ \mathrm{C} & 3.43028 & 1.92635 & -0.72747 \\ \mathrm{C} & 3.82321 & 0.60075 & -1.17494 \\ \mathrm{C} & 4.07052 & -0.21677 & -0.00005 \\ \mathrm{C} & 3.82325 & 0.60081 & 1.17481 \\ \mathrm{C} & 3.43030 & 1.92639 & 0.72727 \\ \mathrm{C} & 2.45319 & 2.63965 & 1.42451 \\ \mathrm{C} & 1.44417 & 3.39164 & 0.69773 \\ \mathrm{C} & 0.18861 & 3.25874 & 1.41869 \\ \mathrm{C} & -1.01073 & 3.14083 & 0.72391 \\ \mathrm{C} & -1.01076 & 3.14078 & -0.72401 \\ \mathrm{C} & -2.06766 & 2.22958 & -1.14522 \\ \mathrm{C} & -2.89346 & 1.84169 & 0.00003 \\ \mathrm{C} & -2.06761 & 2.22966 & 1.14523 \\ \mathrm{C} & -1.80912 & 1.37183 & 2.21832 \\ \mathrm{C} & -6.12752 & 0.34018 & -0.00013 \\ \mathrm{C} & -5.74343 & -0.97667 & -0.00014 \\ \mathrm{H} & -5.67873 & -1.54448 & -0.92375 \\ \mathrm{H} & -5.67886 & -1.54453 & 0.92346 \\ \mathrm{H} & -6.35696 & 0.86723 & -0.92306 \\ \mathrm{H} & -6.35710 & 0.86718 & 0.92279\end{array}$

(6) Intermediate IM6 $\left(\mathrm{C}_{2} \mathrm{H}_{4} \_2 \mathrm{H} \_\mathrm{C}_{59} \mathrm{Ni}\right)$

$\begin{array}{cccc}\mathrm{Ni} & -3.97265 & -0.52038 & -0.37409 \\ \mathrm{C} & -2.62996 & -0.84316 & -1.70605 \\ \mathrm{C} & -1.94314 & -2.09434 & -1.45521 \\ \mathrm{C} & -2.06275 & -2.51018 & -0.08839 \\ \mathrm{C} & -3.03584 & -1.72458 & 0.78523 \\ \mathrm{C} & -2.30465 & -0.99864 & 1.88528 \\ \mathrm{C} & -1.22709 & -1.71999 & 2.54763 \\ \mathrm{C} & -0.59069 & -2.77184 & 1.88012 \\ \mathrm{C} & -1.00759 & -3.17100 & 0.53095 \\ \mathrm{C} & 0.18582 & -3.51777 & -0.20111 \\ \mathrm{C} & 0.29435 & -3.18828 & -1.54973 \\ \mathrm{C} & -0.77863 & -2.45246 & -2.17478 \\ \mathrm{C} & -0.19813 & -1.51243 & -3.09385 \\ \mathrm{C} & -0.77126 & -0.23768 & -3.20703 \\ \mathrm{C} & -1.97630 & 0.10557 & -2.49083 \\ \mathrm{C} & -1.86299 & 1.55306 & -2.12603 \\ \mathrm{C} & -0.57678 & 2.01937 & -2.64469 \\ \mathrm{C} & 0.08003 & 0.92646 & -3.32923 \\ \mathrm{C} & 1.46912 & 0.78849 & -3.30151\end{array}$




\begin{tabular}{|c|c|c|c|}
\hline C & 2.06396 & -0.52868 & -3.14107 \\
\hline $\mathrm{C}$ & 1.24503 & -1.65451 & -3.04506 \\
\hline $\mathrm{C}$ & 1.55329 & -2.69512 & -2.07665 \\
\hline $\mathrm{C}$ & 2.65851 & -2.56390 & -1.23457 \\
\hline $\mathrm{C}$ & 2.54540 & -2.91792 & 0.17333 \\
\hline $\mathrm{C}$ & 1.33360 & -3.38321 & 0.67969 \\
\hline $\mathrm{C}$ & 0.85136 & -2.92399 & 1.96997 \\
\hline $\mathrm{C}$ & 1.60964 & -2.01124 & 2.70721 \\
\hline $\mathrm{C}$ & 0.95357 & -0.91210 & 3.39399 \\
\hline $\mathrm{C}$ & -0.43365 & -0.76855 & 3.29648 \\
\hline $\mathrm{C}$ & -1.02103 & 0.53136 & 3.08057 \\
\hline C & -0.18900 & 1.64020 & 3.01139 \\
\hline $\mathrm{C}$ & 1.24809 & 1.51228 & 3.12318 \\
\hline $\mathrm{C}$ & 1.81450 & 0.24767 & 3.29960 \\
\hline $\mathrm{C}$ & 3.00466 & -0.12477 & 2.55318 \\
\hline $\mathrm{C}$ & 2.87829 & -1.52724 & 2.18743 \\
\hline $\mathrm{C}$ & 3.33025 & -1.96430 & 0.94166 \\
\hline $\mathrm{C}$ & 3.92717 & -1.02254 & 0.00883 \\
\hline $\mathrm{C}$ & 3.50514 & -1.39017 & -1.33126 \\
\hline $\mathrm{C}$ & 3.21709 & -0.39351 & -2.27201 \\
\hline $\mathrm{C}$ & 3.34558 & 1.00606 & -1.90613 \\
\hline $\mathrm{C}$ & 2.27243 & 1.73961 & -2.55504 \\
\hline $\mathrm{C}$ & 1.65029 & 2.79933 & -1.89682 \\
\hline $\mathrm{C}$ & 0.20571 & 2.95815 & -1.96356 \\
\hline $\mathrm{C}$ & -0.24750 & 3.43252 & -0.68096 \\
\hline $\mathrm{C}$ & 0.90169 & 3.54815 & 0.19500 \\
\hline $\mathrm{C}$ & 2.08250 & 3.16952 & -0.55619 \\
\hline $\mathrm{C}$ & 3.10727 & 2.45938 & 0.07339 \\
\hline $\mathrm{C}$ & 3.75536 & 1.35794 & -0.61621 \\
\hline $\mathrm{C}$ & 4.04681 & 0.32367 & 0.35915 \\
\hline $\mathrm{C}$ & 3.57445 & 0.78038 & 1.65685 \\
\hline $\mathrm{C}$ & 2.99407 & 2.10201 & 1.48118 \\
\hline $\mathrm{C}$ & 1.85668 & 2.46387 & 2.20274 \\
\hline $\mathrm{C}$ & 0.79247 & 3.20150 & 1.54494 \\
\hline $\mathrm{C}$ & -0.46245 & 2.68002 & 2.03209 \\
\hline $\mathrm{C}$ & -1.54999 & 2.55036 & 1.18477 \\
\hline $\mathrm{C}$ & -1.46886 & 2.96923 & -0.18992 \\
\hline C & -2.36225 & 2.10402 & -0.94552 \\
\hline C & -3.25097 & 1.39259 & 0.03274 \\
\hline $\mathrm{C}$ & -2.50553 & 1.44469 & 1.26854 \\
\hline $\mathrm{C}$ & -2.18655 & 0.37872 & 2.18309 \\
\hline $\mathrm{C}$ & -4.80880 & 1.46827 & 0.01654 \\
\hline $\mathrm{C}$ & -5.31255 & 0.28587 & 0.81925 \\
\hline $\mathrm{H}$ & -3.78098 & -2.39348 & 1.23438 \\
\hline $\mathrm{H}$ & -4.82901 & -1.70315 & -0.56946 \\
\hline $\mathrm{H}$ & -5.15906 & 1.40495 & -1.03179 \\
\hline $\mathrm{H}$ & -5.13172 & 2.46604 & 0.35991 \\
\hline $\mathrm{H}$ & -5.05884 & 0.32181 & 1.87833 \\
\hline $\mathrm{H}$ & -6.34150 & -0.02281 & 0.64349 \\
\hline
\end{tabular}


(7) Intermediate IM7 $\left(\mathrm{C}_{2} \mathrm{H}_{5} \_\mathrm{HC} 1 \mathrm{C}_{59} \mathrm{Ni}\right)$

\begin{tabular}{|c|c|c|c|}
\hline $\mathrm{Ni}$ & -3.81123 & -0.73349 & -0.51760 \\
\hline $\mathrm{C}$ & -2.47357 & -0.98548 & -1.73072 \\
\hline $\mathrm{C}$ & -1.74061 & -2.22846 & -1.54118 \\
\hline $\mathrm{C}$ & -1.83292 & -2.79289 & -0.19236 \\
\hline C & -2.80162 & -2.10354 & 0.69048 \\
\hline C & -2.24904 & -1.46050 & 1.82962 \\
\hline C & -1.03321 & -2.01453 & 2.37922 \\
\hline $\mathrm{C}$ & -0.30433 & -3.01013 & 1.72945 \\
\hline $\mathrm{C}$ & -0.68148 & -3.34308 & 0.36709 \\
\hline $\mathrm{C}$ & 0.53673 & -3.52364 & -0.38888 \\
\hline $\mathrm{C}$ & 0.59736 & -3.09325 & -1.70811 \\
\hline $\mathrm{C}$ & -0.55150 & -2.42090 & -2.28651 \\
\hline C & -0.05169 & -1.37972 & -3.15142 \\
\hline C & -0.72927 & -0.15952 & -3.23391 \\
\hline C & -1.94904 & 0.04485 & -2.49497 \\
\hline C & -1.96891 & 1.48017 & -2.07005 \\
\hline $\mathrm{C}$ & -0.74123 & 2.08339 & -2.55131 \\
\hline $\mathrm{C}$ & 0.01394 & 1.08610 & -3.28182 \\
\hline $\mathrm{C}$ & 1.40634 & 1.07391 & -3.23824 \\
\hline $\mathrm{C}$ & 2.11414 & -0.19258 & -3.13956 \\
\hline C & 1.39932 & -1.39287 & -3.10634 \\
\hline C & 1.80387 & -2.45286 & -2.20191 \\
\hline C & 2.89596 & -2.26620 & -1.35093 \\
\hline $\mathrm{C}$ & 2.82663 & -2.71550 & 0.03178 \\
\hline $\mathrm{C}$ & 1.66792 & -3.33241 & 0.50128 \\
\hline $\mathrm{C}$ & 1.14216 & -3.00371 & 1.81522 \\
\hline C & 1.79932 & -2.05595 & 2.60534 \\
\hline C & 1.02699 & -1.05979 & 3.32630 \\
\hline $\mathrm{C}$ & -0.36274 & -1.03588 & 3.19343 \\
\hline C & -1.09330 & 0.20231 & 3.06086 \\
\hline C & -0.37149 & 1.39731 & 3.08211 \\
\hline C & 1.07623 & 1.39346 & 3.20102 \\
\hline $\mathrm{C}$ & 1.76722 & 0.18605 & 3.31418 \\
\hline $\mathrm{C}$ & 2.99153 & -0.02739 & 2.56491 \\
\hline $\mathrm{C}$ & 3.00714 & -1.41468 & 2.12020 \\
\hline $\mathrm{C}$ & 3.51319 & -1.73735 & 0.85880 \\
\hline $\mathrm{C}$ & 4.01044 & -0.68607 & -0.01354 \\
\hline $\mathrm{C}$ & 3.63043 & -1.01476 & -1.37706 \\
\hline $\mathrm{C}$ & 3.24860 & 0.00098 & -2.25878 \\
\hline $\mathrm{C}$ & 3.24489 & 1.38699 & -1.81477 \\
\hline $\mathrm{C}$ & 2.11162 & 2.05328 & -2.42810 \\
\hline $\mathrm{C}$ & 1.39176 & 3.01523 & -1.71750 \\
\hline C & -0.05953 & 3.04394 & -1.79232 \\
\hline $\mathrm{C}$ & -0.56578 & 3.39109 & -0.48739 \\
\hline
\end{tabular}




$\begin{array}{lrrr}\mathrm{C} & 0.56206 & 3.56456 & 0.40852 \\ \mathrm{C} & 1.77765 & 3.34182 & -0.35236 \\ \mathrm{C} & 2.86240 & 2.69415 & 0.24277 \\ \mathrm{C} & 3.61371 & 1.70050 & -0.50418 \\ \mathrm{C} & 3.99979 & 0.64322 & 0.41396 \\ \mathrm{C} & 3.47943 & 0.97909 & 1.72910 \\ \mathrm{C} & 2.77469 & 2.24491 & 1.62404 \\ \mathrm{C} & 1.59809 & 2.45076 & 2.34960 \\ \mathrm{C} & 0.47188 & 3.12195 & 1.72753 \\ \mathrm{C} & -0.74010 & 2.46303 & 2.18629 \\ \mathrm{C} & -1.80762 & 2.26234 & 1.31839 \\ \mathrm{C} & -1.74300 & 2.78680 & -0.03636 \\ \mathrm{C} & -2.51472 & 1.90014 & -0.86901 \\ \mathrm{C} & -3.38934 & 1.04962 & 0.01803 \\ \mathrm{C} & -2.61661 & 1.04850 & 1.32293 \\ \mathrm{C} & -2.25170 & -0.01094 & 2.17823 \\ \mathrm{C} & -4.87674 & 1.47348 & 0.12664 \\ \mathrm{C} & -5.73601 & 0.41357 & 0.84508 \\ \mathrm{H} & -3.88987 & -2.31890 & 0.55877 \\ \mathrm{H} & -5.27663 & 1.66497 & -0.87551 \\ \mathrm{H} & -4.94801 & 2.42195 & 0.68015 \\ \mathrm{H} & -5.76201 & -0.56294 & 0.31435 \\ \mathrm{H} & -6.78449 & 0.72518 & 0.89346 \\ \mathrm{H} & -5.38258 & 0.22987 & 1.86456\end{array}$

(8) Intermediate IM8 $\left(\mathrm{C}_{2} \mathrm{H}_{6} \_\mathrm{C}_{59} \mathrm{Ni}\right)$

$\begin{array}{cccc}\mathrm{Ni} & 3.80030 & 0.24296 & 0.08134 \\ \mathrm{C} & 2.74915 & -0.80725 & 1.23297 \\ \mathrm{C} & 2.28210 & -2.05994 & 0.69744 \\ \mathrm{C} & 2.30464 & -2.00282 & -0.75663 \\ \mathrm{C} & 2.78483 & -0.71167 & -1.18005 \\ \mathrm{C} & 2.13853 & -0.01696 & -2.18893 \\ \mathrm{C} & 1.11817 & -0.69120 & -2.96458 \\ \mathrm{C} & 0.71064 & -1.98808 & -2.63180 \\ \mathrm{C} & 1.29954 & -2.65151 & -1.49381 \\ \mathrm{C} & 0.24678 & -3.38669 & -0.81177 \\ \mathrm{C} & 0.22387 & -3.43971 & 0.58028 \\ \mathrm{C} & 1.25287 & -2.75995 & 1.35010 \\ \mathrm{C} & 0.62672 & -2.18214 & 2.51441 \\ \mathrm{C} & 1.02211 & -0.91312 & 2.95472 \\ \mathrm{C} & 2.06788 & -0.18582 & 2.26616 \\ \mathrm{C} & 1.71794 & 1.26140 & 2.31416 \\ \mathrm{C} & 0.46136 & 1.36607 & 3.02226 \\ \mathrm{C} & 0.03390 & 0.03818 & 3.42232 \\ \mathrm{C} & -1.31803 & -0.30093 & 3.42883 \\ \mathrm{C} & -1.73632 & -1.60603 & 2.94030\end{array}$




\begin{tabular}{|c|c|c|c|}
\hline C & -0.78394 & -2.52617 & 2.49459 \\
\hline $\mathrm{C}$ & -1.03538 & -3.30180 & 1.29174 \\
\hline $\mathrm{C}$ & -2.22448 & -3.12387 & 0.58225 \\
\hline $\mathrm{C}$ & -2.20079 & -3.06969 & -0.87104 \\
\hline $\mathrm{C}$ & -0.98861 & -3.19484 & -1.55225 \\
\hline $\mathrm{C}$ & -0.69920 & -2.33197 & -2.68456 \\
\hline $\mathrm{C}$ & -1.63809 & -1.38113 & -3.09290 \\
\hline $\mathrm{C}$ & -1.20609 & -0.04360 & -3.46950 \\
\hline $\mathrm{C}$ & 0.14442 & 0.29273 & -3.39348 \\
\hline $\mathrm{C}$ & 0.55650 & 1.58709 & -2.88323 \\
\hline $\mathrm{C}$ & -0.41897 & 2.51323 & -2.48764 \\
\hline $\mathrm{C}$ & -1.82175 & 2.15612 & -2.53665 \\
\hline $\mathrm{C}$ & -2.21196 & 0.90301 & -3.01871 \\
\hline $\mathrm{C}$ & -3.25373 & 0.15585 & -2.34436 \\
\hline $\mathrm{C}$ & -2.89976 & -1.25664 & -2.39111 \\
\hline $\mathrm{C}$ & -3.17598 & -2.08239 & -1.29830 \\
\hline $\mathrm{C}$ & -3.80564 & -1.52810 & -0.11197 \\
\hline $\mathrm{C}$ & -3.21395 & -2.16958 & 1.04946 \\
\hline $\mathrm{C}$ & -2.97419 & -1.42773 & 2.20892 \\
\hline $\mathrm{C}$ & -3.32820 & -0.01527 & 2.25584 \\
\hline $\mathrm{C}$ & -2.30966 & 0.67806 & 3.01715 \\
\hline $\mathrm{C}$ & -1.90541 & 1.96249 & 2.64235 \\
\hline $\mathrm{C}$ & -0.50199 & 2.32040 & 2.66548 \\
\hline $\mathrm{C}$ & -0.24316 & 3.19426 & 1.54536 \\
\hline $\mathrm{C}$ & -1.48392 & 3.37068 & 0.80641 \\
\hline $\mathrm{C}$ & -2.51506 & 2.60719 & 1.48856 \\
\hline $\mathrm{C}$ & -3.48967 & 1.93316 & 0.75001 \\
\hline $\mathrm{C}$ & -3.90645 & 0.59750 & 1.14147 \\
\hline $\mathrm{C}$ & -4.14267 & -0.17313 & -0.06703 \\
\hline $\mathrm{C}$ & -3.86799 & 0.68488 & -1.20660 \\
\hline $\mathrm{C}$ & -3.46601 & 1.98719 & -0.70368 \\
\hline $\mathrm{C}$ & -2.46886 & 2.71337 & -1.35794 \\
\hline $\mathrm{C}$ & -1.46163 & 3.42302 & -0.58770 \\
\hline $\mathrm{C}$ & -0.19749 & 3.30103 & -1.29706 \\
\hline $\mathrm{C}$ & 0.98811 & 3.13552 & -0.58490 \\
\hline $\mathrm{C}$ & 0.96520 & 3.08073 & 0.86152 \\
\hline $\mathrm{C}$ & 2.00169 & 2.14144 & 1.26598 \\
\hline $\mathrm{C}$ & 2.87171 & 1.84189 & 0.11947 \\
\hline $\mathrm{C}$ & 2.03800 & 2.22807 & -1.02537 \\
\hline $\mathrm{C}$ & 1.78911 & 1.42890 & -2.14445 \\
\hline $\mathrm{C}$ & 6.37635 & -0.69197 & 0.22769 \\
\hline $\mathrm{C}$ & 7.46748 & 0.22510 & -0.32972 \\
\hline $\mathrm{H}$ & 7.94346 & -0.22012 & -1.20988 \\
\hline $\mathrm{H}$ & 8.24662 & 0.40466 & 0.41875 \\
\hline $\mathrm{H}$ & 7.05631 & 1.19657 & -0.62482 \\
\hline $\mathrm{H}$ & 6.76549 & -1.66877 & 0.53617 \\
\hline $\mathrm{H}$ & 5.60961 & -0.90591 & -0.53875 \\
\hline $\mathrm{H}$ & 5.90260 & -0.25030 & 1.11822 \\
\hline
\end{tabular}




\section{Intermediate structures observed over Pd/CNT during acetylene hydrogenation.}

(0) Pd/CNT

\begin{tabular}{|c|c|c|c|}
\hline $\mathrm{H}$ & -4.49124 & 2.15503 & -3.49135 \\
\hline $\mathrm{H}$ & -5.06194 & 0.05737 & -3.51398 \\
\hline C & -4.85070 & -0.42626 & -2.56550 \\
\hline C & -4.48543 & -1.73989 & -2.55277 \\
\hline $\mathrm{H}$ & -4.41269 & -2.27980 & -3.49157 \\
\hline & -2.83808 & -3.77276 & -3.43596 \\
\hline $\mathrm{C}$ & -2.33569 & -3.80044 & -2.47428 \\
\hline C & -1.01134 & -4.12525 & -2.42302 \\
\hline $\mathrm{H}$ & -0.48706 & -4.35313 & -3.34561 \\
\hline $\mathrm{H}$ & 1.57222 & -3.76286 & -3.26883 \\
\hline C & 1.86081 & -3.34180 & -2.31129 \\
\hline C & 2.86063 & -2.40991 & -2.27016 \\
\hline $\mathrm{H}$ & 3.34371 & -2.11372 & -3.19582 \\
\hline $\mathrm{H}$ & 4.02297 & -0.04265 & -3.19165 \\
\hline $\mathrm{C}$ & 3.72983 & 0.43193 & -2.26063 \\
\hline $\mathrm{C}$ & 3.28303 & 1.71840 & -2.27482 \\
\hline $\mathrm{H}$ & 3.24142 & 2.25139 & -3.21902 \\
\hline $\mathrm{H}$ & 1.68710 & 3.71430 & -3.27470 \\
\hline $\mathrm{C}$ & 1.11656 & 3.75449 & -2.35254 \\
\hline $\mathrm{C}$ & -0.19933 & 4.10846 & -2.40007 \\
\hline $\mathrm{H}$ & -0.65131 & 4.34031 & -3.35915 \\
\hline $\mathrm{H}$ & -2.74944 & 3.84147 & -3.42958 \\
\hline $\mathrm{C}$ & -3.08741 & 3.39326 & -2.50068 \\
\hline $\mathrm{C}$ & -4.06729 & 2.44585 & -2.53543 \\
\hline $\mathrm{C}$ & -4.43587 & 1.72891 & -1.34264 \\
\hline $\mathrm{C}$ & -4.81169 & 0.35579 & -1.35722 \\
\hline $\mathrm{C}$ & -4.82520 & -0.35690 & -0.12170 \\
\hline $\mathrm{C}$ & -4.44100 & -1.74441 & -0.10882 \\
\hline $\mathrm{C}$ & -4.05595 & -2.36928 & -1.33131 \\
\hline $\mathrm{C}$ & -3.02670 & -3.35401 & -1.29437 \\
\hline $\mathrm{C}$ & -2.43352 & -3.66965 & -0.03594 \\
\hline $\mathrm{C}$ & -1.03567 & -4.01003 & 0.01631 \\
\hline $\mathrm{C}$ & -0.27798 & -4.03106 & -1.18978 \\
\hline $\mathrm{C}$ & 1.09798 & -3.65226 & -1.13707 \\
\hline $\mathrm{C}$ & 1.65238 & -3.26969 & 0.12158 \\
\hline $\mathrm{C}$ & 2.70039 & -2.28431 & 0.16213 \\
\hline $\mathrm{C}$ & 3.17964 & -1.72421 & -1.05422 \\
\hline $\mathrm{C}$ & 3.67912 & -0.36709 & -1.04228 \\
\hline $\mathrm{C}$ & 3.58814 & 0.38035 & 0.20043 \\
\hline $\mathrm{C}$ & 3.09135 & 1.74929 & 0.17089 \\
\hline $\mathrm{C}$ & 2.75756 & 2.33792 & -1.08190 \\
\hline $\mathrm{C}$ & 1.71548 & 3.30782 & -1.12281 \\
\hline $\mathrm{C}$ & 1.03685 & 3.63632 & 0.08843 \\
\hline $\mathrm{C}$ & -0.35376 & 4.00465 & 0.03805 \\
\hline
\end{tabular}




\begin{tabular}{clll}
$\mathrm{C}$ & -1.02205 & 4.03862 & -1.22214 \\
$\mathrm{C}$ & -2.40313 & 3.69564 & -1.27058 \\
$\mathrm{C}$ & -3.05448 & 3.32488 & -0.05695 \\
$\mathrm{C}$ & -4.08969 & 2.32474 & -0.09374 \\
$\mathrm{C}$ & -4.52131 & 1.72630 & 1.12747 \\
$\mathrm{C}$ & -4.89880 & 0.35435 & 1.11297 \\
$\mathrm{C}$ & -5.02707 & -0.42961 & 2.31458 \\
$\mathrm{C}$ & -4.66375 & -1.74332 & 2.32681 \\
$\mathrm{C}$ & -4.14561 & -2.37142 & 1.13867 \\
$\mathrm{C}$ & -3.11954 & -3.35613 & 1.17571 \\
$\mathrm{C}$ & -2.52100 & -3.80970 & 2.40460 \\
$\mathrm{C}$ & -1.19743 & -4.13159 & 2.45437 \\
$\mathrm{C}$ & -0.37049 & -4.02820 & 1.28016 \\
$\mathrm{C}$ & 0.99827 & -3.64918 & 1.33415 \\
$\mathrm{C}$ & 1.67214 & -3.34335 & 2.56938 \\
$\mathrm{C}$ & 2.65427 & -2.39926 & 2.61088 \\
$\mathrm{C}$ & 3.05014 & -1.69332 & 1.42165 \\
$\mathrm{C}$ & 3.49769 & -0.34883 & 1.44463 \\
$\mathrm{C}$ & 3.51362 & 0.44753 & 2.65184 \\
$\mathrm{C}$ & 3.12168 & 1.75039 & 2.62127 \\
$\mathrm{C}$ & 2.67736 & 2.35955 & 1.38782 \\
$\mathrm{C}$ & 1.63249 & 3.32469 & 1.34774 \\
$\mathrm{C}$ & 0.94657 & 3.77909 & 2.52871 \\
$\mathrm{C}$ & -0.37159 & 4.12433 & 2.48077 \\
$\mathrm{C}$ & -1.10957 & 4.04303 & 1.24813 \\
$\mathrm{C}$ & -2.48909 & 3.69536 & 1.19960 \\
$\mathrm{C}$ & -3.25645 & 3.38935 & 2.37911 \\
$\mathrm{C}$ & -4.23518 & 2.44091 & 2.34447 \\
$\mathrm{H}$ & -4.72286 & 2.14725 & 3.26859 \\
$\mathrm{H}$ & -5.30767 & 0.05335 & 3.24531 \\
$\mathrm{H}$ & -4.66148 & -2.28507 & 3.26734 \\
$\mathrm{H}$ & -3.09823 & -3.79134 & 3.32357 \\
$\mathrm{H}$ & -0.74455 & -4.36533 & 3.41263 \\
$\mathrm{H}$ & 1.32481 & -3.78837 & 3.49616 \\
$\mathrm{H}$ & 3.06990 & -2.10780 & 3.57036 \\
$\mathrm{H}$ & 3.04028 & 2.30204 & 3.55227 \\
$\mathrm{H}$ & 1.45132 & 3.74919 & 3.48892 \\
$\mathrm{H}$ & -0.89094 & 4.36142 & 3.40383 \\
$\mathrm{H}$ & -2.98307 & 3.83508 & 3.33018 \\
$\mathrm{H}$ & 3.72610 & -0.02450 & 3.60600 \\
\hline
\end{tabular}

(1) Intermediate IM1 $\left(\mathrm{C}_{2} \mathrm{H}_{2} \mathrm{Pd} / \mathrm{CNT}\right)$

$\begin{array}{llll}\mathrm{H} & -4.91601 & 1.08544 & -3.73150 \\ \mathrm{H} & -4.91608 & -1.08540 & -3.73151 \\ \mathrm{C} & -4.64923 & -1.48815 & -2.75957\end{array}$




\begin{tabular}{|c|c|c|c|}
\hline C & -3.95887 & -2.66141 & -2.69007 \\
\hline $\mathrm{H}$ & -3.68795 & -3.17348 & -3.60792 \\
\hline $\mathrm{H}$ & -1.79996 & -4.22126 & -3.41675 \\
\hline $\mathrm{C}$ & -1.37015 & -4.11099 & -2.42625 \\
\hline $\mathrm{C}$ & -0.01485 & -4.09862 & -2.28548 \\
\hline $\mathrm{H}$ & 0.61044 & -4.20172 & -3.16648 \\
\hline $\mathrm{H}$ & 2.45241 & -3.13227 & -2.97553 \\
\hline $\mathrm{C}$ & 2.55911 & -2.63475 & -2.01715 \\
\hline $\mathrm{C}$ & 3.28972 & -1.49097 & -1.94515 \\
\hline $\mathrm{H}$ & 3.74776 & -1.09417 & -2.84442 \\
\hline $\mathrm{H}$ & 3.74739 & 1.09389 & -2.84450 \\
\hline $\mathrm{C}$ & 3.28954 & 1.49076 & -1.94516 \\
\hline $\mathrm{C}$ & 2.55893 & 2.63456 & -2.01715 \\
\hline $\mathrm{H}$ & 2.45215 & 3.13201 & -2.97555 \\
\hline $\mathrm{H}$ & 0.61042 & 4.20183 & -3.16642 \\
\hline $\mathrm{C}$ & -0.01487 & 4.09868 & -2.28542 \\
\hline $\mathrm{C}$ & -1.37017 & 4.11110 & -2.42619 \\
\hline $\mathrm{H}$ & -1.79999 & 4.22145 & -3.41668 \\
\hline $\mathrm{H}$ & -3.68791 & 3.17353 & -3.60787 \\
\hline $\mathrm{C}$ & -3.95886 & 2.66147 & -2.69003 \\
\hline $\mathrm{C}$ & -4.64921 & 1.48820 & -2.75955 \\
\hline $\mathrm{C}$ & -4.89572 & 0.71139 & -1.57208 \\
\hline $\mathrm{C}$ & -4.89572 & -0.71134 & -1.57209 \\
\hline $\mathrm{C}$ & -4.80598 & -1.39090 & -0.32056 \\
\hline $\mathrm{C}$ & -4.07828 & -2.63125 & -0.24710 \\
\hline $\mathrm{C}$ & -3.46428 & -3.14743 & -1.42775 \\
\hline $\mathrm{C}$ & -2.22813 & -3.83966 & -1.30161 \\
\hline $\mathrm{C}$ & -1.66222 & -3.99265 & -0.00045 \\
\hline $\mathrm{C}$ & -0.23043 & -3.98247 & 0.14926 \\
\hline $\mathrm{C}$ & 0.58996 & -3.81639 & -1.00869 \\
\hline $\mathrm{C}$ & 1.82030 & -3.11941 & -0.87766 \\
\hline $\mathrm{C}$ & 2.18912 & -2.61493 & 0.40765 \\
\hline $\mathrm{C}$ & 2.95082 & -1.39827 & 0.49559 \\
\hline $\mathrm{C}$ & 3.36761 & -0.72807 & -0.71277 \\
\hline $\mathrm{C}$ & 3.36749 & 0.72792 & -0.71279 \\
\hline $\mathrm{C}$ & 2.95070 & 1.39810 & 0.49558 \\
\hline $\mathrm{C}$ & 2.18903 & 2.61476 & 0.40766 \\
\hline $\mathrm{C}$ & 1.82023 & 3.11928 & -0.87764 \\
\hline $\mathrm{C}$ & 0.58993 & 3.81636 & -1.00865 \\
\hline $\mathrm{C}$ & -0.23044 & 3.98244 & 0.14931 \\
\hline $\mathrm{C}$ & -1.66224 & 3.99267 & -0.00039 \\
\hline $\mathrm{C}$ & -2.22816 & 3.83972 & -1.30155 \\
\hline $\mathrm{C}$ & -3.46430 & 3.14749 & -1.42770 \\
\hline $\mathrm{C}$ & -4.07829 & 2.63129 & -0.24706 \\
\hline $\mathrm{C}$ & -4.80598 & 1.39093 & -0.32054 \\
\hline $\mathrm{C}$ & -5.14628 & 0.71231 & 0.88694 \\
\hline $\mathrm{C}$ & -5.14628 & -0.71230 & 0.88693 \\
\hline $\mathrm{C}$ & -5.14361 & -1.48966 & 2.09829 \\
\hline $\mathrm{C}$ & -4.45639 & -2.66565 & 2.16732 \\
\hline
\end{tabular}




$\begin{array}{clll}\mathrm{C} & -3.71972 & -3.15239 & 1.03074 \\ \mathrm{C} & -2.48391 & -3.85094 & 1.15684 \\ \mathrm{C} & -1.87711 & -4.13276 & 2.43000 \\ \mathrm{C} & -0.52022 & -4.12748 & 2.57052 \\ \mathrm{C} & 0.33362 & -3.83929 & 1.44932 \\ \mathrm{C} & 1.56886 & -3.13761 & 1.58237 \\ \mathrm{C} & 2.05036 & -2.65745 & 2.84745 \\ \mathrm{C} & 2.74314 & -1.48374 & 2.92471 \\ \mathrm{C} & 3.02120 & -0.71704 & 1.74247 \\ \mathrm{C} & 3.02115 & 0.71686 & 1.74246 \\ \mathrm{C} & 2.74307 & 1.48355 & 2.92469 \\ \mathrm{C} & 2.05029 & 2.65727 & 2.84746 \\ \mathrm{C} & 1.56881 & 3.13746 & 1.58240 \\ \mathrm{C} & 0.33361 & 3.83921 & 1.44936 \\ \mathrm{C} & -0.52022 & 4.12743 & 2.57057 \\ \mathrm{C} & -1.87710 & 4.13275 & 2.43006 \\ \mathrm{C} & -2.48392 & 3.85095 & 1.15690 \\ \mathrm{C} & -3.71973 & 3.15240 & 1.03079 \\ \mathrm{C} & -4.45639 & 2.66564 & 2.16737 \\ \mathrm{C} & -5.14361 & 1.48965 & 2.09831 \\ \mathrm{H} & -5.59684 & 1.08626 & 2.99827 \\ \mathrm{H} & -5.59684 & -1.08629 & 2.99825 \\ \mathrm{H} & -4.37527 & -3.17738 & 3.12104 \\ \mathrm{H} & -2.50097 & -4.24286 & 3.31131 \\ \mathrm{H} & -0.09045 & -4.23604 & 3.56114 \\ \mathrm{H} & 1.76074 & -3.16274 & 3.76302 \\ \mathrm{H} & 2.98744 & -1.07597 & 3.90040 \\ \mathrm{H} & 1.76065 & 3.16251 & 3.76305 \\ \mathrm{H} & -0.09043 & 4.23600 & 3.56119 \\ \mathrm{H} & -2.50097 & 4.24289 & 3.31137 \\ \mathrm{H} & -4.37526 & 3.17734 & 3.12109 \\ \mathrm{Pd} & 5.53801 & 0.00011 & -0.49534 \\ \mathrm{C} & 7.56670 & 0.00043 & -1.13735 \\ \mathrm{C} & 7.57740 & 0.00012 & 0.10819 \\ \mathrm{H} & 7.92140 & 0.00062 & -2.14970 \\ \mathrm{H} & 7.94908 & -0.00007 & 1.11440 \\ \mathrm{H} & 2.98732 & 1.07574 & 3.90038 \\ & & & \end{array}$

(2) Intermediate IM2 $\left(\mathrm{C}_{2} \mathrm{H}_{2} \_2 \mathrm{H} \_\mathrm{Pd} / \mathrm{CNT}\right)$

$\begin{array}{llll}\mathrm{H} & 4.91671 & -1.89144 & -3.56008 \\ \mathrm{H} & 5.22091 & 0.27524 & -3.60831 \\ \mathrm{C} & 4.97901 & 0.73461 & -2.65503 \\ \mathrm{C} & 4.44226 & 1.98963 & -2.63573 \\ \mathrm{H} & 4.27281 & 2.50579 & -3.57518 \\ \mathrm{H} & 2.62563 & 3.85938 & -3.48793 \\ \mathrm{C} & 2.12859 & 3.85847 & -2.52325\end{array}$




\begin{tabular}{|c|c|c|c|}
\hline $\mathrm{C}$ & 0.79567 & 4.14058 & -2.46124 \\
\hline $\mathrm{H}$ & 0.26260 & 4.37071 & -3.37811 \\
\hline $\mathrm{H}$ & -1.61912 & 3.49619 & -3.29856 \\
\hline $\mathrm{C}$ & -2.00994 & 3.28661 & -2.30765 \\
\hline $\mathrm{C}$ & -2.93993 & 2.22804 & -2.22688 \\
\hline $\mathrm{H}$ & -3.17277 & 1.73004 & -3.16321 \\
\hline $\mathrm{H}$ & -3.77881 & -0.28380 & -3.04791 \\
\hline $\mathrm{C}$ & -3.39337 & -0.67979 & -2.11456 \\
\hline $\mathrm{C}$ & -2.81372 & -1.91701 & -2.10247 \\
\hline $\mathrm{H}$ & -2.77131 & -2.48397 & -3.02674 \\
\hline $\mathrm{H}$ & -1.10909 & -3.79966 & -3.12354 \\
\hline $\mathrm{C}$ & -0.50843 & -3.79936 & -2.21979 \\
\hline $\mathrm{C}$ & 0.81874 & -4.10705 & -2.30610 \\
\hline $\mathrm{H}$ & 1.24644 & -4.33912 & -3.27628 \\
\hline $\mathrm{H}$ & 3.32981 & -3.72438 & -3.42759 \\
\hline $\mathrm{C}$ & 3.65965 & -3.23925 & -2.51431 \\
\hline $\mathrm{C}$ & 4.55146 & -2.21003 & -2.58878 \\
\hline $\mathrm{C}$ & 4.88435 & -1.44767 & -1.41335 \\
\hline $\mathrm{C}$ & 5.08187 & -0.03535 & -1.44530 \\
\hline $\mathrm{C}$ & 5.02568 & 0.68067 & -0.21355 \\
\hline $\mathrm{C}$ & 4.44593 & 1.99684 & -0.19585 \\
\hline $\mathrm{C}$ & 3.96739 & 2.56609 & -1.40732 \\
\hline $\mathrm{C}$ & 2.84352 & 3.44744 & -1.35172 \\
\hline $\mathrm{C}$ & 2.23155 & 3.70829 & -0.08972 \\
\hline $\mathrm{C}$ & 0.82522 & 4.02520 & -0.02388 \\
\hline $\mathrm{C}$ & 0.06832 & 4.05614 & -1.22610 \\
\hline $\mathrm{C}$ & -1.33351 & 3.76143 & -1.18667 \\
\hline $\mathrm{C}$ & -2.05471 & 3.68484 & 0.13101 \\
\hline $\mathrm{C}$ & -3.16367 & 2.42638 & 0.22860 \\
\hline $\mathrm{C}$ & -3.24958 & 1.60349 & -1.02522 \\
\hline $\mathrm{C}$ & -3.39223 & 0.16876 & -0.93542 \\
\hline $\mathrm{C}$ & -3.12861 & -0.49120 & 0.31906 \\
\hline $\mathrm{C}$ & -2.47891 & -1.79199 & 0.31346 \\
\hline $\mathrm{C}$ & -2.17084 & -2.42533 & -0.92436 \\
\hline $\mathrm{C}$ & -1.07889 & -3.34348 & -0.98196 \\
\hline $\mathrm{C}$ & -0.34467 & -3.60637 & 0.20819 \\
\hline $\mathrm{C}$ & 1.05026 & -3.94447 & 0.12031 \\
\hline $\mathrm{C}$ & 1.67680 & -3.98498 & -1.15998 \\
\hline $\mathrm{C}$ & 3.03974 & -3.57717 & -1.25936 \\
\hline $\mathrm{C}$ & 3.70538 & -3.15259 & -0.07351 \\
\hline $\mathrm{C}$ & 4.64806 & -2.06633 & -0.15239 \\
\hline $\mathrm{C}$ & 5.04553 & -1.41136 & 1.05225 \\
\hline $\mathrm{C}$ & 5.23139 & -0.00428 & 1.02182 \\
\hline $\mathrm{C}$ & 5.27238 & 0.79845 & 2.21902 \\
\hline $\mathrm{C}$ & 4.71656 & 2.04142 & 2.23701 \\
\hline $\mathrm{C}$ & 4.08611 & 2.58226 & 1.05827 \\
\hline $\mathrm{C}$ & 2.95997 & 3.43949 & 1.11067 \\
\hline $\mathrm{C}$ & 2.35398 & 3.84193 & 2.35712 \\
\hline $\mathrm{C}$ & 1.02655 & 4.11056 & 2.43172 \\
\hline
\end{tabular}




$\begin{array}{llll}\mathrm{C} & 0.17105 & 4.03477 & 1.26706 \\ \mathrm{C} & -1.20236 & 3.81229 & 1.36417 \\ \mathrm{C} & -1.84927 & 3.49010 & 2.60179 \\ \mathrm{C} & -2.74900 & 2.45432 & 2.67993 \\ \mathrm{C} & -3.07237 & 1.66688 & 1.52622 \\ \mathrm{C} & -3.11371 & 0.27650 & 1.55573 \\ \mathrm{C} & -2.94286 & -0.48295 & 2.77876 \\ \mathrm{C} & -2.41553 & -1.73145 & 2.76537 \\ \mathrm{C} & -1.97527 & -2.33862 & 1.52902 \\ \mathrm{C} & -0.90310 & -3.26541 & 1.47918 \\ \mathrm{C} & -0.17054 & -3.67966 & 2.64851 \\ \mathrm{C} & 1.14945 & -4.00630 & 2.56494 \\ \mathrm{C} & 1.84544 & -3.93575 & 1.30590 \\ \mathrm{C} & 3.20543 & -3.53923 & 1.20619 \\ \mathrm{C} & 3.99685 & -3.17670 & 2.35643 \\ \mathrm{C} & 4.88598 & -2.14804 & 2.28220 \\ \mathrm{H} & 5.38991 & -1.81066 & 3.18255 \\ \mathrm{H} & 5.64726 & 0.36871 & 3.14261 \\ \mathrm{H} & 4.66296 & 2.58500 & 3.17472 \\ \mathrm{H} & 2.94844 & 3.84693 & 3.26489 \\ \mathrm{H} & 0.58388 & 4.32493 & 3.39882 \\ \mathrm{H} & -1.49163 & 3.92498 & 3.53111 \\ \mathrm{H} & -3.05940 & 2.12189 & 3.66692 \\ \mathrm{H} & -2.24135 & -2.24483 & 3.70522 \\ \mathrm{H} & -0.64560 & -3.64105 & 3.62327 \\ \mathrm{H} & 1.70337 & -4.21601 & 3.47443 \\ \mathrm{H} & 3.80333 & -3.64770 & 3.31507 \\ \mathrm{Pd} & -5.44228 & -0.61471 & -0.40771 \\ \mathrm{C} & -7.46725 & -1.18462 & -0.63112 \\ \mathrm{C} & -7.35860 & -0.62691 & 0.48192 \\ \mathrm{H} & -7.93738 & -1.69854 & -1.44779 \\ \mathrm{H} & -7.64873 & -0.24209 & 1.44105 \\ \mathrm{H} & -3.17421 & -0.01302 & 3.72918 \\ \mathrm{H} & -4.10012 & 2.98829 & 0.28179 \\ \mathrm{H} & -2.73137 & 4.54407 & 0.14874\end{array}$

(3) Intermediate IM3 $\left(\mathrm{C}_{2} \mathrm{H}_{3} \_\mathrm{H} \_\mathrm{Pd} / \mathrm{CNT}\right)$

$\begin{array}{llll}\mathrm{H} & 4.96969 & -2.30642 & -3.40034 \\ \mathrm{H} & 5.56013 & -0.18676 & -3.40251 \\ \mathrm{C} & 5.30831 & 0.28989 & -2.46027 \\ \mathrm{C} & 4.91337 & 1.59456 & -2.45447 \\ \mathrm{H} & 4.86253 & 2.13793 & -3.39263 \\ \mathrm{H} & 3.23660 & 3.53795 & -3.38565 \\ \mathrm{C} & 2.71718 & 3.60201 & -2.43504 \\ \mathrm{C} & 1.40272 & 3.96755 & -2.42096 \\ \mathrm{H} & 0.90562 & 4.18728 & -3.36026\end{array}$




\begin{tabular}{|c|c|c|c|}
\hline $\mathrm{H}$ & -1.25580 & 4.11821 & -3.29833 \\
\hline $\mathrm{C}$ & -1.57828 & 3.61914 & -2.38947 \\
\hline $\mathrm{C}$ & -2.66571 & 2.81197 & -2.41669 \\
\hline $\mathrm{H}$ & -3.19061 & 2.63933 & -3.35272 \\
\hline $\mathrm{H}$ & -3.56117 & 0.40541 & -3.39264 \\
\hline $\mathrm{C}$ & -3.29635 & -0.07054 & -2.45348 \\
\hline $\mathrm{C}$ & -2.85633 & -1.34971 & -2.44358 \\
\hline $\mathrm{H}$ & -2.79503 & -1.89711 & -3.37840 \\
\hline $\mathrm{H}$ & -1.28887 & -3.36228 & -3.38720 \\
\hline $\mathrm{C}$ & -0.75704 & -3.45060 & -2.44576 \\
\hline $\mathrm{C}$ & 0.52508 & -3.91441 & -2.45097 \\
\hline $\mathrm{H}$ & 0.98881 & -4.17825 & -3.39610 \\
\hline $\mathrm{H}$ & 3.13264 & -3.89526 & -3.39457 \\
\hline $\mathrm{C}$ & 3.46299 & -3.45975 & -2.45684 \\
\hline $\mathrm{C}$ & 4.49531 & -2.56989 & -2.46015 \\
\hline $\mathrm{C}$ & 4.85155 & -1.86478 & -1.25544 \\
\hline $\mathrm{C}$ & 5.24179 & -0.49682 & -1.25562 \\
\hline $\mathrm{C}$ & 5.21465 & 0.21274 & -0.01877 \\
\hline $\mathrm{C}$ & 4.79723 & 1.59021 & -0.01291 \\
\hline $\mathrm{C}$ & 4.42801 & 2.20723 & -1.24473 \\
\hline $\mathrm{C}$ & 3.37711 & 3.16805 & -1.23457 \\
\hline $\mathrm{C}$ & 2.74941 & 3.48184 & 0.00965 \\
\hline $\mathrm{C}$ & 1.36460 & 3.86418 & 0.02456 \\
\hline $\mathrm{C}$ & 0.64018 & 3.93778 & -1.20390 \\
\hline $\mathrm{C}$ & -0.76898 & 3.74680 & -1.17677 \\
\hline $\mathrm{C}$ & -1.39499 & 3.47977 & 0.05282 \\
\hline $\mathrm{C}$ & -2.79845 & 2.84498 & 0.06787 \\
\hline $\mathrm{C}$ & -3.02479 & 2.07016 & -1.21262 \\
\hline $\mathrm{C}$ & -3.29186 & 0.72153 & -1.22388 \\
\hline $\mathrm{C}$ & -3.41502 & -0.05788 & 0.03017 \\
\hline $\mathrm{C}$ & -2.76300 & -1.38658 & 0.01338 \\
\hline $\mathrm{C}$ & -2.37490 & -1.96781 & -1.22076 \\
\hline $\mathrm{C}$ & -1.36444 & -2.96815 & -1.23424 \\
\hline $\mathrm{C}$ & -0.74713 & -3.33994 & -0.00311 \\
\hline $\mathrm{C}$ & 0.60600 & -3.82260 & -0.00796 \\
\hline $\mathrm{C}$ & 1.30957 & -3.92262 & -1.24646 \\
\hline $\mathrm{C}$ & 2.71622 & -3.70687 & -1.24964 \\
\hline $\mathrm{C}$ & 3.35525 & -3.38687 & -0.01557 \\
\hline $\mathrm{C}$ & 4.44770 & -2.44969 & -0.01876 \\
\hline $\mathrm{C}$ & 4.86214 & -1.86779 & 1.21562 \\
\hline $\mathrm{C}$ & 5.25835 & -0.50153 & 1.21517 \\
\hline $\mathrm{C}$ & 5.34813 & 0.28062 & 2.42121 \\
\hline $\mathrm{C}$ & 4.95858 & 1.58681 & 2.42630 \\
\hline $\mathrm{C}$ & 4.45253 & 2.20398 & 1.22730 \\
\hline $\mathrm{C}$ & 3.40276 & 3.16555 & 1.23996 \\
\hline $\mathrm{C}$ & 2.76827 & 3.59705 & 2.45471 \\
\hline $\mathrm{C}$ & 1.45309 & 3.95892 & 2.46881 \\
\hline $\mathrm{C}$ & 0.66547 & 3.93069 & 1.26767 \\
\hline $\mathrm{C}$ & -0.74346 & 3.73220 & 1.27207 \\
\hline
\end{tabular}




$\begin{array}{crrr}\mathrm{C} & -1.51513 & 3.56454 & 2.50440 \\ \mathrm{C} & -2.58088 & 2.72887 & 2.54080 \\ \mathrm{C} & -2.97734 & 2.02583 & 1.32786 \\ \mathrm{C} & -3.28352 & 0.69058 & 1.30224 \\ \mathrm{C} & -3.31385 & -0.13129 & 2.50846 \\ \mathrm{C} & -2.89278 & -1.41534 & 2.46917 \\ \mathrm{C} & -2.38699 & -1.99897 & 1.23562 \\ \mathrm{C} & -1.36648 & -2.98793 & 1.23395 \\ \mathrm{C} & -0.74976 & -3.47654 & 2.43873 \\ \mathrm{C} & 0.53680 & -3.92842 & 2.43483 \\ \mathrm{C} & 1.31618 & -3.92646 & 1.22656 \\ \mathrm{C} & 2.72248 & -3.70736 & 1.22212 \\ \mathrm{C} & 3.47631 & -3.46007 & 2.42489 \\ \mathrm{C} & 4.51093 & -2.57268 & 2.42206 \\ \mathrm{H} & 4.99020 & -2.30961 & 3.35985 \\ \mathrm{H} & 5.61540 & -0.20005 & 3.35716 \\ \mathrm{H} & 4.92695 & 2.12773 & 3.36668 \\ \mathrm{H} & 3.30822 & 3.53360 & 3.39384 \\ \mathrm{H} & 0.97547 & 4.17734 & 3.41829 \\ \mathrm{H} & -1.17330 & 4.04398 & 3.41637 \\ \mathrm{H} & -3.06728 & 2.51421 & 3.48913 \\ \mathrm{H} & -2.85787 & -1.99462 & 3.38629 \\ \mathrm{H} & -1.27690 & -3.39719 & 3.38357 \\ \mathrm{H} & 1.00719 & -4.19328 & 3.37643 \\ \mathrm{H} & 3.14986 & -3.89346 & 3.36497 \\ \mathrm{Pd} & -5.52147 & 0.00636 & -0.21441 \\ \mathrm{C} & -7.66689 & -1.90993 & 0.30078 \\ \mathrm{C} & -6.14640 & -1.85691 & 0.38536 \\ \mathrm{H} & -5.66183 & -2.57477 & -0.28368 \\ \mathrm{H} & -5.77615 & -2.00169 & 1.40460 \\ \mathrm{H} & -3.59737 & 0.31896 & 3.45505 \\ \mathrm{H} & -3.53456 & 3.67572 & 0.09602 \\ \mathrm{H} & -8.03241 & -1.74166 & -0.71995 \\ & -8.14480 & -1.17310 & 0.95834 \\ & -8.04070 & -2.90072 & 0.61076\end{array}$

(4) Intermediate IM4 ( $\left.\mathrm{CHCH}_{3} \_\mathrm{Pd} / \mathrm{CNT}\right)$

$\begin{array}{lrrr}\mathrm{H} & 4.91228 & 2.13347 & 3.38211 \\ \mathrm{H} & 5.47150 & 0.03501 & 3.38177 \\ \mathrm{C} & 5.22420 & -0.44738 & 2.44140 \\ \mathrm{C} & 4.85635 & -1.76017 & 2.44160 \\ \mathrm{H} & 4.81697 & -2.30058 & 3.38203 \\ \mathrm{H} & 3.24858 & -3.80111 & 3.38173 \\ \mathrm{C} & 2.70810 & -3.82322 & 2.44077 \\ \mathrm{C} & 1.38343 & -4.14750 & 2.44084 \\ \mathrm{H} & 0.89434 & -4.37864 & 3.38172 \\ \mathrm{H} & -1.17352 & -3.77901 & 3.38384\end{array}$




\begin{tabular}{|c|c|c|c|}
\hline $\mathrm{C}$ & -1.48521 & -3.34221 & 2.44057 \\
\hline $\mathrm{C}$ & -2.46632 & -2.39238 & 2.44112 \\
\hline $\mathrm{H}$ & -2.91523 & -2.09271 & 3.38230 \\
\hline $\mathrm{H}$ & -3.55723 & -0.00588 & 3.39017 \\
\hline $\mathrm{C}$ & -3.28861 & 0.46753 & 2.45156 \\
\hline $\mathrm{C}$ & -2.87656 & 1.76379 & 2.44600 \\
\hline $\mathrm{H}$ & -2.82724 & 2.30702 & 3.38412 \\
\hline $\mathrm{H}$ & -1.24844 & 3.77580 & 3.38364 \\
\hline $\mathrm{C}$ & -0.70915 & 3.80699 & 2.44242 \\
\hline $\mathrm{C}$ & 0.61082 & 4.14849 & 2.44225 \\
\hline $\mathrm{H}$ & 1.09833 & 4.38121 & 3.38359 \\
\hline $\mathrm{H}$ & 3.18937 & 3.83975 & 3.38189 \\
\hline $\mathrm{C}$ & 3.49034 & 3.38958 & 2.44130 \\
\hline $\mathrm{C}$ & 4.45959 & 2.43073 & 2.44152 \\
\hline $\mathrm{C}$ & 4.77933 & 1.71157 & 1.23585 \\
\hline $\mathrm{C}$ & 5.14535 & 0.33600 & 1.23577 \\
\hline $\mathrm{C}$ & 5.11128 & -0.37567 & -0.00003 \\
\hline C & 4.72194 & -1.76170 & -0.00002 \\
\hline $\mathrm{C}$ & 4.38108 & -2.38763 & 1.23587 \\
\hline C & 3.35362 & -3.37331 & 1.23570 \\
\hline $\mathrm{C}$ & 2.71407 & -3.68842 & 0.00001 \\
\hline $\mathrm{C}$ & 1.31521 & -4.02808 & 0.00002 \\
\hline C & 0.60295 & -4.04580 & 1.23614 \\
\hline C & -0.76879 & -3.66122 & 1.23649 \\
\hline C & -1.36902 & -3.27610 & 0.00004 \\
\hline $\mathrm{C}$ & -2.40074 & -2.27502 & 0.00004 \\
\hline $\mathrm{C}$ & -2.80445 & -1.68848 & 1.23701 \\
\hline C & -3.23944 & -0.32628 & 1.24021 \\
\hline $\mathrm{C}$ & -3.21306 & 0.40276 & 0.00003 \\
\hline $\mathrm{C}$ & -2.75758 & 1.78204 & 0.00002 \\
\hline $\mathrm{C}$ & -2.39200 & 2.38522 & 1.23514 \\
\hline $\mathrm{C}$ & -1.35301 & 3.35804 & 1.23603 \\
\hline C & -0.71536 & 3.67740 & -0.00000 \\
\hline $\mathrm{C}$ & 0.67869 & 4.03634 & -0.00002 \\
\hline $\mathrm{C}$ & 1.39128 & 4.06501 & 1.23604 \\
\hline $\mathrm{C}$ & 2.76815 & 3.70314 & 1.23582 \\
\hline $\mathrm{C}$ & 3.37222 & 3.32513 & -0.00003 \\
\hline $\mathrm{C}$ & 4.39597 & 2.31254 & -0.00003 \\
\hline $\mathrm{C}$ & 4.77931 & 1.71156 & -1.23592 \\
\hline $\mathrm{C}$ & 5.14533 & 0.33599 & -1.23583 \\
\hline C & 5.22417 & -0.44741 & -2.44145 \\
\hline C & 4.85631 & -1.76019 & -2.44163 \\
\hline $\mathrm{C}$ & 4.38106 & -2.38764 & -1.23589 \\
\hline $\mathrm{C}$ & 3.35360 & -3.37332 & -1.23569 \\
\hline $\mathrm{C}$ & 2.70806 & -3.82325 & -2.44075 \\
\hline $\mathrm{C}$ & 1.38339 & -4.14753 & -2.44080 \\
\hline $\mathrm{C}$ & 0.60293 & -4.04581 & -1.23608 \\
\hline $\mathrm{C}$ & -0.76880 & -3.66124 & -1.23642 \\
\hline $\mathrm{C}$ & -1.48525 & -3.34224 & -2.44050 \\
\hline
\end{tabular}




$\begin{array}{cccc}\mathrm{C} & -2.46635 & -2.39241 & -2.44103 \\ \mathrm{C} & -2.80447 & -1.68850 & -1.23693 \\ \mathrm{C} & -3.23946 & -0.32630 & -1.24014 \\ \mathrm{C} & -3.28864 & 0.46750 & -2.45150 \\ \mathrm{C} & -2.87660 & 1.76376 & -2.44596 \\ \mathrm{C} & -2.39202 & 2.38521 & -1.23511 \\ \mathrm{C} & -1.35303 & 3.35803 & -1.23603 \\ \mathrm{C} & -0.70919 & 3.80697 & -2.44243 \\ \mathrm{C} & 0.61079 & 4.14846 & -2.44228 \\ \mathrm{C} & 1.39126 & 4.06500 & -1.23608 \\ \mathrm{C} & 2.76813 & 3.70313 & -1.23588 \\ \mathrm{C} & 3.49031 & 3.38956 & -2.44137 \\ \mathrm{C} & 4.45955 & 2.43071 & -2.44159 \\ \mathrm{H} & 4.91224 & 2.13344 & -3.38218 \\ \mathrm{H} & 5.47145 & 0.03497 & -3.38182 \\ \mathrm{H} & 4.81692 & -2.30061 & -3.38206 \\ \mathrm{H} & 3.24853 & -3.80114 & -3.38172 \\ \mathrm{H} & 0.89429 & -4.37868 & -3.38166 \\ \mathrm{H} & -1.17357 & -3.77904 & -3.38376 \\ \mathrm{H} & -2.91529 & -2.09275 & -3.38221 \\ \mathrm{H} & -2.82728 & 2.30698 & -3.38408 \\ \mathrm{H} & -1.24849 & 3.77576 & -3.38364 \\ \mathrm{H} & 1.09828 & 4.38118 & -3.38363 \\ \mathrm{H} & 3.18933 & 3.83971 & -3.38195 \\ \mathrm{Pd} & -5.45460 & -0.12070 & -0.00001 \\ \mathrm{C} & -8.48059 & 0.60819 & -0.00007 \\ \mathrm{C} & -7.36286 & -0.37949 & -0.00002 \\ \mathrm{H} & -8.16063 & 1.65195 & -0.00006 \\ \mathrm{H} & -9.12973 & 0.43112 & 0.87592 \\ \mathrm{H} & -9.12967 & 0.43112 & -0.87609 \\ \mathrm{H} & -7.79679 & -1.39303 & -0.00009 \\ \mathrm{H} & -3.55727 & -0.00591 & -3.39010\end{array}$

(6) Intermediate IM6 $\left(\mathrm{C}_{2} \mathrm{H}_{4} \_2 \mathrm{H} \_\mathrm{Pd} / \mathrm{CNT}\right)$

$\begin{array}{lrrr}\mathrm{H} & 5.19953 & -1.82620 & -3.38033 \\ \mathrm{H} & 5.46960 & 0.34812 & -3.38295 \\ \mathrm{C} & 5.15853 & 0.79092 & -2.44199 \\ \mathrm{C} & 4.60436 & 2.03651 & -2.44068 \\ \mathrm{H} & 4.48930 & 2.56556 & -3.38115 \\ \mathrm{H} & 2.79959 & 3.86372 & -3.37987 \\ \mathrm{C} & 2.25276 & 3.85644 & -2.44262 \\ \mathrm{C} & 0.91950 & 4.12319 & -2.44943 \\ \mathrm{H} & 0.43094 & 4.34365 & -3.39281 \\ \mathrm{H} & -1.54735 & 3.68720 & -3.42395 \\ \mathrm{C} & -1.92443 & 3.36865 & -2.45663 \\ \mathrm{C} & -2.83834 & 2.31910 & -2.45727\end{array}$




\begin{tabular}{|c|c|c|c|}
\hline $\mathrm{H}$ & -3.10309 & 1.90088 & -3.42383 \\
\hline $\mathrm{H}$ & -3.45493 & -0.17588 & -3.40562 \\
\hline $\mathrm{C}$ & -3.13651 & -0.60521 & -2.46140 \\
\hline $\mathrm{C}$ & -2.57405 & -1.84082 & -2.44797 \\
\hline $\mathrm{H}$ & -2.45681 & -2.37770 & -3.38340 \\
\hline $\mathrm{H}$ & -0.83607 & -3.73554 & -3.38171 \\
\hline $\mathrm{C}$ & -0.29645 & -3.74929 & -2.44050 \\
\hline $\mathrm{C}$ & 1.03152 & -4.05823 & -2.44159 \\
\hline $\mathrm{H}$ & 1.52458 & -4.27756 & -3.38332 \\
\hline $\mathrm{H}$ & 3.61660 & -3.66906 & -3.38017 \\
\hline $\mathrm{C}$ & 3.87716 & -3.18994 & -2.44167 \\
\hline C & 4.76541 & -2.15661 & -2.44185 \\
\hline C & 5.00950 & -1.40597 & -1.23575 \\
\hline C & 5.19497 & 0.00490 & -1.23607 \\
\hline $\mathrm{C}$ & 5.06080 & 0.70470 & -0.00024 \\
\hline C & 4.47194 & 2.01718 & -0.00019 \\
\hline C & 4.04809 & 2.59171 & -1.23415 \\
\hline $\mathrm{C}$ & 2.91659 & 3.45406 & -1.23214 \\
\hline $\mathrm{C}$ & 2.24405 & 3.71536 & -0.00006 \\
\hline $\mathrm{C}$ & 0.83350 & 4.02481 & -0.00002 \\
\hline $\mathrm{C}$ & 0.12711 & 4.03922 & -1.24774 \\
\hline $\mathrm{C}$ & -1.26043 & 3.77367 & -1.27742 \\
\hline $\mathrm{C}$ & -2.04427 & 3.66483 & 0.00008 \\
\hline $\mathrm{C}$ & -3.15451 & 2.39657 & 0.00020 \\
\hline C & -3.15248 & 1.60713 & -1.27913 \\
\hline $\mathrm{C}$ & -3.23354 & 0.19459 & -1.25572 \\
\hline $\mathrm{C}$ & -3.12107 & -0.52135 & 0.00040 \\
\hline $\mathrm{C}$ & -2.45957 & -1.82492 & 0.00040 \\
\hline C & -2.04211 & -2.40409 & -1.23119 \\
\hline C & -0.95240 & -3.31898 & -1.23371 \\
\hline $\mathrm{C}$ & -0.30338 & -3.61775 & 0.00024 \\
\hline $\mathrm{C}$ & 1.09737 & -3.94559 & 0.00011 \\
\hline $\mathrm{C}$ & 1.80996 & -3.95582 & -1.23582 \\
\hline $\mathrm{C}$ & 3.17219 & -3.54468 & -1.23569 \\
\hline C & 3.75466 & -3.13595 & -0.00014 \\
\hline $\mathrm{C}$ & 4.69502 & -2.04460 & -0.00022 \\
\hline $\mathrm{C}$ & 5.00970 & -1.40597 & 1.23526 \\
\hline C & 5.19514 & 0.00489 & 1.23557 \\
\hline C & 5.15885 & 0.79090 & 2.44151 \\
\hline C & 4.60462 & 2.03645 & 2.44030 \\
\hline $\mathrm{C}$ & 4.04817 & 2.59165 & 1.23384 \\
\hline $\mathrm{C}$ & 2.91665 & 3.45393 & 1.23197 \\
\hline C & 2.25285 & 3.85616 & 2.44254 \\
\hline $\mathrm{C}$ & 0.91961 & 4.12289 & 2.44947 \\
\hline $\mathrm{C}$ & 0.12713 & 4.03909 & 1.24779 \\
\hline $\mathrm{C}$ & -1.26035 & 3.77378 & 1.27754 \\
\hline C & -1.92455 & 3.36917 & 2.45688 \\
\hline $\mathrm{C}$ & -2.83839 & 2.31966 & 2.45770 \\
\hline $\mathrm{C}$ & -3.15231 & 1.60729 & 1.27963 \\
\hline
\end{tabular}




\begin{tabular}{cccc}
$\mathrm{C}$ & -3.23306 & 0.19482 & 1.25650 \\
$\mathrm{C}$ & -3.13562 & -0.60476 & 2.46229 \\
$\mathrm{C}$ & -2.57330 & -1.84042 & 2.44884 \\
$\mathrm{C}$ & -2.04178 & -2.40393 & 1.23195 \\
$\mathrm{C}$ & -0.95214 & -3.31888 & 1.23430 \\
$\mathrm{C}$ & -0.29598 & -3.74921 & 2.44099 \\
$\mathrm{C}$ & 1.03197 & -4.05821 & 2.44183 \\
$\mathrm{C}$ & 1.81019 & -3.95582 & 1.23590 \\
$\mathrm{C}$ & 3.17241 & -3.54469 & 1.23551 \\
$\mathrm{C}$ & 3.87761 & -3.18997 & 2.44138 \\
$\mathrm{C}$ & 4.76586 & -2.15664 & 2.44141 \\
$\mathrm{H}$ & 5.20016 & -1.82625 & 3.37980 \\
$\mathrm{H}$ & 5.47011 & 0.34810 & 3.38242 \\
$\mathrm{H}$ & 4.48969 & 2.56550 & 3.38079 \\
$\mathrm{H}$ & 2.79974 & 3.86334 & 3.37976 \\
$\mathrm{H}$ & 0.43108 & 4.34317 & 3.39290 \\
$\mathrm{H}$ & -1.54778 & 3.68829 & 3.42414 \\
$\mathrm{H}$ & -3.10356 & 1.90186 & 3.42434 \\
$\mathrm{H}$ & -2.45571 & -2.37713 & 3.38432 \\
$\mathrm{H}$ & -0.83544 & -3.73549 & 3.38229 \\
$\mathrm{H}$ & 1.52520 & -4.27760 & 3.38345 \\
$\mathrm{H}$ & 3.61723 & -3.66911 & 3.37991 \\
$\mathrm{Pd}$ & -5.35942 & -0.57942 & -0.00036 \\
$\mathrm{C}$ & -7.40840 & -0.86238 & -0.69460 \\
$\mathrm{C}$ & -7.40816 & -0.86298 & 0.69392 \\
$\mathrm{H}$ & -7.42156 & -1.79627 & -1.25274 \\
$\mathrm{H}$ & -7.69091 & 0.02674 & -1.25407 \\
$\mathrm{H}$ & -7.42100 & -1.79753 & 1.25100 \\
$\mathrm{H}$ & -7.69060 & 0.02546 & 1.25450 \\
$\mathrm{H}$ & -3.45356 & -0.17522 & 3.40657 \\
$\mathrm{H}$ & -4.09109 & 2.95865 & 0.00022 \\
$\mathrm{H}$ & -2.72996 & 4.51626 & 0.00009 \\
& & & \\
\hline & & & \\
& &
\end{tabular}

(7) Intermediate IM7 $\left(\mathrm{C}_{2} \mathrm{H}_{5} \_\mathrm{HC} 8 \_\mathrm{Pd} / \mathrm{CNT}\right)$

$\begin{array}{lrrr}\mathrm{H} & 5.37817 & -1.62712 & -3.16604 \\ \mathrm{H} & 5.60490 & 0.53664 & -3.12826 \\ \mathrm{C} & 5.25216 & 0.96821 & -2.19691 \\ \mathrm{C} & 4.69774 & 2.21926 & -2.21165 \\ \mathrm{H} & 4.62684 & 2.75188 & -3.15462 \\ \mathrm{H} & 2.95745 & 4.10233 & -3.23095 \\ \mathrm{C} & 2.35238 & 4.06250 & -2.33077 \\ \mathrm{C} & 1.00451 & 4.30592 & -2.42455 \\ \mathrm{H} & 0.57940 & 4.54008 & -3.39563 \\ \mathrm{H} & -1.25336 & 3.48782 & -3.58536 \\ \mathrm{C} & -1.73055 & 3.21715 & -2.64774 \\ \mathrm{C} & -2.63087 & 2.11007 & -2.70301 \\ \mathrm{H} & -2.84973 & 1.70039 & -3.68364 \\ \mathrm{H} & -3.18713 & -0.42795 & -3.73794\end{array}$




\begin{tabular}{|c|c|c|c|}
\hline $\mathrm{C}$ & -2.95340 & -0.86687 & -2.77294 \\
\hline $\mathrm{C}$ & -2.39296 & -2.11228 & -2.72466 \\
\hline $\mathrm{H}$ & -2.19948 & -2.63803 & -3.65393 \\
\hline $\mathrm{H}$ & -0.51555 & -3.95269 & -3.53928 \\
\hline $\mathrm{C}$ & -0.03570 & -3.93068 & -2.56614 \\
\hline $\mathrm{C}$ & 1.31063 & -4.14820 & -2.48401 \\
\hline $\mathrm{H}$ & 1.87311 & -4.33388 & -3.39347 \\
\hline $\mathrm{H}$ & 3.89170 & -3.54012 & -3.27254 \\
\hline $\mathrm{C}$ & 4.08300 & -3.07110 & -2.31259 \\
\hline $\mathrm{C}$ & 4.92013 & -1.99338 & -2.25265 \\
\hline $\mathrm{C}$ & 5.07085 & -1.25224 & -1.03097 \\
\hline $\mathrm{C}$ & 5.22590 & 0.17067 & -1.00629 \\
\hline $\mathrm{C}$ & 4.99539 & 0.85142 & 0.22500 \\
\hline $\mathrm{C}$ & 4.39602 & 2.15685 & 0.20235 \\
\hline $\mathrm{C}$ & 4.07362 & 2.75843 & -1.03957 \\
\hline $\mathrm{C}$ & 2.92735 & 3.62729 & -1.10294 \\
\hline $\mathrm{C}$ & 2.14804 & 3.81302 & 0.07838 \\
\hline $\mathrm{C}$ & 0.74101 & 4.06058 & -0.02946 \\
\hline $\mathrm{C}$ & 0.14129 & 4.13000 & -1.30207 \\
\hline $\mathrm{C}$ & -1.24016 & 3.69259 & -1.45339 \\
\hline $\mathrm{C}$ & -2.09159 & 3.39973 & -0.22961 \\
\hline $\mathrm{C}$ & -2.69477 & 1.98848 & -0.27749 \\
\hline $\mathrm{C}$ & -2.90764 & 1.36518 & -1.56262 \\
\hline $\mathrm{C}$ & -3.10617 & -0.08150 & -1.57837 \\
\hline $\mathrm{C}$ & -3.03333 & -0.77885 & -0.34287 \\
\hline $\mathrm{C}$ & -2.41011 & -2.07849 & -0.28375 \\
\hline $\mathrm{C}$ & -1.91820 & -2.65977 & -1.48456 \\
\hline $\mathrm{C}$ & -0.78672 & -3.53335 & -1.40937 \\
\hline $\mathrm{C}$ & -0.19818 & -3.77004 & -0.13610 \\
\hline $\mathrm{C}$ & 1.21695 & -4.01551 & -0.04832 \\
\hline $\mathrm{C}$ & 2.00317 & -3.98743 & -1.23723 \\
\hline $\mathrm{C}$ & 3.33714 & -3.48133 & -1.15493 \\
\hline $\mathrm{C}$ & 3.81601 & -3.04324 & 0.11290 \\
\hline $\mathrm{C}$ & 4.70059 & -1.90985 & 0.17516 \\
\hline $\mathrm{C}$ & 4.91727 & -1.27087 & 1.43661 \\
\hline $\mathrm{C}$ & 5.06364 & 0.13764 & 1.46131 \\
\hline $\mathrm{C}$ & 4.94564 & 0.91092 & 2.67482 \\
\hline $\mathrm{C}$ & 4.36492 & 2.14090 & 2.65727 \\
\hline $\mathrm{C}$ & 3.86379 & 2.69938 & 1.42349 \\
\hline $\mathrm{C}$ & 2.72593 & 3.52322 & 1.35858 \\
\hline $\mathrm{C}$ & 1.95307 & 3.88295 & 2.53284 \\
\hline $\mathrm{C}$ & 0.61748 & 4.08282 & 2.45091 \\
\hline $\mathrm{C}$ & -0.08323 & 3.95903 & 1.18270 \\
\hline $\mathrm{C}$ & -1.40546 & 3.61669 & 1.09638 \\
\hline $\mathrm{C}$ & -2.22344 & 3.22666 & 2.24645 \\
\hline $\mathrm{C}$ & -3.03219 & 2.14924 & 2.13883 \\
\hline $\mathrm{C}$ & -3.08224 & 1.37605 & 0.89047 \\
\hline $\mathrm{C}$ & -3.39513 & -0.06348 & 0.88119 \\
\hline $\mathrm{C}$ & -3.29420 & -0.83217 & 2.11792 \\
\hline
\end{tabular}




$\begin{array}{cccc}\mathrm{C} & -2.65825 & -2.02993 & 2.16277 \\ \mathrm{C} & -2.04322 & -2.61119 & 0.98872 \\ \mathrm{C} & -0.93095 & -3.48484 & 1.06141 \\ \mathrm{C} & -0.32846 & -3.88524 & 2.30779 \\ \mathrm{C} & 1.00918 & -4.12803 & 2.38864 \\ \mathrm{C} & 1.85313 & -3.97947 & 1.23033 \\ \mathrm{C} & 3.18362 & -3.49336 & 1.31222 \\ \mathrm{C} & 3.80097 & -3.11809 & 2.56010 \\ \mathrm{C} & 4.63862 & -2.04635 & 2.62012 \\ \mathrm{H} & 5.00584 & -1.70916 & 3.58431 \\ \mathrm{H} & 5.22312 & 0.46516 & 3.62488 \\ \mathrm{H} & 4.18947 & 2.66060 & 3.59372 \\ \mathrm{H} & 2.44015 & 3.91388 & 3.50234 \\ \mathrm{H} & 0.04490 & 4.26736 & 3.35446 \\ \mathrm{H} & -2.12025 & 3.73752 & 3.20033 \\ \mathrm{H} & -3.61412 & 1.81330 & 2.99126 \\ \mathrm{H} & -2.54999 & -2.53030 & 3.11940 \\ \mathrm{H} & -0.92632 & -3.90195 & 3.21317 \\ \mathrm{H} & 1.45593 & -4.32717 & 3.35751 \\ \mathrm{H} & 3.51221 & -3.62157 & 3.47734 \\ \mathrm{Pd} & -5.32807 & -0.30990 & -0.07001 \\ \mathrm{C} & -8.13187 & -0.63906 & 0.73834 \\ \mathrm{C} & -6.78860 & -0.33862 & 1.39775 \\ \mathrm{H} & -6.50528 & -1.10633 & 2.12576 \\ \mathrm{H} & -6.78218 & 0.64161 & 1.88915 \\ \mathrm{H} & -3.68460 & -0.39962 & 3.03309 \\ \mathrm{H} & -2.95470 & 4.10204 & -0.26490 \\ \mathrm{H} & -8.13462 & -1.61383 & 0.23414 \\ \mathrm{H} & -8.40940 & 0.12304 & -0.00121 \\ \mathrm{H} & -8.94660 & -0.66651 & 1.48236\end{array}$

(8) Intermediate IM8 $\left(\mathrm{C}_{2} \mathrm{H}_{5} \_\mathrm{HC} 1 \_\mathrm{Pd} / \mathrm{CNT}\right)$

$\begin{array}{lrrr}\mathrm{H} & 4.96969 & -2.30642 & -3.40034 \\ \mathrm{H} & 5.56013 & -0.18676 & -3.40251 \\ \mathrm{C} & 5.30831 & 0.28989 & -2.46027 \\ \mathrm{C} & 4.91337 & 1.59456 & -2.45447 \\ \mathrm{H} & 4.86253 & 2.13793 & -3.39263 \\ \mathrm{H} & 3.23660 & 3.53795 & -3.38565 \\ \mathrm{C} & 2.71718 & 3.60201 & -2.43504 \\ \mathrm{C} & 1.40272 & 3.96755 & -2.42096 \\ \mathrm{H} & 0.90562 & 4.18728 & -3.36026 \\ \mathrm{H} & -1.25580 & 4.11821 & -3.29833 \\ \mathrm{C} & -1.57828 & 3.61914 & -2.38947 \\ \mathrm{C} & -2.66571 & 2.81197 & -2.41669 \\ \mathrm{H} & -3.19061 & 2.63933 & -3.35272 \\ \mathrm{H} & -3.56117 & 0.40541 & -3.39264 \\ \mathrm{C} & -3.29635 & -0.07054 & -2.45348\end{array}$




\begin{tabular}{|c|c|c|c|}
\hline $\mathrm{C}$ & -2.85633 & -1.34971 & -2.44358 \\
\hline $\mathrm{H}$ & -2.79503 & -1.89711 & -3.37840 \\
\hline $\mathrm{H}$ & -1.28887 & -3.36228 & -3.38720 \\
\hline $\mathrm{C}$ & -0.75704 & -3.45060 & -2.44576 \\
\hline $\mathrm{C}$ & 0.52508 & -3.91441 & -2.45097 \\
\hline $\mathrm{H}$ & 0.98881 & -4.17825 & -3.39610 \\
\hline $\mathrm{H}$ & 3.13264 & -3.89526 & -3.39457 \\
\hline $\mathrm{C}$ & 3.46299 & -3.45975 & -2.45684 \\
\hline $\mathrm{C}$ & 4.49531 & -2.56989 & -2.46015 \\
\hline $\mathrm{C}$ & 4.85155 & -1.86478 & -1.25544 \\
\hline C & 5.24179 & -0.49682 & -1.25562 \\
\hline $\mathrm{C}$ & 5.21465 & 0.21274 & -0.01877 \\
\hline $\mathrm{C}$ & 4.79723 & 1.59021 & -0.01291 \\
\hline $\mathrm{C}$ & 4.42801 & 2.20723 & -1.24473 \\
\hline $\mathrm{C}$ & 3.37711 & 3.16805 & -1.23457 \\
\hline $\mathrm{C}$ & 2.74941 & 3.48184 & 0.00965 \\
\hline $\mathrm{C}$ & 1.36460 & 3.86418 & 0.02456 \\
\hline $\mathrm{C}$ & 0.64018 & 3.93778 & -1.20390 \\
\hline $\mathrm{C}$ & -0.76898 & 3.74680 & -1.17677 \\
\hline $\mathrm{C}$ & -1.39499 & 3.47977 & 0.05282 \\
\hline $\mathrm{C}$ & -2.79845 & 2.84498 & 0.06787 \\
\hline $\mathrm{C}$ & -3.02479 & 2.07016 & -1.21262 \\
\hline $\mathrm{C}$ & -3.29186 & 0.72153 & -1.22388 \\
\hline $\mathrm{C}$ & -3.41502 & -0.05788 & 0.03017 \\
\hline $\mathrm{C}$ & -2.76300 & -1.38658 & 0.01338 \\
\hline $\mathrm{C}$ & -2.37490 & -1.96781 & -1.22076 \\
\hline $\mathrm{C}$ & -1.36444 & -2.96815 & -1.23424 \\
\hline $\mathrm{C}$ & -0.74713 & -3.33994 & -0.00311 \\
\hline $\mathrm{C}$ & 0.60600 & -3.82260 & -0.00796 \\
\hline $\mathrm{C}$ & 1.30957 & -3.92262 & -1.24646 \\
\hline $\mathrm{C}$ & 2.71622 & -3.70687 & -1.24964 \\
\hline $\mathrm{C}$ & 3.35525 & -3.38687 & -0.01557 \\
\hline $\mathrm{C}$ & 4.44770 & -2.44969 & -0.01876 \\
\hline $\mathrm{C}$ & 4.86214 & -1.86779 & 1.21562 \\
\hline $\mathrm{C}$ & 5.25835 & -0.50153 & 1.21517 \\
\hline $\mathrm{C}$ & 5.34813 & 0.28062 & 2.42121 \\
\hline $\mathrm{C}$ & 4.95858 & 1.58681 & 2.42630 \\
\hline $\mathrm{C}$ & 4.45253 & 2.20398 & 1.22730 \\
\hline $\mathrm{C}$ & 3.40276 & 3.16555 & 1.23996 \\
\hline $\mathrm{C}$ & 2.76827 & 3.59705 & 2.45471 \\
\hline $\mathrm{C}$ & 1.45309 & 3.95892 & 2.46881 \\
\hline $\mathrm{C}$ & 0.66547 & 3.93069 & 1.26767 \\
\hline $\mathrm{C}$ & -0.74346 & 3.73220 & 1.27207 \\
\hline $\mathrm{C}$ & -1.51513 & 3.56454 & 2.50440 \\
\hline $\mathrm{C}$ & -2.58088 & 2.72887 & 2.54080 \\
\hline $\mathrm{C}$ & -2.97734 & 2.02583 & 1.32786 \\
\hline $\mathrm{C}$ & -3.28352 & 0.69058 & 1.30224 \\
\hline $\mathrm{C}$ & -3.31385 & -0.13129 & 2.50846 \\
\hline $\mathrm{C}$ & -2.89278 & -1.41534 & 2.46917 \\
\hline
\end{tabular}




$\begin{array}{crrr}\mathrm{C} & -2.38699 & -1.99897 & 1.23562 \\ \mathrm{C} & -1.36648 & -2.98793 & 1.23395 \\ \mathrm{C} & -0.74976 & -3.47654 & 2.43873 \\ \mathrm{C} & 0.53680 & -3.92842 & 2.43483 \\ \mathrm{C} & 1.31618 & -3.92646 & 1.22656 \\ \mathrm{C} & 2.72248 & -3.70736 & 1.22212 \\ \mathrm{C} & 3.47631 & -3.46007 & 2.42489 \\ \mathrm{C} & 4.51093 & -2.57268 & 2.42206 \\ \mathrm{H} & 4.99020 & -2.30961 & 3.35985 \\ \mathrm{H} & 5.61540 & -0.20005 & 3.35716 \\ \mathrm{H} & 4.92695 & 2.12773 & 3.36668 \\ \mathrm{H} & 3.30822 & 3.53360 & 3.39384 \\ \mathrm{H} & 0.97547 & 4.17734 & 3.41829 \\ \mathrm{H} & -1.17330 & 4.04398 & 3.41637 \\ \mathrm{H} & -3.06728 & 2.51421 & 3.48913 \\ \mathrm{H} & -2.85787 & -1.99462 & 3.38629 \\ \mathrm{H} & -1.27690 & -3.39719 & 3.38357 \\ \mathrm{H} & 1.00719 & -4.19328 & 3.37643 \\ \mathrm{H} & 3.14986 & -3.89346 & 3.36497 \\ \mathrm{Pd} & -5.52147 & 0.00636 & -0.21441 \\ \mathrm{C} & -7.66689 & -1.90993 & 0.30078 \\ \mathrm{C} & -6.14640 & -1.85691 & 0.38536 \\ \mathrm{H} & -5.66183 & -2.57477 & -0.28368 \\ \mathrm{H} & -5.77615 & -2.00169 & 1.40460 \\ \mathrm{H} & -3.59737 & 0.31896 & 3.45505 \\ \mathrm{H} & -3.53456 & 3.67572 & 0.09602 \\ \mathrm{H} & -8.03241 & -1.74166 & -0.71995 \\ \mathrm{H} & -8.14480 & -1.17310 & 0.95834 \\ \mathrm{H} & -8.04070 & -2.90072 & 0.61076\end{array}$

(9) Intermediate IM9 $\left(\mathrm{C}_{2} \mathrm{H}_{6} \_\mathrm{Pd} / \mathrm{CNT}\right)$

$\begin{array}{llll}\mathrm{H} & 5.72670 & -0.12567 & 3.15432 \\ \mathrm{H} & 5.10524 & -2.20811 & 3.19778 \\ \mathrm{C} & 4.59388 & -2.49700 & 2.28502 \\ \mathrm{C} & 3.60074 & -3.42915 & 2.34342 \\ \mathrm{H} & 3.33995 & -3.86706 & 3.30165 \\ \mathrm{H} & 1.24126 & -4.35689 & 3.42082 \\ \mathrm{C} & 0.70693 & -4.11574 & 2.50743 \\ \mathrm{C} & -0.60410 & -3.74845 & 2.58075 \\ \mathrm{H} & -1.08816 & -3.70543 & 3.55107 \\ \mathrm{H} & -2.65566 & -2.23541 & 3.63982 \\ \mathrm{C} & -2.74320 & -1.68431 & 2.70900 \\ \mathrm{C} & -3.10778 & -0.37375 & 2.74301 \\ \mathrm{H} & -3.29389 & 0.10455 & 3.69954 \\ \mathrm{H} & -2.60350 & 2.17554 & 3.64734 \\ \mathrm{C} & -2.20277 & 2.45829 & 2.67895\end{array}$




\begin{tabular}{|c|c|c|c|}
\hline $\mathrm{C}$ & -1.20738 & 3.38683 & 2.61502 \\
\hline $\mathrm{H}$ & -0.83439 & 3.82886 & 3.53325 \\
\hline $\mathrm{H}$ & 1.24366 & 4.36904 & 3.40491 \\
\hline $\mathrm{C}$ & 1.67219 & 4.12592 & 2.43785 \\
\hline $\mathrm{C}$ & 2.98858 & 3.78120 & 2.36085 \\
\hline $\mathrm{H}$ & 3.58475 & 3.75455 & 3.26744 \\
\hline $\mathrm{H}$ & 5.12138 & 2.22369 & 3.18231 \\
\hline C & 5.09389 & 1.67904 & 2.24385 \\
\hline C & 5.43429 & 0.35924 & 2.22825 \\
\hline $\mathrm{C}$ & 5.26666 & -0.42606 & 1.03237 \\
\hline $\mathrm{C}$ & 4.86626 & -1.79130 & 1.05971 \\
\hline $\mathrm{C}$ & 4.39935 & -2.38646 & -0.15007 \\
\hline C & 3.34955 & -3.37007 & -0.08815 \\
\hline $\mathrm{C}$ & 2.80437 & -3.72704 & 1.18117 \\
\hline C & 1.42062 & -4.05223 & 1.25954 \\
\hline C & 0.64079 & -4.00650 & 0.06504 \\
\hline C & -0.74234 & -3.61517 & 0.14237 \\
\hline C & -1.30682 & -3.28819 & 1.41200 \\
\hline $\mathrm{C}$ & -2.33420 & -2.30513 & 1.46899 \\
\hline C & -2.76071 & -1.69069 & 0.25870 \\
\hline C & -3.22709 & -0.31044 & 0.29296 \\
\hline $\mathrm{C}$ & -3.09898 & 0.41870 & 1.53373 \\
\hline C & -2.63357 & 1.75576 & 1.49934 \\
\hline $\mathrm{C}$ & -2.30264 & 2.34099 & 0.23181 \\
\hline C & -1.24071 & 3.31017 & 0.16738 \\
\hline $\mathrm{C}$ & -0.55447 & 3.67983 & 1.36505 \\
\hline $\mathrm{C}$ & 0.81897 & 4.03492 & 1.28147 \\
\hline $\mathrm{C}$ & 1.45707 & 4.00289 & 0.00375 \\
\hline $\mathrm{C}$ & 2.84711 & 3.63709 & -0.07709 \\
\hline $\mathrm{C}$ & 3.55308 & 3.31468 & 1.12070 \\
\hline $\mathrm{C}$ & 4.56104 & 2.31239 & 1.06500 \\
\hline C & 4.81888 & 1.67663 & -0.18644 \\
\hline C & 5.17880 & 0.28258 & -0.20286 \\
\hline $\mathrm{C}$ & 5.12674 & -0.43361 & -1.43534 \\
\hline $\mathrm{C}$ & 4.72881 & -1.80031 & -1.40802 \\
\hline C & 4.32421 & -2.51522 & -2.59030 \\
\hline $\mathrm{C}$ & 3.33060 & -3.44704 & -2.53173 \\
\hline $\mathrm{C}$ & 2.66742 & -3.73465 & -1.28660 \\
\hline C & 1.28244 & -4.05584 & -1.20846 \\
\hline $\mathrm{C}$ & 0.43466 & -4.11679 & -2.36900 \\
\hline C & -0.87386 & -3.74074 & -2.29584 \\
\hline C & -1.43977 & -3.27941 & -1.05594 \\
\hline $\mathrm{C}$ & -2.46246 & -2.28973 & -0.99782 \\
\hline $\mathrm{C}$ & -2.99639 & -1.66194 & -2.18242 \\
\hline $\mathrm{C}$ & -3.41601 & -0.36645 & -2.16245 \\
\hline C & -3.32876 & 0.43309 & -0.94691 \\
\hline C & -2.81452 & 1.78549 & -0.97224 \\
\hline $\mathrm{C}$ & -2.51209 & 2.46448 & -2.19589 \\
\hline $\mathrm{C}$ & -1.49947 & 3.38137 & -2.26021 \\
\hline
\end{tabular}




$\begin{array}{cccc}\mathrm{C} & -0.70687 & 3.68140 & -1.10328 \\ \mathrm{C} & 0.67460 & 4.03519 & -1.18599 \\ \mathrm{C} & 1.38301 & 4.11188 & -2.43479 \\ \mathrm{C} & 2.69974 & 3.76237 & -2.51317 \\ \mathrm{C} & 3.40768 & 3.30737 & -1.34682 \\ \mathrm{C} & 4.41863 & 2.30452 & -1.40242 \\ \mathrm{C} & 4.81076 & 1.66393 & -2.63061 \\ \mathrm{C} & 5.15293 & 0.34408 & -2.64674 \\ \mathrm{H} & 5.33522 & -0.14579 & -3.59799 \\ \mathrm{H} & 4.73272 & -2.23433 & -3.55588 \\ \mathrm{H} & 2.96651 & -3.89311 & -3.45176 \\ \mathrm{H} & 0.86291 & -4.35985 & -3.33616 \\ \mathrm{H} & -1.46272 & -3.69471 & -3.20616 \\ \mathrm{H} & -2.98190 & -2.19677 & -3.12640 \\ \mathrm{H} & -3.71578 & 0.11275 & -3.08884 \\ \mathrm{H} & -1.22533 & 3.79672 & -3.22449 \\ \mathrm{H} & 0.84328 & 4.34599 & -3.34683 \\ \mathrm{H} & 3.18063 & 3.72192 & -3.48534 \\ \mathrm{H} & 4.72711 & 2.20210 & -3.56951 \\ \mathrm{Pd} & -5.37390 & 0.12917 & -0.16399 \\ \mathrm{C} & -7.96671 & 0.17858 & 0.06274 \\ \mathrm{C} & -8.95599 & -0.53200 & -0.86502 \\ \mathrm{H} & -3.01958 & 2.17436 & -3.11031 \\ \mathrm{H} & -9.56821 & -1.25117 & -0.30971 \\ \mathrm{H} & -8.43214 & -1.07800 & -1.65624 \\ \mathrm{H} & -9.63239 & 0.18585 & -1.34220 \\ \mathrm{H} & -7.30834 & -0.56253 & 0.56286 \\ \mathrm{H} & -8.46664 & 0.73072 & 0.86496 \\ \mathrm{H} & -7.37699 & 0.92940 & -0.50413\end{array}$

Transition state structures observed over $\mathrm{C}_{60}$ during acetylene hydrogenation.

(1) Transition state (TS12)

$\begin{array}{lrrr}\mathrm{C} & -1.57098 & 3.22977 & -0.50375 \\ \mathrm{C} & -1.81675 & 2.32172 & -1.70332 \\ \mathrm{C} & -2.80672 & 1.34022 & -1.36407 \\ \mathrm{C} & -3.07637 & 1.42601 & 0.06349 \\ \mathrm{C} & -2.23777 & 2.45076 & 0.61938 \\ \mathrm{C} & -1.66727 & 2.26837 & 1.86627 \\ \mathrm{C} & -1.96202 & 1.07364 & 2.62784 \\ \mathrm{C} & -2.81518 & 0.09306 & 2.10714 \\ \mathrm{C} & -3.38892 & 0.27403 & 0.79402 \\ \mathrm{C} & -3.44032 & -1.01943 & 0.13298 \\ \mathrm{C} & -3.17201 & -1.10256 & -1.23370 \\ \mathrm{C} & -2.87405 & 0.09175 & -2.00356\end{array}$




\begin{tabular}{|c|c|c|c|}
\hline $\mathrm{C}$ & -1.85769 & -0.24096 & -2.96756 \\
\hline $\mathrm{C}$ & -0.83853 & 0.67784 & -3.24151 \\
\hline $\mathrm{C}$ & -0.79325 & 1.97011 & -2.57277 \\
\hline $\mathrm{C}$ & 0.60227 & 2.35416 & -2.42680 \\
\hline $\mathrm{C}$ & 1.39374 & 1.25031 & -2.84151 \\
\hline $\mathrm{C}$ & 0.51689 & 0.22740 & -3.40740 \\
\hline $\mathrm{C}$ & 0.83553 & -1.12428 & -3.28901 \\
\hline $\mathrm{C}$ & -0.21388 & -2.08197 & -2.98396 \\
\hline $\mathrm{C}$ & -1.53286 & -1.64753 & -2.82486 \\
\hline $\mathrm{C}$ & -2.34932 & -2.18568 & -1.74931 \\
\hline $\mathrm{C}$ & -1.81171 & -3.13059 & -0.87422 \\
\hline $\mathrm{C}$ & -2.08231 & -3.03863 & 0.55193 \\
\hline $\mathrm{C}$ & -2.87699 & -2.00001 & 1.04357 \\
\hline $\mathrm{C}$ & -2.50046 & -1.31404 & 2.27065 \\
\hline $\mathrm{C}$ & -1.33941 & -1.69340 & 2.94980 \\
\hline $\mathrm{C}$ & -0.45665 & -0.67881 & 3.50241 \\
\hline $\mathrm{C}$ & -0.75931 & 0.67287 & 3.33720 \\
\hline $\mathrm{C}$ & 0.29204 & 1.62091 & 3.01775 \\
\hline $\mathrm{C}$ & 1.61023 & 1.17806 & 2.87813 \\
\hline $\mathrm{C}$ & 1.92886 & -0.23017 & 3.04244 \\
\hline $\mathrm{C}$ & 0.91436 & -1.13982 & 3.34240 \\
\hline $\mathrm{C}$ & 0.87564 & -2.42996 & 2.68011 \\
\hline $\mathrm{C}$ & -0.51688 & -2.77467 & 2.44407 \\
\hline $\mathrm{C}$ & -0.87706 & -3.42775 & 1.26117 \\
\hline $\mathrm{C}$ & 0.13602 & -3.77103 & 0.27841 \\
\hline $\mathrm{C}$ & -0.44006 & -3.58180 & -1.04272 \\
\hline $\mathrm{C}$ & 0.34195 & -3.06728 & -2.07808 \\
\hline $\mathrm{C}$ & 1.73910 & -2.72067 & -1.83911 \\
\hline $\mathrm{C}$ & 2.05037 & -1.53478 & -2.60571 \\
\hline $\mathrm{C}$ & 2.89169 & -0.54754 & -2.07064 \\
\hline $\mathrm{C}$ & 2.61781 & 0.86098 & -2.24671 \\
\hline $\mathrm{C}$ & 2.94078 & 1.50843 & -0.99090 \\
\hline $\mathrm{C}$ & 3.49429 & 0.52740 & -0.07683 \\
\hline $\mathrm{C}$ & 3.46250 & -0.75457 & -0.74500 \\
\hline $\mathrm{C}$ & 3.17060 & -1.90888 & -0.01527 \\
\hline $\mathrm{C}$ & 2.29685 & -2.92259 & -0.57176 \\
\hline $\mathrm{C}$ & 1.47348 & -3.44209 & 0.50662 \\
\hline $\mathrm{C}$ & 1.85153 & -2.76276 & 1.73569 \\
\hline $\mathrm{C}$ & 2.89723 & -1.81129 & 1.41309 \\
\hline $\mathrm{C}$ & 2.94376 & -0.57512 & 2.06060 \\
\hline $\mathrm{C}$ & 3.23733 & 0.62101 & 1.29252 \\
\hline $\mathrm{C}$ & 2.42711 & 1.70080 & 1.81172 \\
\hline $\mathrm{C}$ & 1.87673 & 2.63418 & 0.92037 \\
\hline $\mathrm{C}$ & 2.22781 & 2.59622 & -0.47756 \\
\hline $\mathrm{C}$ & 1.01747 & 3.20941 & -1.30258 \\
\hline $\mathrm{C}$ & -0.02287 & 3.66393 & -0.20236 \\
\hline $\mathrm{C}$ & 0.54620 & 3.12961 & 1.09524 \\
\hline $\mathrm{C}$ & -0.24732 & 2.60293 & 2.10020 \\
\hline $\mathrm{C}$ & -0.56012 & 5.08657 & -0.28060 \\
\hline
\end{tabular}




$\begin{array}{lrrr}\mathrm{C} & -1.81931 & 4.73437 & -0.55831 \\ \mathrm{H} & -0.05874 & 6.03727 & -0.11941 \\ \mathrm{H} & -2.73940 & 5.28662 & -0.72210 \\ \mathrm{H} & 1.36702 & 4.36710 & -1.77224 \\ \mathrm{H} & 2.11635 & 3.89221 & -1.32096\end{array}$

(2) Transition state (TS23)

\begin{tabular}{|c|c|c|c|}
\hline $\mathrm{C}$ & -2.25018 & 2.03694 & 1.92637 \\
\hline $\mathrm{C}$ & -2.67830 & 1.98522 & 0.46384 \\
\hline $\mathrm{C}$ & -3.32567 & 0.72736 & 0.19051 \\
\hline $\mathrm{C}$ & -3.14426 & -0.14220 & 1.34175 \\
\hline $\mathrm{C}$ & -2.40392 & 0.57465 & 2.34628 \\
\hline $\mathrm{C}$ & -1.41895 & -0.07601 & 3.06334 \\
\hline $\mathrm{C}$ & -1.20261 & -1.49464 & 2.87391 \\
\hline $\mathrm{C}$ & -1.96113 & -2.20927 & 1.93988 \\
\hline $\mathrm{C}$ & -2.95289 & -1.51681 & 1.15018 \\
\hline $\mathrm{C}$ & -2.92876 & -2.06858 & -0.19523 \\
\hline $\mathrm{C}$ & -3.11096 & -1.23513 & -1.29890 \\
\hline $\mathrm{C}$ & -3.30986 & 0.19124 & -1.10163 \\
\hline C & -2.61685 & 0.89577 & -2.15782 \\
\hline $\mathrm{C}$ & -1.95858 & 2.10062 & -1.86523 \\
\hline $\mathrm{C}$ & -1.98427 & 2.63858 & -0.53262 \\
\hline $\mathrm{C}$ & -0.66798 & 3.27391 & -0.27613 \\
\hline $\mathrm{C}$ & 0.13181 & 3.11945 & -1.46645 \\
\hline $\mathrm{C}$ & -0.64860 & 2.37614 & -2.43168 \\
\hline $\mathrm{C}$ & -0.05322 & 1.43463 & -3.27874 \\
\hline $\mathrm{C}$ & -0.72343 & 0.18498 & -3.55643 \\
\hline $\mathrm{C}$ & -1.99106 & -0.08120 & -3.01795 \\
\hline $\mathrm{C}$ & -2.28612 & -1.40260 & -2.48325 \\
\hline $\mathrm{C}$ & -1.31420 & -2.40345 & -2.51695 \\
\hline $\mathrm{C}$ & -1.12466 & -3.27301 & -1.36789 \\
\hline $\mathrm{C}$ & -1.92139 & -3.11478 & -0.22889 \\
\hline $\mathrm{C}$ & -1.32086 & -3.19818 & 1.09015 \\
\hline $\mathrm{C}$ & 0.05036 & -3.44220 & 1.21658 \\
\hline $\mathrm{C}$ & 0.83534 & -2.71062 & 2.19846 \\
\hline $\mathrm{C}$ & 0.22108 & -1.75438 & 3.00429 \\
\hline $\mathrm{C}$ & 0.88857 & -0.49169 & 3.27820 \\
\hline $\mathrm{C}$ & 2.15335 & -0.24226 & 2.72246 \\
\hline $\mathrm{C}$ & 2.78736 & -1.23162 & 1.88169 \\
\hline $\mathrm{C}$ & 2.14705 & -2.44765 & 1.62289 \\
\hline $\mathrm{C}$ & 2.17069 & -3.00926 & 0.29104 \\
\hline $\mathrm{C}$ & 0.87120 & -3.61834 & 0.03615 \\
\hline $\mathrm{C}$ & 0.29501 & -3.53792 & -1.23362 \\
\hline $\mathrm{C}$ & 0.98998 & -2.83319 & -2.30000 \\
\hline $\mathrm{C}$ & -0.00556 & -2.12799 & -3.09061 \\
\hline $\mathrm{C}$ & 0.28103 & -0.86340 & -3.60523 \\
\hline $\mathrm{C}$ & 1.58047 & -0.25579 & -3.35910 \\
\hline
\end{tabular}




$\begin{array}{lrrr}\mathrm{C} & 1.37702 & 1.16337 & -3.15541 \\ \mathrm{C} & 2.14116 & 1.85129 & -2.22070 \\ \mathrm{C} & 1.52213 & 2.84554 & -1.33763 \\ \mathrm{C} & 2.14474 & 2.78789 & -0.08002 \\ \mathrm{C} & 3.10786 & 1.72521 & -0.09969 \\ \mathrm{C} & 3.13667 & 1.15124 & -1.44311 \\ \mathrm{C} & 3.33077 & -0.21737 & -1.62389 \\ \mathrm{C} & 2.53910 & -0.93678 & -2.60431 \\ \mathrm{C} & 2.23307 & -2.24849 & -2.05855 \\ \mathrm{C} & 2.83833 & -2.33530 & -0.73765 \\ \mathrm{C} & 3.51730 & -1.08498 & -0.47154 \\ \mathrm{C} & 3.49254 & -0.53221 & 0.81616 \\ \mathrm{C} & 3.30721 & 0.88950 & 1.00760 \\ \mathrm{C} & 2.45753 & 1.06195 & 2.18767 \\ \mathrm{C} & 1.50085 & 2.06923 & 2.23789 \\ \mathrm{C} & 1.42022 & 3.19474 & 1.19709 \\ \mathrm{C} & -0.10791 & 3.24932 & 0.98218 \\ \mathrm{C} & -0.81351 & 2.66318 & 2.12268 \\ \mathrm{C} & 0.21366 & 1.79324 & 2.77380 \\ \mathrm{C} & -0.09947 & 0.54708 & 3.31603 \\ \mathrm{C} & -1.69534 & 3.76871 & 3.27961 \\ \mathrm{C} & -2.85599 & 3.17701 & 2.70922 \\ \mathrm{H} & -1.24748 & 3.27631 & 4.14901 \\ \mathrm{H} & -3.78966 & 3.67311 & 2.45951 \\ \mathrm{H} & 1.80913 & 4.14836 & 1.59413 \\ \mathrm{H} & -1.61387 & 4.85816 & 3.37110\end{array}$

(3) Transition state (TS34)

$\begin{array}{lrrr}C & -2.15865 & 1.69821 & 2.81012 \\ C & -2.21800 & 2.39029 & 1.52190 \\ C & -3.08158 & 1.63248 & 0.66381 \\ C & -3.18935 & 0.30615 & 1.26395 \\ C & -2.33997 & 0.25631 & 2.46693 \\ C & -1.58418 & -0.90420 & 2.71717 \\ C & -1.81410 & -2.05394 & 1.84096 \\ C & -2.68826 & -2.01156 & 0.74464 \\ C & -3.37813 & -0.78886 & 0.43618 \\ C & -3.42531 & -0.63323 & -1.01472 \\ C & -3.23705 & 0.62703 & -1.58001 \\ C & -3.04307 & 1.77509 & -0.71513 \\ C & -2.01102 & 2.61167 & -1.27651 \\ C & -1.03533 & 3.17986 & -0.43052 \\ C & -1.07259 & 3.00798 & 1.01512 \\ C & 0.36990 & 2.93462 & 1.50944 \\ C & 1.17985 & 3.06156 & 0.32313 \\ C & 0.33176 & 3.22920 & -0.85519 \\ C & 0.70711 & 2.72794 & -2.10462\end{array}$




\begin{tabular}{|c|c|c|c|}
\hline $\mathrm{C}$ & -0.28462 & 2.08683 & -2.95241 \\
\hline $\mathrm{C}$ & -1.61630 & 2.02511 & -2.54086 \\
\hline $\mathrm{C}$ & -2.37142 & 0.79144 & -2.72996 \\
\hline $\mathrm{C}$ & -1.75785 & -0.32348 & -3.30264 \\
\hline $\mathrm{C}$ & -1.96900 & -1.64442 & -2.72755 \\
\hline $\mathrm{C}$ & -2.77516 & -1.79041 & -1.59469 \\
\hline $\mathrm{C}$ & -2.34440 & -2.65699 & -0.50568 \\
\hline $\mathrm{C}$ & -1.14946 & -3.36733 & -0.61531 \\
\hline $\mathrm{C}$ & -0.27218 & -3.47546 & 0.53451 \\
\hline $\mathrm{C}$ & -0.60002 & -2.82213 & 1.72424 \\
\hline $\mathrm{C}$ & 0.41788 & -2.18765 & 2.53520 \\
\hline $\mathrm{C}$ & 1.75957 & -2.22979 & 2.09056 \\
\hline $\mathrm{C}$ & 2.09208 & -2.84746 & 0.81502 \\
\hline $\mathrm{C}$ & 1.10030 & -3.46179 & 0.05533 \\
\hline $\mathrm{C}$ & 1.06447 & -3.28852 & -1.38528 \\
\hline $\mathrm{C}$ & -0.32814 & -3.24169 & -1.80242 \\
\hline $\mathrm{C}$ & -0.72137 & -2.38072 & -2.83178 \\
\hline $\mathrm{C}$ & 0.25748 & -1.52169 & -3.47480 \\
\hline $\mathrm{C}$ & -0.37836 & -0.24550 & -3.74960 \\
\hline $\mathrm{C}$ & 0.34045 & 0.94279 & -3.58456 \\
\hline $\mathrm{C}$ & 1.73047 & 0.90028 & -3.15488 \\
\hline $\mathrm{C}$ & 1.96043 & 2.01851 & -2.26244 \\
\hline $\mathrm{C}$ & 2.79801 & 1.87396 & -1.15658 \\
\hline $\mathrm{C}$ & 2.43765 & 2.43503 & 0.12860 \\
\hline $\mathrm{C}$ & 2.90038 & 1.52908 & 1.13595 \\
\hline $\mathrm{C}$ & 3.53699 & 0.38761 & 0.50082 \\
\hline $\mathrm{C}$ & 3.47225 & 0.60055 & -0.92958 \\
\hline $\mathrm{C}$ & 3.23915 & -0.48149 & -1.77971 \\
\hline $\mathrm{C}$ & 2.34980 & -0.33065 & -2.91860 \\
\hline $\mathrm{C}$ & 1.59360 & -1.56053 & -3.06691 \\
\hline $\mathrm{C}$ & 2.00838 & -2.47111 & -2.01277 \\
\hline $\mathrm{C}$ & 3.03431 & -1.81301 & -1.22334 \\
\hline $\mathrm{C}$ & 3.08778 & -2.01281 & 0.15603 \\
\hline $\mathrm{C}$ & 3.36372 & -0.88986 & 1.03205 \\
\hline $\mathrm{C}$ & 2.57347 & -1.05956 & 2.22984 \\
\hline $\mathrm{C}$ & 2.01720 & 0.05595 & 2.85804 \\
\hline $\mathrm{C}$ & 2.15460 & 1.35520 & 2.28983 \\
\hline $\mathrm{C}$ & 0.94422 & 2.16907 & 2.63080 \\
\hline $\mathrm{C}$ & 0.30832 & 1.36352 & 3.75005 \\
\hline $\mathrm{C}$ & 0.74366 & 0.00866 & 3.53729 \\
\hline $\mathrm{C}$ & -0.14589 & -1.04411 & 3.21371 \\
\hline $\mathrm{C}$ & -0.77161 & 1.69033 & 4.76315 \\
\hline $\mathrm{C}$ & -1.77364 & 2.36774 & 3.90621 \\
\hline $\mathrm{H}$ & -1.77027 & 3.45458 & 3.86533 \\
\hline $\mathrm{H}$ & -1.11770 & 0.73961 & 5.18668 \\
\hline $\mathrm{H}$ & -0.40547 & 2.29970 & 5.59627 \\
\hline $\mathrm{H}$ & 1.25809 & 2.50550 & 3.82241 \\
\hline
\end{tabular}


(4) Transition state (TS45)

\begin{tabular}{|c|c|c|c|}
\hline C & 0.22037 & 2.72317 & -2.07847 \\
\hline $\mathrm{C}$ & 1.57876 & 2.52221 & -1.54219 \\
\hline $\mathrm{C}$ & 2.12947 & 1.35345 & -2.24677 \\
\hline $\mathrm{C}$ & 1.11874 & 0.81945 & -3.13701 \\
\hline $\mathrm{C}$ & -0.06078 & 1.62842 & -2.97438 \\
\hline C & -1.32900 & 1.05998 & -3.02341 \\
\hline $\mathrm{C}$ & -1.46184 & -0.37101 & -3.22566 \\
\hline $\mathrm{C}$ & -0.32264 & -1.16093 & -3.40105 \\
\hline $\mathrm{C}$ & 0.99711 & -0.55115 & -3.36542 \\
\hline C & 1.90396 & -1.47038 & -2.70641 \\
\hline $\mathrm{C}$ & 2.89344 & -0.96896 & -1.86060 \\
\hline $\mathrm{C}$ & 3.00956 & 0.46460 & -1.63355 \\
\hline $\mathrm{C}$ & 3.38040 & 0.67190 & -0.25194 \\
\hline $\mathrm{C}$ & 2.85148 & 1.75705 & 0.44323 \\
\hline $\mathrm{C}$ & 1.94190 & 2.72533 & -0.18988 \\
\hline C & 1.01067 & 3.16522 & 0.86497 \\
\hline $\mathrm{C}$ & 1.28617 & 2.38138 & 2.04387 \\
\hline $\mathrm{C}$ & 2.44946 & 1.56322 & 1.82157 \\
\hline $\mathrm{C}$ & 2.55697 & 0.32080 & 2.44630 \\
\hline $\mathrm{C}$ & 3.08888 & -0.80802 & 1.70835 \\
\hline $\mathrm{C}$ & 3.49636 & -0.63190 & 0.38590 \\
\hline $\mathrm{C}$ & 3.18672 & -1.64433 & -0.60854 \\
\hline $\mathrm{C}$ & 2.46581 & -2.78464 & -0.24396 \\
\hline $\mathrm{C}$ & 1.42048 & -3.29287 & -1.11733 \\
\hline $\mathrm{C}$ & 1.14673 & -2.65095 & -2.32539 \\
\hline $\mathrm{C}$ & -0.23086 & -2.45719 & -2.75108 \\
\hline $\mathrm{C}$ & -1.27916 & -2.91148 & -1.94688 \\
\hline $\mathrm{C}$ & -2.45798 & -2.08317 & -1.75549 \\
\hline $\mathrm{C}$ & -2.54298 & -0.83973 & -2.37787 \\
\hline $\mathrm{C}$ & -3.05128 & 0.30555 & -1.64015 \\
\hline $\mathrm{C}$ & -3.45457 & 0.17524 & -0.31839 \\
\hline $\mathrm{C}$ & -3.39137 & -1.12885 & 0.32255 \\
\hline $\mathrm{C}$ & -2.89710 & -2.23213 & -0.37723 \\
\hline $\mathrm{C}$ & -1.99674 & -3.16369 & 0.28035 \\
\hline $\mathrm{C}$ & -0.99331 & -3.57837 & -0.68778 \\
\hline $\mathrm{C}$ & 0.32907 & -3.76838 & -0.28312 \\
\hline $\mathrm{C}$ & 0.70190 & -3.55996 & 1.10600 \\
\hline $\mathrm{C}$ & 2.02378 & -2.95561 & 1.13051 \\
\hline $\mathrm{C}$ & 2.33129 & -1.98877 & 2.08813 \\
\hline $\mathrm{C}$ & 1.32952 & -1.58492 & 3.06274 \\
\hline $\mathrm{C}$ & 1.47316 & -0.15703 & 3.29003 \\
\hline $\mathrm{C}$ & 0.33978 & 0.63615 & 3.48696 \\
\hline $\mathrm{C}$ & 0.25070 & 1.94300 & 2.86209 \\
\hline $\mathrm{C}$ & -1.11507 & 2.14199 & 2.40952 \\
\hline $\mathrm{C}$ & -1.86776 & 0.96718 & 2.76935 \\
\hline $\mathrm{C}$ & -0.98033 & 0.03386 & 3.44435 \\
\hline C & -1.12027 & -1.33533 & 3.22878 \\
\hline
\end{tabular}




$\begin{array}{lrrr}\mathrm{C} & 0.05821 & -2.16384 & 3.03610 \\ \mathrm{C} & -0.26197 & -3.16953 & 2.03717 \\ \mathrm{C} & -1.63863 & -2.96349 & 1.61468 \\ \mathrm{C} & -2.16654 & -1.82372 & 2.34480 \\ \mathrm{C} & -3.02011 & -0.92131 & 1.70583 \\ \mathrm{C} & -2.85661 & 0.50951 & 1.90950 \\ \mathrm{C} & -3.09859 & 1.19931 & 0.65168 \\ \mathrm{C} & -2.37503 & 2.35777 & 0.28366 \\ \mathrm{C} & -1.37838 & 2.87320 & 1.24617 \\ \mathrm{C} & -0.28511 & 3.64889 & 0.66306 \\ \mathrm{C} & -0.84148 & 3.33789 & -1.40889 \\ \mathrm{C} & -2.01973 & 2.51475 & -1.14265 \\ \mathrm{C} & -2.30770 & 1.47531 & -2.03373 \\ \mathrm{C} & -0.63454 & 4.52062 & -0.50797 \\ \mathrm{C} & 0.40218 & 5.57067 & -0.94415 \\ \mathrm{H} & -1.60139 & 4.99479 & -0.31939 \\ \mathrm{H} & -0.05657 & 6.56319 & -0.97056 \\ \mathrm{H} & 0.79583 & 5.35759 & -1.94187 \\ \mathrm{H} & 1.24885 & 5.61194 & -0.25335\end{array}$

(5) Transition state (TS56)

\begin{tabular}{|c|c|c|c|}
\hline $\mathrm{C}$ & 0.11451 & 3.95260 & -0.00036 \\
\hline $\mathrm{C}$ & 2.07719 & 2.88668 & 0.26369 \\
\hline $\mathrm{C}$ & 2.23719 & 2.35120 & -1.04801 \\
\hline $\mathrm{C}$ & 1.05847 & 2.53433 & -1.90788 \\
\hline $\mathrm{C}$ & -0.06765 & 3.18609 & -1.26739 \\
\hline $\mathrm{C}$ & -1.34794 & 2.70210 & -1.48883 \\
\hline $\mathrm{C}$ & -1.60053 & 1.69327 & -2.47958 \\
\hline $\mathrm{C}$ & -0.54187 & 1.15798 & -3.21106 \\
\hline $\mathrm{C}$ & 0.80785 & 1.57457 & -2.90755 \\
\hline $\mathrm{C}$ & 1.66798 & 0.41481 & -3.08404 \\
\hline $\mathrm{C}$ & 2.75859 & 0.22795 & -2.24554 \\
\hline $\mathrm{C}$ & 3.04151 & 1.20798 & -1.21609 \\
\hline $\mathrm{C}$ & 3.51166 & 0.49336 & -0.04852 \\
\hline $\mathrm{C}$ & 3.12894 & 0.92240 & 1.22791 \\
\hline $\mathrm{C}$ & 2.34019 & 2.12119 & 1.36816 \\
\hline $\mathrm{C}$ & 1.34187 & 1.88015 & 2.44518 \\
\hline $\mathrm{C}$ & 1.61770 & 0.55096 & 2.97693 \\
\hline $\mathrm{C}$ & 2.71721 & -0.03469 & 2.23108 \\
\hline $\mathrm{C}$ & 2.74014 & -1.39896 & 1.94758 \\
\hline $\mathrm{C}$ & 3.14289 & -1.85362 & 0.62402 \\
\hline $\mathrm{C}$ & 3.51736 & -0.92658 & -0.35243 \\
\hline $\mathrm{C}$ & 3.05832 & -1.09256 & -1.71973 \\
\hline $\mathrm{C}$ & 2.25370 & -2.17962 & -2.06301 \\
\hline $\mathrm{C}$ & 1.12192 & -1.98918 & -2.95516 \\
\hline $\mathrm{C}$ & 0.83908 & -0.71819 & -3.45958 \\
\hline $\mathrm{C}$ & -0.53313 & -0.25580 & -3.53731 \\
\hline
\end{tabular}




\begin{tabular}{|c|c|c|c|}
\hline C & -1.57471 & -1.07969 & -3.10284 \\
\hline $\mathrm{C}$ & -2.67345 & -0.51952 & -2.33193 \\
\hline $\mathrm{C}$ & -2.68009 & 0.83981 & -2.02371 \\
\hline $\mathrm{C}$ & -3.08514 & 1.29875 & -0.70853 \\
\hline $\mathrm{C}$ & -3.46128 & 0.36074 & 0.25937 \\
\hline $\mathrm{C}$ & -3.44524 & -1.05842 & -0.05154 \\
\hline $\mathrm{C}$ & -3.05961 & -1.49159 & -1.32213 \\
\hline $\mathrm{C}$ & -2.19804 & -2.64940 & -1.46600 \\
\hline $\mathrm{C}$ & -1.28218 & -2.39653 & -2.56972 \\
\hline $\mathrm{C}$ & 0.03954 & -2.84219 & -2.49530 \\
\hline $\mathrm{C}$ & 0.50044 & -3.55533 & -1.31578 \\
\hline $\mathrm{C}$ & 1.86865 & -3.14416 & -1.04849 \\
\hline $\mathrm{C}$ & 2.30555 & -2.98353 & 0.26916 \\
\hline $\mathrm{C}$ & 1.38854 & -3.23111 & 1.37275 \\
\hline $\mathrm{C}$ & 1.65897 & -2.25274 & 2.40807 \\
\hline $\mathrm{C}$ & 0.60820 & -1.70246 & 3.14384 \\
\hline $\mathrm{C}$ & 0.59188 & -0.27917 & 3.43541 \\
\hline $\mathrm{C}$ & -0.77151 & 0.17780 & 3.34934 \\
\hline $\mathrm{C}$ & -1.61594 & -0.95809 & 3.01393 \\
\hline $\mathrm{C}$ & -0.75844 & -2.12187 & 2.87948 \\
\hline $\mathrm{C}$ & -1.01952 & -3.06701 & 1.88437 \\
\hline $\mathrm{C}$ & 0.07539 & -3.63103 & 1.11463 \\
\hline $\mathrm{C}$ & -0.37701 & -3.79513 & -0.25647 \\
\hline $\mathrm{C}$ & -1.75360 & -3.33557 & -0.33340 \\
\hline $\mathrm{C}$ & -2.15223 & -2.88730 & 0.98935 \\
\hline $\mathrm{C}$ & -2.98136 & -1.77221 & 1.12755 \\
\hline $\mathrm{C}$ & -2.70514 & -0.78996 & 2.16135 \\
\hline $\mathrm{C}$ & -3.00039 & 0.52356 & 1.61699 \\
\hline $\mathrm{C}$ & -2.18258 & 1.61254 & 1.93388 \\
\hline $\mathrm{C}$ & -1.03442 & 1.43583 & 2.80152 \\
\hline $\mathrm{C}$ & 0.02652 & 2.31497 & 2.35748 \\
\hline $\mathrm{C}$ & -0.56765 & 3.32586 & 1.37518 \\
\hline $\mathrm{C}$ & -1.85501 & 2.61216 & 0.95517 \\
\hline $\mathrm{C}$ & -2.27478 & 2.44918 & -0.35273 \\
\hline $\mathrm{C}$ & -0.80861 & 4.79966 & 1.81405 \\
\hline $\mathrm{C}$ & -0.66517 & 5.24829 & 0.35044 \\
\hline $\mathrm{H}$ & -1.62755 & 5.23289 & -0.17817 \\
\hline $\mathrm{H}$ & -0.15044 & 6.18297 & 0.13631 \\
\hline $\mathrm{H}$ & -1.75544 & 4.99833 & 2.32288 \\
\hline $\mathrm{H}$ & 0.01950 & 5.15828 & 2.43322 \\
\hline $\mathrm{H}$ & 1.46980 & 5.08949 & -0.69923 \\
\hline $\mathrm{H}$ & 1.54197 & 4.15674 & 0.02158 \\
\hline
\end{tabular}

(6) Transition state (TS67)

$\begin{array}{lllr}\mathrm{C} & 0.44521 & 3.27863 & 0.18579 \\ \mathrm{C} & 1.80125 & 2.86983 & 0.08471 \\ \mathrm{C} & 2.22218 & 2.65136 & -1.37827\end{array}$




\begin{tabular}{|c|c|c|c|}
\hline C & 0.83290 & 2.62738 & -2.08077 \\
\hline $\mathrm{C}$ & -0.21544 & 3.26662 & -1.15251 \\
\hline $\mathrm{C}$ & -1.74172 & 2.87102 & -1.28645 \\
\hline $\mathrm{C}$ & -1.96319 & 1.63349 & -2.14685 \\
\hline $\mathrm{C}$ & -0.98884 & 1.10747 & -2.96359 \\
\hline $\mathrm{C}$ & 0.41985 & 1.56904 & -2.89358 \\
\hline $\mathrm{C}$ & 1.26427 & 0.45439 & -3.17814 \\
\hline $\mathrm{C}$ & 2.46594 & 0.32132 & -2.41971 \\
\hline $\mathrm{C}$ & 2.91285 & 1.29704 & -1.51879 \\
\hline $\mathrm{C}$ & 3.47379 & 0.61711 & -0.38561 \\
\hline $\mathrm{C}$ & 3.29913 & 1.06701 & 0.92857 \\
\hline C & 2.39708 & 2.20230 & 1.15097 \\
\hline $\mathrm{C}$ & 1.64151 & 1.95155 & 2.34919 \\
\hline $\mathrm{C}$ & 2.03896 & 0.66485 & 2.87485 \\
\hline $\mathrm{C}$ & 3.04865 & 0.11141 & 1.98416 \\
\hline $\mathrm{C}$ & 3.05999 & -1.26985 & 1.73534 \\
\hline $\mathrm{C}$ & 3.28002 & -1.73795 & 0.37872 \\
\hline $\mathrm{C}$ & 3.48664 & -0.82142 & -0.65167 \\
\hline $\mathrm{C}$ & 2.85202 & -1.01001 & -1.92979 \\
\hline $\mathrm{C}$ & 2.03686 & -2.11299 & -2.15185 \\
\hline $\mathrm{C}$ & 0.78939 & -1.97460 & -2.89989 \\
\hline $\mathrm{C}$ & 0.41594 & -0.71394 & -3.36081 \\
\hline $\mathrm{C}$ & -0.97416 & -0.30412 & -3.26261 \\
\hline $\mathrm{C}$ & -1.92868 & -1.15573 & -2.69753 \\
\hline $\mathrm{C}$ & -2.92185 & -0.60874 & -1.80064 \\
\hline $\mathrm{C}$ & -2.92326 & 0.76260 & -1.52646 \\
\hline $\mathrm{C}$ & -3.15348 & 1.23570 & -0.17322 \\
\hline $\mathrm{C}$ & -3.37653 & 0.31345 & 0.85550 \\
\hline $\mathrm{C}$ & -3.36567 & -1.11343 & 0.56984 \\
\hline $\mathrm{C}$ & -3.15032 & -1.56665 & -0.73170 \\
\hline C & -2.28109 & -2.70797 & -0.97379 \\
\hline $\mathrm{C}$ & -1.53811 & -2.46092 & -2.19693 \\
\hline $\mathrm{C}$ & -0.19577 & -2.84509 & -2.28328 \\
\hline $\mathrm{C}$ & 0.43878 & -3.52466 & -1.16942 \\
\hline $\mathrm{C}$ & 1.82120 & -3.07644 & -1.09201 \\
\hline $\mathrm{C}$ & 2.42835 & -2.89125 & 0.15328 \\
\hline $\mathrm{C}$ & 1.67291 & -3.13424 & 1.36981 \\
\hline $\mathrm{C}$ & 2.06102 & -2.12215 & 2.34290 \\
\hline $\mathrm{C}$ & 1.10040 & -1.58844 & 3.20986 \\
\hline C & 1.09017 & -0.16863 & 3.47535 \\
\hline $\mathrm{C}$ & -0.30021 & 0.26242 & 3.56911 \\
\hline $\mathrm{C}$ & -1.14752 & -0.90040 & 3.36258 \\
\hline C & -0.28370 & -2.03933 & 3.12744 \\
\hline $\mathrm{C}$ & -0.65125 & -3.01315 & 2.19610 \\
\hline $\mathrm{C}$ & 0.34896 & -3.56684 & 1.29637 \\
\hline $\mathrm{C}$ & -0.28116 & -3.77086 & 0.00208 \\
\hline C & -1.66829 & -3.35231 & 0.10375 \\
\hline C & -1.89383 & -2.87863 & 1.45973 \\
\hline $\mathrm{C}$ & -2.73553 & -1.78595 & 1.69355 \\
\hline
\end{tabular}




$\begin{array}{lrrr}\mathrm{C} & -2.35087 & -0.77442 & 2.66027 \\ \mathrm{C} & -2.75110 & 0.52149 & 2.14022 \\ \mathrm{C} & -1.92950 & 1.63738 & 2.33148 \\ \mathrm{C} & -0.68292 & 1.50158 & 3.06309 \\ \mathrm{C} & 0.30360 & 2.36967 & 2.43937 \\ \mathrm{C} & -0.30925 & 3.04059 & 1.33642 \\ \mathrm{C} & -1.71848 & 2.58536 & 1.25695 \\ \mathrm{C} & -2.34726 & 2.40929 & 0.04373 \\ \mathrm{C} & -2.20184 & 4.24444 & -1.85817 \\ \mathrm{C} & -0.86941 & 4.97688 & -1.67331 \\ \mathrm{H} & -0.73756 & 5.54872 & -0.75668 \\ \mathrm{H} & -0.47604 & 5.53767 & -2.53208 \\ \mathrm{H} & -3.03410 & 4.69730 & -1.31293 \\ \mathrm{H} & -2.48357 & 4.16602 & -2.91171 \\ \mathrm{H} & 2.85342 & 3.47795 & -1.74146 \\ \mathrm{H} & 0.22017 & 3.98331 & -2.10762\end{array}$

(7) Transition state (TS78)

$\begin{array}{llll}\mathrm{C} & -3.58375 & -0.18191 & -0.41458 \\ \mathrm{C} & -2.78407 & -0.69059 & -1.64329 \\ \mathrm{C} & -2.24888 & -2.00456 & -1.38687 \\ \mathrm{C} & -2.40002 & -2.29060 & 0.02861 \\ \mathrm{C} & -3.03907 & -1.16206 & 0.65894 \\ \mathrm{C} & -2.61034 & -0.77008 & 1.91445 \\ \mathrm{C} & -1.58071 & -1.51894 & 2.60641 \\ \mathrm{C} & -1.00167 & -2.64612 & 2.01329 \\ \mathrm{C} & -1.42398 & -3.04429 & 0.69230 \\ \mathrm{C} & -0.25379 & -3.52014 & -0.03456 \\ \mathrm{C} & -0.11556 & -3.25443 & -1.39370 \\ \mathrm{C} & -1.13958 & -2.48651 & -2.08930 \\ \mathrm{C} & -0.48752 & -1.63590 & -3.05870 \\ \mathrm{C} & -0.98338 & -0.34846 & -3.28544 \\ \mathrm{C} & -2.14794 & 0.12490 & -2.56331 \\ \mathrm{C} & -1.96146 & 1.55493 & -2.31055 \\ \mathrm{C} & -0.69541 & 1.93846 & -2.80024 \\ \mathrm{C} & -0.07489 & 0.76496 & -3.42981 \\ \mathrm{C} & 1.29700 & 0.56008 & -3.33180 \\ \mathrm{C} & 1.81847 & -0.77353 & -3.09326 \\ \mathrm{C} & 0.93948 & -1.85530 & -2.96157 \\ \mathrm{C} & 1.17382 & -2.85603 & -1.93197 \\ \mathrm{C} & 2.27549 & -2.73726 & -1.08341 \\ \mathrm{C} & 2.13422 & -3.01987 & 0.33666 \\ \mathrm{C} & 0.89377 & -3.40552 & 0.84995 \\ \mathrm{C} & 0.42836 & -2.86405 & 2.11514 \\ \mathrm{C} & 1.22387 & -1.94733 & 2.81096 \\ \mathrm{C} & 0.61658 & -0.78180 & 3.43490 \\ \mathrm{C} & -0.75615 & -0.57388 & 3.32902 \\ \mathrm{C} & -1.28731 & 0.76838 & 3.08582\end{array}$




$\begin{array}{llll}\mathrm{C} & -0.38437 & 1.85595 & 2.96612 \\ \mathrm{C} & 1.04521 & 1.61880 & 3.05999 \\ \mathrm{C} & 1.54096 & 0.33650 & 3.30044 \\ \mathrm{C} & 2.70546 & -0.13861 & 2.57947 \\ \mathrm{C} & 2.51233 & -1.55192 & 2.28192 \\ \mathrm{C} & 2.96082 & -2.07581 & 1.06586 \\ \mathrm{C} & 3.62065 & -1.21350 & 0.10009 \\ \mathrm{C} & 3.18978 & -1.61753 & -1.22821 \\ \mathrm{C} & 2.96688 & -0.65682 & -2.21689 \\ \mathrm{C} & 3.16255 & 0.75508 & -1.91565 \\ \mathrm{C} & 2.14148 & 1.51330 & -2.60841 \\ \mathrm{C} & 1.57004 & 2.63314 & -2.00747 \\ \mathrm{C} & 0.13457 & 2.86849 & -2.10195 \\ \mathrm{C} & -0.29948 & 3.39838 & -0.85947 \\ \mathrm{C} & 0.83780 & 3.51345 & 0.03925 \\ \mathrm{C} & 2.00671 & 3.04093 & -0.68282 \\ \mathrm{C} & 2.98554 & 2.30845 & -0.01118 \\ \mathrm{C} & 3.57943 & 1.14308 & -0.63939 \\ \mathrm{C} & 3.80583 & 0.14019 & 0.38854 \\ \mathrm{C} & 3.34808 & 0.68653 & 1.65406 \\ \mathrm{C} & 2.83744 & 2.02485 & 1.40839 \\ \mathrm{C} & 1.71524 & 2.48187 & 2.09998 \\ \mathrm{C} & 0.69090 & 3.23515 & 1.39710 \\ \mathrm{C} & -0.60455 & 2.83954 & 1.94817 \\ \mathrm{C} & -1.71066 & 2.71937 & 1.09237 \\ \mathrm{C} & -1.55532 & 3.03406 & -0.29801 \\ \mathrm{C} & -2.45318 & 2.04863 & -1.04716 \\ \mathrm{C} & -3.10894 & 1.22898 & -0.07480 \\ \mathrm{C} & -2.61798 & 1.59180 & 1.20254 \\ \mathrm{C} & -2.43069 & 0.65451 & 2.24886 \\ \mathrm{C} & -5.96741 & 0.08031 & 0.56888 \\ \mathrm{C} & -5.11854 & -0.30945 & -0.64361 \\ \mathrm{H} & -5.33586 & -1.34473 & -0.93786 \\ \mathrm{H} & -5.37890 & 0.32325 & -1.50207 \\ \mathrm{H} & -5.74995 & -0.56030 & 1.43072 \\ \mathrm{H} & -7.03346 & -0.01857 & 0.33727 \\ \mathrm{H} & -5.78165 & 1.11839 & 0.86538 \\ \mathrm{H} & -2.71315 & 3.30061 & -0.98021\end{array}$

(8) Transition state (TS89)
C $\quad-1.28709$
3.35772
0.34332
C $\quad-0.01822$
$3.26297-0.54053$
C $\quad-0.35401$
$2.73333-1.83546$
C $\quad-1.71036$
$2.21514-1.78671$
$\begin{array}{llll}\text { C } & -2.23129 & 2.41855 & -0.45789\end{array}$
$\begin{array}{llll}\text { C } & -3.00495 & 1.43009 & 0.11835\end{array}$
$\begin{array}{llll}\text { C } & -3.33968 & 0.23285 & -0.62446\end{array}$ 


\begin{tabular}{|c|c|c|c|}
\hline C & -2.87774 & 0.06069 & -1.93481 \\
\hline $\mathrm{C}$ & -2.04423 & 1.07692 & -2.52966 \\
\hline $\mathrm{C}$ & -1.03407 & 0.41623 & -3.34839 \\
\hline $\mathrm{C}$ & 0.26070 & 0.92503 & -3.40362 \\
\hline $\mathrm{C}$ & 0.60829 & 2.11715 & -2.64128 \\
\hline $\mathrm{C}$ & 1.95245 & 1.95756 & -2.13930 \\
\hline $\mathrm{C}$ & 2.27379 & 2.43225 & -0.86394 \\
\hline $\mathrm{C}$ & 1.26641 & 3.09061 & -0.05196 \\
\hline $\mathrm{C}$ & 1.52680 & 2.75364 & 1.34594 \\
\hline $\mathrm{C}$ & 2.61413 & 1.83293 & 1.38529 \\
\hline $\mathrm{C}$ & 3.10528 & 1.64622 & 0.01847 \\
\hline C & 3.58193 & 0.40405 & -0.39773 \\
\hline $\mathrm{C}$ & 3.24305 & -0.09760 & -1.72058 \\
\hline $\mathrm{C}$ & 2.44563 & 0.66726 & -2.57686 \\
\hline $\mathrm{C}$ & 1.40021 & 0.02606 & -3.35937 \\
\hline $\mathrm{C}$ & 1.19743 & -1.35142 & -3.25610 \\
\hline $\mathrm{C}$ & -0.15495 & -1.88343 & -3.19707 \\
\hline $\mathrm{C}$ & -1.24847 & -1.01560 & -3.24491 \\
\hline $\mathrm{C}$ & -2.38937 & -1.23442 & -2.36924 \\
\hline $\mathrm{C}$ & -2.38318 & -2.31066 & -1.47811 \\
\hline $\mathrm{C}$ & -2.86928 & -2.13147 & -0.11837 \\
\hline $\mathrm{C}$ & -3.33089 & -0.88459 & 0.29650 \\
\hline $\mathrm{C}$ & -2.99218 & -0.37816 & 1.62445 \\
\hline $\mathrm{C}$ & -2.19126 & -1.16581 & 2.48295 \\
\hline $\mathrm{C}$ & -1.70949 & -2.46150 & 2.03850 \\
\hline $\mathrm{C}$ & -2.03929 & -2.93591 & 0.76770 \\
\hline $\mathrm{C}$ & -1.03760 & -3.59754 & -0.04501 \\
\hline $\mathrm{C}$ & -1.25177 & -3.21557 & -1.43318 \\
\hline $\mathrm{C}$ & -0.15530 & -3.00402 & -2.27376 \\
\hline $\mathrm{C}$ & 1.19604 & -3.16950 & -1.76684 \\
\hline $\mathrm{C}$ & 2.03076 & -2.14405 & -2.36935 \\
\hline $\mathrm{C}$ & 3.03585 & -1.52835 & -1.61897 \\
\hline $\mathrm{C}$ & 3.24798 & -1.91199 & -0.22980 \\
\hline $\mathrm{C}$ & 3.59674 & -0.72192 & 0.52289 \\
\hline $\mathrm{C}$ & 3.11982 & -0.55350 & 1.82624 \\
\hline $\mathrm{C}$ & 2.63745 & 0.73659 & 2.27904 \\
\hline $\mathrm{C}$ & 1.50234 & 0.50589 & 3.13910 \\
\hline $\mathrm{C}$ & 1.28316 & -0.92948 & 3.24429 \\
\hline $\mathrm{C}$ & 2.29057 & -1.58684 & 2.42888 \\
\hline $\mathrm{C}$ & 1.95979 & -2.73217 & 1.70547 \\
\hline $\mathrm{C}$ & 2.44839 & -2.90057 & 0.34713 \\
\hline $\mathrm{C}$ & 1.39961 & -3.53426 & -0.43379 \\
\hline $\mathrm{C}$ & 0.26236 & -3.75696 & 0.44181 \\
\hline $\mathrm{C}$ & 0.60725 & -3.26563 & 1.76509 \\
\hline $\mathrm{C}$ & -0.35878 & -2.63602 & 2.54900 \\
\hline $\mathrm{C}$ & -0.01206 & -1.44356 & 3.30634 \\
\hline $\mathrm{C}$ & -1.15415 & -0.54736 & 3.25955 \\
\hline $\mathrm{C}$ & -0.92897 & 0.83449 & 3.12769 \\
\hline $\mathrm{C}$ & 0.39750 & 1.36220 & 3.09686 \\
\hline
\end{tabular}




$\begin{array}{lrrr}\mathrm{C} & 0.39765 & 2.53894 & 2.20429 \\ \mathrm{C} & -0.98942 & 2.68542 & 1.68115 \\ \mathrm{C} & -1.76915 & 1.64051 & 2.26396 \\ \mathrm{C} & -2.80564 & 1.03424 & 1.53191 \\ \mathrm{C} & -3.13102 & 4.96115 & 1.23071 \\ \mathrm{C} & -1.82023 & 4.81588 & 0.45352 \\ \mathrm{H} & -1.94295 & 5.20118 & -0.56652 \\ \mathrm{H} & -1.03339 & 5.42259 & 0.92181 \\ \mathrm{H} & -3.93847 & 4.39080 & 0.75891 \\ \mathrm{H} & -3.43794 & 6.01182 & 1.26550 \\ \mathrm{H} & -3.02885 & 4.60805 & 2.26281 \\ \mathrm{H} & -0.21011 & 3.50935 & 2.68146\end{array}$

Transition state structures observed over $\mathrm{C}_{59} \mathrm{Pd}$ during acetylene hydrogenation.

(1) Transition state (TS12)

\begin{tabular}{cccc} 
Pd & -0.64927 & 3.95429 & -0.19898 \\
C & 0.41939 & 2.83699 & 1.12810 \\
C & 1.75963 & 2.48055 & 0.71201 \\
$\mathrm{C}$ & 1.91926 & 2.42213 & -0.73590 \\
$\mathrm{C}$ & 0.72148 & 2.85360 & -1.43725 \\
$\mathrm{C}$ & 0.15128 & 1.98312 & -2.37040 \\
$\mathrm{C}$ & 0.99548 & 0.95539 & -2.94463 \\
$\mathrm{C}$ & 2.28653 & 0.72358 & -2.46080 \\
$\mathrm{C}$ & 2.74631 & 1.44901 & -1.29929 \\
$\mathrm{C}$ & 3.51303 & 0.53711 & -0.47894 \\
$\mathrm{C}$ & 3.39851 & 0.60574 & 0.90451 \\
$\mathrm{C}$ & 2.49930 & 1.57805 & 1.50600 \\
$\mathrm{C}$ & 1.88686 & 0.95319 & 2.65111 \\
$\mathrm{C}$ & 0.54053 & 1.20519 & 2.92766 \\
$\mathrm{C}$ & -0.22368 & 2.13372 & 2.12526 \\
$\mathrm{C}$ & -1.62209 & 1.60125 & 2.05526 \\
$\mathrm{C}$ & -1.64274 & 0.36870 & 2.82494 \\
$\mathrm{C}$ & -0.32487 & 0.13447 & 3.37539 \\
$\mathrm{C}$ & 0.17395 & -1.16035 & 3.51346 \\
$\mathrm{C}$ & 1.56489 & -1.43481 & 3.19249 \\
$\mathrm{C}$ & 2.40233 & -0.39843 & 2.77425 \\
$\mathrm{C}$ & 3.33677 & -0.61757 & 1.68494 \\
$\mathrm{C}$ & 3.38838 & -1.85904 & 1.04672 \\
$\mathrm{C}$ & 3.49933 & -1.92660 & -0.40256 \\
$\mathrm{C}$ & 3.55812 & -0.75030 & -1.14993 \\
$\mathrm{C}$ & 2.80035 & -0.63246 & -2.38449 \\
$\mathrm{C}$ & 2.01390 & -1.69975 & -2.82090 \\
$\mathrm{C}$ & 0.68539 & -1.45283 & -3.35804 \\
$\mathrm{C}$ & 0.18754 & -0.15010 & -3.39482 \\
& & & \\
\hline
\end{tabular}




$\begin{array}{lrrr}\mathrm{C} & -1.18554 & 0.14069 & -3.04751 \\ \mathrm{C} & -2.02121 & -0.92110 & -2.68680 \\ \mathrm{C} & -1.50994 & -2.27843 & -2.61900 \\ \mathrm{C} & -0.17843 & -2.54127 & -2.94346 \\ \mathrm{C} & 0.60574 & -3.45122 & -2.13025 \\ \mathrm{C} & 1.96272 & -2.92973 & -2.05380 \\ \mathrm{C} & 2.68691 & -3.03881 & -0.86403 \\ \mathrm{C} & 2.08050 & -3.66485 & 0.29947 \\ \mathrm{C} & 2.51288 & -2.93359 & 1.47856 \\ \mathrm{C} & 1.62140 & -2.72524 & 2.53434 \\ \mathrm{C} & 0.26692 & -3.25353 & 2.45816 \\ \mathrm{C} & -0.62550 & -2.29071 & 3.07376 \\ \mathrm{C} & -1.89891 & -2.07460 & 2.54453 \\ \mathrm{C} & -2.42670 & -0.72550 & 2.43766 \\ \mathrm{C} & -3.18878 & -0.64132 & 1.21756 \\ \mathrm{C} & -3.12341 & -1.92795 & 0.54678 \\ \mathrm{C} & -2.32985 & -2.82038 & 1.37152 \\ \mathrm{C} & -1.46689 & -3.74000 & 0.77138 \\ \mathrm{C} & -0.14382 & -3.96422 & 1.32736 \\ \mathrm{C} & 0.77997 & -4.16698 & 0.22493 \\ \mathrm{C} & 0.02821 & -4.06186 & -1.01507 \\ \mathrm{C} & -1.36016 & -3.79901 & -0.67889 \\ \mathrm{C} & -2.11765 & -2.93069 & -1.46919 \\ \mathrm{C} & -3.01903 & -1.98203 & -0.84248 \\ \mathrm{C} & -2.95844 & -0.74736 & -1.60552 \\ \mathrm{C} & -3.01792 & 0.48568 & -0.96642 \\ \mathrm{C} & -3.15372 & 0.54376 & 0.48178 \\ \mathrm{C} & -2.43986 & 1.71361 & 0.94377 \\ \mathrm{C} & -2.24486 & 2.60686 & -0.25913 \\ \mathrm{C} & -2.22702 & 1.62611 & -1.39891 \\ \mathrm{C} & -1.25753 & 1.44861 & -2.39460 \\ \mathrm{C} & -3.03024 & 3.88738 & -0.32255 \\ \mathrm{C} & -2.36808 & 4.87563 & 0.32758 \\ \mathrm{H} & -3.94344 & 3.97035 & -0.92051 \\ \mathrm{H} & -2.70603 & 5.89323 & 0.50095 \\ & 0.70965 & 4.25522 & -1.29345\end{array}$

(2) Transition state (TS23)

$\begin{array}{cccc}\text { Pd } & -4.01861 & -0.46201 & -0.22316 \\ \text { C } & -2.51507 & -0.86341 & -1.51694 \\ \text { C } & -1.84297 & -2.12584 & -1.28099 \\ \text { C } & -1.91601 & -2.53767 & 0.08767 \\ \text { C } & -2.84983 & -1.71225 & 0.96100 \\ \text { C } & -2.07968 & -0.95214 & 1.99847 \\ \text { C } & -0.94910 & -1.64514 & 2.60266 \\ \text { C } & -0.35231 & -2.74393 & 1.97932\end{array}$




\begin{tabular}{|c|c|c|c|}
\hline C & -0.82681 & -3.17588 & 0.66306 \\
\hline C & 0.33024 & -3.53823 & -0.11643 \\
\hline C & 0.37745 & -3.22881 & -1.47425 \\
\hline C & -0.71873 & -2.49145 & -2.05641 \\
\hline $\mathrm{C}$ & -0.17689 & -1.55729 & -3.00290 \\
\hline $\mathrm{C}$ & -0.74150 & -0.27864 & -3.09762 \\
\hline $\mathrm{C}$ & -1.90354 & 0.09649 & -2.32192 \\
\hline C & -1.76053 & 1.56302 & -2.01510 \\
\hline C & -0.49141 & 1.98978 & -2.60745 \\
\hline C & 0.11481 & 0.87244 & -3.29175 \\
\hline C & 1.50179 & 0.71906 & -3.33723 \\
\hline C & 2.08989 & -0.60118 & -3.18330 \\
\hline C & 1.26587 & -1.71591 & -3.02905 \\
\hline $\mathrm{C}$ & 1.61242 & -2.74818 & -2.06530 \\
\hline $\mathrm{C}$ & 2.75568 & -2.61069 & -1.27593 \\
\hline C & 2.70547 & -2.94056 & 0.14221 \\
\hline $\mathrm{C}$ & 1.51561 & -3.39043 & 0.71067 \\
\hline $\mathrm{C}$ & 1.08982 & -2.89986 & 2.01126 \\
\hline $\mathrm{C}$ & 1.88460 & -1.97571 & 2.69456 \\
\hline C & 1.26300 & -0.85443 & 3.37177 \\
\hline C & -0.12555 & -0.69129 & 3.30460 \\
\hline $\mathrm{C}$ & -0.71364 & 0.60465 & 3.09110 \\
\hline $\mathrm{C}$ & 0.13071 & 1.70248 & 2.98460 \\
\hline C & 1.57076 & 1.55932 & 3.04814 \\
\hline C & 2.13195 & 0.29373 & 3.22613 \\
\hline $\mathrm{C}$ & 3.28863 & -0.10493 & 2.44224 \\
\hline $\mathrm{C}$ & 3.13334 & -1.51294 & 2.11011 \\
\hline $\mathrm{C}$ & 3.53134 & -1.97995 & 0.85713 \\
\hline C & 4.09243 & -1.05988 & -0.11914 \\
\hline $\mathrm{C}$ & 3.60787 & -1.44749 & -1.43197 \\
\hline $\mathrm{C}$ & 3.28616 & -0.46390 & -2.37552 \\
\hline $\mathrm{C}$ & 3.44936 & 0.94078 & -2.04483 \\
\hline $\mathrm{C}$ & 2.35380 & 1.67553 & -2.65426 \\
\hline $\mathrm{C}$ & 1.77983 & 2.75955 & -1.99265 \\
\hline $\mathrm{C}$ & 0.33610 & 2.93817 & -1.99493 \\
\hline C & -0.04657 & 3.45755 & -0.70553 \\
\hline $\mathrm{C}$ & 1.14271 & 3.56938 & 0.11544 \\
\hline C & 2.27920 & 3.15121 & -0.68054 \\
\hline C & 3.31964 & 2.43519 & -0.08417 \\
\hline $\mathrm{C}$ & 3.92021 & 1.31096 & -0.78158 \\
\hline $\mathrm{C}$ & 4.24237 & 0.29111 & 0.19953 \\
\hline $\mathrm{C}$ & 3.83184 & 0.77764 & 1.50728 \\
\hline $\mathrm{C}$ & 3.26091 & 2.10316 & 1.33245 \\
\hline $\mathrm{C}$ & 2.15688 & 2.49129 & 2.09414 \\
\hline C & 1.07989 & 3.23820 & 1.47113 \\
\hline C & -0.16483 & 2.74291 & 2.01897 \\
\hline $\mathrm{C}$ & -1.28461 & 2.61317 & 1.22095 \\
\hline C & -1.25540 & 3.04125 & -0.15545 \\
\hline $\mathrm{C}$ & -2.20752 & 2.19905 & -0.85929 \\
\hline
\end{tabular}




$\begin{array}{llll}\mathrm{C} & -3.10538 & 1.58617 & 0.17754 \\ \mathrm{C} & -2.26471 & 1.52760 & 1.36159 \\ \mathrm{C} & -1.93208 & 0.44860 & 2.25364 \\ \mathrm{C} & -4.59223 & 1.82738 & 0.21904 \\ \mathrm{C} & -5.39828 & 0.82256 & 0.60163 \\ \mathrm{H} & -4.93587 & 2.82613 & -0.07925 \\ \mathrm{H} & -6.46452 & 0.85134 & 0.77335 \\ \mathrm{H} & -5.38043 & -1.12829 & 0.33384 \\ \mathrm{H} & -3.66736 & -2.30521 & 1.39188\end{array}$

(3) Transition state (TS34)

$\begin{array}{cccc}\text { Pd } & -3.96790 & -0.54311 & -0.20209 \\ \mathrm{C} & -2.48327 & -1.07141 & -1.47691 \\ \mathrm{C} & -1.80885 & -2.30854 & -1.12484 \\ \mathrm{C} & -1.88484 & -2.56211 & 0.30872 \\ \mathrm{C} & -2.66073 & -1.53480 & 0.96740 \\ \mathrm{C} & -2.10115 & -0.84320 & 2.02990 \\ \mathrm{C} & -0.95798 & -1.42871 & 2.70534 \\ \mathrm{C} & -0.33718 & -2.58229 & 2.21730 \\ \mathrm{C} & -0.78213 & -3.13205 & 0.95805 \\ \mathrm{C} & 0.38948 & -3.56068 & 0.22038 \\ \mathrm{C} & 0.44453 & -3.36342 & -1.15658 \\ \mathrm{C} & -0.66347 & -2.71025 & -1.83031 \\ \mathrm{C} & -0.11792 & -1.85477 & -2.85299 \\ \mathrm{C} & -0.70104 & -0.60533 & -3.08617 \\ \mathrm{C} & -1.87888 & -0.17873 & -2.35582 \\ \mathrm{C} & -1.75275 & 1.31486 & -2.17884 \\ \mathrm{C} & -0.49242 & 1.70626 & -2.79426 \\ \mathrm{C} & 0.14053 & 0.53884 & -3.36470 \\ \mathrm{C} & 1.52945 & 0.41046 & -3.37680 \\ \mathrm{C} & 2.13687 & -0.87799 & -3.09096 \\ \mathrm{C} & 1.32780 & -1.98720 & -2.83908 \\ \mathrm{C} & 1.67830 & -2.91547 & -1.77725 \\ \mathrm{C} & 2.81141 & -2.68335 & -0.99432 \\ \mathrm{C} & 2.75049 & -2.88147 & 0.44609 \\ \mathrm{C} & 1.56149 & -3.30771 & 1.04018 \\ \mathrm{C} & 1.10832 & -2.69983 & 2.28093 \\ \mathrm{C} & 1.87079 & -1.69520 & 2.88077 \\ \mathrm{C} & 1.21501 & -0.52170 & 3.43040 \\ \mathrm{C} & -0.17025 & -0.38891 & 3.31749 \\ \mathrm{C} & -0.77601 & 0.88083 & 2.98235 \\ \mathrm{C} & 0.05105 & 1.99415 & 2.80832 \\ \mathrm{C} & 1.49473 & 1.86545 & 2.89646 \\ \mathrm{C} & 2.06749 & 0.62821 & 3.19155 \\ \mathrm{C} & 3.23924 & 0.17485 & 2.46830 \\ \mathrm{C} & 3.11447 & -1.26243 & 2.27118 \\ & 3.54542 & -1.84182 & 1.07536\end{array}$




$\begin{array}{lrrr}\mathrm{C} & 4.10385 & -1.00491 & 0.02549 \\ \mathrm{C} & 3.64691 & -1.52456 & -1.25249 \\ \mathrm{C} & 3.31953 & -0.64285 & -2.28601 \\ \mathrm{C} & 3.45254 & 0.79316 & -2.08758 \\ \mathrm{C} & 2.35218 & 1.44397 & -2.77202 \\ \mathrm{C} & 1.75206 & 2.57738 & -2.22257 \\ \mathrm{C} & 0.30686 & 2.72239 & -2.25436 \\ \mathrm{C} & -0.10218 & 3.36208 & -1.02797 \\ \mathrm{C} & 1.07639 & 3.57812 & -0.20854 \\ \mathrm{C} & 2.22824 & 3.10166 & -0.95123 \\ \mathrm{C} & 3.27459 & 2.46429 & -0.28058 \\ \mathrm{C} & 3.90197 & 1.29003 & -0.86152 \\ \mathrm{C} & 4.22802 & 0.37249 & 0.21691 \\ \mathrm{C} & 3.79056 & 0.97518 & 1.46494 \\ \mathrm{C} & 3.20256 & 2.26798 & 1.15966 \\ \mathrm{C} & 2.08198 & 2.71032 & 1.86813 \\ \mathrm{C} & 1.00159 & 3.38032 & 1.16888 \\ \mathrm{C} & -0.24975 & 2.94003 & 1.76540 \\ \mathrm{C} & -1.37795 & 2.73240 & 0.98171 \\ \mathrm{C} & -1.31034 & 2.98408 & -0.45121 \\ \mathrm{C} & -2.21394 & 2.05858 & -1.09743 \\ \mathrm{C} & -3.14323 & 1.57794 & -0.03313 \\ \mathrm{C} & -2.30853 & 1.63494 & 1.22438 \\ \mathrm{C} & -1.96911 & 0.65273 & 2.16447 \\ \mathrm{C} & -4.54991 & 1.61136 & -0.12506 \\ \mathrm{C} & -5.35757 & 0.92571 & 0.84548 \\ \mathrm{H} & -4.99732 & 1.93605 & -1.06230 \\ \mathrm{H} & -6.43325 & 0.91623 & 0.70506 \\ \mathrm{H} & -5.02862 & 0.90807 & 1.88088 \\ \mathrm{H} & -5.28938 & -0.97318 & 0.74997\end{array}$

(4) Transition state (TS45)

$\begin{array}{cccc}\text { Pd } & -1.58702 & 3.61369 & -0.09166 \\ \mathrm{C} & -1.94514 & 2.06716 & -1.35262 \\ \mathrm{C} & -2.83706 & 1.08940 & -0.77409 \\ \mathrm{C} & -2.84382 & 1.12370 & 0.69597 \\ \mathrm{C} & -1.95698 & 2.12749 & 1.23648 \\ \mathrm{C} & -1.08978 & 1.80322 & 2.25571 \\ \mathrm{C} & -1.25670 & 0.56357 & 2.97022 \\ \mathrm{C} & -2.24215 & -0.35015 & 2.58422 \\ \mathrm{C} & -3.03102 & -0.07823 & 1.40531 \\ \mathrm{C} & -3.23576 & -1.33740 & 0.71184 \\ \mathrm{C} & -3.22932 & -1.36986 & -0.67869 \\ \mathrm{C} & -3.01792 & -0.14438 & -1.42824 \\ \mathrm{C} & -2.21821 & -0.47083 & -2.58582 \\ \mathrm{C} & -1.22904 & 0.42411 & -3.00485 \\ \mathrm{C} & -1.06853 & 1.69581 & -2.34746\end{array}$




\begin{tabular}{|c|c|c|c|}
\hline C & 0.40027 & 1.98900 & -2.30546 \\
\hline C & 1.06516 & 0.89269 & -2.98858 \\
\hline C & 0.06652 & -0.06278 & -3.42628 \\
\hline $\mathrm{C}$ & 0.33674 & -1.42953 & -3.42768 \\
\hline $\mathrm{C}$ & -0.68087 & -2.36513 & -2.97433 \\
\hline $\mathrm{C}$ & -1.92986 & -1.89237 & -2.56044 \\
\hline $\mathrm{C}$ & -2.55943 & -2.45297 & -1.37790 \\
\hline $\mathrm{C}$ & -1.91884 & -3.46142 & -0.65563 \\
\hline C & -1.92558 & -3.42747 & 0.79854 \\
\hline $\mathrm{C}$ & -2.57262 & -2.38656 & 1.46700 \\
\hline C & -1.95391 & -1.77126 & 2.62788 \\
\hline C & -0.70891 & -2.22394 & 3.07493 \\
\hline $\mathrm{C}$ & 0.30466 & -1.26799 & 3.49358 \\
\hline $\mathrm{C}$ & 0.03478 & 0.09715 & 3.42593 \\
\hline $\mathrm{C}$ & 1.03767 & 1.03137 & 2.95345 \\
\hline C & 2.29689 & 0.54857 & 2.57950 \\
\hline $\mathrm{C}$ & 2.58061 & -0.87466 & 2.62862 \\
\hline $\mathrm{C}$ & 1.60258 & -1.76727 & 3.07263 \\
\hline C & 1.39255 & -3.02301 & 2.38115 \\
\hline C & -0.03724 & -3.30407 & 2.38117 \\
\hline C & -0.63247 & -3.89563 & 1.26386 \\
\hline $\mathrm{C}$ & 0.17460 & -4.21899 & 0.09926 \\
\hline $\mathrm{C}$ & -0.62157 & -3.95049 & -1.08659 \\
\hline $\mathrm{C}$ & -0.01589 & -3.41157 & -2.22471 \\
\hline $\mathrm{C}$ & 1.41391 & -3.13050 & -2.22455 \\
\hline $\mathrm{C}$ & 1.63059 & -1.90834 & -2.97188 \\
\hline $\mathrm{C}$ & 2.60465 & -0.99577 & -2.56094 \\
\hline C & 2.32077 & 0.42813 & -2.58087 \\
\hline C & 2.95016 & 1.02615 & -1.43211 \\
\hline $\mathrm{C}$ & 3.62026 & -0.02240 & -0.67940 \\
\hline $\mathrm{C}$ & 3.40551 & -1.27608 & -1.37971 \\
\hline $\mathrm{C}$ & 3.19288 & -2.45157 & -0.65541 \\
\hline $\mathrm{C}$ & 2.17818 & -3.39715 & -1.08619 \\
\hline $\mathrm{C}$ & 1.54487 & -3.94947 & 0.09932 \\
\hline $\mathrm{C}$ & 2.16728 & -3.34231 & 1.26360 \\
\hline C & 3.18614 & -2.41764 & 0.79859 \\
\hline $\mathrm{C}$ & 3.39231 & -1.20960 & 1.46922 \\
\hline C & 3.61382 & 0.01010 & 0.71322 \\
\hline C & 2.93702 & 1.09249 & 1.40996 \\
\hline $\mathrm{C}$ & 2.28800 & 2.09725 & 0.69040 \\
\hline $\mathrm{C}$ & 2.29470 & 2.06323 & -0.76624 \\
\hline C & 1.04188 & 2.60586 & -1.24197 \\
\hline $\mathrm{C}$ & 0.45104 & 3.39258 & -0.07707 \\
\hline $\mathrm{C}$ & 1.03097 & 2.66126 & 1.12870 \\
\hline $\mathrm{C}$ & 0.37940 & 2.09448 & 2.21377 \\
\hline $\mathrm{C}$ & 0.63484 & 4.92091 & -0.11199 \\
\hline $\mathrm{C}$ & -0.67442 & 5.70393 & -0.13572 \\
\hline $\mathrm{H}$ & 1.23255 & 5.18375 & -0.99785 \\
\hline $\mathrm{H}$ & 1.22505 & 5.22482 & 0.76573 \\
\hline
\end{tabular}




$\begin{array}{llll}\mathrm{H} & -1.75387 & 5.51368 & -0.13578 \\ \mathrm{H} & -0.53585 & 6.78283 & -0.15942\end{array}$

(5) Transition state (TS56)

$\begin{array}{crrr}\text { Pd } & 0.03537 & 3.98804 & 0.20821 \\ \mathrm{C} & 1.13949 & 2.68691 & 1.54588 \\ \mathrm{C} & 2.26500 & 2.04634 & 0.89250 \\ \mathrm{C} & 2.17238 & 2.11828 & -0.56287 \\ \mathrm{C} & 0.93091 & 2.68324 & -1.06467 \\ \mathrm{C} & 0.26031 & 2.10461 & -2.12237 \\ \mathrm{C} & 0.91127 & 1.06239 & -2.88228 \\ \mathrm{C} & 2.17232 & 0.58908 & -2.51423 \\ \mathrm{C} & 2.80117 & 1.10678 & -1.32529 \\ \mathrm{C} & 3.49689 & 0.00112 & -0.68361 \\ \mathrm{C} & 3.52325 & -0.08553 & 0.70306 \\ \mathrm{C} & 2.88250 & 0.94448 & 1.48749 \\ \mathrm{C} & 2.24557 & 0.30951 & 2.61972 \\ \mathrm{C} & 0.98598 & 0.75669 & 3.02819 \\ \mathrm{C} & 0.37175 & 1.91835 & 2.42075 \\ \mathrm{C} & -1.10440 & 1.63506 & 2.33233 \\ \mathrm{C} & -1.30465 & 0.33529 & 2.98010 \\ \mathrm{C} & -0.02771 & -0.19035 & 3.41325 \\ \mathrm{C} & 0.24072 & -1.55941 & 3.39649 \\ \mathrm{C} & 1.53793 & -2.03091 & 2.93801 \\ \mathrm{C} & 2.52021 & -1.11451 & 2.55725 \\ \mathrm{C} & 3.31475 & -1.36142 & 1.36579 \\ \mathrm{C} & 3.10078 & -2.51163 & 0.60708 \\ \mathrm{C} & 3.08765 & -2.42848 & -0.84572 \\ \mathrm{C} & 3.28091 & -1.19663 & -1.47608 \\ \mathrm{C} & 2.46438 & -0.82927 & -2.61803 \\ \mathrm{C} & 1.49408 & -1.71409 & -3.09454 \\ \mathrm{C} & 0.19135 & -1.21302 & -3.50096 \\ \mathrm{C} & -0.09100 & 0.14773 & -3.39365 \\ \mathrm{C} & -1.38482 & 0.60034 & -2.92987 \\ \mathrm{C} & -2.36260 & -0.34246 & -2.58613 \\ \mathrm{C} & -2.06227 & -1.76121 & -2.66302 \\ \mathrm{C} & -0.81282 & -2.19003 & -3.11545 \\ \mathrm{C} & -0.13281 & -3.28519 & -2.45098 \\ \mathrm{C} & 1.29364 & -2.99308 & -2.44275 \\ \mathrm{C} & 2.07184 & -3.34246 & -1.33578 \\ \mathrm{C} & 1.32251 & -3.23481 & 2.15802 \\ \mathrm{C} & -0.10594 & -3.51732 & 2.15036 \\ \mathrm{C} & -2.48401 & 2.92166 \\ \mathrm{C} & -1.99490 & 2.51757 \\ \mathrm{C} & & -36899 & 2.56126\end{array}$




$\begin{array}{lccc}\mathrm{C} & -3.10735 & -0.24013 & 1.42542 \\ \mathrm{C} & -3.33837 & -1.45131 & 0.65482 \\ \mathrm{C} & -2.65299 & -2.53776 & 1.32946 \\ \mathrm{C} & -2.01062 & -3.52827 & 0.58170 \\ \mathrm{C} & -0.71099 & -4.02429 & 0.99840 \\ \mathrm{C} & 0.08442 & -4.26096 & -0.19544 \\ \mathrm{C} & -0.72434 & -3.90974 & -1.35001 \\ \mathrm{C} & -2.02075 & -3.46160 & -0.87194 \\ \mathrm{C} & -2.67951 & -2.41409 & -1.51818 \\ \mathrm{C} & -3.35568 & -1.39357 & -0.73816 \\ \mathrm{C} & -3.16584 & -0.12103 & -1.40918 \\ \mathrm{C} & -2.96712 & 1.04415 & -0.66581 \\ \mathrm{C} & -2.90678 & 0.97849 & 0.78619 \\ \mathrm{C} & -1.94646 & 1.96826 & 1.26103 \\ \mathrm{C} & -1.74006 & 2.92630 & 0.10905 \\ \mathrm{C} & -2.03982 & 2.06350 & -1.08589 \\ \mathrm{C} & -1.20461 & 1.81365 & -2.15658 \\ \mathrm{C} & -2.45225 & 4.31478 & 0.06156 \\ \mathrm{C} & -1.44656 & 5.13802 & -0.74745 \\ \mathrm{H} & -1.49355 & 4.95643 & -1.82038 \\ \mathrm{H} & -1.37661 & 6.19977 & -0.51190 \\ \mathrm{H} & -3.45916 & 4.22283 & -0.37815 \\ \mathrm{H} & -2.57827 & 4.71144 & 1.07629 \\ \mathrm{H} & 1.28777 & 5.11518 & 0.93446 \\ \mathrm{H} & 1.33282 & 4.07653 & 1.43939\end{array}$

(6) Transition state (TS67)

$\begin{array}{crrr}\text { Pd } & 3.96337 & -0.58879 & 0.04771 \\ \mathrm{C} & 2.45075 & -1.14755 & 1.27819 \\ \mathrm{C} & 1.77870 & -2.35391 & 0.97744 \\ \mathrm{C} & 1.95388 & -2.76603 & -0.48353 \\ \mathrm{C} & 2.55629 & -1.47550 & -1.06088 \\ \mathrm{C} & 1.95531 & -0.77999 & -2.07450 \\ \mathrm{C} & 0.78163 & -1.35557 & -2.72691 \\ \mathrm{C} & 0.16015 & -2.54299 & -2.28884 \\ \mathrm{C} & 0.62854 & -3.13743 & -1.08831 \\ \mathrm{C} & -0.49405 & -3.56998 & -0.32148 \\ \mathrm{C} & -0.48049 & -3.36796 & 1.06810 \\ \mathrm{C} & 0.67005 & -2.76306 & 1.72055 \\ \mathrm{C} & 0.17912 & -1.92208 & 2.77467 \\ \mathrm{C} & 0.79605 & -0.69229 & 3.01758 \\ \mathrm{C} & 1.93195 & -0.25625 & 2.22992 \\ \mathrm{C} & 1.84237 & 1.21885 & 2.10471 \\ \mathrm{C} & 0.62109 & 1.62619 & 2.78501 \\ \mathrm{C} & -0.01218 & 0.45890 & 3.35895 \\ \mathrm{C} & -1.40249 & 0.35140 & 3.42612 \\ \mathrm{C} & -2.04393 & -0.92032 & 3.13378\end{array}$




\begin{tabular}{|c|c|c|c|}
\hline C & -1.27117 & -2.03435 & 2.82317 \\
\hline $\mathrm{C}$ & -1.68044 & -2.92843 & 1.75460 \\
\hline $\mathrm{C}$ & -2.83909 & -2.65796 & 1.02344 \\
\hline $\mathrm{C}$ & -2.85208 & -2.83072 & -0.42616 \\
\hline $\mathrm{C}$ & -1.70373 & -3.26955 & -1.08119 \\
\hline C & -1.29317 & -2.63951 & -2.31774 \\
\hline $\mathrm{C}$ & -2.05577 & -1.60365 & -2.85388 \\
\hline $\mathrm{C}$ & -1.39434 & -0.42159 & -3.39201 \\
\hline $\mathrm{C}$ & -0.00064 & -0.30741 & -3.32624 \\
\hline $\mathrm{C}$ & 0.63418 & 0.95188 & -3.00669 \\
\hline $\mathrm{C}$ & -0.16577 & 2.08171 & -2.80223 \\
\hline $\mathrm{C}$ & -1.61325 & 1.95286 & -2.81176 \\
\hline $\mathrm{C}$ & -2.22226 & 0.73180 & -3.11529 \\
\hline $\mathrm{C}$ & -3.37093 & 0.27960 & -2.35296 \\
\hline $\mathrm{C}$ & -3.26584 & -1.16572 & -2.19428 \\
\hline $\mathrm{C}$ & -3.65550 & -1.76589 & -0.99156 \\
\hline $\mathrm{C}$ & -4.15608 & -0.94580 & 0.10046 \\
\hline $\mathrm{C}$ & -3.65062 & -1.49967 & 1.34550 \\
\hline $\mathrm{C}$ & -3.25667 & -0.64579 & 2.37916 \\
\hline $\mathrm{C}$ & -3.37292 & 0.79295 & 2.22234 \\
\hline $\mathrm{C}$ & -2.23033 & 1.40681 & 2.87466 \\
\hline $\mathrm{C}$ & -1.63008 & 2.54127 & 2.32312 \\
\hline $\mathrm{C}$ & -0.18547 & 2.66343 & 2.29939 \\
\hline $\mathrm{C}$ & 0.18102 & 3.30945 & 1.06307 \\
\hline $\mathrm{C}$ & -1.02809 & 3.57670 & 0.30680 \\
\hline $\mathrm{C}$ & -2.15355 & 3.10045 & 1.08532 \\
\hline $\mathrm{C}$ & -3.24533 & 2.50041 & 0.44796 \\
\hline $\mathrm{C}$ & -3.86826 & 1.32588 & 1.02829 \\
\hline $\mathrm{C}$ & -4.25576 & 0.43722 & -0.05472 \\
\hline $\mathrm{C}$ & -3.86251 & 1.06174 & -1.30785 \\
\hline $\mathrm{C}$ & -3.23832 & 2.33745 & -0.99621 \\
\hline $\mathrm{C}$ & -2.14457 & 2.78204 & -1.74376 \\
\hline $\mathrm{C}$ & -1.02008 & 3.41684 & -1.07991 \\
\hline $\mathrm{C}$ & 0.19440 & 2.99448 & -1.75040 \\
\hline $\mathrm{C}$ & 1.36181 & 2.75666 & -1.02446 \\
\hline $\mathrm{C}$ & 1.34574 & 2.91170 & 0.41276 \\
\hline $\mathrm{C}$ & 2.25896 & 1.91911 & 0.96463 \\
\hline $\mathrm{C}$ & 3.15699 & 1.43852 & -0.10997 \\
\hline $\mathrm{C}$ & 2.27757 & 1.65708 & -1.31774 \\
\hline $\mathrm{C}$ & 1.85130 & 0.70849 & -2.24282 \\
\hline $\mathrm{C}$ & 4.64383 & 1.99312 & -0.14592 \\
\hline $\mathrm{C}$ & 5.58386 & 0.80409 & -0.04158 \\
\hline $\mathrm{H}$ & 4.77717 & 2.56086 & -1.07663 \\
\hline $\mathrm{H}$ & 4.77226 & 2.69871 & 0.68355 \\
\hline $\mathrm{H}$ & 2.68110 & -3.58676 & -0.58986 \\
\hline $\mathrm{H}$ & 6.18652 & 0.75189 & 0.86604 \\
\hline $\mathrm{H}$ & 6.19139 & 0.58585 & -0.92094 \\
\hline $\mathrm{H}$ & 4.99783 & -1.85886 & 0.05564 \\
\hline
\end{tabular}


(7) Transition state (TS78)

\begin{tabular}{|c|c|c|c|}
\hline $\mathrm{Pd}$ & -3.83670 & -0.75326 & -0.32888 \\
\hline $\mathrm{C}$ & -2.36559 & -1.15905 & -1.54817 \\
\hline $\mathrm{C}$ & -1.63984 & -2.36694 & -1.21442 \\
\hline $\mathrm{C}$ & -1.71691 & -2.78187 & 0.20568 \\
\hline $\mathrm{C}$ & -2.73336 & -1.85828 & 0.97926 \\
\hline C & -2.05914 & -1.14323 & 2.03642 \\
\hline $\mathrm{C}$ & -0.86412 & -1.64554 & 2.63315 \\
\hline $\mathrm{C}$ & -0.14641 & -2.74580 & 2.11699 \\
\hline $\mathrm{C}$ & -0.52833 & -3.23294 & 0.83519 \\
\hline $\mathrm{C}$ & 0.66409 & -3.55034 & 0.08348 \\
\hline $\mathrm{C}$ & 0.69494 & -3.27833 & -1.27606 \\
\hline $\mathrm{C}$ & -0.46171 & -2.66324 & -1.92371 \\
\hline C & 0.03982 & -1.75286 & -2.91600 \\
\hline C & -0.61936 & -0.53850 & -3.13483 \\
\hline $\mathrm{C}$ & -1.82539 & -0.21259 & -2.41414 \\
\hline $\mathrm{C}$ & -1.80432 & 1.26949 & -2.17959 \\
\hline $\mathrm{C}$ & -0.56554 & 1.76862 & -2.75477 \\
\hline $\mathrm{C}$ & 0.15083 & 0.66902 & -3.36127 \\
\hline $\mathrm{C}$ & 1.54446 & 0.62903 & -3.35234 \\
\hline $\mathrm{C}$ & 2.22953 & -0.62910 & -3.10498 \\
\hline C & 1.49271 & -1.79530 & -2.90050 \\
\hline C & 1.89992 & -2.73941 & -1.87711 \\
\hline C & 3.01436 & -2.47011 & -1.07771 \\
\hline $\mathrm{C}$ & 2.97058 & -2.73850 & 0.35227 \\
\hline $\mathrm{C}$ & 1.81408 & -3.27215 & 0.92178 \\
\hline $\mathrm{C}$ & 1.31238 & -2.76000 & 2.18533 \\
\hline $\mathrm{C}$ & 1.99271 & -1.72628 & 2.82183 \\
\hline $\mathrm{C}$ & 1.24347 & -0.61887 & 3.41046 \\
\hline $\mathrm{C}$ & -0.14431 & -0.57764 & 3.29617 \\
\hline $\mathrm{C}$ & -0.84049 & 0.64710 & 3.00123 \\
\hline C & -0.09345 & 1.82003 & 2.87838 \\
\hline C & 1.35245 & 1.79269 & 2.97524 \\
\hline $\mathrm{C}$ & 2.01592 & 0.59122 & 3.23368 \\
\hline $\mathrm{C}$ & 3.22319 & 0.25354 & 2.50270 \\
\hline $\mathrm{C}$ & 3.20560 & -1.17925 & 2.24418 \\
\hline $\mathrm{C}$ & 3.68815 & -1.67494 & 1.02990 \\
\hline $\mathrm{C}$ & 4.19030 & -0.75612 & 0.01919 \\
\hline $\mathrm{C}$ & 3.77462 & -1.24988 & -1.28275 \\
\hline $\mathrm{C}$ & 3.38999 & -0.34953 & -2.27994 \\
\hline $\mathrm{C}$ & 3.42524 & 1.08213 & -2.02407 \\
\hline $\mathrm{C}$ & 2.29161 & 1.68743 & -2.69636 \\
\hline $\mathrm{C}$ & 1.60823 & 2.75257 & -2.10560 \\
\hline $\mathrm{C}$ & 0.15699 & 2.80889 & -2.15603 \\
\hline $\mathrm{C}$ & -0.30951 & 3.34676 & -0.90140 \\
\hline $\mathrm{C}$ & 0.84173 & 3.60656 & -0.05806 \\
\hline $\mathrm{C}$ & 2.03355 & 3.24674 & -0.80484 \\
\hline $\mathrm{C}$ & 3.11660 & 2.65562 & -0.14939 \\
\hline
\end{tabular}




$\begin{array}{llll}\mathrm{C} & 3.82903 & 1.55513 & -0.77205 \\ \mathrm{C} & 4.21000 & 0.61629 & 0.26965 \\ \mathrm{C} & 3.72128 & 1.13225 & 1.53905 \\ \mathrm{C} & 3.04344 & 2.39099 & 1.28042 \\ \mathrm{C} & 1.88765 & 2.72209 & 1.99185 \\ \mathrm{C} & 0.76719 & 3.34001 & 1.30762 \\ \mathrm{C} & -0.45265 & 2.77993 & 1.86660 \\ \mathrm{C} & -1.54367 & 2.50414 & 1.05395 \\ \mathrm{C} & -1.49199 & 2.84650 & -0.35592 \\ \mathrm{C} & -2.31973 & 1.88016 & -1.04585 \\ \mathrm{C} & -3.25042 & 1.25067 & -0.04408 \\ \mathrm{C} & -2.39892 & 1.32495 & 1.22068 \\ \mathrm{C} & -2.01764 & 0.35044 & 2.16982 \\ \mathrm{C} & -4.63649 & 1.93122 & 0.07828 \\ \mathrm{C} & -5.59754 & 1.19178 & 1.02459 \\ \mathrm{H} & -4.50451 & 2.96402 & 0.44261 \\ \mathrm{H} & -5.08408 & 2.01255 & -0.91895 \\ \mathrm{H} & -2.80209 & -3.18284 & 0.68289 \\ \mathrm{H} & -5.84648 & 0.17271 & 0.67230 \\ \mathrm{H} & -6.55526 & 1.71836 & 1.09954 \\ \mathrm{H} & -5.17810 & 1.10059 & 2.03185\end{array}$

(8) Transition state (TS89)

$\begin{array}{crrr}\text { Pd } & -3.88451 & -0.79366 & 0.05708 \\ \mathrm{C} & -2.48054 & -1.85438 & -0.84468 \\ \mathrm{C} & -1.73325 & -2.63373 & 0.10764 \\ \mathrm{C} & -1.76067 & -2.06061 & 1.45758 \\ \mathrm{C} & -2.47896 & -0.80637 & 1.53680 \\ \mathrm{C} & -1.99025 & 0.23802 & 2.28675 \\ \mathrm{C} & -0.84743 & 0.03670 & 3.14423 \\ \mathrm{C} & -0.20353 & -1.20367 & 3.17707 \\ \mathrm{C} & -0.65684 & -2.26442 & 2.31080 \\ \mathrm{C} & 0.51451 & -2.99423 & 1.85607 \\ \mathrm{C} & 0.55947 & -3.48923 & 0.55743 \\ \mathrm{C} & -0.57153 & -3.29428 & -0.32815 \\ \mathrm{C} & -0.05705 & -3.04741 & -1.65495 \\ \mathrm{C} & -0.68397 & -2.08525 & -2.45166 \\ \mathrm{C} & -1.89406 & -1.43024 & -2.01487 \\ \mathrm{C} & -1.81413 & -0.00637 & -2.46017 \\ \mathrm{C} & -0.58441 & 0.11596 & -3.23308 \\ \mathrm{C} & 0.10245 & -1.15939 & -3.23399 \\ \mathrm{C} & 1.49508 & -1.22551 & -3.20963 \\ \mathrm{C} & 2.15524 & -2.20694 & -2.36404 \\ \mathrm{C} & 1.39253 & -3.09299 & -1.59859 \\ \mathrm{C} & 1.77730 & -3.37575 & -0.22647 \\ \mathrm{C} & 2.90686 & -2.76946 & 0.32441 \\ \mathrm{C} & 2.86369 & -2.25787 & 1.68564\end{array}$




\begin{tabular}{|c|c|c|c|}
\hline $\mathrm{C}$ & 1.69180 & -2.37082 & 2.43608 \\
\hline $\mathrm{C}$ & 1.24488 & -1.26062 & 3.25796 \\
\hline $\mathrm{C}$ & 1.99185 & -0.08047 & 3.30165 \\
\hline $\mathrm{C}$ & 1.31809 & 1.20822 & 3.27340 \\
\hline $\mathrm{C}$ & -0.07224 & 1.26160 & 3.19036 \\
\hline C & -0.72317 & 2.23558 & 2.33675 \\
\hline $\mathrm{C}$ & 0.05597 & 3.13014 & 1.59369 \\
\hline $\mathrm{C}$ & 1.50470 & 3.06729 & 1.66163 \\
\hline $\mathrm{C}$ & 2.12486 & 2.12481 & 2.48485 \\
\hline $\mathrm{C}$ & 3.29023 & 1.40097 & 2.01601 \\
\hline $\mathrm{C}$ & 3.20830 & 0.03772 & 2.52192 \\
\hline $\mathrm{C}$ & 3.63550 & -1.02877 & 1.72685 \\
\hline $\mathrm{C}$ & 4.15341 & -0.77781 & 0.39227 \\
\hline $\mathrm{C}$ & 3.70194 & -1.85403 & -0.47460 \\
\hline $\mathrm{C}$ & 3.33076 & -1.57827 & -1.79277 \\
\hline $\mathrm{C}$ & 3.40683 & -0.21436 & -2.29895 \\
\hline $\mathrm{C}$ & 2.27373 & -0.00002 & -3.17710 \\
\hline $\mathrm{C}$ & 1.62311 & 1.23509 & -3.19499 \\
\hline $\mathrm{C}$ & 0.17269 & 1.29166 & -3.23318 \\
\hline $\mathrm{C}$ & -0.25228 & 2.40126 & -2.42124 \\
\hline $\mathrm{C}$ & 0.92875 & 3.04037 & -1.86118 \\
\hline $\mathrm{C}$ & 2.09101 & 2.31290 & -2.33825 \\
\hline $\mathrm{C}$ & 3.18050 & 2.10565 & -1.48843 \\
\hline $\mathrm{C}$ & 3.84924 & 0.81656 & -1.46693 \\
\hline $\mathrm{C}$ & 4.22802 & 0.52895 & -0.09388 \\
\hline $\mathrm{C}$ & 3.79109 & 1.64025 & 0.73411 \\
\hline $\mathrm{C}$ & 3.14659 & 2.61655 & -0.12687 \\
\hline $\mathrm{C}$ & 2.02781 & 3.31763 & 0.32785 \\
\hline $\mathrm{C}$ & 0.89914 & 3.53489 & -0.55914 \\
\hline $\mathrm{C}$ & -0.31557 & 3.42027 & 0.23113 \\
\hline $\mathrm{C}$ & -1.45053 & 2.80929 & -0.30749 \\
\hline $\mathrm{C}$ & -1.41167 & 2.27047 & -1.65790 \\
\hline $\mathrm{C}$ & -2.24238 & 1.08338 & -1.70753 \\
\hline $\mathrm{C}$ & -3.13488 & 1.12499 & -0.47070 \\
\hline $\mathrm{C}$ & -2.30663 & 1.96100 & 0.48638 \\
\hline $\mathrm{C}$ & -1.91275 & 1.63295 & 1.76879 \\
\hline $\mathrm{C}$ & -4.55783 & 1.66148 & -0.71842 \\
\hline $\mathrm{C}$ & -5.45354 & 1.66948 & 0.53108 \\
\hline $\mathrm{H}$ & -4.45004 & 2.68830 & -1.10072 \\
\hline $\mathrm{H}$ & -5.02771 & 1.08693 & -1.52701 \\
\hline $\mathrm{H}$ & -5.03090 & -1.72338 & -0.79634 \\
\hline $\mathrm{H}$ & -6.42903 & 2.10706 & 0.29658 \\
\hline $\mathrm{H}$ & -4.99894 & 2.24216 & 1.34478 \\
\hline $\mathrm{H}$ & -5.66284 & 0.65444 & 0.91324 \\
\hline
\end{tabular}


Transition state structures observed over $\mathrm{C}_{59} \mathrm{Ni}$ during acetylene hydrogenation.

(1) Transition state (TS12)

\begin{tabular}{|c|c|c|c|}
\hline $\mathrm{Ni}$ & -0.29259 & 4.04483 & 0.05578 \\
\hline $\mathrm{C}$ & 0.69216 & 2.90951 & 1.23162 \\
\hline $\mathrm{C}$ & 1.96868 & 2.47173 & 0.71434 \\
\hline $\mathrm{C}$ & 2.00639 & 2.47365 & -0.74289 \\
\hline $\mathrm{C}$ & 0.78072 & 3.01373 & -1.30489 \\
\hline $\mathrm{C}$ & 0.11587 & 2.27171 & -2.28116 \\
\hline $\mathrm{C}$ & 0.83743 & 1.21942 & -2.96984 \\
\hline $\mathrm{C}$ & 2.12915 & 0.85746 & -2.58089 \\
\hline $\mathrm{C}$ & 2.71752 & 1.47954 & -1.41713 \\
\hline $\mathrm{C}$ & 3.46437 & 0.46798 & -0.69995 \\
\hline $\mathrm{C}$ & 3.45541 & 0.46909 & 0.69068 \\
\hline $\mathrm{C}$ & 2.68954 & 1.47657 & 1.40725 \\
\hline $\mathrm{C}$ & 2.11097 & 0.84218 & 2.56604 \\
\hline $\mathrm{C}$ & 0.81415 & 1.18898 & 2.95508 \\
\hline $\mathrm{C}$ & 0.08124 & 2.21904 & 2.25568 \\
\hline $\mathrm{C}$ & -1.35466 & 1.80985 & 2.25210 \\
\hline $\mathrm{C}$ & -1.43797 & 0.54530 & 2.96276 \\
\hline $\mathrm{C}$ & -0.11073 & 0.17065 & 3.40740 \\
\hline $\mathrm{C}$ & 0.28145 & -1.16717 & 3.43998 \\
\hline $\mathrm{C}$ & 1.61843 & -1.53806 & 3.00534 \\
\hline $\mathrm{C}$ & 2.51262 & -0.55259 & 2.57927 \\
\hline $\mathrm{C}$ & 3.34512 & -0.78657 & 1.41252 \\
\hline $\mathrm{C}$ & 3.24603 & -1.99088 & 0.71220 \\
\hline $\mathrm{C}$ & 3.25153 & -1.98784 & -0.74282 \\
\hline $\mathrm{C}$ & 3.35728 & -0.78091 & -1.43424 \\
\hline $\mathrm{C}$ & 2.53239 & -0.53749 & -2.60557 \\
\hline $\mathrm{C}$ & 1.63326 & -1.51376 & -3.03777 \\
\hline $\mathrm{C}$ & 0.29861 & -1.13237 & -3.47039 \\
\hline $\mathrm{C}$ & -0.08810 & 0.20738 & -3.41986 \\
\hline $\mathrm{C}$ & -1.40919 & 0.58852 & -2.96916 \\
\hline $\mathrm{C}$ & -2.30373 & -0.41677 & -2.58560 \\
\hline $\mathrm{C}$ & -1.90644 & -1.81268 & -2.61351 \\
\hline $\mathrm{C}$ & -0.62605 & -2.16561 & -3.04355 \\
\hline $\mathrm{C}$ & 0.13018 & -3.17783 & -2.33052 \\
\hline $\mathrm{C}$ & 1.52895 & -2.77415 & -2.32671 \\
\hline $\mathrm{C}$ & 2.31903 & -3.00446 & -1.19797 \\
\hline $\mathrm{C}$ & 1.74118 & -3.64011 & -0.02504 \\
\hline $\mathrm{C}$ & 2.31371 & -3.01226 & 1.15370 \\
\hline $\mathrm{C}$ & 1.51751 & -2.78933 & 2.28038 \\
\hline $\mathrm{C}$ & 0.12034 & -3.19862 & 2.27609 \\
\hline $\mathrm{C}$ & -0.64166 & -2.19974 & 3.00047 \\
\hline $\mathrm{C}$ & -1.92330 & -1.84883 & 2.57387 \\
\hline $\mathrm{C}$ & -2.33525 & -0.45552 & 2.57016 \\
\hline $\mathrm{C}$ & -3.16095 & -0.23847 & 1.40991 \\
\hline
\end{tabular}




$\begin{array}{lrrr}\mathrm{C} & -3.25841 & -1.48915 & 0.67622 \\ \mathrm{C} & -2.49498 & -2.48986 & 1.39936 \\ \mathrm{C} & -1.75862 & -3.44605 & 0.69609 \\ \mathrm{C} & -0.42561 & -3.80984 & 1.14465 \\ \mathrm{C} & 0.40053 & -4.02943 & -0.02981 \\ \mathrm{C} & -0.42114 & -3.79573 & -1.20612 \\ \mathrm{C} & -1.75563 & -3.43693 & -0.75898 \\ \mathrm{C} & -2.48682 & -2.46884 & -1.45165 \\ \mathrm{C} & -3.25600 & -1.48111 & -0.71799 \\ \mathrm{C} & -3.14043 & -0.21866 & -1.42724 \\ \mathrm{C} & -3.03899 & 0.97814 & -0.72642 \\ \mathrm{C} & -3.06155 & 0.97558 & 0.72721 \\ \mathrm{C} & -2.20953 & 2.04898 & 1.18879 \\ \mathrm{C} & -1.95893 & 2.95453 & 0.00591 \\ \mathrm{C} & -2.18012 & 2.05977 & -1.16736 \\ \mathrm{C} & -1.32229 & 1.85709 & -2.25139 \\ \mathrm{C} & -2.42474 & 4.36521 & 0.01688 \\ \mathrm{C} & -1.59173 & 5.17013 & 0.72910 \\ \mathrm{H} & -3.25307 & 4.68696 & -0.62204 \\ \mathrm{H} & -1.72731 & 6.21780 & 0.97797 \\ \mathrm{H} & 0.74579 & 5.22256 & -0.30936 \\ \mathrm{H} & 0.85795 & 4.39316 & -1.03580\end{array}$

(2) Transition state (TS23)

$\begin{array}{crrr}\mathrm{Ni} & 0.69652 & 0.02622 & 3.99543 \\ \mathrm{C} & 1.49137 & -1.07938 & 2.65896 \\ \mathrm{C} & 2.56679 & -0.45385 & 1.92360 \\ \mathrm{C} & 2.45835 & 0.99982 & 1.91197 \\ \mathrm{C} & 1.34903 & 1.45614 & 2.73143 \\ \mathrm{C} & 0.43077 & 2.34263 & 2.16871 \\ \mathrm{C} & 0.80535 & 3.06861 & 0.97082 \\ \mathrm{C} & 2.00419 & 2.79608 & 0.30805 \\ \mathrm{C} & 2.83829 & 1.71179 & 0.77293 \\ \mathrm{C} & 3.38751 & 1.04242 & -0.38714 \\ \mathrm{C} & 3.51793 & -0.34209 & -0.37951 \\ \mathrm{C} & 3.09250 & -1.10118 & 0.78572 \\ \mathrm{C} & 2.49703 & -2.32712 & 0.31406 \\ \mathrm{C} & 1.36736 & -2.82956 & 0.96583 \\ \mathrm{C} & 0.83778 & -2.17604 & 2.14043 \\ \mathrm{C} & -0.64735 & -2.32178 & 2.09119 \\ \mathrm{C} & -0.96111 & -3.07138 & 0.88673 \\ \mathrm{C} & 0.27456 & -3.39637 & 0.20323 \\ \mathrm{C} & 0.33449 & -3.42751 & -1.18963 \\ \mathrm{C} & 1.49261 & -2.87658 & -1.87464 \\ \mathrm{C} & 2.55049 & -2.33974 & -1.13641 \\ \mathrm{C} & 3.18139 & -1.10519 & -1.56878 \\ \mathrm{C} & 2.72591 & -0.45076 & -2.71546\end{array}$




\begin{tabular}{|c|c|c|c|}
\hline $\mathrm{C}$ & 2.58659 & 0.99758 & -2.71847 \\
\hline $\mathrm{C}$ & 2.91007 & 1.72861 & -1.57528 \\
\hline $\mathrm{C}$ & 2.05525 & 2.82138 & -1.14309 \\
\hline $\mathrm{C}$ & 0.90905 & 3.13832 & -1.87392 \\
\hline C & -0.33089 & 3.45086 & -1.18203 \\
\hline $\mathrm{C}$ & -0.37671 & 3.39986 & 0.21165 \\
\hline $\mathrm{C}$ & -1.51543 & 2.83483 & 0.90281 \\
\hline $\mathrm{C}$ & -2.58270 & 2.33977 & 0.14532 \\
\hline $\mathrm{C}$ & -2.53789 & 2.36769 & -1.30534 \\
\hline $\mathrm{C}$ & -1.42965 & 2.90910 & -1.95922 \\
\hline $\mathrm{C}$ & -0.87192 & 2.24474 & -3.12217 \\
\hline $\mathrm{C}$ & 0.57606 & 2.38645 & -3.06926 \\
\hline $\mathrm{C}$ & 1.39618 & 1.33306 & -3.48044 \\
\hline $\mathrm{C}$ & 0.80250 & 0.09311 & -3.95350 \\
\hline $\mathrm{C}$ & 1.62411 & -1.00756 & -3.47924 \\
\hline $\mathrm{C}$ & 1.02162 & -2.19907 & -3.06652 \\
\hline $\mathrm{C}$ & -0.42621 & -2.34032 & -3.12527 \\
\hline $\mathrm{C}$ & -0.84913 & -3.10712 & -1.96930 \\
\hline $\mathrm{C}$ & -2.04467 & -2.79633 & -1.31982 \\
\hline $\mathrm{C}$ & -2.10747 & -2.79428 & 0.13177 \\
\hline $\mathrm{C}$ & -2.96832 & -1.71332 & 0.53863 \\
\hline $\mathrm{C}$ & -3.43665 & -1.02675 & -0.65355 \\
\hline $\mathrm{C}$ & -2.86820 & -1.70015 & -1.80705 \\
\hline $\mathrm{C}$ & -2.45765 & -0.95566 & -2.91533 \\
\hline $\mathrm{C}$ & -1.21343 & -1.28371 & -3.58968 \\
\hline $\mathrm{C}$ & -0.58601 & -0.04168 & -4.00655 \\
\hline $\mathrm{C}$ & -1.44058 & 1.05611 & -3.58458 \\
\hline $\mathrm{C}$ & -2.59794 & 0.49267 & -2.91185 \\
\hline $\mathrm{C}$ & -3.14007 & 1.13796 & -1.79771 \\
\hline $\mathrm{C}$ & -3.57169 & 0.36094 & -0.65077 \\
\hline $\mathrm{C}$ & -3.22712 & 1.11216 & 0.54384 \\
\hline $\mathrm{C}$ & -2.77118 & 0.45722 & 1.68268 \\
\hline $\mathrm{C}$ & -2.64842 & -0.99139 & 1.69032 \\
\hline $\mathrm{C}$ & -1.52138 & -1.33949 & 2.52683 \\
\hline $\mathrm{C}$ & -1.17941 & -0.11363 & 3.34079 \\
\hline $\mathrm{C}$ & -1.72559 & 1.00800 & 2.52253 \\
\hline $\mathrm{C}$ & -1.05449 & 2.16357 & 2.11480 \\
\hline $\mathrm{C}$ & -1.28914 & -0.13070 & 4.82229 \\
\hline $\mathrm{C}$ & -0.22024 & -0.73719 & 5.40416 \\
\hline $\mathrm{H}$ & -2.07552 & 0.43393 & 5.33286 \\
\hline $\mathrm{H}$ & -0.07451 & -0.96977 & 6.45421 \\
\hline $\mathrm{H}$ & 1.94603 & 0.52142 & 4.88532 \\
\hline $\mathrm{H}$ & 1.78236 & 1.23285 & 4.05239 \\
\hline
\end{tabular}

(3) Transition state (TS34)

$\begin{array}{cccc}\mathrm{Ni} & 0.42677 & 3.83063 & -1.12701 \\ \mathrm{C} & 0.97183 & 2.99378 & 0.48571\end{array}$




\begin{tabular}{|c|c|c|c|}
\hline C & 2.24995 & 2.31510 & 0.39287 \\
\hline $\mathrm{C}$ & 2.50735 & 1.93415 & -0.98521 \\
\hline $\mathrm{C}$ & 1.42639 & 2.41088 & -1.81115 \\
\hline $\mathrm{C}$ & 0.77593 & 1.53437 & -2.66273 \\
\hline $\mathrm{C}$ & 1.40617 & 0.25658 & -2.95107 \\
\hline $\mathrm{C}$ & 2.57693 & -0.14011 & -2.29738 \\
\hline $\mathrm{C}$ & 3.11752 & 0.70438 & -1.25634 \\
\hline $\mathrm{C}$ & 3.57993 & -0.15043 & -0.17964 \\
\hline $\mathrm{C}$ & 3.38510 & 0.23960 & 1.14388 \\
\hline $\mathrm{C}$ & 2.69927 & 1.48778 & 1.43270 \\
\hline $\mathrm{C}$ & 1.86142 & 1.27948 & 2.58605 \\
\hline C & 0.59553 & 1.87334 & 2.63410 \\
\hline $\mathrm{C}$ & 0.12414 & 2.71773 & 1.55270 \\
\hline $\mathrm{C}$ & -1.36000 & 2.51247 & 1.46365 \\
\hline $\mathrm{C}$ & -1.71440 & 1.51698 & 2.45992 \\
\hline $\mathrm{C}$ & -0.52340 & 1.13699 & 3.18833 \\
\hline $\mathrm{C}$ & -0.35289 & -0.17065 & 3.64221 \\
\hline $\mathrm{C}$ & 0.95233 & -0.80249 & 3.55028 \\
\hline $\mathrm{C}$ & 2.03715 & -0.08836 & 3.03836 \\
\hline $\mathrm{C}$ & 2.97360 & -0.73784 & 2.13569 \\
\hline $\mathrm{C}$ & 2.77344 & -2.06951 & 1.76465 \\
\hline $\mathrm{C}$ & 2.96729 & -2.47416 & 0.38055 \\
\hline $\mathrm{C}$ & 3.35827 & -1.53128 & -0.57190 \\
\hline $\mathrm{C}$ & 2.73555 & -1.52350 & -1.88636 \\
\hline $\mathrm{C}$ & 1.75253 & -2.46632 & -2.19430 \\
\hline $\mathrm{C}$ & 0.56047 & -2.05818 & -2.91666 \\
\hline $\mathrm{C}$ & 0.38849 & -0.71659 & -3.26380 \\
\hline $\mathrm{C}$ & -0.90038 & -0.07192 & -3.13033 \\
\hline $\mathrm{C}$ & -1.98842 & -0.82353 & -2.67765 \\
\hline $\mathrm{C}$ & -1.81786 & -2.21245 & -2.29218 \\
\hline $\mathrm{C}$ & -0.56446 & -2.81623 & -2.39974 \\
\hline $\mathrm{C}$ & -0.07613 & -3.67677 & -1.34000 \\
\hline $\mathrm{C}$ & 1.35717 & -3.45596 & -1.20949 \\
\hline $\mathrm{C}$ & 1.95067 & -3.45890 & 0.05488 \\
\hline $\mathrm{C}$ & 1.13256 & -3.66899 & 1.23855 \\
\hline $\mathrm{C}$ & 1.64061 & -2.80799 & 2.29293 \\
\hline $\mathrm{C}$ & 0.75089 & -2.18743 & 3.17404 \\
\hline C & -0.68085 & -2.41662 & 3.04625 \\
\hline $\mathrm{C}$ & -1.36352 & -1.17293 & 3.34743 \\
\hline C & -2.51574 & -0.81496 & 2.64592 \\
\hline $\mathrm{C}$ & -2.70606 & 0.55875 & 2.21251 \\
\hline $\mathrm{C}$ & -3.35300 & 0.53389 & 0.92266 \\
\hline C & -3.53445 & -0.85179 & 0.52988 \\
\hline $\mathrm{C}$ & -3.02559 & -1.68964 & 1.60062 \\
\hline $\mathrm{C}$ & -2.35919 & -2.87893 & 1.29747 \\
\hline $\mathrm{C}$ & -1.16635 & -3.25236 & 2.03757 \\
\hline C & -0.24102 & -3.88509 & 1.11292 \\
\hline C & -0.85811 & -3.89229 & -0.20275 \\
\hline $\mathrm{C}$ & -2.16684 & -3.27205 & -0.09034 \\
\hline
\end{tabular}




$\begin{array}{llll}\mathrm{C} & -2.64277 & -2.45434 & -1.11845 \\ \mathrm{C} & -3.34049 & -1.22244 & -0.79972 \\ \mathrm{C} & -2.93593 & -0.22185 & -1.77467 \\ \mathrm{C} & -2.75554 & 1.10425 & -1.39747 \\ \mathrm{C} & -3.00653 & 1.49997 & -0.02015 \\ \mathrm{C} & -2.09825 & 2.57838 & 0.29028 \\ \mathrm{C} & -1.57845 & 3.10206 & -1.00465 \\ \mathrm{C} & -1.67855 & 1.92683 & -1.93597 \\ \mathrm{C} & -0.70504 & 1.32222 & -2.74012 \\ \mathrm{C} & -1.50013 & 4.46874 & -1.34294 \\ \mathrm{C} & -0.75671 & 4.89937 & -2.47940 \\ \mathrm{H} & -1.76750 & 5.19427 & -0.57589 \\ \mathrm{H} & -0.64288 & 5.96316 & -2.65792 \\ \mathrm{H} & -0.73312 & 4.27633 & -3.37085 \\ \mathrm{H} & 1.14531 & 4.66935 & -2.17723\end{array}$

(4) Transition state (TS45)

$\begin{array}{rrrr}\mathrm{Ni} & 0.78455 & 3.87969 & -0.10283 \\ \mathrm{C} & 1.41875 & 2.63911 & 1.13657 \\ \mathrm{C} & 2.56505 & 1.88020 & 0.67079 \\ \mathrm{C} & 2.62598 & 1.86905 & -0.79055 \\ \mathrm{C} & 1.56380 & 2.69112 & -1.33293 \\ \mathrm{C} & 0.72764 & 2.12128 & -2.28537 \\ \mathrm{C} & 1.12824 & 0.90209 & -2.95863 \\ \mathrm{C} & 2.29275 & 0.22302 & -2.58764 \\ \mathrm{C} & 3.03573 & 0.69622 & -1.43978 \\ \mathrm{C} & 3.48180 & -0.46282 & -0.69170 \\ \mathrm{C} & 3.47918 & -0.43120 & 0.70109 \\ \mathrm{C} & 3.00179 & 0.75457 & 1.38998 \\ \mathrm{C} & 2.27676 & 0.32437 & 2.56106 \\ \mathrm{C} & 1.11879 & 1.00894 & 2.94408 \\ \mathrm{C} & 0.66848 & 2.16861 & 2.20657 \\ \mathrm{C} & -0.83368 & 2.15077 & 2.25003 \\ \mathrm{C} & -1.22378 & 0.96272 & 2.98857 \\ \mathrm{C} & -0.03016 & 0.27078 & 3.42858 \\ \mathrm{C} & 0.00343 & -1.12120 & 3.49573 \\ \mathrm{C} & 1.19349 & -1.83612 & 3.06651 \\ \mathrm{C} & 2.30696 & -1.12498 & 2.61244 \\ \mathrm{C} & 3.04601 & -1.59551 & 1.45286 \\ \mathrm{C} & 2.63055 & -2.74815 & 0.78235 \\ \mathrm{C} & 2.62502 & -2.77791 & -0.67230 \\ \mathrm{C} & 3.04009 & -1.65610 & -1.39227 \\ \mathrm{C} & 2.29777 & -1.22773 & -2.56659 \\ \mathrm{C} & 1.16963 & -1.94522 & -2.97379 \\ \mathrm{C} & -0.02209 & -1.23345 & -3.40071 \\ \mathrm{C} & -0.04059 & 0.16181 & -3.36674 \\ \mathrm{C} & -1.20608 & 0.88151 & -2.89826\end{array}$




\begin{tabular}{lrrr}
$\mathrm{C}$ & -2.33533 & 0.15549 & -2.50574 \\
$\mathrm{C}$ & -2.32272 & -1.29624 & -2.51867 \\
$\mathrm{C}$ & -1.18279 & -1.97822 & -2.94644 \\
$\mathrm{C}$ & -0.71272 & -3.14091 & -2.21942 \\
$\mathrm{C}$ & 0.74366 & -3.11588 & -2.23065 \\
$\mathrm{C}$ & 1.45635 & -3.52572 & -1.10109 \\
$\mathrm{C}$ & 0.74133 & -3.96181 & 0.08737 \\
$\mathrm{C}$ & 1.46786 & -3.48070 & 1.25017 \\
$\mathrm{C}$ & 0.76603 & -3.03473 & 2.37368 \\
$\mathrm{C}$ & -0.68958 & -3.06838 & 2.38454 \\
$\mathrm{C}$ & -1.16030 & -1.89008 & 3.08620 \\
$\mathrm{C}$ & -2.31680 & -1.23286 & 2.66261 \\
$\mathrm{C}$ & -2.35539 & 0.21922 & 2.62802 \\
$\mathrm{C}$ & -3.12295 & 0.61869 & 1.47333 \\
$\mathrm{C}$ & -3.53554 & -0.57977 & 0.76420 \\
$\mathrm{C}$ & -3.04475 & -1.72800 & 1.50417 \\
$\mathrm{C}$ & -2.58401 & -2.85377 & 0.81749 \\
$\mathrm{C}$ & -1.38526 & -3.53941 & 1.26845 \\
$\mathrm{C}$ & -0.65480 & -3.99051 & 0.09632 \\
$\mathrm{C}$ & -1.39702 & -3.57331 & -1.08097 \\
$\mathrm{C}$ & -2.58933 & -2.87200 & -0.63730 \\
$\mathrm{C}$ & -3.04868 & -1.75902 & -1.34608 \\
$\mathrm{C}$ & -3.53094 & -0.59312 & -0.62910 \\
$\mathrm{C}$ & -3.09262 & 0.58577 & -1.35899 \\
$\mathrm{C}$ & -2.68714 & 1.73037 & -0.68034 \\
$\mathrm{C}$ & -2.73804 & 1.76220 & 0.77471 \\
$\mathrm{C}$ & -1.66574 & 2.61397 & 1.23632 \\
$\mathrm{C}$ & -1.19018 & 3.38771 & 0.05239 \\
$\mathrm{C}$ & -1.56973 & 2.54362 & -1.13465 \\
$\mathrm{C}$ & -0.77441 & 2.08873 & -2.19804 \\
$\mathrm{C}$ & -0.96329 & 4.79545 & 0.02821 \\
$\mathrm{C}$ & -0.10063 & 5.35962 & -1.19775 \\
$\mathrm{H}$ & -1.04388 & 5.42124 & 0.91187 \\
& -1.42472 & 5.43562 & -0.91722 \\
$\mathrm{H}$ & 0.18817 & 6.40313 & -1.12235 \\
\hline
\end{tabular}

(5) Transition state (TS56)

$\begin{array}{rrrr}\mathrm{Ni} & 0.27319 & 3.36140 & 2.13681 \\ \mathrm{C} & 1.49799 & 1.99703 & 2.21056 \\ \mathrm{C} & 2.34724 & 2.00111 & 1.03572 \\ \mathrm{C} & 1.73893 & 2.72196 & -0.08847 \\ \mathrm{C} & 0.40739 & 3.28740 & 0.18528 \\ \mathrm{C} & -0.63307 & 3.00213 & -0.69937 \\ \mathrm{C} & -0.34474 & 2.54839 & -2.02749 \\ \mathrm{C} & 0.96294 & 2.21625 & -2.38793 \\ \mathrm{C} & 2.01252 & 2.27439 & -1.39452\end{array}$




\begin{tabular}{|c|c|c|c|}
\hline C & 2.89906 & 1.15078 & -1.64588 \\
\hline $\mathrm{C}$ & 3.46598 & 0.46836 & -0.57679 \\
\hline $\mathrm{C}$ & 3.16808 & 0.89373 & 0.77411 \\
\hline $\mathrm{C}$ & 3.06459 & -0.29324 & 1.59765 \\
\hline $\mathrm{C}$ & 2.08878 & -0.34741 & 2.59588 \\
\hline $\mathrm{C}$ & 1.26067 & 0.80401 & 2.87842 \\
\hline $\mathrm{C}$ & -0.10510 & 0.30065 & 3.18575 \\
\hline $\mathrm{C}$ & -0.03106 & -1.15624 & 3.14455 \\
\hline $\mathrm{C}$ & 1.31883 & -1.55234 & 2.79284 \\
\hline $\mathrm{C}$ & 1.55564 & -2.67871 & 2.00308 \\
\hline $\mathrm{C}$ & 2.56074 & -2.62543 & 0.95512 \\
\hline $\mathrm{C}$ & 3.29428 & -1.45344 & 0.75526 \\
\hline $\mathrm{C}$ & 3.54902 & -0.98271 & -0.59499 \\
\hline $\mathrm{C}$ & 3.06105 & -1.69542 & -1.69033 \\
\hline $\mathrm{C}$ & 2.47191 & -0.98246 & -2.81326 \\
\hline $\mathrm{C}$ & 2.39485 & 0.41109 & -2.78993 \\
\hline $\mathrm{C}$ & 1.19428 & 1.07934 & -3.25609 \\
\hline $\mathrm{C}$ & 0.11216 & 0.32697 & -3.72440 \\
\hline $\mathrm{C}$ & -1.24907 & 0.69294 & -3.35996 \\
\hline $\mathrm{C}$ & -1.46922 & 1.78306 & -2.52264 \\
\hline $\mathrm{C}$ & -2.46524 & 1.73189 & -1.47044 \\
\hline $\mathrm{C}$ & -3.20957 & 0.55851 & -1.30315 \\
\hline $\mathrm{C}$ & -2.97184 & -0.59217 & -2.15972 \\
\hline $\mathrm{C}$ & -2.01234 & -0.52799 & -3.17133 \\
\hline $\mathrm{C}$ & -1.12132 & -1.64886 & -3.40652 \\
\hline $\mathrm{C}$ & 0.19018 & -1.11859 & -3.75163 \\
\hline $\mathrm{C}$ & 1.34662 & -1.76168 & -3.29808 \\
\hline $\mathrm{C}$ & 1.23863 & -2.95541 & -2.47802 \\
\hline $\mathrm{C}$ & 2.29809 & -2.91364 & -1.48199 \\
\hline $\mathrm{C}$ & 2.05327 & -3.36802 & -0.18487 \\
\hline $\mathrm{C}$ & 0.74036 & -3.89130 & 0.16508 \\
\hline $\mathrm{C}$ & 0.43823 & -3.46687 & 1.51819 \\
\hline $\mathrm{C}$ & -0.86571 & -3.10515 & 1.86483 \\
\hline $\mathrm{C}$ & -1.09988 & -1.93968 & 2.69575 \\
\hline $\mathrm{C}$ & -2.30088 & -1.29422 & 2.22626 \\
\hline $\mathrm{C}$ & -2.81660 & -2.04783 & 1.09456 \\
\hline $\mathrm{C}$ & -1.92413 & -3.16868 & 0.86802 \\
\hline C & -1.63219 & -3.57374 & -0.43656 \\
\hline $\mathrm{C}$ & -0.27337 & -3.94016 & -0.79511 \\
\hline $\mathrm{C}$ & -0.01930 & -3.46019 & -2.14280 \\
\hline $\mathrm{C}$ & -1.22216 & -2.79622 & -2.61731 \\
\hline $\mathrm{C}$ & -2.22086 & -2.86838 & -1.56464 \\
\hline C & -3.08083 & -1.79048 & -1.34235 \\
\hline $\mathrm{C}$ & -3.38645 & -1.37630 & 0.01474 \\
\hline $\mathrm{C}$ & -3.46314 & 0.07412 & 0.03071 \\
\hline $\mathrm{C}$ & -2.97407 & 0.79587 & 1.12145 \\
\hline $\mathrm{C}$ & -2.36225 & 0.09507 & 2.23476 \\
\hline C & -1.29822 & 0.92749 & 2.77915 \\
\hline $\mathrm{C}$ & -1.43871 & 2.28306 & 2.18608 \\
\hline
\end{tabular}




$\begin{array}{lrrr}\mathrm{C} & -2.27465 & 2.05235 & 0.96103 \\ \mathrm{C} & -1.98317 & 2.49117 & -0.32718 \\ \mathrm{C} & -1.65678 & 3.56960 & 3.01552 \\ \mathrm{C} & -1.23850 & 4.76906 & 2.16852 \\ \mathrm{H} & -0.02771 & 4.34954 & 0.90749 \\ \mathrm{H} & 1.49515 & 4.13714 & 2.61945 \\ \mathrm{H} & -1.07742 & 3.51946 & 3.95718 \\ \mathrm{H} & -2.70694 & 3.63935 & 3.34840 \\ \mathrm{H} & -1.88981 & 4.94392 & 1.31252 \\ \mathrm{H} & -0.97010 & 5.68250 & 2.69346\end{array}$

(6) Transition state (TS67)

$\begin{array}{crrr}\mathrm{Ni} & -1.51535 & 2.15699 & 3.07036 \\ \mathrm{C} & -0.90626 & 0.36067 & 3.12623 \\ \mathrm{C} & 0.54398 & 0.29092 & 3.15125 \\ \mathrm{C} & 1.15539 & 1.48388 & 2.62918 \\ \mathrm{C} & 0.21569 & 2.65323 & 2.34876 \\ \mathrm{C} & 0.25532 & 3.00068 & 0.89148 \\ \mathrm{C} & 1.50739 & 2.94677 & 0.18261 \\ \mathrm{C} & 2.53324 & 2.13627 & 0.69661 \\ \mathrm{C} & 2.36551 & 1.39231 & 1.94270 \\ \mathrm{C} & 3.04629 & 0.12933 & 1.77483 \\ \mathrm{C} & 2.49379 & -1.02823 & 2.31129 \\ \mathrm{C} & 1.21893 & -0.94390 & 2.98917 \\ \mathrm{C} & 0.47059 & -2.12801 & 2.67253 \\ \mathrm{C} & -0.91035 & -2.00831 & 2.45927 \\ \mathrm{C} & -1.59924 & -0.75940 & 2.66816 \\ \mathrm{C} & -2.68521 & -0.69467 & 1.63989 \\ \mathrm{C} & -2.61577 & -1.93474 & 0.86959 \\ \mathrm{C} & -1.54074 & -2.75229 & 1.39093 \\ \mathrm{C} & -0.77737 & -3.56407 & 0.55032 \\ \mathrm{C} & 0.66091 & -3.65788 & 0.74294 \\ \mathrm{C} & 1.26818 & -2.95386 & 1.78385 \\ \mathrm{C} & 2.52885 & -2.26737 & 1.55464 \\ \mathrm{C} & 3.12712 & -2.30114 & 0.29375 \\ \mathrm{C} & 3.71056 & -1.08962 & -0.26295 \\ \mathrm{C} & 3.66958 & 0.09958 & 0.46137 \\ \mathrm{C} & 3.35930 & 1.35124 & -0.20355 \\ \mathrm{C} & 3.09817 & 1.36297 & -1.57615 \\ \mathrm{C} & 2.02120 & 2.18434 & -2.11296 \\ \mathrm{C} & 1.23893 & 2.93969 & -1.24215 \\ \mathrm{C} & -0.19648 & 2.99626 & -1.40465 \\ \mathrm{C} & -0.77845 & 2.30889 & -2.46370 \\ \mathrm{C} & 0.02338 & 1.52346 & -3.38043 \\ \mathrm{C} & 1.40664 & 1.45408 & -3.20177 \\ \mathrm{C} & 2.09654 & 0.18342 & -3.34317 \\ \mathrm{C} & 3.14921 & 0.13109 & -2.33977\end{array}$




$\begin{array}{lrrr}\mathrm{C} & 3.43536 & -1.07280 & -1.69116 \\ \mathrm{C} & 2.68662 & -2.27330 & -2.01800 \\ \mathrm{C} & 2.48977 & -3.02567 & -0.78956 \\ \mathrm{C} & 1.28203 & -3.69576 & -0.56713 \\ \mathrm{C} & 0.22972 & -3.64058 & -1.56856 \\ \mathrm{C} & -1.04357 & -3.57331 & -0.87620 \\ \mathrm{C} & -2.07897 & -2.79127 & -1.38914 \\ \mathrm{C} & -2.89497 & -1.97923 & -0.50192 \\ \mathrm{C} & -3.20291 & -0.75086 & -1.18770 \\ \mathrm{C} & -2.56532 & -0.78140 & -2.49048 \\ \mathrm{C} & -1.87895 & -2.05083 & -2.62633 \\ \mathrm{C} & -0.65145 & -2.10926 & -3.29008 \\ \mathrm{C} & 0.42418 & -2.92282 & -2.75373 \\ \mathrm{C} & 1.67456 & -2.22250 & -2.97887 \\ \mathrm{C} & 1.37419 & -0.96899 & -3.65438 \\ \mathrm{C} & -0.06468 & -0.89881 & -3.84911 \\ \mathrm{C} & -0.72698 & 0.32236 & -3.71876 \\ \mathrm{C} & -2.00306 & 0.37942 & -3.02767 \\ \mathrm{C} & -2.02141 & 1.59344 & -2.24368 \\ \mathrm{C} & -2.61728 & 1.61906 & -0.98846 \\ \mathrm{C} & -3.24265 & 0.43204 & -0.45092 \\ \mathrm{C} & -3.09054 & 0.47433 & 0.99221 \\ \mathrm{C} & -2.67516 & 1.86329 & 1.35622 \\ \mathrm{C} & -2.06801 & 2.38240 & 0.12671 \\ \mathrm{C} & -0.81469 & 3.02705 & -0.06983 \\ \mathrm{C} & -3.30803 & 2.66983 & 2.42104 \\ \mathrm{C} & -2.59544 & 3.88585 & 2.72122 \\ \mathrm{H} & 0.57587 & 3.54101 & 2.89740 \\ \mathrm{H} & -1.05162 & 2.41816 & 4.44685 \\ \mathrm{H} & -2.94066 & 1.81618 & 3.61940 \\ \mathrm{H} & -4.35199 & 2.48856 & 2.68230 \\ \mathrm{H} & -2.11279 & 4.42411 & 1.91308 \\ \mathrm{H} & -2.93196 & 4.49248 & 3.55583 \\ & & & \end{array}$

(7) Transition state (TS89)

$\begin{array}{crrr}\mathrm{Ni} & 1.27003 & 3.70420 & 0.40678 \\ \mathrm{C} & 2.01943 & 2.26922 & 1.31320 \\ \mathrm{C} & 2.90522 & 1.47245 & 0.50606 \\ \mathrm{C} & 2.61914 & 1.67630 & -0.89909 \\ \mathrm{C} & 1.50330 & 2.57819 & -1.06627 \\ \mathrm{C} & 0.51935 & 2.32081 & -2.00730 \\ \mathrm{C} & 0.71997 & 1.23727 & -2.95142 \\ \mathrm{C} & 1.84359 & 0.40844 & -2.85904 \\ \mathrm{C} & 2.80510 & 0.62255 & -1.80642 \\ \mathrm{C} & 3.26103 & -0.67691 & -1.34192 \\ \mathrm{C} & 3.49948 & -0.88115 & 0.01446 \\ \mathrm{C} & 3.30663 & 0.20867 & 0.95341\end{array}$




\begin{tabular}{|c|c|c|c|}
\hline $\mathrm{C}$ & 2.72968 & -0.33507 & 2.16051 \\
\hline $\mathrm{C}$ & 1.73518 & 0.38692 & 2.82728 \\
\hline C & 1.31717 & 1.70123 & 2.36123 \\
\hline C & -0.14722 & 1.80768 & 2.58780 \\
\hline $\mathrm{C}$ & -0.56529 & 0.56492 & 3.22217 \\
\hline $\mathrm{C}$ & 0.58646 & -0.30003 & 3.37416 \\
\hline $\mathrm{C}$ & 0.46219 & -1.68448 & 3.23726 \\
\hline $\mathrm{C}$ & 1.47719 & -2.43303 & 2.51551 \\
\hline $\mathrm{C}$ & 2.58593 & -1.77024 & 1.98638 \\
\hline $\mathrm{C}$ & 3.06302 & -2.10927 & 0.65490 \\
\hline C & 2.41154 & -3.09129 & -0.09328 \\
\hline C & 2.16679 & -2.88073 & -1.51204 \\
\hline $\mathrm{C}$ & 2.58136 & -1.69549 & -2.12233 \\
\hline $\mathrm{C}$ & 1.70841 & -1.02219 & -3.06989 \\
\hline $\mathrm{C}$ & 0.46118 & -1.56772 & -3.37809 \\
\hline C & -0.70098 & -0.70286 & -3.50001 \\
\hline C & -0.57187 & 0.67007 & -3.28649 \\
\hline $\mathrm{C}$ & -1.58671 & 1.40017 & -2.55344 \\
\hline $\mathrm{C}$ & -2.70765 & 0.71904 & -2.06471 \\
\hline C & -2.83278 & -0.71479 & -2.26018 \\
\hline C & -1.85042 & -1.41281 & -2.96427 \\
\hline $\mathrm{C}$ & -1.39568 & -2.70763 & -2.49345 \\
\hline $\mathrm{C}$ & 0.03336 & -2.80440 & -2.75159 \\
\hline C & 0.86712 & -3.44453 & -1.83129 \\
\hline $\mathrm{C}$ & 0.30824 & -4.00573 & -0.61313 \\
\hline $\mathrm{C}$ & 1.26135 & -3.78267 & 0.46186 \\
\hline $\mathrm{C}$ & 0.80555 & -3.46129 & 1.74179 \\
\hline $\mathrm{C}$ & -0.62308 & -3.35984 & 2.00046 \\
\hline C & -0.83240 & -2.26584 & 2.92955 \\
\hline $\mathrm{C}$ & -1.95055 & -1.44071 & 2.79962 \\
\hline $\mathrm{C}$ & -1.81569 & -0.00460 & 2.96177 \\
\hline $\mathrm{C}$ & -2.69822 & 0.63486 & 2.01863 \\
\hline $\mathrm{C}$ & -3.37793 & -0.39648 & 1.25228 \\
\hline $\mathrm{C}$ & -2.91197 & -1.68368 & 1.73464 \\
\hline $\mathrm{C}$ & -2.70506 & -2.73111 & 0.83401 \\
\hline $\mathrm{C}$ & -1.53826 & -3.58547 & 0.96960 \\
\hline $\mathrm{C}$ & -1.06191 & -3.91076 & -0.36447 \\
\hline $\mathrm{C}$ & -1.93246 & -3.25336 & -1.32479 \\
\hline $\mathrm{C}$ & -2.95032 & -2.52719 & -0.58570 \\
\hline $\mathrm{C}$ & -3.39584 & -1.28627 & -1.04643 \\
\hline $\mathrm{C}$ & -3.61789 & -0.20229 & -0.10689 \\
\hline $\mathrm{C}$ & -3.19835 & 1.03400 & -0.74489 \\
\hline $\mathrm{C}$ & -2.56450 & 2.03271 & -0.00396 \\
\hline $\mathrm{C}$ & -2.28969 & 1.81588 & 1.40398 \\
\hline $\mathrm{C}$ & -1.02640 & 2.45738 & 1.72126 \\
\hline $\mathrm{C}$ & -0.67601 & 3.38343 & 0.58999 \\
\hline $\mathrm{C}$ & -1.47327 & 2.81772 & -0.54410 \\
\hline C & -0.94315 & 2.43822 & -1.77018 \\
\hline $\mathrm{C}$ & -0.60297 & 4.88523 & 0.88816 \\
\hline
\end{tabular}




$\begin{array}{lrrr}\mathrm{C} & -0.81174 & 5.83853 & -0.31132 \\ \mathrm{H} & -1.26932 & 5.12760 & 1.72387 \\ \mathrm{H} & 0.40519 & 5.12985 & 1.37087 \\ \mathrm{H} & 2.67116 & 4.26780 & 0.60387 \\ \mathrm{H} & -1.62163 & 6.54414 & -0.10493 \\ \mathrm{H} & -1.07853 & 5.28726 & -1.21715 \\ \mathrm{H} & 0.08626 & 6.42601 & -0.52767\end{array}$

Transition state structures observed over Pd/CNT during acetylene hydrogenation.

(1) Transition state (TS12)

$\begin{array}{llll}\mathrm{H} & -2.60870 & -6.08186 & -0.45545 \\ \mathrm{H} & -2.97042 & -5.13763 & -2.39434 \\ \mathrm{C} & -2.15846 & -4.47593 & -2.67903 \\ \mathrm{C} & -2.42988 & -3.36227 & -3.41251 \\ \mathrm{H} & -3.45433 & -3.15572 & -3.70501 \\ \mathrm{H} & -3.84159 & -1.05836 & -3.87307 \\ \mathrm{C} & -2.97222 & -0.44572 & -3.65702 \\ \mathrm{C} & -3.14573 & 0.80243 & -3.16113 \\ \mathrm{H} & -4.15128 & 1.17040 & -2.98502 \\ \mathrm{H} & -4.29062 & 2.69914 & -1.47805 \\ \mathrm{C} & -3.30689 & 2.83502 & -1.03657 \\ \mathrm{C} & -3.20787 & 3.01023 & 0.31080 \\ \mathrm{H} & -4.11114 & 3.02142 & 0.91324 \\ \mathrm{H} & -3.75560 & 1.89355 & 2.70007 \\ \mathrm{C} & -2.72823 & 1.57490 & 2.84010 \\ \mathrm{C} & -2.46840 & 0.48899 & 3.61129 \\ \mathrm{H} & -3.29328 & -0.04118 & 4.07604 \\ \mathrm{H} & -2.88706 & -2.10638 & 4.43521 \\ \mathrm{C} & -1.90837 & -2.39813 & 4.06842 \\ \mathrm{C} & -1.71840 & -3.66695 & 3.61309 \\ \mathrm{H} & -2.54926 & -4.36535 & 3.61975 \\ \mathrm{H} & -2.44017 & -5.77465 & 1.95018 \\ \mathrm{C} & -1.58268 & -5.55567 & 1.32164 \\ \mathrm{C} & -1.67700 & -5.72759 & -0.02555 \\ \mathrm{C} & -0.60427 & -5.30225 & -0.89167 \\ \mathrm{C} & -0.83315 & -4.70600 & -2.15730 \\ \mathrm{C} & 0.24469 & -4.01267 & -2.78810 \\ \mathrm{C} & -0.03930 & -2.84233 & -3.57297 \\ \mathrm{C} & -1.39639 & -2.39440 & -3.69152 \\ \mathrm{C} & -1.64795 & -1.01021 & -3.80556 \\ \mathrm{C} & -0.54475 & -0.09851 & -3.81313 \\ \mathrm{C} & -0.71670 & 1.24208 & -3.31592 \\ \mathrm{C} & -2.01463 & 1.62728 & -2.77670 \\ \mathrm{C} & -2.11618 & 2.62579 & -1.82805 \\ & -0.91648 & 3.45057 & -1.40874\end{array}$




\begin{tabular}{|c|c|c|c|}
\hline $\mathrm{C}$ & -0.78860 & 3.54539 & 0.19211 \\
\hline $\mathrm{C}$ & -1.91029 & 2.98233 & 0.95563 \\
\hline $\mathrm{C}$ & -1.67293 & 2.25725 & 2.12294 \\
\hline $\mathrm{C}$ & -0.31614 & 1.99345 & 2.55292 \\
\hline $\mathrm{C}$ & -0.05104 & 0.82595 & 3.35498 \\
\hline $\mathrm{C}$ & -1.12813 & -0.03582 & 3.73005 \\
\hline $\mathrm{C}$ & -0.86582 & -1.40932 & 3.94605 \\
\hline $\mathrm{C}$ & 0.46935 & -1.89224 & 3.75910 \\
\hline $\mathrm{C}$ & 0.66676 & -3.23107 & 3.27089 \\
\hline $\mathrm{C}$ & -0.47324 & -4.04428 & 2.99129 \\
\hline $\mathrm{C}$ & -0.40894 & -4.94494 & 1.89721 \\
\hline $\mathrm{C}$ & 0.79005 & -4.98379 & 1.12162 \\
\hline $\mathrm{C}$ & 0.69089 & -5.16768 & -0.30314 \\
\hline $\mathrm{C}$ & 1.81883 & -4.87079 & -1.11919 \\
\hline $\mathrm{C}$ & 1.59021 & -4.27906 & -2.39946 \\
\hline $\mathrm{C}$ & 2.62776 & -3.63106 & -3.15051 \\
\hline $\mathrm{C}$ & 2.35350 & -2.52781 & -3.90914 \\
\hline $\mathrm{C}$ & 1.02406 & -1.99003 & -3.96901 \\
\hline $\mathrm{C}$ & 0.76302 & -0.58589 & -4.10368 \\
\hline $\mathrm{C}$ & 1.80360 & 0.38430 & -4.23160 \\
\hline $\mathrm{C}$ & 1.63676 & 1.65328 & -3.74947 \\
\hline $\mathrm{C}$ & 0.41943 & 2.05570 & -3.11214 \\
\hline $\mathrm{C}$ & 0.39654 & 3.06955 & -2.07727 \\
\hline $\mathrm{C}$ & 1.55729 & 3.51507 & -1.48943 \\
\hline $\mathrm{C}$ & 1.64036 & 3.82855 & -0.09123 \\
\hline $\mathrm{C}$ & 0.55973 & 3.43171 & 0.76014 \\
\hline $\mathrm{C}$ & 0.76618 & 2.71435 & 2.00209 \\
\hline $\mathrm{C}$ & 2.07254 & 2.46445 & 2.52476 \\
\hline $\mathrm{C}$ & 2.32823 & 1.35769 & 3.28669 \\
\hline $\mathrm{C}$ & 1.29555 & 0.40956 & 3.56898 \\
\hline $\mathrm{C}$ & 1.56203 & -0.98428 & 3.77014 \\
\hline $\mathrm{C}$ & 2.88130 & -1.54263 & 3.67162 \\
\hline $\mathrm{C}$ & 3.06939 & -2.80810 & 3.19285 \\
\hline $\mathrm{C}$ & 1.95074 & -3.60884 & 2.78090 \\
\hline $\mathrm{C}$ & 2.01381 & -4.50967 & 1.67433 \\
\hline $\mathrm{C}$ & 3.19927 & -4.67514 & 0.87617 \\
\hline $\mathrm{C}$ & 3.10470 & -4.84961 & -0.47423 \\
\hline $\mathrm{H}$ & 4.00954 & -4.85087 & -1.07384 \\
\hline $\mathrm{H}$ & 3.65933 & -3.94536 & -3.02726 \\
\hline $\mathrm{H}$ & 3.17402 & -1.99213 & -4.37567 \\
\hline $\mathrm{H}$ & 2.77105 & 0.09380 & -4.62835 \\
\hline $\mathrm{H}$ & 2.47402 & 2.34314 & -3.78488 \\
\hline $\mathrm{H}$ & 2.50685 & 3.40224 & -2.00355 \\
\hline $\mathrm{H}$ & 2.63924 & 3.86439 & 0.32592 \\
\hline $\mathrm{H}$ & 3.34943 & 1.15206 & 3.59015 \\
\hline $\mathrm{H}$ & 3.74753 & -0.92038 & 3.87141 \\
\hline $\mathrm{H}$ & 4.07998 & -3.16292 & 3.01704 \\
\hline $\mathrm{H}$ & 4.17735 & -4.54008 & 1.32711 \\
\hline Pd & 0.23049 & 5.60764 & 0.48086 \\
\hline
\end{tabular}




$\begin{array}{rrrr}\mathrm{C} & 1.14635 & 7.51677 & 0.47517 \\ \mathrm{C} & -0.09651 & 7.68109 & 0.58146 \\ \mathrm{H} & 2.16402 & 7.84892 & 0.37945 \\ \mathrm{H} & -0.99460 & 8.26288 & 0.67298 \\ \mathrm{H} & 2.89445 & 3.11710 & 2.24990 \\ \mathrm{H} & -1.35368 & 5.20127 & 0.52807 \\ \mathrm{H} & -1.13182 & 4.47262 & -1.73846\end{array}$

(2) Transition state (TS23)

\begin{tabular}{|c|c|c|c|}
\hline $\mathrm{H}$ & -1.15029 & -6.10902 & -2.34573 \\
\hline $\mathrm{H}$ & -2.82908 & -4.91508 & -3.04907 \\
\hline $\mathrm{C}$ & -2.54045 & -3.88719 & -3.24447 \\
\hline $\mathrm{C}$ & -3.44146 & -2.88935 & -3.03677 \\
\hline $\mathrm{H}$ & -4.43651 & -3.13593 & -2.68100 \\
\hline $\mathrm{H}$ & -5.26958 & -1.57530 & -1.46259 \\
\hline $\mathrm{C}$ & -4.60360 & -0.72496 & -1.35720 \\
\hline $\mathrm{C}$ & -4.65534 & 0.02932 & -0.23540 \\
\hline $\mathrm{H}$ & -5.35658 & -0.22446 & 0.55323 \\
\hline $\mathrm{H}$ & -4.73879 & 0.47229 & 2.56889 \\
\hline $\mathrm{C}$ & -3.73916 & 0.88456 & 2.45990 \\
\hline $\mathrm{C}$ & -2.81591 & 0.68328 & 3.43236 \\
\hline $\mathrm{H}$ & -3.08825 & 0.13723 & 4.33032 \\
\hline $\mathrm{H}$ & -1.49143 & -1.25734 & 4.77521 \\
\hline $\mathrm{C}$ & -0.56132 & -1.06414 & 4.25119 \\
\hline $\mathrm{C}$ & 0.33392 & -2.08123 & 4.09181 \\
\hline $\mathrm{H}$ & 0.09515 & -3.06139 & 4.49180 \\
\hline $\mathrm{H}$ & 1.02593 & -4.67255 & 3.41488 \\
\hline $\mathrm{C}$ & 1.58322 & -4.31222 & 2.55609 \\
\hline $\mathrm{C}$ & 1.65900 & -5.08839 & 1.43806 \\
\hline $\mathrm{H}$ & 1.15737 & -6.05078 & 1.42415 \\
\hline $\mathrm{H}$ & 0.44478 & -6.57461 & -0.57411 \\
\hline $\mathrm{C}$ & 0.64599 & -5.77083 & -1.27537 \\
\hline $\mathrm{C}$ & -0.24827 & -5.51013 & -2.26909 \\
\hline $\mathrm{C}$ & -0.07971 & -4.35722 & -3.11847 \\
\hline $\mathrm{C}$ & -1.17158 & -3.58244 & -3.58409 \\
\hline $\mathrm{C}$ & -0.90685 & -2.28781 & -4.12688 \\
\hline $\mathrm{C}$ & -1.86117 & -1.23345 & -3.92299 \\
\hline $\mathrm{C}$ & -3.04767 & -1.50622 & -3.16053 \\
\hline $\mathrm{C}$ & -3.59582 & -0.47741 & -2.37042 \\
\hline $\mathrm{C}$ & -2.97186 & 0.81186 & -2.37591 \\
\hline $\mathrm{C}$ & -3.04634 & 1.64826 & -1.20962 \\
\hline $\mathrm{C}$ & -3.71676 & 1.11808 & -0.02219 \\
\hline $\mathrm{C}$ & -3.33380 & 1.53265 & 1.22729 \\
\hline $\mathrm{C}$ & -2.30796 & 2.63152 & 1.43106 \\
\hline $\mathrm{C}$ & -1.14799 & 2.13654 & 2.34783 \\
\hline $\mathrm{C}$ & -1.41782 & 1.06030 & 3.21992 \\
\hline
\end{tabular}




\begin{tabular}{|c|c|c|c|}
\hline C & -0.35042 & 0.21261 & 3.62277 \\
\hline $\mathrm{C}$ & 0.96811 & 0.49912 & 3.15519 \\
\hline $\mathrm{C}$ & 1.91757 & -0.56980 & 2.99992 \\
\hline $\mathrm{C}$ & 1.51720 & -1.90894 & 3.29272 \\
\hline $\mathrm{C}$ & 2.11261 & -2.97319 & 2.55889 \\
\hline $\mathrm{C}$ & 3.07534 & -2.65438 & 1.55396 \\
\hline $\mathrm{C}$ & 3.15023 & -3.46974 & 0.37037 \\
\hline $\mathrm{C}$ & 2.26338 & -4.58007 & 0.23362 \\
\hline $\mathrm{C}$ & 1.77531 & -4.90061 & -1.06197 \\
\hline $\mathrm{C}$ & 2.18072 & -4.08256 & -2.15912 \\
\hline $\mathrm{C}$ & 1.23479 & -3.80186 & -3.20685 \\
\hline $\mathrm{C}$ & 1.50214 & -2.74061 & -4.11751 \\
\hline $\mathrm{C}$ & 0.40166 & -1.96096 & -4.59113 \\
\hline $\mathrm{C}$ & 0.56972 & -0.68571 & -5.22636 \\
\hline $\mathrm{C}$ & -0.35020 & 0.31065 & -5.04922 \\
\hline $\mathrm{C}$ & -1.50187 & 0.10672 & -4.21900 \\
\hline $\mathrm{C}$ & -2.08901 & 1.16129 & -3.43945 \\
\hline $\mathrm{C}$ & -1.62693 & 2.51128 & -3.47785 \\
\hline $\mathrm{C}$ & -1.71625 & 3.31090 & -2.36913 \\
\hline $\mathrm{C}$ & -2.26368 & 2.81943 & -1.14183 \\
\hline $\mathrm{C}$ & -1.75632 & 3.25401 & 0.15403 \\
\hline $\mathrm{C}$ & -0.56660 & 3.94658 & 0.23355 \\
\hline $\mathrm{C}$ & 0.39602 & 3.76293 & 1.29889 \\
\hline $\mathrm{C}$ & 0.17717 & 2.59073 & 2.15177 \\
\hline $\mathrm{C}$ & 1.26029 & 1.77614 & 2.59941 \\
\hline $\mathrm{C}$ & 2.63358 & 2.05677 & 2.28328 \\
\hline $\mathrm{C}$ & 3.53994 & 1.04617 & 2.14543 \\
\hline $\mathrm{C}$ & 3.13992 & -0.32402 & 2.30652 \\
\hline $\mathrm{C}$ & 3.73266 & -1.38955 & 1.56877 \\
\hline $\mathrm{C}$ & 4.76901 & -1.17652 & 0.59412 \\
\hline $\mathrm{C}$ & 4.83193 & -1.94371 & -0.53204 \\
\hline $\mathrm{C}$ & 3.86497 & -2.98302 & -0.76341 \\
\hline $\mathrm{C}$ & 3.36215 & -3.29062 & -2.06160 \\
\hline $\mathrm{C}$ & 3.76709 & -2.57213 & -3.24028 \\
\hline $\mathrm{C}$ & 2.86819 & -2.30512 & -4.23205 \\
\hline $\mathrm{H}$ & 3.16190 & -1.66086 & -5.05480 \\
\hline $\mathrm{H}$ & 1.49003 & -0.46560 & -5.75784 \\
\hline $\mathrm{H}$ & -0.13974 & 1.29855 & -5.44622 \\
\hline $\mathrm{H}$ & -1.11561 & 2.88568 & -4.35900 \\
\hline $\mathrm{H}$ & -1.28412 & 4.30609 & -2.40167 \\
\hline $\mathrm{H}$ & -0.20458 & 4.48004 & -0.63899 \\
\hline $\mathrm{H}$ & 1.41586 & 3.95957 & 0.99190 \\
\hline $\mathrm{H}$ & 4.54704 & 1.27826 & 1.81427 \\
\hline $\mathrm{H}$ & 5.43874 & -0.32860 & 0.69598 \\
\hline $\mathrm{H}$ & 5.54863 & -1.69190 & -1.30736 \\
\hline $\mathrm{H}$ & 4.75877 & -2.13387 & -3.29107 \\
\hline Pd & -0.47436 & 5.20165 & 2.64516 \\
\hline $\mathrm{C}$ & 0.60208 & 6.82481 & 3.51507 \\
\hline $\mathrm{C}$ & 1.39246 & 6.29666 & 2.70078 \\
\hline
\end{tabular}




$\begin{array}{rrrr}\mathrm{H} & 0.26652 & 7.52540 & 4.25216 \\ \mathrm{H} & 2.31788 & 6.16938 & 2.17401 \\ \mathrm{H} & 2.93999 & 3.07730 & 2.07998 \\ \mathrm{H} & -1.59366 & 5.95967 & 3.58364 \\ \mathrm{H} & -2.86719 & 3.40923 & 1.96352\end{array}$

(3) Transition state (TS34)

\begin{tabular}{|c|c|c|c|}
\hline $\mathrm{H}$ & -5.11483 & 1.01658 & -3.68613 \\
\hline $\mathrm{H}$ & -5.22866 & -1.17589 & -3.63191 \\
\hline $\mathrm{C}$ & -4.92564 & -1.54757 & -2.65816 \\
\hline $\mathrm{C}$ & -4.22289 & -2.71319 & -2.57535 \\
\hline $\mathrm{H}$ & -3.97757 & -3.25318 & -3.48434 \\
\hline $\mathrm{H}$ & -2.01441 & -4.18236 & -3.32154 \\
\hline $\mathrm{C}$ & -1.56326 & -4.02293 & -2.34759 \\
\hline $\mathrm{C}$ & -0.20517 & -3.92769 & -2.24905 \\
\hline $\mathrm{H}$ & 0.39743 & -4.02488 & -3.14580 \\
\hline $\mathrm{H}$ & 2.08111 & -2.79130 & -3.06270 \\
\hline $\mathrm{C}$ & 2.28270 & -2.33413 & -2.10088 \\
\hline $\mathrm{C}$ & 3.03742 & -1.19662 & -2.05356 \\
\hline $\mathrm{H}$ & 3.40757 & -0.75669 & -2.97186 \\
\hline $\mathrm{H}$ & 4.23841 & 1.41700 & -2.86110 \\
\hline $\mathrm{C}$ & 3.64124 & 1.69488 & -1.99740 \\
\hline $\mathrm{C}$ & 2.78124 & 2.73324 & -2.06592 \\
\hline $\mathrm{H}$ & 2.70077 & 3.30342 & -2.98706 \\
\hline $\mathrm{H}$ & 0.60861 & 3.86823 & -3.26502 \\
\hline $\mathrm{C}$ & -0.00566 & 3.82961 & -2.37129 \\
\hline $\mathrm{C}$ & -1.36435 & 3.83696 & -2.49718 \\
\hline $\mathrm{H}$ & -1.80080 & 3.88238 & -3.48948 \\
\hline $\mathrm{H}$ & -3.75640 & 3.02539 & -3.62564 \\
\hline $\mathrm{C}$ & -4.04091 & 2.54915 & -2.69290 \\
\hline $\mathrm{C}$ & -4.80755 & 1.42215 & -2.72738 \\
\hline $\mathrm{C}$ & -5.07661 & 0.68630 & -1.51969 \\
\hline $\mathrm{C}$ & -5.12880 & -0.73613 & -1.48639 \\
\hline $\mathrm{C}$ & -5.01279 & -1.38380 & -0.22094 \\
\hline $\mathrm{C}$ & -4.26342 & -2.60889 & -0.13403 \\
\hline $\mathrm{C}$ & -3.67494 & -3.14361 & -1.31542 \\
\hline $\mathrm{C}$ & -2.39725 & -3.76719 & -1.20739 \\
\hline $\mathrm{C}$ & -1.78322 & -3.83936 & 0.07842 \\
\hline $\mathrm{C}$ & -0.35360 & -3.74138 & 0.17985 \\
\hline $\mathrm{C}$ & 0.41862 & -3.58278 & -1.00226 \\
\hline $\mathrm{C}$ & 1.63304 & -2.82816 & -0.92772 \\
\hline $\mathrm{C}$ & 2.05875 & -2.32554 & 0.34552 \\
\hline $\mathrm{C}$ & 2.87993 & -1.15622 & 0.40740 \\
\hline $\mathrm{C}$ & 3.25190 & -0.49826 & -0.81286 \\
\hline $\mathrm{C}$ & 3.70299 & 0.86899 & -0.77406 \\
\hline $\mathrm{C}$ & 3.48931 & 1.67900 & 0.49101 \\
\hline
\end{tabular}




\begin{tabular}{|c|c|c|c|}
\hline $\mathrm{C}$ & 2.33471 & 2.68999 & 0.34334 \\
\hline $\mathrm{C}$ & 1.91441 & 3.08118 & -0.93750 \\
\hline $\mathrm{C}$ & 0.61597 & 3.63887 & -1.09055 \\
\hline $\mathrm{C}$ & -0.20040 & 3.80638 & 0.06976 \\
\hline $\mathrm{C}$ & -1.63057 & 3.81600 & -0.06366 \\
\hline $\mathrm{C}$ & -2.21596 & 3.64771 & -1.35532 \\
\hline $\mathrm{C}$ & -3.49458 & 3.02685 & -1.44964 \\
\hline $\mathrm{C}$ & -4.12364 & 2.57868 & -0.25033 \\
\hline $\mathrm{C}$ & -4.92758 & 1.38546 & -0.28638 \\
\hline $\mathrm{C}$ & -5.27611 & 0.74922 & 0.94304 \\
\hline $\mathrm{C}$ & -5.31298 & -0.67139 & 0.97775 \\
\hline $\mathrm{C}$ & -5.27940 & -1.41885 & 2.21035 \\
\hline $\mathrm{C}$ & -4.56871 & -2.57819 & 2.29452 \\
\hline $\mathrm{C}$ & -3.84309 & -3.07297 & 1.15106 \\
\hline $\mathrm{C}$ & -2.57556 & -3.70218 & 1.25868 \\
\hline $\mathrm{C}$ & -1.91580 & -3.91886 & 2.52226 \\
\hline $\mathrm{C}$ & -0.56017 & -3.84660 & 2.62219 \\
\hline $\mathrm{C}$ & 0.24910 & -3.54303 & 1.46714 \\
\hline $\mathrm{C}$ & 1.47094 & -2.84250 & 1.54866 \\
\hline $\mathrm{C}$ & 2.03380 & -2.40538 & 2.80874 \\
\hline $\mathrm{C}$ & 2.78550 & -1.28202 & 2.88077 \\
\hline $\mathrm{C}$ & 3.06611 & -0.49483 & 1.69451 \\
\hline $\mathrm{C}$ & 3.26305 & 0.86877 & 1.73884 \\
\hline $\mathrm{C}$ & 3.01981 & 1.67586 & 2.92351 \\
\hline $\mathrm{C}$ & 2.25819 & 2.79038 & 2.79420 \\
\hline $\mathrm{C}$ & 1.67798 & 3.14709 & 1.49840 \\
\hline $\mathrm{C}$ & 0.38483 & 3.72591 & 1.36905 \\
\hline $\mathrm{C}$ & -0.45944 & 4.01063 & 2.49585 \\
\hline $\mathrm{C}$ & -1.81758 & 4.01771 & 2.36915 \\
\hline $\mathrm{C}$ & -2.44412 & 3.73373 & 1.10798 \\
\hline $\mathrm{C}$ & -3.71666 & 3.10336 & 1.01176 \\
\hline $\mathrm{C}$ & -4.47178 & 2.69172 & 2.16714 \\
\hline $\mathrm{C}$ & -5.22341 & 1.55522 & 2.13533 \\
\hline $\mathrm{H}$ & -5.69193 & 1.19999 & 3.04775 \\
\hline $\mathrm{H}$ & -5.72262 & -0.99887 & 3.10776 \\
\hline $\mathrm{H}$ & -4.45633 & -3.06621 & 3.25745 \\
\hline $\mathrm{H}$ & -2.50923 & -4.04317 & 3.42246 \\
\hline $\mathrm{H}$ & -0.09651 & -3.91803 & 3.60025 \\
\hline $\mathrm{H}$ & 1.78791 & -2.94346 & 3.71808 \\
\hline $\mathrm{H}$ & 3.13330 & -0.92332 & 3.84402 \\
\hline $\mathrm{H}$ & 1.99569 & 3.36830 & 3.67477 \\
\hline $\mathrm{H}$ & -0.01966 & 4.12929 & 3.48065 \\
\hline $\mathrm{H}$ & -2.42959 & 4.13930 & 3.25688 \\
\hline $\mathrm{H}$ & -4.35826 & 3.22676 & 3.10444 \\
\hline $\mathrm{Pd}$ & 5.46342 & -0.35415 & -0.68540 \\
\hline $\mathrm{C}$ & 7.50773 & -0.65718 & -0.57424 \\
\hline $\mathrm{C}$ & 8.21952 & -0.81918 & 0.56338 \\
\hline $\mathrm{H}$ & 8.12126 & -0.61451 & -1.48334 \\
\hline $\mathrm{H}$ & 3.33143 & 1.33654 & 3.90747 \\
\hline
\end{tabular}




$\begin{array}{llll}\mathrm{H} & 7.74499 & -0.90629 & 1.54153 \\ \mathrm{H} & 9.31347 & -0.88428 & 0.58308 \\ \mathrm{H} & 4.42632 & 2.25365 & 0.65040\end{array}$

(4) Transition state (TS45)

\begin{tabular}{|c|c|c|c|}
\hline $\mathrm{H}$ & -0.60804 & -5.25248 & -3.32066 \\
\hline $\mathrm{H}$ & 1.55938 & -5.14327 & -3.35429 \\
\hline $\mathrm{C}$ & 1.96059 & -4.78319 & -2.41229 \\
\hline $\mathrm{C}$ & 3.10278 & -4.03846 & -2.4151 \\
\hline $\mathrm{H}$ & 3.59079 & -3.81903 & -3.3594 \\
\hline $\mathrm{H}$ & 4.55905 & -1.88021 & -3.3361 \\
\hline $\mathrm{C}$ & 4.43840 & -1.37633 & -2.3824 \\
\hline $\mathrm{C}$ & 4.35941 & -0.01473 & -2.3534 \\
\hline $\mathrm{H}$ & 4.42063 & 0.53915 & -3.2847 \\
\hline $\mathrm{H}$ & 3.23401 & 2.33743 & -3.23 \\
\hline $\mathrm{C}$ & 2.73830 & 2.48231 & -2.2783 \\
\hline C & 1.53231 & 3.12411 & -2.24677 \\
\hline $\mathrm{H}$ & 1.09510 & 3.47843 & -3.17 \\
\hline $\mathrm{H}$ & -1.07437 & 3.38141 & -3.16 \\
\hline C & -1.45164 & 3.01397 & -2.2146 \\
\hline C & -2.58069 & 2.23792 & -2.2027 \\
\hline $\mathrm{H}$ & -3.08639 & 2.03596 & -3.1413 \\
\hline $\mathrm{H}$ & -4.05166 & 0.11018 & -3.1622 \\
\hline C & -3.89996 & -0.43071 & -2.2336 \\
\hline $\mathrm{C}$ & -3.82732 & -1.79241 & -2.2602 \\
\hline $\mathrm{H}$ & -3.92043 & -2.30948 & -3.209 \\
\hline $\mathrm{H}$ & -2.75940 & -4.13503 & -3.26 \\
\hline $\mathrm{C}$ & -2.22365 & -4.30208 & -2.335 \\
\hline $\mathrm{C}$ & -1.01402 & -4.93077 & -2.3668 \\
\hline C & -0.21214 & -5.03914 & -1.176 \\
\hline C & 1.20992 & -4.96903 & -1.1980 \\
\hline C & 1.89785 & -4.74578 & 0.0315 \\
\hline C & 3.10362 & -3.95898 & 0.02818 \\
\hline C & 3.58031 & -3.42323 & -1.2044 \\
\hline $\mathrm{C}$ & 4.21813 & -2.14933 & -1.1892 \\
\hline $\mathrm{C}$ & 4.34946 & -1.47002 & 0.0582 \\
\hline $\mathrm{C}$ & 4.26295 & -0.03316 & 0.08778 \\
\hline C & 4.05400 & 0.67702 & -1.1298 \\
\hline $\mathrm{C}$ & 3.27656 & 1.87338 & -1.09475 \\
\hline $\mathrm{C}$ & 2.74436 & 2.30211 & 0.1574 \\
\hline $\mathrm{C}$ & 1.47165 & 2.97398 & 0.18878 \\
\hline $\mathrm{C}$ & 0.77853 & 3.20601 & -1.0300 \\
\hline C & -0.66125 & 3.18704 & -1.0113 \\
\hline $\mathrm{C}$ & -1.32338 & 2.90313 & 0.2334 \\
\hline $\mathrm{C}$ & -2.50587 & 2.07172 & 0.2344 \\
\hline $\mathrm{C}$ & -3.00866 & 1.57312 & -1.0035 \\
\hline
\end{tabular}




\begin{tabular}{|c|c|c|c|}
\hline C & -3.63814 & 0.29434 & -1.01883 \\
\hline $\mathrm{C}$ & -3.72923 & -0.43198 & 0.20499 \\
\hline $\mathrm{C}$ & -3.65438 & -1.86915 & 0.17702 \\
\hline $\mathrm{C}$ & -3.48841 & -2.53340 & -1.07450 \\
\hline $\mathrm{C}$ & -2.72280 & -3.73380 & -1.11021 \\
\hline $\mathrm{C}$ & -2.15771 & -4.21654 & 0.10732 \\
\hline $\mathrm{C}$ & -0.88113 & -4.88184 & 0.07370 \\
\hline $\mathrm{C}$ & -0.17194 & -5.08740 & 1.29459 \\
\hline $\mathrm{C}$ & 1.24933 & -5.01819 & 1.27290 \\
\hline $\mathrm{C}$ & 2.03924 & -4.88175 & 2.46941 \\
\hline $\mathrm{C}$ & 3.18020 & -4.13587 & 2.46635 \\
\hline $\mathrm{C}$ & 3.61857 & -3.47096 & 1.26646 \\
\hline $\mathrm{C}$ & 4.25481 & -2.19838 & 1.28169 \\
\hline $\mathrm{C}$ & 4.51261 & -1.47280 & 2.49880 \\
\hline $\mathrm{C}$ & 4.42888 & -0.11264 & 2.52757 \\
\hline $\mathrm{C}$ & 4.08342 & 0.62722 & 1.34142 \\
\hline $\mathrm{C}$ & 3.30796 & 1.81812 & 1.37670 \\
\hline $\mathrm{C}$ & 2.80630 & 2.38479 & 2.60248 \\
\hline $\mathrm{C}$ & 1.59865 & 3.01557 & 2.63435 \\
\hline $\mathrm{C}$ & 0.79811 & 3.13168 & 1.44327 \\
\hline $\mathrm{C}$ & -0.61856 & 3.09882 & 1.46809 \\
\hline $\mathrm{C}$ & -1.37382 & 2.91054 & 2.68180 \\
\hline $\mathrm{C}$ & -2.50765 & 2.15642 & 2.68003 \\
\hline $\mathrm{C}$ & -2.97091 & 1.52906 & 1.46635 \\
\hline $\mathrm{C}$ & -3.59437 & 0.24980 & 1.45215 \\
\hline $\mathrm{C}$ & -3.81206 & -0.52119 & 2.64780 \\
\hline $\mathrm{C}$ & -3.73978 & -1.88257 & 2.62091 \\
\hline $\mathrm{C}$ & -3.44483 & -2.57953 & 1.39667 \\
\hline $\mathrm{C}$ & -2.68173 & -3.78050 & 1.36090 \\
\hline $\mathrm{C}$ & -2.14375 & -4.39607 & 2.54629 \\
\hline $\mathrm{C}$ & -0.93481 & -5.02541 & 2.51432 \\
\hline $\mathrm{H}$ & -0.49856 & -5.38446 & 3.44102 \\
\hline $\mathrm{H}$ & 1.66844 & -5.28178 & 3.40782 \\
\hline $\mathrm{H}$ & 3.69882 & -3.95501 & 3.40248 \\
\hline $\mathrm{H}$ & 4.66698 & -2.01497 & 3.42628 \\
\hline $\mathrm{H}$ & 4.51786 & 0.40454 & 3.47758 \\
\hline $\mathrm{H}$ & 3.34254 & 2.21794 & 3.53118 \\
\hline $\mathrm{H}$ & 1.19297 & 3.33902 & 3.58758 \\
\hline $\mathrm{H}$ & -3.00379 & 1.93964 & 3.62054 \\
\hline $\mathrm{H}$ & -3.92770 & -0.01496 & 3.60071 \\
\hline $\mathrm{H}$ & -3.79968 & -2.43466 & 3.55336 \\
\hline $\mathrm{H}$ & -2.64912 & -4.26560 & 3.49789 \\
\hline $\mathrm{Pd}$ & -1.76760 & 5.19911 & -0.84154 \\
\hline $\mathrm{C}$ & -1.79691 & 8.30328 & -0.87424 \\
\hline $\mathrm{C}$ & -2.46336 & 6.98305 & -0.63431 \\
\hline $\mathrm{H}$ & -2.39683 & 9.19489 & -0.64661 \\
\hline $\mathrm{H}$ & -1.46562 & 8.35444 & -1.92163 \\
\hline $\mathrm{H}$ & -0.86407 & 8.34298 & -0.29318 \\
\hline $\mathrm{H}$ & -3.48569 & 7.13019 & -0.25143 \\
\hline
\end{tabular}


$\begin{array}{llll}\text { H } & -0.97981 & 3.27875 & 3.62358\end{array}$

(5) Transition state (TS56)

$\begin{array}{lrrr}\mathrm{H} & 1.65247 & -1.82868 & -5.91584 \\ \mathrm{H} & 3.33327 & -0.64713 & -5.18335 \\ \mathrm{C} & 3.53410 & -0.83395 & -4.13316 \\ \mathrm{C} & 4.01329 & 0.18233 & -3.35534 \\ \mathrm{H} & 4.18663 & 1.15415 & -3.80634 \\ \mathrm{H} & 3.95115 & 2.76981 & -2.42284 \\ \mathrm{C} & 3.65150 & 2.43380 & -1.43537 \\ \mathrm{C} & 2.88847 & 3.25810 & -0.65600 \\ \mathrm{H} & 2.60913 & 4.23407 & -1.04027 \\ \mathrm{H} & 0.63402 & 4.57952 & -0.52689 \\ \mathrm{C} & 0.26404 & 4.06709 & 0.35595 \\ \mathrm{C} & -1.15796 & 3.92602 & 0.44146 \\ \mathrm{H} & -1.72259 & 4.29035 & -0.40772 \\ \mathrm{H} & -3.41307 & 3.22213 & -0.95540 \\ \mathrm{C} & -3.37055 & 2.21117 & -0.56399 \\ \mathrm{C} & -3.85001 & 1.18258 & -1.32686 \\ \mathrm{H} & -4.25270 & 1.39985 & -2.31069 \\ \mathrm{H} & -4.03897 & -0.21392 & -3.68495 \\ \mathrm{C} & -3.54729 & -1.07525 & -3.24467 \\ \mathrm{C} & -2.79129 & -1.88719 & -4.04152 \\ \mathrm{H} & -2.69356 & -1.65159 & -5.09653 \\ \mathrm{H} & -0.74106 & -2.22774 & -5.88246 \\ \mathrm{C} & -0.11943 & -2.66135 & -5.10542 \\ \mathrm{C} & 1.22666 & -2.43674 & -5.12386 \\ \mathrm{C} & 2.05283 & -2.86166 & -4.02519 \\ \mathrm{C} & 3.15422 & -2.08798 & -3.54648 \\ \mathrm{C} & 3.67130 & -2.38133 & -2.25065 \\ \mathrm{C} & 4.16005 & -1.30606 & -1.43124 \\ \mathrm{C} & 4.14381 & 0.02100 & -1.93481 \\ \mathrm{C} & 3.94937 & 1.10159 & -1.01268 \\ \mathrm{C} & 3.75664 & 0.80219 & 0.36779 \\ \mathrm{C} & 2.96300 & 1.67994 & 1.18885 \\ \mathrm{C} & 2.37116 & 2.82412 & 0.60765 \\ \mathrm{C} & 1.12834 & 3.33637 & 1.14217 \\ \mathrm{C} & 0.60357 & 2.83367 & 2.47966 \\ \mathrm{C} & -0.96846 & 2.47570 & 2.42984 \\ \mathrm{C} & -1.69095 & 2.83849 & 1.20361 \\ \mathrm{C} & -2.72363 & 1.96382 & 0.68699 \\ \mathrm{C} & -3.39167 & -0.68937 & 1.27499 \\ \mathrm{C} & -0.39761 & 0.47352 \\ \mathrm{C} & -0.17469 & -0.89954 \\ \mathrm{C} & -1.26408 & -1.82129 \\ \mathrm{C} & -2.52642 & -1.32465\end{array}$




\begin{tabular}{|c|c|c|c|}
\hline $\mathrm{C}$ & -2.36945 & -3.39188 & -2.16631 \\
\hline $\mathrm{C}$ & -2.01030 & -2.94998 & -3.47294 \\
\hline $\mathrm{C}$ & -0.73199 & -3.32659 & -3.98635 \\
\hline $\mathrm{C}$ & 0.11449 & -4.12545 & -3.16576 \\
\hline $\mathrm{C}$ & 1.53471 & -3.88799 & -3.18565 \\
\hline $\mathrm{C}$ & 2.33811 & -4.42833 & -2.13491 \\
\hline $\mathrm{C}$ & 3.42452 & -3.65499 & -1.65315 \\
\hline $\mathrm{C}$ & 4.07381 & -3.92795 & -0.39413 \\
\hline $\mathrm{C}$ & 4.52613 & -2.90914 & 0.38666 \\
\hline $\mathrm{C}$ & 4.37479 & -1.53674 & -0.03274 \\
\hline $\mathrm{C}$ & 4.16424 & -0.47346 & 0.87214 \\
\hline $\mathrm{C}$ & 4.08836 & -0.67399 & 2.30299 \\
\hline $\mathrm{C}$ & 3.31940 & 0.12686 & 3.07874 \\
\hline $\mathrm{C}$ & 2.55824 & 1.22497 & 2.51144 \\
\hline $\mathrm{C}$ & 1.41129 & 1.71540 & 3.10694 \\
\hline $\mathrm{C}$ & 0.79417 & 1.08107 & 4.24796 \\
\hline $\mathrm{C}$ & -0.54993 & 0.85516 & 4.26109 \\
\hline $\mathrm{C}$ & -1.36502 & 1.24542 & 3.12964 \\
\hline $\mathrm{C}$ & -2.32751 & 0.39995 & 2.57726 \\
\hline $\mathrm{C}$ & -2.66346 & -0.87997 & 3.16205 \\
\hline $\mathrm{C}$ & -3.15881 & -1.89220 & 2.40599 \\
\hline $\mathrm{C}$ & -3.35142 & -1.73226 & 0.98374 \\
\hline $\mathrm{C}$ & -3.24161 & -2.81205 & 0.07555 \\
\hline $\mathrm{C}$ & -2.93233 & -4.15815 & 0.49012 \\
\hline $\mathrm{C}$ & -2.19813 & -4.97932 & -0.30991 \\
\hline $\mathrm{C}$ & -1.70787 & -4.51537 & -1.58414 \\
\hline $\mathrm{C}$ & -0.44133 & -4.89505 & -2.09845 \\
\hline $\mathrm{C}$ & 0.45447 & -5.77600 & -1.38907 \\
\hline $\mathrm{C}$ & 1.79687 & -5.54983 & -1.40629 \\
\hline $\mathrm{H}$ & 2.44813 & -6.14934 & -0.77792 \\
\hline $\mathrm{H}$ & 4.10470 & -4.94440 & -0.01461 \\
\hline $\mathrm{H}$ & 4.91490 & -3.13037 & 1.37544 \\
\hline $\mathrm{H}$ & 4.59212 & -1.52444 & 2.75110 \\
\hline $\mathrm{H}$ & 3.21739 & -0.09557 & 4.13593 \\
\hline $\mathrm{H}$ & 1.40789 & 0.63637 & 5.02690 \\
\hline $\mathrm{H}$ & -0.97831 & 0.24794 & 5.05264 \\
\hline $\mathrm{H}$ & -3.33070 & -2.86109 & 2.86336 \\
\hline $\mathrm{H}$ & -3.20017 & -4.48936 & 1.48815 \\
\hline $\mathrm{H}$ & -1.88844 & -5.95005 & 0.06414 \\
\hline $\mathrm{H}$ & 0.05112 & -6.55287 & -0.74714 \\
\hline $\mathrm{Pd}$ & -1.94716 & 4.57328 & 2.58064 \\
\hline $\mathrm{C}$ & -2.60391 & 6.64486 & 2.71363 \\
\hline $\mathrm{C}$ & -2.50190 & 6.11243 & 4.00373 \\
\hline $\mathrm{H}$ & -3.56954 & 6.71943 & 2.21935 \\
\hline $\mathrm{H}$ & -1.81587 & 7.27509 & 2.30926 \\
\hline $\mathrm{H}$ & -3.38693 & 5.77283 & 4.53492 \\
\hline $\mathrm{H}$ & -1.63930 & 6.33206 & 4.62749 \\
\hline $\mathrm{H}$ & -2.44666 & -1.05680 & 4.21006 \\
\hline $\mathrm{H}$ & -1.62143 & 3.46418 & 3.73670 \\
\hline
\end{tabular}


$\begin{array}{llll}\mathrm{H} & 0.68073 & 3.67980 & 3.17039\end{array}$

(7) Transition state (TS78)

$\begin{array}{crrr}\mathrm{H} & -5.32862 & -1.80745 & 3.18703 \\ \mathrm{H} & -5.64029 & 0.35011 & 3.21289 \\ \mathrm{C} & -5.31186 & 0.81730 & 2.28987 \\ \mathrm{C} & -4.79894 & 2.08080 & 2.32714 \\ \mathrm{H} & -4.72993 & 2.59684 & 3.27956 \\ \mathrm{H} & -3.01182 & 3.92111 & 3.35458 \\ \mathrm{C} & -2.44049 & 3.90403 & 2.43227 \\ \mathrm{C} & -1.09099 & 4.09905 & 2.48021 \\ \mathrm{H} & -0.61449 & 4.26692 & 3.44076 \\ \mathrm{H} & 1.48576 & 3.66145 & 3.49394 \\ \mathrm{C} & 1.76824 & 3.16765 & 2.57094 \\ \mathrm{C} & 2.67650 & 2.13303 & 2.62022 \\ \mathrm{H} & 3.07697 & 1.84664 & 3.58839 \\ \mathrm{H} & 3.24440 & -0.38212 & 3.69078 \\ \mathrm{C} & 3.04613 & -0.84447 & 2.72879 \\ \mathrm{C} & 2.49091 & -2.09204 & 2.68694 \\ \mathrm{H} & 2.27295 & -2.59918 & 3.62115 \\ \mathrm{H} & 0.65042 & -3.99934 & 3.48000 \\ \mathrm{C} & 0.16672 & -3.95480 & 2.50935 \\ \mathrm{C} & -1.17817 & -4.17668 & 2.42458 \\ \mathrm{H} & -1.73743 & -4.38864 & 3.33018 \\ \mathrm{H} & -3.76561 & -3.66125 & 3.23267 \\ \mathrm{C} & -3.97565 & -3.16821 & 2.28890 \\ \mathrm{C} & -4.85568 & -2.12594 & 2.26336 \\ \mathrm{C} & -5.04698 & -1.35898 & 1.06187 \\ \mathrm{C} & -5.27355 & 0.04748 & 1.07386 \\ \mathrm{C} & -5.11998 & 0.76922 & -0.14669 \\ \mathrm{C} & -4.58450 & 2.10461 & -0.10857 \\ \mathrm{C} & -4.21769 & 2.67107 & 1.15073 \\ \mathrm{C} & -3.08561 & 3.53210 & 1.20063 \\ \mathrm{C} & -2.37602 & 3.79139 & -0.01247 \\ \mathrm{C} & -0.95043 & 3.97705 & 0.03473 \\ \mathrm{C} & -0.28127 & 3.94263 & 1.30382 \\ \mathrm{C} & 1.05419 & 3.46756 & 1.36616 \\ \mathrm{C} & 1.64214 & 3.00977 & 0.12850 \\ \mathrm{C} & 2.65270 & 1.88542 & 0.19026 \\ \mathrm{C} & 3.00561 & 1.34950 & 1.48182 \\ \mathrm{C} & 3.22209 & -0.08120 & 1.52050 \\ \mathrm{C} & 2.56408 & -2.09631 & 0.24894 \\ \mathrm{C} & 0.32751 & -3.74882 & 0.08164\end{array}$




\begin{tabular}{|c|c|c|c|}
\hline $\mathrm{C}$ & -1.08864 & -3.98188 & -0.00915 \\
\hline $\mathrm{C}$ & -1.87407 & -3.99197 & 1.18300 \\
\hline C & -3.21672 & -3.51490 & 1.11704 \\
\hline $\mathrm{C}$ & -3.71475 & -3.05981 & -0.13766 \\
\hline $\mathrm{C}$ & -4.65434 & -1.96972 & -0.16711 \\
\hline $\mathrm{C}$ & -4.92722 & -1.32005 & -1.40713 \\
\hline $\mathrm{C}$ & -5.17674 & 0.08192 & -1.39673 \\
\hline $\mathrm{C}$ & -5.14109 & 0.88641 & -2.58882 \\
\hline $\mathrm{C}$ & -4.64444 & 2.15642 & -2.55167 \\
\hline $\mathrm{C}$ & -4.14560 & 2.70999 & -1.32150 \\
\hline $\mathrm{C}$ & -3.01035 & 3.57226 & -1.27379 \\
\hline $\mathrm{C}$ & -2.28952 & 3.96664 & -2.45028 \\
\hline $\mathrm{C}$ & -0.93401 & 4.13288 & -2.40596 \\
\hline $\mathrm{C}$ & -0.21237 & 3.94129 & -1.18379 \\
\hline $\mathrm{C}$ & 1.10879 & 3.39084 & -1.15918 \\
\hline C & 1.80153 & 2.93814 & -2.31050 \\
\hline $\mathrm{C}$ & 2.71168 & 1.89568 & -2.24420 \\
\hline $\mathrm{C}$ & 3.05617 & 1.26084 & -1.03591 \\
\hline C & 3.59627 & -0.10272 & -0.93734 \\
\hline C & 3.57940 & -0.93554 & -2.14765 \\
\hline C & 2.92012 & -2.11830 & -2.18558 \\
\hline $\mathrm{C}$ & 2.21843 & -2.64024 & -1.02504 \\
\hline $\mathrm{C}$ & 1.07206 & -3.46348 & -1.11146 \\
\hline C & 0.45643 & -3.81481 & -2.36574 \\
\hline $\mathrm{C}$ & -0.88621 & -4.03182 & -2.44862 \\
\hline $\mathrm{C}$ & -1.72735 & -3.91705 & -1.28477 \\
\hline C & -3.07010 & -3.45401 & -1.35043 \\
\hline C & -3.70293 & -3.06521 & -2.58356 \\
\hline C & -4.60151 & -2.03996 & -2.61073 \\
\hline $\mathrm{H}$ & -4.98179 & -1.69221 & -3.56599 \\
\hline $\mathrm{H}$ & -5.40266 & 0.44522 & -3.54543 \\
\hline $\mathrm{H}$ & -4.51994 & 2.70496 & -3.47991 \\
\hline $\mathrm{H}$ & -2.79951 & 4.01494 & -3.40668 \\
\hline $\mathrm{H}$ & -0.39212 & 4.31656 & -3.32847 \\
\hline $\mathrm{H}$ & 1.50054 & 3.29740 & -3.28782 \\
\hline $\mathrm{H}$ & 3.09418 & 1.48947 & -3.17470 \\
\hline $\mathrm{H}$ & 2.88488 & -2.67496 & -3.11709 \\
\hline $\mathrm{H}$ & 1.04883 & -3.80521 & -3.27485 \\
\hline $\mathrm{H}$ & -1.33761 & -4.19079 & -3.42271 \\
\hline $\mathrm{H}$ & -3.38590 & -3.51742 & -3.51790 \\
\hline $\mathrm{Pd}$ & 5.46100 & -0.16240 & 0.06064 \\
\hline $\mathrm{C}$ & 8.35957 & 0.34161 & -0.52918 \\
\hline $\mathrm{C}$ & 6.99333 & 0.45199 & -1.21127 \\
\hline $\mathrm{H}$ & 6.94965 & -0.18064 & -2.10691 \\
\hline $\mathrm{H}$ & 6.78584 & 1.48570 & -1.51631 \\
\hline $\mathrm{H}$ & 4.06734 & -0.55872 & -3.04146 \\
\hline $\mathrm{H}$ & 2.91464 & 3.18281 & 0.13968 \\
\hline $\mathrm{H}$ & 8.58044 & -0.68695 & -0.21572 \\
\hline $\mathrm{H}$ & 8.42717 & 0.97846 & 0.36217 \\
\hline
\end{tabular}


H $\quad 9.17897 \quad 0.64925 \quad-1.20180$

(8) Transition state (TS89)

\begin{tabular}{|c|c|c|c|}
\hline $\mathrm{H}$ & -0.76300 & -4.96834 & -3.97552 \\
\hline $\mathrm{H}$ & 0.95598 & -5.74505 & -2.84328 \\
\hline C & 0.89574 & -5.46139 & -1.79718 \\
\hline $\mathrm{C}$ & 2.04140 & -5.18864 & -1.11059 \\
\hline $\mathrm{H}$ & 2.99658 & -5.26508 & -1.62024 \\
\hline $\mathrm{H}$ & 4.37254 & -3.78069 & -0.90743 \\
\hline $\mathrm{C}$ & 3.99268 & -3.19317 & -0.07792 \\
\hline $\mathrm{C}$ & 4.45433 & -1.92162 & 0.10030 \\
\hline $\mathrm{H}$ & 5.19173 & -1.52581 & -0.59056 \\
\hline $\mathrm{H}$ & 5.36549 & 0.63237 & -0.61709 \\
\hline $\mathrm{C}$ & 4.50713 & 1.07222 & -0.11819 \\
\hline $\mathrm{C}$ & 3.97395 & 2.22407 & -0.59162 \\
\hline $\mathrm{H}$ & 4.38333 & 2.68537 & -1.48652 \\
\hline $\mathrm{H}$ & 2.56063 & 3.23200 & -2.71892 \\
\hline $\mathrm{C}$ & 1.63412 & 3.09477 & -2.17008 \\
\hline C & 0.49633 & 2.77833 & -2.83059 \\
\hline $\mathrm{H}$ & 0.51807 & 2.68849 & -3.91160 \\
\hline $\mathrm{H}$ & -0.90444 & 1.33804 & -4.66409 \\
\hline $\mathrm{C}$ & -1.53588 & 0.89666 & -3.90029 \\
\hline $\mathrm{C}$ & -2.08056 & -0.33378 & -4.12167 \\
\hline $\mathrm{H}$ & -1.86522 & -0.84823 & -5.05272 \\
\hline $\mathrm{H}$ & -1.88612 & -3.00167 & -4.85295 \\
\hline $\mathrm{C}$ & -2.04854 & -3.29093 & -3.81946 \\
\hline $\mathrm{C}$ & -1.41983 & -4.39592 & -3.32799 \\
\hline $\mathrm{C}$ & -1.49737 & -4.71306 & -1.92500 \\
\hline $\mathrm{C}$ & -0.38993 & -5.22451 & -1.19283 \\
\hline $\mathrm{C}$ & -0.43483 & -5.16028 & 0.23109 \\
\hline $\mathrm{C}$ & 0.77488 & -4.87232 & 0.95589 \\
\hline $\mathrm{C}$ & 1.98464 & -4.66405 & 0.22949 \\
\hline $\mathrm{C}$ & 2.91814 & -3.70936 & 0.72454 \\
\hline $\mathrm{C}$ & 2.60875 & -3.01364 & 1.93307 \\
\hline $\mathrm{C}$ & 3.09232 & -1.67365 & 2.11928 \\
\hline $\mathrm{C}$ & 3.88532 & -1.06233 & 1.10100 \\
\hline $\mathrm{C}$ & 3.88069 & 0.35586 & 0.99350 \\
\hline $\mathrm{C}$ & 3.08661 & 1.10222 & 1.88100 \\
\hline $\mathrm{C}$ & 2.70912 & 2.55310 & 1.52626 \\
\hline $\mathrm{C}$ & 2.75808 & 2.74276 & 0.02558 \\
\hline $\mathrm{C}$ & 1.65684 & 3.12095 & -0.70728 \\
\hline $\mathrm{C}$ & 0.35820 & 3.41596 & -0.05945 \\
\hline $\mathrm{C}$ & -0.83201 & 2.89741 & -0.76414 \\
\hline $\mathrm{C}$ & -0.72575 & 2.46586 & -2.11136 \\
\hline $\mathrm{C}$ & -1.68807 & 1.55522 & -2.63088 \\
\hline $\mathrm{C}$ & -2.71968 & 1.07944 & -1.76940 \\
\hline
\end{tabular}




$\begin{array}{lrrr}\mathrm{C} & -3.28920 & -0.21933 & -1.99861 \\ \mathrm{C} & -2.81197 & -1.01126 & -3.08661 \\ \mathrm{C} & -2.79812 & -2.42743 & -2.94305 \\ \mathrm{C} & -3.24943 & -2.98751 & -1.71199 \\ \mathrm{C} & -2.58861 & -4.15662 & -1.19417 \\ \mathrm{C} & -2.79298 & -4.51826 & 0.17023 \\ \mathrm{C} & -1.68812 & -5.03633 & 0.90109 \\ \mathrm{C} & -1.66996 & -5.09824 & 2.33990 \\ \mathrm{C} & -0.52330 & -4.83187 & 3.02696 \\ \mathrm{C} & 0.68607 & -4.48526 & 2.32555 \\ \mathrm{C} & 1.61870 & -3.53168 & 2.82307 \\ \mathrm{C} & 1.42496 & -2.84250 & 4.06877 \\ \mathrm{C} & 1.88303 & -1.56973 & 4.24482 \\ \mathrm{C} & 2.58305 & -0.88401 & 3.19422 \\ \mathrm{C} & 2.58276 & 0.53285 & 3.06270 \\ \mathrm{C} & 1.88576 & 1.41383 & 4.00088 \\ \mathrm{C} & 1.28676 & 2.54786 & 3.56392 \\ \mathrm{C} & 1.37849 & 2.90558 & 2.15507 \\ \mathrm{C} & 0.30512 & 3.33128 & 1.41759 \\ \mathrm{C} & -1.01528 & 3.54143 & 2.00238 \\ \mathrm{C} & -2.12885 & 3.24044 & 1.29749 \\ \mathrm{C} & -2.03631 & 2.68511 & -0.04431 \\ \mathrm{C} & -2.99391 & 1.76488 & -0.54663 \\ \mathrm{C} & -4.11229 & 1.29829 & 0.23005 \\ \mathrm{C} & -4.64746 & 0.06268 & 0.01653 \\ \mathrm{C} & -4.10782 & -0.81198 & -0.98964 \\ \mathrm{C} & -4.09026 & -2.22736 & -0.84534 \\ \mathrm{C} & -4.60133 & -2.89806 & 0.32314 \\ \mathrm{C} & -3.97606 & -4.00554 & 0.81326 \\ \mathrm{H} & -4.30232 & -4.42740 & 1.75873 \\ \mathrm{H} & -2.59749 & -5.24219 & 2.88528 \\ \mathrm{H} & -0.55492 & -4.77321 & 4.11022 \\ \mathrm{H} & 0.81480 & -3.29558 & 4.84335 \\ \mathrm{H} & 1.62930 & -1.03829 & 5.15612 \\ \mathrm{H} & 1.77047 & 1.10617 & 5.03546 \\ \mathrm{H} & 0.66372 & 3.12578 & 4.24194 \\ \mathrm{H} & -3.10223 & 3.33964 & 1.76715 \\ \mathrm{H} & -4.47589 & 1.89327 & 1.06113 \\ \mathrm{H} & -5.42127 & -0.30142 & 0.68486 \\ \mathrm{H} & -5.41901 & -2.45670 & 0.88415 \\ \mathrm{Pd} & 0.86682 & 5.46196 & -0.33892 \\ \mathrm{C} & -1.73754 & 6.19258 & -1.80008 \\ \mathrm{C} & -0.85633 & 6.54470 & -0.59833 \\ \mathrm{H} & -1.39208 & 6.47987 & 0.35286 \\ \mathrm{H} & -0.40705 & 7.54792 & -0.68653 \\ \mathrm{H} & -1.09678 & 3.86336 & 3.03622 \\ \mathrm{H} & 3.48043 & 3.21352 & 1.97537 \\ \mathrm{H} & -1.34833 & 5.35255 & -2.38685 \\ \mathrm{H} & -1.83072 & 7.04294 & -2.48794 \\ & & & \end{array}$


H $\quad-2.74863 \quad 5.90987 \quad-1.48374$

\section{Coordinates of structures of acetylene, ethylene and ethane over all different active sites of pure and impure fullerene.}

For pure fullerene

(1) $\mathrm{C}_{2} \mathrm{H}_{2} \_\mathrm{C}_{60} \_\mathrm{T}$

\begin{tabular}{|c|c|c|c|}
\hline $\mathrm{C}$ & -3.00042 & 1.57067 & 0.01352 \\
\hline C & -2.37231 & 2.10193 & 1.18042 \\
\hline & -1.37388 & 3.08274 & 0.76352 \\
\hline & -1.36945 & 3.10422 & -0.68309 \\
\hline & -2.36487 & 2.13608 & -1.13416 \\
\hline & -2.11348 & 1.36430 & -2.27809 \\
\hline & -0.87171 & 1.52441 & -2.99253 \\
\hline & 0.08711 & 2.45351 & -2.55888 \\
\hline & -0.17191 & 3.25780 & -1.37622 \\
\hline & 1.07620 & 3.40169 & -0.64595 \\
\hline 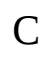 & 1.07193 & 3.38103 & 0.74968 \\
\hline & -0.18057 & 3.21558 & 1.46784 \\
\hline & 0.07156 & 2.37696 & 2.62797 \\
\hline 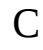 & -0.88970 & 1.43595 & 3.02923 \\
\hline & -2.12736 & 1.29661 & 2.30321 \\
\hline $\bar{T}$ & -2.48539 & -0.13254 & 2.28123 \\
\hline C & -1.45081 & -0.84967 & 2.99648 \\
\hline C & -0.46611 & 0.11180 & 3.45828 \\
\hline $\mathrm{C}$ & 0.88756 & -0.22211 & 3.47124 \\
\hline C & 1.87859 & 0.75147 & 3.04436 \\
\hline $\mathrm{C}$ & 1.47445 & 2.02602 & 2.63117 \\
\hline C & 2.09708 & 2.64335 & 1.46940 \\
\hline $\mathrm{C}$ & 3.08739 & 1.95995 & 0.76436 \\
\hline C & 3.09159 & 1.98108 & -0.69003 \\
\hline C & 2.10563 & 2.68503 & -1.38076 \\
\hline C & 1.49011 & 2.10228 & -2.56394 \\
\hline C & 1.89656 & 0.84013 & -3.01162 \\
\hline C & 0.90791 & -0.12049 & -3.47217 \\
\hline C & -0.44574 & 0.21317 & -3.45707 \\
\hline C & -1.43309 & -0.76123 & -3.02882 \\
\hline C & -1.02547 & -2.03869 & -2.63051 \\
\hline C & 0.38516 & -2.38437 & -2.63444 \\
\hline C & 1.33320 & -1.44591 & -3.04725 \\
\hline C & 2.57690 & -1.30111 & -2.31621 \\
\hline C & 2.92513 & 0.11237 & -2.29422 \\
\hline C & 3.51150 & 0.66881 & -1.1555 \\
\hline C & 3.76678 & -0.16059 & 0.0080 \\
\hline & 3.50466 & 0.63455 & 1.1938 \\
\hline
\end{tabular}




$\begin{array}{lrrr}\mathrm{C} & 2.91163 & 0.04513 & 2.31233 \\ \mathrm{C} & 2.56346 & -1.36844 & 2.29105 \\ \mathrm{C} & 1.31552 & -1.53449 & 3.01035 \\ \mathrm{C} & 0.37011 & -2.46067 & 2.56519 \\ \mathrm{C} & -1.04067 & -2.11487 & 2.56318 \\ \mathrm{C} & -1.64128 & -2.68733 & 1.38313 \\ \mathrm{C} & -0.60804 & -3.39615 & 0.64587 \\ \mathrm{C} & 0.63829 & -3.25746 & 1.37860 \\ \mathrm{C} & 1.84075 & -3.09819 & 0.68682 \\ \mathrm{C} & 2.82315 & -2.13500 & 1.15172 \\ \mathrm{C} & 3.43382 & -1.51858 & -0.01272 \\ \mathrm{C} & 2.83005 & -2.10073 & -1.19810 \\ \mathrm{C} & 1.84502 & -3.07695 & -0.76719 \\ \mathrm{C} & 0.64654 & -3.21557 & -1.47027 \\ \mathrm{C} & -0.60397 & -3.37566 & -0.74923 \\ \mathrm{C} & -1.63304 & -2.64542 & -1.47144 \\ \mathrm{C} & -2.61997 & -1.94383 & -0.76420 \\ \mathrm{C} & -2.62402 & -1.96527 & 0.69100 \\ \mathrm{C} & -3.08439 & -0.69513 & 1.16910 \\ \mathrm{C} & -3.62763 & 0.15895 & -0.00843 \\ \mathrm{C} & -3.07713 & -0.65986 & -1.20744 \\ \mathrm{C} & -2.47174 & -0.06539 & -2.29925 \\ \mathrm{C} & -6.08616 & -0.48419 & 0.00361 \\ \mathrm{C} & -5.09858 & 0.39021 & -0.00626 \\ \mathrm{H} & -7.16721 & -0.35631 & 0.01150 \\ \mathrm{H} & -5.35137 & 1.48513 & -0.01010\end{array}$

(2) $\mathrm{C}_{2} \mathrm{H}_{2} \_\mathrm{C}_{60} \_\mathrm{PH}$

$\begin{array}{lrrr}\mathrm{C} & 3.55332 & -0.81670 & -0.00014 \\ \mathrm{C} & 2.83994 & -1.46055 & 1.17964 \\ \mathrm{C} & 2.08105 & -2.59721 & 0.72662 \\ \mathrm{C} & 2.08101 & -2.59715 & -0.72694 \\ \mathrm{C} & 2.83986 & -1.46045 & -1.17990 \\ \mathrm{C} & 2.39928 & -0.74195 & -2.27558 \\ \mathrm{C} & 1.22066 & -1.17581 & -2.99672 \\ \mathrm{C} & 0.51375 & -2.31407 & -2.59101 \\ \mathrm{C} & 0.95327 & -3.04170 & -1.42481 \\ \mathrm{C} & -0.22225 & -3.49322 & -0.69742 \\ \mathrm{C} & -0.22221 & -3.49328 & 0.69715 \\ \mathrm{C} & 0.95335 & -3.04183 & 1.42451 \\ \mathrm{C} & 0.51389 & -2.31428 & 2.59079 \\ \mathrm{C} & 1.22083 & -1.17606 & 2.99656 \\ \mathrm{C} & 2.39941 & -0.74214 & 2.27539 \\ \mathrm{C} & 2.39940 & 0.74194 & 2.27544 \\ \mathrm{C} & 1.22083 & 1.17581 & 2.99666 \\ \mathrm{C} & 0.49547 & -0.00014 & 3.44226 \\ \mathrm{C} & -0.89942 & -0.00014 & 3.46448\end{array}$




$\begin{array}{lrrr}\mathrm{C} & -1.63178 & -1.17722 & 3.02519 \\ \mathrm{C} & -0.93818 & -2.31114 & 2.59798 \\ \mathrm{C} & -1.39457 & -3.04057 & 1.42522 \\ \mathrm{C} & -2.52291 & -2.60482 & 0.72710 \\ \mathrm{C} & -2.52295 & -2.60476 & -0.72718 \\ \mathrm{C} & -1.39465 & -3.04045 & -1.42539 \\ \mathrm{C} & -0.93832 & -2.31092 & -2.59812 \\ \mathrm{C} & -1.63194 & -1.17697 & -3.02521 \\ \mathrm{C} & -0.89960 & 0.00014 & -3.46444 \\ \mathrm{C} & 0.49529 & 0.00014 & -3.44229 \\ \mathrm{C} & 1.22067 & 1.17606 & -2.99663 \\ \mathrm{C} & 0.51375 & 2.31428 & -2.59082 \\ \mathrm{C} & -0.93832 & 2.31114 & -2.59793 \\ \mathrm{C} & -1.63194 & 1.17722 & -3.02511 \\ \mathrm{C} & -2.80697 & 0.72797 & -2.30336 \\ \mathrm{C} & -2.80697 & -0.72778 & -2.30342 \\ \mathrm{C} & -3.24372 & -1.42613 & -1.17545 \\ \mathrm{C} & -3.69127 & -0.69798 & 0.00007 \\ \mathrm{C} & -3.24366 & -1.42622 & 1.17551 \\ \mathrm{C} & -2.80684 & -0.72797 & 2.30351 \\ \mathrm{C} & -2.80684 & 0.72778 & 2.30357 \\ \mathrm{C} & -1.63178 & 1.17697 & 3.02529 \\ \mathrm{C} & -0.93818 & 2.31092 & 2.59817 \\ \mathrm{C} & 0.51389 & 2.31407 & 2.59098 \\ \mathrm{C} & 0.95335 & 3.04170 & 1.42476 \\ \mathrm{C} & -0.22221 & 3.49322 & 0.69743 \\ \mathrm{C} & -1.39457 & 3.04045 & 1.42547 \\ \mathrm{C} & -2.52291 & 2.60476 & 0.72732 \\ \mathrm{C} & -3.24366 & 1.42613 & 1.17562 \\ \mathrm{C} & -3.69127 & 0.69798 & 0.00013 \\ \mathrm{C} & -3.24372 & 1.42622 & -1.17534 \\ \mathrm{C} & -2.52295 & 2.60482 & -0.72697 \\ \mathrm{C} & -1.39465 & 3.04057 & -1.42515 \\ \mathrm{C} & -0.22225 & 3.49328 & -0.69714 \\ \mathrm{C} & 0.95328 & 3.04183 & -1.42457 \\ \mathrm{C} & 2.08101 & 2.59721 & -0.72674 \\ \mathrm{C} & 2.08105 & 2.59715 & 0.72683 \\ \mathrm{C} & 2.83992 & 1.46044 & 1.17974 \\ \mathrm{C} & 3.55332 & 0.81670 & -0.00006 \\ \mathrm{C} & 2.83988 & 1.46055 & -1.17980 \\ \mathrm{C} & 2.39929 & 0.74214 & -2.27552 \\ \mathrm{C} & 5.07179 & 0.66836 & -0.00001 \\ \mathrm{C} & 5.07179 & -0.66836 & -0.00017 \\ \mathrm{H} & 5.84953 & 1.42585 & 0.00012 \\ \mathrm{H} & 5.84954 & -1.42583 & -0.00023 \\ & & & \end{array}$

(3) $\mathrm{C}_{2} \mathrm{H}_{2} \mathrm{C}_{60} \mathrm{HH}$ 


\begin{tabular}{|c|c|c|c|}
\hline $\mathrm{C}$ & 3.65582 & -1.18869 & -0.06805 \\
\hline $\mathrm{C}$ & 2.92757 & -1.27012 & -1.34768 \\
\hline $\mathrm{C}$ & 2.65460 & 0.00000 & -2.06820 \\
\hline $\mathrm{C}$ & 2.92757 & 1.27012 & -1.34768 \\
\hline $\mathrm{C}$ & 3.65582 & 1.18869 & -0.06805 \\
\hline C & 2.92290 & 1.56321 & 1.07584 \\
\hline $\mathrm{C}$ & 1.95448 & 2.61871 & 0.93611 \\
\hline $\mathrm{C}$ & 1.58116 & 3.08333 & -0.31807 \\
\hline $\mathrm{C}$ & 2.01688 & 2.32541 & -1.48171 \\
\hline $\mathrm{C}$ & 0.91803 & 2.30329 & -2.40817 \\
\hline $\mathrm{C}$ & 0.70311 & 1.16114 & -3.16311 \\
\hline $\mathrm{C}$ & 1.56009 & 0.00000 & -2.97692 \\
\hline $\mathrm{C}$ & 0.70311 & -1.16114 & -3.16311 \\
\hline $\mathrm{C}$ & 0.91803 & -2.30329 & -2.40817 \\
\hline $\mathrm{C}$ & 2.01688 & -2.32541 & -1.48171 \\
\hline $\mathrm{C}$ & 1.58116 & -3.08333 & -0.31806 \\
\hline $\mathrm{C}$ & 0.21330 & -3.51420 & -0.53071 \\
\hline $\mathrm{C}$ & -0.19686 & -3.04467 & -1.84014 \\
\hline $\mathrm{C}$ & -1.50154 & -2.60894 & -2.05383 \\
\hline $\mathrm{C}$ & -1.73920 & -1.42340 & -2.86186 \\
\hline $\mathrm{C}$ & -0.66224 & -0.72248 & -3.41068 \\
\hline $\mathrm{C}$ & -0.66224 & 0.72248 & -3.41068 \\
\hline $\mathrm{C}$ & -1.73920 & 1.42339 & -2.86186 \\
\hline $\mathrm{C}$ & -1.50154 & 2.60894 & -2.05383 \\
\hline $\mathrm{C}$ & -0.19686 & 3.04467 & -1.84014 \\
\hline $\mathrm{C}$ & 0.21330 & 3.51420 & -0.53071 \\
\hline $\mathrm{C}$ & -0.69733 & 3.50201 & 0.53011 \\
\hline $\mathrm{C}$ & -0.27801 & 3.04000 & 1.84383 \\
\hline $\mathrm{C}$ & 1.02789 & 2.58836 & 2.03097 \\
\hline $\mathrm{C}$ & 1.33214 & 1.40305 & 2.80476 \\
\hline $\mathrm{C}$ & 0.26899 & 0.72249 & 3.39170 \\
\hline $\mathrm{C}$ & -1.10323 & 1.17487 & 3.20480 \\
\hline $\mathrm{C}$ & -1.37639 & 2.31008 & 2.44147 \\
\hline $\mathrm{C}$ & -2.48102 & 2.30979 & 1.49646 \\
\hline $\mathrm{C}$ & -2.05784 & 3.04245 & 0.31229 \\
\hline $\mathrm{C}$ & -2.45412 & 2.60834 & -0.95562 \\
\hline $\mathrm{C}$ & -3.28742 & 1.42554 & -1.09069 \\
\hline $\mathrm{C}$ & -2.85060 & 0.69809 & -2.27222 \\
\hline $\mathrm{C}$ & -2.85060 & -0.69809 & -2.27222 \\
\hline $\mathrm{C}$ & -3.28742 & -1.42554 & -1.09069 \\
\hline $\mathrm{C}$ & -2.45412 & -2.60834 & -0.95562 \\
\hline $\mathrm{C}$ & -2.05784 & -3.04245 & 0.31229 \\
\hline $\mathrm{C}$ & -0.69733 & -3.50201 & 0.53011 \\
\hline $\mathrm{C}$ & -0.27801 & -3.04000 & 1.84383 \\
\hline $\mathrm{C}$ & -1.37639 & -2.31008 & 2.44147 \\
\hline $\mathrm{C}$ & -2.48102 & -2.30979 & 1.49646 \\
\hline $\mathrm{C}$ & -3.28231 & -1.17563 & 1.36535 \\
\hline $\mathrm{C}$ & -3.69737 & -0.72639 & 0.04630 \\
\hline $\mathrm{C}$ & -3.69737 & 0.72639 & 0.04630 \\
\hline
\end{tabular}




$\begin{array}{lrrr}\mathrm{C} & -3.28231 & 1.17563 & 1.36535 \\ \mathrm{C} & -3.01657 & 0.00000 & 2.17839 \\ \mathrm{C} & -1.94728 & 0.00000 & 3.07860 \\ \mathrm{C} & -1.10323 & -1.17487 & 3.20480 \\ \mathrm{C} & 0.26899 & -0.72249 & 3.39171 \\ \mathrm{C} & 1.33214 & -1.40305 & 2.80476 \\ \mathrm{C} & 1.02789 & -2.58836 & 2.03097 \\ \mathrm{C} & 1.95448 & -2.61871 & 0.93611 \\ \mathrm{C} & 2.92290 & -1.56321 & 1.07584 \\ \mathrm{C} & 2.49370 & -0.71188 & 2.20294 \\ \mathrm{C} & 2.49370 & 0.71188 & 2.20294 \\ \mathrm{C} & 5.04128 & 0.65983 & 0.00539 \\ \mathrm{C} & 5.04128 & -0.65983 & 0.00539 \\ \mathrm{H} & 5.89694 & 1.33250 & 0.04983 \\ \mathrm{H} & 5.89694 & -1.33250 & 0.04983\end{array}$

(4) $\mathrm{C}_{2} \mathrm{H}_{4} \_\mathrm{C}_{60} \_\mathrm{T}$

$\begin{array}{lrrr}\mathrm{C} & 2.85965 & -1.07528 & 1.15229 \\ \mathrm{C} & 3.16014 & -0.66333 & -0.20906 \\ \mathrm{C} & 3.13121 & 0.78912 & -0.25542 \\ \mathrm{C} & 2.81313 & 1.27483 & 1.07688 \\ \mathrm{C} & 2.64483 & 0.12276 & 1.94693 \\ \mathrm{C} & 1.66437 & 0.13509 & 2.93999 \\ \mathrm{C} & 0.81106 & 1.30019 & 3.10453 \\ \mathrm{C} & 0.97260 & 2.40673 & 2.26936 \\ \mathrm{C} & 1.99423 & 2.39392 & 1.23502 \\ \mathrm{C} & 1.45829 & 3.07246 & 0.06655 \\ \mathrm{C} & 1.76425 & 2.60665 & -1.21298 \\ \mathrm{C} & 2.61730 & 1.44121 & -1.37687 \\ \mathrm{C} & 2.11085 & 0.66799 & -2.49876 \\ \mathrm{C} & 2.13868 & -0.72662 & -2.45449 \\ \mathrm{C} & 2.67399 & -1.40611 & -1.28615 \\ \mathrm{C} & 1.86722 & -2.59141 & -1.04672 \\ \mathrm{C} & 0.83310 & -2.64437 & -2.06715 \\ \mathrm{C} & 1.00097 & -1.49181 & -2.93684 \\ \mathrm{C} & -0.11898 & -0.83193 & -3.44514 \\ \mathrm{C} & -0.14769 & 0.62051 & -3.49178 \\ \mathrm{C} & 0.94462 & 1.35535 & -3.02787 \\ \mathrm{C} & 0.73026 & 2.55366 & -2.23353 \\ \mathrm{C} & -0.56817 & 2.96862 & -1.93352 \\ \mathrm{C} & -0.88646 & 3.45461 & -0.60116 \\ \mathrm{C} & 0.10628 & 3.50456 & 0.37853 \\ \mathrm{C} & -0.19405 & 3.09319 & 1.73966 \\ \mathrm{C} & -1.47546 & 2.64772 & 2.06747 \\ \mathrm{C} & -1.64331 & 1.49515 & 2.93714 \\ \mathrm{C} & -0.52338 & 0.83522 & 3.44527\end{array}$




$\begin{array}{lrrr}\mathrm{C} & -0.49469 & -0.61731 & 3.49197 \\ \mathrm{C} & -1.58702 & -1.35224 & 3.02832 \\ \mathrm{C} & -2.75331 & -0.66481 & 2.49922 \\ \mathrm{C} & -2.78094 & 0.72985 & 2.45459 \\ \mathrm{C} & -3.31602 & 1.40930 & 1.28634 \\ \mathrm{C} & -2.50958 & 2.59477 & 1.04702 \\ \mathrm{C} & -2.22065 & 2.98956 & -0.26022 \\ \mathrm{C} & -2.72707 & 2.21628 & -1.38197 \\ \mathrm{C} & -1.70575 & 2.20336 & -2.41626 \\ \mathrm{C} & -1.49985 & 1.05324 & -3.17951 \\ \mathrm{C} & -2.30672 & -0.13179 & -2.93983 \\ \mathrm{C} & -1.45339 & -1.29690 & -3.10425 \\ \mathrm{C} & -1.61496 & -2.40354 & -2.26918 \\ \mathrm{C} & -0.44831 & -3.08994 & -1.73938 \\ \mathrm{C} & -0.74861 & -3.50133 & -0.37828 \\ \mathrm{C} & -2.10058 & -3.06907 & -0.06624 \\ \mathrm{C} & -2.63651 & -2.39069 & -1.23482 \\ \mathrm{C} & -3.45552 & -1.27159 & -1.07689 \\ \mathrm{C} & -3.28732 & -0.11940 & -1.94687 \\ \mathrm{C} & -3.50196 & 1.07855 & -1.15209 \\ \mathrm{C} & -3.80238 & 0.66677 & 0.20921 \\ \mathrm{C} & -3.77342 & -0.78571 & 0.25570 \\ \mathrm{C} & -3.25957 & -1.43802 & 1.37744 \\ \mathrm{C} & -2.40661 & -2.60350 & 1.21339 \\ \mathrm{C} & -1.37265 & -2.55055 & 2.23400 \\ \mathrm{C} & -0.07419 & -2.96535 & 1.93386 \\ \mathrm{C} & 0.24410 & -3.45127 & 0.60141 \\ \mathrm{C} & 1.57833 & -2.98637 & 0.26051 \\ \mathrm{C} & 2.08472 & -2.21299 & 1.38222 \\ \mathrm{C} & 1.06339 & -2.20017 & 2.41659 \\ \mathrm{C} & 0.85745 & -1.04997 & 3.17968 \\ \mathrm{C} & 7.51239 & 0.56437 & 0.01009 \\ \mathrm{C} & 6.94010 & -0.63766 & -0.01600 \\ \mathrm{H} & 6.78200 & -1.21795 & 0.89003 \\ \mathrm{H} & 6.60508 & -1.09425 & -0.94418 \\ \mathrm{H} & 7.84742 & 1.02066 & 0.93863 \\ \mathrm{H} & 7.67105 & 1.14427 & -0.89611\end{array}$

(5) $\mathrm{C}_{2} \mathrm{H}_{4} \_\mathrm{C}_{60} \_\mathrm{PH}$
$\begin{array}{llll}\text { C } & -3.54518 & -0.82037 & 0.01093\end{array}$
$\begin{array}{llll}\text { C } & -2.84785 & -1.18125 & 1.31967\end{array}$
$\begin{array}{llll}\text { C } & -2.57250 & -0.00006 & 2.09594\end{array}$
$\begin{array}{llll}\text { C } & -2.84785 & 1.18118 & 1.31974\end{array}$
$\begin{array}{llll}\text { C } & -3.54517 & 0.82037 & 0.01097\end{array}$
$\begin{array}{llll}\text { C } & -2.83207 & 1.45173 & -1.14703\end{array}$
C $\quad-1.95686 \quad 2.61219 \quad-0.98894$
$\begin{array}{llll}\text { C } & -1.57844 & 3.04177 & 0.27228\end{array}$ 


\begin{tabular}{|c|c|c|c|}
\hline C & -2.02608 & 2.28631 & 1.45677 \\
\hline $\mathrm{C}$ & -0.93570 & 2.28637 & 2.40415 \\
\hline $\mathrm{C}$ & -0.69761 & 1.16825 & 3.20706 \\
\hline $\mathrm{C}$ & -1.53004 & -0.00009 & 3.04793 \\
\hline $\mathrm{C}$ & -0.69761 & -1.16843 & 3.20700 \\
\hline $\mathrm{C}$ & -0.93570 & -2.28650 & 2.40402 \\
\hline $\mathrm{C}$ & -2.02608 & -2.28640 & 1.45665 \\
\hline $\mathrm{C}$ & -1.57844 & -3.04179 & 0.27211 \\
\hline $\mathrm{C}$ & -0.21701 & -3.48385 & 0.51206 \\
\hline $\mathrm{C}$ & 0.17891 & -3.02374 & 1.83042 \\
\hline $\mathrm{C}$ & 1.48699 & -2.60120 & 2.06579 \\
\hline C & 1.73440 & -1.42522 & 2.88664 \\
\hline $\mathrm{C}$ & 0.66421 & -0.72738 & 3.44906 \\
\hline $\mathrm{C}$ & 0.66421 & 0.72719 & 3.44910 \\
\hline $\mathrm{C}$ & 1.73440 & 1.42505 & 2.88672 \\
\hline $\mathrm{C}$ & 1.48700 & 2.60108 & 2.06593 \\
\hline $\mathrm{C}$ & 0.17891 & 3.02363 & 1.83059 \\
\hline $\mathrm{C}$ & -0.21701 & 3.48382 & 0.51226 \\
\hline $\mathrm{C}$ & 0.71645 & 3.49724 & -0.52927 \\
\hline $\mathrm{C}$ & 0.31911 & 3.05129 & -1.85059 \\
\hline $\mathrm{C}$ & -0.99267 & 2.61816 & -2.07077 \\
\hline $\mathrm{C}$ & -1.24682 & 1.43452 & -2.85723 \\
\hline $\mathrm{C}$ & -0.18741 & 0.73562 & -3.41165 \\
\hline $\mathrm{C}$ & 1.17460 & 1.17915 & -3.20185 \\
\hline $\mathrm{C}$ & 1.42667 & 2.31786 & -2.42842 \\
\hline $\mathrm{C}$ & 2.51796 & 2.31130 & -1.46796 \\
\hline $\mathrm{C}$ & 2.07676 & 3.04115 & -0.28819 \\
\hline $\mathrm{C}$ & 2.45533 & 2.60644 & 0.98095 \\
\hline $\mathrm{C}$ & 3.29287 & 1.42506 & 1.12856 \\
\hline $\mathrm{C}$ & 2.84777 & 0.70054 & 2.30336 \\
\hline $\mathrm{C}$ & 2.84776 & -0.70067 & 2.30332 \\
\hline $\mathrm{C}$ & 3.29287 & -1.42513 & 1.12848 \\
\hline $\mathrm{C}$ & 2.45532 & -2.60649 & 0.98080 \\
\hline $\mathrm{C}$ & 2.07675 & -3.04114 & -0.28835 \\
\hline $\mathrm{C}$ & 0.71644 & -3.49721 & -0.52946 \\
\hline $\mathrm{C}$ & 0.31911 & -3.05119 & -1.85076 \\
\hline $\mathrm{C}$ & 1.42666 & -2.31772 & -2.42855 \\
\hline C & 2.51796 & -2.31122 & -1.46809 \\
\hline C & 3.31695 & -1.17693 & -1.32751 \\
\hline $\mathrm{C}$ & 3.71698 & -0.72519 & -0.00365 \\
\hline $\mathrm{C}$ & 3.71698 & 0.72518 & -0.00361 \\
\hline $\mathrm{C}$ & 3.31695 & 1.17700 & -1.32744 \\
\hline $\mathrm{C}$ & 3.06506 & 0.00006 & -2.14356 \\
\hline $\mathrm{C}$ & 2.01344 & 0.00009 & -3.05999 \\
\hline $\mathrm{C}$ & 1.17460 & -1.17897 & -3.20191 \\
\hline $\mathrm{C}$ & -0.18741 & -0.73543 & -3.41169 \\
\hline C & -1.24682 & -1.43436 & -2.85731 \\
\hline $\mathrm{C}$ & -0.99268 & -2.61804 & -2.07091 \\
\hline $\mathrm{C}$ & -1.95687 & -2.61213 & -0.98909 \\
\hline
\end{tabular}




$\begin{array}{lrrr}\mathrm{C} & -2.83208 & -1.45167 & -1.14711 \\ \mathrm{C} & -2.38716 & -0.72418 & -2.24352 \\ \mathrm{C} & -2.38716 & 0.72431 & -2.24348 \\ \mathrm{C} & -5.11149 & 0.77614 & 0.01656 \\ \mathrm{C} & -5.11150 & -0.77614 & 0.01657 \\ \mathrm{H} & -5.54950 & -1.22463 & -0.87930 \\ \mathrm{H} & -5.55982 & -1.24782 & 0.89583 \\ \mathrm{H} & -5.54942 & 1.22459 & -0.87938 \\ \mathrm{H} & -5.55989 & 1.24787 & 0.89575\end{array}$

(6) $\mathrm{C}_{2} \mathrm{H}_{4} \_\mathrm{C}_{60} \_\mathrm{HH}$

\begin{tabular}{lrrr}
$\mathrm{C}$ & -2.80568 & 1.45195 & 1.17548 \\
$\mathrm{C}$ & -2.05086 & 2.59251 & 0.72612 \\
$\mathrm{C}$ & -2.05086 & 2.59247 & -0.72625 \\
$\mathrm{C}$ & -2.80569 & 1.45189 & -1.17556 \\
$\mathrm{C}$ & -3.52711 & 0.80655 & -0.00002 \\
$\mathrm{C}$ & -3.52711 & -0.80655 & 0.00002 \\
$\mathrm{C}$ & -2.80568 & -1.45195 & -1.17549 \\
$\mathrm{C}$ & -2.36729 & -0.74220 & -2.27548 \\
$\mathrm{C}$ & -2.36729 & 0.74208 & -2.27551 \\
$\mathrm{C}$ & -1.19023 & 1.17621 & -2.99824 \\
$\mathrm{C}$ & -0.48400 & 2.31462 & -2.59238 \\
$\mathrm{C}$ & -0.92463 & 3.04013 & -1.42523 \\
$\mathrm{C}$ & 0.25048 & 3.49231 & -0.69743 \\
$\mathrm{C}$ & 0.25048 & 3.49236 & 0.69725 \\
$\mathrm{C}$ & -0.92463 & 3.04020 & 1.42508 \\
$\mathrm{C}$ & -0.48400 & 2.31475 & 2.59227 \\
$\mathrm{C}$ & 0.96781 & 2.31133 & 2.59864 \\
$\mathrm{C}$ & 1.42340 & 3.04037 & 1.42523 \\
$\mathrm{C}$ & 2.55165 & 2.60466 & 0.72707 \\
$\mathrm{C}$ & 2.55165 & 2.60462 & -0.72720 \\
$\mathrm{C}$ & 1.42340 & 3.04030 & -1.42538 \\
$\mathrm{C}$ & 0.96781 & 2.31120 & -2.59875 \\
$\mathrm{C}$ & 1.66166 & 1.17719 & -3.02597 \\
$\mathrm{C}$ & 0.92971 & -0.00009 & -3.46577 \\
$\mathrm{C}$ & -0.46535 & -0.00009 & -3.44384 \\
$\mathrm{C}$ & -1.19023 & -1.17636 & -2.99818 \\
$\mathrm{C}$ & -0.48400 & -2.31475 & -2.59227 \\
$\mathrm{C}$ & -0.92463 & -3.04020 & -1.42508 \\
$\mathrm{C}$ & -2.05086 & -2.59251 & -0.72612 \\
$\mathrm{C}$ & -2.05086 & -2.59247 & 0.72625 \\
$\mathrm{C}$ & -0.92463 & -3.04013 & 1.42523 \\
$\mathrm{C}$ & 0.25048 & -3.49231 & 0.69743 \\
$\mathrm{C}$ & 0.25048 & -3.49235 & -0.69725 \\
$\mathrm{C}$ & 1.42340 & -3.04037 & -1.42523 \\
$\mathrm{C}$ & 0.96782 & -2.31133 & -2.59864 \\
& 1.66166 & -1.17734 & -3.02591 \\
\hline
\end{tabular}




$\begin{array}{lrrr}\mathrm{C} & 2.83621 & -0.72796 & -2.30357 \\ \mathrm{C} & 2.83621 & 0.72785 & -2.30360 \\ \mathrm{C} & 3.27276 & 1.42615 & -1.17552 \\ \mathrm{C} & 3.72020 & 0.69804 & -0.00002 \\ \mathrm{C} & 3.27276 & 1.42621 & 1.17545 \\ \mathrm{C} & 2.83621 & 0.72796 & 2.30357 \\ \mathrm{C} & 1.66166 & 1.17734 & 3.02592 \\ \mathrm{C} & 0.92971 & 0.00009 & 3.46576 \\ \mathrm{C} & 1.66165 & -1.17719 & 3.02598 \\ \mathrm{C} & 2.83621 & -0.72785 & 2.30361 \\ \mathrm{C} & 3.27276 & -1.42615 & 1.17553 \\ \mathrm{C} & 3.72020 & -0.69804 & 0.00002 \\ \mathrm{C} & 3.27276 & -1.42621 & -1.17545 \\ \mathrm{C} & 2.55165 & -2.60466 & -0.72707 \\ \mathrm{C} & 2.55165 & -2.60462 & 0.72720 \\ \mathrm{C} & 1.42340 & -3.04030 & 1.42538 \\ \mathrm{C} & 0.96781 & -2.31120 & 2.59875 \\ \mathrm{C} & -0.48400 & -2.31462 & 2.59238 \\ \mathrm{C} & -1.19023 & -1.17621 & 2.99824 \\ \mathrm{C} & -0.46535 & 0.00009 & 3.44384 \\ \mathrm{C} & -1.19023 & 1.17636 & 2.99818 \\ \mathrm{C} & -2.36729 & 0.74220 & 2.27547 \\ \mathrm{C} & -2.36729 & -0.74208 & 2.27551 \\ \mathrm{C} & -2.80569 & -1.45189 & 1.17555 \\ \mathrm{C} & -5.09433 & -0.77469 & -0.00001 \\ \mathrm{C} & -5.09433 & 0.77469 & 0.00001 \\ \mathrm{H} & -5.53691 & 1.23472 & 0.88751 \\ \mathrm{H} & -5.53699 & 1.23476 & -0.88743 \\ \mathrm{H} & -5.53698 & -1.23477 & 0.88743 \\ \mathrm{H} & -5.53692 & -1.23472 & -0.88751\end{array}$

(7) $\mathrm{C}_{2} \mathrm{H}_{6} \_\mathrm{C}_{60} \_\mathrm{T}$

\begin{tabular}{|c|c|c|c|}
\hline C & 3.15490 & -0.19497 & 0.11756 \\
\hline $\mathrm{C}$ & 2.92309 & 0.18771 & -1.26558 \\
\hline $\mathrm{C}$ & 2.51801 & 1.58384 & -1.28383 \\
\hline $\mathrm{C}$ & 2.49896 & 2.06386 & 0.08808 \\
\hline $\mathrm{C}$ & 2.89223 & 0.96454 & 0.95396 \\
\hline C & 2.28022 & 0.80328 & 2.19794 \\
\hline C & 1.25044 & 1.73427 & 2.62773 \\
\hline C & 0.87301 & 2.78992 & 1.79623 \\
\hline C & 1.51004 & 2.95831 & 0.50054 \\
\hline $\mathrm{C}$ & 0.49911 & 3.40944 & -0.44140 \\
\hline $\mathrm{C}$ & 0.51691 & 2.94788 & -1.75831 \\
\hline C & 1.54648 & 2.01613 & -2.18762 \\
\hline C & 0.94100 & 1.07195 & -3.11189 \\
\hline $\mathrm{C}$ & 1.32968 & -0.26826 & -3.09377 \\
\hline $\mathrm{C}$ & 2.34071 & -0.71930 & -2.15225 \\
\hline
\end{tabular}




\begin{tabular}{|c|c|c|c|}
\hline C & 1.96685 & -2.04700 & -1.69359 \\
\hline $\mathrm{C}$ & 0.72434 & -2.41610 & -2.35143 \\
\hline $\mathrm{C}$ & 0.33086 & -1.31689 & -3.21713 \\
\hline $\mathrm{C}$ & -1.01743 & -0.98313 & -3.35300 \\
\hline $\mathrm{C}$ & -1.42284 & 0.41282 & -3.37166 \\
\hline $\mathrm{C}$ & -0.46334 & 1.41958 & -3.25355 \\
\hline $\mathrm{C}$ & -0.72545 & 2.57903 & -2.41685 \\
\hline $\mathrm{C}$ & -1.93652 & 2.68517 & -1.73114 \\
\hline $\mathrm{C}$ & -1.95556 & 3.16569 & -0.35949 \\
\hline $\mathrm{C}$ & -0.76256 & 3.52009 & 0.27226 \\
\hline $\mathrm{C}$ & -0.53121 & 3.13782 & 1.65545 \\
\hline $\mathrm{C}$ & -1.50202 & 2.41579 & 2.35145 \\
\hline $\mathrm{C}$ & -1.10864 & 1.31651 & 3.21718 \\
\hline $\mathrm{C}$ & 0.23956 & 0.98282 & 3.35295 \\
\hline $\mathrm{C}$ & 0.64495 & -0.41312 & 3.37153 \\
\hline $\mathrm{C}$ & -0.31451 & -1.41983 & 3.25344 \\
\hline $\mathrm{C}$ & -1.71884 & -1.07232 & 3.11197 \\
\hline $\mathrm{C}$ & -2.10754 & 0.26796 & 3.09389 \\
\hline $\mathrm{C}$ & -3.11836 & 0.71913 & 2.15236 \\
\hline $\mathrm{C}$ & -2.74441 & 2.04672 & 1.69361 \\
\hline $\mathrm{C}$ & -2.96645 & 2.41375 & 0.36560 \\
\hline $\mathrm{C}$ & -3.57236 & 1.46898 & -0.55837 \\
\hline $\mathrm{C}$ & -2.93617 & 1.63707 & -1.85454 \\
\hline $\mathrm{C}$ & -2.68433 & 0.52375 & -2.65778 \\
\hline $\mathrm{C}$ & -3.05828 & -0.80352 & -2.19801 \\
\hline $\mathrm{C}$ & -2.02831 & -1.73454 & -2.62771 \\
\hline $\mathrm{C}$ & -1.65084 & -2.79019 & -1.79624 \\
\hline $\mathrm{C}$ & -0.24653 & -3.13809 & -1.65547 \\
\hline $\mathrm{C}$ & -0.01510 & -3.52040 & -0.27233 \\
\hline $\mathrm{C}$ & -1.27677 & -3.40978 & 0.44137 \\
\hline $\mathrm{C}$ & -2.28773 & -2.95864 & -0.50046 \\
\hline $\mathrm{C}$ & -3.27674 & -2.06434 & -0.08779 \\
\hline $\mathrm{C}$ & -3.66971 & -0.96480 & -0.95371 \\
\hline $\mathrm{C}$ & -3.93263 & 0.19461 & -0.11747 \\
\hline $\mathrm{C}$ & -3.70081 & -0.18803 & 1.26560 \\
\hline $\mathrm{C}$ & -3.29590 & -1.58413 & 1.28410 \\
\hline $\mathrm{C}$ & -2.32447 & -2.01668 & 2.18779 \\
\hline $\mathrm{C}$ & -1.29476 & -2.94833 & 1.75841 \\
\hline $\mathrm{C}$ & -0.05239 & -2.57931 & 2.41679 \\
\hline $\mathrm{C}$ & 1.15860 & -2.68530 & 1.73093 \\
\hline $\mathrm{C}$ & 1.17797 & -3.16596 & 0.35934 \\
\hline $\mathrm{C}$ & 2.18914 & -2.41420 & -0.36562 \\
\hline $\mathrm{C}$ & 2.79520 & -1.46957 & 0.55856 \\
\hline $\mathrm{C}$ & 2.15796 & -1.63713 & 1.85422 \\
\hline $\mathrm{C}$ & 1.90636 & -0.52398 & 2.65764 \\
\hline $\mathrm{C}$ & 8.54262 & -0.02576 & -0.00487 \\
\hline $\mathrm{C}$ & 7.01296 & 0.03198 & 0.00446 \\
\hline $\mathrm{H}$ & 6.64825 & 0.85147 & 0.63460 \\
\hline $\mathrm{H}$ & 6.57942 & -0.89779 & 0.39049 \\
\hline
\end{tabular}




$\begin{array}{rrrr}\mathrm{H} & 6.61232 & 0.18749 & -1.00405 \\ \mathrm{H} & 8.94194 & -0.18347 & 1.00385 \\ \mathrm{H} & 8.97468 & 0.90567 & -0.38899 \\ \mathrm{H} & 8.90752 & -0.84414 & -0.63637\end{array}$

(8) $\mathrm{C}_{2} \mathrm{H}_{6} \_\mathrm{C}_{60} \_\mathrm{PH}$

\begin{tabular}{lrrr}
$C$ & -3.15116 & 0.11641 & -0.25421 \\
$\mathrm{C}$ & -2.74110 & 0.68558 & -1.52750 \\
$\mathrm{C}$ & -2.23434 & -0.39306 & -2.35947 \\
$\mathrm{C}$ & -2.33000 & -1.62912 & -1.60068 \\
$\mathrm{C}$ & -2.89612 & -1.31458 & -0.29922 \\
$\mathrm{C}$ & -2.43779 & -1.97790 & 0.83984 \\
$\mathrm{C}$ & -1.39535 & -2.98449 & 0.72505 \\
$\mathrm{C}$ & -0.85181 & -3.28655 & -0.52460 \\
$\mathrm{C}$ & -1.32818 & -2.59481 & -1.71075 \\
$\mathrm{C}$ & -0.18985 & -2.36563 & -2.58524 \\
$\mathrm{C}$ & -0.09827 & -1.17896 & -3.31410 \\
$\mathrm{C}$ & -1.14119 & -0.17273 & -3.19868 \\
$\mathrm{C}$ & -0.50930 & 1.13553 & -3.24084 \\
$\mathrm{C}$ & -0.99622 & 2.17131 & -2.44189 \\
$\mathrm{C}$ & -2.13495 & 1.94196 & -1.56808 \\
$\mathrm{C}$ & -1.91205 & 2.68150 & -0.33644 \\
$\mathrm{C}$ & -0.63548 & 3.36756 & -0.44904 \\
$\mathrm{C}$ & -0.06981 & 3.05260 & -1.75044 \\
$\mathrm{C}$ & 1.30630 & 2.86302 & -1.88544 \\
$\mathrm{C}$ & 1.81362 & 1.78449 & -2.71754 \\
$\mathrm{C}$ & 0.92397 & 0.93816 & -3.38170 \\
$\mathrm{C}$ & 1.17796 & -0.49237 & -3.42671 \\
$\mathrm{C}$ & 2.31154 & -1.01979 & -2.80615 \\
$\mathrm{C}$ & 2.21586 & -2.25549 & -2.04675 \\
$\mathrm{C}$ & 0.99057 & -2.91501 & -1.93886 \\
$\mathrm{C}$ & 0.58148 & -3.48424 & -0.66532 \\
$\mathrm{C}$ & 1.41404 & -3.37101 & 0.44910 \\
$\mathrm{C}$ & 0.84827 & -3.05603 & 1.75044 \\
$\mathrm{C}$ & -0.52778 & -2.86633 & 1.88543 \\
$\mathrm{C}$ & -1.03500 & -1.78777 & 2.71756 \\
$\mathrm{C}$ & -0.14561 & -0.94149 & 3.38153 \\
$\mathrm{C}$ & 1.28758 & -1.13889 & 3.24092 \\
$\mathrm{C}$ & 1.77450 & -2.17472 & 2.44191 \\
$\mathrm{C}$ & 2.91328 & -1.94554 & 1.56815 \\
$\mathrm{C}$ & 2.69075 & -2.68509 & 0.33657 \\
$\mathrm{C}$ & 3.08393 & -2.13876 & -0.88643 \\
$\mathrm{C}$ & 3.71540 & -0.82993 & -0.92822 \\
$\mathrm{C}$ & 2.99390 & 1.23507 & -2.07112 \\
$\mathrm{C}$ & -0.13858 & -2.11458 \\
$\mathrm{C}$ & -2.97450 & -0.83974 \\
$\mathrm{C}$ & & -0.72501 \\
\hline
\end{tabular}




$\begin{array}{lrrr}\mathrm{C} & 1.63031 & 3.28313 & 0.52469 \\ \mathrm{C} & 0.19705 & 3.48077 & 0.66538 \\ \mathrm{C} & -0.21201 & 2.91170 & 1.93900 \\ \mathrm{C} & 0.96831 & 2.36239 & 2.58546 \\ \mathrm{C} & 2.10665 & 2.59147 & 1.71092 \\ \mathrm{C} & 3.10826 & 1.62579 & 1.60089 \\ \mathrm{C} & 3.67419 & 1.31082 & 0.29947 \\ \mathrm{C} & 3.92834 & -0.11973 & 0.25433 \\ \mathrm{C} & 3.51889 & -0.68882 & 1.52747 \\ \mathrm{C} & 3.01243 & 0.38983 & 2.35980 \\ \mathrm{C} & 1.91941 & 0.16944 & 3.19892 \\ \mathrm{C} & 0.87654 & 1.17571 & 3.31433 \\ \mathrm{C} & -0.39963 & 0.48905 & 3.42678 \\ \mathrm{C} & -1.53306 & 1.01651 & 2.80632 \\ \mathrm{C} & -1.43736 & 2.25228 & 2.04700 \\ \mathrm{C} & -2.30531 & 2.13564 & 0.88669 \\ \mathrm{C} & -2.93688 & 0.82679 & 0.92859 \\ \mathrm{C} & -2.45953 & 0.13534 & 2.11488 \\ \mathrm{C} & -2.21515 & -1.23828 & 2.07098 \\ \mathrm{C} & -7.40651 & 0.69941 & -0.01494 \\ \mathrm{C} & -8.16090 & -0.63246 & 0.01230 \\ \mathrm{H} & -9.24560 & -0.47707 & 0.04472 \\ \mathrm{H} & -7.88659 & -1.22827 & 0.89073 \\ \mathrm{H} & -7.94029 & -1.23470 & -0.87693 \\ \mathrm{H} & -7.63566 & 1.30547 & 0.86963 \\ \mathrm{H} & -7.67299 & 1.29119 & -0.89848 \\ \mathrm{H} & -6.32139 & 0.54360 & -0.03574 \\ & & & \end{array}$

(9) $\mathrm{C}_{2} \mathrm{H}_{6} \mathrm{C}_{60} \mathrm{HH}$

$\begin{array}{lrrr}\mathrm{C} & -3.12365 & 0.38398 & -0.33759 \\ \mathrm{C} & -2.84009 & 1.22477 & 0.81422 \\ \mathrm{C} & -2.17613 & 2.42731 & 0.33998 \\ \mathrm{C} & -2.04817 & 2.32989 & -1.10471 \\ \mathrm{C} & -2.63330 & 1.06725 & -1.52360 \\ \mathrm{C} & -2.04773 & 0.33627 & -2.55821 \\ \mathrm{C} & -0.85341 & 0.83787 & -3.21722 \\ \mathrm{C} & -0.29185 & 2.05068 & -2.81553 \\ \mathrm{C} & -0.90117 & 2.81156 & -1.73750 \\ \mathrm{C} & 0.16513 & 3.41087 & -0.95215 \\ \mathrm{C} & 0.04210 & 3.50424 & 0.43483 \\ \mathrm{C} & -1.15243 & 3.00290 & 1.09412 \\ \mathrm{C} & -0.75033 & 2.39910 & 2.35361 \\ \mathrm{C} & -1.38789 & 1.24395 & 2.80865 \\ \mathrm{C} & -2.45423 & 0.64499 & 2.02351 \\ \mathrm{C} & -2.33433 & -0.79964 & 2.13162 \\ \mathrm{C} & -1.19428 & -1.09372 & 2.98391 \\ \mathrm{C} & -0.60923 & 0.16960 & 3.40244\end{array}$




\begin{tabular}{|c|c|c|c|}
\hline C & 0.77614 & 0.29300 & 3.51700 \\
\hline $\mathrm{C}$ & 1.44049 & 1.49597 & 3.04288 \\
\hline $\mathrm{C}$ & 0.69283 & 2.52732 & 2.47266 \\
\hline $\mathrm{C}$ & 1.18243 & 3.21021 & 1.28690 \\
\hline $\mathrm{C}$ & 2.40049 & 2.83522 & 0.71823 \\
\hline $\mathrm{C}$ & 2.52886 & 2.73779 & -0.72650 \\
\hline $\mathrm{C}$ & 1.43392 & 3.02019 & -1.54476 \\
\hline $\mathrm{C}$ & 1.15118 & 2.17944 & -2.69617 \\
\hline $\mathrm{C}$ & 1.97483 & 1.09000 & -2.98399 \\
\hline $\mathrm{C}$ & 1.38970 & -0.17335 & -3.40245 \\
\hline $\mathrm{C}$ & 0.00432 & -0.29675 & -3.51690 \\
\hline C & -0.65999 & -1.49970 & -3.04275 \\
\hline $\mathrm{C}$ & 0.08767 & -2.53104 & -2.47255 \\
\hline $\mathrm{C}$ & 1.53083 & -2.40288 & -2.35358 \\
\hline $\mathrm{C}$ & 2.16837 & -1.24769 & -2.80863 \\
\hline $\mathrm{C}$ & 3.23478 & -0.64870 & -2.02357 \\
\hline $\mathrm{C}$ & 3.11500 & 0.79596 & -2.13186 \\
\hline $\mathrm{C}$ & 3.38674 & 1.60336 & -1.02621 \\
\hline $\mathrm{C}$ & 3.78840 & 0.99938 & 0.23373 \\
\hline $\mathrm{C}$ & 3.17877 & 1.76065 & 1.31159 \\
\hline $\mathrm{C}$ & 2.70873 & 1.10479 & 2.45045 \\
\hline $\mathrm{C}$ & 2.82822 & -0.33994 & 2.55832 \\
\hline $\mathrm{C}$ & 1.63387 & -0.84161 & 3.21725 \\
\hline $\mathrm{C}$ & 1.07236 & -2.05446 & 2.81554 \\
\hline $\mathrm{C}$ & -0.37065 & -2.18315 & 2.69607 \\
\hline $\mathrm{C}$ & -0.65343 & -3.02399 & 1.54470 \\
\hline $\mathrm{C}$ & 0.61533 & -3.41474 & 0.95215 \\
\hline $\mathrm{C}$ & 1.68168 & -2.81540 & 1.73751 \\
\hline $\mathrm{C}$ & 2.82865 & -2.33377 & 1.10474 \\
\hline $\mathrm{C}$ & 3.41357 & -1.07083 & 1.52343 \\
\hline C & 3.90319 & -0.38759 & 0.33748 \\
\hline C & 3.62044 & -1.22833 & -0.81408 \\
\hline $\mathrm{C}$ & 2.95663 & -2.43118 & -0.33999 \\
\hline $\mathrm{C}$ & 1.93294 & -3.00675 & -1.09412 \\
\hline $\mathrm{C}$ & 0.73836 & -3.50812 & -0.43482 \\
\hline $\mathrm{C}$ & -0.40196 & -3.21403 & -1.28684 \\
\hline $\mathrm{C}$ & -1.62007 & -2.83911 & -0.71822 \\
\hline C & -1.74841 & -2.74165 & 0.72649 \\
\hline C & -2.60632 & -1.60725 & 1.02621 \\
\hline C & -3.00827 & -1.00345 & -0.23371 \\
\hline C & -2.39845 & -1.76460 & -1.31158 \\
\hline $\mathrm{C}$ & -1.92816 & -1.10848 & -2.45018 \\
\hline C & -7.39337 & 0.68351 & 0.00735 \\
\hline $\mathrm{C}$ & -8.21525 & -0.60793 & -0.00754 \\
\hline $\mathrm{H}$ & -9.28534 & -0.40158 & 0.11129 \\
\hline $\mathrm{H}$ & -7.91641 & -1.28041 & 0.80501 \\
\hline $\mathrm{H}$ & -8.08648 & -1.15186 & -0.95056 \\
\hline $\mathrm{H}$ & -7.52224 & 1.22724 & 0.95054 \\
\hline $\mathrm{H}$ & -7.69274 & 1.35609 & -0.80498 \\
\hline
\end{tabular}


$\begin{array}{llll}\mathrm{H} & -6.32341 & 0.47709 & -0.11169\end{array}$

For impure fullerene

For palladium doped fullerene

\section{Acetylene}

(1) $\mathrm{C}_{2} \mathrm{H}_{2} \mathrm{C}_{59} \mathrm{Pd} \_\mathrm{TM}$

$\begin{array}{cccc}\text { Pd } & -3.95579 & -0.31326 & 0.00011 \\ \mathrm{C} & -2.69569 & 0.70660 & 1.23746 \\ \mathrm{C} & -2.23385 & 1.97203 & 0.72728 \\ \mathrm{C} & -2.23387 & 1.97179 & -0.72772 \\ \mathrm{C} & -2.69573 & 0.70617 & -1.23752 \\ \mathrm{C} & -2.01441 & 0.05219 & -2.24503 \\ \mathrm{C} & -0.98348 & 0.77378 & -2.96620 \\ \mathrm{C} & -0.60628 & 2.06236 & -2.57751 \\ \mathrm{C} & -1.22368 & 2.66360 & -1.42067 \\ \mathrm{C} & -0.19748 & 3.39314 & -0.69648 \\ \mathrm{C} & -0.19745 & 3.39335 & 0.69561 \\ \mathrm{C} & -1.22363 & 2.66402 & 1.42003 \\ \mathrm{C} & -0.60622 & 2.06308 & 2.57702 \\ \mathrm{C} & -0.98341 & 0.77458 & 2.96605 \\ \mathrm{C} & -2.01436 & 0.05281 & 2.24509 \\ \mathrm{C} & -1.63691 & -1.38459 & 2.24223 \\ \mathrm{C} & -0.38500 & -1.49300 & 2.96153 \\ \mathrm{C} & 0.01359 & -0.17445 & 3.41571 \\ \mathrm{C} & 1.35954 & 0.18921 & 3.45363 \\ \mathrm{C} & 1.76070 & 1.51877 & 3.02045 \\ \mathrm{C} & 0.79806 & 2.43612 & 2.59351 \\ \mathrm{C} & 1.05270 & 3.25847 & 1.42357 \\ \mathrm{C} & 2.25579 & 3.13136 & 0.72710 \\ \mathrm{C} & 2.25576 & 3.13117 & -0.72796 \\ \mathrm{C} & 1.05265 & 3.25808 & -1.42444 \\ \mathrm{C} & 0.79800 & 2.43541 & -2.59415 \\ \mathrm{C} & 1.76063 & 1.51797 & -3.02088 \\ \mathrm{C} & 1.35946 & 0.18829 & -3.45371 \\ \mathrm{C} & 0.01352 & -0.17536 & -3.41566 \\ \mathrm{C} & -0.38506 & -1.49379 & -2.96114 \\ \mathrm{C} & 0.60161 & -2.41457 & -2.58097 \\ \mathrm{C} & 1.99865 & -2.03115 & -2.59233 \\ \mathrm{C} & 2.37518 & -0.75573 & -3.02230 \\ \mathrm{C} & 3.39282 & -0.01574 & -2.30287 \\ \mathrm{C} & 3.01377 & 1.39046 & -2.30289 \\ \mathrm{C} & 3.25686 & 2.17931 & -1.17558 \\ \mathrm{C} & 3.87830 & 1.59308 & -0.00025\end{array}$




$\begin{array}{lrrr}\mathrm{C} & 3.25689 & 2.17963 & 1.17494 \\ \mathrm{C} & 3.01382 & 1.39107 & 2.30246 \\ \mathrm{C} & 3.39287 & -0.01513 & 2.30281 \\ \mathrm{C} & 2.37524 & -0.75493 & 3.02244 \\ \mathrm{C} & 1.99870 & -2.03045 & 2.59282 \\ \mathrm{C} & 0.60166 & -2.41387 & 2.58160 \\ \mathrm{C} & 0.37605 & -3.24644 & 1.42259 \\ \mathrm{C} & 1.63053 & -3.37470 & 0.69820 \\ \mathrm{C} & 2.63728 & -2.62028 & 1.42507 \\ \mathrm{C} & 3.61069 & -1.90244 & 0.72766 \\ \mathrm{C} & 3.99777 & -0.57531 & 1.17525 \\ \mathrm{C} & 4.23895 & 0.24376 & -0.00007 \\ \mathrm{C} & 3.99774 & -0.57562 & -1.17518 \\ \mathrm{C} & 3.61068 & -1.90263 & -0.72723 \\ \mathrm{C} & 2.63725 & -2.62066 & -1.42443 \\ \mathrm{C} & 1.63052 & -3.37490 & -0.69734 \\ \mathrm{C} & 0.37603 & -3.24681 & -1.42173 \\ \mathrm{C} & -0.82196 & -3.12595 & -0.72280 \\ \mathrm{C} & -0.82196 & -3.12575 & 0.72367 \\ \mathrm{C} & -1.88135 & -2.21947 & 1.14747 \\ \mathrm{C} & -2.72483 & -1.89231 & 0.00021 \\ \mathrm{C} & -1.88133 & -2.21978 & -1.14684 \\ \mathrm{C} & -1.63695 & -1.38523 & -2.24188 \\ \mathrm{C} & -5.65695 & 1.23943 & -0.00005 \\ \mathrm{C} & -6.24896 & 0.15852 & -0.00042 \\ \mathrm{H} & -5.41271 & 2.28258 & 0.00026 \\ \mathrm{H} & -6.95789 & -0.64394 & -0.00092 \\ & & & \end{array}$

(2) $\mathrm{C}_{2} \mathrm{H}_{2} \mathrm{C}_{59} \mathrm{Pd}$-TC1

$\begin{array}{cccc}\mathrm{Pd} & 3.96908 & -0.60462 & -0.02801 \\ \mathrm{C} & 3.20157 & 1.37321 & -0.19813 \\ \mathrm{C} & 2.40137 & 1.53636 & 1.09439 \\ \mathrm{C} & 2.20781 & 0.40228 & 1.96114 \\ \mathrm{C} & 2.59120 & -0.87857 & 1.41341 \\ \mathrm{C} & 1.79892 & -1.99363 & 1.60300 \\ \mathrm{C} & 0.71385 & -1.93733 & 2.55849 \\ \mathrm{C} & 0.43002 & -0.75776 & 3.24769 \\ \mathrm{C} & 1.17452 & 0.43485 & 2.92863 \\ \mathrm{C} & 0.27915 & 1.55726 & 3.01576 \\ \mathrm{C} & 0.42137 & 2.60437 & 2.10562 \\ \mathrm{C} & 1.48600 & 2.58366 & 1.13963 \\ \mathrm{C} & 0.96677 & 3.20228 & -0.09960 \\ \mathrm{C} & 1.34265 & 2.67447 & -1.32526 \\ \mathrm{C} & 2.32219 & 1.58175 & -1.39022 \\ \mathrm{C} & 1.86065 & 0.64499 & -2.31453 \\ \mathrm{C} & 0.65700 & 1.21046 & -2.97678 \\ \mathrm{C} & 0.34478 & 2.46857 & -2.35063\end{array}$




\begin{tabular}{|c|c|c|c|}
\hline $\mathrm{C}$ & -0.98744 & 2.84631 & -2.14257 \\
\hline $\mathrm{C}$ & -1.37980 & 3.41098 & -0.86767 \\
\hline $\mathrm{C}$ & -0.41836 & 3.56571 & 0.13726 \\
\hline C & -0.75151 & 3.21589 & 1.50556 \\
\hline $\mathrm{C}$ & -2.02112 & 2.73843 & 1.82813 \\
\hline $\mathrm{C}$ & -2.16921 & 1.63823 & 2.77141 \\
\hline $\mathrm{C}$ & -1.04086 & 1.06472 & 3.35860 \\
\hline $\mathrm{C}$ & -0.94643 & -0.37922 & 3.50466 \\
\hline $\mathrm{C}$ & -1.98894 & -1.19411 & 3.05956 \\
\hline $\mathrm{C}$ & -1.69260 & -2.43042 & 2.35298 \\
\hline $\mathrm{C}$ & -0.36804 & -2.79394 & 2.10867 \\
\hline $\mathrm{C}$ & 0.02095 & -3.36943 & 0.83818 \\
\hline $\mathrm{C}$ & -0.94677 & -3.55378 & -0.15642 \\
\hline $\mathrm{C}$ & -2.32467 & -3.15240 & 0.08498 \\
\hline $\mathrm{C}$ & -2.69225 & -2.60764 & 1.31364 \\
\hline $\mathrm{C}$ & -3.59661 & -1.46820 & 1.36972 \\
\hline $\mathrm{C}$ & -3.16197 & -0.60019 & 2.44713 \\
\hline $\mathrm{C}$ & -3.25115 & 0.79238 & 2.30520 \\
\hline $\mathrm{C}$ & -3.77284 & 1.36332 & 1.07890 \\
\hline $\mathrm{C}$ & -3.01748 & 2.57295 & 0.78339 \\
\hline $\mathrm{C}$ & -2.70054 & 2.89662 & -0.53506 \\
\hline $\mathrm{C}$ & -3.12522 & 2.02004 & -1.61713 \\
\hline $\mathrm{C}$ & -2.05874 & 1.98884 & -2.60298 \\
\hline $\mathrm{C}$ & -1.75325 & 0.79151 & -3.25808 \\
\hline $\mathrm{C}$ & -0.37104 & 0.40475 & -3.43715 \\
\hline $\mathrm{C}$ & -0.29095 & -1.05297 & -3.30384 \\
\hline $\mathrm{C}$ & -1.61828 & -1.55236 & -3.02592 \\
\hline $\mathrm{C}$ & -2.52003 & -0.41317 & -2.97917 \\
\hline $\mathrm{C}$ & -3.54680 & -0.37898 & -2.03563 \\
\hline $\mathrm{C}$ & -3.84726 & 0.86032 & -1.33555 \\
\hline $\mathrm{C}$ & -4.18292 & 0.52521 & 0.03925 \\
\hline $\mathrm{C}$ & -4.09354 & -0.91487 & 0.18719 \\
\hline $\mathrm{C}$ & -3.70501 & -1.47853 & -1.09631 \\
\hline $\mathrm{C}$ & -2.84467 & -2.57543 & -1.14713 \\
\hline $\mathrm{C}$ & -1.78400 & -2.62179 & -2.13840 \\
\hline $\mathrm{C}$ & -0.62075 & -3.23164 & -1.53014 \\
\hline $\mathrm{C}$ & 0.66049 & -2.76385 & -1.83481 \\
\hline $\mathrm{C}$ & 0.81612 & -1.62345 & -2.70807 \\
\hline $\mathrm{C}$ & 1.94815 & -0.81412 & -2.20255 \\
\hline $\mathrm{C}$ & 2.54316 & -1.50150 & -1.15415 \\
\hline $\mathrm{C}$ & 1.68655 & -2.63937 & -0.81647 \\
\hline $\mathrm{C}$ & 1.35858 & -2.90234 & 0.50645 \\
\hline $\mathrm{C}$ & 4.63926 & 1.79862 & -0.21049 \\
\hline $\mathrm{C}$ & 5.38471 & 0.84782 & 0.40658 \\
\hline $\mathrm{H}$ & 4.94895 & 2.69550 & -0.75650 \\
\hline $\mathrm{H}$ & 6.47295 & 0.90004 & 0.45868 \\
\hline
\end{tabular}

(3) $\mathrm{C}_{2} \mathrm{H}_{2} \mathrm{C}_{59} \mathrm{Pd} \_\mathrm{TC} 5$ 


\begin{tabular}{|c|c|c|c|}
\hline $\mathrm{Pd}$ & 3.93034 & -0.58400 & 0.06259 \\
\hline $\mathrm{C}$ & 2.50741 & -1.38844 & 1.29835 \\
\hline $\mathrm{C}$ & 1.78337 & -2.51930 & 0.76427 \\
\hline $\mathrm{C}$ & 1.82275 & -2.50137 & -0.69327 \\
\hline $\mathrm{C}$ & 2.56292 & -1.36396 & -1.18673 \\
\hline $\mathrm{C}$ & 2.04905 & -0.56623 & -2.18483 \\
\hline $\mathrm{C}$ & 0.90491 & -1.03781 & -2.92967 \\
\hline $\mathrm{C}$ & 0.26266 & -2.23116 & -2.58096 \\
\hline $\mathrm{C}$ & 0.71298 & -2.96266 & -1.42198 \\
\hline $\mathrm{C}$ & -0.45996 & -3.48099 & -0.73842 \\
\hline $\mathrm{C}$ & -0.49804 & -3.50062 & 0.65318 \\
\hline $\mathrm{C}$ & 0.63553 & -3.00145 & 1.41139 \\
\hline $\mathrm{C}$ & 0.12472 & -2.29982 & 2.56413 \\
\hline $\mathrm{C}$ & 0.74971 & -1.11806 & 2.97806 \\
\hline $\mathrm{C}$ & 1.93473 & -0.63229 & 2.30477 \\
\hline $\mathrm{C}$ & 1.85219 & 0.87003 & 2.30683 \\
\hline $\mathrm{C}$ & 0.62131 & 1.21951 & 2.99586 \\
\hline $\mathrm{C}$ & -0.04807 & 0.00569 & 3.41577 \\
\hline $\mathrm{C}$ & -1.43972 & -0.07606 & 3.42725 \\
\hline $\mathrm{C}$ & -2.09284 & -1.28933 & 2.96377 \\
\hline $\mathrm{C}$ & -1.32422 & -2.37664 & 2.54077 \\
\hline $\mathrm{C}$ & -1.71243 & -3.11913 & 1.35320 \\
\hline $\mathrm{C}$ & -2.84548 & -2.73856 & 0.63123 \\
\hline $\mathrm{C}$ & -2.80593 & -2.71897 & -0.82290 \\
\hline $\mathrm{C}$ & -1.63482 & -3.08046 & -1.49225 \\
\hline $\mathrm{C}$ & -1.18500 & -2.30742 & -2.63770 \\
\hline $\mathrm{C}$ & -1.93004 & -1.20766 & -3.07152 \\
\hline $\mathrm{C}$ & -1.25459 & 0.01809 & -3.46793 \\
\hline $\mathrm{C}$ & 0.13390 & 0.09969 & -3.38124 \\
\hline $\mathrm{C}$ & 0.77946 & 1.30108 & -2.89289 \\
\hline $\mathrm{C}$ & -0.00798 & 2.39882 & -2.52586 \\
\hline $\mathrm{C}$ & -1.45597 & 2.31153 & -2.58466 \\
\hline $\mathrm{C}$ & -2.06893 & 1.14417 & -3.04417 \\
\hline $\mathrm{C}$ & -3.23553 & 0.61673 & -2.36570 \\
\hline $\mathrm{C}$ & -3.14892 & -0.83752 & -2.38240 \\
\hline $\mathrm{C}$ & -3.57830 & -1.57625 & -1.27643 \\
\hline $\mathrm{C}$ & -4.09819 & -0.89021 & -0.10584 \\
\hline $\mathrm{C}$ & -3.64221 & -1.60788 & 1.07288 \\
\hline $\mathrm{C}$ & -3.27375 & -0.89986 & 2.21940 \\
\hline $\mathrm{C}$ & -3.35951 & 0.55446 & 2.23620 \\
\hline $\mathrm{C}$ & -2.23048 & 1.06132 & 2.98955 \\
\hline $\mathrm{C}$ & -1.59500 & 2.24106 & 2.59645 \\
\hline $\mathrm{C}$ & -0.14600 & 2.32749 & 2.61669 \\
\hline $\mathrm{C}$ & 0.27075 & 3.11814 & 1.48602 \\
\hline $\mathrm{C}$ & -0.91278 & 3.50344 & 0.73578 \\
\hline $\mathrm{C}$ & -2.06987 & 2.96289 & 1.42595 \\
\hline $\mathrm{C}$ & -3.15059 & 2.46766 & 0.69238 \\
\hline $\mathrm{C}$ & -3.80893 & 1.24140 & 1.10592 \\
\hline
\end{tabular}




$\begin{array}{lrrr}\mathrm{C} & -4.18080 & 0.50371 & -0.08958 \\ \mathrm{C} & -3.74622 & 1.27294 & -1.24325 \\ \mathrm{C} & -3.11153 & 2.48732 & -0.76171 \\ \mathrm{C} & -1.99341 & 3.00172 & -1.42243 \\ \mathrm{C} & -0.87464 & 3.52245 & -0.65726 \\ \mathrm{C} & 0.34813 & 3.15379 & -1.35247 \\ \mathrm{C} & 1.48869 & 2.79012 & -0.63514 \\ \mathrm{C} & 1.44661 & 2.77297 & 0.82194 \\ \mathrm{C} & 2.29984 & 1.70071 & 1.28761 \\ \mathrm{C} & 3.21141 & 1.36326 & 0.13308 \\ \mathrm{C} & 2.35914 & 1.72040 & -1.07565 \\ \mathrm{C} & 1.96972 & 0.93185 & -2.14644 \\ \mathrm{C} & 4.66092 & 1.75283 & 0.13552 \\ \mathrm{C} & 5.38380 & 0.79008 & -0.49236 \\ \mathrm{H} & 4.99718 & 2.64478 & 0.67512 \\ \mathrm{H} & 6.47166 & 0.83605 & -0.55760\end{array}$

(4) $\mathrm{C}_{2} \mathrm{H}_{2} \_\mathrm{C}_{59} \mathrm{Pd} \_\mathrm{HH}$-M-C5

$\begin{array}{cccc}\text { Pd } & -3.93027 & -0.58408 & -0.06245 \\ \text { C } & -2.50719 & -1.38749 & -1.29897 \\ \text { C } & -1.78313 & -2.51904 & -0.76625 \\ \text { C } & -1.82239 & -2.50256 & 0.69136 \\ \text { C } & -2.56254 & -1.36553 & 1.18556 \\ \text { C } & -2.04885 & -0.56846 & 2.18431 \\ \mathrm{C} & -0.90458 & -1.04058 & 2.92875 \\ \mathrm{C} & -0.26226 & -2.23362 & 2.57912 \\ \mathrm{C} & -0.71255 & -2.96426 & 1.41954 \\ \mathrm{C} & 0.46043 & -3.48181 & 0.73539 \\ \mathrm{C} & 0.49836 & -3.50014 & -0.65623 \\ \mathrm{C} & -0.63536 & -3.00038 & -1.41394 \\ \mathrm{C} & -0.12472 & -2.29762 & -2.56608 \\ \mathrm{C} & -0.74982 & -1.11549 & -2.97872 \\ \mathrm{C} & -1.93479 & -0.63033 & -2.30473 \\ \mathrm{C} & -1.85236 & 0.87199 & -2.30579 \\ \mathrm{C} & -0.62158 & 1.22209 & -2.99468 \\ \mathrm{C} & 0.04779 & 0.00866 & -3.41566 \\ \mathrm{C} & 1.43946 & -0.07296 & -3.42734 \\ \mathrm{C} & 2.09273 & -1.28657 & -2.96500 \\ \mathrm{C} & 1.32426 & -2.37432 & -2.54288 \\ \mathrm{C} & 1.71263 & -3.11785 & -1.35602 \\ \mathrm{C} & 2.84571 & -2.73779 & -0.63379 \\ \mathrm{C} & 2.80627 & -2.71950 & 0.82035 \\ \mathrm{C} & 1.63528 & -3.08173 & 1.48949 \\ \mathrm{C} & 1.18543 & -2.30971 & 2.63563 \\ \mathrm{C} & 1.93037 & -1.21025 & 3.07036 \\ \mathrm{C} & 1.25481 & 0.01509 & 3.46782 \\ \mathrm{C} & -0.13370 & 0.09660 & 3.38124\end{array}$




\begin{tabular}{lrrr}
$\mathrm{C}$ & -0.77937 & 1.29834 & 2.89388 \\
$\mathrm{C}$ & 0.00788 & 2.39655 & 2.52792 \\
$\mathrm{C}$ & 1.45591 & 2.30932 & 2.58659 \\
$\mathrm{C}$ & 2.06900 & 1.14163 & 3.04503 \\
$\mathrm{C}$ & 3.23563 & 0.61489 & 2.36604 \\
$\mathrm{C}$ & 3.14919 & -0.83936 & 2.38148 \\
$\mathrm{C}$ & 3.57856 & -1.57709 & 1.27484 \\
$\mathrm{C}$ & 4.09829 & -0.88997 & 0.10480 \\
$\mathrm{C}$ & 3.64229 & -1.60665 & -1.07451 \\
$\mathrm{C}$ & 3.27367 & -0.89764 & -2.22038 \\
$\mathrm{C}$ & 3.35930 & 0.55668 & -2.23592 \\
$\mathrm{C}$ & 2.23015 & 1.06413 & -2.98874 \\
$\mathrm{C}$ & 1.59463 & 2.24347 & -2.59454 \\
$\mathrm{C}$ & 0.14561 & 2.32984 & -2.61464 \\
$\mathrm{C}$ & -0.27111 & 3.11950 & -1.48326 \\
$\mathrm{C}$ & 0.91242 & 3.50420 & -0.73276 \\
$\mathrm{C}$ & 2.06954 & 2.96434 & -1.42346 \\
$\mathrm{C}$ & 3.15032 & 2.46853 & -0.69040 \\
$\mathrm{C}$ & 3.80874 & 1.24268 & -1.10508 \\
$\mathrm{C}$ & 4.18076 & 0.50397 & 0.08976 \\
$\mathrm{C}$ & 3.74617 & 1.27215 & 1.24414 \\
$\mathrm{C}$ & 3.11134 & 2.48691 & 0.76371 \\
$\mathrm{C}$ & 1.99320 & 3.00061 & 1.42495 \\
$\mathrm{C}$ & 0.87434 & 3.52194 & 0.66030 \\
$\mathrm{C}$ & -0.34836 & 3.15256 & 1.35526 \\
$\mathrm{C}$ & -1.48901 & 2.78958 & 0.63772 \\
$\mathrm{C}$ & -1.44695 & 2.77379 & -0.81939 \\
$\mathrm{C}$ & -2.29997 & 1.70176 & -1.28594 \\
$\mathrm{C}$ & -3.21165 & 1.36322 & -0.13180 \\
$\mathrm{C}$ & -2.35944 & 1.71935 & 1.07730 \\
$\mathrm{C}$ & -1.96962 & 0.92966 & 2.14712 \\
$\mathrm{C}$ & -4.66112 & 1.75286 & -0.13536 \\
$\mathrm{C}$ & -5.38447 & 0.78974 & 0.49137 \\
$\mathrm{H}$ & -4.99672 & 2.64500 & -0.67509 \\
& -6.47251 & 0.83483 & 0.55471 \\
\hline & & &
\end{tabular}

(5) $\mathrm{C}_{2} \mathrm{H}_{2} \_\mathrm{C}_{59} \mathrm{Pd} \_\mathrm{PH} \_\mathrm{M}-\mathrm{C} 1$

$\begin{array}{cccc}\text { Pd } & -3.96909 & -0.60464 & -0.02803 \\ \text { C } & -2.59116 & -0.87877 & 1.41330 \\ \text { C } & -2.20776 & 0.40198 & 1.96126 \\ \text { C } & -2.40137 & 1.53620 & 1.09468 \\ \text { C } & -3.20153 & 1.37346 & -0.19772 \\ \text { C } & -2.32224 & 1.58224 & -1.38989 \\ \text { C } & -1.34260 & 2.67471 & -1.32471 \\ \text { C } & -0.96672 & 3.20239 & -0.09896 \\ \text { C } & -1.48588 & 2.58344 & 1.14010 \\ \text { C } & -0.42127 & 2.60394 & 2.10610\end{array}$




\begin{tabular}{|c|c|c|c|}
\hline $\mathrm{C}$ & -0.27905 & 1.55668 & 3.01606 \\
\hline $\mathrm{C}$ & -1.17445 & 0.43431 & 2.92876 \\
\hline $\mathrm{C}$ & -0.42997 & -0.75836 & 3.24757 \\
\hline $\mathrm{C}$ & -0.71383 & -1.93782 & 2.55818 \\
\hline $\mathrm{C}$ & -1.79891 & -1.99391 & 1.60272 \\
\hline $\mathrm{C}$ & -1.35861 & -2.90237 & 0.50596 \\
\hline $\mathrm{C}$ & -0.02099 & -3.36957 & 0.83757 \\
\hline $\mathrm{C}$ & 0.36805 & -2.79436 & 2.10816 \\
\hline $\mathrm{C}$ & 1.69262 & -2.43091 & 2.35250 \\
\hline $\mathrm{C}$ & 1.98899 & -1.19473 & 3.05930 \\
\hline $\mathrm{C}$ & 0.94651 & -0.37991 & 3.50458 \\
\hline $\mathrm{C}$ & 1.04096 & 1.06406 & 3.35878 \\
\hline $\mathrm{C}$ & 2.16930 & 1.63765 & 2.77167 \\
\hline $\mathrm{C}$ & 2.02123 & 2.73805 & 1.82861 \\
\hline $\mathrm{C}$ & 0.75162 & 3.21558 & 1.50615 \\
\hline $\mathrm{C}$ & 0.41845 & 3.56570 & 0.13793 \\
\hline $\mathrm{C}$ & 1.37987 & 3.41116 & -0.86703 \\
\hline $\mathrm{C}$ & 0.98748 & 2.84673 & -2.14203 \\
\hline $\mathrm{C}$ & -0.34474 & 2.46902 & -2.35011 \\
\hline $\mathrm{C}$ & -0.65704 & 1.21106 & -2.97648 \\
\hline $\mathrm{C}$ & 0.37097 & 0.40541 & -3.43705 \\
\hline $\mathrm{C}$ & 1.75319 & 0.79212 & -3.25795 \\
\hline $\mathrm{C}$ & 2.05873 & 1.98931 & -2.60263 \\
\hline $\mathrm{C}$ & 3.12523 & 2.02030 & -1.61680 \\
\hline $\mathrm{C}$ & 2.70059 & 2.89670 & -0.53456 \\
\hline $\mathrm{C}$ & 3.01757 & 2.57276 & 0.78383 \\
\hline $\mathrm{C}$ & 3.77289 & 1.36304 & 1.07909 \\
\hline $\mathrm{C}$ & 3.25121 & 0.79187 & 2.30529 \\
\hline $\mathrm{C}$ & 3.16201 & -0.60072 & 2.44695 \\
\hline $\mathrm{C}$ & 3.59661 & -1.46853 & 1.36937 \\
\hline $\mathrm{C}$ & 2.69222 & -2.60794 & 1.31309 \\
\hline $\mathrm{C}$ & 2.32461 & -3.15246 & 0.08434 \\
\hline $\mathrm{C}$ & 0.94668 & -3.55375 & -0.15711 \\
\hline $\mathrm{C}$ & 0.62064 & -3.23134 & -1.53077 \\
\hline $\mathrm{C}$ & 1.78389 & -2.62140 & -2.13893 \\
\hline $\mathrm{C}$ & 2.84460 & -2.57527 & -1.14767 \\
\hline $\mathrm{C}$ & 3.70494 & -1.47839 & -1.09666 \\
\hline $\mathrm{C}$ & 4.09352 & -0.91498 & 0.18694 \\
\hline $\mathrm{C}$ & 4.18293 & 0.52513 & 0.03927 \\
\hline C & 3.84725 & 0.86050 & -1.33546 \\
\hline $\mathrm{C}$ & 3.54674 & -0.37865 & -2.03577 \\
\hline $\mathrm{C}$ & 2.51996 & -0.41264 & -2.97930 \\
\hline C & 1.61818 & -1.55181 & -3.02624 \\
\hline $\mathrm{C}$ & 0.29086 & -1.05231 & -3.30401 \\
\hline $\mathrm{C}$ & -0.81625 & -1.62287 & -2.70835 \\
\hline $\mathrm{C}$ & -0.66059 & -2.76346 & -1.83531 \\
\hline C & -1.68660 & -2.63913 & -0.81691 \\
\hline C & -2.54317 & -1.50120 & -1.15430 \\
\hline $\mathrm{C}$ & -1.94816 & -0.81355 & -2.20262 \\
\hline
\end{tabular}




$\begin{array}{llll}\mathrm{C} & -1.86069 & 0.64549 & -2.31431 \\ \mathrm{C} & -4.63927 & 1.79866 & -0.21030 \\ \mathrm{C} & -5.38485 & 0.84778 & 0.40642 \\ \mathrm{H} & -4.94908 & 2.69560 & -0.75618 \\ \mathrm{H} & -6.47311 & 0.89973 & 0.45812\end{array}$

(6) $\mathrm{C}_{2} \mathrm{H}_{2} \_\mathrm{C}_{59} \mathrm{Pd} \_\mathrm{HH} \_\mathrm{C} 1-\mathrm{C} 2$

$\begin{array}{lrrr}\text { Pd } & -3.88876 & -0.83073 & 0.10170 \\ \mathrm{C} & -2.49897 & -1.23631 & 1.48423 \\ \mathrm{C} & -2.10658 & -0.10974 & 2.30108 \\ \mathrm{C} & -2.39344 & 1.16109 & 1.68873 \\ \mathrm{C} & -3.24126 & 1.11320 & 0.45942 \\ \mathrm{C} & -2.66763 & 1.77367 & -0.90835 \\ \mathrm{C} & -1.54282 & 2.76357 & -0.60947 \\ \mathrm{C} & -1.09122 & 3.03816 & 0.67183 \\ \mathrm{C} & -1.49837 & 2.21067 & 1.83159 \\ \mathrm{C} & -0.35130 & 2.08556 & 2.70121 \\ \mathrm{C} & -0.10947 & 0.88847 & 3.37445 \\ \mathrm{C} & -0.99115 & -0.23530 & 3.15658 \\ \mathrm{C} & -0.19309 & -1.43309 & 3.13412 \\ \mathrm{C} & -0.50780 & -2.44248 & 2.21857 \\ \mathrm{C} & -1.65393 & -2.32258 & 1.34347 \\ \mathrm{C} & -1.28990 & -2.96699 & 0.04542 \\ \mathrm{C} & 0.06654 & -3.44354 & 0.17721 \\ \mathrm{C} & 0.54665 & -3.13901 & 1.51173 \\ \mathrm{C} & 1.88092 & -2.80004 & 1.73268 \\ \mathrm{C} & 2.20919 & -1.72721 & 2.66028 \\ \mathrm{C} & 1.19299 & -1.06222 & 3.35098 \\ \mathrm{C} & 1.24538 & 0.38294 & 3.49544 \\ \mathrm{C} & 2.30825 & 1.10233 & 2.94568 \\ \mathrm{C} & 2.05611 & 2.35740 & 2.25262 \\ \mathrm{C} & 0.75113 & 2.83234 & 2.12756 \\ \mathrm{C} & 0.29512 & 3.41365 & 0.87821 \\ \mathrm{C} & 1.18032 & 3.50033 & -0.20161 \\ \mathrm{C} & 0.71168 & 3.18303 & -1.52944 \\ \mathrm{C} & -0.61745 & 2.78245 & -1.70303 \\ \mathrm{C} & -0.94110 & 1.67925 & -2.59231 \\ \mathrm{C} & 0.09003 & 1.06073 & -3.31765 \\ \mathrm{C} & 1.46769 & 1.46010 & -3.11717 \\ \mathrm{C} & 1.77805 & 2.50275 & -2.24136 \\ \mathrm{C} & 2.90785 & 2.38438 & -1.34206 \\ \mathrm{C} & 2.53966 & 3.00467 & -0.07665 \\ \mathrm{C} & 3.96919 & 2.44020 & 1.12494 \\ \mathrm{C} & 3.36727 & 0.40877 & 2.23853 \\ \mathrm{C} & & -0.98341 & 2.10151 \\ \mathrm{C} & -1.60182 & 0.83588\end{array}$




$\begin{array}{lrrr}\mathrm{C} & 2.80436 & -2.73342 & 0.61420 \\ \mathrm{C} & 2.35060 & -3.02894 & -0.67345 \\ \mathrm{C} & 0.97272 & -3.41719 & -0.89714 \\ \mathrm{C} & 0.54656 & -2.84034 & -2.14837 \\ \mathrm{C} & 1.65557 & -2.08529 & -2.71076 \\ \mathrm{C} & 2.77566 & -2.20345 & -1.79744 \\ \mathrm{C} & 3.62054 & -1.11221 & -1.58160 \\ \mathrm{C} & 4.08591 & -0.80565 & -0.23962 \\ \mathrm{C} & 4.12960 & 0.63862 & -0.09789 \\ \mathrm{C} & 3.69090 & 1.22699 & -1.35140 \\ \mathrm{C} & 3.37575 & 0.14477 & -2.26885 \\ \mathrm{C} & 2.29125 & 0.26072 & -3.14158 \\ \mathrm{C} & 1.41626 & -0.87629 & -3.36740 \\ \mathrm{C} & 0.05893 & -0.36825 & -3.47718 \\ \mathrm{C} & -0.99581 & -1.08322 & -2.91805 \\ \mathrm{C} & -0.75291 & -2.34642 & -2.25910 \\ \mathrm{C} & -1.71896 & -2.44964 & -1.17883 \\ \mathrm{C} & -2.76252 & -1.45014 & -1.40072 \\ \mathrm{C} & -2.10637 & -0.43330 & -2.23095 \\ \mathrm{C} & -2.06584 & 0.95299 & -2.06729 \\ \mathrm{C} & -4.07454 & 2.38030 & -0.97487 \\ \mathrm{C} & -4.48751 & 1.88822 & 0.21363 \\ \mathrm{H} & -4.51261 & 3.05933 & -1.70075 \\ \mathrm{H} & -5.37538 & 2.03541 & 0.82051\end{array}$

(7) $\mathrm{C}_{2} \mathrm{H}_{2} \_\mathrm{C}_{59} \mathrm{Pd} \_\mathrm{PH} \_\mathrm{C} 2-\mathrm{C} 3$

$\begin{array}{cccc}\mathrm{Pd} & 3.86079 & -1.00858 & -0.12873 \\ \mathrm{C} & 2.29892 & -2.02874 & -0.85539 \\ \mathrm{C} & 1.83887 & -1.40684 & -2.06963 \\ \mathrm{C} & 2.18646 & 0.00991 & -2.18043 \\ \mathrm{C} & 3.17078 & 0.53051 & -1.27753 \\ \mathrm{C} & 2.86122 & 1.84233 & -0.56681 \\ \mathrm{C} & 1.65245 & 2.57741 & -1.13142 \\ \mathrm{C} & 1.02619 & 2.18226 & -2.29831 \\ \mathrm{C} & 1.30033 & 0.86156 & -2.85962 \\ \mathrm{C} & 0.08655 & 0.36782 & -3.44856 \\ \mathrm{C} & -0.23680 & -0.99069 & -3.37387 \\ \mathrm{C} & 0.65291 & -1.88389 & -2.66002 \\ \mathrm{C} & -0.15398 & -2.86573 & -1.97850 \\ \mathrm{C} & 0.23586 & -3.32076 & -0.71701 \\ \mathrm{C} & 1.47110 & -2.86048 & -0.12549 \\ \mathrm{C} & 1.23649 & -2.74057 & 1.33604 \\ \mathrm{C} & -0.13743 & -3.13435 & 1.59118 \\ \mathrm{C} & -0.74952 & -3.50414 & 0.33019 \\ \mathrm{C} & -2.09094 & -3.21587 & 0.07961 \\ \mathrm{C} & -2.49790 & -2.73265 & -1.23100 \\ \mathrm{C} & -1.54970 & -2.56830 & -2.24056\end{array}$




\begin{tabular}{|c|c|c|c|}
\hline $\mathrm{C}$ & -1.59767 & -1.39736 & -3.10441 \\
\hline $\mathrm{C}$ & -2.60019 & -0.43836 & -2.92509 \\
\hline $\mathrm{C}$ & -2.26737 & 0.97410 & -3.00441 \\
\hline $\mathrm{C}$ & -0.95425 & 1.36825 & -3.26436 \\
\hline $\mathrm{C}$ & -0.37031 & 2.47955 & -2.54059 \\
\hline $\mathrm{C}$ & -1.12009 & 3.16137 & -1.58044 \\
\hline $\mathrm{C}$ & -0.49580 & 3.52503 & -0.32872 \\
\hline $\mathrm{C}$ & 0.85235 & 3.17737 & -0.09780 \\
\hline $\mathrm{C}$ & 1.26768 & 2.69492 & 1.18735 \\
\hline $\mathrm{C}$ & 0.32549 & 2.54491 & 2.18750 \\
\hline $\mathrm{C}$ & -1.06146 & 2.87858 & 1.96455 \\
\hline $\mathrm{C}$ & -1.47054 & 3.36454 & 0.72018 \\
\hline $\mathrm{C}$ & -2.70600 & 2.88339 & 0.12734 \\
\hline $\mathrm{C}$ & -2.48574 & 2.75299 & -1.30534 \\
\hline $\mathrm{C}$ & -3.04790 & 1.68018 & -2.00046 \\
\hline $\mathrm{C}$ & -3.85662 & 0.70090 & -1.29770 \\
\hline $\mathrm{C}$ & -3.57873 & -0.60572 & -1.86734 \\
\hline $\mathrm{C}$ & -3.53437 & -1.73352 & -1.03800 \\
\hline $\mathrm{C}$ & -3.76560 & -1.59709 & 0.38669 \\
\hline $\mathrm{C}$ & -2.87901 & -2.52024 & 1.08132 \\
\hline $\mathrm{C}$ & -2.29706 & -2.15684 & 2.29494 \\
\hline $\mathrm{C}$ & -0.90448 & -2.47763 & 2.56214 \\
\hline $\mathrm{C}$ & -0.32500 & -1.37368 & 3.29625 \\
\hline $\mathrm{C}$ & -1.34518 & -0.36346 & 3.47491 \\
\hline $\mathrm{C}$ & -2.57116 & -0.84480 & 2.86586 \\
\hline $\mathrm{C}$ & -3.41222 & 0.04378 & 2.19520 \\
\hline $\mathrm{C}$ & -4.02878 & -0.33976 & 0.93545 \\
\hline $\mathrm{C}$ & -4.07195 & 0.83036 & 0.07883 \\
\hline $\mathrm{C}$ & -3.48400 & 1.94251 & 0.80340 \\
\hline $\mathrm{C}$ & -3.06861 & 1.45398 & 2.10988 \\
\hline $\mathrm{C}$ & -1.88206 & 1.91559 & 2.67972 \\
\hline $\mathrm{C}$ & -1.00177 & 0.99238 & 3.37648 \\
\hline $\mathrm{C}$ & 0.35925 & 1.37157 & 3.07585 \\
\hline $\mathrm{C}$ & 1.32487 & 0.40418 & 2.88118 \\
\hline $\mathrm{C}$ & 0.99483 & -0.99437 & 3.03379 \\
\hline $\mathrm{C}$ & 1.80542 & -1.71080 & 2.07053 \\
\hline $\mathrm{C}$ & 2.71185 & -0.73789 & 1.46265 \\
\hline $\mathrm{C}$ & 2.35820 & 0.53438 & 1.83663 \\
\hline $\mathrm{C}$ & 2.54478 & 1.84687 & 1.09062 \\
\hline $\mathrm{C}$ & 4.06565 & 2.69095 & -0.14426 \\
\hline $\mathrm{C}$ & 3.84583 & 2.65133 & 1.17201 \\
\hline $\mathrm{H}$ & 4.81784 & 3.16561 & -0.76677 \\
\hline $\mathrm{H}$ & 4.34080 & 3.08167 & 2.03705 \\
\hline
\end{tabular}

(8) $\mathrm{C}_{2} \mathrm{H}_{2} \_\mathrm{C}_{59} \mathrm{Pd}$-HH_C3-C4
$\begin{array}{llll}\mathrm{Pd} & -3.82095 & -1.03046 & -0.14619\end{array}$
C $\quad-2.25063 \quad-2.20996 \quad-0.55665$ 


\begin{tabular}{|c|c|c|c|}
\hline $\mathrm{C}$ & -1.58468 & -2.75621 & 0.60338 \\
\hline $\mathrm{C}$ & -1.82128 & -1.94813 & 1.79124 \\
\hline $\mathrm{C}$ & -2.68967 & -0.83164 & 1.50475 \\
\hline $\mathrm{C}$ & -2.35340 & 0.44235 & 1.90640 \\
\hline $\mathrm{C}$ & -1.27851 & 0.59786 & 2.84741 \\
\hline $\mathrm{C}$ & -0.54094 & -0.49965 & 3.30561 \\
\hline $\mathrm{C}$ & -0.79697 & -1.79996 & 2.74016 \\
\hline $\mathrm{C}$ & 0.48084 & -2.46736 & 2.57260 \\
\hline $\mathrm{C}$ & 0.70572 & -3.24852 & 1.44219 \\
\hline $\mathrm{C}$ & -0.33599 & -3.38952 & 0.43967 \\
\hline $\mathrm{C}$ & 0.29883 & -3.40655 & -0.85443 \\
\hline $\mathrm{C}$ & -0.30966 & -2.75444 & -1.93033 \\
\hline $\mathrm{C}$ & -1.58514 & -2.08436 & -1.76267 \\
\hline $\mathrm{C}$ & -1.57166 & -0.87597 & -2.62003 \\
\hline $\mathrm{C}$ & -0.28711 & -0.83335 & -3.26883 \\
\hline $\mathrm{C}$ & 0.48935 & -1.99117 & -2.86420 \\
\hline $\mathrm{C}$ & 1.86980 & -1.89483 & -2.69579 \\
\hline $\mathrm{C}$ & 2.50332 & -2.55164 & -1.56337 \\
\hline $\mathrm{C}$ & 1.73439 & -3.29379 & -0.66588 \\
\hline $\mathrm{C}$ & 1.98656 & -3.19152 & 0.76240 \\
\hline $\mathrm{C}$ & 2.99220 & -2.34521 & 1.23452 \\
\hline $\mathrm{C}$ & 2.75697 & -1.52271 & 2.40960 \\
\hline $\mathrm{C}$ & 1.52306 & -1.58177 & 3.06187 \\
\hline $\mathrm{C}$ & 0.88704 & -0.36121 & 3.52205 \\
\hline $\mathrm{C}$ & 1.51193 & 0.87162 & 3.31524 \\
\hline $\mathrm{C}$ & 0.73193 & 2.02139 & 2.88495 \\
\hline $\mathrm{C}$ & -0.63628 & 1.87703 & 2.63767 \\
\hline $\mathrm{C}$ & -1.28420 & 2.52793 & 1.53500 \\
\hline $\mathrm{C}$ & -0.52173 & 3.27941 & 0.65723 \\
\hline $\mathrm{C}$ & 0.90763 & 3.40285 & 0.86921 \\
\hline $\mathrm{C}$ & 1.52761 & 2.79738 & 1.96840 \\
\hline $\mathrm{C}$ & 2.80574 & 2.12780 & 1.81136 \\
\hline $\mathrm{C}$ & 2.79483 & 0.93458 & 2.64784 \\
\hline $\mathrm{C}$ & 3.40453 & -0.24066 & 2.19690 \\
\hline $\mathrm{C}$ & 4.04554 & -0.27102 & 0.89460 \\
\hline $\mathrm{C}$ & 3.78912 & -1.57063 & 0.29864 \\
\hline $\mathrm{C}$ & 3.55170 & -1.67501 & -1.07390 \\
\hline $\mathrm{C}$ & 3.56939 & -0.48359 & -1.90953 \\
\hline $\mathrm{C}$ & 2.54010 & -0.62421 & -2.91860 \\
\hline $\mathrm{C}$ & 1.79909 & 0.48946 & -3.32632 \\
\hline $\mathrm{C}$ & 0.37077 & 0.37873 & -3.52845 \\
\hline $\mathrm{C}$ & -0.23733 & 1.60713 & -3.07897 \\
\hline $\mathrm{C}$ & 0.79663 & 2.48212 & -2.58432 \\
\hline $\mathrm{C}$ & 2.06641 & 1.79502 & -2.73604 \\
\hline $\mathrm{C}$ & 3.04902 & 1.92360 & -1.75380 \\
\hline $\mathrm{C}$ & 3.82056 & 0.76768 & -1.33582 \\
\hline $\mathrm{C}$ & 4.05563 & 0.87427 & 0.09332 \\
\hline $\mathrm{C}$ & 3.42552 & 2.09605 & 0.56226 \\
\hline $\mathrm{C}$ & 2.79587 & 2.74208 & -0.57739 \\
\hline
\end{tabular}




$\begin{array}{lrrr}\mathrm{C} & 1.56482 & 3.38229 & -0.42478 \\ \mathrm{C} & 0.54217 & 3.25586 & -1.44707 \\ \mathrm{C} & -0.75338 & 3.21276 & -0.79912 \\ \mathrm{C} & -1.76151 & 2.41873 & -1.31291 \\ \mathrm{C} & -1.47174 & 1.56663 & -2.42664 \\ \mathrm{C} & -2.19877 & 0.32190 & -2.23387 \\ \mathrm{C} & -3.23627 & 0.51287 & -1.26354 \\ \mathrm{C} & -2.89289 & 1.81734 & -0.49703 \\ \mathrm{C} & -2.56257 & 1.77688 & 1.15776 \\ \mathrm{C} & -3.84998 & 2.58774 & 1.26171 \\ \mathrm{C} & -4.06356 & 2.68494 & -0.05227 \\ \mathrm{H} & -4.34404 & 2.98671 & 2.14222 \\ \mathrm{H} & -4.80513 & 3.19628 & -0.65803\end{array}$

(9) $\mathrm{C}_{2} \mathrm{H}_{2} \_\mathrm{C}_{59} \mathrm{Pd}$ PH_C4-C5

$\begin{array}{crrr}\text { Pd } & -3.88679 & -0.87201 & -0.06716 \\ \mathrm{C} & -2.42108 & -1.69100 & -1.14947 \\ \mathrm{C} & -1.67859 & -2.67145 & -0.38630 \\ \mathrm{C} & -1.74939 & -2.43561 & 1.04645 \\ \mathrm{C} & -2.64550 & -1.35679 & 1.42921 \\ \mathrm{C} & -2.09776 & -0.30452 & 2.20087 \\ \mathrm{C} & -0.94517 & -0.63000 & 3.06183 \\ \mathrm{C} & -0.25074 & -1.82122 & 2.88996 \\ \mathrm{C} & -0.64714 & -2.75271 & 1.84858 \\ \mathrm{C} & 0.55804 & -3.31279 & 1.27510 \\ \mathrm{C} & 0.63358 & -3.55539 & -0.09372 \\ \mathrm{C} & -0.49784 & -3.20744 & -0.93521 \\ \mathrm{C} & 0.01488 & -2.69162 & -2.17982 \\ \mathrm{C} & -0.64483 & -1.63099 & -2.81058 \\ \mathrm{C} & -1.86390 & -1.08414 & -2.25673 \\ \mathrm{C} & -1.84194 & 0.38916 & -2.51870 \\ \mathrm{C} & -0.61175 & 0.67557 & -3.21684 \\ \mathrm{C} & 0.11898 & -0.56108 & -3.41553 \\ \mathrm{C} & 1.51120 & -0.57916 & -3.36782 \\ \mathrm{C} & 2.19928 & -1.67128 & -2.69738 \\ \mathrm{C} & 1.46439 & -2.71094 & -2.12237 \\ \mathrm{C} & 1.84727 & -3.23515 & -0.82061 \\ \mathrm{C} & 2.94675 & -2.69616 & -0.14886 \\ \mathrm{C} & 2.86934 & -2.44903 & 1.28310 \\ \mathrm{C} & 1.69999 & -2.75293 & 1.98106 \\ \mathrm{C} & 1.19554 & -1.83251 & 2.98487 \\ \mathrm{C} & 1.88397 & -0.64590 & 3.25607 \\ \mathrm{C} & 1.14935 & 0.59126 & 3.44917 \\ \mathrm{C} & -0.24132 & 0.58725 & 3.33297 \\ \mathrm{C} & -0.94634 & 1.64854 & 2.62761 \\ \mathrm{C} & -0.20116 & 2.72173 & 2.11383 \\ \mathrm{C} & 1.24261 & 2.70582 & 2.20138\end{array}$




$\begin{array}{lrrr}\mathrm{C} & 1.91758 & 1.67193 & 2.86543 \\ \mathrm{C} & 3.11939 & 1.10255 & 2.28828 \\ \mathrm{C} & 3.10353 & -0.33433 & 2.53455 \\ \mathrm{C} & 3.58447 & -1.21643 & 1.56617 \\ \mathrm{C} & 4.10526 & -0.70289 & 0.30785 \\ \mathrm{C} & 3.70588 & -1.61390 & -0.74856 \\ \mathrm{C} & 3.34596 & -1.11604 & -2.00628 \\ \mathrm{C} & 3.36778 & 0.31702 & -2.24893 \\ \mathrm{C} & 2.24139 & 0.64967 & -3.09974 \\ \mathrm{C} & 1.54725 & 1.84495 & -2.91060 \\ \mathrm{C} & 0.09556 & 1.86227 & -2.97589 \\ \mathrm{C} & -0.38734 & 2.78279 & -1.98287 \\ \mathrm{C} & 0.75009 & 3.33558 & -1.28409 \\ \mathrm{C} & 1.95420 & 2.76143 & -1.85703 \\ \mathrm{C} & 3.02868 & 2.43650 & -1.02836 \\ \mathrm{C} & 3.75257 & 1.19426 & -1.22942 \\ \mathrm{C} & 4.12330 & 0.67356 & 0.07444 \\ \mathrm{C} & 3.62922 & 1.59519 & 1.08579 \\ \mathrm{C} & 2.94533 & 2.67993 & 0.40522 \\ \mathrm{C} & 1.78141 & 3.22437 & 0.95214 \\ \mathrm{C} & 0.66376 & 3.55349 & 0.08957 \\ \mathrm{C} & -0.56185 & 3.24329 & 0.80254 \\ \mathrm{C} & -1.67253 & 2.75711 & 0.13083 \\ \mathrm{C} & -1.56490 & 2.47917 & -1.27650 \\ \mathrm{C} & -2.35904 & 1.32179 & -1.63051 \\ \mathrm{C} & -3.28859 & 1.06349 & -0.50313 \\ \mathrm{C} & -2.71265 & 1.83529 & 0.79027 \\ \mathrm{C} & -2.10664 & 1.10189 & 1.97398 \\ \mathrm{C} & -4.12869 & 2.45897 & 0.79729 \\ \mathrm{C} & -4.56839 & 1.81884 & -0.30759 \\ \mathrm{H} & -4.53129 & 3.27093 & 1.39681 \\ \mathrm{H} & -5.45260 & 1.91411 & -0.92948\end{array}$

(10) $\mathrm{C}_{2} \mathrm{H}_{2}$ CC59Pd_HH_C6-C7

$\begin{array}{cccc}\text { Pd } & -3.87944 & -0.82558 & -0.20386 \\ \mathrm{C} & -3.24704 & 0.87210 & 0.82582 \\ \mathrm{C} & -2.74925 & 1.96842 & -0.30294 \\ \mathrm{C} & -2.34849 & 1.24114 & -1.58461 \\ \mathrm{C} & -2.61091 & -0.15198 & -1.59349 \\ \mathrm{C} & -1.78788 & -1.06031 & -2.26379 \\ \mathrm{C} & -0.76399 & -0.54062 & -3.12491 \\ \mathrm{C} & -0.53045 & 0.84216 & -3.18762 \\ \mathrm{C} & -1.31286 & 1.73094 & -2.38844 \\ \mathrm{C} & -0.42535 & 2.81294 & -1.93213 \\ \mathrm{C} & -0.57386 & 3.31310 & -0.64921 \\ \mathrm{C} & -1.64236 & 2.82755 & 0.21175 \\ \mathrm{C} & -1.12224 & 2.69141 & 1.50796\end{array}$




\begin{tabular}{|c|c|c|c|}
\hline C & -1.44652 & 1.57439 & 2.34747 \\
\hline $\mathrm{C}$ & -2.33689 & 0.55876 & 1.93888 \\
\hline $\mathrm{C}$ & -1.83412 & -0.72457 & 2.39920 \\
\hline $\mathrm{C}$ & -0.65057 & -0.44797 & 3.20292 \\
\hline $\mathrm{C}$ & -0.40853 & 0.97427 & 3.17676 \\
\hline $\mathrm{C}$ & 0.88987 & 1.46996 & 3.17479 \\
\hline $\mathrm{C}$ & 1.23490 & 2.60141 & 2.30880 \\
\hline $\mathrm{C}$ & 0.26714 & 3.19409 & 1.51183 \\
\hline $\mathrm{C}$ & 0.59721 & 3.58268 & 0.16131 \\
\hline $\mathrm{C}$ & 1.87809 & 3.35092 & -0.34993 \\
\hline $\mathrm{C}$ & 2.03362 & 2.83067 & -1.69599 \\
\hline C & 0.89766 & 2.55977 & -2.46540 \\
\hline $\mathrm{C}$ & 0.83186 & 1.34346 & -3.25654 \\
\hline $\mathrm{C}$ & 1.90167 & 0.45030 & -3.25817 \\
\hline $\mathrm{C}$ & 1.65670 & -0.98597 & -3.20826 \\
\hline $\mathrm{C}$ & 0.34963 & -1.46600 & -3.13900 \\
\hline $\mathrm{C}$ & 0.01218 & -2.57946 & -2.27205 \\
\hline $\mathrm{C}$ & 1.02340 & -3.19302 & -1.51866 \\
\hline $\mathrm{C}$ & 2.37562 & -2.66940 & -1.56110 \\
\hline $\mathrm{C}$ & 2.69345 & -1.59240 & -2.39713 \\
\hline $\mathrm{C}$ & 3.56334 & -0.53295 & -1.92316 \\
\hline $\mathrm{C}$ & 3.08288 & 0.72966 & -2.46382 \\
\hline $\mathrm{C}$ & 3.14430 & 1.89253 & -1.69055 \\
\hline $\mathrm{C}$ & 3.68089 & 1.83860 & -0.34282 \\
\hline $\mathrm{C}$ & 2.88810 & 2.73553 & 0.48713 \\
\hline $\mathrm{C}$ & 2.57377 & 2.37579 & 1.79954 \\
\hline $\mathrm{C}$ & 3.05017 & 1.11229 & 2.34407 \\
\hline $\mathrm{C}$ & 2.01449 & 0.56855 & 3.20439 \\
\hline $\mathrm{C}$ & 1.78657 & -0.81445 & 3.24348 \\
\hline $\mathrm{C}$ & 0.43706 & -1.32365 & 3.26327 \\
\hline $\mathrm{C}$ & 0.40678 & -2.53798 & 2.46957 \\
\hline $\mathrm{C}$ & 1.74209 & -2.77920 & 1.94884 \\
\hline $\mathrm{C}$ & 2.59739 & -1.70454 & 2.42580 \\
\hline $\mathrm{C}$ & 3.58398 & -1.17714 & 1.59260 \\
\hline $\mathrm{C}$ & 3.81739 & 0.25779 & 1.55232 \\
\hline $\mathrm{C}$ & 4.12916 & 0.62437 & 0.17948 \\
\hline $\mathrm{C}$ & 4.08368 & -0.58312 & -0.62738 \\
\hline C & 3.75048 & -1.69663 & 0.24252 \\
\hline C & 2.92376 & -2.72380 & -0.21708 \\
\hline C & 1.90197 & -3.28023 & 0.65736 \\
\hline $\mathrm{C}$ & 0.73535 & -3.57185 & -0.15461 \\
\hline $\mathrm{C}$ & -0.54241 & -3.35363 & 0.36127 \\
\hline C & -0.70448 & -2.80950 & 1.68610 \\
\hline $\mathrm{C}$ & -1.89070 & -1.93067 & 1.65780 \\
\hline $\mathrm{C}$ & -2.61276 & -2.20883 & 0.44759 \\
\hline $\mathrm{C}$ & -1.62100 & -2.79807 & -0.44680 \\
\hline C & -1.31651 & -2.36886 & -1.73871 \\
\hline C & -4.14520 & 2.53514 & -0.07451 \\
\hline $\mathrm{C}$ & -4.50605 & 1.64543 & 0.88614 \\
\hline
\end{tabular}




$\begin{array}{llll}\mathrm{H} & -4.61568 & 3.41954 & -0.49325 \\ \mathrm{H} & -5.36708 & 1.56118 & 1.54310\end{array}$

(11) $\mathrm{C}_{2} \mathrm{H}_{2} \_\mathrm{C} 599 \mathrm{Pd} \_\mathrm{HH} \_\mathrm{C} 7-\mathrm{C} 8$

\begin{tabular}{|c|c|c|c|}
\hline $\mathrm{Ni}$ & -3.60892 & -1.52946 & 0.00269 \\
\hline $\mathrm{C}$ & -3.14350 & -0.30892 & 1.43286 \\
\hline $\mathrm{C}$ & -3.39828 & 1.08814 & 1.30793 \\
\hline $\mathrm{C}$ & -3.39737 & 1.08204 & -1.31244 \\
\hline $\mathrm{C}$ & -3.14231 & -0.31549 & -1.43235 \\
\hline $\mathrm{C}$ & -2.13583 & -0.78235 & -2.28004 \\
\hline $\mathrm{C}$ & -1.38062 & 0.19422 & -3.02871 \\
\hline $\mathrm{C}$ & -1.42429 & 1.53144 & -2.62086 \\
\hline $\mathrm{C}$ & -2.33549 & 1.95709 & -1.61494 \\
\hline C & -1.58922 & 2.90113 & -0.71309 \\
\hline $\mathrm{C}$ & -1.58947 & 2.90413 & 0.70185 \\
\hline $\mathrm{C}$ & -2.33629 & 1.96408 & 1.60740 \\
\hline $\mathrm{C}$ & -1.42532 & 1.54193 & 2.61494 \\
\hline $\mathrm{C}$ & -1.38181 & 0.20609 & 3.02747 \\
\hline $\mathrm{C}$ & -2.13689 & -0.77295 & 2.28207 \\
\hline C & -1.30074 & -1.99498 & 2.21370 \\
\hline C & -0.07773 & -1.72544 & 2.95547 \\
\hline C & -0.12143 & -0.36341 & 3.43762 \\
\hline C & 1.03479 & 0.41796 & 3.46755 \\
\hline C & 0.98094 & 1.80350 & 3.02232 \\
\hline $\mathrm{C}$ & -0.22619 & 2.33168 & 2.57079 \\
\hline $\mathrm{C}$ & -0.30968 & 3.16165 & 1.38740 \\
\hline $\mathrm{C}$ & 0.87737 & 3.44833 & 0.71341 \\
\hline $\mathrm{C}$ & 0.87757 & 3.44553 & -0.72614 \\
\hline $\mathrm{C}$ & -0.30928 & 3.15610 & -1.39932 \\
\hline $\mathrm{C}$ & -0.22535 & 2.32160 & -2.57947 \\
\hline $\mathrm{C}$ & 0.98196 & 1.79189 & -3.02874 \\
\hline C & 1.03610 & 0.40475 & -3.46886 \\
\hline C & -0.12005 & -0.37662 & -3.43652 \\
\hline $\mathrm{C}$ & -0.07634 & -1.73692 & -2.94953 \\
\hline $\mathrm{C}$ & 1.14762 & -2.29153 & -2.56891 \\
\hline $\mathrm{C}$ & 2.35205 & -1.48728 & -2.59182 \\
\hline C & 2.29496 & -0.15627 & -3.02398 \\
\hline C & 3.01909 & 0.87631 & -2.30660 \\
\hline $\mathrm{C}$ & 2.20173 & 2.08018 & -2.30842 \\
\hline C & 2.14782 & 2.89696 & -1.17859 \\
\hline C & 2.92887 & 2.55204 & -0.00434 \\
\hline C & 2.14744 & 2.90144 & 1.16833 \\
\hline C & 2.20095 & 2.08895 & 2.30129 \\
\hline $\mathrm{C}$ & 3.01824 & 0.88505 & 2.30427 \\
\hline $\mathrm{C}$ & 2.29373 & -0.14480 & 3.02519 \\
\hline $\mathrm{C}$ & 2.35088 & -1.47741 & 2.59808 \\
\hline $\mathrm{C}$ & 1.14639 & -2.28162 & 2.57762 \\
\hline C & 1.20005 & -3.15059 & 1.41985 \\
\hline
\end{tabular}




$\begin{array}{lrrr}\mathrm{C} & 2.43162 & -2.87134 & 0.70292 \\ \mathrm{C} & 3.14118 & -1.82930 & 1.42993 \\ \mathrm{C} & 3.82744 & -0.83456 & 0.72962 \\ \mathrm{C} & 3.76514 & 0.54758 & 1.17379 \\ \mathrm{C} & 3.72382 & 1.39953 & -0.00202 \\ \mathrm{C} & 3.76560 & 0.54313 & -1.17460 \\ \mathrm{C} & 3.82775 & -0.83731 & -0.72516 \\ \mathrm{C} & 3.14182 & -1.83471 & -1.42198 \\ \mathrm{C} & 2.43198 & -2.87401 & -0.69133 \\ \mathrm{C} & 1.20078 & -3.15608 & -1.40776 \\ \mathrm{C} & 0.02606 & -3.42545 & -0.71518 \\ \mathrm{C} & 0.02562 & -3.42254 & 0.72777 \\ \mathrm{C} & -1.27557 & -2.89730 & 1.14571 \\ \mathrm{C} & -2.18259 & -2.82066 & 0.00431 \\ \mathrm{C} & -1.27487 & -2.90199 & -1.13591 \\ \mathrm{C} & -1.29962 & -2.00390 & -2.20735 \\ \mathrm{C} & -4.63490 & 1.57324 & -0.66521 \\ \mathrm{C} & -4.63526 & 1.57670 & 0.65776 \\ \mathrm{H} & -5.45801 & 1.91299 & -1.29191 \\ \mathrm{H} & -5.45867 & 1.91977 & 1.28225\end{array}$

\section{Ethylene}

(1) $\mathrm{C}_{2} \mathrm{H}_{4} \mathrm{C}_{59} \mathrm{Pd} \_\mathrm{TM}$

\begin{tabular}{|c|c|c|c|}
\hline $\mathrm{Pd}$ & 3.91817 & 0.35515 & -0.00045 \\
\hline $\mathrm{C}$ & 2.67160 & -0.67836 & 1.23726 \\
\hline C & 2.22161 & -1.94696 & 0.72505 \\
\hline $\mathrm{C}$ & 2.22142 & -1.94581 & -0.72878 \\
\hline C & 2.67130 & -0.67640 & -1.23918 \\
\hline C & 1.98260 & -0.02638 & -2.24454 \\
\hline C & 0.96075 & -0.75812 & -2.96838 \\
\hline C & 0.59531 & -2.05020 & -2.57877 \\
\hline C & 1.21853 & -2.64692 & -1.42289 \\
\hline C & 0.19899 & -3.38641 & -0.69867 \\
\hline C & 0.19917 & -3.38751 & 0.69325 \\
\hline C & 1.21890 & -2.64914 & 1.41834 \\
\hline C & 0.59601 & -2.05422 & 2.57531 \\
\hline C & 0.96155 & -0.76271 & 2.96678 \\
\hline C & 1.98322 & -0.02989 & 2.24381 \\
\hline C & 1.59554 & 1.40390 & 2.24522 \\
\hline C & 0.34339 & 1.50024 & 2.96365 \\
\hline C & -0.04350 & 0.17741 & 3.41708 \\
\hline C & -1.38620 & -0.19841 & 3.45376 \\
\hline C & -1.77517 & -1.53129 & 3.01918 \\
\hline C & -0.80458 & -2.44029 & 2.59210 \\
\hline C & -1.05192 & -3.26408 & 1.42153 \\
\hline C & -2.25623 & -3.14715 & 0.72530 \\
\hline
\end{tabular}




\begin{tabular}{|c|c|c|c|}
\hline $\mathrm{C}$ & -2.25642 & -3.14603 & -0.72969 \\
\hline $\mathrm{C}$ & -1.05230 & -3.26186 & -1.42643 \\
\hline $\mathrm{C}$ & -0.80528 & -2.43626 & -2.59577 \\
\hline $\mathrm{C}$ & -1.77600 & -1.52663 & -3.02122 \\
\hline $\mathrm{C}$ & -1.38715 & -0.19308 & -3.45386 \\
\hline $\mathrm{C}$ & -0.04444 & 0.18267 & -3.41696 \\
\hline $\mathrm{C}$ & 0.34255 & 1.50480 & -2.96160 \\
\hline $\mathrm{C}$ & -0.65263 & 2.41696 & -2.58110 \\
\hline $\mathrm{C}$ & -2.04573 & 2.01979 & -2.59123 \\
\hline $\mathrm{C}$ & -2.41145 & 0.74149 & -3.02234 \\
\hline $\mathrm{C}$ & -3.42167 & -0.00819 & -2.30252 \\
\hline $\mathrm{C}$ & -3.03031 & -1.41110 & -2.30352 \\
\hline $\mathrm{C}$ & -3.26595 & -2.20266 & -1.17648 \\
\hline $\mathrm{C}$ & -3.89291 & -1.62323 & -0.00080 \\
\hline $\mathrm{C}$ & -3.26563 & -2.20447 & 1.17382 \\
\hline $\mathrm{C}$ & -3.02968 & -1.41464 & 2.30201 \\
\hline $\mathrm{C}$ & -3.42104 & -0.01173 & 2.30327 \\
\hline $\mathrm{C}$ & -2.41061 & 0.73683 & 3.02395 \\
\hline $\mathrm{C}$ & -2.04500 & 2.01578 & 2.59471 \\
\hline $\mathrm{C}$ & -0.65190 & 2.41297 & 2.58482 \\
\hline $\mathrm{C}$ & -0.43336 & 3.24615 & 1.42534 \\
\hline $\mathrm{C}$ & -1.68869 & 3.36358 & 0.70051 \\
\hline $\mathrm{C}$ & -2.68889 & 2.60023 & 1.42707 \\
\hline $\mathrm{C}$ & -3.65579 & 1.87417 & 0.72932 \\
\hline $\mathrm{C}$ & -4.03124 & 0.54351 & 1.17614 \\
\hline $\mathrm{C}$ & -4.26494 & -0.27694 & 0.00029 \\
\hline $\mathrm{C}$ & -4.03155 & 0.54532 & -1.17436 \\
\hline $\mathrm{C}$ & -3.65599 & 1.87528 & -0.72559 \\
\hline $\mathrm{C}$ & -2.68929 & 2.60242 & -1.42249 \\
\hline $\mathrm{C}$ & -1.68890 & 3.36466 & -0.69503 \\
\hline $\mathrm{C}$ & -0.43377 & 3.24835 & -1.42038 \\
\hline $\mathrm{C}$ & 0.76529 & 3.13756 & -0.72075 \\
\hline $\mathrm{C}$ & 0.76552 & 3.13644 & 0.72520 \\
\hline $\mathrm{C}$ & 1.83244 & 2.23826 & 1.14812 \\
\hline $\mathrm{C}$ & 2.67993 & 1.92565 & 0.00097 \\
\hline $\mathrm{C}$ & 1.83206 & 2.24002 & -1.14535 \\
\hline $\mathrm{C}$ & 1.59488 & 1.40736 & -2.24368 \\
\hline $\mathrm{C}$ & 5.65554 & -1.30903 & 0.00269 \\
\hline $\mathrm{C}$ & 6.27393 & -0.08957 & -0.00076 \\
\hline $\mathrm{H}$ & 6.59663 & 0.38221 & -0.92590 \\
\hline $\mathrm{H}$ & 6.59763 & 0.38689 & 0.92163 \\
\hline $\mathrm{H}$ & 5.47674 & -1.85369 & -0.91957 \\
\hline $\mathrm{H}$ & 5.47756 & -1.84875 & 0.92800 \\
\hline
\end{tabular}

(2) $\mathrm{C}_{2} \mathrm{H}_{4} \mathrm{C}_{59} \mathrm{Pd} \_\mathrm{TC} 1$

$\begin{array}{cccc}\mathrm{Ni} & 3.94103 & -0.28535 & -0.00004 \\ \mathrm{C} & 2.84963 & 0.71559 & -1.18821\end{array}$




\begin{tabular}{|c|c|c|c|}
\hline $\mathrm{C}$ & 2.38857 & 2.00043 & -0.72636 \\
\hline $\mathrm{C}$ & 2.38874 & 2.00104 & 0.72458 \\
\hline $\mathrm{C}$ & 2.84990 & 0.71659 & 1.18726 \\
\hline $\mathrm{C}$ & 2.18449 & 0.06769 & 2.21676 \\
\hline $\mathrm{C}$ & 1.15507 & 0.78195 & 2.95068 \\
\hline $\mathrm{C}$ & 0.76658 & 2.07120 & 2.57453 \\
\hline $\mathrm{C}$ & 1.37869 & 2.68536 & 1.42080 \\
\hline $\mathrm{C}$ & 0.34708 & 3.40480 & 0.69622 \\
\hline $\mathrm{C}$ & 0.34696 & 3.40428 & -0.69864 \\
\hline $\mathrm{C}$ & 1.37844 & 2.68431 & -1.42289 \\
\hline $\mathrm{C}$ & 0.76612 & 2.06938 & -2.57609 \\
\hline $\mathrm{C}$ & 1.15451 & 0.77988 & -2.95144 \\
\hline $\mathrm{C}$ & 2.18403 & 0.06613 & -2.21721 \\
\hline $\mathrm{C}$ & 1.80896 & -1.37256 & -2.21803 \\
\hline $\mathrm{C}$ & 0.56217 & -1.48974 & -2.94986 \\
\hline $\mathrm{C}$ & 0.16110 & -0.17430 & -3.40459 \\
\hline $\mathrm{C}$ & -1.18665 & 0.18072 & -3.45026 \\
\hline $\mathrm{C}$ & -1.59834 & 1.50745 & -3.02108 \\
\hline $\mathrm{C}$ & -0.64145 & 2.42999 & -2.59274 \\
\hline $\mathrm{C}$ & -0.90276 & 3.25445 & -1.42504 \\
\hline $\mathrm{C}$ & -2.10484 & 3.11842 & -0.72851 \\
\hline $\mathrm{C}$ & -2.10472 & 3.11892 & 0.72668 \\
\hline $\mathrm{C}$ & -0.90252 & 3.25547 & 1.42292 \\
\hline $\mathrm{C}$ & -0.64100 & 2.43181 & 2.59116 \\
\hline $\mathrm{C}$ & -1.59780 & 1.50952 & 3.02023 \\
\hline $\mathrm{C}$ & -1.18602 & 0.18307 & 3.45023 \\
\hline $\mathrm{C}$ & 0.16174 & -0.17196 & 3.40460 \\
\hline $\mathrm{C}$ & 0.56273 & -1.48768 & 2.95062 \\
\hline $\mathrm{C}$ & -0.41622 & -2.41497 & 2.57358 \\
\hline $\mathrm{C}$ & -1.81652 & -2.04370 & 2.59133 \\
\hline $\mathrm{C}$ & -2.19600 & -0.76830 & 3.01798 \\
\hline $\mathrm{C}$ & -3.22090 & -0.03553 & 2.30216 \\
\hline $\mathrm{C}$ & -2.84953 & 1.37262 & 2.30144 \\
\hline $\mathrm{C}$ & -3.09886 & 2.16010 & 1.17465 \\
\hline $\mathrm{C}$ & -3.71510 & 1.56763 & -0.00025 \\
\hline $\mathrm{C}$ & -3.09908 & 2.15930 & -1.17566 \\
\hline $\mathrm{C}$ & -2.84994 & 1.37105 & -2.30196 \\
\hline $\mathrm{C}$ & -3.22132 & -0.03709 & -2.30166 \\
\hline $\mathrm{C}$ & -2.19657 & -0.77036 & -3.01718 \\
\hline $\mathrm{C}$ & -1.81702 & -2.04548 & -2.58974 \\
\hline $\mathrm{C}$ & -0.41672 & -2.41676 & -2.57200 \\
\hline $\mathrm{C}$ & -0.18873 & -3.25924 & -1.41770 \\
\hline $\mathrm{C}$ & -1.44425 & -3.39189 & -0.69658 \\
\hline $\mathrm{C}$ & -2.45331 & -2.64010 & -1.42353 \\
\hline $\mathrm{C}$ & -3.43037 & -1.92660 & -0.72645 \\
\hline $\mathrm{C}$ & -3.82333 & -0.60117 & -1.17436 \\
\hline $\mathrm{C}$ & -4.07050 & 0.21679 & 0.00025 \\
\hline $\mathrm{C}$ & -3.82313 & -0.60037 & 1.17537 \\
\hline $\mathrm{C}$ & -3.43024 & -1.92612 & 0.72828 \\
\hline
\end{tabular}




$\begin{array}{lrrr}\mathrm{C} & -2.45304 & -2.63914 & 1.42567 \\ \mathrm{C} & -1.44412 & -3.39141 & 0.69905 \\ \mathrm{C} & -0.18846 & -3.25823 & 1.41983 \\ \mathrm{C} & 1.01079 & -3.14059 & 0.72487 \\ \mathrm{C} & 1.01067 & -3.14113 & -0.72308 \\ \mathrm{C} & 2.06751 & -2.23013 & -1.14475 \\ \mathrm{C} & 2.89352 & -1.84186 & 0.00025 \\ \mathrm{C} & 2.06772 & -2.22929 & 1.14569 \\ \mathrm{C} & 1.80939 & -1.37098 & 2.21848 \\ \mathrm{C} & 6.12737 & -0.34011 & -0.00003 \\ \mathrm{C} & 5.74350 & 0.97671 & -0.00003 \\ \mathrm{H} & 5.67880 & 1.54447 & -0.92361 \\ \mathrm{H} & 5.67883 & 1.54441 & 0.92359 \\ \mathrm{H} & 6.35684 & -0.86712 & -0.92298 \\ \mathrm{H} & 6.35692 & -0.86712 & 0.92290\end{array}$

(3) $\mathrm{C}_{2} \mathrm{H}_{4} \_\mathrm{C}_{59} \mathrm{Pd} \_\mathrm{TC} 5$

$\begin{array}{cccc}\text { Pd } & 4.02094 & -0.96241 & 0.00018 \\ \mathrm{C} & 2.56081 & -1.44785 & 1.23666 \\ \mathrm{C} & 1.79111 & -2.57169 & 0.73085 \\ \mathrm{C} & 1.79122 & -2.57316 & -0.72700 \\ \mathrm{C} & 2.56115 & -1.45050 & -1.23472 \\ \mathrm{C} & 1.99680 & -0.59539 & -2.17368 \\ \mathrm{C} & 0.82123 & -1.06837 & -2.88706 \\ \mathrm{C} & 0.18270 & -2.26777 & -2.54790 \\ \mathrm{C} & 0.65070 & -3.01371 & -1.40755 \\ \mathrm{C} & -0.50626 & -3.51913 & -0.69393 \\ \mathrm{C} & -0.50633 & -3.51801 & 0.69892 \\ \mathrm{C} & 0.65052 & -3.01128 & 1.41188 \\ \mathrm{C} & 0.18233 & -2.26380 & 2.55110 \\ \mathrm{C} & 0.82076 & -1.06396 & 2.88865 \\ \mathrm{C} & 1.99639 & -0.59181 & 2.17468 \\ \mathrm{C} & 1.90735 & 0.92242 & 2.13855 \\ \mathrm{C} & 0.67800 & 1.26278 & 2.86620 \\ \mathrm{C} & 0.03467 & 0.05967 & 3.33329 \\ \mathrm{C} & -1.35672 & -0.03011 & 3.42103 \\ \mathrm{C} & -2.02350 & -1.25288 & 3.00907 \\ \mathrm{C} & -1.26719 & -2.34481 & 2.58273 \\ \mathrm{C} & -1.69583 & -3.11545 & 1.42603 \\ \mathrm{C} & -2.84892 & -2.74649 & 0.72964 \\ \mathrm{C} & -2.84883 & -2.74757 & -0.72604 \\ \mathrm{C} & -1.69568 & -3.11759 & -1.42175 \\ \mathrm{C} & -1.26682 & -2.34867 & -2.57952 \\ \mathrm{C} & -2.02301 & -1.25729 & -3.00751 \\ \mathrm{C} & -1.35612 & -0.03511 & -3.42106 \\ \mathrm{C} & 0.03527 & 0.05473 & -3.33321 \\ \mathrm{C} & 0.67858 & 1.25841 & -2.86754\end{array}$




\begin{tabular}{lrrr}
$\mathrm{C}$ & -0.11347 & 2.36428 & -2.54716 \\
$\mathrm{C}$ & -1.56161 & 2.26878 & -2.58446 \\
$\mathrm{C}$ & -2.17353 & 1.08942 & -3.00859 \\
$\mathrm{C}$ & -3.32658 & 0.56746 & -2.30051 \\
$\mathrm{C}$ & -3.23272 & -0.88495 & -2.29922 \\
$\mathrm{C}$ & -3.63346 & -1.61072 & -1.17421 \\
$\mathrm{C}$ & -4.12542 & -0.91051 & 0.00037 \\
$\mathrm{C}$ & -3.63365 & -1.60901 & 1.17605 \\
$\mathrm{C}$ & -3.23310 & -0.88159 & 2.30006 \\
$\mathrm{C}$ & -3.32701 & 0.57082 & 2.29927 \\
$\mathrm{C}$ & -2.17410 & 1.09384 & 3.00681 \\
$\mathrm{C}$ & -1.56216 & 2.27264 & 2.58111 \\
$\mathrm{C}$ & -0.11402 & 2.36820 & 2.54408 \\
$\mathrm{C}$ & 0.25052 & 3.17267 & 1.40583 \\
$\mathrm{C}$ & -0.96078 & 3.53560 & 0.69413 \\
$\mathrm{C}$ & -2.08639 & 2.98278 & 1.42305 \\
$\mathrm{C}$ & -3.18181 & 2.46678 & 0.72603 \\
$\mathrm{C}$ & -3.81632 & 1.23959 & 1.17429 \\
$\mathrm{C}$ & -4.21516 & 0.48297 & -0.00064 \\
$\mathrm{C}$ & -3.81606 & 1.23788 & -1.17659 \\
$\mathrm{C}$ & -3.18164 & 2.46570 & -0.73000 \\
$\mathrm{C}$ & -2.08603 & 2.98064 & -1.42755 \\
$\mathrm{C}$ & -0.96059 & 3.53449 & -0.69917 \\
$\mathrm{C}$ & 0.25089 & 3.17035 & -1.41000 \\
$\mathrm{C}$ & 1.42023 & 2.86307 & -0.73153 \\
$\mathrm{C}$ & 1.42004 & 2.86449 & 0.72808 \\
$\mathrm{C}$ & 2.34616 & 1.82526 & 1.17187 \\
$\mathrm{C}$ & 3.32698 & 1.76036 & -0.00037 \\
$\mathrm{C}$ & 2.34640 & 1.82282 & -1.17334 \\
$\mathrm{C}$ & 1.90783 & 0.91895 & -2.13915 \\
$\mathrm{C}$ & 4.39757 & 2.85299 & -0.00097 \\
$\mathrm{C}$ & 4.80981 & 1.40834 & 0.00045 \\
$\mathrm{H}$ & 5.26939 & 1.04573 & 0.92250 \\
& 5.27007 & 1.04500 & -0.92112 \\
& 4.53350 & 3.41932 & -0.91757 \\
$\mathrm{H}$ & 4.53269 & 3.42070 & 0.91491 \\
\hline
\end{tabular}

(4) $\mathrm{C}_{2} \mathrm{H}_{4} \_\mathrm{C} 599 \mathrm{Pd} \_\mathrm{HH} \_\mathrm{M}-\mathrm{C} 5$

$\begin{array}{cccc}\text { Pd } & 3.91778 & -0.35581 & 0.00002 \\ \mathrm{C} & 2.67180 & 0.67765 & -1.23799 \\ \mathrm{C} & 2.22182 & 1.94667 & -0.72687 \\ \mathrm{C} & 2.22181 & 1.94663 & 0.72699 \\ \mathrm{C} & 2.67178 & 0.67759 & 1.23806 \\ \mathrm{C} & 1.98311 & 0.02802 & 2.24384 \\ \mathrm{C} & 0.96133 & 0.76027 & 2.96731 \\ \mathrm{C} & 0.59587 & 2.05215 & 2.57700 \\ \mathrm{C} & 1.21892 & 2.64814 & 1.42070\end{array}$




\begin{tabular}{|c|c|c|c|}
\hline $\mathrm{C}$ & 0.19928 & 3.38707 & 0.69605 \\
\hline $\mathrm{C}$ & 0.19929 & 3.38710 & -0.69588 \\
\hline C & 1.21894 & 2.64821 & -1.42055 \\
\hline C & 0.59590 & 2.05227 & -2.57689 \\
\hline $\mathrm{C}$ & 0.96136 & 0.76041 & -2.96726 \\
\hline $\mathrm{C}$ & 1.98314 & 0.02813 & -2.24382 \\
\hline $\mathrm{C}$ & 1.59529 & -1.40563 & -2.24403 \\
\hline C & 0.34304 & -1.50240 & -2.96232 \\
\hline C & -0.04380 & -0.17996 & -3.41674 \\
\hline C & -1.38647 & 0.19588 & -3.45371 \\
\hline C & -1.77537 & 1.52910 & -3.02014 \\
\hline C & -0.80470 & 2.43835 & -2.59382 \\
\hline C & -1.05190 & 3.26308 & -1.42390 \\
\hline $\mathrm{C}$ & -2.25612 & 3.14672 & -0.72743 \\
\hline $\mathrm{C}$ & -2.25613 & 3.14668 & 0.72756 \\
\hline $\mathrm{C}$ & -1.05192 & 3.26302 & 1.42405 \\
\hline C & -0.80473 & 2.43823 & 2.59394 \\
\hline $\mathrm{C}$ & -1.77540 & 1.52896 & 3.02020 \\
\hline $\mathrm{C}$ & -1.38651 & 0.19571 & 3.45371 \\
\hline $\mathrm{C}$ & -0.04384 & -0.18012 & 3.41674 \\
\hline C & 0.34300 & -1.50254 & 2.96226 \\
\hline C & -0.65227 & -2.41497 & 2.58275 \\
\hline $\mathrm{C}$ & -2.04536 & -2.01775 & 2.59286 \\
\hline $\mathrm{C}$ & -2.41093 & -0.73912 & 3.02305 \\
\hline $\mathrm{C}$ & -3.42125 & 0.01006 & 2.30287 \\
\hline $\mathrm{C}$ & -3.02984 & 1.41294 & 2.30277 \\
\hline $\mathrm{C}$ & -3.26561 & 2.20369 & 1.17519 \\
\hline $\mathrm{C}$ & -3.89273 & 1.62339 & 0.00002 \\
\hline $\mathrm{C}$ & -3.26560 & 2.20374 & -1.17512 \\
\hline $\mathrm{C}$ & -3.02981 & 1.41305 & -2.30274 \\
\hline $\mathrm{C}$ & -3.42123 & 0.01017 & -2.30290 \\
\hline $\mathrm{C}$ & -2.41090 & -0.73898 & -3.02311 \\
\hline $\mathrm{C}$ & -2.04533 & -2.01763 & -2.59297 \\
\hline $\mathrm{C}$ & -0.65224 & -2.41485 & -2.58287 \\
\hline $\mathrm{C}$ & -0.43369 & -3.24735 & -1.42288 \\
\hline $\mathrm{C}$ & -1.68895 & -3.36412 & -0.69786 \\
\hline $\mathrm{C}$ & -2.68915 & -2.60119 & -1.42485 \\
\hline $\mathrm{C}$ & -3.65590 & -1.87454 & -0.72752 \\
\hline $\mathrm{C}$ & -4.03132 & -0.54421 & -1.17529 \\
\hline $\mathrm{C}$ & -4.26484 & 0.27713 & -0.00002 \\
\hline $\mathrm{C}$ & -4.03133 & -0.54426 & 1.17522 \\
\hline $\mathrm{C}$ & -3.65591 & -1.87458 & 0.72740 \\
\hline $\mathrm{C}$ & -2.68916 & -2.60126 & 1.42470 \\
\hline $\mathrm{C}$ & -1.68896 & -3.36415 & 0.69769 \\
\hline $\mathrm{C}$ & -0.43371 & -3.24741 & 1.42273 \\
\hline $\mathrm{C}$ & 0.76531 & -3.13742 & 0.72294 \\
\hline $\mathrm{C}$ & 0.76532 & -3.13739 & -0.72307 \\
\hline C & 1.83235 & -2.23974 & -1.14677 \\
\hline $\mathrm{C}$ & 2.68033 & -1.92654 & -0.00003 \\
\hline
\end{tabular}




$\begin{array}{lrrc}\mathrm{C} & 1.83234 & -2.23979 & 1.14669 \\ \mathrm{C} & 1.59526 & -1.40573 & 2.24399 \\ \mathrm{C} & 6.27295 & 0.09122 & -0.00003 \\ \mathrm{C} & 5.65336 & 1.31019 & 0.00001 \\ \mathrm{H} & 5.47456 & 1.85229 & -0.92370 \\ \mathrm{H} & 5.47462 & 1.85225 & 0.92376 \\ \mathrm{H} & 6.59654 & -0.38256 & -0.92384 \\ \mathrm{H} & 6.59659 & -0.38261 & 0.92373\end{array}$

(5) $\mathrm{C}_{2} \mathrm{H}_{4} \_\mathrm{C}_{59} \mathrm{Pd} \_\mathrm{PH}$ _M-C1

$\begin{array}{crrr}\text { Pd } & -3.91697 & -0.69851 & 0.00993 \\ \mathrm{C} & -2.54853 & -0.71751 & 1.47839 \\ \mathrm{C} & -2.20867 & 0.63402 & 1.86547 \\ \mathrm{C} & -2.43211 & 1.63017 & 0.85757 \\ \mathrm{C} & -3.22697 & 1.25410 & -0.38148 \\ \mathrm{C} & -2.32429 & 1.24477 & -1.58462 \\ \mathrm{C} & -1.38581 & 2.37560 & -1.69166 \\ \mathrm{C} & -1.03397 & 3.11361 & -0.56913 \\ \mathrm{C} & -1.54514 & 2.69577 & 0.75312 \\ \mathrm{C} & -0.48301 & 2.87838 & 1.70351 \\ \mathrm{C} & -0.32212 & 1.97645 & 2.75600 \\ \mathrm{C} & -1.18707 & 0.82830 & 2.82542 \\ \mathrm{C} & -0.41761 & -0.28771 & 3.30783 \\ \mathrm{C} & -0.66873 & -1.55953 & 2.78954 \\ \mathrm{C} & -1.73511 & -1.78140 & 1.83123 \\ \mathrm{C} & -1.25982 & -2.84918 & 0.90390 \\ \mathrm{C} & 0.08529 & -3.21870 & 1.31557 \\ \mathrm{C} & 0.44092 & -2.44573 & 2.48518 \\ \mathrm{C} & 1.75361 & -2.01734 & 2.68610 \\ \mathrm{C} & 2.01217 & -0.68354 & 3.20467 \\ \mathrm{C} & 0.94654 & 0.15961 & 3.52003 \\ \mathrm{C} & 1.00710 & 1.56967 & 3.16910 \\ \mathrm{C} & 2.12488 & 2.07928 & 2.50675 \\ \mathrm{C} & 1.95569 & 3.02320 & 1.40982 \\ \mathrm{C} & 0.67747 & 3.41936 & 1.01807 \\ \mathrm{C} & 0.34092 & 3.54700 & -0.38920 \\ \mathrm{C} & 1.31130 & 3.26295 & -1.35604 \\ \mathrm{C} & 0.94104 & 2.49793 & -2.52883 \\ \mathrm{C} & -0.37741 & 2.04946 & -2.67421 \\ \mathrm{C} & -0.64720 & 0.70386 & -3.10887 \\ \mathrm{C} & 0.40782 & -0.12427 & -3.45282 \\ \mathrm{C} & 1.77675 & 0.32828 & -3.32908 \\ \mathrm{C} & 2.04178 & 1.61738 & -2.85800 \\ \mathrm{C} & 3.10059 & 1.82702 & -1.88620 \\ \mathrm{C} & 2.64435 & 2.84290 & -0.94824 \\ \mathrm{C} & 2.96241 & 2.72969 & 0.40426 \\ & 3.74817 & 1.59900 & 0.87891\end{array}$




$\begin{array}{lrrr}\mathrm{C} & 3.23118 & 1.20186 & 2.17404 \\ \mathrm{C} & 3.17701 & -0.15586 & 2.52019 \\ \mathrm{C} & 3.64420 & -1.16197 & 1.58620 \\ \mathrm{C} & 2.77100 & -2.32177 & 1.69491 \\ \mathrm{C} & 2.43357 & -3.05621 & 0.55982 \\ \mathrm{C} & 1.07146 & -3.53176 & 0.37166 \\ \mathrm{C} & 0.75380 & -3.43327 & -1.03841 \\ \mathrm{C} & 1.90375 & -2.88119 & -1.72205 \\ \mathrm{C} & 2.95050 & -2.65391 & -0.74160 \\ \mathrm{C} & 3.77846 & -1.53659 & -0.84975 \\ \mathrm{C} & 4.13815 & -0.77697 & 0.33757 \\ \mathrm{C} & 4.18875 & 0.62747 & -0.02275 \\ \mathrm{C} & 3.85523 & 0.74444 & -1.43342 \\ \mathrm{C} & 3.59675 & -0.59321 & -1.94257 \\ \mathrm{C} & 2.57884 & -0.79739 & -2.87485 \\ \mathrm{C} & 1.71392 & -1.95944 & -2.75847 \\ \mathrm{C} & 0.37417 & -1.54958 & -3.11406 \\ \mathrm{C} & -0.72039 & -2.06613 & -2.45108 \\ \mathrm{C} & -0.53710 & -3.06148 & -1.42115 \\ \mathrm{C} & -1.58312 & -2.82147 & -0.44411 \\ \mathrm{C} & -2.49334 & -1.80116 & -0.95686 \\ \mathrm{C} & -1.88945 & -1.23550 & -2.07369 \\ \mathrm{C} & -1.83716 & 0.20097 & -2.37158 \\ \mathrm{C} & -4.63422 & 1.87979 & -0.43789 \\ \mathrm{C} & -5.34959 & 0.83263 & 0.42662 \\ \mathrm{H} & -6.31009 & 0.48775 & 0.02947 \\ \mathrm{H} & -5.40921 & 1.08139 & 1.48592 \\ \mathrm{H} & -5.00334 & 1.87394 & -1.46887 \\ \mathrm{H} & -4.64670 & 2.91753 & -0.07653\end{array}$

(6) $\mathrm{C}_{2} \mathrm{H}_{4}$-C $599 \mathrm{Pd}$-HH_C1-C2

$\begin{array}{crrr}\text { Pd } & -3.84564 & -0.93015 & 0.11368 \\ \mathrm{C} & -2.44073 & -1.31202 & 1.47183 \\ \mathrm{C} & -2.06732 & -0.19094 & 2.30001 \\ \mathrm{C} & -2.37496 & 1.07617 & 1.69692 \\ \mathrm{C} & -3.23795 & 1.03320 & 0.46699 \\ \mathrm{C} & -2.68447 & 1.73069 & -0.85879 \\ \mathrm{C} & -1.57171 & 2.73195 & -0.54344 \\ \mathrm{C} & -1.11643 & 3.00134 & 0.73417 \\ \mathrm{C} & -1.50514 & 2.13999 & 1.87516 \\ \mathrm{C} & -0.35548 & 2.02005 & 2.74089 \\ \mathrm{C} & -0.08908 & 0.81574 & 3.39283 \\ \mathrm{C} & -0.95114 & -0.31645 & 3.15495 \\ \mathrm{C} & -0.13411 & -1.50174 & 3.10720 \\ \mathrm{C} & -0.43445 & -2.50179 & 2.17684 \\ \mathrm{C} & -1.58374 & -2.38438 & 1.30366 \\ \mathrm{C} & -1.21609 & -3.00097 & -0.00522\end{array}$




\begin{tabular}{|c|c|c|c|}
\hline C & 0.14914 & -3.45524 & 0.11452 \\
\hline $\mathrm{C}$ & 0.62867 & -3.16692 & 1.45315 \\
\hline $\mathrm{C}$ & 1.95769 & -2.80772 & 1.67525 \\
\hline $\mathrm{C}$ & 2.27082 & -1.74642 & 2.62095 \\
\hline $\mathrm{C}$ & 1.24619 & -1.11174 & 3.32762 \\
\hline $\mathrm{C}$ & 1.27454 & 0.33136 & 3.49956 \\
\hline $\mathrm{C}$ & 2.32232 & 1.07906 & 2.95905 \\
\hline $\mathrm{C}$ & 2.04588 & 2.34277 & 2.29064 \\
\hline $\mathrm{C}$ & 0.73226 & 2.79795 & 2.17905 \\
\hline $\mathrm{C}$ & 0.26277 & 3.39752 & 0.94332 \\
\hline $\mathrm{C}$ & 1.14186 & 3.51879 & -0.13866 \\
\hline C & 0.67092 & 3.21466 & -1.46878 \\
\hline $\mathrm{C}$ & -0.65177 & 2.79162 & -1.64259 \\
\hline $\mathrm{C}$ & -0.95668 & 1.69926 & -2.54940 \\
\hline $\mathrm{C}$ & 0.07865 & 1.11311 & -3.29314 \\
\hline $\mathrm{C}$ & 1.45008 & 1.53333 & -3.09179 \\
\hline $\mathrm{C}$ & 1.74542 & 2.56486 & -2.19812 \\
\hline $\mathrm{C}$ & 2.88131 & 2.45014 & -1.30646 \\
\hline $\mathrm{C}$ & 2.50821 & 3.04148 & -0.02833 \\
\hline $\mathrm{C}$ & 2.95266 & 2.46206 & 1.16122 \\
\hline $\mathrm{C}$ & 3.78254 & 1.27117 & 1.12697 \\
\hline C & 3.38946 & 0.41699 & 2.23453 \\
\hline $\mathrm{C}$ & 3.36561 & -0.97306 & 2.07206 \\
\hline $\mathrm{C}$ & 3.73667 & -1.56150 & 0.79414 \\
\hline $\mathrm{C}$ & 2.87530 & -2.70402 & 0.55469 \\
\hline $\mathrm{C}$ & 2.42208 & -2.98410 & -0.73645 \\
\hline $\mathrm{C}$ & 1.05039 & -3.39268 & -0.96218 \\
\hline $\mathrm{C}$ & 0.60897 & -2.80015 & -2.20072 \\
\hline $\mathrm{C}$ & 1.70202 & -2.01558 & -2.75309 \\
\hline $\mathrm{C}$ & 2.82823 & -2.13091 & -1.84684 \\
\hline $\mathrm{C}$ & 3.65420 & -1.02893 & -1.61379 \\
\hline $\mathrm{C}$ & 4.11942 & -0.73863 & -0.26801 \\
\hline $\mathrm{C}$ & 4.13846 & 0.70324 & -0.09990 \\
\hline $\mathrm{C}$ & 3.68426 & 1.30681 & -1.34045 \\
\hline $\mathrm{C}$ & 3.38434 & 0.23612 & -2.27645 \\
\hline $\mathrm{C}$ & 2.29404 & 0.34923 & -3.14224 \\
\hline $\mathrm{C}$ & 1.43792 & -0.79875 & -3.38566 \\
\hline C & 0.07135 & -0.31344 & -3.48033 \\
\hline $\mathrm{C}$ & -0.96753 & -1.05751 & -2.93041 \\
\hline $\mathrm{C}$ & -0.70059 & -2.32982 & -2.29958 \\
\hline $\mathrm{C}$ & -1.66195 & -2.47261 & -1.21912 \\
\hline $\mathrm{C}$ & -2.72411 & -1.48656 & -1.41898 \\
\hline C & -2.08564 & -0.44090 & -2.22232 \\
\hline $\mathrm{C}$ & -2.05900 & 0.94034 & -2.02218 \\
\hline $\mathrm{C}$ & -4.12251 & 2.34224 & -1.05324 \\
\hline $\mathrm{C}$ & -4.54292 & 1.86292 & 0.35467 \\
\hline $\mathrm{H}$ & -5.49644 & 1.31961 & 0.42289 \\
\hline $\mathrm{H}$ & -4.56690 & 2.65497 & 1.11262 \\
\hline $\mathrm{H}$ & -4.66855 & 1.84664 & -1.86092 \\
\hline
\end{tabular}


$\begin{array}{llll}\text { H } & -4.14378 & 3.42169 & -1.22110\end{array}$

(7) $\mathrm{C}_{2} \mathrm{H}_{4} \_\mathrm{C}_{59} \mathrm{Pd}$ PHH_C2-C3

\begin{tabular}{|c|c|c|c|}
\hline $\mathrm{Pd}$ & 3.81437 & -1.12579 & -0.12976 \\
\hline $\mathrm{C}$ & 2.22183 & -2.12087 & -0.82393 \\
\hline $\mathrm{C}$ & 1.77398 & -1.50657 & -2.04558 \\
\hline $\mathrm{C}$ & 2.15628 & -0.09928 & -2.18140 \\
\hline $\mathrm{C}$ & 3.15218 & 0.42273 & -1.28800 \\
\hline C & 2.87743 & 1.75707 & -0.60868 \\
\hline $\mathrm{C}$ & 1.67917 & 2.49062 & -1.18635 \\
\hline C & 1.04589 & 2.09556 & -2.34795 \\
\hline C & 1.28673 & 0.75640 & -2.87779 \\
\hline C & 0.05990 & 0.28070 & -3.45470 \\
\hline $\mathrm{C}$ & -0.29651 & -1.06747 & -3.35195 \\
\hline $\mathrm{C}$ & 0.57449 & -1.96724 & -2.62359 \\
\hline C & -0.25412 & -2.91548 & -1.91979 \\
\hline $\mathrm{C}$ & 0.12836 & -3.35695 & -0.65127 \\
\hline C & 1.37718 & -2.91732 & -0.07485 \\
\hline C & 1.15329 & -2.76003 & 1.38443 \\
\hline $\mathrm{C}$ & -0.22888 & -3.11252 & 1.65456 \\
\hline C & -0.85635 & -3.49246 & 0.40415 \\
\hline $\mathrm{C}$ & -2.19077 & -3.17484 & 0.15343 \\
\hline $\mathrm{C}$ & -2.59078 & -2.70819 & -1.16539 \\
\hline C & -1.64308 & -2.58819 & -2.18192 \\
\hline C & -1.66600 & -1.43420 & -3.06893 \\
\hline $\mathrm{C}$ & -2.64425 & -0.44761 & -2.90572 \\
\hline $\mathrm{C}$ & -2.27744 & 0.95422 & -3.01587 \\
\hline $\mathrm{C}$ & -0.95601 & 1.31013 & -3.28906 \\
\hline $\mathrm{C}$ & -0.34312 & 2.42267 & -2.59166 \\
\hline $\mathrm{C}$ & -1.07345 & 3.14341 & -1.64427 \\
\hline C & -0.43647 & 3.51694 & -0.40266 \\
\hline $\mathrm{C}$ & 0.90340 & 3.14083 & -0.16902 \\
\hline C & 1.32209 & 2.67633 & 1.11998 \\
\hline C & 0.38053 & 2.57039 & 2.12899 \\
\hline $\mathrm{C}$ & -1.00004 & 2.93172 & 1.90480 \\
\hline $\mathrm{C}$ & -1.40759 & 3.40358 & 0.65412 \\
\hline $\mathrm{C}$ & -2.65693 & 2.93993 & 0.07643 \\
\hline $\mathrm{C}$ & -2.44649 & 2.77401 & -1.35455 \\
\hline $\mathrm{C}$ & -3.03721 & 1.70028 & -2.02461 \\
\hline $\mathrm{C}$ & -3.86556 & 0.75593 & -1.29799 \\
\hline $\mathrm{C}$ & -3.62199 & -0.56898 & -1.84121 \\
\hline $\mathrm{C}$ & -3.60099 & -1.67989 & -0.98874 \\
\hline $\mathrm{C}$ & -3.82242 & -1.50834 & 0.43389 \\
\hline $\mathrm{C}$ & -2.95626 & -2.43900 & 1.14393 \\
\hline $\mathrm{C}$ & -2.35922 & -2.06530 & 2.34725 \\
\hline $\mathrm{C}$ & -0.97391 & -2.41579 & 2.61472 \\
\hline $\mathrm{C}$ & -0.36264 & -1.31042 & 3.32104 \\
\hline
\end{tabular}




$\begin{array}{lrrr}\mathrm{C} & -1.35612 & -0.27095 & 3.48341 \\ \mathrm{C} & -2.59698 & -0.73454 & 2.89110 \\ \mathrm{C} & -3.41928 & 0.16056 & 2.20593 \\ \mathrm{C} & -4.05182 & -0.23381 & 0.95742 \\ \mathrm{C} & -4.07092 & 0.91917 & 0.07674 \\ \mathrm{C} & -3.45269 & 2.03118 & 0.77514 \\ \mathrm{C} & -3.04130 & 1.55976 & 2.08937 \\ \mathrm{C} & -1.84058 & 2.00378 & 2.64354 \\ \mathrm{C} & -0.98017 & 1.07402 & 3.35555 \\ \mathrm{C} & 0.38759 & 1.41341 & 3.03807 \\ \mathrm{C} & 1.32493 & 0.41757 & 2.85310 \\ \mathrm{C} & 0.96395 & -0.96891 & 3.04077 \\ \mathrm{C} & 1.74998 & -1.72586 & 2.08906 \\ \mathrm{C} & 2.67321 & -0.78687 & 1.45128 \\ \mathrm{C} & 2.34524 & 0.50130 & 1.79248 \\ \mathrm{C} & 2.57805 & 1.79540 & 1.02896 \\ \mathrm{C} & 3.93702 & 2.55698 & 1.22002 \\ \mathrm{C} & 4.14064 & 2.64982 & -0.30909 \\ \mathrm{H} & 5.06417 & 2.19883 & -0.68094 \\ \mathrm{H} & 4.04877 & 3.66033 & -0.71634 \\ \mathrm{H} & 4.68081 & 1.93202 & 1.72292 \\ \mathrm{H} & 3.85544 & 3.50424 & 1.76015\end{array}$

(8) $\mathrm{C}_{2} \mathrm{H}_{4} \_\mathrm{C} 599 \mathrm{Pd}$-HH_C3-C4

$\begin{array}{cccc}\text { Pd } & -3.77357 & -1.14261 & -0.16661 \\ \mathrm{C} & -2.17411 & -2.29246 & -0.54621 \\ \mathrm{C} & -1.50015 & -2.81423 & 0.62005 \\ \mathrm{C} & -1.76097 & -1.99457 & 1.79335 \\ \mathrm{C} & -2.64696 & -0.89896 & 1.47803 \\ \mathrm{C} & -2.33256 & 0.38614 & 1.85618 \\ \mathrm{C} & -1.28243 & 0.57872 & 2.81546 \\ \mathrm{C} & -0.52637 & -0.49586 & 3.29609 \\ \mathrm{C} & -0.74770 & -1.80922 & 2.74641 \\ \mathrm{C} & 0.54721 & -2.44739 & 2.59479 \\ \mathrm{C} & 0.79851 & -3.23523 & 1.47439 \\ \mathrm{C} & -0.23423 & -3.41408 & 0.46815 \\ \mathrm{C} & 0.40627 & -3.42321 & -0.82321 \\ \mathrm{C} & -0.21401 & -2.79185 & -1.90572 \\ \mathrm{C} & -1.50616 & -2.15303 & -1.74836 \\ \mathrm{C} & -1.52047 & -0.94485 & -2.60907 \\ \mathrm{C} & -0.23380 & -0.87822 & -3.25572 \\ \mathrm{C} & 0.56900 & -2.01449 & -2.84080 \\ \mathrm{C} & 1.94659 & -1.88550 & -2.66907 \\ \mathrm{C} & 2.59125 & -2.51783 & -1.52882 \\ \mathrm{C} & 1.83746 & -3.27220 & -0.62879 \\ \mathrm{C} & 2.08047 & -3.15142 & 0.80005 \\ \mathrm{C} & 3.06178 & -2.27523 & 1.26924\end{array}$




\begin{tabular}{|c|c|c|c|}
\hline $\mathrm{C}$ & 2.79942 & -1.44765 & 2.43493 \\
\hline C & 1.56388 & -1.53086 & 3.08118 \\
\hline $\mathrm{C}$ & 0.89519 & -0.32090 & 3.52412 \\
\hline $\mathrm{C}$ & 1.48904 & 0.92525 & 3.30794 \\
\hline $\mathrm{C}$ & 0.68455 & 2.05311 & 2.86236 \\
\hline $\mathrm{C}$ & -0.67891 & 1.87523 & 2.60456 \\
\hline $\mathrm{C}$ & -1.34175 & 2.50586 & 1.49498 \\
\hline $\mathrm{C}$ & -0.58061 & 3.26408 & 0.62149 \\
\hline $\mathrm{C}$ & 0.84448 & 3.42248 & 0.84004 \\
\hline $\mathrm{C}$ & 1.47081 & 2.84009 & 1.94749 \\
\hline $\mathrm{C}$ & 2.76484 & 2.19851 & 1.80344 \\
\hline $\mathrm{C}$ & 2.77528 & 1.01234 & 2.64926 \\
\hline $\mathrm{C}$ & 3.41651 & -0.15195 & 2.21395 \\
\hline $\mathrm{C}$ & 4.06583 & -0.17909 & 0.91544 \\
\hline $\mathrm{C}$ & 3.84422 & -1.48970 & 0.33033 \\
\hline $\mathrm{C}$ & 3.61597 & -1.61200 & -1.04247 \\
\hline $\mathrm{C}$ & 3.61014 & -0.42835 & -1.88883 \\
\hline $\mathrm{C}$ & 2.58898 & -0.60215 & -2.90092 \\
\hline $\mathrm{C}$ & 1.82481 & 0.48961 & -3.32308 \\
\hline $\mathrm{C}$ & 0.39948 & 0.34403 & -3.52859 \\
\hline $\mathrm{C}$ & -0.23663 & 1.56203 & -3.09407 \\
\hline $\mathrm{C}$ & 0.77503 & 2.46702 & -2.60684 \\
\hline $\mathrm{C}$ & 2.05982 & 1.80693 & -2.74461 \\
\hline $\mathrm{C}$ & 3.03450 & 1.96809 & -1.75866 \\
\hline $\mathrm{C}$ & 3.82915 & 0.83391 & -1.32554 \\
\hline $\mathrm{C}$ & 4.05382 & 0.95886 & 0.10394 \\
\hline $\mathrm{C}$ & 3.39327 & 2.16980 & 0.55836 \\
\hline $\mathrm{C}$ & 2.75680 & 2.79168 & -0.59077 \\
\hline $\mathrm{C}$ & 1.51109 & 3.40582 & -0.45022 \\
\hline $\mathrm{C}$ & 0.49945 & 3.24751 & -1.47842 \\
\hline $\mathrm{C}$ & -0.79571 & 3.18102 & -0.83746 \\
\hline $\mathrm{C}$ & -1.77250 & 2.34553 & -1.33531 \\
\hline $\mathrm{C}$ & -1.47065 & 1.49547 & -2.44192 \\
\hline $\mathrm{C}$ & -2.17767 & 0.24208 & -2.23557 \\
\hline $\mathrm{C}$ & -3.22587 & 0.43340 & -1.26588 \\
\hline $\mathrm{C}$ & -2.90025 & 1.75131 & -0.51641 \\
\hline $\mathrm{C}$ & -2.59655 & 1.70840 & 1.11109 \\
\hline $\mathrm{C}$ & -3.97633 & 2.41613 & 1.32928 \\
\hline $\mathrm{C}$ & -4.10523 & 2.69681 & -0.18087 \\
\hline $\mathrm{H}$ & -3.88363 & 3.73477 & -0.44540 \\
\hline $\mathrm{H}$ & -5.04764 & 2.40541 & -0.65062 \\
\hline $\mathrm{H}$ & -3.95738 & 3.28212 & 1.99649 \\
\hline $\mathrm{H}$ & -4.72530 & 1.70887 & 1.69844 \\
\hline
\end{tabular}

(9) $\mathrm{C}_{2} \mathrm{H}_{4} \_\mathrm{C}_{59} \mathrm{Pd} \_\mathrm{PH}$ _C4-C5

$\begin{array}{cccc}\text { Pd } & -3.84661 & -0.96155 & -0.07016 \\ \text { C } & -2.35821 & -1.77168 & -1.10451\end{array}$ 


\begin{tabular}{|c|c|c|c|}
\hline $\mathrm{C}$ & -1.61248 & -2.71198 & -0.30084 \\
\hline $\mathrm{C}$ & -1.69711 & -2.42868 & 1.12327 \\
\hline $\mathrm{C}$ & -2.62131 & -1.36023 & 1.47382 \\
\hline $\mathrm{C}$ & -2.07985 & -0.26625 & 2.19886 \\
\hline $\mathrm{C}$ & -0.92234 & -0.54525 & 3.07587 \\
\hline $\mathrm{C}$ & -0.21073 & -1.73017 & 2.94729 \\
\hline $\mathrm{C}$ & -0.59096 & -2.70221 & 1.93573 \\
\hline $\mathrm{C}$ & 0.62327 & -3.26302 & 1.38494 \\
\hline $\mathrm{C}$ & 0.70881 & -3.55437 & 0.02535 \\
\hline $\mathrm{C}$ & -0.42394 & -3.25172 & -0.83056 \\
\hline $\mathrm{C}$ & 0.08486 & -2.77041 & -2.09090 \\
\hline $\mathrm{C}$ & -0.58667 & -1.74138 & -2.76150 \\
\hline $\mathrm{C}$ & -1.81238 & -1.19273 & -2.22892 \\
\hline $\mathrm{C}$ & -1.81719 & 0.27032 & -2.52994 \\
\hline $\mathrm{C}$ & -0.58873 & 0.55257 & -3.23857 \\
\hline $\mathrm{C}$ & 0.16139 & -0.67922 & -3.39830 \\
\hline $\mathrm{C}$ & 1.55365 & -0.67568 & -3.34534 \\
\hline $\mathrm{C}$ & 2.25494 & -1.73552 & -2.63610 \\
\hline $\mathrm{C}$ & 1.53411 & -2.76621 & -2.02847 \\
\hline $\mathrm{C}$ & 1.91976 & -3.24084 & -0.70785 \\
\hline $\mathrm{C}$ & 3.00879 & -2.66293 & -0.05139 \\
\hline C & 2.92299 & -2.36871 & 1.37096 \\
\hline $\mathrm{C}$ & 1.75503 & -2.66422 & 2.07506 \\
\hline $\mathrm{C}$ & 1.23579 & -1.71863 & 3.04732 \\
\hline $\mathrm{C}$ & 1.90745 & -0.51529 & 3.28196 \\
\hline $\mathrm{C}$ & 1.15539 & 0.71724 & 3.43337 \\
\hline $\mathrm{C}$ & -0.23636 & 0.68975 & 3.31288 \\
\hline $\mathrm{C}$ & -0.95093 & 1.71533 & 2.57058 \\
\hline $\mathrm{C}$ & -0.22208 & 2.77661 & 2.02144 \\
\hline $\mathrm{C}$ & 1.21961 & 2.78762 & 2.11288 \\
\hline $\mathrm{C}$ & 1.90842 & 1.78693 & 2.81475 \\
\hline $\mathrm{C}$ & 3.12021 & 1.21692 & 2.25831 \\
\hline $\mathrm{C}$ & 3.12456 & -0.21099 & 2.55265 \\
\hline $\mathrm{C}$ & 3.62000 & -1.11751 & 1.61473 \\
\hline $\mathrm{C}$ & 4.13633 & -0.63865 & 0.34094 \\
\hline $\mathrm{C}$ & 3.75331 & -1.59014 & -0.68525 \\
\hline $\mathrm{C}$ & 3.39049 & -1.14007 & -1.96014 \\
\hline $\mathrm{C}$ & 3.39238 & 0.28364 & -2.25127 \\
\hline $\mathrm{C}$ & 2.26569 & 0.57127 & -3.11683 \\
\hline $\mathrm{C}$ & 1.55316 & 1.76238 & -2.97188 \\
\hline $\mathrm{C}$ & 0.10198 & 1.75561 & -3.04233 \\
\hline $\mathrm{C}$ & -0.39637 & 2.69965 & -2.07899 \\
\hline $\mathrm{C}$ & 0.72764 & 3.29445 & -1.39807 \\
\hline $\mathrm{C}$ & 1.94183 & 2.71943 & -1.94704 \\
\hline $\mathrm{C}$ & 3.01783 & 2.43722 & -1.10381 \\
\hline $\mathrm{C}$ & 3.76057 & 1.20082 & -1.26024 \\
\hline $\mathrm{C}$ & 4.13481 & 0.72937 & 0.06152 \\
\hline $\mathrm{C}$ & 3.62492 & 1.67637 & 1.04059 \\
\hline $\mathrm{C}$ & 2.92582 & 2.72669 & 0.32110 \\
\hline
\end{tabular}




$\begin{array}{lrrr}\mathrm{C} & 1.75227 & 3.26992 & 0.84649 \\ \mathrm{C} & 0.63215 & 3.55381 & -0.03020 \\ \mathrm{C} & -0.58910 & 3.25099 & 0.68770 \\ \mathrm{C} & -1.68159 & 2.71544 & 0.03097 \\ \mathrm{C} & -1.57018 & 2.39876 & -1.36371 \\ \mathrm{C} & -2.34720 & 1.21571 & -1.66147 \\ \mathrm{C} & -3.27969 & 0.98133 & -0.51893 \\ \mathrm{C} & -2.72096 & 1.81452 & 0.71724 \\ \mathrm{C} & -2.09887 & 1.12451 & 1.91919 \\ \mathrm{C} & -4.65504 & 1.71465 & -0.42156 \\ \mathrm{C} & -4.16200 & 2.45820 & 0.84229 \\ \mathrm{H} & -4.67244 & 2.15463 & 1.75978 \\ \mathrm{H} & -4.16575 & 3.54830 & 0.77164 \\ \mathrm{H} & -5.53649 & 1.07255 & -0.25785 \\ \mathrm{H} & -4.86915 & 2.33590 & -1.29685\end{array}$

(10) $\mathrm{C}_{2} \mathrm{H}_{4} \_\mathrm{C}_{59} \mathrm{Pd} \_\mathrm{HH}$ _C6-C7

$\begin{array}{cccc}\text { Pd } & -3.82620 & -0.92698 & -0.22315 \\ \mathrm{C} & -3.25410 & 0.78203 & 0.80338 \\ \mathrm{C} & -2.79398 & 1.90528 & -0.27900 \\ \mathrm{C} & -2.34020 & 1.20780 & -1.55265 \\ \mathrm{C} & -2.58185 & -0.19385 & -1.59471 \\ \mathrm{C} & -1.74092 & -1.07192 & -2.28427 \\ \mathrm{C} & -0.72450 & -0.51579 & -3.12971 \\ \mathrm{C} & -0.52087 & 0.87178 & -3.17191 \\ \mathrm{C} & -1.32127 & 1.72955 & -2.35678 \\ \mathrm{C} & -0.46425 & 2.82658 & -1.88911 \\ \mathrm{C} & -0.63112 & 3.30273 & -0.59945 \\ \mathrm{C} & -1.69220 & 2.78135 & 0.25156 \\ \mathrm{C} & -1.16679 & 2.63535 & 1.53649 \\ \mathrm{C} & -1.46511 & 1.48365 & 2.34792 \\ \mathrm{C} & -2.32168 & 0.46445 & 1.90874 \\ \mathrm{C} & -1.78912 & -0.81981 & 2.36565 \\ \mathrm{C} & -0.62026 & -0.52905 & 3.18482 \\ \mathrm{C} & -0.41269 & 0.89620 & 3.17600 \\ \mathrm{C} & 0.86924 & 1.42509 & 3.19245 \\ \mathrm{C} & 1.19053 & 2.58182 & 2.34645 \\ \mathrm{C} & 0.21264 & 3.16718 & 1.55741 \\ \mathrm{C} & 0.53572 & 3.58515 & 0.21612 \\ \mathrm{C} & 1.82270 & 3.39073 & -0.29557 \\ \mathrm{C} & 1.99386 & 2.89544 & -1.64997 \\ \mathrm{C} & 0.86600 & 2.61141 & -2.42546 \\ \mathrm{C} & 0.82877 & 1.40610 & -3.23447 \\ \mathrm{C} & 1.91940 & 0.53729 & -3.24825 \\ \mathrm{C} & 1.70666 & -0.90435 & -3.21907 \\ \mathrm{C} & 0.41181 & -1.41448 & -3.15558 \\ \mathrm{C} & 0.09906 & -2.54850 & -2.30766\end{array}$




$\begin{array}{lrrr}\mathrm{C} & 1.12362 & -3.15217 & -1.56455 \\ \mathrm{C} & 2.46431 & -2.59800 & -1.59748 \\ \mathrm{C} & 2.75698 & -1.50000 & -2.41492 \\ \mathrm{C} & 3.60170 & -0.42964 & -1.92495 \\ \mathrm{C} & 3.09159 & 0.83062 & -2.44709 \\ \mathrm{C} & 3.12526 & 1.98253 & -1.65609 \\ \mathrm{C} & 3.66007 & 1.91934 & -0.30758 \\ \mathrm{C} & 2.84438 & 2.78464 & 0.53343 \\ \mathrm{C} & 2.53376 & 2.39475 & 1.83901 \\ \mathrm{C} & 3.04005 & 1.13419 & 2.36541 \\ \mathrm{C} & 2.01710 & 0.55073 & 3.21328 \\ \mathrm{C} & 1.82534 & -0.83754 & 3.23367 \\ \mathrm{C} & 0.48783 & -1.38089 & 3.24149 \\ \mathrm{C} & 0.49035 & -2.58406 & 2.43445 \\ \mathrm{C} & 1.83223 & -2.78268 & 1.90980 \\ \mathrm{C} & 2.65998 & -1.69448 & 2.40428 \\ \mathrm{C} & 3.63355 & -1.12878 & 1.58047 \\ \mathrm{C} & 3.83094 & 0.31180 & 1.56270 \\ \mathrm{C} & 4.13643 & 0.70810 & 0.19672 \\ \mathrm{C} & 4.12105 & -0.48757 & -0.62880 \\ \mathrm{C} & 3.81436 & -1.62217 & 0.22267 \\ \mathrm{C} & 3.01272 & -2.66185 & -0.25419 \\ \mathrm{C} & 2.00460 & -3.25741 & 0.61021 \\ \mathrm{C} & 0.84513 & -3.56243 & -0.20879 \\ \mathrm{C} & -0.43789 & -3.38279 & 0.30844 \\ \mathrm{C} & -0.61401 & -2.86927 & 1.64437 \\ \mathrm{C} & -1.81880 & -2.02116 & 1.62835 \\ \mathrm{C} & -2.54830 & -2.30970 & 0.41534 \\ \mathrm{C} & -1.52903 & -2.83969 & -0.49099 \\ \mathrm{C} & -1.23554 & -2.37762 & -1.77391 \\ \mathrm{C} & -4.26232 & 2.46276 & -0.19140 \\ \mathrm{H} & -4.57909 & 1.55050 & 1.02219 \\ \mathrm{H} & -5.51427 & 0.97192 & 0.98503 \\ & -4.57049 & 2.07812 & 1.98372 \\ \mathrm{H} & -4.33230 & 3.53788 & -0.00953\end{array}$

\section{(11) $\mathrm{C}_{2} \mathrm{H}_{4} \_\mathrm{C}_{59} \mathrm{Pd} \_\mathrm{HH}$ _C7-C8}

$\begin{array}{cccc}\text { Pd } & -3.91813 & -0.35517 & -0.00001 \\ \mathrm{C} & -2.67144 & 0.67727 & 1.23826 \\ \mathrm{C} & -2.22160 & 1.94631 & 0.72701 \\ \mathrm{C} & -2.22148 & 1.94638 & -0.72680 \\ \mathrm{C} & -2.67142 & 0.67743 & -1.23821 \\ \mathrm{C} & -1.98290 & 0.02825 & -2.24420 \\ \mathrm{C} & -0.96113 & 0.76058 & -2.96756 \\ \mathrm{C} & -0.59567 & 2.05236 & -2.57692 \\ \mathrm{C} & -1.21873 & 2.64811 & -1.42047\end{array}$




\begin{tabular}{|c|c|c|c|}
\hline C & -0.19910 & 3.38701 & -0.69577 \\
\hline $\mathrm{C}$ & -0.19912 & 3.38691 & 0.69615 \\
\hline $\mathrm{C}$ & -1.21873 & 2.64788 & 1.42072 \\
\hline $\mathrm{C}$ & -0.59571 & 2.05203 & 2.57714 \\
\hline $\mathrm{C}$ & -0.96118 & 0.76021 & 2.96763 \\
\hline $\mathrm{C}$ & -1.98295 & 0.02796 & 2.24419 \\
\hline $\mathrm{C}$ & -1.59522 & -1.40580 & 2.24438 \\
\hline $\mathrm{C}$ & -0.34299 & -1.50272 & 2.96254 \\
\hline $\mathrm{C}$ & 0.04394 & -0.18026 & 3.41702 \\
\hline $\mathrm{C}$ & 1.38664 & 0.19554 & 3.45383 \\
\hline $\mathrm{C}$ & 1.77554 & 1.52879 & 3.02031 \\
\hline C & 0.80488 & 2.43811 & 2.59408 \\
\hline $\mathrm{C}$ & 1.05207 & 3.26289 & 1.42417 \\
\hline $\mathrm{C}$ & 2.25629 & 3.14656 & 0.72769 \\
\hline $\mathrm{C}$ & 2.25630 & 3.14665 & -0.72729 \\
\hline $\mathrm{C}$ & 1.05209 & 3.26308 & -1.42379 \\
\hline $\mathrm{C}$ & 0.80493 & 2.43844 & -2.59379 \\
\hline $\mathrm{C}$ & 1.77558 & 1.52915 & -3.02011 \\
\hline $\mathrm{C}$ & 1.38669 & 0.19596 & -3.45380 \\
\hline $\mathrm{C}$ & 0.04399 & -0.17984 & -3.41705 \\
\hline $\mathrm{C}$ & -0.34294 & -1.50235 & -2.96274 \\
\hline $\mathrm{C}$ & 0.65230 & -2.41481 & -2.58312 \\
\hline $\mathrm{C}$ & 2.04540 & -2.01762 & -2.59308 \\
\hline $\mathrm{C}$ & 2.41106 & -0.73896 & -3.02318 \\
\hline $\mathrm{C}$ & 3.42136 & 0.01013 & -2.30287 \\
\hline $\mathrm{C}$ & 3.02999 & 1.41303 & -2.30266 \\
\hline $\mathrm{C}$ & 3.26578 & 2.20366 & -1.17500 \\
\hline $\mathrm{C}$ & 3.89289 & 1.62326 & 0.00012 \\
\hline $\mathrm{C}$ & 3.26576 & 2.20352 & 1.17531 \\
\hline $\mathrm{C}$ & 3.02996 & 1.41275 & 2.30287 \\
\hline $\mathrm{C}$ & 3.42133 & 0.00985 & 2.30292 \\
\hline $\mathrm{C}$ & 2.41101 & -0.73933 & 3.02312 \\
\hline $\mathrm{C}$ & 2.04536 & -2.01793 & 2.59286 \\
\hline $\mathrm{C}$ & 0.65226 & -2.41513 & 2.58283 \\
\hline $\mathrm{C}$ & 0.43358 & -3.24733 & 1.42267 \\
\hline $\mathrm{C}$ & 1.68881 & -3.36415 & 0.69758 \\
\hline $\mathrm{C}$ & 2.68909 & -2.60139 & 1.42464 \\
\hline C & 3.65589 & -1.87474 & 0.72737 \\
\hline C & 4.03138 & -0.54445 & 1.17525 \\
\hline $\mathrm{C}$ & 4.26492 & 0.27698 & 0.00005 \\
\hline $\mathrm{C}$ & 4.03140 & -0.54431 & -1.17526 \\
\hline $\mathrm{C}$ & 3.65590 & -1.87465 & -0.72754 \\
\hline $\mathrm{C}$ & 2.68912 & -2.60121 & -1.42492 \\
\hline $\mathrm{C}$ & 1.68882 & -3.36407 & -0.69797 \\
\hline $\mathrm{C}$ & 0.43360 & -3.24716 & -1.42307 \\
\hline $\mathrm{C}$ & -0.76538 & -3.13697 & -0.72318 \\
\hline C & -0.76539 & -3.13705 & 0.72278 \\
\hline C & -1.83224 & -2.23923 & 1.14658 \\
\hline $\mathrm{C}$ & -2.67993 & -1.92567 & -0.00012 \\
\hline
\end{tabular}




$\begin{array}{lrrr}\mathrm{C} & -1.83222 & -2.23909 & -1.14689 \\ \mathrm{C} & -1.59518 & -1.40553 & -2.24457 \\ \mathrm{C} & -6.27391 & 0.08963 & -0.00005 \\ \mathrm{C} & -5.65550 & 1.30907 & -0.00003 \\ \mathrm{H} & -5.47750 & 1.85125 & 0.92383 \\ \mathrm{H} & -5.47679 & 1.85129 & -0.92375 \\ \mathrm{H} & -6.59710 & -0.38425 & 0.92387 \\ \mathrm{H} & -6.59724 & -0.38467 & -0.92367\end{array}$

\section{Ethane}

(1) $\mathrm{C}_{2} \mathrm{H}_{6} \mathrm{C}_{59} \mathrm{Pd}$-TM

$\begin{array}{rrrr}\text { Pd } & 3.76229 & 0.24698 & -0.27016 \\ \text { C } & 2.61831 & -0.81740 & 0.96690 \\ \text { C } & 2.09604 & -2.05267 & 0.42845 \\ \text { C } & 2.00123 & -1.96286 & -1.02276 \\ \text { C } & 2.45891 & -0.66652 & -1.46900 \\ \text { C } & 1.71961 & 0.04371 & -2.36881 \\ \mathrm{C} & 0.62492 & -0.60653 & -3.04540 \\ \mathrm{C} & 0.26529 & -1.89793 & -2.73456 \\ \mathrm{C} & 0.94788 & -2.57944 & -1.66880 \\ \mathrm{C} & -0.03615 & -3.34258 & -0.93114 \\ \mathrm{C} & 0.05279 & -3.42657 & 0.43355 \\ \mathrm{C} & 1.13010 & -2.75159 & 1.12526 \\ \mathrm{C} & 0.59671 & -2.21045 & 2.34558 \\ \mathrm{C} & 1.00382 & -0.96364 & 2.76218 \\ \mathrm{C} & 2.00713 & -0.22730 & 2.03415 \\ \mathrm{C} & 1.62955 & 1.22999 & 2.12468 \\ \mathrm{C} & 0.42404 & 1.29020 & 2.92592 \\ \mathrm{C} & 0.04900 & -0.04397 & 3.32786 \\ \mathrm{C} & -1.27133 & -0.39814 & 3.44756 \\ \mathrm{C} & -1.71216 & -1.69347 & 2.97158 \\ \mathrm{C} & -0.80288 & -2.57212 & 2.43413 \\ \mathrm{C} & -1.14182 & -3.32410 & 1.24512 \\ \mathrm{C} & -2.36755 & -3.15016 & 0.64851 \\ \mathrm{C} & -2.46172 & -3.06140 & -0.79570 \\ \mathrm{C} & -1.32491 & -3.15149 & -1.56266 \\ \mathrm{C} & -1.13675 & -2.25743 & -2.68486 \\ \mathrm{C} & -2.10102 & -1.32718 & -2.98894 \\ \mathrm{C} & -1.71533 & 0.01997 & -3.35657 \\ \mathrm{C} & -0.38787 & 0.36739 & -3.36655 \\ \mathrm{C} & 0.04650 & 1.64565 & -2.85704 \\ \mathrm{C} & -0.89402 & 2.53477 & -2.38279 \\ \mathrm{C} & -2.29202 & 2.16350 & -2.33843 \\ \mathrm{C} & -2.69221 & 0.93651 & -2.80851 \\ \mathrm{C} & -3.66901 & 0.16018 & -2.07944 \\ \mathrm{C} & -3.30188 & -1.23918 & -2.18935\end{array}$




$\begin{array}{lrrr}\mathrm{C} & -3.47710 & -2.08237 & -1.11765 \\ \mathrm{C} & -4.01402 & -1.56666 & 0.12535 \\ \mathrm{C} & -3.32486 & -2.22579 & 1.21616 \\ \mathrm{C} & -3.00565 & -1.51817 & 2.35090 \\ \mathrm{C} & -3.37273 & -0.11880 & 2.46089 \\ \mathrm{C} & -2.30358 & 0.57060 & 3.14651 \\ \mathrm{C} & -1.95774 & 1.84886 & 2.78167 \\ \mathrm{C} & -0.56286 & 2.22299 & 2.68952 \\ \mathrm{C} & -0.41714 & 3.13680 & 1.59166 \\ \mathrm{C} & -1.71317 & 3.30600 & 0.96347 \\ \mathrm{C} & -2.66808 & 2.50988 & 1.70460 \\ \mathrm{C} & -3.67420 & 1.84851 & 1.04069 \\ \mathrm{C} & -4.03628 & 0.50159 & 1.42942 \\ \mathrm{C} & -4.36149 & -0.24158 & 0.22947 \\ \mathrm{C} & -4.18846 & 0.64490 & -0.90285 \\ \mathrm{C} & -3.76845 & 1.93721 & -0.40296 \\ \mathrm{C} & -2.85156 & 2.68257 & -1.10592 \\ \mathrm{C} & -1.80245 & 3.39000 & -0.40318 \\ \mathrm{C} & -0.60013 & 3.30896 & -1.20986 \\ \mathrm{C} & 0.62301 & 3.15685 & -0.61360 \\ \mathrm{C} & 0.71717 & 3.06800 & 0.82797 \\ \mathrm{C} & 1.80198 & 2.15855 & 1.12768 \\ \mathrm{C} & 2.64106 & 2.03141 & -0.08824 \\ \mathrm{C} & 1.65254 & 2.29936 & -1.16025 \\ \mathrm{C} & 1.34499 & 1.49782 & -2.23209 \\ \mathrm{C} & 8.78671 & -0.40706 & 0.45947 \\ \mathrm{C} & 7.28680 & -0.21195 & 0.24136 \\ \mathrm{H} & 7.07073 & 0.80951 & -0.06008 \\ \mathrm{H} & 6.73378 & -0.41814 & 1.15372 \\ \mathrm{H} & 6.91668 & -0.87895 & -0.53281 \\ \mathrm{H} & 9.15914 & 0.25628 & 1.23328 \\ \mathrm{H} & 9.34250 & -0.20183 & -0.44958 \\ \mathrm{H} & 9.00657 & -1.42584 & 0.76103 \\ & & & \\ & & & \\ & & \end{array}$

(2) $\mathrm{C}_{2} \mathrm{H}_{6} \_\mathrm{C}_{59} \mathrm{Pd}$ _TC5

$\begin{array}{cccc}\text { Pd } & 3.49589 & -1.48725 & 0.08823 \\ \text { C } & 1.91118 & -2.15850 & 1.11918 \\ \text { C } & 1.02335 & -3.00473 & 0.36239 \\ \text { C } & 1.14341 & -2.75938 & -1.06973 \\ \text { C } & 2.11746 & -1.73460 & -1.34933 \\ \text { C } & 1.83436 & -0.68648 & -2.20441 \\ \text { C } & 0.66820 & -0.78868 & -3.05395 \\ \text { C } & -0.22613 & -1.85654 & -2.90929 \\ \text { C } & 0.00029 & -2.84624 & -1.88490 \\ \text { C } & -1.28997 & -3.19999 & -1.31485 \\ \text { C } & -1.40437 & -3.43455 & 0.05141 \\ \text { C } & -0.23349 & -3.32498 & 0.90668\end{array}$




\begin{tabular}{|c|c|c|c|}
\hline $\mathrm{C}$ & -0.64971 & -2.72504 & 2.15038 \\
\hline $\mathrm{C}$ & 0.17971 & -1.79076 & 2.78320 \\
\hline $\mathrm{C}$ & 1.46451 & -1.44538 & 2.21568 \\
\hline $\mathrm{C}$ & 1.70886 & 0.00093 & 2.48125 \\
\hline $\mathrm{C}$ & 0.55149 & 0.49031 & 3.19342 \\
\hline $\mathrm{C}$ & -0.38236 & -0.60441 & 3.39224 \\
\hline $\mathrm{C}$ & -1.75823 & -0.38350 & 3.35179 \\
\hline $\mathrm{C}$ & -2.62247 & -1.33815 & 2.67330 \\
\hline $\mathrm{C}$ & -2.08019 & -2.48551 & 2.08900 \\
\hline $\mathrm{C}$ & -2.54831 & -2.92173 & 0.78481 \\
\hline $\mathrm{C}$ & -3.53647 & -2.19515 & 0.11799 \\
\hline $\mathrm{C}$ & -3.41712 & -1.95008 & -1.31066 \\
\hline $\mathrm{C}$ & -2.31419 & -2.44169 & -2.01139 \\
\hline $\mathrm{C}$ & -1.65397 & -1.61173 & -3.00406 \\
\hline $\mathrm{C}$ & -2.12641 & -0.32084 & -3.25449 \\
\hline $\mathrm{C}$ & -1.19084 & 0.78062 & -3.43021 \\
\hline $\mathrm{C}$ & 0.17888 & 0.54738 & -3.31760 \\
\hline $\mathrm{C}$ & 1.03759 & 1.48800 & -2.61979 \\
\hline $\mathrm{C}$ & 0.47685 & 2.65866 & -2.08488 \\
\hline $\mathrm{C}$ & -0.95061 & 2.88677 & -2.17425 \\
\hline $\mathrm{C}$ & -1.77299 & 1.97143 & -2.83807 \\
\hline $\mathrm{C}$ & -3.05560 & 1.60288 & -2.27413 \\
\hline $\mathrm{C}$ & -3.27682 & 0.18681 & -2.53553 \\
\hline $\mathrm{C}$ & -3.90911 & -0.61002 & -1.57726 \\
\hline $\mathrm{C}$ & -4.33893 & -0.02808 & -0.31748 \\
\hline $\mathrm{C}$ & -4.10197 & -1.00611 & 0.73028 \\
\hline $\mathrm{C}$ & -3.65612 & -0.58954 & 1.98751 \\
\hline $\mathrm{C}$ & -3.43467 & 0.82683 & 2.24876 \\
\hline $\mathrm{C}$ & -2.27039 & 0.95163 & 3.10196 \\
\hline $\mathrm{C}$ & -1.37716 & 2.01175 & 2.92099 \\
\hline $\mathrm{C}$ & 0.05192 & 1.78712 & 2.99312 \\
\hline $\mathrm{C}$ & 0.68582 & 2.63193 & 2.01047 \\
\hline $\mathrm{C}$ & -0.34602 & 3.38236 & 1.31240 \\
\hline $\mathrm{C}$ & -1.62694 & 2.99822 & 1.87955 \\
\hline $\mathrm{C}$ & -2.74549 & 2.87187 & 1.05410 \\
\hline $\mathrm{C}$ & -3.66959 & 1.76690 & 1.24269 \\
\hline $\mathrm{C}$ & -4.12200 & 1.32939 & -0.06647 \\
\hline $\mathrm{C}$ & -3.47665 & 2.16313 & -1.06578 \\
\hline $\mathrm{C}$ & -2.62604 & 3.11724 & -0.37508 \\
\hline $\mathrm{C}$ & -1.39265 & 3.47857 & -0.91947 \\
\hline $\mathrm{C}$ & -0.23125 & 3.61774 & -0.05826 \\
\hline $\mathrm{C}$ & 0.92026 & 3.11246 & -0.78917 \\
\hline $\mathrm{C}$ & 1.90602 & 2.39434 & -0.11516 \\
\hline $\mathrm{C}$ & 1.78747 & 2.15087 & 1.30562 \\
\hline $\mathrm{C}$ & 2.35745 & 0.83435 & 1.56244 \\
\hline $\mathrm{C}$ & 3.14018 & 0.44744 & 0.38996 \\
\hline $\mathrm{C}$ & 2.54604 & 1.22206 & -0.69796 \\
\hline $\mathrm{C}$ & 2.07818 & 0.75866 & -1.93302 \\
\hline $\mathrm{C}$ & 8.25873 & 1.82313 & 0.38419 \\
\hline
\end{tabular}




$\begin{array}{lllr}\mathrm{C} & 7.23691 & 1.62745 & -0.73912 \\ \mathrm{H} & 8.37245 & 0.91195 & 0.98341 \\ \mathrm{H} & 7.95463 & 2.62820 & 1.06314 \\ \mathrm{H} & 9.24690 & 2.08198 & -0.01382 \\ \mathrm{H} & 6.24931 & 1.36809 & -0.33969 \\ \mathrm{H} & 7.54305 & 0.82356 & -1.41967 \\ \mathrm{H} & 7.12333 & 2.53881 & -1.33782\end{array}$

(3) $\mathrm{C}_{2} \mathrm{H}_{6} \_\mathrm{C}_{59} \mathrm{Pd}$ _TC1

$\begin{array}{cccc}\text { Pd } & 3.73681 & -0.87397 & -0.07127 \\ \mathrm{C} & 2.63205 & 0.12888 & -1.42579 \\ \mathrm{C} & 2.35038 & 1.49566 & -1.06669 \\ \mathrm{C} & 2.41540 & 1.69106 & 0.37567 \\ \mathrm{C} & 2.74146 & 0.46432 & 1.05632 \\ \mathrm{C} & 2.02090 & 0.03573 & 2.15329 \\ \mathrm{C} & 1.12428 & 0.97245 & 2.79608 \\ \mathrm{C} & 0.89330 & 2.23494 & 2.23664 \\ \mathrm{C} & 1.53037 & 2.59393 & 0.99370 \\ \mathrm{C} & 0.57008 & 3.34648 & 0.20195 \\ \mathrm{C} & 0.50916 & 3.16075 & -1.17538 \\ \mathrm{C} & 1.40532 & 2.21468 & -1.82137 \\ \mathrm{C} & 0.66683 & 1.54855 & -2.86546 \\ \mathrm{C} & 0.86359 & 0.18109 & -3.09222 \\ \mathrm{C} & 1.82313 & -0.56427 & -2.30525 \\ \mathrm{C} & 1.28012 & -1.93380 & -2.09837 \\ \mathrm{C} & -0.00580 & -1.97286 & -2.75469 \\ \mathrm{C} & -0.26074 & -0.68461 & -3.37593 \\ \mathrm{C} & -1.55170 & -0.15985 & -3.42320 \\ \mathrm{C} & -1.76491 & 1.25645 & -3.16423 \\ \mathrm{C} & -0.67878 & 2.09296 & -2.89545 \\ \mathrm{C} & -0.77845 & 3.09020 & -1.84400 \\ \mathrm{C} & -1.95650 & 3.21051 & -1.10460 \\ \mathrm{C} & -1.89305 & 3.40426 & 0.33542 \\ \mathrm{C} & -0.65381 & 3.46904 & 0.97485 \\ \mathrm{C} & -0.45109 & 2.78353 & 2.23949 \\ \mathrm{C} & -1.49977 & 2.05947 & 2.81261 \\ \mathrm{C} & -1.24826 & 0.75838 & 3.41548 \\ \mathrm{C} & 0.03957 & 0.22430 & 3.39368 \\ \mathrm{C} & 0.25472 & -1.18451 & 3.11253 \\ \mathrm{C} & -0.85567 & -2.01514 & 2.89306 \\ \mathrm{C} & -2.19105 & -1.45492 & 2.88531 \\ \mathrm{C} & -2.39069 & -0.09599 & 3.14724 \\ \mathrm{C} & -3.33769 & 0.66581 & 2.35815 \\ \mathrm{C} & -2.78977 & 2.00035 & 2.15522 \\ & -2.98281 & 2.65613 & 0.93627 \\ & -3.08568 & 2.34341 & -1.39004\end{array}$




$\begin{array}{lrrr}\mathrm{C} & -2.99184 & 1.38750 & -2.40491 \\ \mathrm{C} & -3.54072 & 0.05327 & -2.20217 \\ \mathrm{C} & -2.65674 & -0.90023 & -2.84211 \\ \mathrm{C} & -2.41962 & -2.14479 & -2.25086 \\ \mathrm{C} & -1.08340 & -2.70315 & -2.22797 \\ \mathrm{C} & -0.90928 & -3.38947 & -0.97070 \\ \mathrm{C} & -2.13546 & -3.25770 & -0.19933 \\ \mathrm{C} & -3.07383 & -2.48543 & -0.99548 \\ \mathrm{C} & -3.91958 & -1.56280 & -0.37733 \\ \mathrm{C} & -4.16132 & -0.26887 & -0.99282 \\ \mathrm{C} & -4.24582 & 0.72350 & 0.06496 \\ \mathrm{C} & -4.05682 & 0.04372 & 1.33483 \\ \mathrm{C} & -3.85526 & -1.36933 & 1.06344 \\ \mathrm{C} & -2.94770 & -2.10663 & 1.82607 \\ \mathrm{C} & -2.07389 & -3.07221 & 1.18235 \\ \mathrm{C} & -0.78378 & -3.01068 & 1.85119 \\ \mathrm{C} & 0.38763 & -3.13710 & 1.10678 \\ \mathrm{C} & 0.32401 & -3.32978 & -0.32465 \\ \mathrm{C} & 1.46772 & -2.63340 & -0.90047 \\ \mathrm{C} & 2.40229 & -2.32589 & 0.17728 \\ \mathrm{C} & 1.56882 & -2.32677 & 1.37643 \\ \mathrm{C} & 1.47766 & -1.33428 & 2.35935 \\ \mathrm{C} & 6.44271 & 0.52316 & 0.01072 \\ \mathrm{C} & 6.59518 & 2.03094 & 0.22645 \\ \mathrm{H} & 6.09408 & 0.02577 & 0.92672 \\ \mathrm{H} & 7.38632 & 0.04490 & -0.27444 \\ \mathrm{H} & 5.73254 & 0.32138 & -0.81093 \\ \mathrm{H} & 7.31523 & 2.23992 & 1.02495 \\ \mathrm{H} & 5.64111 & 2.48975 & 0.50721 \\ \mathrm{H} & 6.95065 & 2.52566 & -0.68371\end{array}$

(4) $\mathrm{C}_{2} \mathrm{H}_{6} \_\mathrm{C}_{59} \mathrm{Pd} \_\mathrm{HH} \_\mathrm{M}-\mathrm{C} 5$

$\begin{array}{cccc}\text { Pd } & 3.76229 & 0.24698 & -0.27016 \\ \text { C } & 2.61831 & -0.81740 & 0.96690 \\ \text { C } & 2.09604 & -2.05267 & 0.42845 \\ \text { C } & 2.00123 & -1.96286 & -1.02276 \\ \text { C } & 2.45891 & -0.66652 & -1.46900 \\ \text { C } & 1.71961 & 0.04371 & -2.36881 \\ \text { C } & 0.62492 & -0.60653 & -3.04540 \\ \text { C } & 0.26529 & -1.89793 & -2.73456 \\ \text { C } & 0.94788 & -2.57944 & -1.66880 \\ \text { C } & -0.03615 & -3.34258 & -0.93114 \\ \text { C } & 0.05279 & -3.42657 & 0.43355 \\ \text { C } & 1.13010 & -2.75159 & 1.12526 \\ \text { C } & 0.59671 & -2.21045 & 2.34558 \\ \text { C } & 1.00382 & -0.96364 & 2.76218 \\ \text { C } & 2.00713 & -0.22730 & 2.03415\end{array}$




\begin{tabular}{|c|c|c|c|}
\hline $\mathrm{C}$ & 1.62955 & 1.22999 & 2.12468 \\
\hline $\mathrm{C}$ & 0.42404 & 1.29020 & 2.92592 \\
\hline $\mathrm{C}$ & 0.04900 & -0.04397 & 3.32786 \\
\hline $\mathrm{C}$ & -1.27133 & -0.39814 & 3.44756 \\
\hline $\mathrm{C}$ & -1.71216 & -1.69347 & 2.97158 \\
\hline C & -0.80288 & -2.57212 & 2.43413 \\
\hline $\mathrm{C}$ & -1.14182 & -3.32410 & 1.24512 \\
\hline $\mathrm{C}$ & -2.36755 & -3.15016 & 0.64851 \\
\hline $\mathrm{C}$ & -2.46172 & -3.06140 & -0.79570 \\
\hline $\mathrm{C}$ & -1.32491 & -3.15149 & -1.56266 \\
\hline $\mathrm{C}$ & -1.13675 & -2.25743 & -2.68486 \\
\hline $\mathrm{C}$ & -2.10102 & -1.32718 & -2.98894 \\
\hline $\mathrm{C}$ & -1.71533 & 0.01997 & -3.35657 \\
\hline $\mathrm{C}$ & -0.38787 & 0.36739 & -3.36655 \\
\hline $\mathrm{C}$ & 0.04650 & 1.64565 & -2.85704 \\
\hline $\mathrm{C}$ & -0.89402 & 2.53477 & -2.38279 \\
\hline $\mathrm{C}$ & -2.29202 & 2.16350 & -2.33843 \\
\hline $\mathrm{C}$ & -2.69221 & 0.93651 & -2.80851 \\
\hline $\mathrm{C}$ & -3.66901 & 0.16018 & -2.07944 \\
\hline $\mathrm{C}$ & -3.30188 & -1.23918 & -2.18935 \\
\hline $\mathrm{C}$ & -3.47710 & -2.08237 & -1.11765 \\
\hline $\mathrm{C}$ & -4.01402 & -1.56666 & 0.12535 \\
\hline $\mathrm{C}$ & -3.32486 & -2.22579 & 1.21616 \\
\hline $\mathrm{C}$ & -3.00565 & -1.51817 & 2.35090 \\
\hline $\mathrm{C}$ & -3.37273 & -0.11880 & 2.46089 \\
\hline $\mathrm{C}$ & -2.30358 & 0.57060 & 3.14651 \\
\hline $\mathrm{C}$ & -1.95774 & 1.84886 & 2.78167 \\
\hline $\mathrm{C}$ & -0.56286 & 2.22299 & 2.68952 \\
\hline $\mathrm{C}$ & -0.41714 & 3.13680 & 1.59166 \\
\hline $\mathrm{C}$ & -1.71317 & 3.30600 & 0.96347 \\
\hline $\mathrm{C}$ & -2.66808 & 2.50988 & 1.70460 \\
\hline $\mathrm{C}$ & -3.67420 & 1.84851 & 1.04069 \\
\hline $\mathrm{C}$ & -4.03628 & 0.50159 & 1.42942 \\
\hline $\mathrm{C}$ & -4.36149 & -0.24158 & 0.22947 \\
\hline $\mathrm{C}$ & -4.18846 & 0.64490 & -0.90285 \\
\hline $\mathrm{C}$ & -3.76845 & 1.93721 & -0.40296 \\
\hline $\mathrm{C}$ & -2.85156 & 2.68257 & -1.10592 \\
\hline $\mathrm{C}$ & -1.80245 & 3.39000 & -0.40318 \\
\hline $\mathrm{C}$ & -0.60013 & 3.30896 & -1.20986 \\
\hline $\mathrm{C}$ & 0.62301 & 3.15685 & -0.61360 \\
\hline $\mathrm{C}$ & 0.71717 & 3.06800 & 0.82797 \\
\hline $\mathrm{C}$ & 1.80198 & 2.15855 & 1.12768 \\
\hline $\mathrm{C}$ & 2.64106 & 2.03141 & -0.08824 \\
\hline $\mathrm{C}$ & 1.65254 & 2.29936 & -1.16025 \\
\hline $\mathrm{C}$ & 1.34499 & 1.49782 & -2.23209 \\
\hline $\mathrm{C}$ & 8.78671 & -0.40706 & 0.45947 \\
\hline $\mathrm{C}$ & 7.28680 & -0.21195 & 0.24136 \\
\hline $\mathrm{H}$ & 7.07073 & 0.80951 & -0.06008 \\
\hline $\mathrm{H}$ & 6.73378 & -0.41814 & 1.15372 \\
\hline
\end{tabular}




$\begin{array}{llll}\mathrm{H} & 6.91668 & -0.87895 & -0.53281 \\ \mathrm{H} & 9.15914 & 0.25628 & 1.23328 \\ \mathrm{H} & 9.34250 & -0.20183 & -0.44958 \\ \mathrm{H} & 9.00657 & -1.42584 & 0.76103\end{array}$

(5) $\mathrm{C}_{2} \mathrm{H}_{6} \_\mathrm{C}_{59} \mathrm{Pd} \_\mathrm{HH} \_\mathrm{M}-\mathrm{C} 1$

$\begin{array}{cccc}\text { Pd } & 3.73610 & -0.88000 & -0.00221 \\ \mathrm{C} & 2.68946 & 0.29541 & -1.25898 \\ \mathrm{C} & 2.39274 & 1.60509 & -0.73745 \\ \mathrm{C} & 2.39453 & 1.61003 & 0.71954 \\ \mathrm{C} & 2.69250 & 0.30391 & 1.24917 \\ \mathrm{C} & 1.92485 & -0.25807 & 2.24999 \\ \mathrm{C} & 1.00229 & 0.59305 & 2.97109 \\ \mathrm{C} & 0.79543 & 1.91838 & 2.56980 \\ \mathrm{C} & 1.48500 & 2.43095 & 1.41123 \\ \mathrm{C} & 0.55959 & 3.28426 & 0.68324 \\ \mathrm{C} & 0.55793 & 3.27957 & -0.70796 \\ \mathrm{C} & 1.48153 & 2.42133 & -1.43244 \\ \mathrm{C} & 0.78922 & 1.90106 & -2.58591 \\ \mathrm{C} & 0.99514 & 0.57307 & -2.97883 \\ \mathrm{C} & 1.91943 & -0.27322 & -2.25423 \\ \mathrm{C} & 1.36861 & -1.65505 & -2.24890 \\ \mathrm{C} & 0.11228 & -1.60197 & -2.95904 \\ \mathrm{C} & -0.11569 & -0.24319 & -3.41947 \\ \mathrm{C} & -1.40364 & 0.29020 & -3.45374 \\ \mathrm{C} & -1.62758 & 1.66200 & -3.02211 \\ \mathrm{C} & -0.55437 & 2.45175 & -2.60215 \\ \mathrm{C} & -0.69934 & 3.30401 & -1.43470 \\ \mathrm{C} & -1.90806 & 3.33391 & -0.73672 \\ \mathrm{C} & -1.90633 & 3.33882 & 0.71754 \\ \mathrm{C} & -0.69593 & 3.31358 & 1.41282 \\ \mathrm{C} & -0.54813 & 2.46915 & 2.58558 \\ \mathrm{C} & -1.62032 & 1.68221 & 3.01337 \\ \mathrm{C} & -1.39533 & 0.31334 & 3.45368 \\ \mathrm{C} & -0.10746 & -0.22028 & 3.41983 \\ \mathrm{C} & 0.11942 & -1.58212 & 2.96801 \\ \mathrm{C} & -0.98022 & -2.37163 & 2.59539 \\ \mathrm{C} & -2.31460 & -1.80830 & 2.60303 \\ \mathrm{C} & -2.52519 & -0.49326 & 3.02920 \\ \mathrm{C} & -3.43683 & 0.36951 & 2.30485 \\ \mathrm{C} & -2.88094 & 1.71628 & 2.29984 \\ \mathrm{C} & -3.71651 & 2.02248 & -0.00301 \\ \mathrm{C} & -2.88648 & 1.70083 & -2.30579 \\ \mathrm{C} & -2.359 & -0.51353 & -3.02117\end{array}$




$\begin{array}{lrrr}\mathrm{C} & -2.32086 & -1.82568 & -2.58672 \\ \mathrm{C} & -0.98645 & -2.38897 & -2.57854 \\ \mathrm{C} & -0.86644 & -3.23273 & -1.41411 \\ \mathrm{C} & -2.12433 & -3.19504 & -0.68523 \\ \mathrm{C} & -3.02779 & -2.32221 & -1.41439 \\ \mathrm{C} & -3.89868 & -1.48229 & -0.71847 \\ \mathrm{C} & -4.11372 & -0.11841 & -1.17060 \\ \mathrm{C} & -4.24490 & 0.72911 & 0.00198 \\ \mathrm{C} & -4.11093 & -0.11056 & 1.17987 \\ \mathrm{C} & -3.89693 & -1.47743 & 0.73631 \\ \mathrm{C} & -3.02433 & -2.31264 & 1.43572 \\ \mathrm{C} & -2.12265 & -3.19036 & 0.71025 \\ \mathrm{C} & -0.86302 & -3.22317 & 1.43634 \\ \mathrm{C} & 0.33932 & -3.25802 & 0.73251 \\ \mathrm{C} & 0.33759 & -3.26290 & -0.71293 \\ \mathrm{C} & 1.50488 & -2.50400 & -1.14406 \\ \mathrm{C} & 2.39152 & -2.34270 & 0.00424 \\ \mathrm{C} & 1.50766 & -2.49628 & 1.15576 \\ \mathrm{C} & 1.37403 & -1.63993 & 2.25521 \\ \mathrm{C} & 6.43087 & 0.52081 & 0.00302 \\ \mathrm{C} & 6.61180 & 2.04104 & 0.00416 \\ \mathrm{H} & 7.16398 & 2.37113 & -0.88222 \\ \mathrm{H} & 7.16892 & 2.36906 & 0.88823 \\ \mathrm{H} & 5.64491 & 2.55549 & 0.00751 \\ \mathrm{H} & 7.38754 & -0.01316 & -0.00063 \\ \mathrm{H} & 5.88383 & 0.19446 & -0.89586 \\ \mathrm{H} & 5.88889 & 0.19218 & 0.90392\end{array}$

(6) $\mathrm{C}_{2} \mathrm{H}_{6} \_\mathrm{C}_{59} \mathrm{Pd}$-HH_C1-C2

$\begin{array}{cccc}\mathrm{Pd} & 3.73672 & -0.87423 & -0.07150 \\ \mathrm{C} & 2.63185 & 0.12788 & -1.42646 \\ \mathrm{C} & 2.35032 & 1.49488 & -1.06808 \\ \mathrm{C} & 2.41561 & 1.69112 & 0.37417 \\ \mathrm{C} & 2.74172 & 0.46476 & 1.05548 \\ \mathrm{C} & 2.02129 & 0.03684 & 2.15281 \\ \mathrm{C} & 1.12483 & 0.97397 & 2.79521 \\ \mathrm{C} & 0.89384 & 2.23615 & 2.23508 \\ \mathrm{C} & 1.53072 & 2.59438 & 0.99182 \\ \mathrm{C} & 0.57033 & 3.34654 & 0.19981 \\ \mathrm{C} & 0.50916 & 3.16000 & -1.17740 \\ \mathrm{C} & 1.40516 & 2.21352 & -1.82300 \\ \mathrm{C} & 0.66643 & 1.54681 & -2.86656 \\ \mathrm{C} & 0.86308 & 0.17921 & -3.09257 \\ \mathrm{C} & 1.82272 & -0.56575 & -2.30535 \\ \mathrm{C} & 1.27967 & -1.93513 & -2.09758 \\ \mathrm{C} & -0.00637 & -1.97451 & -2.75366 \\ \mathrm{C} & -0.26135 & -0.68659 & -3.37558\end{array}$




\begin{tabular}{|c|c|c|c|}
\hline C & -1.55228 & -0.16178 & -3.42294 \\
\hline $\mathrm{C}$ & -1.76537 & 1.25468 & -3.16476 \\
\hline $\mathrm{C}$ & -0.67914 & 2.09129 & -2.89665 \\
\hline $\mathrm{C}$ & -0.77857 & 3.08913 & -1.84575 \\
\hline $\mathrm{C}$ & -1.95648 & 3.20994 & -1.10622 \\
\hline $\mathrm{C}$ & -1.89278 & 3.40454 & 0.33368 \\
\hline $\mathrm{C}$ & -0.65342 & 3.46962 & 0.97285 \\
\hline $\mathrm{C}$ & -0.45052 & 2.78482 & 2.23785 \\
\hline $\mathrm{C}$ & -1.49914 & 2.06116 & 2.81158 \\
\hline $\mathrm{C}$ & -1.24760 & 0.76040 & 3.41515 \\
\hline $\mathrm{C}$ & 0.04020 & 0.22623 & 3.39343 \\
\hline $\mathrm{C}$ & 0.25522 & -1.18276 & 3.11306 \\
\hline $\mathrm{C}$ & -0.85525 & -2.01345 & 2.89427 \\
\hline $\mathrm{C}$ & -2.19060 & -1.45316 & 2.88642 \\
\hline $\mathrm{C}$ & -2.39012 & -0.09406 & 3.14761 \\
\hline $\mathrm{C}$ & -3.33722 & 0.66732 & 2.35825 \\
\hline $\mathrm{C}$ & -2.78925 & 2.00173 & 2.15445 \\
\hline $\mathrm{C}$ & -2.98247 & 2.65681 & 0.93515 \\
\hline $\mathrm{C}$ & -3.72413 & 2.00497 & -0.13053 \\
\hline $\mathrm{C}$ & -3.08576 & 2.34274 & -1.39096 \\
\hline $\mathrm{C}$ & -2.99216 & 1.38623 & -2.40529 \\
\hline $\mathrm{C}$ & -3.54108 & 0.05215 & -2.20169 \\
\hline $\mathrm{C}$ & -2.65727 & -0.90177 & -2.84123 \\
\hline $\mathrm{C}$ & -2.42011 & -2.14600 & -2.24930 \\
\hline $\mathrm{C}$ & -1.08392 & -2.70443 & -2.22633 \\
\hline $\mathrm{C}$ & -0.90962 & -3.39004 & -0.96868 \\
\hline $\mathrm{C}$ & -2.13566 & -3.25774 & -0.19718 \\
\hline $\mathrm{C}$ & -3.07412 & -2.48586 & -0.99361 \\
\hline $\mathrm{C}$ & -3.91970 & -1.56283 & -0.37585 \\
\hline $\mathrm{C}$ & -4.16147 & -0.26924 & -0.99203 \\
\hline $\mathrm{C}$ & -4.24573 & 0.72374 & 0.06517 \\
\hline $\mathrm{C}$ & -4.05655 & 0.04469 & 1.33541 \\
\hline $\mathrm{C}$ & -3.85513 & -1.36854 & 1.06481 \\
\hline $\mathrm{C}$ & -2.94747 & -2.10545 & 1.82770 \\
\hline $\mathrm{C}$ & -2.07384 & -3.07145 & 1.18439 \\
\hline $\mathrm{C}$ & -0.78360 & -3.00960 & 1.85295 \\
\hline $\mathrm{C}$ & 0.38767 & -3.13651 & 1.10841 \\
\hline $\mathrm{C}$ & 0.32379 & -3.33003 & -0.32289 \\
\hline $\mathrm{C}$ & 1.46744 & -2.63405 & -0.89933 \\
\hline $\mathrm{C}$ & 2.40222 & -2.32600 & 0.17811 \\
\hline $\mathrm{C}$ & 1.56895 & -2.32610 & 1.37739 \\
\hline $\mathrm{C}$ & 1.47802 & -1.33302 & 2.35975 \\
\hline $\mathrm{C}$ & 6.44270 & 0.52367 & 0.01061 \\
\hline $\mathrm{C}$ & 6.59450 & 2.03135 & 0.22737 \\
\hline $\mathrm{H}$ & 7.38640 & 0.04610 & -0.27541 \\
\hline $\mathrm{H}$ & 5.73218 & 0.32211 & -0.81080 \\
\hline $\mathrm{H}$ & 6.09478 & 0.02541 & 0.92639 \\
\hline $\mathrm{H}$ & 6.95118 & 2.52664 & -0.68200 \\
\hline $\mathrm{H}$ & 7.31333 & 2.24006 & 1.02703 \\
\hline
\end{tabular}


$\begin{array}{llll}\text { H } & 5.63992 & 2.48978 & 0.50699\end{array}$

(7) $\mathrm{C}_{2} \mathrm{H}_{6} \_\mathrm{C}_{59} \mathrm{Pd}$ PPH_C2-C3

\begin{tabular}{|c|c|c|c|}
\hline $\mathrm{Pd}$ & 3.73670 & -0.87438 & -0.07160 \\
\hline $\mathrm{C}$ & 2.63177 & 0.12744 & -1.42671 \\
\hline $\mathrm{C}$ & 2.35031 & 1.49454 & -1.06861 \\
\hline $\mathrm{C}$ & 2.41571 & 1.69110 & 0.37359 \\
\hline $\mathrm{C}$ & 2.74183 & 0.46489 & 1.05517 \\
\hline C & 2.02146 & 0.03721 & 2.15263 \\
\hline $\mathrm{C}$ & 1.12508 & 0.97451 & 2.79489 \\
\hline C & 0.89408 & 2.23658 & 2.23449 \\
\hline C & 1.53089 & 2.59452 & 0.99111 \\
\hline C & 0.57047 & 3.34654 & 0.19900 \\
\hline C & 0.50920 & 3.15971 & -1.17816 \\
\hline $\mathrm{C}$ & 1.40512 & 2.21305 & -1.82362 \\
\hline C & 0.66629 & 1.54615 & -2.86698 \\
\hline $\mathrm{C}$ & 0.86287 & 0.17850 & -3.09271 \\
\hline $\mathrm{C}$ & 1.82254 & -0.56633 & -2.30540 \\
\hline $\mathrm{C}$ & 1.27946 & -1.93565 & -2.09732 \\
\hline C & -0.00662 & -1.97512 & -2.75330 \\
\hline C & -0.26160 & -0.68733 & -3.37546 \\
\hline $\mathrm{C}$ & -1.55252 & -0.16248 & -3.42284 \\
\hline $\mathrm{C}$ & -1.76554 & 1.25404 & -3.16494 \\
\hline $\mathrm{C}$ & -0.67926 & 2.09067 & -2.89709 \\
\hline $\mathrm{C}$ & -0.77858 & 3.08875 & -1.84640 \\
\hline $\mathrm{C}$ & -1.95643 & 3.20975 & -1.10681 \\
\hline $\mathrm{C}$ & -1.89262 & 3.40465 & 0.33305 \\
\hline $\mathrm{C}$ & -0.65322 & 3.46982 & 0.97211 \\
\hline $\mathrm{C}$ & -0.45025 & 2.78530 & 2.23725 \\
\hline $\mathrm{C}$ & -1.49886 & 2.06179 & 2.81121 \\
\hline $\mathrm{C}$ & -1.24732 & 0.76115 & 3.41503 \\
\hline $\mathrm{C}$ & 0.04045 & 0.22692 & 3.39333 \\
\hline $\mathrm{C}$ & 0.25541 & -1.18212 & 3.11324 \\
\hline $\mathrm{C}$ & -0.85510 & -2.01283 & 2.89473 \\
\hline C & -2.19043 & -1.45249 & 2.88685 \\
\hline $\mathrm{C}$ & -2.38988 & -0.09334 & 3.14776 \\
\hline C & -3.33702 & 0.66792 & 2.35831 \\
\hline C & -2.78902 & 2.00226 & 2.15419 \\
\hline $\mathrm{C}$ & -2.98229 & 2.65708 & 0.93475 \\
\hline C & -3.72405 & 2.00505 & -0.13074 \\
\hline $\mathrm{C}$ & -3.08577 & 2.34253 & -1.39128 \\
\hline $\mathrm{C}$ & -2.99227 & 1.38580 & -2.40542 \\
\hline $\mathrm{C}$ & -3.54122 & 0.05178 & -2.20149 \\
\hline $\mathrm{C}$ & -2.65749 & -0.90230 & -2.84089 \\
\hline C & -2.42033 & -2.14641 & -2.24871 \\
\hline C & -1.08416 & -2.70489 & -2.22572 \\
\hline C & -0.90979 & -3.39023 & -0.96794 \\
\hline
\end{tabular}




$\begin{array}{lrrr}\mathrm{C} & -2.13577 & -3.25773 & -0.19637 \\ \mathrm{C} & -3.07426 & -2.48598 & -0.99289 \\ \mathrm{C} & -3.91977 & -1.56280 & -0.37527 \\ \mathrm{C} & -4.16154 & -0.26934 & -0.99172 \\ \mathrm{C} & -4.24568 & 0.72388 & 0.06528 \\ \mathrm{C} & -4.05644 & 0.04509 & 1.33565 \\ \mathrm{C} & -3.85509 & -1.36820 & 1.06533 \\ \mathrm{C} & -2.94740 & -2.10498 & 1.82832 \\ \mathrm{C} & -2.07384 & -3.07115 & 1.18515 \\ \mathrm{C} & -0.78356 & -3.00920 & 1.85361 \\ \mathrm{C} & 0.38766 & -3.13630 & 1.10901 \\ \mathrm{C} & 0.32367 & -3.33012 & -0.32225 \\ \mathrm{C} & 1.46730 & -2.63431 & -0.89892 \\ \mathrm{C} & 2.40217 & -2.32605 & 0.17836 \\ \mathrm{C} & 1.56898 & -2.32586 & 1.37771 \\ \mathrm{C} & 1.47814 & -1.33258 & 2.35987 \\ \mathrm{C} & 6.44245 & 0.52392 & 0.01070 \\ \mathrm{C} & 6.59428 & 2.03158 & 0.22764 \\ \mathrm{H} & 5.73176 & 0.32252 & -0.81060 \\ \mathrm{H} & 6.09468 & 0.02552 & 0.92648 \\ \mathrm{H} & 7.38610 & 0.04641 & -0.27557 \\ \mathrm{H} & 5.63971 & 2.48998 & 0.50739 \\ \mathrm{H} & 6.95090 & 2.52698 & -0.68170 \\ \mathrm{H} & 7.31316 & 2.24018 & 1.02728\end{array}$

(8) $\mathrm{C}_{2} \mathrm{H}_{6} \_\mathrm{C}_{59} \mathrm{Pd} \_\mathrm{HH}$-C3-C4

$\begin{array}{cccc}\text { Pd } & -3.44393 & -1.78328 & -0.15796 \\ \mathrm{C} & -1.68219 & -2.65299 & -0.56838 \\ \mathrm{C} & -0.93380 & -3.06001 & 0.59422 \\ \mathrm{C} & -1.32128 & -2.29478 & 1.77264 \\ \mathrm{C} & -2.34980 & -1.33450 & 1.46290 \\ \mathrm{C} & -2.25062 & -0.01784 & 1.87047 \\ \mathrm{C} & -1.26066 & 0.31990 & 2.86951 \\ \mathrm{C} & -0.33452 & -0.63599 & 3.30515 \\ \mathrm{C} & -0.34796 & -1.95868 & 2.73091 \\ \mathrm{C} & 1.03195 & -2.38642 & 2.56556 \\ \mathrm{C} & 1.40186 & -3.11660 & 1.44075 \\ \mathrm{C} & 0.40786 & -3.45032 & 0.43316 \\ \mathrm{C} & 1.03591 & -3.33871 & -0.86045 \\ \mathrm{C} & 0.32043 & -2.79811 & -1.93601 \\ \mathrm{C} & -1.05382 & -2.37904 & -1.76850 \\ \mathrm{C} & -1.27250 & -1.17465 & -2.61871 \\ \mathrm{C} & -0.01281 & -0.89372 & -3.26763 \\ \mathrm{C} & 0.96268 & -1.89131 & -2.86320 \\ \mathrm{C} & 2.30290 & -1.54853 & -2.69006 \\ \mathrm{C} & 3.04442 & -2.08692 & -1.55895 \\ \mathrm{C} & 2.42556 & -2.96608 & -0.66630\end{array}$




\begin{tabular}{|c|c|c|c|}
\hline C & 2.65163 & -2.82300 & 0.76141 \\
\hline $\mathrm{C}$ & 3.48489 & -1.81000 & 1.23941 \\
\hline $\mathrm{C}$ & 3.09814 & -1.04691 & 2.41552 \\
\hline $\mathrm{C}$ & 1.89475 & -1.32982 & 3.06450 \\
\hline $\mathrm{C}$ & 1.04610 & -0.24542 & 3.52788 \\
\hline $\mathrm{C}$ & 1.43836 & 1.07979 & 3.32192 \\
\hline $\mathrm{C}$ & 0.46538 & 2.07461 & 2.89397 \\
\hline $\mathrm{C}$ & -0.85539 & 1.69354 & 2.66174 \\
\hline $\mathrm{C}$ & -1.58791 & 2.21239 & 1.51988 \\
\hline $\mathrm{C}$ & -0.96005 & 3.13276 & 0.66536 \\
\hline $\mathrm{C}$ & 0.42161 & 3.50541 & 0.88872 \\
\hline $\mathrm{C}$ & 1.12425 & 2.99411 & 1.98389 \\
\hline $\mathrm{C}$ & 2.49610 & 2.55494 & 1.82806 \\
\hline $\mathrm{C}$ & 2.69230 & 1.37422 & 2.65882 \\
\hline $\mathrm{C}$ & 3.50623 & 0.33121 & 2.20894 \\
\hline $\mathrm{C}$ & 4.15078 & 0.42111 & 0.91002 \\
\hline $\mathrm{C}$ & 4.13090 & -0.90154 & 0.30905 \\
\hline $\mathrm{C}$ & 3.91716 & -1.04171 & -1.06476 \\
\hline $\mathrm{C}$ & 3.72094 & 0.13906 & -1.89533 \\
\hline $\mathrm{C}$ & 2.73314 & -0.17904 & -2.90634 \\
\hline $\mathrm{C}$ & 1.80163 & 0.78385 & -3.30609 \\
\hline $\mathrm{C}$ & 0.41530 & 0.42025 & -3.51669 \\
\hline $\mathrm{C}$ & -0.40596 & 1.51669 & -3.06359 \\
\hline $\mathrm{C}$ & 0.46413 & 2.56739 & -2.55892 \\
\hline $\mathrm{C}$ & 1.83498 & 2.11229 & -2.71146 \\
\hline $\mathrm{C}$ & 2.78323 & 2.41416 & -1.73273 \\
\hline $\mathrm{C}$ & 3.74773 & 1.41046 & -1.31767 \\
\hline $\mathrm{C}$ & 3.95756 & 1.55206 & 0.11270 \\
\hline $\mathrm{C}$ & 3.12237 & 2.64354 & 0.58276 \\
\hline $\mathrm{C}$ & 2.39657 & 3.17827 & -0.55660 \\
\hline $\mathrm{C}$ & 1.07692 & 3.60805 & -0.40748 \\
\hline $\mathrm{C}$ & 0.09294 & 3.29993 & -1.43054 \\
\hline $\mathrm{C}$ & -1.16379 & 3.01150 & -0.75760 \\
\hline $\mathrm{C}$ & -1.99332 & 2.00370 & -1.24498 \\
\hline $\mathrm{C}$ & -1.60854 & 1.24487 & -2.41455 \\
\hline $\mathrm{C}$ & -2.09569 & -0.11479 & -2.22129 \\
\hline $\mathrm{C}$ & -3.07461 & -0.09030 & -1.13616 \\
\hline $\mathrm{C}$ & -2.70727 & 1.09244 & -0.36046 \\
\hline $\mathrm{C}$ & -2.46827 & 1.18359 & 1.01629 \\
\hline $\mathrm{C}$ & -6.44791 & 2.35620 & 1.09029 \\
\hline $\mathrm{C}$ & -6.52294 & 2.55286 & -0.42616 \\
\hline $\mathrm{H}$ & -6.90324 & 3.19675 & 1.62701 \\
\hline $\mathrm{H}$ & -5.40800 & 2.27135 & 1.42652 \\
\hline $\mathrm{H}$ & -6.97280 & 1.44452 & 1.40048 \\
\hline $\mathrm{H}$ & -6.00963 & 3.47141 & -0.73362 \\
\hline $\mathrm{H}$ & -7.56173 & 2.62427 & -0.76936 \\
\hline $\mathrm{H}$ & -6.05189 & 1.71810 & -0.95811 \\
\hline
\end{tabular}


(9) $\mathrm{C}_{2} \mathrm{H}_{6} \_\mathrm{C}_{59} \mathrm{Pd} \_\mathrm{PH}$ _C4-C5

\begin{tabular}{|c|c|c|c|}
\hline $\mathrm{Pd}$ & -3.24632 & -2.20865 & -0.02249 \\
\hline $\mathrm{C}$ & -1.73252 & -2.32558 & -1.33436 \\
\hline $\mathrm{C}$ & -0.61909 & -3.12539 & -0.88975 \\
\hline $\mathrm{C}$ & -0.57532 & -3.21719 & 0.56432 \\
\hline C & -1.65749 & -2.48362 & 1.17123 \\
\hline $\mathrm{C}$ & -1.43443 & -1.60513 & 2.21461 \\
\hline $\mathrm{C}$ & -0.15929 & -1.65633 & 2.89543 \\
\hline C & 0.87057 & -2.47620 & 2.41772 \\
\hline C & 0.66970 & -3.25194 & 1.21845 \\
\hline C & 1.90746 & -3.22011 & 0.45560 \\
\hline $\mathrm{C}$ & 1.86556 & -3.13341 & -0.93216 \\
\hline $\mathrm{C}$ & 0.58398 & -3.07417 & -1.61685 \\
\hline $\mathrm{C}$ & 0.71508 & -2.15492 & -2.72031 \\
\hline C & -0.33873 & -1.28688 & -3.03113 \\
\hline $\mathrm{C}$ & -1.57034 & -1.32497 & -2.27350 \\
\hline $\mathrm{C}$ & -2.08684 & 0.06921 & -2.16857 \\
\hline C & -1.13888 & 0.91303 & -2.85853 \\
\hline C & -0.07344 & 0.08718 & -3.39913 \\
\hline C & 1.23825 & 0.55701 & -3.44645 \\
\hline $\mathrm{C}$ & 2.33597 & -0.33379 & -3.09926 \\
\hline C & 2.08022 & -1.66189 & -2.74870 \\
\hline C & 2.79304 & -2.26490 & -1.63582 \\
\hline $\mathrm{C}$ & 3.73028 & -1.51827 & -0.91963 \\
\hline $\mathrm{C}$ & 3.77400 & -1.60852 & 0.53114 \\
\hline $\mathrm{C}$ & 2.87923 & -2.44219 & 1.20440 \\
\hline $\mathrm{C}$ & 2.23685 & -1.98477 & 2.42428 \\
\hline $\mathrm{C}$ & 2.51830 & -0.70933 & 2.92091 \\
\hline $\mathrm{C}$ & 1.44659 & 0.12742 & 3.44063 \\
\hline C & 0.13273 & -0.33775 & 3.41459 \\
\hline C & -0.96006 & 0.54464 & 3.04485 \\
\hline C & -0.68283 & 1.88791 & 2.74554 \\
\hline $\mathrm{C}$ & 0.68522 & 2.36364 & 2.74216 \\
\hline C & 1.73356 & 1.50626 & 3.08878 \\
\hline C & 2.96749 & 1.52125 & 2.32958 \\
\hline C & 3.45566 & 0.15204 & 2.22961 \\
\hline C & 4.06930 & -0.28625 & 1.05313 \\
\hline C & 4.21456 & 0.62083 & -0.07230 \\
\hline $\mathrm{C}$ & 3.99871 & -0.14026 & -1.29071 \\
\hline C & 3.31649 & 0.43854 & -2.36420 \\
\hline C & 2.82869 & 1.80806 & -2.26462 \\
\hline C & 1.55092 & 1.88224 & -2.94360 \\
\hline C & 0.52864 & 2.68710 & -2.43197 \\
\hline $\mathrm{C}$ & -0.83898 & 2.20991 & -2.41174 \\
\hline C & -1.45960 & 2.68879 & -1.20073 \\
\hline C & -0.47867 & 3.45997 & -0.45297 \\
\hline C & 0.75543 & 3.46023 & -1.21905 \\
\hline $\mathrm{C}$ & 1.98468 & 3.38086 & -0.56217 \\
\hline
\end{tabular}




$\begin{array}{lrrr}\mathrm{C} & 3.04359 & 2.54122 & -1.09526 \\ \mathrm{C} & 3.74164 & 1.93202 & 0.02361 \\ \mathrm{C} & 3.11446 & 2.39475 & 1.24946 \\ \mathrm{C} & 2.02838 & 3.28980 & 0.88926 \\ \mathrm{C} & 0.84130 & 3.28233 & 1.62368 \\ \mathrm{C} & -0.43668 & 3.37292 & 0.93914 \\ \mathrm{C} & -1.37329 & 2.51099 & 1.64291 \\ \mathrm{C} & -2.31114 & 1.77470 & 0.92188 \\ \mathrm{C} & -2.35464 & 1.86462 & -0.52116 \\ \mathrm{C} & -2.72852 & 0.54756 & -1.02050 \\ \mathrm{C} & -3.26482 & -0.22200 & 0.10096 \\ \mathrm{C} & -2.65940 & 0.40474 & 1.27534 \\ \mathrm{C} & -1.95092 & -0.21047 & 2.31389 \\ \mathrm{C} & -6.39931 & 2.42481 & 0.59119 \\ \mathrm{C} & -6.17594 & 3.38018 & -0.58420 \\ \mathrm{H} & -5.91643 & 2.79603 & 1.50280 \\ \mathrm{H} & -5.98653 & 1.43114 & 0.38081 \\ \mathrm{H} & -7.46686 & 2.30295 & 0.80948 \\ \mathrm{H} & -5.10843 & 3.49291 & -0.80635 \\ \mathrm{H} & -6.57763 & 4.37783 & -0.37125 \\ \mathrm{H} & -6.66537 & 3.01420 & -1.49442\end{array}$

(10) $\mathrm{C}_{2} \mathrm{H}_{6} \_\mathrm{C} 59 \mathrm{Pd}$ _HH_C6-C7

$\begin{array}{cccc}\text { Pd } & -3.73672 & -0.87419 & -0.07147 \\ \mathrm{C} & -2.74170 & 0.46479 & 1.05549 \\ \mathrm{C} & -2.41558 & 1.69113 & 0.37413 \\ \mathrm{C} & -2.35031 & 1.49486 & -1.06811 \\ \mathrm{C} & -2.63186 & 0.12785 & -1.42647 \\ \mathrm{C} & -1.82271 & -0.56578 & -2.30535 \\ \mathrm{C} & -0.86307 & 0.17916 & -3.09258 \\ \mathrm{C} & -0.66642 & 1.54676 & -2.86659 \\ \mathrm{C} & -1.40514 & 2.21348 & -1.82304 \\ \mathrm{C} & -0.50913 & 3.15998 & -1.17746 \\ \mathrm{C} & -0.57030 & 3.34654 & 0.19975 \\ \mathrm{C} & -1.53069 & 2.59440 & 0.99177 \\ \mathrm{C} & -0.89382 & 2.23620 & 2.23504 \\ \mathrm{C} & -1.12482 & 0.97403 & 2.79520 \\ \mathrm{C} & -2.02128 & 0.03689 & 2.15283 \\ \mathrm{C} & -1.47802 & -1.33297 & 2.35979 \\ \mathrm{C} & -0.25522 & -1.18270 & 3.11309 \\ \mathrm{C} & -0.04019 & 0.22629 & 3.39344 \\ \mathrm{C} & 1.24761 & 0.76046 & 3.41514 \\ \mathrm{C} & 1.49916 & 2.06120 & 2.81154 \\ \mathrm{C} & 0.45054 & 2.78487 & 2.23780 \\ \mathrm{C} & 0.65344 & 3.46963 & 0.97279 \\ \mathrm{C} & 1.89280 & 3.40454 & 0.33362 \\ \mathrm{C} & 1.95650 & 3.20991 & -1.10628\end{array}$




\begin{tabular}{|c|c|c|c|}
\hline $\mathrm{C}$ & 0.77859 & 3.08909 & -1.84581 \\
\hline $\mathrm{C}$ & 0.67916 & 2.09124 & -2.89669 \\
\hline $\mathrm{C}$ & 1.76538 & 1.25462 & -3.16479 \\
\hline C & 1.55229 & -0.16185 & -3.42294 \\
\hline $\mathrm{C}$ & 0.26135 & -0.68666 & -3.37557 \\
\hline $\mathrm{C}$ & 0.00636 & -1.97455 & -2.75363 \\
\hline $\mathrm{C}$ & 1.08391 & -2.70447 & -2.22628 \\
\hline $\mathrm{C}$ & 2.42011 & -2.14605 & -2.24926 \\
\hline C & 2.65727 & -0.90183 & -2.84121 \\
\hline $\mathrm{C}$ & 3.54108 & 0.05210 & -2.20169 \\
\hline $\mathrm{C}$ & 2.99217 & 1.38618 & -2.40532 \\
\hline C & 3.08578 & 2.34270 & -1.39100 \\
\hline $\mathrm{C}$ & 3.72415 & 2.00496 & -0.13057 \\
\hline $\mathrm{C}$ & 2.98249 & 2.65681 & 0.93510 \\
\hline $\mathrm{C}$ & 2.78927 & 2.00176 & 2.15441 \\
\hline $\mathrm{C}$ & 3.33723 & 0.66736 & 2.35824 \\
\hline C & 2.39013 & -0.09402 & 3.14762 \\
\hline C & 2.19060 & -1.45311 & 2.88645 \\
\hline $\mathrm{C}$ & 0.85525 & -2.01340 & 2.89432 \\
\hline $\mathrm{C}$ & 0.78360 & -3.00956 & 1.85301 \\
\hline C & 2.07383 & -3.07143 & 1.18444 \\
\hline C & 2.94747 & -2.10542 & 1.82774 \\
\hline $\mathrm{C}$ & 3.85513 & -1.36853 & 1.06483 \\
\hline C & 4.05656 & 0.04470 & 1.33541 \\
\hline C & 4.24573 & 0.72373 & 0.06516 \\
\hline C & 4.16148 & -0.26928 & -0.99203 \\
\hline $\mathrm{C}$ & 3.91970 & -1.56285 & -0.37582 \\
\hline $\mathrm{C}$ & 3.07411 & -2.48589 & -0.99356 \\
\hline $\mathrm{C}$ & 2.13564 & -3.25774 & -0.19712 \\
\hline $\mathrm{C}$ & 0.90961 & -3.39005 & -0.96862 \\
\hline $\mathrm{C}$ & -0.32380 & -3.33002 & -0.32283 \\
\hline $\mathrm{C}$ & -0.38768 & -3.13648 & 1.10848 \\
\hline $\mathrm{C}$ & -1.56895 & -2.32605 & 1.37743 \\
\hline $\mathrm{C}$ & -2.40223 & -2.32596 & 0.17815 \\
\hline C & -1.46745 & -2.63405 & -0.89928 \\
\hline C & -1.27967 & -1.93516 & -2.09755 \\
\hline $\mathrm{C}$ & -6.59480 & 2.03122 & 0.22756 \\
\hline $\mathrm{C}$ & -6.44274 & 0.52362 & 0.01031 \\
\hline $\mathrm{H}$ & -5.73214 & 0.32249 & -0.81113 \\
\hline $\mathrm{H}$ & -7.38633 & 0.04595 & -0.27588 \\
\hline $\mathrm{H}$ & -6.09476 & 0.02515 & 0.92597 \\
\hline $\mathrm{H}$ & -6.95183 & 2.52672 & -0.68156 \\
\hline $\mathrm{H}$ & -5.64023 & 2.48974 & 0.50706 \\
\hline $\mathrm{H}$ & -7.31346 & 2.23953 & 1.02748 \\
\hline
\end{tabular}

(11) $\mathrm{C}_{2} \mathrm{H}_{6} \_\mathrm{C} 599 \mathrm{Pd} \_\mathrm{HH} \_\mathrm{C} 7-\mathrm{C} 8$

Pd $\quad-3.73673 \quad-0.87419 \quad-0.07151$ 


\begin{tabular}{|c|c|c|c|}
\hline $\mathrm{C}$ & -2.74172 & 0.46477 & 1.05550 \\
\hline $\mathrm{C}$ & -2.41559 & 1.69114 & 0.37421 \\
\hline $\mathrm{C}$ & -2.35031 & 1.49493 & -1.06804 \\
\hline $\mathrm{C}$ & -2.63185 & 0.12793 & -1.42644 \\
\hline $\mathrm{C}$ & -1.82273 & -0.56567 & -2.30536 \\
\hline $\mathrm{C}$ & -0.86308 & 0.17930 & -3.09256 \\
\hline $\mathrm{C}$ & -0.66642 & 1.54689 & -2.86652 \\
\hline $\mathrm{C}$ & -1.40514 & 2.21358 & -1.82294 \\
\hline $\mathrm{C}$ & -0.50913 & 3.16004 & -1.17732 \\
\hline $\mathrm{C}$ & -0.57030 & 3.34654 & 0.19989 \\
\hline $\mathrm{C}$ & -1.53070 & 2.59438 & 0.99189 \\
\hline $\mathrm{C}$ & -0.89382 & 2.23611 & 2.23514 \\
\hline $\mathrm{C}$ & -1.12483 & 0.97391 & 2.79524 \\
\hline $\mathrm{C}$ & -2.02129 & 0.03681 & 2.15282 \\
\hline $\mathrm{C}$ & -1.47803 & -1.33307 & 2.35972 \\
\hline $\mathrm{C}$ & -0.25523 & -1.18283 & 3.11303 \\
\hline $\mathrm{C}$ & -0.04019 & 0.22614 & 3.39344 \\
\hline $\mathrm{C}$ & 1.24761 & 0.76031 & 3.41516 \\
\hline $\mathrm{C}$ & 1.49916 & 2.06108 & 2.81162 \\
\hline $\mathrm{C}$ & 0.45054 & 2.78477 & 2.23792 \\
\hline $\mathrm{C}$ & 0.65345 & 3.46959 & 0.97294 \\
\hline $\mathrm{C}$ & 1.89281 & 3.40452 & 0.33377 \\
\hline $\mathrm{C}$ & 1.95651 & 3.20996 & -1.10614 \\
\hline $\mathrm{C}$ & 0.77860 & 3.08918 & -1.84567 \\
\hline $\mathrm{C}$ & 0.67915 & 2.09136 & -2.89660 \\
\hline $\mathrm{C}$ & 1.76538 & 1.25475 & -3.16473 \\
\hline $\mathrm{C}$ & 1.55228 & -0.16170 & -3.42294 \\
\hline $\mathrm{C}$ & 0.26134 & -0.68651 & -3.37559 \\
\hline $\mathrm{C}$ & 0.00635 & -1.97443 & -2.75371 \\
\hline $\mathrm{C}$ & 1.08390 & -2.70438 & -2.22639 \\
\hline $\mathrm{C}$ & 2.42009 & -2.14596 & -2.24935 \\
\hline $\mathrm{C}$ & 2.65726 & -0.90171 & -2.84125 \\
\hline $\mathrm{C}$ & 3.54108 & 0.05218 & -2.20169 \\
\hline $\mathrm{C}$ & 2.99217 & 1.38628 & -2.40526 \\
\hline $\mathrm{C}$ & 3.08579 & 2.34275 & -1.39090 \\
\hline $\mathrm{C}$ & 3.72415 & 2.00495 & -0.13049 \\
\hline $\mathrm{C}$ & 2.98249 & 2.65676 & 0.93521 \\
\hline $\mathrm{C}$ & 2.78927 & 2.00165 & 2.15449 \\
\hline $\mathrm{C}$ & 3.33722 & 0.66724 & 2.35826 \\
\hline $\mathrm{C}$ & 2.39012 & -0.09416 & 3.14760 \\
\hline $\mathrm{C}$ & 2.19059 & -1.45324 & 2.88639 \\
\hline $\mathrm{C}$ & 0.85524 & -2.01353 & 2.89422 \\
\hline $\mathrm{C}$ & 0.78358 & -3.00965 & 1.85288 \\
\hline $\mathrm{C}$ & 2.07381 & -3.07149 & 1.18431 \\
\hline $\mathrm{C}$ & 2.94746 & -2.10551 & 1.82764 \\
\hline $\mathrm{C}$ & 3.85512 & -1.36859 & 1.06477 \\
\hline $\mathrm{C}$ & 4.05656 & 0.04462 & 1.33541 \\
\hline $\mathrm{C}$ & 4.24573 & 0.72371 & 0.06519 \\
\hline $\mathrm{C}$ & 4.16147 & -0.26925 & -0.99204 \\
\hline
\end{tabular}




$\begin{array}{lrrr}\mathrm{C} & 3.91969 & -1.56285 & -0.37589 \\ \mathrm{C} & 3.07410 & -2.48586 & -0.99367 \\ \mathrm{C} & 2.13563 & -3.25775 & -0.19726 \\ \mathrm{C} & 0.90959 & -3.39001 & -0.96877 \\ \mathrm{C} & -0.32382 & -3.33001 & -0.32297 \\ \mathrm{C} & -0.38769 & -3.13653 & 1.10834 \\ \mathrm{C} & -1.56897 & -2.32612 & 1.37733 \\ \mathrm{C} & -2.40225 & -2.32598 & 0.17805 \\ \mathrm{C} & -1.46747 & -2.63402 & -0.89939 \\ \mathrm{C} & -1.27968 & -1.93506 & -2.09762 \\ \mathrm{C} & -6.44257 & 0.52360 & 0.01068 \\ \mathrm{C} & -6.59461 & 2.03129 & 0.22729 \\ \mathrm{H} & -6.09442 & 0.02553 & 0.92649 \\ \mathrm{H} & -5.73212 & 0.32212 & -0.81079 \\ \mathrm{H} & -7.38621 & 0.04582 & -0.27513 \\ \mathrm{H} & -5.64005 & 2.48989 & 0.50674 \\ \mathrm{H} & -7.31337 & 2.23998 & 1.02702 \\ \mathrm{H} & -6.95148 & 2.52642 & -0.68209\end{array}$

For Nickel doped fullerene

\section{Acetylene}

(1) $\mathrm{C}_{2} \mathrm{H}_{2} \mathrm{C}_{59} \mathrm{Ni}$ _TM

$\begin{array}{cccc}\mathrm{Ni} & -3.99892 & -0.14814 & 0.00000 \\ \mathrm{C} & -2.87242 & 0.81443 & 1.18648 \\ \mathrm{C} & -2.36445 & 2.08235 & 0.72482 \\ \mathrm{C} & -2.36439 & 2.08191 & -0.72603 \\ \mathrm{C} & -2.87227 & 0.81369 & -1.18703 \\ \mathrm{C} & -2.22461 & 0.14538 & -2.21855 \\ \mathrm{C} & -1.16541 & 0.82518 & -2.94632 \\ \mathrm{C} & -0.73921 & 2.10263 & -2.57668 \\ \mathrm{C} & -1.33219 & 2.73344 & -1.42052 \\ \mathrm{C} & -0.28200 & 3.42306 & -0.69905 \\ \mathrm{C} & -0.28205 & 3.42343 & 0.69728 \\ \mathrm{C} & -1.33228 & 2.73420 & 1.41903 \\ \mathrm{C} & -0.73940 & 2.10397 & 2.57556 \\ \mathrm{C} & -1.16563 & 0.82671 & 2.94583 \\ \mathrm{C} & -2.22484 & 0.14655 & 2.21834 \\ \mathrm{C} & -1.88170 & -1.30043 & 2.20932 \\ \mathrm{C} & -0.63576 & -1.45497 & 2.94630 \\ \mathrm{C} & -0.19822 & -0.15327 & 3.40052 \\ \mathrm{C} & 1.15897 & 0.16324 & 3.45023 \\ \mathrm{C} & 1.60964 & 1.47687 & 3.02249 \\ \mathrm{C} & 0.67853 & 2.42335 & 2.59123 \\ \mathrm{C} & 0.96337 & 3.24020 & 1.42367\end{array}$




\begin{tabular}{|c|c|c|c|}
\hline $\mathrm{C}$ & 2.16081 & 3.07021 & 0.72704 \\
\hline $\mathrm{C}$ & 2.16086 & 3.06983 & -0.72845 \\
\hline $\mathrm{C}$ & 0.96347 & 3.23946 & -1.42525 \\
\hline $\mathrm{C}$ & 0.67872 & 2.42201 & -2.59241 \\
\hline $\mathrm{C}$ & 1.60987 & 1.47534 & -3.02314 \\
\hline $\mathrm{C}$ & 1.15924 & 0.16149 & -3.45026 \\
\hline $\mathrm{C}$ & -0.19795 & -0.15501 & -3.40050 \\
\hline $\mathrm{C}$ & -0.63551 & -1.45650 & -2.94570 \\
\hline $\mathrm{C}$ & 0.31520 & -2.40866 & -2.56523 \\
\hline $\mathrm{C}$ & 1.72690 & -2.08230 & -2.58869 \\
\hline $\mathrm{C}$ & 2.14077 & -0.81795 & -3.01480 \\
\hline $\mathrm{C}$ & 3.18866 & -0.11476 & -2.30159 \\
\hline $\mathrm{C}$ & 2.85645 & 1.30255 & -2.30172 \\
\hline $\mathrm{C}$ & 3.12831 & 2.08386 & -1.17577 \\
\hline $\mathrm{C}$ & 3.72595 & 1.47379 & -0.00025 \\
\hline $\mathrm{C}$ & 3.12822 & 2.08445 & 1.17494 \\
\hline $\mathrm{C}$ & 2.85628 & 1.30372 & 2.30125 \\
\hline $\mathrm{C}$ & 3.18848 & -0.11360 & 2.30185 \\
\hline $\mathrm{C}$ & 2.14052 & -0.81641 & 3.01533 \\
\hline $\mathrm{C}$ & 1.72668 & -2.08098 & 2.58982 \\
\hline $\mathrm{C}$ & 0.31498 & -2.40732 & 2.56639 \\
\hline $\mathrm{C}$ & 0.06583 & -3.25363 & 1.41512 \\
\hline $\mathrm{C}$ & 1.31849 & -3.42044 & 0.69876 \\
\hline $\mathrm{C}$ & 2.34692 & -2.69525 & 1.42551 \\
\hline $\mathrm{C}$ & 3.34399 & -2.01065 & 0.72800 \\
\hline $\mathrm{C}$ & 3.77378 & -0.69572 & 1.17498 \\
\hline $\mathrm{C}$ & 4.04525 & 0.11373 & 0.00011 \\
\hline $\mathrm{C}$ & 3.77387 & -0.69631 & -1.17437 \\
\hline $\mathrm{C}$ & 3.34405 & -2.01101 & -0.72676 \\
\hline $\mathrm{C}$ & 2.34705 & -2.69597 & -1.42401 \\
\hline $\mathrm{C}$ & 1.31856 & -3.42081 & -0.69698 \\
\hline $\mathrm{C}$ & 0.06596 & -3.25438 & -1.41355 \\
\hline $\mathrm{C}$ & -1.13074 & -3.11230 & -0.72389 \\
\hline $\mathrm{C}$ & -1.13080 & -3.11189 & 0.72529 \\
\hline $\mathrm{C}$ & -2.16613 & -2.17247 & 1.14885 \\
\hline $\mathrm{C}$ & -2.93408 & -1.71801 & 0.00025 \\
\hline $\mathrm{C}$ & -2.16601 & -2.17313 & -1.14805 \\
\hline $\mathrm{C}$ & -1.88150 & -1.30163 & -2.20891 \\
\hline $\mathrm{C}$ & -5.69405 & 0.79371 & -0.00009 \\
\hline $\mathrm{C}$ & -5.94876 & -0.44533 & -0.00002 \\
\hline $\mathrm{H}$ & -6.00221 & 1.82677 & -0.00011 \\
\hline $\mathrm{H}$ & -6.60183 & -1.30048 & -0.00002 \\
\hline
\end{tabular}

(2) $\mathrm{C}_{2} \mathrm{H}_{2} \_\mathrm{C}_{59} \mathrm{Ni} \_\mathrm{TC} 5$

$\begin{array}{cccc}\mathrm{Ni} & 3.95331 & -0.67095 & 0.14333 \\ \mathrm{C} & 2.65048 & -1.26477 & 1.41127 \\ \mathrm{C} & 1.93388 & -2.44761 & 0.99102\end{array}$




\begin{tabular}{|c|c|c|c|}
\hline $\mathrm{C}$ & 1.98911 & -2.57353 & -0.46259 \\
\hline $\mathrm{C}$ & 2.74621 & -1.49055 & -1.03868 \\
\hline $\mathrm{C}$ & 2.25366 & -0.79795 & -2.12125 \\
\hline $\mathrm{C}$ & 1.10816 & -1.31592 & -2.82893 \\
\hline $\mathrm{C}$ & 0.44859 & -2.46637 & -2.38074 \\
\hline $\mathrm{C}$ & 0.88341 & -3.09443 & -1.15577 \\
\hline $\mathrm{C}$ & -0.30002 & -3.53403 & -0.43582 \\
\hline $\mathrm{C}$ & -0.34745 & -3.42918 & 0.95247 \\
\hline $\mathrm{C}$ & 0.78425 & -2.87308 & 1.67457 \\
\hline $\mathrm{C}$ & 0.26964 & -2.07576 & 2.76205 \\
\hline $\mathrm{C}$ & 0.90374 & -0.87193 & 3.08870 \\
\hline C & 2.09261 & -0.45220 & 2.38115 \\
\hline $\mathrm{C}$ & 2.02890 & 1.04117 & 2.27119 \\
\hline $\mathrm{C}$ & 0.80049 & 1.46417 & 2.91516 \\
\hline $\mathrm{C}$ & 0.11290 & 0.29378 & 3.42483 \\
\hline $\mathrm{C}$ & -1.27913 & 0.22779 & 3.42115 \\
\hline $\mathrm{C}$ & -1.94067 & -1.01386 & 3.05311 \\
\hline $\mathrm{C}$ & -1.17931 & -2.14078 & 2.73022 \\
\hline $\mathrm{C}$ & -1.56397 & -2.97729 & 1.60567 \\
\hline $\mathrm{C}$ & -2.68739 & -2.64888 & 0.84407 \\
\hline $\mathrm{C}$ & -2.63691 & -2.75637 & -0.60558 \\
\hline C & -1.46568 & -3.18865 & -1.23074 \\
\hline $\mathrm{C}$ & -0.99899 & -2.52600 & -2.43723 \\
\hline $\mathrm{C}$ & -1.72715 & -1.45878 & -2.97197 \\
\hline $\mathrm{C}$ & -1.03302 & -0.28002 & -3.46703 \\
\hline $\mathrm{C}$ & 0.35572 & -0.20985 & -3.38106 \\
\hline $\mathrm{C}$ & 1.01593 & 1.02147 & -2.99020 \\
\hline $\mathrm{C}$ & 0.23983 & 2.15436 & -2.71585 \\
\hline $\mathrm{C}$ & -1.20870 & 2.08337 & -2.78137 \\
\hline $\mathrm{C}$ & -1.83442 & 0.88899 & -3.14460 \\
\hline C & -3.01250 & 0.43727 & -2.43379 \\
\hline $\mathrm{C}$ & -2.94446 & -1.01465 & -2.32541 \\
\hline $\mathrm{C}$ & -3.39205 & -1.64933 & -1.16339 \\
\hline $\mathrm{C}$ & -3.91186 & -0.85821 & -0.06105 \\
\hline $\mathrm{C}$ & -3.47500 & -1.47651 & 1.17965 \\
\hline $\mathrm{C}$ & -3.10842 & -0.67658 & 2.26493 \\
\hline $\mathrm{C}$ & -3.17759 & 0.77495 & 2.15558 \\
\hline $\mathrm{C}$ & -2.05082 & 1.33253 & 2.87483 \\
\hline $\mathrm{C}$ & -1.39776 & 2.46747 & 2.38783 \\
\hline $\mathrm{C}$ & 0.05118 & 2.53896 & 2.41973 \\
\hline $\mathrm{C}$ & 0.49192 & 3.21641 & 1.22571 \\
\hline $\mathrm{C}$ & -0.67837 & 3.55055 & 0.43084 \\
\hline $\mathrm{C}$ & -1.85029 & 3.08860 & 1.15280 \\
\hline $\mathrm{C}$ & -2.93010 & 2.54463 & 0.45360 \\
\hline C & -3.60747 & 1.36633 & 0.96504 \\
\hline $\mathrm{C}$ & -3.97775 & 0.53295 & -0.16580 \\
\hline C & -3.52339 & 1.19443 & -1.37696 \\
\hline C & -2.87690 & 2.43793 & -0.99615 \\
\hline $\mathrm{C}$ & -1.74502 & 2.87732 & -1.68728 \\
\hline
\end{tabular}




$\begin{array}{lrrr}\mathrm{C} & -0.62555 & 3.44534 & -0.95762 \\ \mathrm{C} & 0.59784 & 2.99673 & -1.60482 \\ \mathrm{C} & 1.72167 & 2.67083 & -0.84376 \\ \mathrm{C} & 1.66630 & 2.79163 & 0.60606 \\ \mathrm{C} & 2.49729 & 1.75724 & 1.17749 \\ \mathrm{C} & 3.37084 & 1.23681 & 0.07524 \\ \mathrm{C} & 2.57561 & 1.55084 & -1.17919 \\ \mathrm{C} & 2.19073 & 0.69586 & -2.20256 \\ \mathrm{C} & 4.84985 & 1.34053 & 0.10414 \\ \mathrm{C} & 5.43438 & 0.28879 & -0.53803 \\ \mathrm{H} & 5.32731 & 2.11384 & 0.71556 \\ \mathrm{H} & 6.51775 & 0.19075 & -0.61798\end{array}$

(3) $\mathrm{C}_{2} \mathrm{H}_{2} \mathrm{C}_{59} \mathrm{Ni}$-TC1

$\begin{array}{lrrr}\mathrm{Ni} & 4.01231 & -0.70010 & -0.11061 \\ \mathrm{C} & 3.35158 & 1.36620 & -0.10947 \\ \mathrm{C} & 2.60204 & 1.41009 & 1.19957 \\ \mathrm{C} & 2.43566 & 0.18198 & 1.96875 \\ \mathrm{C} & 2.81818 & -1.03217 & 1.28875 \\ \mathrm{C} & 2.02436 & -2.16277 & 1.38704 \\ \mathrm{C} & 0.94075 & -2.21216 & 2.33693 \\ \mathrm{C} & 0.66253 & -1.1144 & 3.14583 \\ \mathrm{C} & 1.40319 & 0.11245 & 2.93759 \\ \mathrm{C} & 0.49728 & 1.21149 & 3.14879 \\ \mathrm{C} & 0.62182 & 2.35233 & 2.35787 \\ \mathrm{C} & 1.66505 & 2.43259 & 1.37198 \\ \mathrm{C} & 1.13510 & 3.19702 & 0.23624 \\ \mathrm{C} & 1.49706 & 2.79418 & -1.03791 \\ \mathrm{C} & 2.51714 & 1.77228 & -1.24451 \\ \mathrm{C} & 2.03475 & 0.92351 & -2.27774 \\ \mathrm{C} & 0.81461 & 1.53956 & -2.85057 \\ \mathrm{C} & 0.49421 & 2.70640 & -2.07383 \\ \mathrm{C} & -0.83395 & 3.05860 & -1.81490 \\ \mathrm{C} & -1.21720 & 3.48002 & -0.48252 \\ \mathrm{C} & -0.24687 & 3.52666 & 0.52448 \\ \mathrm{C} & -0.56145 & 3.02455 & 1.84876 \\ \mathrm{C} & -1.82545 & 2.50853 & 2.12966 \\ \mathrm{C} & -1.95592 & 1.30987 & 2.94607 \\ \mathrm{C} & -0.81777 & 0.68187 & 3.45439 \\ \mathrm{C} & -0.71509 & -0.76721 & 3.44400 \\ \mathrm{C} & -1.76067 & -1.53597 & 2.92705 \\ \mathrm{C} & -1.46630 & -2.68635 & 2.08752 \\ \mathrm{C} & -0.14300 & -3.01605 & 1.79860 \\ \mathrm{C} & 0.24085 & -3.43923 & 0.46782 \\ \mathrm{C} & -0.73000 & -3.50706 & -0.53838 \\ \mathrm{C} & -2.10905 & -3.15355 & -0.24734 \\ \mathrm{C} & -2.46936 & -2.74894 & 1.03813\end{array}$




$\begin{array}{lrrr}\mathrm{C} & -3.38169 & -1.63234 & 1.22637 \\ \mathrm{C} & -2.93897 & -0.88360 & 2.38908 \\ \mathrm{C} & -3.03926 & 0.51426 & 2.40228 \\ \mathrm{C} & -3.57456 & 1.21206 & 1.24875 \\ \mathrm{C} & -2.83263 & 2.45326 & 1.08306 \\ \mathrm{C} & -2.52888 & 2.91951 & -0.19639 \\ \mathrm{C} & -2.96049 & 2.16424 & -1.36423 \\ \mathrm{C} & -1.90428 & 2.24671 & -2.35720 \\ \mathrm{C} & -1.59997 & 1.13325 & -3.14658 \\ \mathrm{C} & -0.21664 & 0.77645 & -3.38237 \\ \mathrm{C} & -0.12535 & -0.68172 & -3.41738 \\ \mathrm{C} & -1.44664 & -1.21947 & -3.17559 \\ \mathrm{C} & -2.35631 & -0.09992 & -2.99349 \\ \mathrm{C} & -3.37185 & -0.17727 & -2.04024 \\ \mathrm{C} & -3.67252 & 0.97514 & -1.20447 \\ \mathrm{C} & -3.99302 & 0.49029 & 0.12734 \\ \mathrm{C} & -3.89255 & -0.95703 & 0.11589 \\ \mathrm{C} & -3.51343 & -1.37378 & -1.22564 \\ \mathrm{C} & -2.64387 & -2.44976 & -1.40430 \\ \mathrm{C} & -1.59338 & -2.37753 & -2.40537 \\ \mathrm{C} & -0.41726 & -3.03476 & -1.87469 \\ \mathrm{C} & 0.85484 & -2.52202 & -2.13169 \\ \mathrm{C} & 0.99489 & -1.29466 & -2.88795 \\ \mathrm{C} & 2.11423 & -0.52633 & -2.30903 \\ \mathrm{C} & 2.68539 & -1.29451 & -1.28822 \\ \mathrm{C} & 1.88254 & -2.49747 & -1.10765 \\ \mathrm{C} & 1.57014 & -2.91616 & 0.18657 \\ \mathrm{C} & 4.83646 & 1.36315 & -0.16283 \\ \mathrm{C} & 5.46197 & 0.33706 & 0.48106 \\ \mathrm{H} & 5.30189 & 2.13178 & -0.78941 \\ \mathrm{H} & 6.54762 & 0.28523 & 0.56440\end{array}$

(4) $\mathrm{C}_{2} \mathrm{H}_{2} \_\mathrm{C}_{59} \mathrm{Ni} \_\mathrm{HH} \_\mathrm{M}-\mathrm{C} 5$

$\begin{array}{cccr}\mathrm{Ni} & -3.95335 & -0.67118 & -0.14296 \\ \mathrm{C} & -2.65029 & -1.26526 & -1.41088 \\ \mathrm{C} & -1.93350 & -2.44811 & -0.99104 \\ \mathrm{C} & -1.98863 & -2.57413 & 0.46255 \\ \mathrm{C} & -2.74582 & -1.49120 & 1.03845 \\ \mathrm{C} & -2.25341 & -0.79837 & 2.12098 \\ \mathrm{C} & -1.10789 & -1.31622 & 2.82873 \\ \mathrm{C} & -0.44813 & -2.46663 & 2.38062 \\ \mathrm{C} & -0.88286 & -3.09480 & 1.15570 \\ \mathrm{C} & 0.30062 & -3.53419 & 0.43568 \\ \mathrm{C} & 0.34799 & -3.42923 & -0.95261 \\ \mathrm{C} & -0.78383 & -2.87329 & -1.67461 \\ \mathrm{C} & -0.26943 & -2.07576 & -2.76205 \\ \mathrm{C} & -0.90374 & -0.87200 & -3.08849\end{array}$




\begin{tabular}{|c|c|c|c|}
\hline $\mathrm{C}$ & -2.09268 & -0.45246 & -2.38077 \\
\hline $\mathrm{C}$ & -2.02910 & 1.04089 & -2.27081 \\
\hline $\mathrm{C}$ & -0.80078 & 1.46404 & -2.91487 \\
\hline $\mathrm{C}$ & -0.11308 & 0.29379 & -3.42464 \\
\hline $\mathrm{C}$ & 1.27898 & 0.22803 & -3.42111 \\
\hline $\mathrm{C}$ & 1.94073 & -1.01354 & -3.05321 \\
\hline $\mathrm{C}$ & 1.17953 & -2.14055 & -2.73030 \\
\hline $\mathrm{C}$ & 1.56440 & -2.97709 & -1.60584 \\
\hline $\mathrm{C}$ & 2.68778 & -2.64853 & -0.84426 \\
\hline $\mathrm{C}$ & 2.63738 & -2.75608 & 0.60539 \\
\hline $\mathrm{C}$ & 1.46624 & -3.18860 & 1.23058 \\
\hline $\mathrm{C}$ & 0.99946 & -2.52602 & 2.43709 \\
\hline $\mathrm{C}$ & 1.72746 & -1.45872 & 2.97185 \\
\hline $\mathrm{C}$ & 1.03314 & -0.28005 & 3.46692 \\
\hline $\mathrm{C}$ & -0.35560 & -0.21006 & 3.38094 \\
\hline $\mathrm{C}$ & -1.01597 & 1.02117 & 2.99010 \\
\hline $\mathrm{C}$ & -0.24003 & 2.15419 & 2.71589 \\
\hline $\mathrm{C}$ & 1.20851 & 2.08338 & 2.78139 \\
\hline $\mathrm{C}$ & 1.83440 & 0.88907 & 3.14455 \\
\hline $\mathrm{C}$ & 3.01253 & 0.43754 & 2.43371 \\
\hline $\mathrm{C}$ & 2.94469 & -1.01438 & 2.32527 \\
\hline $\mathrm{C}$ & 3.39235 & -1.64895 & 1.16322 \\
\hline $\mathrm{C}$ & 3.91201 & -0.85771 & 0.06090 \\
\hline $\mathrm{C}$ & 3.47520 & -1.47603 & -1.17982 \\
\hline $\mathrm{C}$ & 3.10847 & -0.67612 & -2.26504 \\
\hline $\mathrm{C}$ & 3.17744 & 0.77541 & -2.15565 \\
\hline $\mathrm{C}$ & 2.05054 & 1.33285 & -2.87482 \\
\hline $\mathrm{C}$ & 1.39736 & 2.46769 & -2.38776 \\
\hline $\mathrm{C}$ & -0.05159 & 2.53897 & -2.41955 \\
\hline $\mathrm{C}$ & -0.49234 & 3.21644 & -1.22551 \\
\hline $\mathrm{C}$ & 0.67794 & 3.55069 & -0.43072 \\
\hline $\mathrm{C}$ & 1.84988 & 3.08887 & -1.15274 \\
\hline $\mathrm{C}$ & 2.92977 & 2.54501 & -0.45359 \\
\hline $\mathrm{C}$ & 3.60727 & 1.36681 & -0.96510 \\
\hline $\mathrm{C}$ & 3.97770 & 0.53345 & 0.16570 \\
\hline $\mathrm{C}$ & 3.52328 & 1.19482 & 1.37690 \\
\hline $\mathrm{C}$ & 2.87661 & 2.43825 & 0.99616 \\
\hline $\mathrm{C}$ & 1.74471 & 2.87746 & 1.68735 \\
\hline $\mathrm{C}$ & 0.62516 & 3.44539 & 0.95774 \\
\hline $\mathrm{C}$ & -0.59818 & 2.99662 & 1.60494 \\
\hline $\mathrm{C}$ & -1.72200 & 2.67068 & 0.84396 \\
\hline $\mathrm{C}$ & -1.66662 & 2.79153 & -0.60585 \\
\hline $\mathrm{C}$ & -2.49759 & 1.75711 & -1.17728 \\
\hline $\mathrm{C}$ & -3.37128 & 1.23684 & -0.07519 \\
\hline $\mathrm{C}$ & -2.57599 & 1.55060 & 1.17935 \\
\hline $\mathrm{C}$ & -2.19077 & 0.69545 & 2.20244 \\
\hline $\mathrm{C}$ & -4.85023 & 1.34039 & -0.10471 \\
\hline $\mathrm{C}$ & -5.43492 & 0.28882 & 0.53765 \\
\hline $\mathrm{H}$ & -5.32725 & 2.11344 & -0.71665 \\
\hline
\end{tabular}


H $\quad-6.51837 \quad 0.19045 \quad 0.61631$

(5) $\mathrm{C}_{2} \mathrm{H}_{2} \_\mathrm{C}_{59} \mathrm{Ni} \_\mathrm{HH} \_\mathrm{M}-\mathrm{C} 1$

\begin{tabular}{|c|c|c|c|}
\hline $\mathrm{Ni}$ & -4.01267 & -0.70040 & -0.11042 \\
\hline $\mathrm{C}$ & -2.81737 & -1.03336 & 1.28838 \\
\hline $\mathrm{C}$ & -2.43539 & 0.18083 & 1.96874 \\
\hline $\mathrm{C}$ & -2.60220 & 1.40873 & 1.19988 \\
\hline $\mathrm{C}$ & -3.35211 & 1.36484 & -0.10936 \\
\hline $\mathrm{C}$ & -2.51736 & 1.77080 & -1.24453 \\
\hline $\mathrm{C}$ & -1.49816 & 2.79401 & -1.03807 \\
\hline $\mathrm{C}$ & -1.13633 & 3.19648 & 0.23619 \\
\hline C & -1.66600 & 2.43174 & 1.37222 \\
\hline C & -0.62264 & 2.35190 & 2.35794 \\
\hline C & -0.49757 & 1.21112 & 3.14890 \\
\hline C & -1.40301 & 0.11166 & 2.93765 \\
\hline C & -0.66192 & -1.11191 & 3.14578 \\
\hline $\mathrm{C}$ & -0.93969 & -2.21258 & 2.33661 \\
\hline $\mathrm{C}$ & -2.02324 & -2.16353 & 1.38659 \\
\hline $\mathrm{C}$ & -1.56891 & -2.91742 & 0.18630 \\
\hline C & -0.23939 & -3.43974 & 0.46765 \\
\hline C & 0.14425 & -3.01621 & 1.79838 \\
\hline C & 1.46746 & -2.68602 & 2.08734 \\
\hline $\mathrm{C}$ & 1.76140 & -1.53552 & 2.92687 \\
\hline $\mathrm{C}$ & 0.71554 & -0.76718 & 3.44393 \\
\hline $\mathrm{C}$ & 0.81764 & 0.68197 & 3.45435 \\
\hline C & 1.95554 & 1.31041 & 2.94597 \\
\hline C & 1.82452 & 2.50902 & 2.12954 \\
\hline $\mathrm{C}$ & 0.56033 & 3.02460 & 1.84874 \\
\hline $\mathrm{C}$ & 0.24553 & 3.52663 & 0.52446 \\
\hline C & 1.21587 & 3.48037 & -0.48259 \\
\hline C & 0.83280 & 3.05898 & -1.81497 \\
\hline C & -0.49535 & 2.70645 & -2.07394 \\
\hline $\mathrm{C}$ & -0.81529 & 1.53932 & -2.85051 \\
\hline $\mathrm{C}$ & 0.21619 & 0.77655 & -3.38214 \\
\hline $\mathrm{C}$ & 1.59936 & 1.13378 & -3.14653 \\
\hline $\mathrm{C}$ & 1.90336 & 2.24742 & -2.35723 \\
\hline $\mathrm{C}$ & 2.95967 & 2.16531 & -1.36433 \\
\hline $\mathrm{C}$ & 2.52783 & 2.92038 & -0.19646 \\
\hline $\mathrm{C}$ & 2.83171 & 2.45418 & 1.08294 \\
\hline $\mathrm{C}$ & 3.57416 & 1.21326 & 1.24863 \\
\hline $\mathrm{C}$ & 3.03916 & 0.51525 & 2.40213 \\
\hline $\mathrm{C}$ & 2.93940 & -0.88270 & 2.38890 \\
\hline $\mathrm{C}$ & 3.38240 & -1.63124 & 1.22622 \\
\hline $\mathrm{C}$ & 2.47060 & -2.74832 & 1.03800 \\
\hline $\mathrm{C}$ & 2.11042 & -3.15309 & -0.24743 \\
\hline $\mathrm{C}$ & 0.73154 & -3.50741 & -0.53850 \\
\hline C & 0.41848 & -3.03503 & -1.87464 \\
\hline
\end{tabular}




$\begin{array}{lrrr}\mathrm{C} & 1.59420 & -2.37714 & -2.40532 \\ \mathrm{C} & 2.64483 & -2.44895 & -1.40438 \\ \mathrm{C} & 3.51393 & -1.37259 & -1.22576 \\ \mathrm{C} & 3.89295 & -0.95569 & 0.11574 \\ \mathrm{C} & 3.99286 & 0.49167 & 0.12720 \\ \mathrm{C} & 3.67206 & 0.97642 & -1.20459 \\ \mathrm{C} & 3.37179 & -0.17612 & -2.04033 \\ \mathrm{C} & 2.35612 & -0.09916 & -2.99345 \\ \mathrm{C} & 1.44689 & -1.21904 & -3.17544 \\ \mathrm{C} & 0.12538 & -0.68183 & -3.41699 \\ \mathrm{C} & -0.99443 & -1.29534 & -2.88747 \\ \mathrm{C} & -0.85390 & -2.52266 & -2.13147 \\ \mathrm{C} & -1.88153 & -2.49850 & -1.10751 \\ \mathrm{C} & -2.68569 & -1.29598 & -1.28834 \\ \mathrm{C} & -2.11432 & -0.52763 & -2.30837 \\ \mathrm{C} & -2.03508 & 0.92271 & -2.27745 \\ \mathrm{C} & -4.83650 & 1.36377 & -0.16236 \\ \mathrm{C} & -5.46188 & 0.33730 & 0.48133 \\ \mathrm{H} & -5.30302 & 2.13123 & -0.78968 \\ \mathrm{H} & -6.54760 & 0.28421 & 0.56310\end{array}$

(6) $\mathrm{C}_{2} \mathrm{H}_{2}$ - ${ }_{59} \mathrm{Ni} \_\mathrm{HH} \_\mathrm{C} 1-\mathrm{C} 2$

$\begin{array}{cccc}\mathrm{Ni} & -3.78125 & -1.10025 & 0.35473 \\ \mathrm{C} & -2.53119 & -1.15487 & 1.75273 \\ \mathrm{C} & -2.23752 & 0.11756 & 2.37423 \\ \mathrm{C} & -2.64932 & 1.21251 & 1.53714 \\ \mathrm{C} & -3.47713 & 0.79201 & 0.37530 \\ \mathrm{C} & -3.01561 & 1.23997 & -1.11643 \\ \mathrm{C} & -1.99755 & 2.38121 & -1.06271 \\ \mathrm{C} & -1.54913 & 2.94538 & 0.12291 \\ \mathrm{C} & -1.86134 & 2.34673 & 1.44224 \\ \mathrm{C} & -0.69154 & 2.49363 & 2.27784 \\ \mathrm{C} & -0.32961 & 1.47968 & 3.16683 \\ \mathrm{C} & -1.10721 & 0.26082 & 3.20418 \\ \mathrm{C} & -0.20250 & -0.84448 & 3.38154 \\ \mathrm{C} & -0.44017 & -2.03423 & 2.68428 \\ \mathrm{C} & -1.60128 & -2.18198 & 1.83214 \\ \mathrm{C} & -1.18977 & -3.03994 & 0.67932 \\ \mathrm{C} & 0.20552 & -3.36861 & 0.86568 \\ \mathrm{C} & 0.66838 & -2.76346 & 2.09862 \\ \mathrm{C} & 1.96882 & -2.27341 & 2.20756 \\ \mathrm{C} & 2.21106 & -1.01441 & 2.89593 \\ \mathrm{C} & 1.14708 & -0.31792 & 3.47356 \\ \mathrm{C} & 1.06791 & 1.12703 & 3.33320 \\ \mathrm{C} & 2.05182 & 1.81361 & 2.61792 \\ \mathrm{C} & 1.67351 & 2.87857 & 1.70062 \\ \mathrm{C} & 0.32762 & 3.20052 & 1.52804\end{array}$




\begin{tabular}{|c|c|c|c|}
\hline $\mathrm{C}$ & -0.20024 & 3.47524 & 0.20330 \\
\hline $\mathrm{C}$ & 0.65617 & 3.42726 & -0.90124 \\
\hline $\mathrm{C}$ & 0.19847 & 2.81213 & -2.12371 \\
\hline C & -1.08900 & 2.26518 & -2.16750 \\
\hline $\mathrm{C}$ & -1.31342 & 0.98430 & -2.81452 \\
\hline $\mathrm{C}$ & -0.24102 & 0.32706 & -3.43628 \\
\hline $\mathrm{C}$ & 1.09435 & 0.88093 & -3.36914 \\
\hline $\mathrm{C}$ & 1.31342 & 2.09905 & -2.72107 \\
\hline C & 2.46387 & 2.26460 & -1.85610 \\
\hline $\mathrm{C}$ & 2.05774 & 3.08822 & -0.72617 \\
\hline $\mathrm{C}$ & 2.55631 & 2.81497 & 0.54802 \\
\hline $\mathrm{C}$ & 3.47417 & 1.70788 & 0.74915 \\
\hline $\mathrm{C}$ & 3.15908 & 1.08891 & 2.02550 \\
\hline $\mathrm{C}$ & 3.23841 & -0.30132 & 2.16515 \\
\hline $\mathrm{C}$ & 3.64174 & -1.12316 & 1.03425 \\
\hline $\mathrm{C}$ & 2.86593 & -2.34820 & 1.06702 \\
\hline $\mathrm{C}$ & 2.42807 & -2.93409 & -0.12283 \\
\hline $\mathrm{C}$ & 1.08733 & -3.47430 & -0.22131 \\
\hline $\mathrm{C}$ & 0.59417 & -3.20729 & -1.55202 \\
\hline $\mathrm{C}$ & 1.62141 & -2.48295 & -2.28471 \\
\hline $\mathrm{C}$ & 2.75911 & -2.31587 & -1.40053 \\
\hline $\mathrm{C}$ & 3.50071 & -1.13222 & -1.43000 \\
\hline $\mathrm{C}$ & 3.95279 & -0.52505 & -0.18943 \\
\hline $\mathrm{C}$ & 3.86267 & 0.91656 & -0.33559 \\
\hline $\mathrm{C}$ & 3.35172 & 1.20251 & -1.66446 \\
\hline $\mathrm{C}$ & 3.12897 & -0.06353 & -2.34206 \\
\hline $\mathrm{C}$ & 2.02702 & -0.22105 & -3.18582 \\
\hline $\mathrm{C}$ & 1.26140 & -1.45503 & -3.15844 \\
\hline $\mathrm{C}$ & -0.14020 & -1.10493 & -3.32076 \\
\hline $\mathrm{C}$ & -1.11839 & -1.78954 & -2.60677 \\
\hline $\mathrm{C}$ & -0.74813 & -2.87051 & -1.71776 \\
\hline $\mathrm{C}$ & -1.68407 & -2.84013 & -0.60876 \\
\hline $\mathrm{C}$ & -2.81040 & -1.95781 & -0.93561 \\
\hline $\mathrm{C}$ & -2.27032 & -1.11711 & -2.02032 \\
\hline $\mathrm{C}$ & -2.34844 & 0.27220 & -2.11641 \\
\hline $\mathrm{C}$ & -4.49674 & 1.60726 & -1.25636 \\
\hline $\mathrm{C}$ & -4.83350 & 1.29227 & 0.01398 \\
\hline $\mathrm{H}$ & -5.03483 & 2.05027 & -2.08982 \\
\hline $\mathrm{H}$ & -5.74204 & 1.40654 & 0.59719 \\
\hline
\end{tabular}

(7) $\mathrm{C}_{2} \mathrm{H}_{2} \mathrm{C}_{59} \mathrm{Ni} \_\mathrm{PH}$ _C2-C3

$\begin{array}{cccc}\mathrm{Ni} & 3.67860 & -1.49126 & -0.31137 \\ \mathrm{C} & 2.15782 & -2.29404 & -1.04644 \\ \mathrm{C} & 1.77706 & -1.54133 & -2.21770 \\ \mathrm{C} & 2.32122 & -0.18277 & -2.23426 \\ \mathrm{C} & 3.35218 & 0.08778 & -1.26940 \\ \mathrm{C} & 3.25643 & 1.39041 & -0.49803\end{array}$




\begin{tabular}{|c|c|c|c|}
\hline C & 2.16834 & 2.34024 & -0.96946 \\
\hline $\mathrm{C}$ & 1.48339 & 2.14149 & -2.15234 \\
\hline $\mathrm{C}$ & 1.55839 & 0.83820 & -2.81637 \\
\hline $\mathrm{C}$ & 0.28125 & 0.57159 & -3.42104 \\
\hline $\mathrm{C}$ & -0.22680 & -0.73093 & -3.43967 \\
\hline $\mathrm{C}$ & 0.53447 & -1.80049 & -2.82632 \\
\hline $\mathrm{C}$ & -0.39839 & -2.71528 & -2.21565 \\
\hline $\mathrm{C}$ & -0.06477 & -3.33085 & -1.00733 \\
\hline $\mathrm{C}$ & 1.22614 & -3.08997 & -0.40286 \\
\hline $\mathrm{C}$ & 1.02114 & -3.07579 & 1.06585 \\
\hline $\mathrm{C}$ & -0.39015 & -3.29381 & 1.31811 \\
\hline C & -1.05881 & -3.46264 & 0.04210 \\
\hline $\mathrm{C}$ & -2.34698 & -2.96445 & -0.15281 \\
\hline $\mathrm{C}$ & -2.69273 & -2.31726 & -1.40871 \\
\hline $\mathrm{C}$ & -1.73984 & -2.20208 & -2.42087 \\
\hline $\mathrm{C}$ & -1.63045 & -0.96635 & -3.18203 \\
\hline $\mathrm{C}$ & -2.48181 & 0.10622 & -2.89978 \\
\hline $\mathrm{C}$ & -1.95650 & 1.46118 & -2.87391 \\
\hline $\mathrm{C}$ & -0.60441 & 1.68848 & -3.13165 \\
\hline $\mathrm{C}$ & 0.13898 & 2.64557 & -2.33507 \\
\hline $\mathrm{C}$ & -0.49719 & 3.33908 & -1.30308 \\
\hline C & 0.18640 & 3.50347 & -0.03927 \\
\hline $\mathrm{C}$ & 1.47431 & 2.95380 & 0.13009 \\
\hline $\mathrm{C}$ & 1.83138 & 2.30166 & 1.35699 \\
\hline $\mathrm{C}$ & 0.89133 & 2.19813 & 2.36402 \\
\hline $\mathrm{C}$ & -0.43678 & 2.74250 & 2.20306 \\
\hline $\mathrm{C}$ & -0.78859 & 3.39010 & 1.01588 \\
\hline $\mathrm{C}$ & -2.08612 & 3.13850 & 0.41347 \\
\hline $\mathrm{C}$ & -1.90340 & 3.10231 & -1.03008 \\
\hline $\mathrm{C}$ & -2.61922 & 2.18128 & -1.79831 \\
\hline $\mathrm{C}$ & -3.55043 & 1.26862 & -1.16028 \\
\hline $\mathrm{C}$ & -3.46623 & -0.01100 & -1.84120 \\
\hline $\mathrm{C}$ & -3.57162 & -1.20023 & -1.10959 \\
\hline $\mathrm{C}$ & -3.76782 & -1.15549 & 0.32594 \\
\hline $\mathrm{C}$ & -3.01631 & -2.25136 & 0.92107 \\
\hline $\mathrm{C}$ & -2.37656 & -2.07900 & 2.14805 \\
\hline $\mathrm{C}$ & -1.04286 & -2.61723 & 2.35714 \\
\hline C & -0.30055 & -1.67150 & 3.16442 \\
\hline $\mathrm{C}$ & -1.16252 & -0.54414 & 3.44691 \\
\hline $\mathrm{C}$ & -2.45214 & -0.79275 & 2.82961 \\
\hline $\mathrm{C}$ & -3.16578 & 0.25986 & 2.25504 \\
\hline $\mathrm{C}$ & -3.84396 & 0.07521 & 0.98205 \\
\hline C & -3.73040 & 1.30909 & 0.22676 \\
\hline $\mathrm{C}$ & -2.98118 & 2.26121 & 1.02706 \\
\hline $\mathrm{C}$ & -2.62464 & 1.60889 & 2.27782 \\
\hline $\mathrm{C}$ & -1.37691 & 1.84668 & 2.85474 \\
\hline C & -0.62867 & 0.75214 & 3.44943 \\
\hline C & 0.76965 & 0.95917 & 3.14993 \\
\hline $\mathrm{C}$ & 1.58374 & -0.11581 & 2.85132 \\
\hline
\end{tabular}




$\begin{array}{llll}\mathrm{C} & 1.05668 & -1.46156 & 2.90101 \\ \mathrm{C} & 1.73970 & -2.19871 & 1.86102 \\ \mathrm{C} & 2.75554 & -1.32257 & 1.27678 \\ \mathrm{C} & 2.60180 & -0.05086 & 1.78442 \\ \mathrm{C} & 2.96817 & 1.28795 & 1.15583 \\ \mathrm{C} & 4.58156 & 2.00906 & -0.03352 \\ \mathrm{C} & 4.37545 & 1.87981 & 1.27871 \\ \mathrm{H} & 5.38858 & 2.41807 & -0.63304 \\ \mathrm{H} & 4.94417 & 2.14070 & 2.16582\end{array}$

(8) $\mathrm{C}_{2} \mathrm{H}_{2} \mathrm{C}_{59} \mathrm{Ni} \_\mathrm{HH}$-C3-C4

$\begin{array}{crrr}\mathrm{Ni} & -3.62407 & -1.51080 & -0.35634 \\ \mathrm{C} & -2.08023 & -2.46743 & -0.83201 \\ \mathrm{C} & -1.35549 & -3.07761 & 0.26081 \\ \mathrm{C} & -1.72305 & -2.44243 & 1.51684 \\ \mathrm{C} & -2.72944 & -1.43706 & 1.28024 \\ \mathrm{C} & -2.59713 & -0.18098 & 1.83920 \\ \mathrm{C} & -1.57708 & 0.02929 & 2.83157 \\ \mathrm{C} & -0.69563 & -0.99276 & 3.20455 \\ \mathrm{C} & -0.75089 & -2.25416 & 2.50883 \\ \mathrm{C} & 0.61253 & -2.71137 & 2.31012 \\ \mathrm{C} & 0.96468 & -3.33280 & 1.11308 \\ \mathrm{C} & -0.03181 & -3.51298 & 0.07006 \\ \mathrm{C} & 0.61662 & -3.30120 & -1.20169 \\ \mathrm{C} & -0.06710 & -2.62801 & -2.21832 \\ \mathrm{C} & -1.42585 & -2.16058 & -2.01442 \\ \mathrm{C} & -1.56473 & -0.87745 & -2.74871 \\ \mathrm{C} & -0.29262 & -0.59365 & -3.36022 \\ \mathrm{C} & 0.63186 & -1.66800 & -3.04634 \\ \mathrm{C} & 1.98158 & -1.39877 & -2.83073 \\ \mathrm{C} & 2.68693 & -2.07365 & -1.75413 \\ \mathrm{C} & 2.01765 & -3.00611 & -0.95963 \\ \mathrm{C} & 2.23213 & -3.02025 & 0.47916 \\ \mathrm{C} & 3.09892 & -2.09350 & 1.06376 \\ \mathrm{C} & 2.72977 & -1.43900 & 2.30755 \\ \mathrm{C} & 1.50795 & -1.74032 & 2.91432 \\ \mathrm{C} & 0.69446 & -0.67504 & 3.47231 \\ \mathrm{C} & 1.13839 & 0.64845 & 3.41111 \\ \mathrm{C} & 0.20763 & 1.71362 & 3.07306 \\ \mathrm{C} & -1.12078 & 1.40135 & 2.76899 \\ \mathrm{C} & -1.83506 & 2.06604 & 1.71666 \\ \mathrm{C} & -1.17468 & 3.00749 & 0.94615 \\ \mathrm{C} & 0.21762 & 3.31081 & 1.21485 \\ \mathrm{C} & 0.89907 & 2.68598 & 2.26549 \\ \mathrm{C} & 2.26289 & 2.22457 & 2.08204 \\ \mathrm{C} & 2.40929 & 0.96062 & 2.79215 \\ & 3.18990 & -0.06327 & 2.24549\end{array}$




$\begin{array}{lrrr}\mathrm{C} & 3.84775 & 0.13347 & 0.96618 \\ \mathrm{C} & 3.79042 & -1.12080 & 0.23509 \\ \mathrm{C} & 3.59037 & -1.11356 & -1.14704 \\ \mathrm{C} & 3.45089 & 0.14924 & -1.85624 \\ \mathrm{C} & 2.46455 & -0.02780 & -2.90129 \\ \mathrm{C} & 1.58498 & 1.00948 & -3.22205 \\ \mathrm{C} & 0.18743 & 0.72038 & -3.47085 \\ \mathrm{C} & -0.59649 & 1.80459 & -2.93295 \\ \mathrm{C} & 0.29678 & 2.75589 & -2.31812 \\ \mathrm{C} & 1.65377 & 2.27062 & -2.49659 \\ \mathrm{C} & 2.59142 & 2.43232 & -1.47587 \\ \mathrm{C} & 3.51060 & 1.35696 & -1.15225 \\ \mathrm{C} & 3.70740 & 1.34669 & 0.28650 \\ \mathrm{C} & 2.90198 & 2.41225 & 0.85661 \\ \mathrm{C} & 2.20550 & 3.07981 & -0.23102 \\ \mathrm{C} & 0.89295 & 3.52037 & -0.05339 \\ \mathrm{C} & -0.08317 & 3.36036 & -1.11557 \\ \mathrm{C} & -1.36971 & 3.06340 & -0.51628 \\ \mathrm{C} & -2.24969 & 2.20088 & -1.14101 \\ \mathrm{C} & -1.82436 & 1.52287 & -2.32867 \\ \mathrm{C} & -2.37124 & 0.17785 & -2.28788 \\ \mathrm{C} & -3.39300 & 0.06638 & -1.28213 \\ \mathrm{C} & -3.28326 & 1.35128 & -0.42293 \\ \mathrm{C} & -2.98404 & 1.18357 & 1.22367 \\ \mathrm{C} & -4.38032 & 1.77953 & 1.36841 \\ \mathrm{C} & -4.58499 & 1.97686 & 0.06403 \\ \mathrm{H} & -4.94743 & 2.00089 & 2.26738 \\ \mathrm{H} & -5.38729 & 2.42543 & -0.51321\end{array}$

(9) $\mathrm{C}_{2} \mathrm{H}_{2} \_\mathrm{C}_{59} \mathrm{Ni}$-PH_C4-C5

$\begin{array}{crrr}\mathrm{Ni} & -3.78100 & -1.18135 & -0.21523 \\ \mathrm{C} & -2.42405 & -1.75672 & -1.36594 \\ \mathrm{C} & -1.60518 & -2.77906 & -0.73771 \\ \mathrm{C} & -1.71138 & -2.74217 & 0.71075 \\ \mathrm{C} & -2.72274 & -1.79806 & 1.17058 \\ \mathrm{C} & -2.27448 & -0.82651 & 2.11070 \\ \mathrm{C} & -1.10559 & -1.13450 & 2.95734 \\ \mathrm{C} & -0.29810 & -2.22217 & 2.65461 \\ \mathrm{C} & -0.59674 & -3.05038 & 1.49201 \\ \mathrm{C} & 0.66038 & -3.42862 & 0.88495 \\ \mathrm{C} & 0.76901 & -3.49205 & -0.50267 \\ \mathrm{C} & -0.37960 & -3.14902 & -1.32298 \\ \mathrm{C} & 0.10061 & -2.45189 & -2.48960 \\ \mathrm{C} & -0.64565 & -1.39021 & -3.01103 \\ \mathrm{C} & -1.91111 & -1.01632 & -2.41700 \\ \mathrm{C} & -2.01969 & 0.46783 & -2.53343 \\ \mathrm{C} & -0.81448 & 0.94323 & -3.16244\end{array}$




\begin{tabular}{|c|c|c|c|}
\hline $\mathrm{C}$ & 0.02767 & -0.19489 & -3.47848 \\
\hline $\mathrm{C}$ & 1.41501 & -0.09239 & -3.39707 \\
\hline $\mathrm{C}$ & 2.19014 & -1.18806 & -2.83760 \\
\hline $\mathrm{C}$ & 1.54445 & -2.34735 & -2.40050 \\
\hline $\mathrm{C}$ & 1.95813 & -2.98506 & -1.16014 \\
\hline $\mathrm{C}$ & 2.99635 & -2.43340 & -0.40582 \\
\hline $\mathrm{C}$ & 2.88185 & -2.36518 & 1.04304 \\
\hline $\mathrm{C}$ & 1.73811 & -2.85411 & 1.67524 \\
\hline $\mathrm{C}$ & 1.14189 & -2.11051 & 2.77184 \\
\hline $\mathrm{C}$ & 1.71498 & -0.90609 & 3.19421 \\
\hline $\mathrm{C}$ & 0.86548 & 0.22567 & 3.51493 \\
\hline $\mathrm{C}$ & -0.51771 & 0.10564 & 3.37354 \\
\hline $\mathrm{C}$ & -1.30565 & 1.16793 & 2.76830 \\
\hline $\mathrm{C}$ & -0.66509 & 2.34956 & 2.38148 \\
\hline $\mathrm{C}$ & 0.77181 & 2.47051 & 2.50918 \\
\hline $\mathrm{C}$ & 1.53118 & 1.43311 & 3.06588 \\
\hline $\mathrm{C}$ & 2.79051 & 1.05008 & 2.45749 \\
\hline $\mathrm{C}$ & 2.90671 & -0.40070 & 2.53765 \\
\hline $\mathrm{C}$ & 3.47607 & -1.11422 & 1.48284 \\
\hline $\mathrm{C}$ & 3.96038 & -0.41108 & 0.30380 \\
\hline $\mathrm{C}$ & 3.65982 & -1.22469 & -0.85916 \\
\hline $\mathrm{C}$ & 3.27006 & -0.61672 & -2.05810 \\
\hline $\mathrm{C}$ & 3.16360 & 0.83083 & -2.13527 \\
\hline $\mathrm{C}$ & 2.02386 & 1.15820 & -2.97067 \\
\hline $\mathrm{C}$ & 1.21942 & 2.25645 & -2.66166 \\
\hline $\mathrm{C}$ & -0.22604 & 2.15354 & -2.76561 \\
\hline $\mathrm{C}$ & -0.80853 & 2.89919 & -1.68072 \\
\hline $\mathrm{C}$ & 0.26218 & 3.46178 & -0.89118 \\
\hline $\mathrm{C}$ & 1.52382 & 3.07220 & -1.49640 \\
\hline $\mathrm{C}$ & 2.61288 & 2.75190 & -0.68562 \\
\hline $\mathrm{C}$ & 3.45245 & 1.61346 & -1.01194 \\
\hline $\mathrm{C}$ & 3.85426 & 0.97912 & 0.23055 \\
\hline $\mathrm{C}$ & 3.26050 & 1.72468 & 1.32981 \\
\hline $\mathrm{C}$ & 2.48717 & 2.81580 & 0.76364 \\
\hline $\mathrm{C}$ & 1.26935 & 3.17930 & 1.34118 \\
\hline $\mathrm{C}$ & 0.13497 & 3.50288 & 0.49611 \\
\hline $\mathrm{C}$ & -1.06495 & 2.99499 & 1.13490 \\
\hline $\mathrm{C}$ & -2.11260 & 2.48619 & 0.38395 \\
\hline $\mathrm{C}$ & -1.96473 & 2.40681 & -1.04636 \\
\hline $\mathrm{C}$ & -2.63250 & 1.23056 & -1.55303 \\
\hline $\mathrm{C}$ & -3.50628 & 0.68887 & -0.49039 \\
\hline $\mathrm{C}$ & -3.04925 & 1.37705 & 0.89776 \\
\hline $\mathrm{C}$ & -2.38886 & 0.58260 & 2.01394 \\
\hline $\mathrm{C}$ & -4.54174 & 1.78493 & 0.95719 \\
\hline $\mathrm{C}$ & -4.88981 & 1.21641 & -0.21515 \\
\hline $\mathrm{H}$ & -5.05609 & 2.44369 & 1.65163 \\
\hline $\mathrm{H}$ & -5.79093 & 1.23916 & -0.81942 \\
\hline
\end{tabular}


(10) $\mathrm{C}_{2} \mathrm{H}_{2}$-C $59 \mathrm{Ni}$-HH_C6-C7

$\begin{array}{cccc}\mathrm{Ni} & -3.77221 & -1.15889 & -0.32531 \\ \mathrm{C} & -3.47173 & 0.40686 & 0.74901 \\ \mathrm{C} & -3.09996 & 1.66369 & -0.23789 \\ \mathrm{C} & -2.60872 & 1.07891 & -1.55806 \\ \mathrm{C} & -2.73259 & -0.33061 & -1.63064 \\ \mathrm{C} & -1.83168 & -1.10103 & -2.37636 \\ \mathrm{C} & -0.85385 & -0.43162 & -3.18809 \\ \mathrm{C} & -0.74807 & 0.96747 & -3.15068 \\ \mathrm{C} & -1.62365 & 1.72371 & -2.31039 \\ \mathrm{C} & -0.84714 & 2.84172 & -1.75335 \\ \mathrm{C} & -1.06320 & 3.23470 & -0.44111 \\ \mathrm{C} & -2.09237 & 2.58785 & 0.36085 \\ \mathrm{C} & -1.58045 & 2.40791 & 1.65371 \\ \mathrm{C} & -1.80647 & 1.19980 & 2.39591 \\ \mathrm{C} & -2.58529 & 0.14054 & 1.89457 \\ \mathrm{C} & -1.96787 & -1.11858 & 2.28415 \\ \mathrm{C} & -0.83295 & -0.79257 & 3.13845 \\ \mathrm{C} & -0.72796 & 0.64330 & 3.21170 \\ \mathrm{C} & 0.51462 & 1.25753 & 3.27441 \\ \mathrm{C} & 0.76429 & 2.47648 & 2.49727 \\ \mathrm{C} & -0.24305 & 3.03268 & 1.72301 \\ \mathrm{C} & 0.06749 & 3.54770 & 0.41171 \\ \mathrm{C} & 1.37254 & 3.47331 & -0.08658 \\ \mathrm{C} & 1.59603 & 3.06783 & -1.46219 \\ \mathrm{C} & 0.50309 & 2.75102 & -2.27415 \\ \mathrm{C} & 0.56340 & 1.59454 & -3.15088 \\ \mathrm{C} & 1.71216 & 0.80690 & -3.19364 \\ \mathrm{C} & 1.60165 & -0.64474 & -3.25091 \\ \mathrm{C} & 0.34436 & -1.24665 & -3.24323 \\ \mathrm{C} & 0.10239 & -2.44553 & -2.46280 \\ \mathrm{C} & 1.15233 & -3.01551 & -1.73112 \\ \mathrm{C} & 2.45232 & -2.37142 & -1.70943 \\ \mathrm{C} & 2.67658 & -1.21009 & -2.45820 \\ \mathrm{C} & 3.43839 & -0.11347 & -1.89343 \\ \mathrm{C} & 2.84890 & 1.13495 & -2.35353 \\ \mathrm{C} & 2.79106 & 2.23982 & -1.50000 \\ \mathrm{C} & 3.30918 & 2.13677 & -0.14765 \\ \mathrm{C} & 2.42305 & 2.89440 & 0.72571 \\ \mathrm{C} & 2.12497 & 2.41240 & 2.00256 \\ \mathrm{C} & 2.71188 & 1.16294 & 2.46723 \\ \mathrm{C} & 1.72087 & 0.46329 & 3.26406 \\ \mathrm{C} & 1.62631 & -0.93359 & 3.20200 \\ \mathrm{C} & 0.32879 & -1.56640 & 3.15619 \\ \mathrm{C} & 0.42271 & -2.71928 & 2.27992 \\ \mathrm{C} & 1.78241 & -2.79608 & 1.77102 \\ \mathrm{C} & 2.52712 & -1.68380 & 2.34036 \\ \mathrm{C} & 3.47003 & -1.00650 & 1.56665\end{array}$




$\begin{array}{lccc}\mathrm{C} & 3.56731 & 0.44265 & 1.63323 \\ \mathrm{C} & 3.86391 & 0.93583 & 0.29698 \\ \mathrm{C} & 3.94285 & -0.20940 & -0.59384 \\ \mathrm{C} & 3.70381 & -1.40889 & 0.18738 \\ \mathrm{C} & 2.98297 & -2.47191 & -0.36113 \\ \mathrm{C} & 2.00562 & -3.18317 & 0.45007 \\ \mathrm{C} & 0.88148 & -3.52092 & -0.40448 \\ \mathrm{C} & -0.41915 & -3.45914 & 0.09755 \\ \mathrm{C} & -0.64893 & -3.03347 & 1.45571 \\ \mathrm{C} & -1.90473 & -2.26210 & 1.46050 \\ \mathrm{C} & -2.59328 & -2.47036 & 0.20339 \\ \mathrm{C} & -1.53238 & -2.94384 & -0.68955 \\ \mathrm{C} & -1.24569 & -2.39205 & -1.93771 \\ \mathrm{C} & -4.57150 & 2.00771 & -0.03530 \\ \mathrm{C} & -4.84734 & 0.96804 & 0.79059 \\ \mathrm{H} & -5.15009 & 2.85087 & -0.40126 \\ \mathrm{H} & -5.72947 & 0.67473 & 1.35306\end{array}$

(11) $\mathrm{C}_{2} \mathrm{H}_{2} \_\mathrm{C}_{59} \mathrm{Ni} \_\mathrm{HH} \_\mathrm{C} 7-\mathrm{C} 8$

$\begin{array}{crrr}\mathrm{Ni} & -3.60892 & -1.52946 & 0.00269 \\ \mathrm{C} & -3.14350 & -0.30892 & 1.43286 \\ \mathrm{C} & -3.39828 & 1.08814 & 1.30793 \\ \mathrm{C} & -3.39737 & 1.08204 & -1.31244 \\ \mathrm{C} & -3.14231 & -0.31549 & -1.43235 \\ \mathrm{C} & -2.13583 & -0.78235 & -2.28004 \\ \mathrm{C} & -1.38062 & 0.19422 & -3.02871 \\ \mathrm{C} & -1.42429 & 1.53144 & -2.62086 \\ \mathrm{C} & -2.33549 & 1.95709 & -1.61494 \\ \mathrm{C} & -1.58922 & 2.90113 & -0.71309 \\ \mathrm{C} & -1.58947 & 2.90413 & 0.70185 \\ \mathrm{C} & -2.33629 & 1.96408 & 1.60740 \\ \mathrm{C} & -1.42532 & 1.54193 & 2.61494 \\ \mathrm{C} & -1.38181 & 0.20609 & 3.02747 \\ \mathrm{C} & -2.13689 & -0.77295 & 2.28207 \\ \mathrm{C} & -1.30074 & -1.99498 & 2.21370 \\ \mathrm{C} & -0.07773 & -1.72544 & 2.95547 \\ \mathrm{C} & -0.12143 & -0.36341 & 3.43762 \\ \mathrm{C} & 1.03479 & 0.41796 & 3.46755 \\ \mathrm{C} & 0.98094 & 1.80350 & 3.02232 \\ \mathrm{C} & -0.22619 & 2.33168 & 2.57079 \\ \mathrm{C} & -0.30968 & 3.16165 & 1.38740 \\ \mathrm{C} & 0.87737 & 3.44833 & 0.71341 \\ \mathrm{C} & 0.87757 & 3.44553 & -0.72614 \\ \mathrm{C} & -0.30928 & 3.15610 & -1.39932 \\ \mathrm{C} & -0.22535 & 2.32160 & -2.57947 \\ \mathrm{C} & 0.98196 & 1.79189 & -3.02874 \\ \mathrm{C} & 1.03610 & 0.40475 & -3.46886\end{array}$




\begin{tabular}{lrrr}
$\mathrm{C}$ & -0.12005 & -0.37662 & -3.43652 \\
$\mathrm{C}$ & -0.07634 & -1.73692 & -2.94953 \\
$\mathrm{C}$ & 1.14762 & -2.29153 & -2.56891 \\
$\mathrm{C}$ & 2.35205 & -1.48728 & -2.59182 \\
$\mathrm{C}$ & 2.29496 & -0.15627 & -3.02398 \\
$\mathrm{C}$ & 3.01909 & 0.87631 & -2.30660 \\
$\mathrm{C}$ & 2.20173 & 2.08018 & -2.30842 \\
$\mathrm{C}$ & 2.14782 & 2.89696 & -1.17859 \\
$\mathrm{C}$ & 2.92887 & 2.55204 & -0.00434 \\
$\mathrm{C}$ & 2.14744 & 2.90144 & 1.16833 \\
$\mathrm{C}$ & 2.20095 & 2.08895 & 2.30129 \\
$\mathrm{C}$ & 3.01824 & 0.88505 & 2.30427 \\
$\mathrm{C}$ & 2.29373 & -0.14480 & 3.02519 \\
$\mathrm{C}$ & 2.35088 & -1.47741 & 2.59808 \\
$\mathrm{C}$ & 1.14639 & -2.28162 & 2.57762 \\
$\mathrm{C}$ & 1.20005 & -3.15059 & 1.41985 \\
$\mathrm{C}$ & 2.43162 & -2.87134 & 0.70292 \\
$\mathrm{C}$ & 3.14118 & -1.82930 & 1.42993 \\
$\mathrm{C}$ & 3.82744 & -0.83456 & 0.72962 \\
$\mathrm{C}$ & 3.76514 & 0.54758 & 1.17379 \\
$\mathrm{C}$ & 3.72382 & 1.39953 & -0.00202 \\
$\mathrm{C}$ & 3.76560 & 0.54313 & -1.17460 \\
$\mathrm{C}$ & 3.82775 & -0.83731 & -0.72516 \\
$\mathrm{C}$ & 3.14182 & -1.83471 & -1.42198 \\
$\mathrm{C}$ & 2.43198 & -2.87401 & -0.69133 \\
$\mathrm{C}$ & 1.20078 & -3.15608 & -1.40776 \\
$\mathrm{C}$ & 0.02606 & -3.42545 & -0.71518 \\
$\mathrm{C}$ & 0.02562 & -3.42254 & 0.72777 \\
$\mathrm{C}$ & -1.27557 & -2.89730 & 1.14571 \\
$\mathrm{C}$ & -2.18259 & -2.82066 & 0.00431 \\
$\mathrm{C}$ & -1.27487 & -2.90199 & -1.13591 \\
$\mathrm{C}$ & -1.29962 & -2.00390 & -2.20735 \\
$\mathrm{C}$ & -4.63490 & 1.57324 & -0.66521 \\
& -4.63526 & 1.57670 & 0.65776 \\
$\mathrm{H}$ & -5.45867 & 1.91977 & 1.28225 \\
\hline
\end{tabular}

\section{Ethylene}

(1) $\mathrm{C}_{2} \mathrm{H}_{4} \mathrm{C}_{59} \mathrm{Ni}$-TM

$\begin{array}{crrr}\mathrm{Ni} & -3.94124 & 0.28522 & 0.00005 \\ \mathrm{C} & -2.84956 & -0.71598 & -1.18756 \\ \mathrm{C} & -2.38862 & -2.00074 & -0.72538 \\ \mathrm{C} & -2.38859 & -2.00068 & 0.72558 \\ \mathrm{C} & -2.84949 & -0.71588 & 1.18769 \\ \mathrm{C} & -2.18418 & -0.06679 & 2.21705 \\ \mathrm{C} & -1.15471 & -0.78084 & 2.95117\end{array}$




\begin{tabular}{|c|c|c|c|}
\hline $\mathrm{C}$ & -0.76629 & -2.07020 & 2.57544 \\
\hline $\mathrm{C}$ & -1.37852 & -2.68478 & 1.42197 \\
\hline $\mathrm{C}$ & -0.34699 & -3.40449 & 0.69754 \\
\hline $\mathrm{C}$ & -0.34701 & -3.40453 & -0.69734 \\
\hline $\mathrm{C}$ & -1.37857 & -2.68487 & -1.42177 \\
\hline $\mathrm{C}$ & -0.76637 & -2.07034 & -2.57529 \\
\hline $\mathrm{C}$ & -1.15482 & -0.78100 & -2.95107 \\
\hline $\mathrm{C}$ & -2.18427 & -0.06693 & -2.21696 \\
\hline $\mathrm{C}$ & -1.80920 & 1.37170 & -2.21829 \\
\hline $\mathrm{C}$ & -0.56249 & 1.48864 & -2.95033 \\
\hline $\mathrm{C}$ & -0.16148 & 0.17304 & -3.40466 \\
\hline $\mathrm{C}$ & 1.18629 & -0.18197 & -3.45029 \\
\hline $\mathrm{C}$ & 1.59804 & -1.50855 & -3.02066 \\
\hline $\mathrm{C}$ & 0.64120 & -2.43095 & -2.59191 \\
\hline $\mathrm{C}$ & 0.90264 & -3.25497 & -1.42391 \\
\hline $\mathrm{C}$ & 2.10478 & -3.11865 & -0.72754 \\
\hline $\mathrm{C}$ & 2.10480 & -3.11861 & 0.72765 \\
\hline $\mathrm{C}$ & 0.90268 & -3.25490 & 1.42407 \\
\hline $\mathrm{C}$ & 0.64129 & -2.43081 & 2.59203 \\
\hline $\mathrm{C}$ & 1.59814 & -1.50839 & 3.02071 \\
\hline $\mathrm{C}$ & 1.18641 & -0.18179 & 3.45028 \\
\hline $\mathrm{C}$ & -0.16136 & 0.17322 & 3.40469 \\
\hline $\mathrm{C}$ & -0.56238 & 1.48881 & 2.95030 \\
\hline $\mathrm{C}$ & 0.41652 & 2.41592 & 2.57277 \\
\hline $\mathrm{C}$ & 1.81682 & 2.04467 & 2.59050 \\
\hline $\mathrm{C}$ & 2.19635 & 0.76942 & 3.01758 \\
\hline $\mathrm{C}$ & 3.22117 & 0.03639 & 2.30189 \\
\hline $\mathrm{C}$ & 2.84980 & -1.37175 & 2.30171 \\
\hline $\mathrm{C}$ & 3.09901 & -2.15964 & 1.17518 \\
\hline $\mathrm{C}$ & 3.71511 & -1.56760 & -0.00001 \\
\hline $\mathrm{C}$ & 3.09897 & -2.15970 & -1.17515 \\
\hline $\mathrm{C}$ & 2.84972 & -1.37187 & -2.30172 \\
\hline $\mathrm{C}$ & 3.22109 & 0.03627 & -2.30198 \\
\hline $\mathrm{C}$ & 2.19624 & 0.76926 & -3.01767 \\
\hline $\mathrm{C}$ & 1.81673 & 2.04453 & -2.59063 \\
\hline $\mathrm{C}$ & 0.41643 & 2.41578 & -2.57288 \\
\hline $\mathrm{C}$ & 0.18856 & 3.25866 & -1.41884 \\
\hline $\mathrm{C}$ & 1.44414 & 3.39160 & -0.69793 \\
\hline $\mathrm{C}$ & 2.45314 & 2.63957 & -1.42470 \\
\hline $\mathrm{C}$ & 3.43028 & 1.92635 & -0.72747 \\
\hline $\mathrm{C}$ & 3.82321 & 0.60075 & -1.17494 \\
\hline $\mathrm{C}$ & 4.07052 & -0.21677 & -0.00005 \\
\hline $\mathrm{C}$ & 3.82325 & 0.60081 & 1.17481 \\
\hline $\mathrm{C}$ & 3.43030 & 1.92639 & 0.72727 \\
\hline $\mathrm{C}$ & 2.45319 & 2.63965 & 1.42451 \\
\hline $\mathrm{C}$ & 1.44417 & 3.39164 & 0.69773 \\
\hline $\mathrm{C}$ & 0.18861 & 3.25874 & 1.41869 \\
\hline $\mathrm{C}$ & -1.01073 & 3.14083 & 0.72391 \\
\hline $\mathrm{C}$ & -1.01076 & 3.14078 & -0.72401 \\
\hline
\end{tabular}




$\begin{array}{lrrr}\mathrm{C} & -2.06766 & 2.22958 & -1.14522 \\ \mathrm{C} & -2.89346 & 1.84169 & 0.00003 \\ \mathrm{C} & -2.06761 & 2.22966 & 1.14523 \\ \mathrm{C} & -1.80912 & 1.37183 & 2.21832 \\ \mathrm{C} & -6.12752 & 0.34018 & -0.00013 \\ \mathrm{C} & -5.74343 & -0.97667 & -0.00014 \\ \mathrm{H} & -5.67873 & -1.54448 & -0.92375 \\ \mathrm{H} & -5.67886 & -1.54453 & 0.92346 \\ \mathrm{H} & -6.35696 & 0.86723 & -0.92306 \\ \mathrm{H} & -6.35710 & 0.86718 & 0.92279\end{array}$

(2) $\mathrm{C}_{2} \mathrm{H}_{4} \_\mathrm{C}_{59} \mathrm{Ni} \_\mathrm{TC} 5$

$\begin{array}{rrrr}\mathrm{Ni} & 3.72754 & -1.16400 & -0.00008 \\ \mathrm{C} & 2.50708 & -1.80726 & 1.19864 \\ \mathrm{C} & 1.62268 & -2.85138 & 0.72694 \\ \mathrm{C} & 1.62271 & -2.85059 & -0.72908 \\ \mathrm{C} & 2.50696 & -1.80583 & -1.19973 \\ \mathrm{C} & 2.08190 & -0.94030 & -2.20033 \\ \mathrm{C} & 0.86395 & -1.25687 & -2.92393 \\ \mathrm{C} & 0.07107 & -2.35491 & -2.56411 \\ \mathrm{C} & 0.44106 & -3.15004 & -1.41746 \\ \mathrm{C} & -0.77208 & -3.49615 & -0.69861 \\ \mathrm{C} & -0.77211 & -3.49676 & 0.69581 \\ \mathrm{C} & 0.44100 & -3.15134 & 1.41501 \\ \mathrm{C} & 0.07100 & -2.35709 & 2.56227 \\ \mathrm{C} & 0.86390 & -1.25934 & 2.92297 \\ \mathrm{C} & 2.08189 & -0.94230 & 2.19968 \\ \mathrm{C} & 2.19694 & 0.56250 & 2.16747 \\ \mathrm{C} & 1.03605 & 1.07159 & 2.89686 \\ \mathrm{C} & 0.23438 & -0.03651 & 3.36341 \\ \mathrm{C} & -1.15641 & 0.06491 & 3.43336 \\ \mathrm{C} & -1.97948 & -1.05557 & 3.01364 \\ \mathrm{C} & -1.37527 & -2.23895 & 2.58439 \\ \mathrm{C} & -1.89915 & -2.93993 & 1.42203 \\ \mathrm{C} & -2.99137 & -2.41677 & 0.72647 \\ \mathrm{C} & -2.99134 & -2.41617 & -0.72846 \\ \mathrm{C} & -1.89910 & -2.93875 & -1.42442 \\ \mathrm{C} & -1.37521 & -2.23681 & -2.58618 \\ \mathrm{C} & -1.97945 & -1.05311 & -3.01451 \\ \mathrm{C} & -1.15640 & 0.06771 & -3.43332 \\ \mathrm{C} & 0.23440 & -0.03372 & -3.36348 \\ \mathrm{C} & 1.03602 & 1.07405 & -2.89612 \\ \mathrm{C} & 0.40750 & 2.27413 & -2.55402 \\ \mathrm{C} & -1.04076 & 2.37752 & -2.58526 \\ \mathrm{C} & -1.80914 & 1.29348 & -3.01055 \\ \mathrm{C} & -3.02004 & 0.93198 & -2.29995 \\ \mathrm{C} & -3.12439 & -0.52000 & -2.30130\end{array}$




$\begin{array}{lrrr}\mathrm{C} & -3.61792 & -1.18520 & -1.17616 \\ \mathrm{C} & -4.00976 & -0.42597 & -0.00019 \\ \mathrm{C} & -3.61794 & -1.18615 & 1.17518 \\ \mathrm{C} & -3.12441 & -0.52187 & 2.30086 \\ \mathrm{C} & -3.02004 & 0.93010 & 2.30067 \\ \mathrm{C} & -1.80913 & 1.29102 & 3.01155 \\ \mathrm{C} & -1.04074 & 2.37538 & 2.58713 \\ \mathrm{C} & 0.40752 & 2.27195 & 2.55577 \\ \mathrm{C} & 0.88509 & 3.00866 & 1.41241 \\ \mathrm{C} & -0.26393 & 3.53519 & 0.69785 \\ \mathrm{C} & -1.45749 & 3.14753 & 1.42650 \\ \mathrm{C} & -2.61371 & 2.78943 & 0.72899 \\ \mathrm{C} & -3.41105 & 1.66044 & 1.17565 \\ \mathrm{C} & -3.90993 & 0.96677 & 0.00038 \\ \mathrm{C} & -3.41105 & 1.66139 & -1.17433 \\ \mathrm{C} & -2.61371 & 2.79003 & -0.72675 \\ \mathrm{C} & -1.45751 & 3.14871 & -1.42399 \\ \mathrm{C} & -0.26394 & 3.53579 & -0.69504 \\ \mathrm{C} & 0.88507 & 3.00992 & -1.41007 \\ \mathrm{C} & 2.00164 & 2.53779 & -0.72933 \\ \mathrm{C} & 2.00163 & 2.53707 & 0.73127 \\ \mathrm{C} & 2.75392 & 1.37509 & 1.18372 \\ \mathrm{C} & 3.68667 & 1.09418 & 0.00025 \\ \mathrm{C} & 2.75394 & 1.37628 & -1.18286 \\ \mathrm{C} & 2.19692 & 0.56442 & -2.16714 \\ \mathrm{C} & 4.98783 & 1.87532 & 0.00039 \\ \mathrm{C} & 5.12394 & 0.38389 & -0.00040 \\ \mathrm{H} & 5.49808 & -0.07629 & 0.92096 \\ \mathrm{H} & 5.49718 & -0.07535 & -0.92260 \\ \mathrm{H} & 5.25483 & 2.39543 & 0.91544 \\ \mathrm{H} & 5.25471 & 2.39662 & -0.91402\end{array}$

(3) $\mathrm{C}_{2} \mathrm{H}_{4}{ }_{-} \mathrm{C}_{59} \mathrm{Ni}$-TC1

$\begin{array}{cccc}\mathrm{Ni} & 3.94103 & -0.28535 & -0.00004 \\ \mathrm{C} & 2.84963 & 0.71559 & -1.18821 \\ \mathrm{C} & 2.38857 & 2.00043 & -0.72636 \\ \mathrm{C} & 2.38874 & 2.00104 & 0.72458 \\ \mathrm{C} & 2.84990 & 0.71659 & 1.18726 \\ \mathrm{C} & 2.18449 & 0.06769 & 2.21676 \\ \mathrm{C} & 1.15507 & 0.78195 & 2.95068 \\ \mathrm{C} & 0.76658 & 2.07120 & 2.57453 \\ \mathrm{C} & 1.37869 & 2.68536 & 1.42080 \\ \mathrm{C} & 0.34708 & 3.40480 & 0.69622 \\ \mathrm{C} & 0.34696 & 3.40428 & -0.69864 \\ \mathrm{C} & 1.37844 & 2.68431 & -1.42289 \\ \mathrm{C} & 0.76612 & 2.06938 & -2.57609 \\ \mathrm{C} & 1.15451 & 0.77988 & -2.95144\end{array}$




\begin{tabular}{|c|c|c|c|}
\hline $\mathrm{C}$ & 2.18403 & 0.06613 & -2.21721 \\
\hline $\mathrm{C}$ & 1.80896 & -1.37256 & -2.21803 \\
\hline C & 0.56217 & -1.48974 & -2.94986 \\
\hline $\mathrm{C}$ & 0.16110 & -0.17430 & -3.40459 \\
\hline $\mathrm{C}$ & -1.18665 & 0.18072 & -3.45026 \\
\hline $\mathrm{C}$ & -1.59834 & 1.50745 & -3.02108 \\
\hline $\mathrm{C}$ & -0.64145 & 2.42999 & -2.59274 \\
\hline $\mathrm{C}$ & -0.90276 & 3.25445 & -1.42504 \\
\hline $\mathrm{C}$ & -2.10484 & 3.11842 & -0.72851 \\
\hline $\mathrm{C}$ & -2.10472 & 3.11892 & 0.72668 \\
\hline C & -0.90252 & 3.25547 & 1.42292 \\
\hline C & -0.64100 & 2.43181 & 2.59116 \\
\hline $\mathrm{C}$ & -1.59780 & 1.50952 & 3.02023 \\
\hline $\mathrm{C}$ & -1.18602 & 0.18307 & 3.45023 \\
\hline C & 0.16174 & -0.17196 & 3.40460 \\
\hline C & 0.56273 & -1.48768 & 2.95062 \\
\hline C & -0.41622 & -2.41497 & 2.57358 \\
\hline $\mathrm{C}$ & -1.81652 & -2.04370 & 2.59133 \\
\hline $\mathrm{C}$ & -2.19600 & -0.76830 & 3.01798 \\
\hline C & -3.22090 & -0.03553 & 2.30216 \\
\hline C & -2.84953 & 1.37262 & 2.30144 \\
\hline $\mathrm{C}$ & -3.09886 & 2.16010 & 1.17465 \\
\hline $\mathrm{C}$ & -3.71510 & 1.56763 & -0.00025 \\
\hline $\mathrm{C}$ & -3.09908 & 2.15930 & -1.17566 \\
\hline $\mathrm{C}$ & -2.84994 & 1.37105 & -2.30196 \\
\hline $\mathrm{C}$ & -3.22132 & -0.03709 & -2.30166 \\
\hline $\mathrm{C}$ & -2.19657 & -0.77036 & -3.01718 \\
\hline $\mathrm{C}$ & -1.81702 & -2.04548 & -2.58974 \\
\hline $\mathrm{C}$ & -0.41672 & -2.41676 & -2.57200 \\
\hline $\mathrm{C}$ & -0.18873 & -3.25924 & -1.41770 \\
\hline $\mathrm{C}$ & -1.44425 & -3.39189 & -0.69658 \\
\hline $\mathrm{C}$ & -2.45331 & -2.64010 & -1.42353 \\
\hline $\mathrm{C}$ & -3.43037 & -1.92660 & -0.72645 \\
\hline $\mathrm{C}$ & -3.82333 & -0.60117 & -1.17436 \\
\hline $\mathrm{C}$ & -4.07050 & 0.21679 & 0.00025 \\
\hline $\mathrm{C}$ & -3.82313 & -0.60037 & 1.17537 \\
\hline $\mathrm{C}$ & -3.43024 & -1.92612 & 0.72828 \\
\hline $\mathrm{C}$ & -2.45304 & -2.63914 & 1.42567 \\
\hline $\mathrm{C}$ & -1.44412 & -3.39141 & 0.69905 \\
\hline $\mathrm{C}$ & -0.18846 & -3.25823 & 1.41983 \\
\hline $\mathrm{C}$ & 1.01079 & -3.14059 & 0.72487 \\
\hline C & 1.01067 & -3.14113 & -0.72308 \\
\hline $\mathrm{C}$ & 2.06751 & -2.23013 & -1.14475 \\
\hline $\mathrm{C}$ & 2.89352 & -1.84186 & 0.00025 \\
\hline $\mathrm{C}$ & 2.06772 & -2.22929 & 1.14569 \\
\hline $\mathrm{C}$ & 1.80939 & -1.37098 & 2.21848 \\
\hline $\mathrm{C}$ & 6.12737 & -0.34011 & -0.00003 \\
\hline C & 5.74350 & 0.97671 & -0.00003 \\
\hline $\mathrm{H}$ & 5.67880 & 1.54447 & -0.92361 \\
\hline
\end{tabular}




$\begin{array}{rrrc}\mathrm{H} & 5.67883 & 1.54441 & 0.92359 \\ \mathrm{H} & 6.35684 & -0.86712 & -0.92298 \\ \mathrm{H} & 6.35692 & -0.86712 & 0.92290\end{array}$

(4) $\mathrm{C}_{2} \mathrm{H}_{4}{ }_{-} \mathrm{C}_{59} \mathrm{Ni} \_\mathrm{HH}$-M-C5

\begin{tabular}{|c|c|c|c|}
\hline $\mathrm{Ni}$ & -3.94124 & 0.28522 & 0.00005 \\
\hline $\mathrm{C}$ & -2.84956 & -0.71598 & -1.18756 \\
\hline C & -2.38862 & -2.00074 & -0.72538 \\
\hline $\mathrm{C}$ & -2.38859 & -2.00068 & 0.72558 \\
\hline C & -2.84949 & -0.71588 & 1.18769 \\
\hline C & -2.18418 & -0.06679 & 2.21705 \\
\hline C & -1.15471 & -0.78084 & 2.95117 \\
\hline $\mathrm{C}$ & -0.76629 & -2.07020 & 2.57544 \\
\hline $\mathrm{C}$ & -1.37852 & -2.68478 & 1.42197 \\
\hline C & -0.34699 & -3.40449 & 0.69754 \\
\hline C & -0.34701 & -3.40453 & -0.69734 \\
\hline C & -1.37857 & -2.68487 & -1.42177 \\
\hline $\mathrm{C}$ & -0.76637 & -2.07034 & -2.57529 \\
\hline C & -1.15482 & -0.78100 & -2.95107 \\
\hline C & -2.18427 & -0.06693 & -2.21696 \\
\hline C & -1.80920 & 1.37170 & -2.21829 \\
\hline C & -0.56249 & 1.48864 & -2.95033 \\
\hline C & -0.16148 & 0.17304 & -3.40466 \\
\hline C & 1.18629 & -0.18197 & -3.45029 \\
\hline $\mathrm{C}$ & 1.59804 & -1.50855 & -3.02066 \\
\hline $\mathrm{C}$ & 0.64120 & -2.43095 & -2.59191 \\
\hline $\mathrm{C}$ & 0.90264 & -3.25497 & -1.42391 \\
\hline C & 2.10478 & -3.11865 & -0.72754 \\
\hline $\mathrm{C}$ & 2.10480 & -3.11861 & 0.72765 \\
\hline $\mathrm{C}$ & 0.90268 & -3.25490 & 1.42407 \\
\hline $\mathrm{C}$ & 0.64129 & -2.43081 & 2.59203 \\
\hline C & 1.59814 & -1.50839 & 3.02071 \\
\hline C & 1.18641 & -0.18179 & 3.45028 \\
\hline C & -0.16136 & 0.17322 & 3.40469 \\
\hline C & -0.56238 & 1.48881 & 2.95030 \\
\hline C & 0.41652 & 2.41592 & 2.57277 \\
\hline C & 1.81682 & 2.04467 & 2.59050 \\
\hline C & 2.19635 & 0.76942 & 3.01758 \\
\hline C & 3.22117 & 0.03639 & 2.30189 \\
\hline $\mathrm{C}$ & 2.84980 & -1.37175 & 2.30171 \\
\hline C & 3.09901 & -2.15964 & 1.17518 \\
\hline C & 3.71511 & -1.56760 & -0.00001 \\
\hline C & 3.09897 & -2.15970 & -1.17515 \\
\hline C & 2.84972 & -1.37187 & -2.30172 \\
\hline C & 3.22109 & 0.03627 & -2.30198 \\
\hline $\mathrm{C}$ & 2.19624 & 0.76926 & -3.01767 \\
\hline C & 1.81673 & 2.04453 & -2.59063 \\
\hline C & 0.41643 & 2.41578 & -2.57288 \\
\hline $\mathrm{C}$ & 0.18856 & 3.25866 & -1.41884 \\
\hline
\end{tabular}




$\begin{array}{lrrr}\mathrm{C} & 1.44414 & 3.39160 & -0.69793 \\ \mathrm{C} & 2.45314 & 2.63957 & -1.42470 \\ \mathrm{C} & 3.43028 & 1.92635 & -0.72747 \\ \mathrm{C} & 3.82321 & 0.60075 & -1.17494 \\ \mathrm{C} & 4.07052 & -0.21677 & -0.00005 \\ \mathrm{C} & 3.82325 & 0.60081 & 1.17481 \\ \mathrm{C} & 3.43030 & 1.92639 & 0.72727 \\ \mathrm{C} & 2.45319 & 2.63965 & 1.42451 \\ \mathrm{C} & 1.44417 & 3.39164 & 0.69773 \\ \mathrm{C} & 0.18861 & 3.25874 & 1.41869 \\ \mathrm{C} & -1.01073 & 3.14083 & 0.72391 \\ \mathrm{C} & -1.01076 & 3.14078 & -0.72401 \\ \mathrm{C} & -2.06766 & 2.22958 & -1.14522 \\ \mathrm{C} & -2.89346 & 1.84169 & 0.00003 \\ \mathrm{C} & -2.06761 & 2.22966 & 1.14523 \\ \mathrm{C} & -1.80912 & 1.37183 & 2.21832 \\ \mathrm{C} & -6.12752 & 0.34018 & -0.00013 \\ \mathrm{C} & -5.74343 & -0.97667 & -0.00014 \\ \mathrm{H} & -5.67873 & -1.54448 & -0.92375 \\ \mathrm{H} & -5.67886 & -1.54453 & 0.92346 \\ \mathrm{H} & -6.35696 & 0.86723 & -0.92306 \\ \mathrm{H} & -6.35710 & 0.86718 & 0.92279\end{array}$

(5) $\mathrm{C}_{2} \mathrm{H}_{4}$-C ${ }_{59} \mathrm{Ni}$-PH_M-C1

$\begin{array}{crrr}\mathrm{Ni} & -3.93814 & -0.77234 & -0.02857 \\ \mathrm{C} & -2.82509 & -0.66861 & 1.46142 \\ \mathrm{C} & -2.47261 & 0.68350 & 1.81548 \\ \mathrm{C} & -2.62893 & 1.62793 & 0.72900 \\ \mathrm{C} & -3.33110 & 1.13711 & -0.52032 \\ \mathrm{C} & -2.46440 & 1.22588 & -1.71436 \\ \mathrm{C} & -1.49232 & 2.32210 & -1.78568 \\ \mathrm{C} & -1.17416 & 3.08341 & -0.67365 \\ \mathrm{C} & -1.72683 & 2.68300 & 0.62758 \\ \mathrm{C} & -0.70376 & 2.90086 & 1.61628 \\ \mathrm{C} & -0.57976 & 2.02861 & 2.69784 \\ \mathrm{C} & -1.46495 & 0.89594 & 2.78440 \\ \mathrm{C} & -0.71408 & -0.21840 & 3.30960 \\ \mathrm{C} & -0.95824 & -1.49652 & 2.79904 \\ \mathrm{C} & -2.01274 & -1.72912 & 1.83837 \\ \mathrm{C} & -1.51470 & -2.78696 & 0.90219 \\ \mathrm{C} & -0.18594 & -3.17505 & 1.35694 \\ \mathrm{C} & 0.15020 & -2.39432 & 2.52814 \\ \mathrm{C} & 1.46077 & -1.97738 & 2.75816 \\ \mathrm{C} & 1.71849 & -0.63829 & 3.26341 \\ \mathrm{C} & 0.65044 & 0.21999 & 3.53380 \\ \mathrm{C} & 0.73465 & 1.62109 & 3.15345 \\ \mathrm{C} & 1.87574 & 2.10820 & 2.51378\end{array}$




\begin{tabular}{|c|c|c|c|}
\hline C & 1.74564 & 3.03104 & 1.39440 \\
\hline $\mathrm{C}$ & 0.48101 & 3.42353 & 0.95713 \\
\hline $\mathrm{C}$ & 0.19185 & 3.52354 & -0.46210 \\
\hline $\mathrm{C}$ & 1.18905 & 3.22282 & -1.39539 \\
\hline $\mathrm{C}$ & 0.85021 & 2.44184 & -2.56744 \\
\hline C & -0.46324 & 1.99338 & -2.74465 \\
\hline $\mathrm{C}$ & -0.73391 & 0.65368 & -3.19294 \\
\hline $\mathrm{C}$ & 0.32909 & -0.19382 & -3.47664 \\
\hline $\mathrm{C}$ & 1.69550 & 0.25109 & -3.30899 \\
\hline $\mathrm{C}$ & 1.95357 & 1.54618 & -2.84699 \\
\hline $\mathrm{C}$ & 2.98196 & 1.76567 & -1.84526 \\
\hline $\mathrm{C}$ & 2.50459 & 2.80103 & -0.93944 \\
\hline $\mathrm{C}$ & 2.77995 & 2.71200 & 0.42470 \\
\hline $\mathrm{C}$ & 3.53992 & 1.58334 & 0.94302 \\
\hline $\mathrm{C}$ & 2.98368 & 1.21681 & 2.23109 \\
\hline $\mathrm{C}$ & 2.90413 & -0.13397 & 2.59940 \\
\hline $\mathrm{C}$ & 3.39027 & -1.16171 & 1.69711 \\
\hline $\mathrm{C}$ & 2.50044 & -2.30753 & 1.79918 \\
\hline $\mathrm{C}$ & 2.18655 & -3.05739 & 0.66698 \\
\hline $\mathrm{C}$ & 0.82310 & -3.51154 & 0.44570 \\
\hline $\mathrm{C}$ & 0.55201 & -3.44003 & -0.97550 \\
\hline $\mathrm{C}$ & 1.73279 & -2.92211 & -1.63458 \\
\hline $\mathrm{C}$ & 2.74964 & -2.68822 & -0.62441 \\
\hline $\mathrm{C}$ & 3.59503 & -1.58320 & -0.72677 \\
\hline $\mathrm{C}$ & 3.92540 & -0.80512 & 0.45721 \\
\hline $\mathrm{C}$ & 4.00079 & 0.59194 & 0.07307 \\
\hline $\mathrm{C}$ & 3.71154 & 0.68508 & -1.34848 \\
\hline $\mathrm{C}$ & 3.45823 & -0.65942 & -1.84235 \\
\hline $\mathrm{C}$ & 2.47181 & -0.87250 & -2.80559 \\
\hline $\mathrm{C}$ & 1.59223 & -2.02427 & -2.69861 \\
\hline $\mathrm{C}$ & 0.27060 & -1.61209 & -3.11546 \\
\hline $\mathrm{C}$ & -0.85454 & -2.09848 & -2.47864 \\
\hline $\mathrm{C}$ & -0.72017 & -3.05663 & -1.40252 \\
\hline $\mathrm{C}$ & -1.79192 & -2.77512 & -0.46358 \\
\hline $\mathrm{C}$ & -2.64221 & -1.71669 & -1.02294 \\
\hline $\mathrm{C}$ & -2.01605 & -1.24047 & -2.17051 \\
\hline $\mathrm{C}$ & -1.95426 & 0.17370 & -2.50596 \\
\hline $\mathrm{C}$ & -4.86422 & 1.31397 & -0.54487 \\
\hline $\mathrm{C}$ & -5.34752 & 0.39303 & 0.58724 \\
\hline $\mathrm{H}$ & -6.29614 & -0.11978 & 0.38064 \\
\hline $\mathrm{H}$ & -5.33850 & 0.84123 & 1.57877 \\
\hline $\mathrm{H}$ & -5.25763 & 1.02303 & -1.52711 \\
\hline $\mathrm{H}$ & -5.09942 & 2.38280 & -0.41543 \\
\hline
\end{tabular}

(6) $\mathrm{C}_{2} \mathrm{H}_{4} \_\mathrm{C}_{59} \mathrm{Ni} \_\mathrm{HH} \_\mathrm{C} 1-\mathrm{C} 2$

$\begin{array}{cccc}\mathrm{Ni} & -3.73471 & -1.22780 & 0.26126 \\ \mathrm{C} & -2.47066 & -1.40587 & 1.61413\end{array}$




\begin{tabular}{|c|c|c|c|}
\hline $\mathrm{C}$ & -2.19240 & -0.21330 & 2.37675 \\
\hline $\mathrm{C}$ & -2.61781 & 0.96199 & 1.66456 \\
\hline $\mathrm{C}$ & -3.44564 & 0.66228 & 0.45189 \\
\hline $\mathrm{C}$ & -3.01126 & 1.33875 & -0.92292 \\
\hline $\mathrm{C}$ & -1.99584 & 2.46464 & -0.73179 \\
\hline $\mathrm{C}$ & -1.54876 & 2.88935 & 0.50565 \\
\hline $\mathrm{C}$ & -1.84745 & 2.11011 & 1.72901 \\
\hline $\mathrm{C}$ & -0.67868 & 2.16747 & 2.57762 \\
\hline $\mathrm{C}$ & -0.29785 & 1.05546 & 3.33103 \\
\hline $\mathrm{C}$ & -1.06157 & -0.16529 & 3.21740 \\
\hline $\mathrm{C}$ & -0.14364 & -1.27540 & 3.24789 \\
\hline $\mathrm{C}$ & -0.37031 & -2.37416 & 2.41101 \\
\hline $\mathrm{C}$ & -1.53336 & -2.42602 & 1.55136 \\
\hline $\mathrm{C}$ & -1.12574 & -3.12546 & 0.29558 \\
\hline $\mathrm{C}$ & 0.27425 & -3.45710 & 0.43033 \\
\hline $\mathrm{C}$ & 0.74059 & -3.00877 & 1.72850 \\
\hline $\mathrm{C}$ & 2.03544 & -2.51843 & 1.88969 \\
\hline $\mathrm{C}$ & 2.26783 & -1.35400 & 2.73122 \\
\hline $\mathrm{C}$ & 1.20033 & -0.74904 & 3.39938 \\
\hline $\mathrm{C}$ & 1.10440 & 0.70139 & 3.44486 \\
\hline $\mathrm{C}$ & 2.07546 & 1.48468 & 2.81715 \\
\hline $\mathrm{C}$ & 1.67928 & 2.65382 & 2.04568 \\
\hline $\mathrm{C}$ & 0.32881 & 2.98016 & 1.92298 \\
\hline $\mathrm{C}$ & -0.20793 & 3.42205 & 0.64818 \\
\hline $\mathrm{C}$ & 0.64106 & 3.52378 & -0.45947 \\
\hline $\mathrm{C}$ & 0.18007 & 3.06262 & -1.74718 \\
\hline $\mathrm{C}$ & -1.10123 & 2.50865 & -1.85213 \\
\hline $\mathrm{C}$ & -1.32030 & 1.31988 & -2.65575 \\
\hline $\mathrm{C}$ & -0.24708 & 0.75807 & -3.36224 \\
\hline $\mathrm{C}$ & 1.08364 & 1.31328 & -3.23196 \\
\hline $\mathrm{C}$ & 1.29706 & 2.44232 & -2.43717 \\
\hline $\mathrm{C}$ & 2.45205 & 2.50848 & -1.56527 \\
\hline $\mathrm{C}$ & 2.04573 & 3.17796 & -0.33688 \\
\hline $\mathrm{C}$ & 2.55484 & 2.74828 & 0.88947 \\
\hline $\mathrm{C}$ & 3.48509 & 1.63517 & 0.94187 \\
\hline $\mathrm{C}$ & 3.18539 & 0.85416 & 2.13009 \\
\hline $\mathrm{C}$ & 3.28137 & -0.54129 & 2.09109 \\
\hline $\mathrm{C}$ & 3.68559 & -1.20737 & 0.86237 \\
\hline $\mathrm{C}$ & 2.92390 & -2.43586 & 0.74329 \\
\hline $\mathrm{C}$ & 2.48404 & -2.87136 & -0.50862 \\
\hline $\mathrm{C}$ & 1.14873 & -3.41174 & -0.66721 \\
\hline $\mathrm{C}$ & 0.64113 & -2.97955 & -1.94759 \\
\hline $\mathrm{C}$ & 1.65398 & -2.15522 & -2.58811 \\
\hline $\mathrm{C}$ & 2.79772 & -2.08998 & -1.69854 \\
\hline $\mathrm{C}$ & 3.52600 & -0.90368 & -1.58148 \\
\hline $\mathrm{C}$ & 3.98115 & -0.45420 & -0.27656 \\
\hline $\mathrm{C}$ & 3.87523 & 0.99307 & -0.23724 \\
\hline $\mathrm{C}$ & 3.35192 & 1.44037 & -1.51599 \\
\hline $\mathrm{C}$ & 3.13606 & 0.26811 & -2.34755 \\
\hline
\end{tabular}




$\begin{array}{lrrr}\mathrm{C} & 2.02793 & 0.20726 & -3.19614 \\ \mathrm{C} & 1.27531 & -1.02857 & -3.32093 \\ \mathrm{C} & -0.13093 & -0.67656 & -3.42844 \\ \mathrm{C} & -1.09434 & -1.45585 & -2.79701 \\ \mathrm{C} & -0.70684 & -2.63998 & -2.06048 \\ \mathrm{C} & -1.63508 & -2.76625 & -0.95223 \\ \mathrm{C} & -2.77369 & -1.85882 & -1.15938 \\ \mathrm{C} & -2.24679 & -0.87522 & -2.11656 \\ \mathrm{C} & -2.33984 & 0.51489 & -2.03729 \\ \mathrm{C} & -4.51871 & 1.76459 & -1.11091 \\ \mathrm{C} & -4.90361 & 1.15952 & 0.26097 \\ \mathrm{H} & -5.67712 & 0.37395 & 0.20977 \\ \mathrm{H} & -5.21905 & 1.87059 & 1.03160 \\ \mathrm{H} & -4.99634 & 1.27890 & -1.96581 \\ \mathrm{H} & -4.65977 & 2.84505 & -1.19042\end{array}$

(7) $\mathrm{C}_{2} \mathrm{H}_{4} \_\mathrm{C}_{59} \mathrm{Ni} \_\mathrm{PH}$ _C2-C3

$\begin{array}{rrrr}\mathrm{Ni} & 3.60375 & -1.62562 & -0.29947 \\ \mathrm{C} & 2.05264 & -2.40670 & -0.98786 \\ \mathrm{C} & 1.68840 & -1.67611 & -2.17728 \\ \mathrm{C} & 2.27311 & -0.33396 & -2.23687 \\ \mathrm{C} & 3.31698 & -0.05442 & -1.28434 \\ \mathrm{C} & 3.26377 & 1.27438 & -0.55728 \\ \mathrm{C} & 2.19341 & 2.22355 & -1.05753 \\ \mathrm{C} & 1.50172 & 2.01350 & -2.23312 \\ \mathrm{C} & 1.53454 & 0.68681 & -2.85022 \\ \mathrm{C} & 0.24607 & 0.43876 & -3.43961 \\ \mathrm{C} & -0.30046 & -0.84763 & -3.41316 \\ \mathrm{C} & 0.43396 & -1.91870 & -2.77035 \\ \mathrm{C} & -0.52203 & -2.78505 & -2.12409 \\ \mathrm{C} & -0.19916 & -3.37310 & -0.89920 \\ \mathrm{C} & 1.10293 & -3.15370 & -0.31353 \\ \mathrm{C} & 0.91212 & -3.08309 & 1.15516 \\ \mathrm{C} & -0.50237 & -3.24861 & 1.42667 \\ \mathrm{C} & -1.18736 & -3.43914 & 0.16222 \\ \mathrm{C} & -2.46075 & -2.90714 & -0.03908 \\ \mathrm{C} & -2.79659 & -2.29076 & -1.31315 \\ \mathrm{C} & -1.84887 & -2.23778 & -2.33573 \\ \mathrm{C} & -1.70852 & -1.03150 & -3.13747 \\ \mathrm{C} & -2.52577 & 0.07457 & -2.88487 \\ \mathrm{C} & -1.96075 & 1.41334 & -2.90815 \\ \mathrm{C} & -0.60437 & 1.59092 & -3.18273 \\ \mathrm{C} & 0.17232 & 2.55220 & -2.42382 \\ \mathrm{C} & -0.43596 & 3.29949 & -1.41229 \\ \mathrm{C} & 0.26084 & 3.48531 & -0.15945 \\ \mathrm{C} & 1.53296 & 2.90319 & 0.01988 \\ \mathrm{C} & 1.89065 & 2.28343 & 1.26186\end{array}$




$\begin{array}{lrrr}\mathrm{C} & 0.95521 & 2.24107 & 2.28037 \\ \mathrm{C} & -0.35904 & 2.81686 & 2.11061 \\ \mathrm{C} & -0.70569 & 3.43662 & 0.90666 \\ \mathrm{C} & -2.01496 & 3.20337 & 0.32239 \\ \mathrm{C} & -1.84548 & 3.11370 & -1.12096 \\ \mathrm{C} & -2.59385 & 2.18861 & -1.85290 \\ \mathrm{C} & -3.54519 & 1.32562 & -1.17748 \\ \mathrm{C} & -3.50435 & 0.02193 & -1.81588 \\ \mathrm{C} & -3.63899 & -1.13866 & -1.04455 \\ \mathrm{C} & -3.82145 & -1.04060 & 0.39031 \\ \mathrm{C} & -3.09833 & -2.13853 & 1.01581 \\ \mathrm{C} & -2.44248 & -1.94519 & 2.23121 \\ \mathrm{C} & -1.12407 & -2.51717 & 2.44734 \\ \mathrm{C} & -0.34554 & -1.56687 & 3.21467 \\ \mathrm{C} & -1.17050 & -0.40476 & 3.46695 \\ \mathrm{C} & -2.47270 & -0.63508 & 2.86949 \\ \mathrm{C} & -3.16002 & 0.41891 & 2.26620 \\ \mathrm{C} & -3.85523 & 0.21276 & 1.00570 \\ \mathrm{C} & -3.71213 & 1.41738 & 0.20897 \\ \mathrm{C} & -2.92916 & 2.37280 & 0.97151 \\ \mathrm{C} & -2.57950 & 1.75146 & 2.24031 \\ \mathrm{C} & -1.32000 & 1.97113 & 2.79913 \\ \mathrm{C} & -0.59951 & 0.87496 & 3.42383 \\ \mathrm{C} & 0.80140 & 1.03118 & 3.10383 \\ \mathrm{C} & 1.57740 & -0.07694 & 2.82789 \\ \mathrm{C} & 1.01394 & -1.40490 & 2.92928 \\ \mathrm{C} & 1.66236 & -2.19608 & 1.90796 \\ \mathrm{C} & 2.69313 & -1.36712 & 1.27995 \\ \mathrm{C} & 2.57518 & -0.07391 & 1.74097 \\ \mathrm{C} & 2.99369 & 1.23002 & 1.07774 \\ \mathrm{C} & 4.45905 & 1.74986 & 1.29948 \\ \mathrm{C} & 4.65819 & 1.93229 & -0.22194 \\ \mathrm{H} & 5.49586 & 1.37687 & -0.64990 \\ & 4.71577 & 2.97397 & -0.54795 \\ \mathrm{H} & 4.53675 & 2.65126 & 1.91358\end{array}$

(8) $\mathrm{C}_{2} \mathrm{H}_{4} \_\mathrm{C} 59 \mathrm{Ni}$ _HH_C3-C4

$\begin{array}{cccc}\mathrm{Ni} & -3.55067 & -1.64047 & -0.36069 \\ \mathrm{C} & -1.97690 & -2.57032 & -0.78239 \\ \mathrm{C} & -1.24384 & -3.12845 & 0.33131 \\ \mathrm{C} & -1.63980 & -2.45930 & 1.55921 \\ \mathrm{C} & -2.66281 & -1.48294 & 1.27119 \\ \mathrm{C} & -2.56111 & -0.20685 & 1.78628 \\ \mathrm{C} & -1.57420 & 0.05610 & 2.79730 \\ \mathrm{C} & -0.67283 & -0.93123 & 3.21246 \\ \mathrm{C} & -0.68614 & -2.21422 & 2.55569\end{array}$




$\begin{array}{lrrr}\text { C } & 0.69177 & -2.63945 & 2.38525 \\ \mathrm{C} & 1.07429 & -3.28552 & 1.21071 \\ \mathrm{C} & 0.09394 & -3.52738 & 0.16450 \\ \mathrm{C} & 0.74595 & -3.32829 & -1.10739 \\ \mathrm{C} & 0.05066 & -2.70062 & -2.14619 \\ \mathrm{C} & -1.32245 & -2.26823 & -1.96488 \\ \mathrm{C} & -1.49565 & -1.00441 & -2.72768 \\ \mathrm{C} & -0.22608 & -0.70160 & -3.33952 \\ \mathrm{C} & 0.72686 & -1.74099 & -2.99269 \\ \mathrm{C} & 2.06730 & -1.42853 & -2.77545 \\ \mathrm{C} & 2.78299 & -2.05401 & -1.67575 \\ \mathrm{C} & 2.13510 & -2.98445 & -0.86169 \\ \mathrm{C} & 2.33724 & -2.95278 & 0.57886 \\ \mathrm{C} & 3.17130 & -1.98526 & 1.14479 \\ \mathrm{C} & 2.77143 & -1.30722 & 2.36639 \\ \mathrm{C} & 1.55281 & -1.62640 & 2.97052 \\ \mathrm{C} & 0.70379 & -0.56842 & 3.48939 \\ \mathrm{C} & 1.10909 & 0.76514 & 3.39402 \\ \mathrm{C} & 0.15279 & 1.79589 & 3.01955 \\ \mathrm{C} & -1.16434 & 1.44061 & 2.70932 \\ \mathrm{C} & -1.89318 & 2.06050 & 1.63600 \\ \mathrm{C} & -1.24084 & 2.99579 & 0.85125 \\ \mathrm{C} & 0.14055 & 3.34364 & 1.12244 \\ \mathrm{C} & 0.82807 & 2.76527 & 2.19504 \\ \mathrm{C} & 2.20511 & 2.33523 & 2.03532 \\ \mathrm{C} & 2.37802 & 1.09522 & 2.78007 \\ \mathrm{C} & 3.19266 & 0.07909 & 2.26996 \\ \mathrm{C} & 3.85755 & 0.25875 & 0.99150 \\ \mathrm{C} & 3.84186 & -1.01607 & 0.29515 \\ \mathrm{C} & 3.65367 & -1.05243 & -1.08835 \\ \mathrm{C} & 3.48563 & 0.18566 & -1.83351 \\ \mathrm{C} & 2.51369 & -0.04769 & -2.88092 \\ \mathrm{C} & 1.60859 & 0.95483 & -3.23773 \\ \mathrm{C} & 0.22102 & 0.62027 & -3.48790 \\ \mathrm{C} & -0.59496 & 1.69661 & -2.98538 \\ \mathrm{C} & 0.26723 & 2.69098 & -2.39414 \\ \mathrm{C} & 1.63735 & 2.23752 & -2.54762 \\ \mathrm{C} & 2.56153 & 2.45411 & -1.52427 \\ \mathrm{C} & 3.50603 & 1.41373 & -1.16294 \\ \mathrm{C} & 3.68988 & 1.44835 & 0.27714 \\ \mathrm{C} & 2.85091 & 2.50662 & 0.81083 \\ \mathrm{C} & 2.14787 & 3.12574 & -0.30078 \\ \mathrm{C} & 0.82283 & 3.53641 & -0.14577 \\ \mathrm{C} & -0.13723 & 3.32064 & -1.21216 \\ \mathrm{C} & -1.41822 & 3.00690 & -0.61552 \\ \mathrm{C} & -2.25840 & 2.09146 & -1.21086 \\ \mathrm{C} & -1.81680 & 1.39478 & -2.37739 \\ \mathrm{C} & -2.33347 & 0.03989 & -2.29676 \\ \mathrm{C} & -3.36506 & -0.05529 & -1.28868 \\ & & & \end{array}$




$\begin{array}{llll}\mathrm{C} & -3.28388 & 1.25501 & -0.47334 \\ \mathrm{C} & -3.00678 & 1.12411 & 1.14955 \\ \mathrm{C} & -4.48301 & 1.59446 & 1.38573 \\ \mathrm{C} & -4.64299 & 1.94772 & -0.10676 \\ \mathrm{H} & -4.60758 & 3.02285 & -0.30394 \\ \mathrm{H} & -5.51302 & 1.52370 & -0.61350 \\ \mathrm{H} & -4.60179 & 2.41308 & 2.10085 \\ \mathrm{H} & -5.11736 & 0.76142 & 1.70238\end{array}$

(9) $\mathrm{C}_{2} \mathrm{H}_{4} \_\mathrm{C}_{59} \mathrm{Ni}$-PH_C4-C5

$\begin{array}{rrrr}\mathrm{Ni} & 3.74698 & -1.24648 & 0.17602 \\ \mathrm{C} & 2.36622 & -1.88388 & 1.23654 \\ \mathrm{C} & 1.55731 & -2.84482 & 0.51597 \\ \mathrm{C} & 1.68308 & -2.68001 & -0.92236 \\ \mathrm{C} & 2.70659 & -1.70478 & -1.28970 \\ \mathrm{C} & 2.26453 & -0.64792 & -2.14105 \\ \mathrm{C} & 1.09997 & -0.88106 & -3.02185 \\ \mathrm{C} & 0.28716 & -1.98776 & -2.82243 \\ \mathrm{C} & 0.57328 & -2.91554 & -1.73461 \\ \mathrm{C} & -0.69069 & -3.33866 & -1.17120 \\ \mathrm{C} & -0.81475 & -3.52464 & 0.20471 \\ \mathrm{C} & 0.32817 & -3.25934 & 1.06087 \\ \mathrm{C} & -0.15688 & -2.65774 & 2.27855 \\ \mathrm{C} & 0.58920 & -1.64653 & 2.89533 \\ \mathrm{C} & 1.85692 & -1.22879 & 2.34106 \\ \mathrm{C} & 1.98031 & 0.23974 & 2.56991 \\ \mathrm{C} & 0.77300 & 0.66774 & 3.23221 \\ \mathrm{C} & -0.07902 & -0.48841 & 3.45172 \\ \mathrm{C} & -1.46518 & -0.37295 & 3.36839 \\ \mathrm{C} & -2.24128 & -1.41515 & 2.71448 \\ \mathrm{C} & -1.59884 & -2.53750 & 2.18657 \\ \mathrm{C} & -2.00567 & -3.06639 & 0.89307 \\ \mathrm{C} & -3.03497 & -2.44738 & 0.18021 \\ \mathrm{C} & -2.90808 & -2.25802 & -1.25674 \\ \mathrm{C} & -1.75923 & -2.69459 & -1.91829 \\ \mathrm{C} & -1.15183 & -1.86267 & -2.94279 \\ \mathrm{C} & -1.71868 & -0.62599 & -3.26718 \\ \mathrm{C} & -0.86382 & 0.52585 & -3.48706 \\ \mathrm{C} & 0.51955 & 0.39021 & -3.34442 \\ \mathrm{C} & 1.30505 & 1.39783 & -2.65287 \\ \mathrm{C} & 0.66254 & 2.54186 & -2.17311 \\ \mathrm{C} & -0.77143 & 2.67740 & -2.29842 \\ \mathrm{C} & -1.53003 & 1.69256 & -2.94639 \\ \mathrm{C} & -2.79493 & 1.26425 & -2.37904 \\ \mathrm{C} & -2.91481 & -0.17434 & -2.58054 \\ & -3.98549 & -0.36856 & -0.36353\end{array}$




$\begin{array}{lrrr}\mathrm{C} & -3.69655 & -1.27786 & 0.72929 \\ \mathrm{C} & -3.31360 & -0.77502 & 1.97832 \\ \mathrm{C} & -3.20169 & 0.66012 & 2.17728 \\ \mathrm{C} & -2.06743 & 0.91116 & 3.04499 \\ \mathrm{C} & -1.25635 & 2.02784 & 2.83658 \\ \mathrm{C} & 0.18793 & 1.90870 & 2.93938 \\ \mathrm{C} & 0.77865 & 2.73961 & 1.92513 \\ \mathrm{C} & -0.28237 & 3.37596 & 1.18132 \\ \mathrm{C} & -1.54873 & 2.94110 & 1.74206 \\ \mathrm{C} & -2.63289 & 2.69388 & 0.89866 \\ \mathrm{C} & -3.47788 & 1.53583 & 1.12096 \\ \mathrm{C} & -3.87410 & 1.01015 & -0.17324 \\ \mathrm{C} & -3.27114 & 1.84313 & -1.20211 \\ \mathrm{C} & -2.49738 & 2.87914 & -0.53965 \\ \mathrm{C} & -1.27554 & 3.28579 & -1.07643 \\ \mathrm{C} & -0.14654 & 3.53342 & -0.19833 \\ \mathrm{C} & 1.05358 & 3.07707 & -0.86920 \\ \mathrm{C} & 2.08324 & 2.49214 & -0.15691 \\ \mathrm{C} & 1.93458 & 2.29320 & 1.25591 \\ \mathrm{C} & 2.60490 & 1.07391 & 1.64972 \\ \mathrm{C} & 3.45724 & 0.60562 & 0.52795 \\ \mathrm{C} & 3.03075 & 1.44334 & -0.75027 \\ \mathrm{C} & 2.38194 & 0.74755 & -1.93378 \\ \mathrm{C} & 4.95719 & 0.96985 & 0.32105 \\ \mathrm{C} & 4.55536 & 1.88827 & -0.85624 \\ \mathrm{H} & 5.01919 & 1.64537 & -1.81464 \\ \mathrm{H} & 4.68986 & 2.95108 & -0.64453 \\ \mathrm{H} & 5.58088 & 0.11402 & -0.01643 \\ \mathrm{H} & 5.46257 & 1.41700 & 1.18101\end{array}$

(10) $\mathrm{C}_{2} \mathrm{H}_{4} \_\mathrm{C}_{59} \mathrm{Ni}$ _HH_C6-C7

$\begin{array}{cccc}\mathrm{Ni} & -3.70737 & -1.24594 & -0.33750 \\ \mathrm{C} & -3.45652 & 0.32915 & 0.71227 \\ \mathrm{C} & -3.12507 & 1.60310 & -0.21606 \\ \mathrm{C} & -2.59532 & 1.04213 & -1.52864 \\ \mathrm{C} & -2.69306 & -0.37454 & -1.62549 \\ \mathrm{C} & -1.78118 & -1.11572 & -2.38925 \\ \mathrm{C} & -0.81416 & -0.41718 & -3.18739 \\ \mathrm{C} & -0.73622 & 0.98245 & -3.13271 \\ \mathrm{C} & -1.62583 & 1.71174 & -2.28283 \\ \mathrm{C} & -0.87521 & 2.84204 & -1.72173 \\ \mathrm{C} & -1.10318 & 3.21851 & -0.40685 \\ \mathrm{C} & -2.11979 & 2.54129 & 0.38459 \\ \mathrm{C} & -1.60456 & 2.35807 & 1.66891 \\ \mathrm{C} & -1.80719 & 1.12431 & 2.38249 \\ \mathrm{C} & -2.56384 & 0.06586 & 1.86067 \\ \mathrm{C} & -1.92053 & -1.19170 & 2.25070\end{array}$




\begin{tabular}{|c|c|c|c|}
\hline $\mathrm{C}$ & -0.79727 & -0.85321 & 3.11594 \\
\hline $\mathrm{C}$ & -0.72049 & 0.58162 & 3.20188 \\
\hline $\mathrm{C}$ & 0.50589 & 1.22200 & 3.28163 \\
\hline $\mathrm{C}$ & 0.73520 & 2.45867 & 2.52290 \\
\hline $\mathrm{C}$ & -0.27953 & 3.00683 & 1.75360 \\
\hline $\mathrm{C}$ & 0.02280 & 3.54415 & 0.45057 \\
\hline $\mathrm{C}$ & 1.32955 & 3.49847 & -0.04563 \\
\hline $\mathrm{C}$ & 1.56345 & 3.11200 & -1.42584 \\
\hline $\mathrm{C}$ & 0.47834 & 2.78297 & -2.24258 \\
\hline $\mathrm{C}$ & 0.56192 & 1.63680 & -3.12949 \\
\hline $\mathrm{C}$ & 1.72695 & 0.87284 & -3.17992 \\
\hline C & 1.64618 & -0.57967 & -3.25305 \\
\hline $\mathrm{C}$ & 0.40231 & -1.20649 & -3.25148 \\
\hline $\mathrm{C}$ & 0.18419 & -2.41935 & -2.48727 \\
\hline $\mathrm{C}$ & 1.24467 & -2.97711 & -1.76111 \\
\hline $\mathrm{C}$ & 2.53163 & -2.30716 & -1.73016 \\
\hline $\mathrm{C}$ & 2.73244 & -1.13255 & -2.46467 \\
\hline $\mathrm{C}$ & 3.47093 & -0.02789 & -1.88760 \\
\hline $\mathrm{C}$ & 2.85518 & 1.21372 & -2.33464 \\
\hline $\mathrm{C}$ & 2.77431 & 2.30792 & -1.46966 \\
\hline $\mathrm{C}$ & 3.29172 & 2.19970 & -0.11711 \\
\hline $\mathrm{C}$ & 2.38908 & 2.93003 & 0.76187 \\
\hline $\mathrm{C}$ & 2.09673 & 2.42599 & 2.03238 \\
\hline $\mathrm{C}$ & 2.70823 & 1.18298 & 2.48453 \\
\hline $\mathrm{C}$ & 1.72961 & 0.45319 & 3.26873 \\
\hline $\mathrm{C}$ & 1.66505 & -0.94402 & 3.19203 \\
\hline $\mathrm{C}$ & 0.38070 & -1.60440 & 3.13444 \\
\hline $\mathrm{C}$ & 0.50375 & -2.74658 & 2.25122 \\
\hline $\mathrm{C}$ & 1.86643 & -2.78732 & 1.74343 \\
\hline $\mathrm{C}$ & 2.58520 & -1.66549 & 2.32629 \\
\hline $\mathrm{C}$ & 3.51538 & -0.95927 & 1.56231 \\
\hline $\mathrm{C}$ & 3.58122 & 0.49074 & 1.64502 \\
\hline $\mathrm{C}$ & 3.87047 & 1.00568 & 0.31532 \\
\hline $\mathrm{C}$ & 3.97492 & -0.12784 & -0.58786 \\
\hline $\mathrm{C}$ & 3.76054 & -1.34076 & 0.17933 \\
\hline $\mathrm{C}$ & 3.06271 & -2.41274 & -0.38254 \\
\hline $\mathrm{C}$ & 2.10040 & -3.15436 & 0.41885 \\
\hline C & 0.98442 & -3.50656 & -0.44203 \\
\hline $\mathrm{C}$ & -0.31767 & -3.47867 & 0.05779 \\
\hline $\mathrm{C}$ & -0.55957 & -3.07443 & 1.42126 \\
\hline $\mathrm{C}$ & -1.83058 & -2.33261 & 1.43188 \\
\hline $\mathrm{C}$ & -2.51723 & -2.54735 & 0.16789 \\
\hline $\mathrm{C}$ & -1.44056 & -2.98026 & -0.72606 \\
\hline $\mathrm{C}$ & -1.16584 & -2.40033 & -1.96475 \\
\hline $\mathrm{C}$ & -4.65316 & 1.97183 & -0.11712 \\
\hline $\mathrm{C}$ & -4.94073 & 0.76854 & 0.82415 \\
\hline $\mathrm{H}$ & -5.69559 & 0.04775 & 0.46402 \\
\hline $\mathrm{H}$ & -5.23042 & 1.03677 & 1.84613 \\
\hline $\mathrm{H}$ & -5.16639 & 1.93582 & -1.08207 \\
\hline
\end{tabular}


$\begin{array}{llll}\mathrm{H} & -4.83060 & 2.94726 & 0.34185\end{array}$

(11) $\mathrm{C}_{2} \mathrm{H}_{4} \_\mathrm{C}_{59} \mathrm{Ni} \_\mathrm{HH} \_\mathrm{C} 7-\mathrm{C} 8$

\begin{tabular}{|c|c|c|c|}
\hline $\mathrm{Ni}$ & -3.55833 & -1.50646 & 0.05561 \\
\hline $\mathrm{C}$ & -2.87975 & -0.24170 & 1.25790 \\
\hline $\mathrm{C}$ & -2.81515 & 1.11095 & 0.76685 \\
\hline $\mathrm{C}$ & -2.84164 & 1.10846 & -0.68849 \\
\hline $\mathrm{C}$ & -2.92367 & -0.24596 & -1.16950 \\
\hline $\mathrm{C}$ & -2.10287 & -0.68336 & -2.19498 \\
\hline $\mathrm{C}$ & -1.33797 & 0.29227 & -2.94341 \\
\hline $\mathrm{C}$ & -1.33380 & 1.63922 & -2.55969 \\
\hline $\mathrm{C}$ & -2.08383 & 2.05450 & -1.39856 \\
\hline $\mathrm{C}$ & -1.29117 & 3.04504 & -0.68615 \\
\hline $\mathrm{C}$ & -1.27084 & 3.04928 & 0.70725 \\
\hline $\mathrm{C}$ & -2.04266 & 2.06486 & 1.44851 \\
\hline $\mathrm{C}$ & -1.26093 & 1.65943 & 2.59273 \\
\hline $\mathrm{C}$ & -1.25279 & 0.31604 & 2.98661 \\
\hline $\mathrm{C}$ & -2.03676 & -0.66777 & 2.26879 \\
\hline C & -1.26928 & -1.94371 & 2.26609 \\
\hline $\mathrm{C}$ & -0.03318 & -1.69139 & 2.97058 \\
\hline $\mathrm{C}$ & -0.02026 & -0.31081 & 3.41967 \\
\hline $\mathrm{C}$ & 1.16760 & 0.41764 & 3.44052 \\
\hline $\mathrm{C}$ & 1.17029 & 1.80452 & 3.00167 \\
\hline $\mathrm{C}$ & -0.01878 & 2.41170 & 2.58848 \\
\hline $\mathrm{C}$ & -0.02215 & 3.26959 & 1.41557 \\
\hline $\mathrm{C}$ & 1.15858 & 3.48256 & 0.70212 \\
\hline $\mathrm{C}$ & 1.13821 & 3.47605 & -0.75227 \\
\hline $\mathrm{C}$ & -0.06212 & 3.25816 & -1.43087 \\
\hline $\mathrm{C}$ & -0.09143 & 2.38986 & -2.59577 \\
\hline $\mathrm{C}$ & 1.08540 & 1.77872 & -3.03646 \\
\hline $\mathrm{C}$ & 1.07027 & 0.38797 & -3.46359 \\
\hline $\mathrm{C}$ & -0.11722 & -0.33910 & -3.40293 \\
\hline $\mathrm{C}$ & -0.11789 & -1.71468 & -2.93933 \\
\hline $\mathrm{C}$ & 1.09449 & -2.32017 & -2.57863 \\
\hline $\mathrm{C}$ & 2.32523 & -1.55743 & -2.61199 \\
\hline $\mathrm{C}$ & 2.31801 & -0.22914 & -3.04713 \\
\hline $\mathrm{C}$ & 3.09457 & 0.77054 & -2.34322 \\
\hline $\mathrm{C}$ & 2.33268 & 2.01224 & -2.33771 \\
\hline $\mathrm{C}$ & 2.35956 & 2.84230 & -1.21406 \\
\hline $\mathrm{C}$ & 3.13866 & 2.46127 & -0.04869 \\
\hline $\mathrm{C}$ & 2.39276 & 2.85257 & 1.13515 \\
\hline $\mathrm{C}$ & 2.39789 & 2.03248 & 2.26613 \\
\hline $\mathrm{C}$ & 3.15969 & 0.79048 & 2.26071 \\
\hline $\mathrm{C}$ & 2.40325 & -0.20333 & 2.99423 \\
\hline $\mathrm{C}$ & 2.39930 & -1.53580 & 2.57160 \\
\hline $\mathrm{C}$ & 1.16856 & -2.29950 & 2.58009 \\
\hline $\mathrm{C}$ & 1.17236 & -3.16913 & 1.42695 \\
\hline
\end{tabular}




$\begin{array}{lrrr}\mathrm{C} & 2.40086 & -2.93747 & 0.68273 \\ \mathrm{C} & 3.16212 & -1.92526 & 1.39455 \\ \mathrm{C} & 3.88215 & -0.96384 & 0.68294 \\ \mathrm{C} & 3.88382 & 0.42010 & 1.12469 \\ \mathrm{C} & 3.86673 & 1.26907 & -0.05391 \\ \mathrm{C} & 3.85078 & 0.40997 & -1.22541 \\ \mathrm{C} & 3.86157 & -0.97006 & -0.77165 \\ \mathrm{C} & 3.12151 & -1.93735 & -1.45416 \\ \mathrm{C} & 2.38073 & -2.94309 & -0.71229 \\ \mathrm{C} & 1.13105 & -3.17964 & -1.41870 \\ \mathrm{C} & -0.04213 & -3.40404 & -0.70194 \\ \mathrm{C} & -0.02078 & -3.39938 & 0.74557 \\ \mathrm{C} & -1.28698 & -2.83168 & 1.18637 \\ \mathrm{C} & -2.21935 & -2.77655 & 0.05369 \\ \mathrm{C} & -1.32077 & -2.83746 & -1.10919 \\ \mathrm{C} & -1.33423 & -1.95968 & -2.19765 \\ \mathrm{C} & -6.88075 & 0.93929 & -0.60848 \\ \mathrm{C} & -6.63765 & 1.58962 & 0.52784 \\ \mathrm{H} & -7.14341 & 1.32636 & 1.45062 \\ \mathrm{H} & -5.94688 & 2.41866 & 0.57626 \\ \mathrm{H} & -7.58330 & 0.11437 & -0.64649 \\ \mathrm{H} & -6.41270 & 1.19178 & -1.55598\end{array}$

\section{Ethane}

\section{(1) $\mathrm{C}_{2} \mathrm{H}_{6} \mathrm{C}_{59} \mathrm{Ni} \_\mathrm{TM}$}

$\begin{array}{rrrr}\mathrm{Ni} & 3.74006 & -0.84201 & 0.13647 \\ \mathrm{C} & 2.92247 & 0.43635 & -0.97306 \\ \mathrm{C} & 2.59700 & 1.69528 & -0.35413 \\ \mathrm{C} & 2.51248 & 1.51797 & 1.08783 \\ \mathrm{C} & 2.78230 & 0.14200 & 1.42128 \\ \mathrm{C} & 1.98008 & -0.53200 & 2.32717 \\ \mathrm{C} & 1.01132 & 0.21666 & 3.10138 \\ \mathrm{C} & 0.80916 & 1.58119 & 2.86285 \\ \mathrm{C} & 1.55925 & 2.24291 & 1.82301 \\ \mathrm{C} & 0.66705 & 3.17411 & 1.15132 \\ \mathrm{C} & 0.74604 & 3.34199 & -0.22977 \\ \mathrm{C} & 1.72167 & 2.58623 & -0.99854 \\ \mathrm{C} & 1.10278 & 2.19985 & -2.24342 \\ \mathrm{C} & 1.34848 & 0.92688 & -2.77238 \\ \mathrm{C} & 2.23508 & 0.00453 & -2.09451 \\ \mathrm{C} & 1.69355 & -1.36916 & -2.29009 \\ \mathrm{C} & 0.48551 & -1.23589 & -3.07331 \\ \mathrm{C} & 0.27303 & 0.16700 & -3.37721 \\ \mathrm{C} & -1.01597 & 0.69512 & -3.42940 \\ \mathrm{C} & -1.28286 & 2.00302 & -2.85029 \\ \mathrm{C} & -0.24546 & 2.73867 & -2.27142\end{array}$




\begin{tabular}{|c|c|c|c|}
\hline C & -0.46904 & 3.44286 & -1.02016 \\
\hline $\mathrm{C}$ & -1.71682 & 3.37956 & -0.39712 \\
\hline C & -1.79908 & 3.20516 & 1.04433 \\
\hline C & -0.63048 & 3.10165 & 1.80121 \\
\hline $\mathrm{C}$ & -0.54034 & 2.11715 & 2.86597 \\
\hline $\mathrm{C}$ & -1.62654 & 1.28035 & 3.13343 \\
\hline $\mathrm{C}$ & -1.40925 & -0.13062 & 3.41293 \\
\hline $\mathrm{C}$ & -0.11569 & -0.64921 & 3.38499 \\
\hline C & 0.14815 & -1.94392 & 2.78495 \\
\hline $\mathrm{C}$ & -0.91642 & -2.68621 & 2.25395 \\
\hline $\mathrm{C}$ & -2.25714 & -2.13793 & 2.25585 \\
\hline $\mathrm{C}$ & -2.50273 & -0.88461 & 2.82438 \\
\hline $\mathrm{C}$ & -3.38413 & 0.05530 & 2.16256 \\
\hline $\mathrm{C}$ & -2.84281 & 1.39428 & 2.35445 \\
\hline $\mathrm{C}$ & -2.92766 & 2.33631 & 1.32622 \\
\hline $\mathrm{C}$ & -3.54602 & 1.97544 & 0.06203 \\
\hline $\mathrm{C}$ & -2.79414 & 2.61756 & -1.00249 \\
\hline $\mathrm{C}$ & -2.58090 & 1.94544 & -2.20890 \\
\hline $\mathrm{C}$ & -3.12123 & 0.60611 & -2.40093 \\
\hline $\mathrm{C}$ & -2.15823 & -0.16214 & -3.16301 \\
\hline $\mathrm{C}$ & -1.96080 & -1.51749 & -2.88272 \\
\hline $\mathrm{C}$ & -0.62163 & -2.06862 & -2.85829 \\
\hline $\mathrm{C}$ & -0.56213 & -3.05831 & -1.80800 \\
\hline $\mathrm{C}$ & -1.86271 & -3.11481 & -1.15849 \\
\hline $\mathrm{C}$ & -2.72970 & -2.15928 & -1.82664 \\
\hline $\mathrm{C}$ & -3.65011 & -1.41439 & -1.08645 \\
\hline $\mathrm{C}$ & -3.85191 & -0.00587 & -1.37967 \\
\hline $\mathrm{C}$ & -4.06233 & 0.69055 & -0.12233 \\
\hline $\mathrm{C}$ & -3.98713 & -0.28701 & 0.94978 \\
\hline $\mathrm{C}$ & -3.73362 & -1.58850 & 0.35545 \\
\hline $\mathrm{C}$ & -2.89317 & -2.50020 & 0.99750 \\
\hline $\mathrm{C}$ & -1.94268 & -3.28182 & 0.22465 \\
\hline $\mathrm{C}$ & -0.72485 & -3.39891 & 1.01206 \\
\hline $\mathrm{C}$ & 0.51784 & -3.34648 & 0.38501 \\
\hline $\mathrm{C}$ & 0.60079 & -3.17248 & -1.04955 \\
\hline $\mathrm{C}$ & 1.78158 & -2.36140 & -1.30975 \\
\hline $\mathrm{C}$ & 2.60452 & -2.28277 & -0.09395 \\
\hline $\mathrm{C}$ & 1.65022 & -2.63726 & 0.96318 \\
\hline $\mathrm{C}$ & 1.43898 & -1.90507 & 2.13466 \\
\hline $\mathrm{C}$ & 6.83411 & 1.35020 & -0.33246 \\
\hline $\mathrm{C}$ & 6.38055 & -0.05208 & 0.07979 \\
\hline $\mathrm{H}$ & 5.64076 & 0.01025 & 0.89874 \\
\hline $\mathrm{H}$ & 7.20397 & -0.66755 & 0.45804 \\
\hline $\mathrm{H}$ & 5.94965 & -0.59476 & -0.77566 \\
\hline $\mathrm{H}$ & 7.27592 & 1.88584 & 0.51444 \\
\hline $\mathrm{H}$ & 5.99208 & 1.94369 & -0.70397 \\
\hline $\mathrm{H}$ & 7.58629 & 1.29935 & -1.12690 \\
\hline
\end{tabular}


(2) $\mathrm{C}_{2} \mathrm{H}_{6} \mathrm{C}_{59} \mathrm{Ni} \_\mathrm{TC} 5$

$\begin{array}{crrr}\mathrm{Ni} & 2.99861 & -2.47398 & -0.00086 \\ \mathrm{C} & 1.68355 & -2.49544 & 1.32851 \\ \mathrm{C} & 0.47994 & -3.22227 & 1.01360 \\ \mathrm{C} & 0.36668 & -3.38023 & -0.43010 \\ \mathrm{C} & 1.49502 & -2.75878 & -1.07543 \\ \mathrm{C} & 1.31468 & -1.93810 & -2.17721 \\ \mathrm{C} & 0.01718 & -1.90119 & -2.81762 \\ \mathrm{C} & -1.06955 & -2.59135 & -2.26620 \\ \mathrm{C} & -0.89924 & -3.32807 & -1.03728 \\ \mathrm{C} & -2.09426 & -3.13477 & -0.23194 \\ \mathrm{C} & -1.98577 & -2.98363 & 1.14867 \\ \mathrm{C} & -0.67802 & -3.01955 & 1.78278 \\ \mathrm{C} & -0.66941 & -2.03319 & 2.83616 \\ \mathrm{C} & 0.47723 & -1.25871 & 3.05172 \\ \mathrm{C} & 1.66080 & -1.45397 & 2.24217 \\ \mathrm{C} & 2.29830 & -0.11945 & 2.04516 \\ \mathrm{C} & 1.47044 & 0.84515 & 2.73433 \\ \mathrm{C} & 0.35813 & 0.15266 & 3.35828 \\ \mathrm{C} & -0.89848 & 0.75022 & 3.43463 \\ \mathrm{C} & -2.09075 & -0.04341 & 3.17815 \\ \mathrm{C} & -1.97750 & -1.40553 & 2.88754 \\ \mathrm{C} & -2.79270 & -1.99099 & 1.83683 \\ \mathrm{C} & -3.68337 & -1.19209 & 1.11710 \\ \mathrm{C} & -3.79666 & -1.34979 & -0.32454 \\ \mathrm{C} & -3.01463 & -2.29988 & -0.98442 \\ \mathrm{C} & -2.38051 & -1.96750 & -2.24901 \\ \mathrm{C} & -2.55984 & -0.69778 & -2.80402 \\ \mathrm{C} & -1.43470 & 0.00211 & -3.40534 \\ \mathrm{C} & -0.17154 & -0.58641 & -3.39744 \\ \mathrm{C} & 1.01192 & 0.20483 & -3.11573 \\ \mathrm{C} & 0.87847 & 1.58038 & -2.87817 \\ \mathrm{C} & -0.43522 & 2.18983 & -2.85546 \\ \mathrm{C} & -1.57216 & 1.41889 & -3.11419 \\ \mathrm{C} & -2.76710 & 1.59351 & -2.31518 \\ \mathrm{C} & -3.37878 & 0.28493 & -2.12433 \\ \mathrm{C} & -3.98359 & -0.03224 & -0.90529 \\ \mathrm{C} & -3.99114 & 0.93987 & 0.17418 \\ \mathrm{C} & -3.80011 & 0.22232 & 1.42263 \\ \mathrm{C} & -3.02108 & 0.78393 & 2.43732 \\ \mathrm{C} & -2.40967 & 2.09278 & 2.24675 \\ \mathrm{C} & -1.10302 & 2.07387 & 2.87103 \\ \mathrm{C} & -0.03259 & 2.75195 & 2.28043 \\ \mathrm{C} & 1.27914 & 2.13972 & 2.23073 \\ \mathrm{C} & 1.89726 & 2.50402 & 0.97732 \\ \mathrm{C} & 0.96190 & 3.32934 & 0.22875 \\ \mathrm{C} & -0.23428 & 3.48431 & 1.03885 \\ \mathrm{C} & -1.49238 & 3.49227 & 0.43321 \\ & & & \end{array}$




$\begin{array}{lrrr}\mathrm{C} & -2.60223 & 2.78576 & 1.04913 \\ \mathrm{C} & -3.40241 & 2.19381 & -0.00895 \\ \mathrm{C} & -2.78452 & 2.53078 & -1.27952 \\ \mathrm{C} & -1.60526 & 3.33446 & -1.00834 \\ \mathrm{C} & -0.45549 & 3.17525 & -1.78437 \\ \mathrm{C} & 0.85338 & 3.17769 & -1.15398 \\ \mathrm{C} & 1.67557 & 2.19487 & -1.84265 \\ \mathrm{C} & 2.57218 & 1.40944 & -1.12231 \\ \mathrm{C} & 2.68548 & 1.56703 & 0.31312 \\ \mathrm{C} & 2.95201 & 0.24580 & 0.86447 \\ \mathrm{C} & 3.32337 & -0.67103 & -0.22254 \\ \mathrm{C} & 2.77293 & -0.00327 & -1.41066 \\ \mathrm{C} & 1.95224 & -0.60280 & -2.37091 \\ \mathrm{C} & 6.84386 & 2.05187 & 0.59047 \\ \mathrm{C} & 7.04754 & 0.98460 & -0.48834 \\ \mathrm{H} & 6.92140 & 1.62169 & 1.59591 \\ \mathrm{H} & 5.85511 & 2.51810 & 0.50731 \\ \mathrm{H} & 7.59332 & 2.84823 & 0.51287 \\ \mathrm{H} & 6.29010 & 0.19536 & -0.41456 \\ \mathrm{H} & 8.03209 & 0.51041 & -0.39917 \\ \mathrm{H} & 6.97938 & 1.41653 & -1.49378\end{array}$

(3) $\mathrm{C}_{2} \mathrm{H}_{6}{ }_{-} \mathrm{C}_{59} \mathrm{Ni}$-TC1

$\begin{array}{crrr}\mathrm{Ni} & -3.74005 & -0.84218 & 0.13644 \\ \mathrm{C} & -2.78226 & 0.14174 & 1.42131 \\ \mathrm{C} & -2.51251 & 1.51776 & 1.08795 \\ \mathrm{C} & -2.59706 & 1.69517 & -0.35400 \\ \mathrm{C} & -2.92248 & 0.43626 & -0.97299 \\ \mathrm{C} & -2.23510 & 0.00453 & -2.09447 \\ \mathrm{C} & -1.34855 & 0.92694 & -2.77230 \\ \mathrm{C} & -1.10289 & 2.19990 & -2.24328 \\ \mathrm{C} & -1.72179 & 2.58619 & -0.99838 \\ \mathrm{C} & -0.74618 & 3.34195 & -0.22958 \\ \mathrm{C} & -0.66716 & 3.17400 & 1.15150 \\ \mathrm{C} & -1.55930 & 2.24271 & 1.82316 \\ \mathrm{C} & -0.80915 & 1.58097 & 2.86294 \\ \mathrm{C} & -1.01126 & 0.21644 & 3.10143 \\ \mathrm{C} & -1.97999 & -0.53222 & 2.32720 \\ \mathrm{C} & -1.43883 & -1.90526 & 2.13463 \\ \mathrm{C} & -0.14800 & -1.94410 & 2.78489 \\ \mathrm{C} & 0.11581 & -0.64940 & 3.38497 \\ \mathrm{C} & 1.40934 & -0.13076 & 3.41291 \\ \mathrm{C} & 1.62656 & 1.28024 & 3.13347 \\ \mathrm{C} & 0.54033 & 2.11701 & 2.86608 \\ \mathrm{C} & 0.63039 & 3.10156 & 1.80136 \\ \mathrm{C} & 1.79897 & 3.20516 & 1.04447 \\ \mathrm{C} & 1.71668 & 3.37963 & -0.39697\end{array}$




\begin{tabular}{|c|c|c|c|}
\hline $\mathrm{C}$ & 0.46889 & 3.44291 & -1.01999 \\
\hline $\mathrm{C}$ & 0.24532 & 2.73877 & -2.27127 \\
\hline $\mathrm{C}$ & 1.28274 & 2.00321 & -2.85021 \\
\hline $\mathrm{C}$ & 1.01589 & 0.69532 & -3.42938 \\
\hline $\mathrm{C}$ & -0.27308 & 0.16714 & -3.37720 \\
\hline $\mathrm{C}$ & -0.48550 & -1.23577 & -3.07336 \\
\hline $\mathrm{C}$ & 0.62169 & -2.06847 & -2.85839 \\
\hline $\mathrm{C}$ & 1.96083 & -1.51727 & -2.88281 \\
\hline $\mathrm{C}$ & 2.15821 & -0.16190 & -3.16305 \\
\hline $\mathrm{C}$ & 3.12119 & 0.60635 & -2.40095 \\
\hline $\mathrm{C}$ & 2.58079 & 1.94565 & -2.20884 \\
\hline $\mathrm{C}$ & 2.79402 & 2.61771 & -1.00240 \\
\hline $\mathrm{C}$ & 3.54594 & 1.97556 & 0.06208 \\
\hline $\mathrm{C}$ & 2.92760 & 2.33635 & 1.32629 \\
\hline $\mathrm{C}$ & 2.84281 & 1.39427 & 2.35447 \\
\hline $\mathrm{C}$ & 3.38419 & 0.05532 & 2.16251 \\
\hline $\mathrm{C}$ & 2.50285 & -0.88466 & 2.82430 \\
\hline $\mathrm{C}$ & 2.25730 & -2.13796 & 2.25571 \\
\hline $\mathrm{C}$ & 0.91660 & -2.68630 & 2.25382 \\
\hline $\mathrm{C}$ & 0.72503 & -3.39894 & 1.01189 \\
\hline $\mathrm{C}$ & 1.94284 & -3.28176 & 0.22446 \\
\hline $\mathrm{C}$ & 2.89332 & -2.50014 & 0.99733 \\
\hline $\mathrm{C}$ & 3.73373 & -1.58838 & 0.35532 \\
\hline $\mathrm{C}$ & 3.98719 & -0.28691 & 0.94970 \\
\hline $\mathrm{C}$ & 4.06232 & 0.69071 & -0.12236 \\
\hline $\mathrm{C}$ & 3.85190 & -0.00565 & -1.37973 \\
\hline $\mathrm{C}$ & 3.65017 & -1.41419 & -1.08658 \\
\hline $\mathrm{C}$ & 2.72977 & -2.15908 & -1.82679 \\
\hline $\mathrm{C}$ & 1.86284 & -3.11469 & -1.15867 \\
\hline $\mathrm{C}$ & 0.56224 & -3.05820 & -1.80815 \\
\hline $\mathrm{C}$ & -0.60065 & -3.17245 & -1.04968 \\
\hline $\mathrm{C}$ & -0.51768 & -3.34652 & 0.38488 \\
\hline $\mathrm{C}$ & -1.65007 & -2.63738 & 0.96311 \\
\hline $\mathrm{C}$ & -2.60440 & -2.28287 & -0.09400 \\
\hline $\mathrm{C}$ & -1.78148 & -2.36139 & -1.30981 \\
\hline $\mathrm{C}$ & -1.69351 & -1.36913 & -2.29012 \\
\hline $\mathrm{C}$ & -6.38071 & -0.05199 & 0.07962 \\
\hline $\mathrm{C}$ & -6.83468 & 1.35026 & -0.33224 \\
\hline $\mathrm{H}$ & -5.94999 & -0.59446 & -0.77605 \\
\hline $\mathrm{H}$ & -7.20386 & -0.66768 & 0.45810 \\
\hline $\mathrm{H}$ & -5.64062 & 0.01037 & 0.89829 \\
\hline $\mathrm{H}$ & -7.58711 & 1.29940 & -1.12643 \\
\hline $\mathrm{H}$ & -5.99289 & 1.94398 & -0.70393 \\
\hline $\mathrm{H}$ & -7.27630 & 1.88569 & 0.51490 \\
\hline
\end{tabular}

(4) $\mathrm{C}_{2} \mathrm{H}_{6} \_\mathrm{C}_{59} \mathrm{Ni} \_\mathrm{HH} \_\mathrm{M}-\mathrm{C} 5$

Ni $\quad 3.80030 \quad 0.24296 \quad 0.08134$ 


\begin{tabular}{|c|c|c|c|}
\hline C & 2.74915 & -0.80725 & 1.23297 \\
\hline $\mathrm{C}$ & 2.28210 & -2.05994 & 0.69744 \\
\hline $\mathrm{C}$ & 2.30464 & -2.00282 & -0.75663 \\
\hline $\mathrm{C}$ & 2.78483 & -0.71167 & -1.18005 \\
\hline $\mathrm{C}$ & 2.13853 & -0.01696 & -2.18893 \\
\hline $\mathrm{C}$ & 1.11817 & -0.69120 & -2.96458 \\
\hline $\mathrm{C}$ & 0.71064 & -1.98808 & -2.63180 \\
\hline $\mathrm{C}$ & 1.29954 & -2.65151 & -1.49381 \\
\hline $\mathrm{C}$ & 0.24678 & -3.38669 & -0.81177 \\
\hline $\mathrm{C}$ & 0.22387 & -3.43971 & 0.58028 \\
\hline $\mathrm{C}$ & 1.25287 & -2.75995 & 1.35010 \\
\hline $\mathrm{C}$ & 0.62672 & -2.18214 & 2.51441 \\
\hline $\mathrm{C}$ & 1.02211 & -0.91312 & 2.95472 \\
\hline $\mathrm{C}$ & 2.06788 & -0.18582 & 2.26616 \\
\hline $\mathrm{C}$ & 1.71794 & 1.26140 & 2.31416 \\
\hline $\mathrm{C}$ & 0.46136 & 1.36607 & 3.02226 \\
\hline $\mathrm{C}$ & 0.03390 & 0.03818 & 3.42232 \\
\hline $\mathrm{C}$ & -1.31803 & -0.30093 & 3.42883 \\
\hline $\mathrm{C}$ & -1.73632 & -1.60603 & 2.94030 \\
\hline $\mathrm{C}$ & -0.78394 & -2.52617 & 2.49459 \\
\hline $\mathrm{C}$ & -1.03538 & -3.30180 & 1.29174 \\
\hline $\mathrm{C}$ & -2.22448 & -3.12387 & 0.58225 \\
\hline $\mathrm{C}$ & -2.20079 & -3.06969 & -0.87104 \\
\hline $\mathrm{C}$ & -0.98861 & -3.19484 & -1.55225 \\
\hline $\mathrm{C}$ & -0.69920 & -2.33197 & -2.68456 \\
\hline $\mathrm{C}$ & -1.63809 & -1.38113 & -3.09290 \\
\hline $\mathrm{C}$ & -1.20609 & -0.04360 & -3.46950 \\
\hline $\mathrm{C}$ & 0.14442 & 0.29273 & -3.39348 \\
\hline $\mathrm{C}$ & 0.55650 & 1.58709 & -2.88323 \\
\hline $\mathrm{C}$ & -0.41897 & 2.51323 & -2.48764 \\
\hline $\mathrm{C}$ & -1.82175 & 2.15612 & -2.53665 \\
\hline $\mathrm{C}$ & -2.21196 & 0.90301 & -3.01871 \\
\hline $\mathrm{C}$ & -3.25373 & 0.15585 & -2.34436 \\
\hline $\mathrm{C}$ & -2.89976 & -1.25664 & -2.39111 \\
\hline $\mathrm{C}$ & -3.17598 & -2.08239 & -1.29830 \\
\hline $\mathrm{C}$ & -3.80564 & -1.52810 & -0.11197 \\
\hline $\mathrm{C}$ & -3.21395 & -2.16958 & 1.04946 \\
\hline $\mathrm{C}$ & -2.97419 & -1.42773 & 2.20892 \\
\hline $\mathrm{C}$ & -3.32820 & -0.01527 & 2.25584 \\
\hline $\mathrm{C}$ & -2.30966 & 0.67806 & 3.01715 \\
\hline $\mathrm{C}$ & -1.90541 & 1.96249 & 2.64235 \\
\hline $\mathrm{C}$ & -0.50199 & 2.32040 & 2.66548 \\
\hline $\mathrm{C}$ & -0.24316 & 3.19426 & 1.54536 \\
\hline $\mathrm{C}$ & -1.48392 & 3.37068 & 0.80641 \\
\hline $\mathrm{C}$ & -2.51506 & 2.60719 & 1.48856 \\
\hline $\mathrm{C}$ & -3.48967 & 1.93316 & 0.75001 \\
\hline $\mathrm{C}$ & -3.90645 & 0.59750 & 1.14147 \\
\hline $\mathrm{C}$ & -4.14267 & -0.17313 & -0.06703 \\
\hline $\mathrm{C}$ & -3.86799 & 0.68488 & -1.20660 \\
\hline
\end{tabular}




$\begin{array}{lrrr}\mathrm{C} & -3.46601 & 1.98719 & -0.70368 \\ \mathrm{C} & -2.46886 & 2.71337 & -1.35794 \\ \mathrm{C} & -1.46163 & 3.42302 & -0.58770 \\ \mathrm{C} & -0.19749 & 3.30103 & -1.29706 \\ \mathrm{C} & 0.98811 & 3.13552 & -0.58490 \\ \mathrm{C} & 0.96520 & 3.08073 & 0.86152 \\ \mathrm{C} & 2.00169 & 2.14144 & 1.26598 \\ \mathrm{C} & 2.87171 & 1.84189 & 0.11947 \\ \mathrm{C} & 2.03800 & 2.22807 & -1.02537 \\ \mathrm{C} & 1.78911 & 1.42890 & -2.14445 \\ \mathrm{C} & 6.37635 & -0.69197 & 0.22769 \\ \mathrm{C} & 7.46748 & 0.22510 & -0.32972 \\ \mathrm{H} & 7.94346 & -0.22012 & -1.20988 \\ \mathrm{H} & 8.24662 & 0.40466 & 0.41875 \\ \mathrm{H} & 7.05631 & 1.19657 & -0.62482 \\ \mathrm{H} & 6.76549 & -1.66877 & 0.53617 \\ \mathrm{H} & 5.60961 & -0.90591 & -0.53875 \\ \mathrm{H} & 5.90260 & -0.25030 & 1.11822\end{array}$

(5) $\mathrm{C}_{2} \mathrm{H}_{6} \_\mathrm{C}_{59} \mathrm{Ni}$-PH_M-C1

$\begin{array}{rrrr}\mathrm{Ni} & 3.73911 & -0.85861 & 0.10400 \\ \mathrm{C} & 2.91089 & 0.38779 & -1.03366 \\ \mathrm{C} & 2.60041 & 1.66741 & -0.45071 \\ \mathrm{C} & 2.53601 & 1.53657 & 0.99720 \\ \mathrm{C} & 2.80477 & 0.17059 & 1.37018 \\ \mathrm{C} & 2.01230 & -0.47037 & 2.30800 \\ \mathrm{C} & 1.05797 & 0.30710 & 3.07182 \\ \mathrm{C} & 0.85865 & 1.66451 & 2.79345 \\ \mathrm{C} & 1.59651 & 2.28895 & 1.72226 \\ \mathrm{C} & 0.69919 & 3.20271 & 1.03400 \\ \mathrm{C} & 0.75921 & 3.32643 & -0.35270 \\ \mathrm{C} & 1.72044 & 2.54217 & -1.11080 \\ \mathrm{C} & 1.08225 & 2.11985 & -2.33413 \\ \mathrm{C} & 1.31468 & 0.82969 & -2.82617 \\ \mathrm{C} & 2.20649 & -0.07546 & -2.13178 \\ \mathrm{C} & 1.65579 & -1.45196 & -2.27635 \\ \mathrm{C} & 0.43725 & -1.33729 & -3.04619 \\ \mathrm{C} & 0.22709 & 0.05639 & -3.39131 \\ \mathrm{C} & -1.06017 & 0.58897 & -3.44158 \\ \mathrm{C} & -1.31275 & 1.91557 & -2.90020 \\ \mathrm{C} & -0.26389 & 2.66405 & -2.35994 \\ \mathrm{C} & -0.46642 & 3.40869 & -1.12874 \\ \mathrm{C} & -1.70541 & 3.37136 & -0.48624 \\ \mathrm{C} & -1.76793 & 3.24293 & 0.96098 \\ \mathrm{C} & -0.58916 & 3.15755 & 1.70420 \\ \mathrm{C} & -0.48832 & 2.20690 & 2.79837 \\ \mathrm{C} & -1.57434 & 1.38436 & 3.10734\end{array}$




\begin{tabular}{|c|c|c|c|}
\hline C & -1.35965 & -0.01812 & 3.42836 \\
\hline $\mathrm{C}$ & -0.06891 & -0.54376 & 3.39836 \\
\hline $\mathrm{C}$ & 0.18036 & -1.85770 & 2.83528 \\
\hline $\mathrm{C}$ & -0.89495 & -2.61131 & 2.34373 \\
\hline $\mathrm{C}$ & -2.23316 & -2.05678 & 2.34745 \\
\hline $\mathrm{C}$ & -2.46495 & -0.78495 & 2.87949 \\
\hline $\mathrm{C}$ & -3.35116 & 0.13811 & 2.20055 \\
\hline $\mathrm{C}$ & -2.80115 & 1.47974 & 2.34246 \\
\hline $\mathrm{C}$ & -2.89634 & 2.38910 & 1.28610 \\
\hline $\mathrm{C}$ & -3.53454 & 1.99176 & 0.04285 \\
\hline $\mathrm{C}$ & -2.79481 & 2.59614 & -1.05189 \\
\hline $\mathrm{C}$ & -2.60174 & 1.88505 & -2.23910 \\
\hline $\mathrm{C}$ & -3.15092 & 0.54307 & -2.38117 \\
\hline $\mathrm{C}$ & -2.20254 & -0.25368 & -3.13231 \\
\hline $\mathrm{C}$ & -2.00754 & -1.60057 & -2.81230 \\
\hline $\mathrm{C}$ & -0.67058 & -2.15712 & -2.78907 \\
\hline $\mathrm{C}$ & -0.60068 & -3.11352 & -1.70894 \\
\hline $\mathrm{C}$ & -1.89199 & -3.14274 & -1.03950 \\
\hline $\mathrm{C}$ & -2.76402 & -2.20450 & -1.72518 \\
\hline $\mathrm{C}$ & -3.67031 & -1.43214 & -0.99604 \\
\hline $\mathrm{C}$ & -3.87017 & -0.03264 & -1.33115 \\
\hline $\mathrm{C}$ & -4.05926 & 0.70421 & -0.09365 \\
\hline $\mathrm{C}$ & -3.97273 & -0.23927 & 1.00775 \\
\hline $\mathrm{C}$ & -3.73393 & -1.56014 & 0.45168 \\
\hline $\mathrm{C}$ & -2.88864 & -2.45545 & 1.11032 \\
\hline $\mathrm{C}$ & -1.95277 & -3.26556 & 0.34920 \\
\hline $\mathrm{C}$ & -0.72446 & -3.36400 & 1.12264 \\
\hline $\mathrm{C}$ & 0.50935 & -3.33778 & 0.47652 \\
\hline $\mathrm{C}$ & 0.57239 & -3.20950 & -0.96383 \\
\hline $\mathrm{C}$ & 1.75318 & -2.41329 & -1.26646 \\
\hline $\mathrm{C}$ & 2.59401 & -2.30023 & -0.06594 \\
\hline $\mathrm{C}$ & 1.65322 & -2.61632 & 1.01566 \\
\hline $\mathrm{C}$ & 1.46196 & -1.84618 & 2.16595 \\
\hline $\mathrm{C}$ & 6.37442 & -0.06146 & 0.02846 \\
\hline $\mathrm{C}$ & 6.78256 & 1.39169 & -0.22371 \\
\hline $\mathrm{H}$ & 7.45839 & 1.46450 & -1.08245 \\
\hline $\mathrm{H}$ & 7.29845 & 1.81175 & 0.64623 \\
\hline $\mathrm{H}$ & 5.90758 & 2.01688 & -0.43072 \\
\hline $\mathrm{H}$ & 7.23159 & -0.70989 & 0.24005 \\
\hline $\mathrm{H}$ & 5.87085 & -0.48691 & -0.85402 \\
\hline $\mathrm{H}$ & 5.71177 & -0.12922 & 0.90962 \\
\hline
\end{tabular}

(6) $\mathrm{C}_{2} \mathrm{H}_{6} \_\mathrm{C}_{59} \mathrm{Ni} \_\mathrm{HH} \_\mathrm{C} 1-\mathrm{C} 2$
$\mathrm{Ni} \quad 3.74007$
$-0.84189-0.13656$
C 2.78218
$0.14187 \quad-1.42148$
$\begin{array}{llll}\text { C } & 2.51234 & 1.51789 & -1.08822\end{array}$
$\begin{array}{llll}\text { C } & 2.59695 & 1.69544 & 0.35370\end{array}$ 


\begin{tabular}{|c|c|c|c|}
\hline $\mathrm{C}$ & 2.92253 & 0.43663 & 0.97281 \\
\hline $\mathrm{C}$ & 2.23521 & 0.00494 & 2.09435 \\
\hline $\mathrm{C}$ & 1.34861 & 0.92735 & 2.77213 \\
\hline $\mathrm{C}$ & 1.10284 & 2.20024 & 2.24301 \\
\hline $\mathrm{C}$ & 1.72164 & 2.58646 & 0.99804 \\
\hline $\mathrm{C}$ & 0.74594 & 3.34207 & 0.22920 \\
\hline $\mathrm{C}$ & 0.66687 & 3.17398 & -1.15186 \\
\hline $\mathrm{C}$ & 1.55905 & 2.24270 & -1.82346 \\
\hline $\mathrm{C}$ & 0.80892 & 1.58080 & -2.86315 \\
\hline $\mathrm{C}$ & 1.01111 & 0.21625 & -3.10150 \\
\hline $\mathrm{C}$ & 1.97994 & -0.53227 & -2.32724 \\
\hline $\mathrm{C}$ & 1.43888 & -1.90533 & -2.13451 \\
\hline $\mathrm{C}$ & 0.14802 & -1.94431 & -2.78472 \\
\hline $\mathrm{C}$ & -0.11590 & -0.64970 & -3.38491 \\
\hline $\mathrm{C}$ & -1.40947 & -0.13115 & -3.41284 \\
\hline $\mathrm{C}$ & -1.62679 & 1.27986 & -3.13353 \\
\hline $\mathrm{C}$ & -0.54060 & 2.11673 & -2.86627 \\
\hline $\mathrm{C}$ & -0.63070 & 3.10138 & -1.80165 \\
\hline $\mathrm{C}$ & -1.79925 & 3.20497 & -1.04471 \\
\hline $\mathrm{C}$ & -1.71691 & 3.37958 & 0.39670 \\
\hline $\mathrm{C}$ & -0.46910 & 3.44301 & 1.01966 \\
\hline $\mathrm{C}$ & -0.24542 & 2.73902 & 2.27101 \\
\hline $\mathrm{C}$ & -1.28276 & 2.00342 & 2.85005 \\
\hline $\mathrm{C}$ & -1.01579 & 0.69561 & 3.42933 \\
\hline $\mathrm{C}$ & 0.27322 & 0.16752 & 3.37713 \\
\hline $\mathrm{C}$ & 0.48573 & -1.23540 & 3.07341 \\
\hline $\mathrm{C}$ & -0.62140 & -2.06821 & 2.85859 \\
\hline $\mathrm{C}$ & -1.96059 & -1.51711 & 2.88303 \\
\hline $\mathrm{C}$ & -2.15805 & -0.16173 & 3.16314 \\
\hline $\mathrm{C}$ & -3.12112 & 0.60638 & 2.40100 \\
\hline $\mathrm{C}$ & -2.58084 & 1.94570 & 2.20875 \\
\hline $\mathrm{C}$ & -2.79417 & 2.61763 & 1.00225 \\
\hline $\mathrm{C}$ & -3.54609 & 1.97533 & -0.06213 \\
\hline $\mathrm{C}$ & -2.92783 & 2.33604 & -1.32641 \\
\hline $\mathrm{C}$ & -2.84302 & 1.39387 & -2.35450 \\
\hline $\mathrm{C}$ & -3.38429 & 0.05490 & -2.16239 \\
\hline $\mathrm{C}$ & -2.50290 & -0.88508 & -2.82412 \\
\hline $\mathrm{C}$ & -2.25724 & -2.13832 & -2.25543 \\
\hline $\mathrm{C}$ & -0.91650 & -2.68656 & -2.25354 \\
\hline $\mathrm{C}$ & -0.72483 & -3.39907 & -1.01155 \\
\hline $\mathrm{C}$ & -1.94261 & -3.28190 & -0.22408 \\
\hline $\mathrm{C}$ & -2.89317 & -2.50042 & -0.99698 \\
\hline $\mathrm{C}$ & -3.73362 & -1.58865 & -0.35502 \\
\hline $\mathrm{C}$ & -3.98720 & -0.28726 & -0.94952 \\
\hline $\mathrm{C}$ & -4.06237 & 0.69045 & 0.12246 \\
\hline $\mathrm{C}$ & -3.85185 & -0.00577 & 1.37989 \\
\hline $\mathrm{C}$ & -3.65001 & -1.41433 & 1.08685 \\
\hline $\mathrm{C}$ & -2.72953 & -2.15908 & 1.82709 \\
\hline $\mathrm{C}$ & -1.86256 & -3.11469 & 1.15903 \\
\hline
\end{tabular}




$\begin{array}{lrrr}\mathrm{C} & -0.56193 & -3.05805 & 1.80845 \\ \mathrm{C} & 0.60094 & -3.17228 & 1.04993 \\ \mathrm{C} & 0.51790 & -3.34650 & -0.38460 \\ \mathrm{C} & 1.65022 & -2.63734 & -0.96295 \\ \mathrm{C} & 2.60458 & -2.28266 & 0.09406 \\ \mathrm{C} & 1.78172 & -2.36112 & 1.30993 \\ \mathrm{C} & 1.69372 & -1.36875 & 2.29013 \\ \mathrm{C} & 6.38075 & -0.05214 & -0.07984 \\ \mathrm{C} & 6.83453 & 1.34999 & 0.33265 \\ \mathrm{H} & 7.20406 & -0.66768 & -0.45821 \\ \mathrm{H} & 5.64094 & 0.01041 & -0.89874 \\ \mathrm{H} & 5.94979 & -0.59490 & 0.77555 \\ \mathrm{H} & 7.27646 & 1.88570 & -0.51416 \\ \mathrm{H} & 7.58668 & 1.29890 & 1.12710 \\ \mathrm{H} & 5.99258 & 1.94356 & 0.70423\end{array}$

(7) $\mathrm{C}_{2} \mathrm{H}_{6}$-C ${ }_{59} \mathrm{Ni}$-PH_C2-C3

$\begin{array}{crrr}\mathrm{Ni} & 3.29883 & -1.96053 & 0.39224 \\ \mathrm{C} & 1.67375 & -2.84380 & 0.11644 \\ \mathrm{C} & 1.18335 & -2.85574 & -1.23849 \\ \mathrm{C} & 1.81945 & -1.78833 & -1.99859 \\ \mathrm{C} & 2.73260 & -1.06627 & -1.14947 \\ \mathrm{C} & 2.74436 & 0.31936 & -1.14192 \\ \mathrm{C} & 2.00444 & 1.03124 & -2.16232 \\ \mathrm{C} & 1.19689 & 0.33737 & -3.07192 \\ \mathrm{C} & 1.08309 & -1.09806 & -2.97587 \\ \mathrm{C} & -0.30057 & -1.45510 & -3.24361 \\ \mathrm{C} & -0.90891 & -2.47630 & -2.51676 \\ \mathrm{C} & -0.15913 & -3.18352 & -1.49144 \\ \mathrm{C} & -1.05100 & -3.43620 & -0.38547 \\ \mathrm{C} & -0.58031 & -3.30930 & 0.92743 \\ \mathrm{C} & 0.79751 & -2.94870 & 1.18463 \\ \mathrm{C} & 0.81923 & -2.09093 & 2.40558 \\ \mathrm{C} & -0.55198 & -1.95021 & 2.84193 \\ \mathrm{C} & -1.41213 & -2.69920 & 1.94492 \\ \mathrm{C} & -2.68423 & -2.22958 & 1.62324 \\ \mathrm{C} & -3.16357 & -2.32885 & 0.25302 \\ \mathrm{C} & -2.36438 & -2.92131 & -0.72826 \\ \mathrm{C} & -2.27553 & -2.32111 & -2.04874 \\ \mathrm{C} & -2.98289 & -1.15117 & -2.33163 \\ \mathrm{C} & -2.34785 & -0.08514 & -3.09021 \\ \mathrm{C} & -1.03244 & -0.23412 & -3.53327 \\ \mathrm{C} & -0.10176 & 0.87722 & -3.43269 \\ \mathrm{C} & -0.52868 & 2.09518 & -2.89668 \\ \mathrm{C} & 0.32786 & 2.82874 & -1.97743 \\ \mathrm{C} & 1.56244 & 2.29637 & -1.61098 \\ \mathrm{C} & 2.02419 & 2.37556 & -0.23761\end{array}$




$\begin{array}{lrrr}\mathrm{C} & 1.22833 & 3.02144 & 0.71920 \\ \mathrm{C} & -0.06856 & 3.54922 & 0.34875 \\ \mathrm{C} & -0.51362 & 3.45708 & -0.97353 \\ \mathrm{C} & -1.88663 & 3.09376 & -1.25599 \\ \mathrm{C} & -1.89632 & 2.25284 & -2.44601 \\ \mathrm{C} & -2.78963 & 1.18230 & -2.53701 \\ \mathrm{C} & -3.69969 & 0.90291 & -1.43975 \\ \mathrm{C} & -3.81490 & -0.53933 & -1.31123 \\ \mathrm{C} & -3.90631 & -1.12096 & -0.04398 \\ \mathrm{C} & -3.89682 & -0.27999 & 1.14608 \\ \mathrm{C} & -3.14975 & -0.96977 & 2.17764 \\ \mathrm{C} & -2.33138 & -0.24971 & 3.05282 \\ \mathrm{C} & -1.02229 & -0.75698 & 3.40900 \\ \mathrm{C} & -0.12262 & 0.36666 & 3.52628 \\ \mathrm{C} & -0.86401 & 1.58081 & 3.22237 \\ \mathrm{C} & -2.23455 & 1.19745 & 2.93000 \\ \mathrm{C} & -2.94633 & 1.85912 & 1.92753 \\ \mathrm{C} & -3.79619 & 1.10781 & 1.01981 \\ \mathrm{C} & -3.68859 & 1.70849 & -0.29831 \\ \mathrm{C} & -2.76973 & 2.82979 & -0.20630 \\ \mathrm{C} & -2.31116 & 2.92521 & 1.16883 \\ \mathrm{C} & -0.99053 & 3.28543 & 1.44379 \\ \mathrm{C} & -0.25461 & 2.60359 & 2.49461 \\ \mathrm{C} & 1.11993 & 2.45248 & 2.04251 \\ \mathrm{C} & 1.82614 & 1.28946 & 2.33891 \\ \mathrm{C} & 1.19381 & 0.22802 & 3.09421 \\ \mathrm{C} & 1.71307 & -1.02899 & 2.57492 \\ \mathrm{C} & 2.88791 & -0.76186 & 1.73362 \\ \mathrm{C} & 2.71519 & 0.65326 & 1.37735 \\ \mathrm{H} & 2.76455 & 1.17470 & 0.08051 \\ \mathrm{H} & 6.84478 & 1.59359 & 0.36365 \\ \mathrm{H} & 6.88047 & 1.37113 & -1.15076 \\ \mathrm{H} & 6.97789 & 0.65161 & 0.90886 \\ \mathrm{H} & 5.88729 & 2.02349 & 0.67905 \\ \mathrm{H} & 6.073966 & 2.27681 & 0.68617 \\ \mathrm{H} & 0.70239 & -1.47349 \\ \mathrm{H} & -1.92968 & 0.92338 & -1.46764 \\ \mathrm{H} & 2.31511 & -1.69550\end{array}$

(8) $\mathrm{C}_{2} \mathrm{H}_{6} \_\mathrm{C}_{59} \mathrm{Ni} \_\mathrm{HH} \_\mathrm{C} 3-\mathrm{C} 4$

$\begin{array}{cccc}\mathrm{Ni} & -3.36579 & -1.79568 & -0.44097 \\ \mathrm{C} & -1.75000 & -2.64572 & -0.84688 \\ \mathrm{C} & -1.01653 & -3.17695 & 0.27414 \\ \mathrm{C} & -1.46073 & -2.52874 & 1.50067 \\ \mathrm{C} & -2.48898 & -1.56736 & 1.19437 \\ \mathrm{C} & -2.45212 & -0.29549 & 1.74276 \\ \mathrm{C} & -1.51086 & -0.01926 & 2.80653\end{array}$




\begin{tabular}{|c|c|c|c|}
\hline C & -0.57393 & -0.98351 & 3.19871 \\
\hline $\mathrm{C}$ & -0.53149 & -2.25569 & 2.51826 \\
\hline $\mathrm{C}$ & 0.86418 & -2.63049 & 2.35929 \\
\hline $\mathrm{C}$ & 1.28922 & -3.25073 & 1.18636 \\
\hline $\mathrm{C}$ & 0.33658 & -3.52234 & 0.12201 \\
\hline $\mathrm{C}$ & 0.99683 & -3.27530 & -1.13744 \\
\hline $\mathrm{C}$ & 0.29474 & -2.65419 & -2.17798 \\
\hline $\mathrm{C}$ & -1.09315 & -2.27927 & -2.01033 \\
\hline $\mathrm{C}$ & -1.31158 & -1.00778 & -2.76008 \\
\hline $\mathrm{C}$ & -0.04135 & -0.64707 & -3.34923 \\
\hline $\mathrm{C}$ & 0.94366 & -1.65432 & -3.00156 \\
\hline C & 2.26926 & -1.29902 & -2.76085 \\
\hline $\mathrm{C}$ & 2.99161 & -1.91645 & -1.65906 \\
\hline $\mathrm{C}$ & 2.36863 & -2.88410 & -0.86659 \\
\hline $\mathrm{C}$ & 2.54899 & -2.86370 & 0.57505 \\
\hline $\mathrm{C}$ & 3.33921 & -1.87542 & 1.16551 \\
\hline $\mathrm{C}$ & 2.89560 & -1.22819 & 2.39010 \\
\hline $\mathrm{C}$ & 1.68084 & -1.59678 & 2.97166 \\
\hline $\mathrm{C}$ & 0.78815 & -0.57757 & 3.49656 \\
\hline $\mathrm{C}$ & 1.15073 & 0.76970 & 3.42195 \\
\hline $\mathrm{C}$ & 0.16453 & 1.77209 & 3.04821 \\
\hline $\mathrm{C}$ & -1.13451 & 1.37820 & 2.73461 \\
\hline $\mathrm{C}$ & -1.84112 & 1.97958 & 1.61906 \\
\hline $\mathrm{C}$ & -1.21481 & 2.98642 & 0.87067 \\
\hline $\mathrm{C}$ & 0.14652 & 3.37757 & 1.17439 \\
\hline $\mathrm{C}$ & 0.82610 & 2.78353 & 2.24210 \\
\hline $\mathrm{C}$ & 2.21388 & 2.39608 & 2.09704 \\
\hline $\mathrm{C}$ & 2.41517 & 1.15150 & 2.82739 \\
\hline $\mathrm{C}$ & 3.27156 & 0.17235 & 2.31731 \\
\hline $\mathrm{C}$ & 3.95087 & 0.39230 & 1.05192 \\
\hline $\mathrm{C}$ & 3.98771 & -0.87311 & 0.33928 \\
\hline $\mathrm{C}$ & 3.81915 & -0.89687 & -1.04759 \\
\hline $\mathrm{C}$ & 3.61804 & 0.34762 & -1.77855 \\
\hline $\mathrm{C}$ & 2.66801 & 0.09577 & -2.84206 \\
\hline $\mathrm{C}$ & 1.72776 & 1.06992 & -3.19056 \\
\hline $\mathrm{C}$ & 0.35730 & 0.69171 & -3.46926 \\
\hline $\mathrm{C}$ & -0.50746 & 1.73012 & -2.95983 \\
\hline C & 0.32111 & 2.75224 & -2.33929 \\
\hline $\mathrm{C}$ & 1.70735 & 2.34243 & -2.48378 \\
\hline $\mathrm{C}$ & 2.61680 & 2.57889 & -1.45109 \\
\hline $\mathrm{C}$ & 3.59248 & 1.56427 & -1.09253 \\
\hline $\mathrm{C}$ & 3.75490 & 1.58471 & 0.35069 \\
\hline $\mathrm{C}$ & 2.87570 & 2.60975 & 0.88560 \\
\hline $\mathrm{C}$ & 2.17294 & 3.22625 & -0.22628 \\
\hline $\mathrm{C}$ & 0.83830 & 3.61118 & -0.08513 \\
\hline $\mathrm{C}$ & -0.10461 & 3.37337 & -1.16462 \\
\hline $\mathrm{C}$ & -1.37508 & 2.99632 & -0.56461 \\
\hline C & -2.16655 & 2.01998 & -1.16458 \\
\hline $\mathrm{C}$ & -1.72480 & 1.37550 & -2.38380 \\
\hline
\end{tabular}




$\begin{array}{llll}\mathrm{C} & -2.18323 & -0.00527 & -2.32341 \\ \mathrm{C} & -3.16988 & -0.14565 & -1.24337 \\ \mathrm{C} & -2.88309 & 1.01636 & -0.39069 \\ \mathrm{C} & -2.67060 & 0.97506 & 0.99122 \\ \mathrm{C} & -6.87446 & 1.14322 & 1.20092 \\ \mathrm{C} & -7.05352 & 1.40038 & -0.29760 \\ \mathrm{H} & -7.58032 & 1.73292 & 1.79749 \\ \mathrm{H} & -5.86196 & 1.40610 & 1.52897 \\ \mathrm{H} & -7.03859 & 0.08696 & 1.44695 \\ \mathrm{H} & -6.90385 & 2.45911 & -0.53973 \\ \mathrm{H} & -8.06002 & 1.12423 & -0.63340 \\ \mathrm{H} & -6.33310 & 0.82133 & -0.88715\end{array}$

(9) $\mathrm{C}_{2} \mathrm{H}_{6} \_\mathrm{C}_{59} \mathrm{Ni}$-PH_C4-C5

\begin{tabular}{|c|c|c|c|}
\hline $\mathrm{Ni}$ & 2.99901 & -2.47339 & 0.00037 \\
\hline $\mathrm{C}$ & 1.49618 & -2.75767 & 1.07618 \\
\hline $\mathrm{C}$ & 0.36751 & -3.37979 & 0.43207 \\
\hline $\mathrm{C}$ & 0.47977 & -3.22288 & -1.01181 \\
\hline $\mathrm{C}$ & 1.68304 & -2.49608 & -1.32808 \\
\hline $\mathrm{C}$ & 1.65950 & -1.45530 & -2.24249 \\
\hline $\mathrm{C}$ & 0.47535 & -1.26084 & -3.05139 \\
\hline $\mathrm{C}$ & -0.67101 & -2.03535 & -2.83448 \\
\hline $\mathrm{C}$ & -0.67875 & -3.02094 & -1.78037 \\
\hline $\mathrm{C}$ & -1.98607 & -2.98476 & -1.14541 \\
\hline $\mathrm{C}$ & -2.09361 & -3.13488 & 0.23538 \\
\hline C & -0.89802 & -3.32738 & 1.04007 \\
\hline $\mathrm{C}$ & -1.06762 & -2.58976 & 2.26853 \\
\hline $\mathrm{C}$ & 0.01936 & -1.89900 & 2.81870 \\
\hline $\mathrm{C}$ & 1.31644 & -1.93617 & 2.17746 \\
\hline $\mathrm{C}$ & 1.95390 & -0.60062 & 2.36975 \\
\hline $\mathrm{C}$ & 1.01394 & 0.20740 & 3.11460 \\
\hline $\mathrm{C}$ & -0.16920 & -0.58382 & 3.39768 \\
\hline C & -1.43245 & 0.00448 & 3.40599 \\
\hline C & -2.55788 & -0.69605 & 2.80595 \\
\hline C & -2.37871 & -1.96615 & 2.25176 \\
\hline $\mathrm{C}$ & -3.01362 & -2.29959 & 0.98786 \\
\hline C & -3.79626 & -1.35014 & 0.32779 \\
\hline $\mathrm{C}$ & -3.68395 & -1.19348 & -1.11404 \\
\hline $\mathrm{C}$ & -2.79363 & -1.99276 & -1.83377 \\
\hline $\mathrm{C}$ & -1.97924 & -1.40796 & -2.88546 \\
\hline C & -2.09292 & -0.04607 & -3.17701 \\
\hline C & -0.90096 & 0.74757 & -3.43488 \\
\hline C & 0.35581 & 0.15027 & -3.35893 \\
\hline C & 1.46842 & 0.84342 & -2.73624 \\
\hline C & 1.27724 & 2.13834 & -2.23348 \\
\hline C & -0.03464 & 2.75030 & -2.28275 \\
\hline $\mathrm{C}$ & -1.10534 & 2.07160 & -2.87213 \\
\hline
\end{tabular}




$\begin{array}{lrrr}\mathrm{C} & -2.41158 & 2.09076 & -2.24699 \\ \mathrm{C} & -3.02289 & 0.78166 & -2.43618 \\ \mathrm{C} & -3.80115 & 0.22068 & -1.42055 \\ \mathrm{C} & -3.99145 & 0.93913 & -0.17251 \\ \mathrm{C} & -3.98302 & -0.03218 & 0.90768 \\ \mathrm{C} & -3.37745 & 0.28601 & 2.12609 \\ \mathrm{C} & -2.76587 & 1.59484 & 2.31555 \\ \mathrm{C} & -1.57035 & 1.42102 & 3.11387 \\ \mathrm{C} & -0.43372 & 2.19197 & 2.85381 \\ \mathrm{C} & 0.88009 & 1.58276 & 2.87610 \\ \mathrm{C} & 1.67639 & 2.19660 & 1.83959 \\ \mathrm{C} & 0.85357 & 3.17878 & 1.15074 \\ \mathrm{C} & -0.45488 & 3.17659 & 1.78200 \\ \mathrm{C} & -1.60520 & 3.33501 & 1.00662 \\ \mathrm{C} & -2.78414 & 2.53133 & 1.27920 \\ \mathrm{C} & -3.40282 & 2.19330 & 0.00929 \\ \mathrm{C} & -2.60346 & 2.78460 & -1.04977 \\ \mathrm{C} & -1.49331 & 3.49177 & -0.43511 \\ \mathrm{C} & -0.23562 & 3.48356 & -1.04160 \\ \mathrm{C} & 0.96113 & 3.32940 & -0.23218 \\ \mathrm{C} & 1.89613 & 2.50368 & -0.98076 \\ \mathrm{C} & 2.68497 & 1.56733 & -0.31640 \\ \mathrm{C} & 2.57265 & 1.41080 & 1.11923 \\ \mathrm{C} & 2.77385 & -0.00165 & 1.40851 \\ \mathrm{C} & 3.32359 & -0.67022 & 0.22053 \\ \mathrm{C} & 2.95133 & 0.24574 & -0.86693 \\ \mathrm{C} & 2.29691 & -0.12052 & -2.04691 \\ \mathrm{C} & 7.04831 & 0.98462 & 0.48931 \\ \mathrm{C} & 6.84424 & 2.05115 & -0.59018 \\ \mathrm{H} & 8.03257 & 0.50992 & 0.39969 \\ \mathrm{H} & 6.29046 & 0.19566 & 0.41674 \\ \mathrm{H} & 6.98115 & 1.41731 & 1.49449 \\ \mathrm{H} & 6.92085 & 1.62014 & -1.59534 \\ & 7.59409 & 2.84726 & -0.51376 \\ & 5.85575 & 2.51785 & -0.50665\end{array}$

(10) $\mathrm{C}_{2} \mathrm{H}_{6} \_\mathrm{C}_{59} \mathrm{Ni}$ _HH_C6-C7

$\begin{array}{cccc}\mathrm{Ni} & 3.74006 & -0.84201 & 0.13647 \\ \mathrm{C} & 2.92247 & 0.43635 & -0.97306 \\ \mathrm{C} & 2.59700 & 1.69528 & -0.35413 \\ \mathrm{C} & 2.51248 & 1.51797 & 1.08783 \\ \mathrm{C} & 2.78230 & 0.14200 & 1.42128 \\ \mathrm{C} & 1.98008 & -0.53200 & 2.32717 \\ \mathrm{C} & 1.01132 & 0.21666 & 3.10138 \\ \mathrm{C} & 0.80916 & 1.58119 & 2.86285 \\ \mathrm{C} & 1.55925 & 2.24291 & 1.82301 \\ \mathrm{C} & 0.66705 & 3.17411 & 1.15132\end{array}$




\begin{tabular}{|c|c|c|c|}
\hline C & 0.74604 & 3.34199 & -0.22977 \\
\hline $\mathrm{C}$ & 1.72167 & 2.58623 & -0.99854 \\
\hline $\mathrm{C}$ & 1.10278 & 2.19985 & -2.24342 \\
\hline $\mathrm{C}$ & 1.34848 & 0.92688 & -2.77238 \\
\hline $\mathrm{C}$ & 2.23508 & 0.00453 & -2.09451 \\
\hline $\mathrm{C}$ & 1.69355 & -1.36916 & -2.29009 \\
\hline $\mathrm{C}$ & 0.48551 & -1.23589 & -3.07331 \\
\hline $\mathrm{C}$ & 0.27303 & 0.16700 & -3.37721 \\
\hline $\mathrm{C}$ & -1.01597 & 0.69512 & -3.42940 \\
\hline $\mathrm{C}$ & -1.28286 & 2.00302 & -2.85029 \\
\hline $\mathrm{C}$ & -0.24546 & 2.73867 & -2.27142 \\
\hline C & -0.46904 & 3.44286 & -1.02016 \\
\hline $\mathrm{C}$ & -1.71682 & 3.37956 & -0.39712 \\
\hline $\mathrm{C}$ & -1.79908 & 3.20516 & 1.04433 \\
\hline $\mathrm{C}$ & -0.63048 & 3.10165 & 1.80121 \\
\hline $\mathrm{C}$ & -0.54034 & 2.11715 & 2.86597 \\
\hline $\mathrm{C}$ & -1.62654 & 1.28035 & 3.13343 \\
\hline $\mathrm{C}$ & -1.40925 & -0.13062 & 3.41293 \\
\hline $\mathrm{C}$ & -0.11569 & -0.64921 & 3.38499 \\
\hline $\mathrm{C}$ & 0.14815 & -1.94392 & 2.78495 \\
\hline $\mathrm{C}$ & -0.91642 & -2.68621 & 2.25395 \\
\hline $\mathrm{C}$ & -2.25714 & -2.13793 & 2.25585 \\
\hline $\mathrm{C}$ & -2.50273 & -0.88461 & 2.82438 \\
\hline $\mathrm{C}$ & -3.38413 & 0.05530 & 2.16256 \\
\hline $\mathrm{C}$ & -2.84281 & 1.39428 & 2.35445 \\
\hline $\mathrm{C}$ & -2.92766 & 2.33631 & 1.32622 \\
\hline $\mathrm{C}$ & -3.54602 & 1.97544 & 0.06203 \\
\hline $\mathrm{C}$ & -2.79414 & 2.61756 & -1.00249 \\
\hline $\mathrm{C}$ & -2.58090 & 1.94544 & -2.20890 \\
\hline $\mathrm{C}$ & -3.12123 & 0.60611 & -2.40093 \\
\hline C & -2.15823 & -0.16214 & -3.16301 \\
\hline C & -1.96080 & -1.51749 & -2.88272 \\
\hline $\mathrm{C}$ & -0.62163 & -2.06862 & -2.85829 \\
\hline $\mathrm{C}$ & -0.56213 & -3.05831 & -1.80800 \\
\hline $\mathrm{C}$ & -1.86271 & -3.11481 & -1.15849 \\
\hline $\mathrm{C}$ & -2.72970 & -2.15928 & -1.82664 \\
\hline $\mathrm{C}$ & -3.65011 & -1.41439 & -1.08645 \\
\hline C & -3.85191 & -0.00587 & -1.37967 \\
\hline $\mathrm{C}$ & -4.06233 & 0.69055 & -0.12233 \\
\hline C & -3.98713 & -0.28701 & 0.94978 \\
\hline C & -3.73362 & -1.58850 & 0.35545 \\
\hline $\mathrm{C}$ & -2.89317 & -2.50020 & 0.99750 \\
\hline C & -1.94268 & -3.28182 & 0.22465 \\
\hline $\mathrm{C}$ & -0.72485 & -3.39891 & 1.01206 \\
\hline $\mathrm{C}$ & 0.51784 & -3.34648 & 0.38501 \\
\hline $\mathrm{C}$ & 0.60079 & -3.17248 & -1.04955 \\
\hline $\mathrm{C}$ & 1.78158 & -2.36140 & -1.30975 \\
\hline C & 2.60452 & -2.28277 & -0.09395 \\
\hline C & 1.65022 & -2.63726 & 0.96318 \\
\hline
\end{tabular}




$\begin{array}{lrrr}\mathrm{C} & 1.43898 & -1.90507 & 2.13466 \\ \mathrm{C} & 6.83411 & 1.35020 & -0.33246 \\ \mathrm{C} & 6.38055 & -0.05208 & 0.07979 \\ \mathrm{H} & 5.64076 & 0.01025 & 0.89874 \\ \mathrm{H} & 7.20397 & -0.66755 & 0.45804 \\ \mathrm{H} & 5.94965 & -0.59476 & -0.77566 \\ \mathrm{H} & 7.27592 & 1.88584 & 0.51444 \\ \mathrm{H} & 5.99208 & 1.94369 & -0.70397 \\ \mathrm{H} & 7.58629 & 1.29935 & -1.12690\end{array}$

(11) $\mathrm{C}_{2} \mathrm{H}_{6} \_\mathrm{C}_{59} \mathrm{Ni} \_\mathrm{HH}$ _C7-C8

\begin{tabular}{crrr}
$\mathrm{Ni}$ & -3.08246 & -2.35493 & 0.05396 \\
$\mathrm{C}$ & -2.74719 & -0.93573 & 1.22546 \\
$\mathrm{C}$ & -2.97743 & 0.38537 & 0.69897 \\
$\mathrm{C}$ & -2.97021 & 0.34041 & -0.75708 \\
$\mathrm{C}$ & -2.73456 & -1.01061 & -1.19861 \\
$\mathrm{C}$ & -1.81816 & -1.27529 & -2.20329 \\
$\mathrm{C}$ & -1.27805 & -0.17030 & -2.96649 \\
$\mathrm{C}$ & -1.58541 & 1.15208 & -2.62241 \\
$\mathrm{C}$ & -2.43003 & 1.41554 & -1.48223 \\
$\mathrm{C}$ & -1.89541 & 2.57620 & -0.78872 \\
$\mathrm{C}$ & -1.90226 & 2.61910 & 0.60378 \\
$\mathrm{C}$ & -2.44451 & 1.50353 & 1.36213 \\
$\mathrm{C}$ & -1.61159 & 1.31142 & 2.52507 \\
$\mathrm{C}$ & -1.30740 & 0.01292 & 2.95219 \\
$\mathrm{C}$ & -1.84080 & -1.13743 & 2.25401 \\
$\mathrm{C}$ & -0.80296 & -2.20903 & 2.29116 \\
$\mathrm{C}$ & 0.33264 & -1.66654 & 3.00314 \\
$\mathrm{C}$ & 0.02693 & -0.30954 & 3.41593 \\
$\mathrm{C}$ & 1.01931 & 0.66859 & 3.43343 \\
$\mathrm{C}$ & 0.71700 & 2.00989 & 2.95773 \\
$\mathrm{C}$ & -0.57079 & 2.32410 & 2.51563 \\
$\mathrm{C}$ & -0.74765 & 3.13180 & 1.32079 \\
$\mathrm{C}$ & 0.36747 & 3.58906 & 0.61570 \\
$\mathrm{C}$ & 0.37480 & 3.54398 & -0.83790 \\
$\mathrm{C}$ & -0.73336 & 3.04395 & -1.52439 \\
$\mathrm{C}$ & -0.54460 & 2.16361 & -2.66502 \\
$\mathrm{C}$ & 0.74731 & 1.82330 & -3.07436 \\
$\mathrm{C}$ & 1.05364 & 0.45519 & -3.46271 \\
$\mathrm{C}$ & 0.06089 & -0.52035 & -3.39552 \\
$\mathrm{C}$ & 0.36219 & -1.84914 & -2.89601 \\
$\mathrm{C}$ & 1.67382 & -2.15639 & -2.50663 \\
$\mathrm{C}$ & 2.70179 & -1.13636 & -2.54716 \\
$\mathrm{C}$ & 2.40085 & 0.14592 & -3.01563 \\
$\mathrm{C}$ & 2.91990 & 1.31218 & -2.33162 \\
$\mathrm{C}$ & 1.89748 & 2.35005 & -2.36858 \\
& 1.71610 & 3.19152 & -1.26791 \\
\hline & & & \\
\hline
\end{tabular}




\begin{tabular}{lrrr}
$\mathrm{C}$ & 2.53999 & 3.02263 & -0.08348 \\
$\mathrm{C}$ & 1.70425 & 3.26422 & 1.07997 \\
$\mathrm{C}$ & 1.87424 & 2.49234 & 2.23216 \\
$\mathrm{C}$ & 2.89643 & 1.45439 & 2.26951 \\
$\mathrm{C}$ & 2.37087 & 0.33274 & 3.01982 \\
$\mathrm{C}$ & 2.67580 & -0.97593 & 2.63397 \\
$\mathrm{C}$ & 1.64795 & -1.99656 & 2.64616 \\
$\mathrm{C}$ & 1.87080 & -2.87114 & 1.51861 \\
$\mathrm{C}$ & 3.02898 & -2.38569 & 0.78419 \\
$\mathrm{C}$ & 3.52826 & -1.21069 & 1.47754 \\
$\mathrm{C}$ & 4.02493 & -0.12803 & 0.74903 \\
$\mathrm{C}$ & 3.70580 & 1.23044 & 1.15316 \\
$\mathrm{C}$ & 3.51810 & 2.02561 & -0.04773 \\
$\mathrm{C}$ & 3.71773 & 1.15778 & -1.19529 \\
$\mathrm{C}$ & 4.03226 & -0.17302 & -0.70483 \\
$\mathrm{C}$ & 3.54238 & -1.29879 & -1.36995 \\
$\mathrm{C}$ & 3.03581 & -2.42876 & -0.61040 \\
$\mathrm{C}$ & 1.88495 & -2.95907 & -1.32478 \\
$\mathrm{C}$ & 0.78023 & -3.42618 & -0.61693 \\
$\mathrm{C}$ & 0.77303 & -3.38127 & 0.83034 \\
$\mathrm{C}$ & -0.59725 & -3.10731 & 1.23928 \\
$\mathrm{C}$ & -1.49622 & -3.29613 & 0.09233 \\
$\mathrm{C}$ & -0.58564 & -3.17787 & -1.05524 \\
$\mathrm{C}$ & -0.78042 & -2.34687 & -2.16303 \\
$\mathrm{C}$ & -7.01387 & 0.96967 & -0.57212 \\
$\mathrm{C}$ & -6.81184 & 2.01704 & 0.52620 \\
$\mathrm{H}$ & -7.15977 & -0.03064 & -0.14638 \\
$\mathrm{H}$ & -6.14617 & 0.92288 & -1.24051 \\
$\mathrm{H}$ & -7.89319 & 1.19708 & -1.18589 \\
$\mathrm{H}$ & -5.92333 & 1.79468 & 1.12855 \\
$\mathrm{H}$ & -7.67218 & 2.05593 & 1.20433 \\
$\mathrm{H}$ & -6.68019 & 3.01884 & 0.10117 \\
& & & \\
\hline
\end{tabular}

Data for Gibbs free energy in kcal/mol for energy profile diagram shown in figure 9.

$\begin{array}{llllc}\text { Structures } & \mathrm{C}_{60} & \mathrm{C}_{59} \mathrm{Pd} & \mathrm{C}_{59} \mathrm{Ni} & \mathrm{Pd} / \mathrm{CNT} \\ \mathrm{IM} 1+2 \mathrm{H}_{2}(\mathrm{~g}) & -10.12 & -26.24 & -29.82 & -19.41 \\ \mathrm{TS} 12+\mathrm{H}_{2}(\mathrm{~g}) & -82.07 & 6.21 & -7.73 & 47.98 \\ \mathrm{IM} 2+\mathrm{H}_{2}(\mathrm{~g}) & -0.43 & -1.79 & -10.67 & 26.6 \\ \mathrm{TS} 23+\mathrm{H}_{2}(\mathrm{~g}) & 48.61 & 1.40 & -7.73 & 48.32 \\ \mathrm{IM} 3+\mathrm{H}_{2}(\mathrm{~g}) & 42.38 & -64.55 & -72.63 & -0.97 \\ \mathrm{TS} 34+\mathrm{H}_{2}(\mathrm{~g}) & 64.95 & -43.72 & -56.58 & 16.63 \\ \mathrm{IM} 4+\mathrm{H}_{2}(\mathrm{~g}) & -34.85 & -76.76 & -80.76 & -18.46 \\ \mathrm{TS} 45+\mathrm{H}_{2}(\mathrm{~g}) & -32.68 & -9.46 & -23.90 & -16.77 \\ \mathrm{IM} 5+\mathrm{H}_{2}(\mathrm{~g}) & -35.34 & -47.68 & -45.04 & -50.34\end{array}$


Data for Gibbs free energy in kcal/mol for energy profile diagram shown in figure 10.

$\begin{array}{llllc}\text { Structures } & \mathrm{C}_{60} & \mathrm{C}_{59} \mathrm{Pd} & \mathrm{C}_{59} \mathrm{Ni} & \mathrm{Pd} / \mathrm{CNT} \\ \text { IM5 }+\mathrm{H}_{2}(\mathrm{~g}) & 4.18 & -8.15 & -5.51 & -8.6 \\ \text { TS56 } & 149.25 & 25.28 & 28.44 & 51.45 \\ \text { IM6 } & 14.74 & -1.05 & 15.57 & 30.48 \\ \text { TS67 } & 97.57 & -35.74 & - & 53.27 \\ \text { IM7 } & 9.15 & -9.43 & - & 44.25 \\ \text { TS78 } & 49.38 & 28.37 & - & 67.18 \\ \text { TS68 } & - & - & 23.12 & - \\ \text { IM8 } & 23.89 & -34.82 & -33.95 & 28.15 \\ \text { TS89 } & 36.91 & -12.54 & -17.25 & 31.54 \\ \text { IM9 } & -19.18 & -20.93 & -20.97 & -24.23\end{array}$

Data for absolute energy value in Hartree for acetylene, ethylene, ethane, hydrogen, pure fullerene, palladium doped fullerene, nickel doped fulllerene and Pd/CNT are given below.
$\mathrm{C}_{2} \mathrm{H}_{2}-77.3256452931$
$\mathrm{C}_{2} \mathrm{H}_{4}-78.5858248655$
$\mathrm{C}_{2} \mathrm{H}_{6}-79.8290133391$
$\mathrm{H}_{2} \quad-1.1754823877$
$\mathrm{C}_{60} \quad-2285.796048$
$\mathrm{C}_{59} \mathrm{Pd}-2374.66228215$
$\mathrm{C}_{59} \mathrm{Ni}-2416.900041$
$\mathrm{Pd} / \mathrm{CNT}-2427.5781$ 